\title{
Asterinales of India
}

\section{Hosagoudar VB}

Jawarlal Nehru Tropical Botanic Garden and Research Institute, Palode 695 562, Thiruvananthapuram, Kerala, India.

Hosagoudar VB. 2012 - Asterinales of India. Mycosphere 2(5), 617-852, Doi 10.5943 /mycosphere/3/5/9

This paper gives an account of 293 taxa resulting from the examination of 1168 fungal exsiccates belonging to 434 host plants in 82 families. The fungal taxa are distributed among 20 genera in two families, namely, Asterinaceae: Asterina (207 species), Asterolibertia (4), Bheemamyces (2), Gangamyces (2), Ishwaramyces (1), Meliolaster (1), Prillieuxina (11), Symphaster (1), Trichasterina (1), Vishnumyces (1); Lembosiaceae: Cirsosia (5), Echidnodella (5), Echidnoides (1), Eupelte (1), Lembosia (19), Maheshwaramyces (2). The anamorphs are Asterostomella (24), Asterostomula (3), Bramhamyces (1) and Mahanteshamyces (1). A new genus, Gangamyces with its type species, G. miliusae, is proposed to accommodate Asterina-like species possessing appressoria in couplets with a stellate lumen in the hyphal cells. Asterina cassiicola, Asterina clusiacearum, Asterina homaligena, Asterina kannurensis, Asterina kodajadriensis, Asterina mallotigena, Asterina mezonevronis, Asterina murrayicola, Asterina physalidis, Bheemamyces capparidis, Gangamyces miliusae, Gangamyces shoreae, Cirsosia vateriae, Lembosia calamigena, Lembosia garciniae, Lembosia pandanacearum, Maheshwaramyces coculi, Asterostomella anogeissi, Asterostomella flacourtiae-montanae, Asterostomella shoreae, Asterostomella xylosmae, Asterostomella ziziphina and Asterostomula pavettae are new species, while, Asterina munnarensis, Asterina songii and Asterina viburnicola are new names proposed for homonyms. The new combination has effected here as Prillieuxina argyreiae based on Asterinella argyreiae. All the genera, species and infraspecific taxa are arranged alphabetically in the two families (Asterinaceae and Lembosiaceae). Individual taxa are dealt with in detail and supplemented with line drawings and or photomicrographs. Host and fungus indexes are provided.

Key words - Taxonomy- black mildews-asterinaceous fungi-India

\section{Article Information}

Received 15 February 2012

Accepted 28 April 2012

Published online 26 September 2012

*Corresponding author: VB Hosagoudar - e-mail - vbhosagoudar@rediffmail.com

\section{Introduction}

India is located to the north of an equator, lies between $8^{\circ} 4^{\prime}$ and $37^{\circ} 6^{\prime}$ north latitude and $68^{\circ} 7^{\prime}$ and $97^{\circ} 25^{\prime}$ east latitude, measures 3214 kilometers from south to north and $2933 \mathrm{~km}$ from east to west, the total land area being $32,87,263$ square kilometers. It is bounded in the southwest by the Arabian Sea and in the southeast by the Bay of Bengal, while the Himalayan ranges in the north, northeast and northwest separates the country from the other Asian countries. The land narrows towards the southern tip and ends in Indian Ocean. The Andaman and Nicobar Islands are in the Bay of Bengal and the Lakshadweep in the Arabian Sea. It comprises snow clad regions in the Himalayan region, highest rainfall area in Assam, deserts in Rajasthan, hilly tracts in the Eastern and 
Western Ghats of peninsular India and coastlines along the eastern and western sides of peninsular India. It has mainly three seasons in a year viz. rainy season (June to September and October to November), winter season (October to February) and summer season (March to June). The forest cover is $19.27 \%$ of the total geographic area. Of this, $11 \%$ has dense forest, while, the remaining are open forest. The total number of species of Phanerogams in India is estimated to be 45,000 , of which 18,000 have been described. Of these, $40-45 \%$ is endemic to the region and is largely concentrated in the Western Himalayas, Eastern Himalayas and Peninsular India.

\section{BIOGEOGRAPHIC REGIONS OF INDIA}

According to Rogers \& Panwar (1988), India represents ten biogeographic regions:

1. Trans Himalaya (Jammu and Kashmir, Himachal) with an area of 184823 sq. $\mathrm{km}$

2. Himalaya (Uttaranchal, Jammu and Kashmir, Himachal Pradesh, Arunachal Pradesh, Sikkim) with an area of 210662 sq. km

3. Desert (Rajasthan, Gujarat) with an area of 215757 sq. km

4. Semi arid (Punjab, Rajasthan, Gujarat, Haryana, Delhi, Madhya Pradesh, Uttar Pradesh) with an area of 545850 sq. km

5. Western Ghats (Maharashtra, Karnataka, Goa, Kerala, Tamil Nadu) with an area of 132606 sq. km

6. Deccan Peninsula (Maharashtra, Madhya Pradesh, Uttar Pradesh, West Bengal, Andhra Pradesh, Orissa, Tamil $\mathrm{Nadu}$, Bihar) with an area of 1380380 sq. km

7. Gangetic Plain (Uttar Pradesh, West Bengal, Bihar) with an area of 354782 sq. km

8. Coast (Konkan region of Karnataka, Kerala, Goa, North Maharashtra, Orissa, Gujarat, Tamil Nadu, Andhra Pradesh) with an area of 82813 sq. km
9. Northeast (Assam, Nagaland, Manipur, Mizorum, Tripura) with an area of 171341 sq. $\mathrm{km}$

10. Islands (Andaman and Nicobar group of Islands, Lakshadweep) with an area of 8249 sq. km.

India is the mega biodiversity centre with a vegetation rich Western Ghats and north eastern regions, harbours more than 18,000 flowering plants and of which several are Indian origin. If we consider 1:10 ratio of plant to fungus, it has to $1,80,000$ plant parasitic fungi instead of the presently known 27,000 species (Manoharachary et al. 2005). The study of the fungal systematics in India flourished between the years 1950-80, evidenced by the appearance of Monographs on Clavariaceae (Thind, 1961), Indian Cercosporae (Vasudeva, 1963), Mucorales of India (Tandon, 1968), Indian Polyporaceae (Bakshi, 1971), Hyphomycetes (Subramanian, 1971), Myxomycetes (Thind, 1977), Ustilaginales (Mundkur \& Thirumalachar, 1952), Phyllachora (Kamat et al. 1978). Subsequently, the study of systematics started declining and now it has become rare and even we are not in a position to identify the common fungi.

Like rusts, smuts and powdery mildews, black mildews are the parasitic fungi which produce black colonies on the host surfaces. Black mildews are the group of organisms occurring commonly in the tropical and subtropical regions of the world. These black colony forming organisms belong to different taxonomic groups, viz. Meliolaceous fungi, Schiffnerulaceous fungi, Asterinaceous fungi, Hyphomycetous fungi. Meliolaceous fungi are distinct from others in having brown mycelium with bi-cellular appressoria, setae, perithecial appendages and consistent brown septate ascospores (Hansford 1961; Hosagoudar 1996, 2008; Hosagoudar \& Agarwal 2008; Hosagoudar et al. 1997).

Asterinaceous fungi are ectophytic, obligate biotrophs infecting wide range of flowering plants ranging from herbs to trees, weeds to economically important cultivated plants. These fungi produce thin to dense black colonies on the surface of the leaves. Structurally brown superficial mycelium 
produces appressoria which in turn produce haustoria or nutritive hyphae in to the epidermal cells of the host plant for the nourishment. The fruiting body is flattened with radiating cells known as thyriothecium, which splits radially like a star, hence they are known as Asterinaceous fungi. Asterinaceous fungi (s.s. Arx \& Muller, 1975) represent 32 genera, belong to two families, namely, Asterinaceae and Lembosiaceae. The family Asterinaceae consists of more than 800 species and the genus Asterina alone is numbered more than 500 species (Hosagoudar \& Abraham, 2000). Doidge (1942) was the first to give an elaborate account of these fungi for South Africa.

The first report of the genus Asterina from India dates back to Asterina carbonacea Cooke and Asterina congesta Cooke known on coriaceous leaves and Santalum album, respectively, from Belgaum, Karnataka (Cooke, 1884). An intensive mycological exploration in India was between1950-1975. Plant parasitic fungi received much attention from several researchers, such as Fungi of Madras were published by T. S. Ramakrishnan especially in Ascomycetes. E. J. Butler who came to British India in 1901, collected fungi from different region and published "The Fungi Indiae Orientalis" jointly with H. Sydow \& P. Sydow and establishes "Herbarium Crytogamae Indiae Orientalis" at Indian Agriculture Research Institute in Pusha, New Delhi. Thirumalachar made collections of these fungi from Western Ghats region of Karnataka (Hansford \& Thirumalachar, 1948). Kar \& Maity (1970) and Kar \& Ghosh (1986) contributed from West Bengal. Thite \& Kulkarni (1976), Patil \& Thite (1977, 1978, 1981) contributed to this group from Maharashtra. Studies were carried out by Hosagoudar \& Hanlin (1995), Hosagoudar \& Goos (1994, 1996), Hosagoudar and his colleagues contributed much to this group mainly from the Western Ghats of southern India (fide references). Subsequently, Hosagoudar et al. (2011) gave an account of these fungi for Kerala state.

In India, Ramanujam (1963), Ramanujam \& Rao (1973) have reported the occurrence of Asterinaceae members from the Neogene lignite sediments of South India.
Palaeoasterina siwalika has been recorded by Mitra et al. (2002). The in situ occurrence of Palaeoasterina siwalika on the cuticle of Chonemorpha micenica leaf from the upper Siwalik sediments of Arunachal Pradesh was reported by Das et al. (2007).

The present study is the result of the work carried out in the Botanical Survey of India, Southern Circle, Coimbatore since 1981 and subsequently in Jawaharlal Nehru Tropical Botanic Garden and Research Institute, Palode since 1996 to till date. Extensive exploration trips have been conducted to different parts of eastern and Western Ghats in Peninsular India but more intensively studied in Kerala State. In practice, in experience, certain plants can be identified even in their vegetative state based on the infected fungus. However, the speciation here is mostly restricted up to the host family but in some places it is restricted to host genus, where many species are known. The studied material deposited in Herbarium Cryptogamae Indiae Orientalis (HCIO) and Agharkar Mycological Herbarium (AMH). The material received from Prof. Kamal and A.W. Nawami from Uttar Pradesh are deposited in the herbarium of Jawaharlal Nehru Tropical Botanic Garden \& Research Institute (TBGT). The material sent to me for confirmation have been included in the present work.

\section{METHODOLOGY}

Infected plant parts were noticed and collected carefully in the field and notes were made regarding their pathogenicity, nature of colonies, nature of infection, locality, altitude. For each collection, a separate field number was given. Each infected plant parts was collected separately in polythene bags along with a host twig (preferably with the reproductive parts) to facilitate the identity of the corresponding host. These collections were pressed neatly and dried in-between blotting papers. The host plants were identified by matching them with the authentic herbarium material and also by consulting the experts. However, the knowledge of identification of flowering plants is the pre-requisite for this study.

In the laboratory, nail polish technique (Hosagoudar and Kapoor, 1984) was used to 
study the structural and morphological characters of the fungi. Since the desired quality and quantity of nail polish is difficult to procure from the market, this problem eased by preparing a Xylene-thermocol solution. $5 \mathrm{ml}$ or desired quantity of Xyline poured in a container, very bright and clean Thermocol cut into minute pieces, added to Xyline, mixed thoroughly till getting it to a particular consistency and poured it into air tight bottle for the use. A drop of Xyline -Thermocol solution applied on the selected colonies, carefully thinned with the help of a fine brush without disturbing the colonies. Colonies with hyperparasites (wooly nature) were avoided. The treated colonies along with their host plants kept in dust free chamber for half an hour. When the applied solution dried, a thin colourless "film" or "flip" was formed with the colonies firmly embedded in it. For soft host parts, flip was lifted up with a slight pressure on the upper side of the leaves and just below the colonies or an edge of the flip eased and

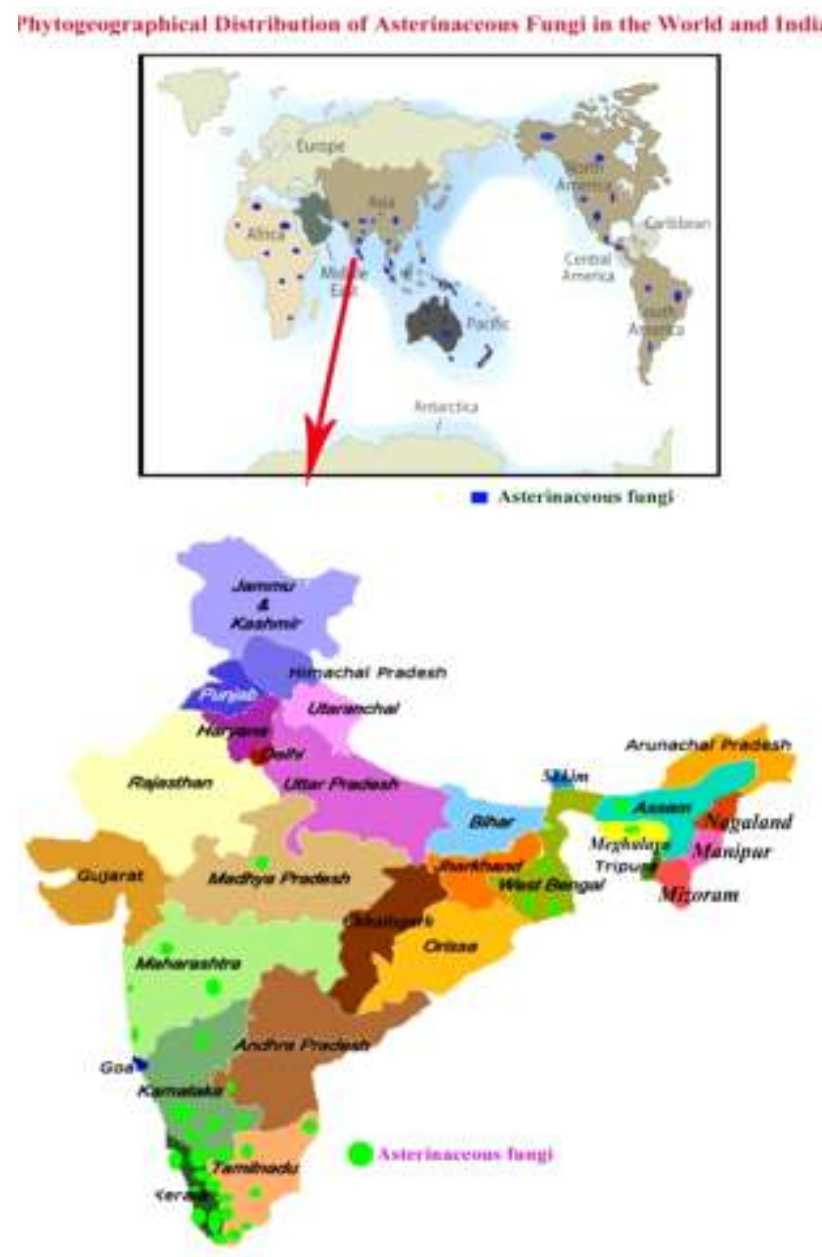

Figure 1 - Study area subsequently the entire flip peeled-off by using the thumb nail and ring finger of the left hand. In case of hard host parts, the flip was eased-off with the help of a razor or scalpel. A drop of DPX was added on clean slide and the flip was spread properly on it. Care was taken to avoid air bubbles while mounting. One or two more drops of DPX was again added on the flip and clean cover glass was placed over it and gentle pressure on the cover glass brings out the excess DPX and it was removed after drying. These slides were labeled and placed in the dust free chamber for one to two days for drying.

In some species, the septa were not visible due to heavy pigmentation. In such cases, scrape was taken directly from the infected host and mounted in $10 \% \mathrm{KOH}$ solution. After 30 minutes, $\mathrm{KOH}$ was replaced by lacto phenol (Rangaswamy, 1975). Both the mountants worked well as clearing agents and made the septa visible.

The individual material was assigned to its taxonomic rank and prepared for herbarium carrying the details of fungus name, host name, date of collection, locality, name of the collector, expert who identified the specimen and its herbarium number. The envelopes were serially arranged in a rack based on their collection number. Part of the herbarium material deposited in the Herbarium Cryptogamae Indiae Orientalis (HCIO), IARI, New Delhi and part of it in the Jawaharlal Nehru Tropical Botanic Garden Travancore herbarium (TBGT), Thiruvananthapuram, Kerala.

\section{Infection patterns}

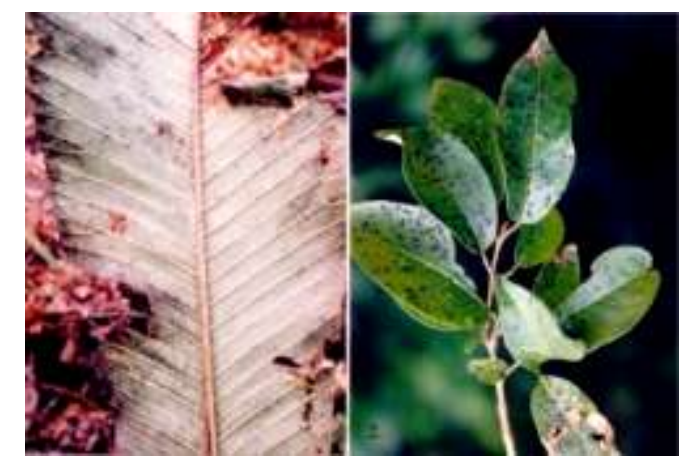

Figure 2 - 1. Asterostomella dilleniacearum on leaves of Dillenia pentagyna, 2. Lembosia terminaliae-chebulae on leaves of Terminalia chebula 

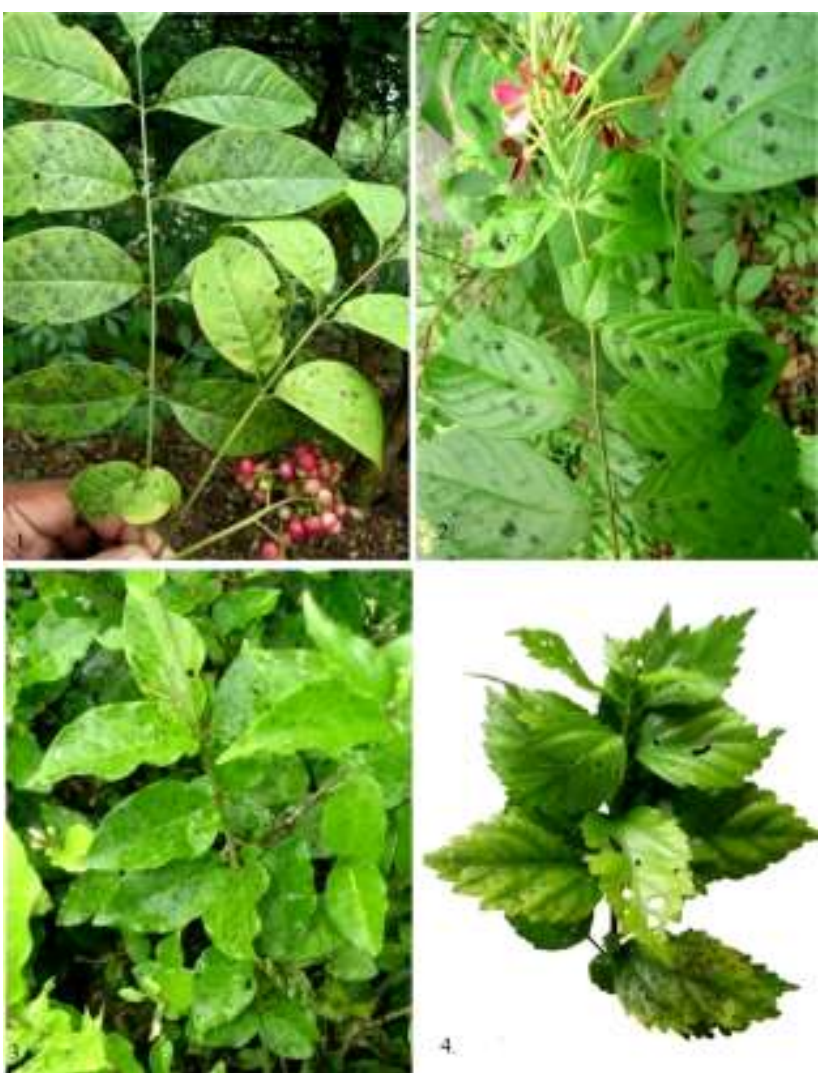

Figure 3 - 1. Asterina cipadessae on leaves of Cipadessa baccifera, 2. Asterina escharoides on leaves of Quisqualis indica, 3. Asterina erysiphoides on leaves of Jasminum sp., 4. Asterina hibisci on Hibiscus rosa-sinensis
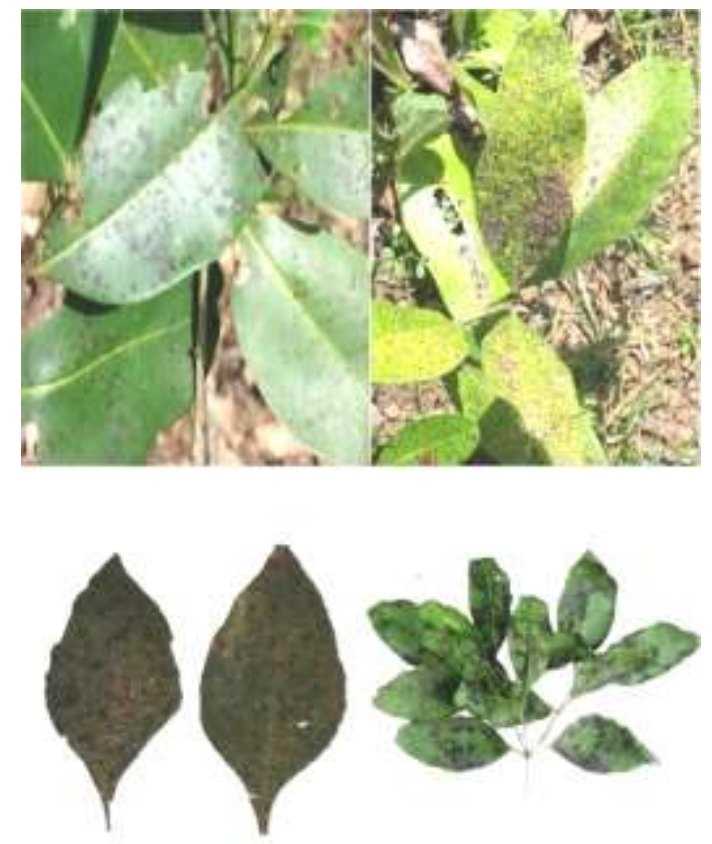

Figure 4 - 1. Lembosia hosagoudarii on leaves of Syzygium cumini, 2. Asterina claviflori on leaves of Syzygium mundagam, 3. Asterina tertia on leaves of Dipteracanthus patalus, 4. Asterina congesta on leaves of Santalum album
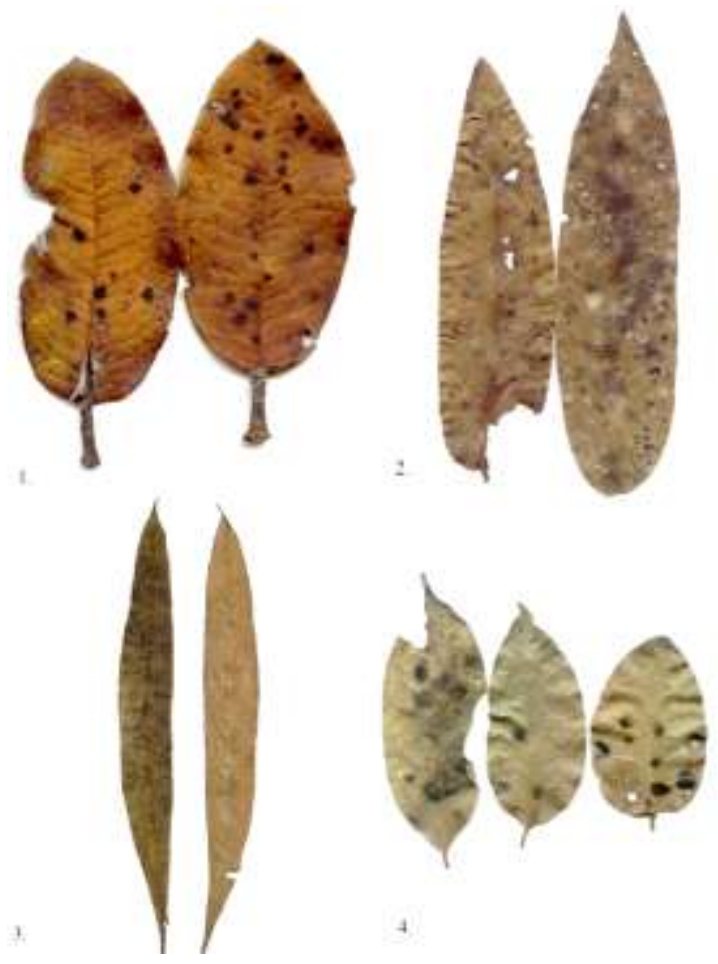

Figure 5, 6 - 1. Asterina hakgalensis on leaves of Rhododendron arboreum, 2. Asterina hopeae on Hopea ponga, 3. Asterina lobeliacearum on leaves of Lobelia nicotinifolia, 4. Asterina olacicola on leaves of Olax scandens
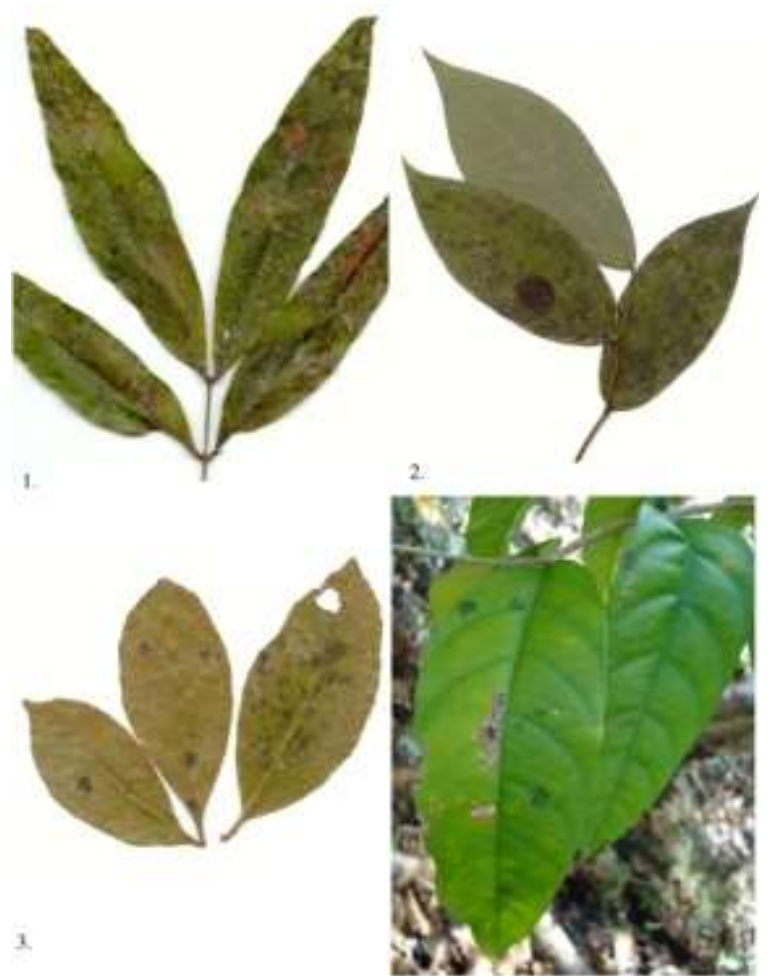

Figure 7 - 1. Asterina saracae on leaves of Saraca asoka, 2. Asterina thotteae on leaves of Thottea silicosa, 3. Asterina toddaliae on leaves of Toddalia asiatica, 4. Asterina wrightii on leaves of Wrightia tinctoria 


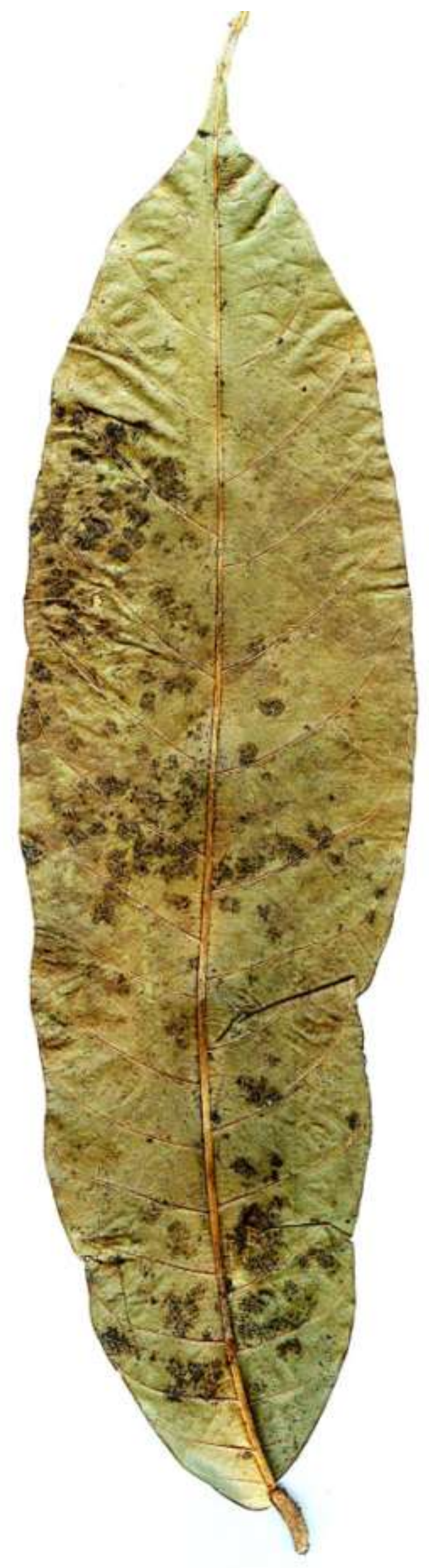

Figure 8 - Asterolibertia vateriae on Vateria indica

\section{TAXONOMIC CHARACTERS}

\section{Steps followed during the identification:} Macroscopic

Host identity: Based on the collected twig, nature of the leaves, field notes, by matching the twig with the probable material in the herbarium and by consulting the angiosperm expert, host identity is confirmed.

1. Position of the colonies on the host epiphyllous, hypophyllous, caulicolous, ramicolous.

2. Nature of the colonies: dense, subdense, thin, very thin, velvety.

3. Pathogenicity: Effect of the fungus on the host plant such as causing wrinkling of the leaves, producing yellow haloes around the colonies.

\section{Microscopic}

\section{Mycelium}

1. Nature of the mycelium: straight, substraight, flexuous, crooked; measurements (length and breadth) of cells

2. Branching pattern: opposite, alternate or irregular at acute or wide angles

\section{Appressoria}

3. Placement of appressoria: alternate, opposite or both or unilateral or in clusters

4. Morphology of appressoria: Unicellular, bicellular or multicellular. Shape of lower (stalk) cells and upper (apical) cells; morphological characters such as entire, angular, crenately lobate, lobate, deeply lobate, sinuately lobate.

5. Nature of the appressoria: straight or curved; antrorse (directed towards growing tip), spreading or recurved; distantly, moderately or densely placed

6. Measurements: length and breadth of lower (stalk) cells and upper (apical) cells

7. Shape of head cells appressoria: Oval, globose, pyriform, cylindrical.

8. Margin of head cells appressoria: Entire, angular, crenately lobate, sublobate, lobate.

9. Shape and type of dehiscence in thyriothecia: Thyriothecia orbicular, oval, elongated, X or Y-shaped; dehisce stellately at the centre or dehisce vertically along the centre

10. Morphology of ascospores: one or two septate, septation at the middle or at the 
extreme end to form pinch-off cell; in case of two septate spores-one cell globose and the cell elongated with a septum at the middle

11. All these observations supplemented with the line drawings/photomicrographs to confirm its correct identity.

\section{Microscopic details}

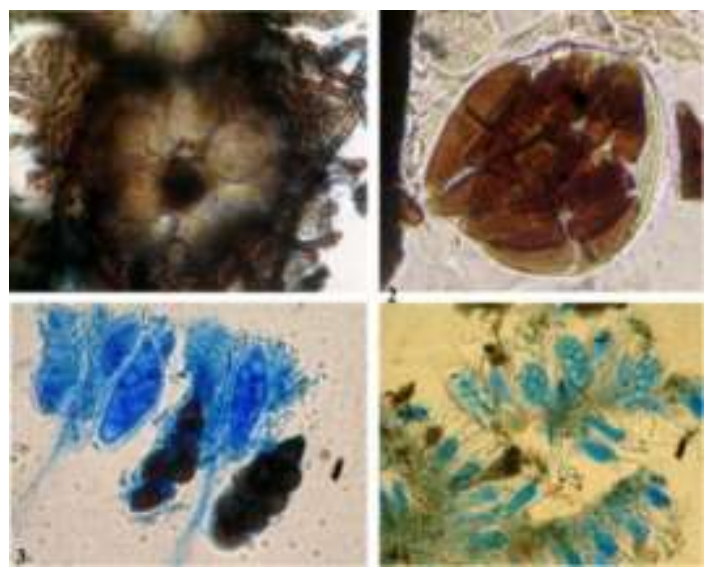

Figure 9 - Asci: 1.Spherical, 2. Ovate, 3- 4. Cylindrical

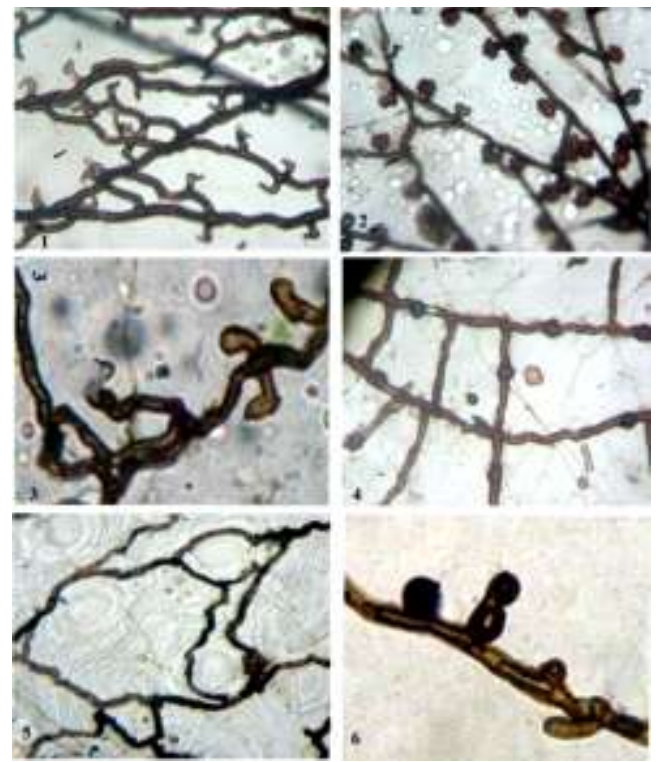

Figure 10 - Morphology and position of appressoria: 1. Hyphae with unicellular appressoria, 2. Hyphae with two celled appressoria, 3. Hyphae with 1-3- septate appressoria, 4. Hyphae with intercalary appressoria, 5. Hyphae without appressoria, 6. Hyphae with conidiophores
12. The identification procedure followed is: scrapes mounted in $10 \% \mathrm{KOH}$ solution, mountant replaced with Lactophenol prepared with a tinge of cotton blue, permanent slide prepared by the nail polish technique, fungus identified up to generic level, corresponding host identified, number of species of the concerned genus of the fungus known on the members of the host family is listed, detailed description, line drawings and photographs of all these taxa are collected, key prepared for all these taxa, then the description of the material to be identified was matched with the known species.

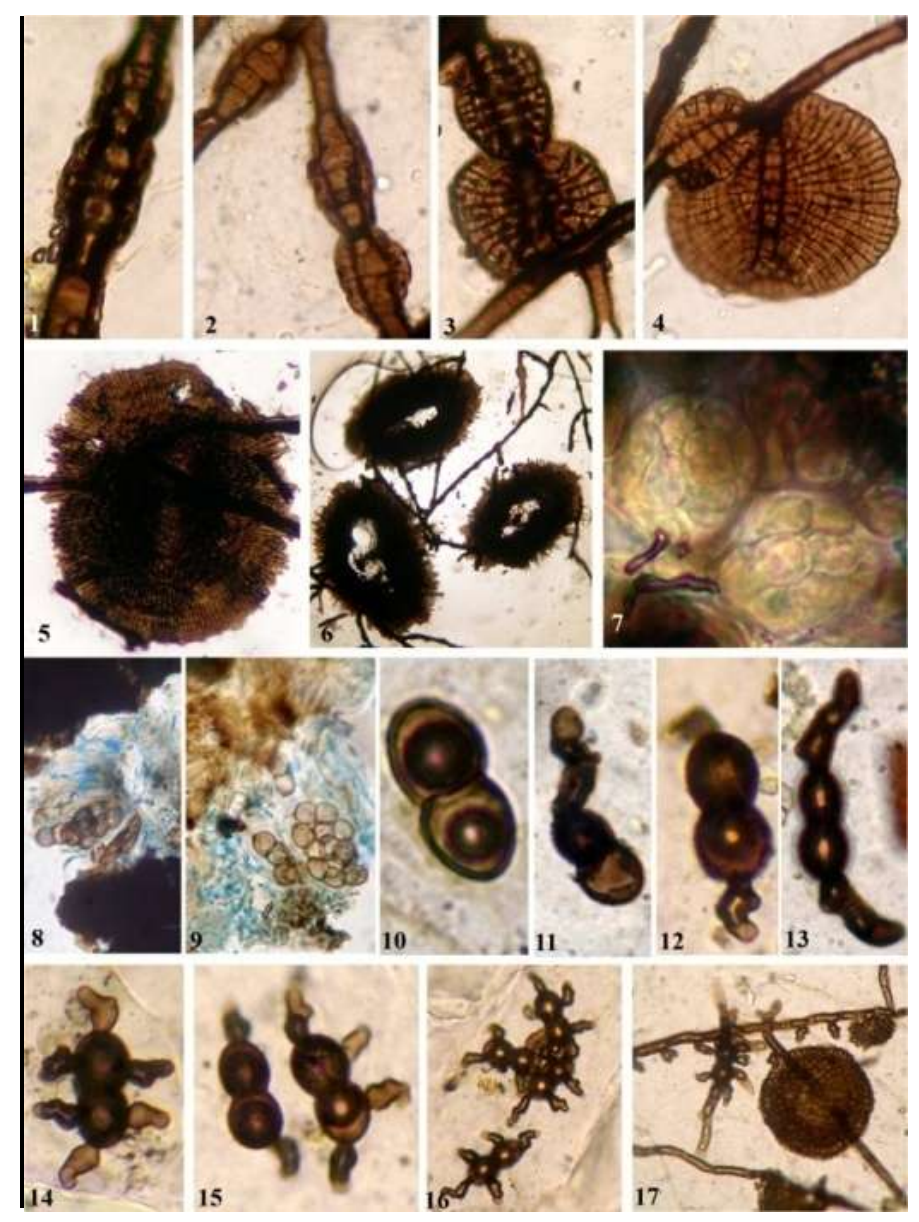

Figure 11 - Development of thyriothecia and ascospore germination: 1-4. Initial stage of development of thyriothecium, 5. Radiate shield of thyriothecium 6. Mature thyriothecia with exposed asci, 7. Asci with ascospores, 8. Mature ascus, 9. Releasing of ascspores from ascus, 10-16. Germinating ascospores, 17. Ascospore forming colony 


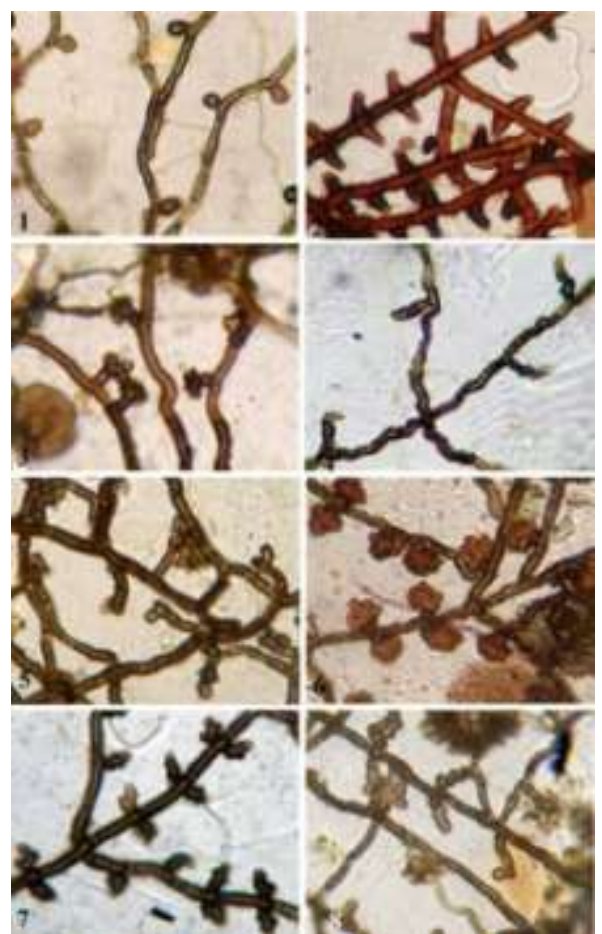

Figure 12 - Morphology and types of appressoria: 1. Unicellular, 2. Opposie, unicellular, ovate to conoid, 3. Uniseptate sublobate, 4, 5. Multicellular, 6. variously lobate, 7. Alternate \& Opposite, 8. Appressoria vary in shape

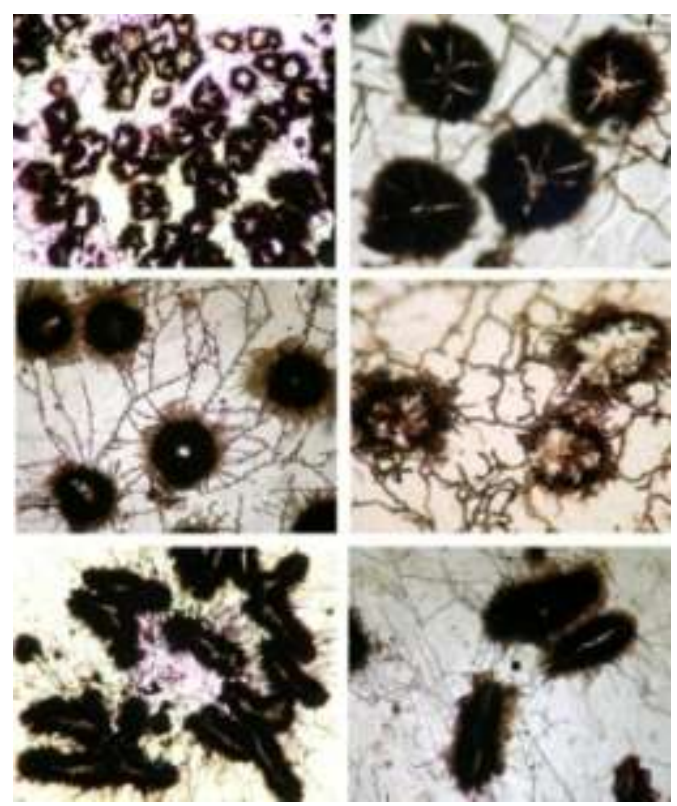

Figure 13 - Morphology of thyriothecia: Thyriothecia - 1, 2. Thyriothecia of Asterina showing stellate dehiscence, 3 . Thyriothecia of Asterina with fimbriate margin, 4. Pycnothyria of Bramhamyces, 5. Thyriothecia of Maheshwaramyces showing longitudinal dehiscence, 6. Thyriothecia of Lembosia showing longitudinal dehiscence

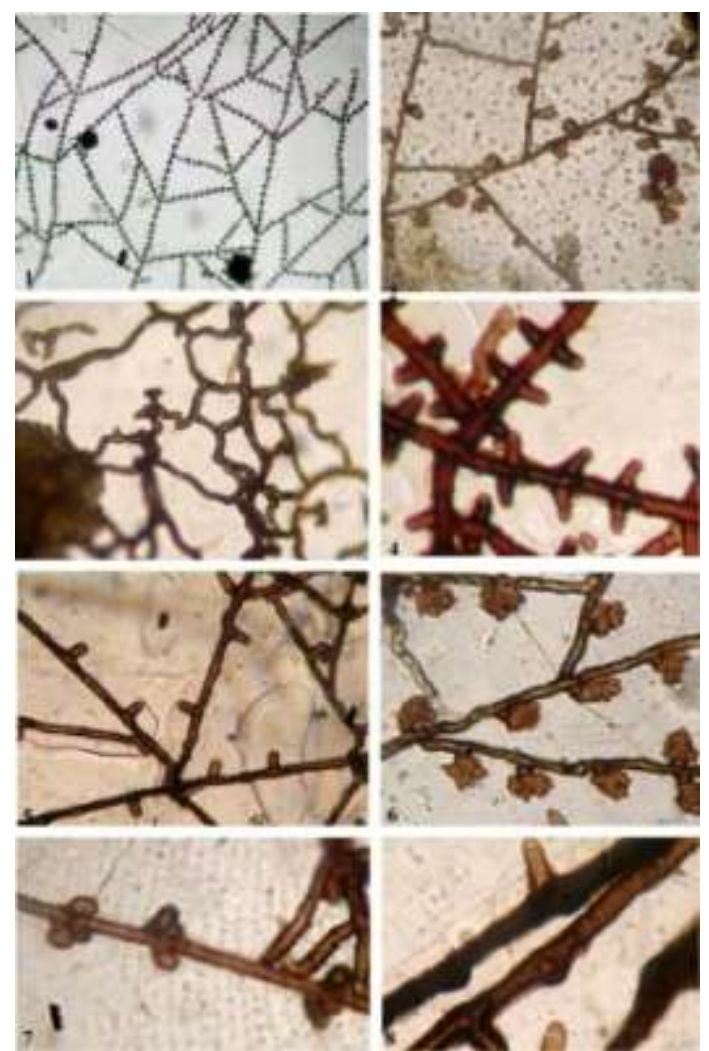

Figure 14 - Mycelial Branching: 1.Opposite, 2. Alternate, 3. Irregular. Arrangement of appressoria-4. Opposite, 5. Alternate and unilateral, 6. Unilateral, 7. Whorled, 8. Intercalary

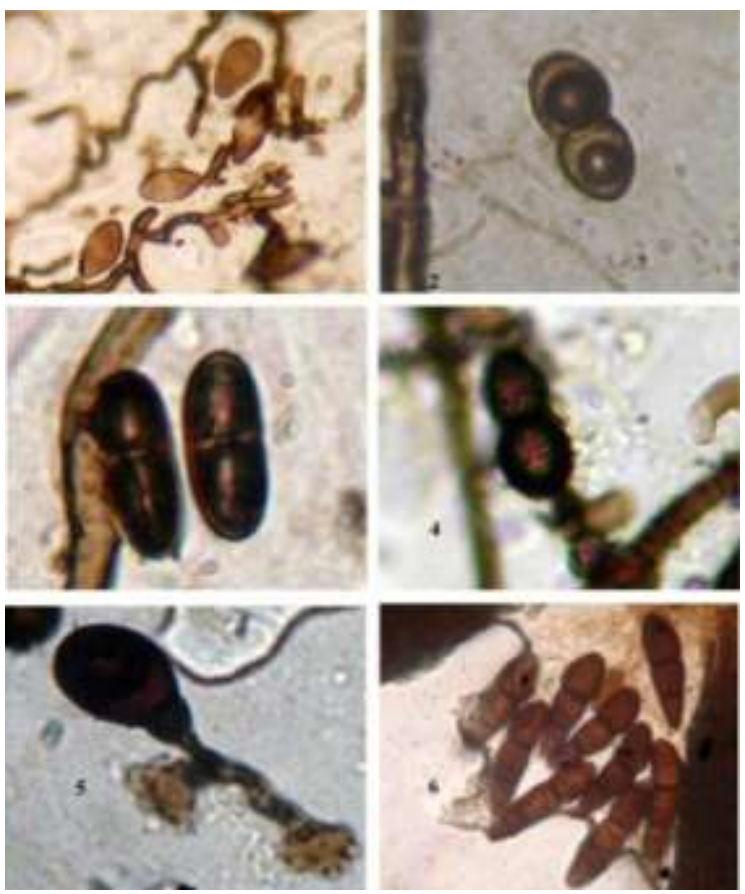

Figure 15 - Spores: 1. Pycnothyriospores, 2. Ascospore constricted at the middle, 3. Ascospores not constricted at the septum, 4. Wall of the ascospore echinulated, 5 . Germinating ascospore of Vishnumyces, 6. Ascospores of Meliolaster 


\section{Order Asterinales}

Leaf parasites. Mycelium ectophytic, with or without appressoria, nutrient mycelium and leaf permeating stroma present. Ascomata ectophytic, dimidiate, orbicular, eilliptic, elongated, X- or Y-shaped, with radiating cells, astomatous, orbicular thyriothecia dehisce stellately at the center, eilliptic, elongated, $\mathrm{X}$ or Y-shaped thyriothecia dehisce vertically with a central suture; asci globose, spherical, oval or rarely cylindrical, octosporous, bitunicate; ascospores two to many septate, conglobate, hyaline, brown at maturity.

Type Family - Asterinaceae.

\section{KEY TO THE FAMILIES OF THE ORDER ASTERINALES}

1. Thyriothecia orbicular, dehisce stellately at the center Asterinaceae 1. Thyriothecia oval to elongated, $\times$ or $Y$ shaped, dehisce longitudinally at the center Lembosiaceae

\section{The Family Asterinaceae}

Asterinaceae Hansf., Mycol. Pap. 15: 189, 1946; Arx \& Muller, Stud. Mycol. 9: 40, 1975; Hosag., Abraham \& C.K. Biju, J. Mycopathol. Res. 39: 62, 2001.

Leaf parasites. Mycelium ectophytic, with or without appressoria, nutrient mycelium and leaf permeating stroma present. Ascomata ectophytic, dimidiate, orbicular with radiating cells, astomatous, dehisce stellately at the center; asci globose, spherical, octosporous, bitunicate; ascospores two to many septate, conglobate, hyaline to brown.

Type Genus - Asterina Lev.

\section{KEY TO THE GENERA}

1. Appressoria present. 2

1. Appressoria absent or rarely observed ....... 9

2. Appressoria in couplets with stellate lumen in the hyphal cell Gangamyces

2. Appressoria not so 3

3. Appressoria both intercalary and lateral

Bheemamyces

3. Appressoria not so 4

4. Appressoria intercalary Asterolibertia

4. Appressoria lateral. 5

5. Appressoria in clusters Ishwaramyces

5. Appressoria not so 6

6. Mycelium/thyriothecia setose
Trichasterina

6. Mycelium/thyriothecia devoid of setae.......7

7. Ascospores one septate ............................ 8

7. Ascospores two septate and one cell taper....

Meliolaster

8. Septa at the middle of ascospores....Asterina

8. Septa at the extreme end by forming one pinched-off cell Vishnumyces

9. Appressoria formed only around the stomata Symphaster

9. Appressoria not formed ............ Prillieuxina

\section{KEY TO THE SPECIES}

(based on host families)

\section{Acanthaceae Asterina}

1. Appressoria entire A. betonicae

1. Appressoria lobed .2

2. Appressoria few .................. A. phlogacanthi

2. Appressoria not so ...3

3. Ascospores smooth ........................... A. tertia

3. Ascospores echinulate. A. tertia var. africana

\section{Alangiaceae \\ Asterina}

1. Ascospores 18-22 $\times 8-10 \mu \mathrm{m} . . . \ldots \ldots . .$. A. balii

1. Ascospores 21-25 $\times 12-16 \mu \mathrm{m}$ A. perpusilla

\section{Anacardiaceae}

Asterina

1. Appressoria alternately arranged, entire ........ A. drimycarpi

1. Appressoria opposite or opposite and alternate, entire to lobate

2. Appressoria alternate and opposite, entire to deeply and irregularly shallowly to deeply lobate, on Nothopegia ............. A. nothopegiae 2. Appressoria opposite, $10 \%$ solitary, entire to variously lobed, on Lannea A. lanneae

\section{Asterolibertia}

1. On Mangifera ....................... A. mangiferae 1. On Nothopegia ..................... A. nothopegiae
Annonaceae... 4
Asterina
1. On Miliusa A. miliusae
1. On other hosts . .2
2. On Polyalthia ........................... clemensis
2. On Saccopetalum A. saccopetali 
Gangagamyces

Single species

G. miliusae

\section{Prillieuxina}

Single species

P. polyalthiae

\section{Trichasterina}

Single species

T. goniothalami

\section{Apiaceae}

\section{Asterina}

Single species

A. hydrocotyles

\section{Apocyanaceae}

1. Appressoria two celled A. wrightiae

1. Appressoria unicellular

2. On Aganosma

2. On Parsonsia A. aganosmae A. parsonsiae

Aquifoliaceae

Asterina

Single species

A. bottomleyae

Prillieuxina

Single species

P. aquifoliacearum

\section{Araliaceae}

Asterina

Single species

A. araliae

\section{Arecaceae}

\section{Asterina}

Single species

A. arecacearum

\section{Aristolochiaceae}

Asterina

1. Appressoria alternate to unilateral, unicellular A. aristolochiae

1. Appressoria alternate and about 3\% opposite, two celled

A. thotteae

\section{Asclepiadaceae}

\section{Asterina}

1. On Tylophora A. prataparajii

1. On other hosts ....................... 2

2. On Wattakakka A. travancorensis

2. On other hosts A. gymnemae

3. On Gymnema

3. On other hosts

4. Appressoria unicellular.

A. tylophorae-indicae

4. Appressoria 2-celled. 5

5. Head cells of appressoria entire to
A. asclepiadis

5. Head cells entire to lobate .......................... 6

6. On Cynanchum ........................................ 7

6. On Toxocarpus ......................... toxocarpi

7. Head cells lobate......................... cynanchi

7. Head cells entire .................A. cynanchicola

Asteraceae

\section{Asterina}

1. On Adenostemma............A. adenostemmatis

1. On other hosts........................................... 2

2. On Blumea .............................. A. blumeae

2. On Eupatorium ......................... A. eupatorii

Averrhoaceae

Asterina

Single species

A. averrhoae

\section{Balsaminaceae}

Single species

A. dhivaharanii

\section{Berberidaceae}

Asterina

Single species

A. goosii

\section{Caesalpiniaceae}

\section{Asterina}

1. On Mezonevron

A. mezonevronis

1. On other hosts

2. On Saraca A. saracae

2. On Cassia A. cassiicola

\section{Capparaceae}

Asterina

1. Appressoria opposite

A. kodajadriensis

1. Both alternate and/or opposite

2. Appressoria alternate

A. capparidis

2. Appressoria alternate and opposite

A. emciciana

\section{Bheemamyces}

Single species

B. capparidis

\section{Caprifoliaceae}

\section{Asterina}

Single species A. viburnicola

\section{Celastraceae}

\section{Asterina}

1. Appressoria in whorls of five to six at each septum

A. gymnosporiae

1. Appressoria alternate or opposite at each septum 
2. Appressoria mammiform, entire to sublobate 3

2. Appressoria clavate, cylindrical, straight to slightly curved, entire.... 4

3. Ascospores more than $30 \mu \mathrm{m}$ long.

A. euonymi

3. Ascospores less than $30 \mu \mathrm{m}$ long A.dissiliens

4. On Glyptopetalum A. glyptopetali

4. On Microtropis 5

5. Appressoria alternate, ascospores less than $30 \mu \mathrm{m}$ long A. microtropidis

5. Appressoria alternate and opposite, ascospores more than $30 \mu \mathrm{m}$ long

A. microtropidicola

\section{Chloranthaceae}

\section{Asterina}

Single species A. sarcandrae

\section{Clusiaceae}

Asterina

1. Appressoria unicellular 2

1. Appressoria 2-celled A. clusiacearum

2. Appressoria oblong, alternate and opposite.. A. garciniicola

2. Appressoria not so A. garciniae

3. Appressoria entire A. morellae

3. Appressoria entire to lobate P. garciniae

\section{Prillieuxina}

Single species

\section{Combretaceae}

\section{Asterina}

1. On Anogeissus A. anogeissi

1. On other hosts .. 2

2. Appressoria unicellular.

A. combreti var. cylindrica

2. Appressoria bicellular 3

3. Appressoria few, distantly placed A. escharoides

3. Appressoria not so A. combreti

\section{Convolvulaceae}

\section{Asterina}

Single species A. argyreiae

\section{Bheemamyces}

Single species B. argyreicola

\section{Prillieuxina}

Single species P. argyreiae

\section{Dichapetalaceae}

Asterina

Single species A. dichapetali

\section{Dilleniaceae}

\section{Asterina}

Single species A. acrotremae

\section{Dipterocarpaceae}

\section{Asterina}

1. On Shorea. A. plurisporus

1. On Hopea 2

2. Appressoria globose, entire ........ A. hopeae

2. Appressoria globose, ovate, attenuated, often angular A. hopiicola

\section{Gangagamyces}

Single species G. shoreae

\section{Ebenaceae}

\section{Asterina}

1. Appressoria alternate and opposite, unicellular A. diospyri 1. Apressoria alternate, 2-celled ........ A. mabae

\section{Prillieuxina}

Single species P. diospyri

\section{Elaegnaceae}

\section{Asterina}

Single species A. elaegni

\section{Prillieuxina}

Single species P. elaegni

\section{Elaeocarpaceae}

\section{Asterina}

1. Appressoria cylindrical, uncinate, forked ..... A. elaeocarpicola

1. Appressoria not so 2

2. Appressoria ovate, oblong, ascospores less than $30 \mu \mathrm{m}$ long.......... A. elaeocarpi var. ovalis 2. Appressoria ovate, conoid, rounded at the apex, ascospores more than $30 \mu \mathrm{m}$ long

A. gamsil

\section{Ericaceae}

\section{Asterino}

Single A. hakgalensis

\section{Erythropalaceae}

\section{Asterina}

Single species A. erythropalicola 


\section{Euphorbiaceae}

Asterina

On Aporusa

1.Appressoria alternate and opposite

A. aporusae

1.Appressoria alternate only

A.aporusae var. cylindrica

\section{On Glochidion}

1.Appressoria alternate. A. lobulifera

1.Appressoria alternate and opposite A. lobulifera var. indica

\section{On Homonoia}

Single species

A. homonoiae

\section{On Mallotus}

1. Appressoria two celled A. malloticola

1. Appressoria unicellular. A. mallotigena

\section{On Phyllanthus}

1. Appressoria lobate... A. phyllanthi-beddomei

1. Appressoria entire ... 2

2. Form mycelial net A. phyllanthigena

2. Not so A. phyllanthicola

\section{Meliolaster}

Single species M. aporusae

\section{Fabaceae}

\section{Asterina}

Single species

A. millettiae

\section{Flacourtiaceae}

\section{Asterina}

1. On Homalium. A. homaligena

1. On other hosts A. granulosa

2. Appressoria unicellular. A. granulosa

2. Appressoria more than one celled

3. Appressoria opposite ........A. talacauveriana

3. Appressoria alternate ... 4

4. Appressoria straight, curved to uncinate

A. hydnocarpi

4. Appressoria not so 5

5. Head cells entire to sinuate A. caseariae-esculentae

5. Head cells obpyriform, frequently 2-3 lobate ........................................................... 6

6. Appressoria distantly placed, stalk cells often multiseptate. A. flacourtiacearum 6. Appressoria numerous, stalk cells not so ..... A. caseariae

\section{Asterolibertia}

1. Colonies crustose, cause pathogenic effect on the host, ascospores 36-39 $\mu \mathrm{m}$ long, on Vateria A. vateriae

1. Colonies not so, ascospores 49-52 $\mu \mathrm{m}$ long, on Hydnocarpus A. hydnocarpi

\section{Ishwaramyces}

Single species I. flacourtiae

\section{Gentianaceae}

Asterina

Single species

A. enicostematis

\section{Hippocrataceae}

\section{Asterina}

Single species A. loeseneriellae

\section{Icacinaceae}

\section{Asterina}

1. Appressoria entire A. gomphandrae

1. Appressoria 2-4 deeply lobed.......A. natsiati

\section{Lamiaceae}

\section{Asterina}

1. On Leucas. A. leucadis

1. On other hosts. .2

2. Head cells of appressoria ovate to globose ... A. hyptidicola

2. Head cells of appressoria ovate to oblong, clavate, hamate A. pogostemonis

\section{Lauraceae}

1. On Cinnamomum .......................................2

1. On other hosts.......................................... 4

2. Appressoria unicellular ............................... 3

2. Appressoria two celled............ A. cinnamomi

3. Appressoria proliferate apically .A. cinnamomicola 3. Appressoria globose, never proliferate A. munnarensis

4. On Neolitsea ....................... A. neolitsiicola

4. On other hosts........................................... 5

5. On Cryptocarya ...............A. cryptocariicola

5. On other hosts 6

6. Appressoria conoid to ampulliform, broadly rounded at the apex......................... A. litseae 6. Appressoria conoid, entire to variously sublobate ........................ litseae-ligustrinae 
Linaceae

Asterina

Single species

A. hugoniae

\section{Lobeliaceae}

\section{Asterina}

Single species

A. lobeliacearum

\section{Loranthaceae}

\section{Asterina}

1. Appressoria alternate only 2

1. Appressoria alternate and opposite ...A. visci

2. Appressoria ovate to globose....A. deightonii

2. Appressoria oblong to clavate

A. loranthigena

\section{Lythraceae}

\section{Asterina}

1. On Lawsonia

A. lawsoniae

1. On Woodfordia. A. woodfordiae

\section{Magnoliaceae}

Asterina

1. Ascospores $22-25 \times 10-13 \mu \mathrm{m}$

A. micheliifolia

1. Ascospores $25-33 \times 15-18 \mu \mathrm{m}$

A. micheliigena

Malvaceae

Asterina

1. On Hibiscus A. hibisci

1. On other hosts... ...2

2. Appressoria angular, sublobate to deeply lobate, bisect, mostly irregularly trilobate

2. Appressoria mammiform to clavate $1-4$ lobed A. pavoniae

\section{Melastomataceae}

\section{Asterina}

1. Appressoria opposite A. madikeriensis

1. Appressoria alternate A. memecylonis

\section{Meliaceae}

\section{Asterina}

1. Appressoria alternate only A. chukrasiae

1. Appressoria alternate and opposite .. 2

2. Appressoria conoid to ampulliform A. cipadessae

2. Appressoria globose A. aglaiae

3. On Aglaia A. trichiliae

Menispermaceae Asterina
1. Appressoria unicellular ... 3

1. Appressoria bicellular 2

2. On piper A. piperina

2. On Lepianthes A. lepianthis

3. On Anamirta A. anamirtae

3. On Tinospora A. tinosporae

\section{Prillieuxina}

Single species P. anamirtae

\section{Moraceae}

Asterina

Single species A. mysorensis

\section{Myristicaceae}

\section{Asterino}

1.Appressoria two celled A. myristicae

1. Appressoria unicellular .2

2. On Knema. A. knemae-attenuatae

2. On Myristica. A. myristicacearum

\section{Myrsinaceae}

\section{Asterina}

1. On Myrsine A. myrsines

1. On Ardisia 2

2. Appressoria cylindrical, entire ....A. ardisiae 2. Appressoria globose, entire, crenately to deeply lobate A. ardisiicola

\section{Prillieuxina}

Single species P. ardisiae

\section{Myrtaceae}

\section{Asterino}

1. On Rhodomyrtus .................... A. rhodomyrti

1. On other hosts........................................... 2

2. Appressoria unicellular .............................. 3

2. Appressoria bicellular ................................4

3. Two dark brown bands present in the middle of each cell ...................... A. gopalakrishnanii

3. Not so A. claviflori

4. On Syzygium henianum .............. A. henianii

4. On other hosts.......................................... 5

5. On Syzygium cumini................ A. jambolana

5. On Eugenia discifera ............... A. disciferae

Olacaceae

Asterina

Single species A. olacicola

Oleaceae

Asterina

1. On Ligustrum .......................... ligustricola

1. On Jasminum spp. ..................................... 2 
2. Appressoria unicellular A. jasmini var. indica

2. Appressoria bicellular

3. Stalk cells gibbous A. pongalaparensis

3. Stalk cells not so A. jasminicola

4. Hyphae tortuous

4. Hyphae not so 5

5. Colonies epiphyllous, appressoria 2-5\% opposite .A. erysiphoides

5. Colonies hypophyllous, appressoria $30 \%$ opposite A. erysiphoides var. indica

\section{Prillieuxina}

Single species .P. jasmini

\section{Opiliaceae}

\section{Asterina}

1. On opilia . A. crebra

1. On cansjera. ... 2

2. Appressoria broad based, lobed, up to $10 \mu \mathrm{m}$ long... A. cansjerae

2. Appressoria entire, more than $10 \mu \mathrm{m}$ long...3

3. Appressoria $5 \%$ opposite, conoid A. cansjericola

3. Appressoria $15 \%$ opposite, ovate to cylindrical ..............A. cansjericola var. indica

\section{Passifloraceae \\ Asterina}

Single species A. adeniicola

\section{Piperaceae}

Asterina

1. On Piper A. piperina

1. On Lepianthes A. lepianthis

\section{Pittosporaceae}

Asterina

Single species A. pittospori

\section{Polygalaceae}

Asterina

Single species A. polygalae

\section{Ranunculaceae \\ Asterina}

1. On Naravelia A. naraveliae

1. On Clematis A. clematidis

\section{Rhamnaceae}

\section{Asterina}

Single species A. rhamni
1. Appressoria in whorls A. ixorae

1. Not so ....................................................... 2

2. Appressoria unicellular ............................. 4

2. Appressoria two celled................................ 3

3. On Plectranthus ...................... A. plectranthi

3. On Canthium ....................... A. canthiigena

4. On Canthium dicoccum.... A. canthii-dicocci

4. On Psychotria A. psychotriicola

\section{Prillieuxina}

Single species P. ixorigena

\section{Rutaceae}

Asterina

1. Appressoria unicellular 3

1. Appressoria two celled................................2

2. On Acronychia. A. rhabdodendri var. levibus

2. On Toddalia. A. toddaliicola

3. Appressoria opposite only........A. zanthoxyli

3. Appressoria alternate and opposite ............. 4

4. Appressoria alternate only..........................5

4. Appressoria alternate and opposite ............7

5. Appressoria antrorse, retrorse, straight, flexuous to curved ........................ A. toddaliae

5. Not so.....

6

6. Appressoria hemispherical.... A. banguiensis

6. Appressoria ovate, oblong, cylindrical

7. On Aegle. A. atalantiae

7. On other hosts A. delicatula

8. Appressoria mostly opposite on Acronychia ........................................... Acronychiae

8. On other hosts 9

9. Appressoria alternate and about $10-12 \%$ opposite, on clausena. A. clausenicola 9. Not so, on other hosts. 10

10. On Melicope A. melicopecola

10. On Murraya 11

11. Appressoria alternate and opposite .A. murrayae 11. Appressoria alternate only.. A. murrayicola

\section{Sabiaceae}

\section{Asterina}

1. On Sabia. A. sabiana

1. On Meliosma 2

2. Appressoria oblong, cylindrical, straight to curved, 10-13 $\mu \mathrm{m}$ long A. meliosmae-simplicifoliae 2. Appressoria mammiform, 13-22 $\mu \mathrm{m}$ long..... A. sabiacearum

\section{Rubiaceae}




\section{Santalaceae}

Asterina

1. Appressoria alternate, entire to sinuately lobate...

A. congesta

1. Appressoria opposite to alternate, entire

A. scleropyri

\section{Sapindaceae}

Vishnumyces

Single species

V. otonephelii

\section{Sapotaceae}

\section{Asterina}

1. Appressoria mostly alternate and opposite...

1. Appressoria alternate to unilateral

$$
\text { A. palaquii }
$$

2. Appressoria unicellular. A. laxiuscula

2. Appressoria two celled ..A. mimusopsidicola

\section{Symphaster}

Single species

S. mimusopsidis

\section{Simaroubaceae}

\section{Asterina}

Single species

A. samaderae

\section{Solanaceae}

\section{Asterina}

1. Appressoria unicellular.

1. Appressoria two celled A. diplopoda

2. Appressoria alternate and opposite

2. Appressoria alternate only

A. balakrishnanii

3. On Lycianthes A. lycianthedis

3. On Physalis A. physalidis

\section{Sterculiaceae}

\section{Asterina}

Single species

A. helicteridis

\section{Prillieuxina}

Single species P. pterigotae

\section{Symplocaceae}

\section{Asterina}

1. Appressoria alternate and opposite; ascospores more than $35 \mu \mathrm{m}$ long.......A. indica 1. Appressoria alternate only; ascospores less than $35 \mu \mathrm{m}$ long A. suttonii

\section{Theaceae}

\section{Asterina}

1. Appressoria alternate 3

1. Appressoria alternate and opposite

2. Appressoria $70 \%$ opposite ..A. schimae
2. Appressoria about 5\% opposite, cylindrical.. A. theacearum

3. Appressoria entire to furcated ........A. songii

3. Appressoria entire only A. cannonil

Thunbergiaceae

\section{Asterina}

Single species

A. thunbergiicola

\section{Tiliaceae}

Asterina

1. On Triumfetta A. triumfetticola

1. On Grewia A. wingfieldii

\section{Ulmaceae}

\section{Asterina}

Single species A. dallasica

\section{Urticaceae}

Asterina

1. Appressoria two celled A. girardiniae

1. Appressoria unicellular 2

2. Appressoria mostly opposite

A. oreocnidegena

2. Appressoria alternate only 3

3.Appressoria crenately lobate

A. elatostematis

3. Appressoria stellately sublobate A. oreocnidecola

Verbenaceae

Asterina

On Premna

1. Appressoria unicellular A. pusilla

1. Appressoria bicellular A. kukkalensis

\section{On Clerodendrum}

Single species

A. kannurensis

\section{Vitaceae}

Asterina

1. On Cissus A. cissi

1. On Vitis A. viticola

\section{Xanthophyllaceae}

Asterina

Single species A. xanthophylli

\section{Abbreviations used in the line drawings}

Unless otherwise mentioned, it is applicable to all the line drawings.
a. Appressoriate mycelium
b. Thyriothecium
c. Ascus
d. Ascospores
e. Pycnothyriospores 


\section{The genus Asterina}

Asterina Lev., Ann. Sci. Nat. Bot. Ser., 3(3):57, 1845; Hansf., Mycol. Pap. 15: 189, 1946b; Arx \& Muller, Stud. Mycol. 9: 42, 1975; Hosag., Abraham \& C.K. Biju, J. Mycopathol. Res. 39: 62, 2001; Hosag., Chandraprabha \& Agarwal, Asterinales of Kerala, p. 32 , 2011. Dimerosporium Fuckel, Symb. Mycol. p.86,1870. Asterella (Sacc.) Speg. ex Sacc., Syll. Fung. 9: 393, 1891 non P. de Beauvois 1805. Myxasterina Hohnel, Sber. Akad. Wiss. Wien 118: 870, 1909. Englerulaster Hohnel, Sber. Akad. Wiss. Wien 119: 454, 1910. Parasterina Theiss., Sydow \& Sydow, Ann. Mycol. 15: 246, 1917. Calothyriolum Speg., Boln Acad. nac. Cien. Cordoba 23: 498, 1919. Opeasterina Speg., Boln Acad. nac. Cien. Cordoba 23: 498, 1919. Englera F. Stev. in Stev. \& Ryan, Illinois. Biol. Monogr. 17: 45, 1939.

Leaf parasites. Mycelium ectophytic, appressoria lateral, setae absent. Thyriothecia orbicular with radiating cells, astomatous, dehisce stellately at the center; asci globose, octosporous, bitunicate; ascospores conglobate, uniseptate, brown.

Type sp.: - A. melastomatis Lév.

Anamorphs: Asterostomella Speg., Clasterosporium Schwein, Mahanteshamyces Hosag.

\section{DESCRIPTIONS OF SPECIES}

Asterina acronychiae Hosag. \& Goos, Mycotaxon 59: 150, 1996; Hosag., H. Biju \& Appaiah, J. Mycopathol. Res. 44: 5, 2006; Hosag., Chandra. \& Agarwal, Asterinales of Kerala, p. 32, 2011. (Fig. 16)

Colonies epiphyllous, dense, crustose, up to $3 \mathrm{~mm}$ in diameter, confluent. Hyphae straight to substraight, branching opposite at acute angles, loosely reticulate, cells 13-21 × 3-7 $\mu \mathrm{m}$. Appressoria mostly opposite, rarely solitary, unicellular, ovate, oblong, entire, angular to slightly lobate, 6-9 $\times 5-8 \mu \mathrm{m}$. Thyriothecia scattered, orbicular, up to 132 $\mu \mathrm{m}$ in diameter; margin fringed, stellately dehisced at the centre; asci numerous, globose, octosporous, $35-42 \mu \mathrm{m}$ in diameter; ascospores brown, conglobate, uniseptate, deeply constricted at the septum, 20-24 × 9-12 $\mu \mathrm{m}$, wall minutely echinulate.

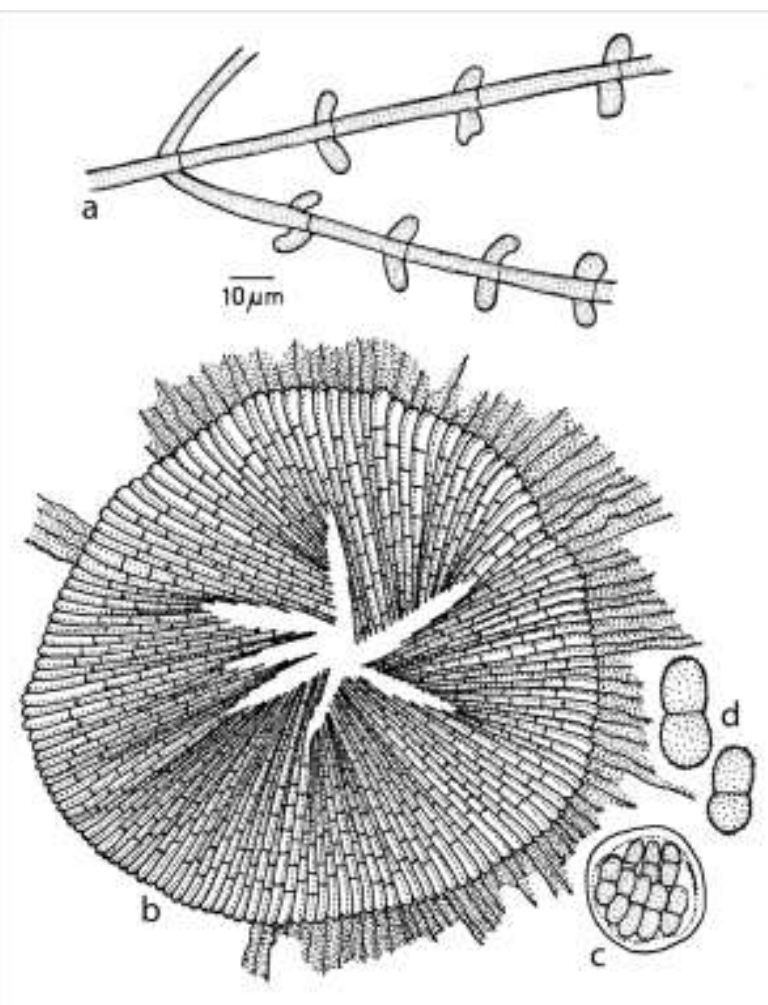

Figure 16 - Asterina acronychiae

Material examined: Tamil Nadu, Tirunelveli, Kakachi forest, on leaves of Acronychia pedunculata (L.) Miq. (Rutaceae), 21 February, 1994, V.B. Hosagoudar HCIO 41628 (holotype); Kerala, Wyanad, Banasuran mala, 19 November, 1999, C.K. Biju HCIO 45148, TBGT 1203; Palghat, Silent valley, Walakad, 6 August 2008, M.C. Riju \& Jacob Thomas TBGT 5547; Palghat, Silent valley, Sairandhri, 16 February, 2007, Girish \& al. TBGT 5633, 5635; Palghat, Silent valley National park, Sairandhri, 22 July 2009, S.S. Shaji \& P.P. Rajesh Kumar TBGT 4543 (as A.longipedunculata); Karnataka, Kodagu, Nishane motta, on leaves of Acronychia sp. (Rutaceae), 11 November 2003, V.B. Hosagoudar \& al. HCIO 45729, TBGT 1478; Kerala, Wyanad, Padinjarethara, 10 November 2007, M. C. Riju HCIO 48324, TBGT 2972; Alapuzha,Vandanam, 3 October 2002, A. Manojkumar HCIO 44614, TBGT 896; Wayanad, Banasuran mala, 16 April 1999, C.K. Biju HCIO 45202, TBGT 1238; Kottayam, Ponthanpuzha reserve forest, 16 December, 2007, P.J. Robin HCIO 49825, TBGT 3977; 14 February, 2007, P.J. Robin HCIO 49988; 
TBGT 4140; Palghat, Silent valley National park, 1 March 2009, S.S. Shaji TBGT 4516. Karnataka, Coorg, 26 November, 2008, V.B. Hosagoudar \& al. HCIO 49164, TBGT 3419; Hakathur, 26 November, 2008 V.B. Hosagoudar \& al. HCIO 49175, TBGT 3430.

This species is similar to Asterina vepridis Doidge in having opposite appressoria but differs from it in having epiphyllous colonies, larger and echinulate ascospores.

Distribution: Karnataka, Kerala, Tamil $\mathrm{Nadu}$

Asterina acrotremae Hosag. \& Chandra. ex Hosag. in Hosag., Chandra. \& Agarwal, Asterinales of Kerala, p.33, 2011.

Asterina acrotremae Hosag. \& Chandra., Advances in Pollen Spore Research 27:156, 2009 (nom. invalid.).

(Fig. 17)
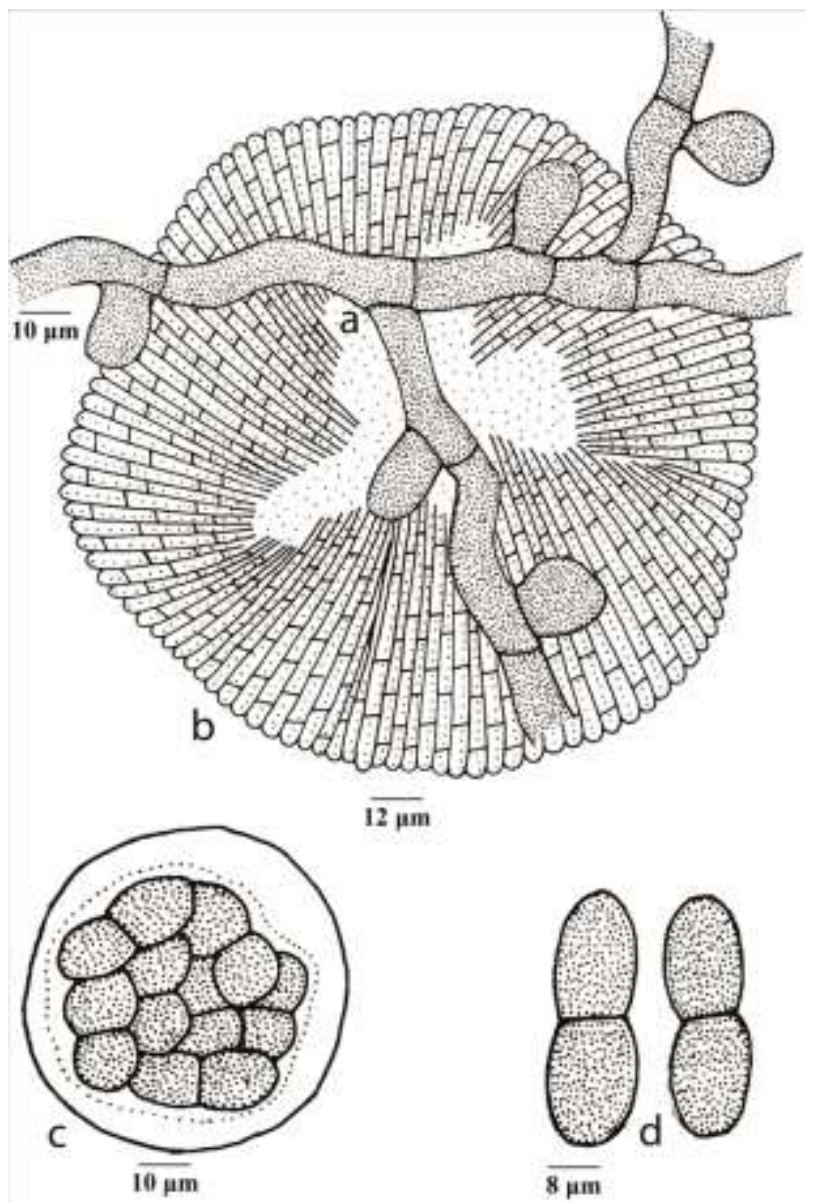

Figure 16 - Asterina acrotremae
Colonies epiphyllous, dense, up to 4 $\mathrm{mm}$ in diameter, confluent. Hyphae crooked, branching irregular at acute to wide angles, loosely to closely reticulate, cells 15-22 × 4-7 $\mu \mathrm{m}$. Appressoria unicellular, alternate, ovate, globose, entire, 7-11 $\times 7-9 \mu \mathrm{m}$. Thyriothecia scattered, orbicular, up to $160 \mu \mathrm{m}$ in diameter, stellately dehisced at the center, margin crenate; asci many, globose, octosporous, up to $33 \mu \mathrm{m}$ in diameter; ascospores oblong, conglobate, brown, 1-septate, constricted at the septum, 18$22 \times 7-9 \mu \mathrm{m}$, wall smooth.

Material examined: Kerala, Thiruvananthapuram, Chemunji hills, on leaves of Acrotrema arnottianum Wight (Dilleniaceae), 11 March 1997, V.B. Hosagoudar HCIO 42955 (holotype), TBGT 256 (isotype).

Hosagoudar \& Chandra Prabha (2009) proposed this species and was validated by Hosagoudar (Hosagoudar et al., 2011).

Distribution: Kerala

Asterina adenostemmatis Kar \& Ghosh, Indian Phytopath. 39: 208, 1986 (adenostemmae).

Colonies amphigenous, mostly epiphyllous, thin, up to $3 \mathrm{~mm}$ in diameter, confluent. Hyphae undulate, closely reticulate, cells $15-57 \times 3-8 \mu \mathrm{m}$. Appressoria alternate to unilateral, unicellular, straight to curved, 2-4times deeply lobate, 6-11 $\times$ 6-10 $\mu \mathrm{m}$. Thyriothecia scattered to grouped, orbicular, $68-183 \mu \mathrm{m}$ in diameter, stellately dehisce at the center; asci many, globose, 8 -spored, 22-35 $\mu \mathrm{m}$ in diameter; ascospores fusiform-elliptic, brown, uniseptate, constricted at the septum, $12-18 \times 6-8 \mu \mathrm{m}$, wall smooth.

West Bengal, Darjeeling, Mirik (3, 937 $\mathrm{ft}$.), on leaves of Adenostemma viscosum Forst. (Asteraceae), 26 November 1977, IMI 225671(holotype).

Distribution: West Bengal

Asterina adeniicola Hosag. \& Kamar., Zoos print J. 21: 2303, 2006; Hosag., 
Chandraprabha \& Agarwal, Asterinales of Kerala, p.35, 2011. (Fig. 18)

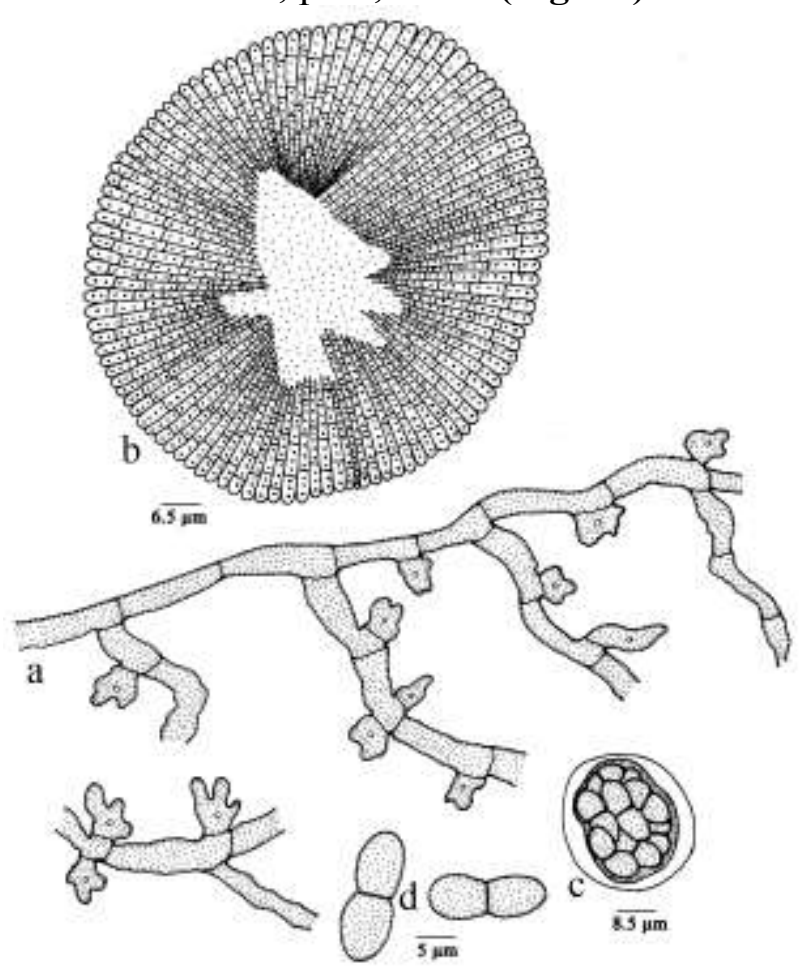

Figure 18 - Asterina adeniicola

Colonies epiphyllous, dense, crustose, up to $1 \mathrm{~mm}$ in diameter, confluent. Hyphae strongly flexuous to rarely crooked, branching alternate to irregular at acute to wide angles, loosely to closely reticulate, cells 11-15 $\times 4-7$ $\mu \mathrm{m}$. Appressoria alternate, about 3\% opposite, unicellular, globose, ovate, sessile to slightly stipitate, mostly 2-3-lobate, often angular to rarely entire, 6-9 $\times 6-8 \mu \mathrm{m}$. Thyriothecia loosely scattered to connate at the centre of the colony, orbicular, stellately dehisced at the centre, up to $75 \mu \mathrm{m}$ in diameter, margin crenate; asci few, globose, octosporous, up to $30 \mu \mathrm{m}$ in diameter; ascospores oblong, conglobate, uniseptate, slightly constricted at the septa, lower cell slightly larger, 14-16 × 6-8 $\mu \mathrm{m}$, wall smooth.

Material examined: Kerala, WyanadPeriya, on leaves of Adenia hondala (Gaertn.) Wilde (Passifloraceae), 17 December 2002, M. Kamarudeen HCIO 44792 (holotype), TBGT 1029 (isotype).

The released ascospores readily germinated and formed colonies. Asterina adeniae Hansf. is known on Adenia lobata from Uganda (Hansford, 1945). However, Asterina adeniicola differs from it in having dense and crustose colonies, 3\% opposite and smaller appressoria, smaller thyriothecia and shorter ascospores.

\section{Distribution: Kerala}

Asterina aganosmae Petrak in Sydow \& Petrak, Ann. Mycol. 29: 238, 1931; Hosag. \& Hanlin, New Botanist 22: 188, 1995.

Colonies amphigenous, often hypophyllous, thin, scattered, up to $2 \mathrm{~mm}$ in diameter, often confluent. Hyphae undulate, irregularly branched, reticulate, cells 10-16 × 3$5 \mu \mathrm{m}$. Appressoria alternate, continuous, more or less clavate, rarely depressed, slightly to distinctly lobate, 6-11 $\times 5-8 \mu \mathrm{m}$. Thyriothecia closely scattered, orbicular to broadly elliptic, $100-160 \mu \mathrm{m}$ diameter, mostly triangularly dehisced at the centre, fimbriate at the margin; asci few, globose to subglobose, broadly ovate to ellipsoidal, broadly rounded at the apex, 4-8 spored, 28-36 $\mu \mathrm{m}$ diameter; ascospores conglobate, elongated, rounded at both ends, straight to substraight, septate at the medium, slightly constricted at the septum, initially hyaline, later olivaceous brown, 16-19 × 7-9 $\mu \mathrm{m}$.

Material examined: Tamil Nadu, Kodaikanal, Bryant Park, on leaves of Aganosma cymosum (Roxb.) G. Don. (Apocynaceae), 2 February1993, V.B. Hosagoudar HCIO 40883.

Petrak in Petrak \& Sydow (1931) reported this species on Aganosma sp. from Philippines and is known from India only by a single collection from this locality. The Material should be collected once again to confirm its correct identity.

Distribution: Tamil Nadu

Asterina aglaiae Hosag., J. Mycopathol. Res. 44: 5, 2006; Hosag., Chandraprabha \& Agarwal, Asterinales of Kerala, p.36, 2011.

(Fig. 19)

Colonies epiphyllous, thin to subdense, up to $3 \mathrm{~mm}$ in diameter, rarely confluent. Hyphae straight to slightly crooked, branching 
irregular at acute to wide angles, loosely to closely reticulate, cells 16-23 $\times$ 4-6 $\mu \mathrm{m}$. Appressoria alternate, opposite to subopposite, unicellular, ovate, conoid, ampulliform, antrorse, subantrorse to retrorse, straight to curved, entire to rarely angular to sublobate, 8$13 \times 5-15 \mu \mathrm{m}$. Thyriothecia scattered, orbicular, up to $120 \mu \mathrm{m}$ in diameter, stellately dehisced at the centre, margin crenate to fimbriate, fringed hyphae few, crooked; asci globose, octosporous, up to $30 \mu \mathrm{m}$ in diameter; ascospores conglobate, oblong, brown, uniseptate, deeply constricted at the septum, 20-28 $\times 11-13 \mu \mathrm{m}$, wall smooth.

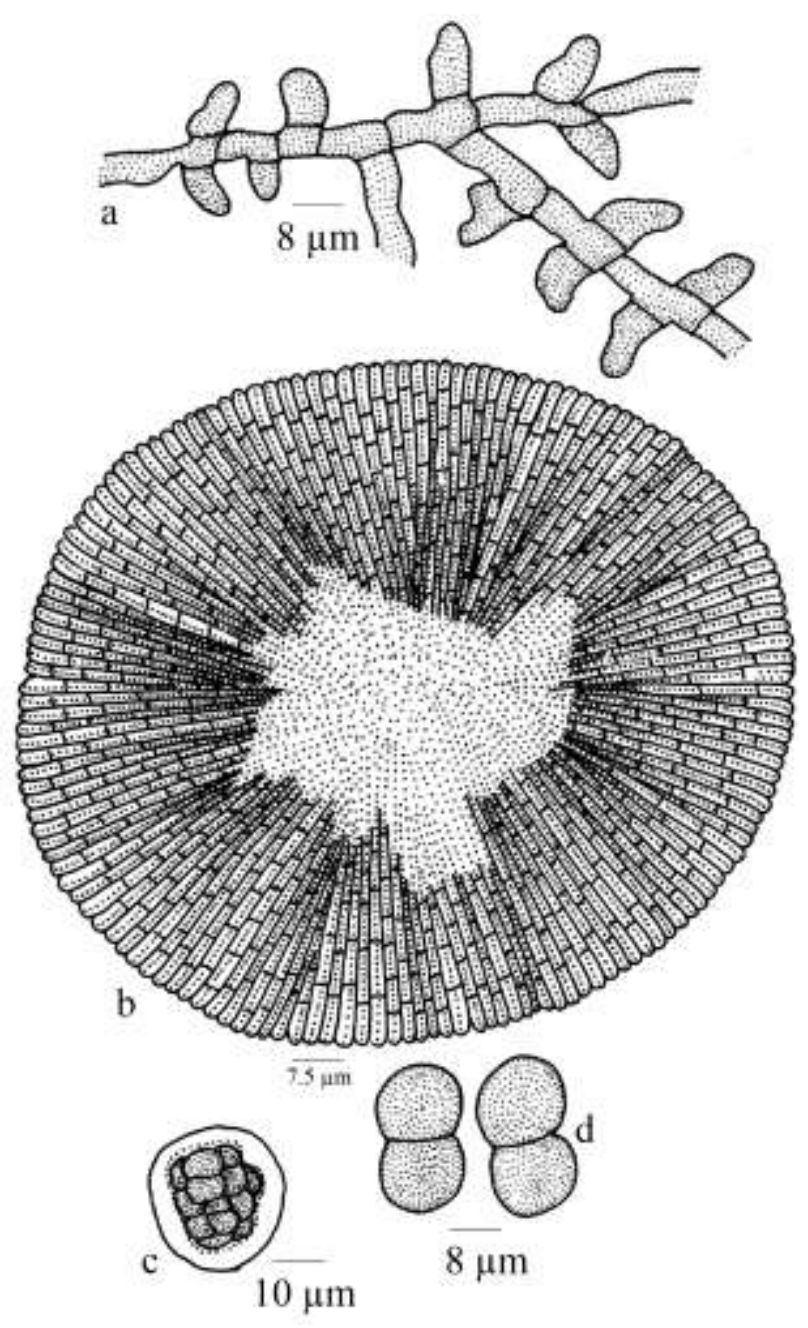

Figure 19 - Asterina aglaiae

\section{Material examined: Karnataka,} Kodagu (Coorg), Madikeri, Jodupal, on leaves of Aglaia sp. (Meliaceae), 12 November 2003, V.B. Hosagoudar \& al. HCIO 45756 (holotype), TBGT 1505 (isotype); Kerala, Thrissur, Malakkapara, 18 October, 2007, Jacob Thomas HCIO 48258, TBGT 2997;
Kottayam, Ponthanpuzha, 17 November 2008, P.J. Robin \& al. HCIO 49461, TBGT 3703.

Based on the morphology and arrangement of appressoria and measurements of the ascospores, A. aglaiae is similar to $A$. silvatica Speg. known on Trichilia sp. from Brazil (Theissen, 1913) but differs from it in having ovoid to ampulliform appressoria and larger ascospores.

Distribution: Karnataka, Kerala

Asterina anamirtae Hosag. in Hosag., Balakr. \& Goos, Mycotaxon 59: 167, 1996. (Fig. 20)
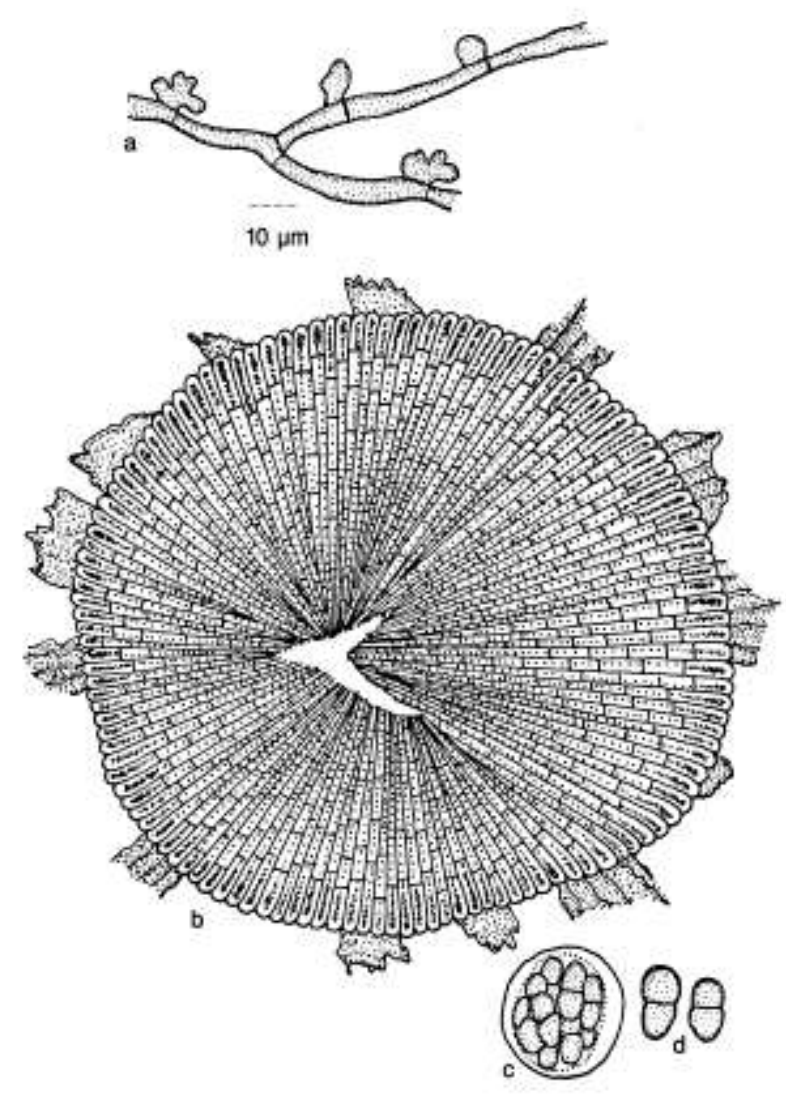

Figure 20 - Asterina anamirtae

Colonies epiphyllous, thin, up to $2 \mathrm{~mm}$ in diameter, widely confluent. Hyphae pale brown, substraight to flexuous, branching alternate to irregular at acute to wide angles, loosely reticulate, cells $12-16 \times 5-7 \mu \mathrm{m}$. Appressoria alternate, unicellular, pale brown, globose to ovate, entire to irregularly sublobate, 9-10 $\times 6-7 \mu \mathrm{m}$. Thyriothecia scattered, round, up to $78 \mu \mathrm{m}$ in diameter, dehisce stellately at the center and later opened widely, margin serrate to fimbriate, fringed hyphae flexuous; 
asci globose, eight spored, 18-22 $\mu \mathrm{m}$ in diameter; ascospores conglobate, cinnamon brown, 1-septate, deeply constricted at the septum, one cell slightly larger, 14-16 × 9-10 $\mu \mathrm{m}$, wall echinulate.

Material examined: Tamil Nadu, Coimbatore, Anamalai, near Nirar Dam, on leaves of Anamirta cocculus (L.) Wight \& Arn. (Menispermaceae), 27 March 1990, V.B. Hosagoudar HCIO 30952 (holotype).

This is the only species of the genus Asterina on this host genus (Hansford, 1944, 1946, 1949; Hansford \& Thirumalachar, 1948; Stevens \& Ryan, 1939).

\section{Distribution: Tamil Nadu}

Asterina anogeissi Rajak, J. Econ. Taxon. Bot. 7: 30, 1985.

Colonies effuse, epiphyllous, black, covering almost whole lamina, easily detachable. Mycelium brown, septate, branched, appressoriate, loosely reticulate. Thyriothecia subglobose, brown, opaque, dimidiate, superficial, opening by radiating fissures, bearing asci basally, 220-425 $\mu \mathrm{m}$ diameter; asci globular, hyaline, membranous, aparaphysate, 8-spored, 35-65 $\mu \mathrm{m}$ in diameter; ascospores hyaline, becoming brown at maturity, 2-celled, constricted at the septum, cells unequal, smooth walled, ends subacute to rounded, 26-33.5 × 14-19 $\mu \mathrm{m}$.

Madhya Pradesh, Mandla forest, on living leaves of Anogeissus latifolia Wall. (Combretaceae), January 1977, R.C. Rajak IMI 212442 (holotype).

Distribution: Madhya Pradesh

Asterina aporusae Hansf., Reinwardtia 3: 129, 1954; Hosag. \& Agarwal, Indian Phytopath. 56: 98, 2003; Hosag. \& Appaiah, J. Mycopathol. Res. 43:168, 2005; Hosag., Chandraprabha \& Agarwal, Asterinales of Kerala, p. 37, 2011.

(Figs 21-22)
Colonies amphigenous, minute, thin, up to $2 \mathrm{~mm}$ in diameter, confluent. Hyphae straight, flexuous to crooked, branching alternate, opposite to irregular at acute angles, loosely to closely reticulate, cells $10-12 \times 3-5$ $\mu \mathrm{m}$.

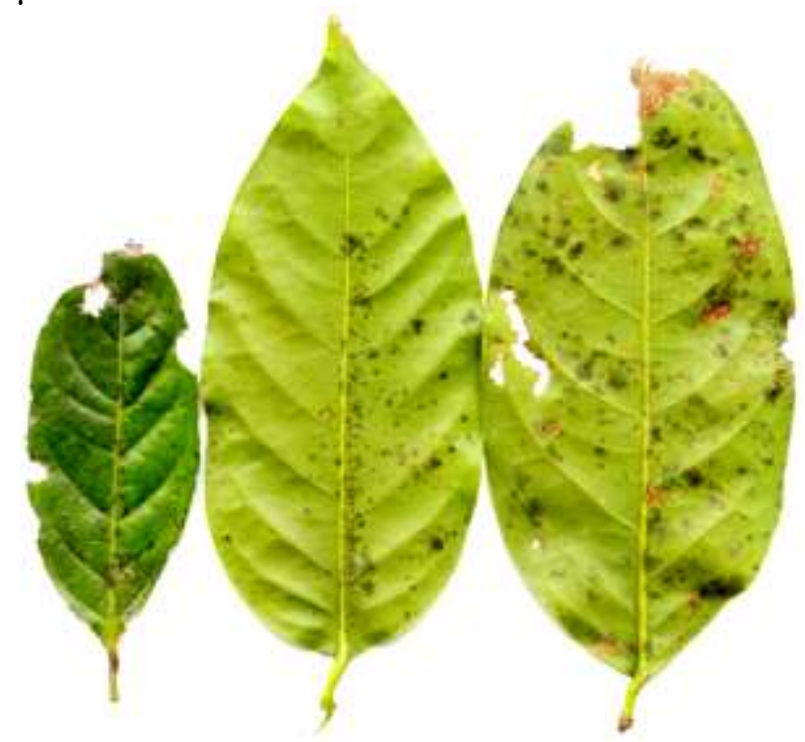

Figure 21 - Asterina aporusae-Infected leaves

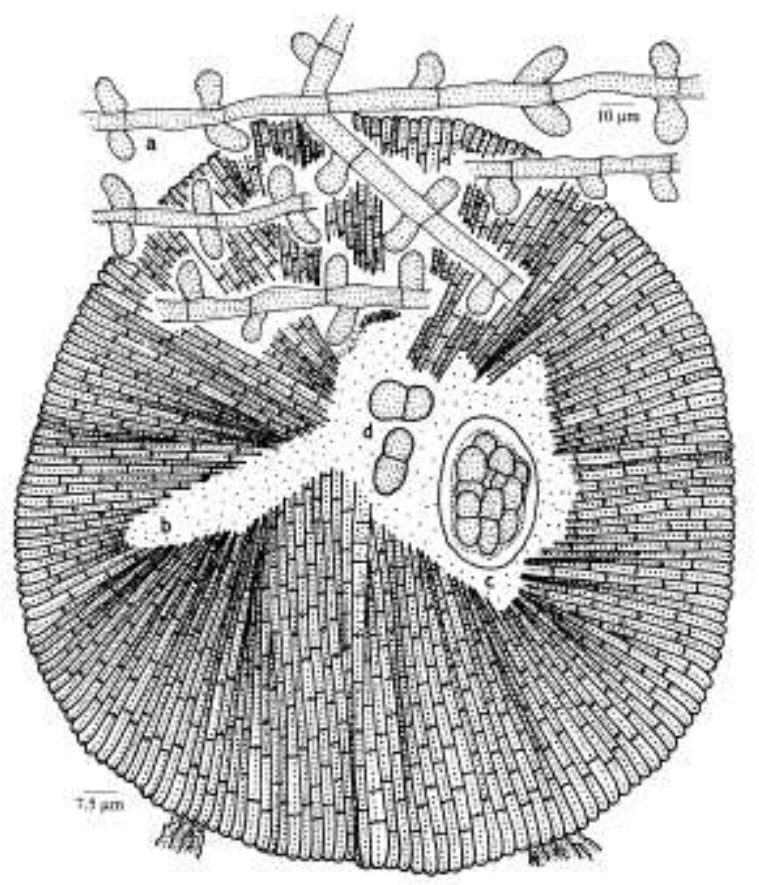

Figure 22 - Asterina aporusae

Appressoria opposite, subopposite, unilateral to rarely alternate, cylindrical, ovate, oblong, entire, rounded at the apex, 8-10 × 3-5 $\mu \mathrm{m}$. Thyriothecia scattered to grouped, orbicular, up to $160 \mu \mathrm{m}$ in diameter, crenate to slightly fimbriate at the margin, fringed hyphae flexuous and devoid of appressoria, 
thyriothecia initially longitudinally dehisced but later and frequently stellately dehisced at the centre; asci globose to ovate, octosporous, up to $30 \mu \mathrm{m}$ in diameter; ascospores brown, oblong, conglobate, 1-septate, constricted at the septum, $11-13 \times 4-5 \mu \mathrm{m}$, wall smooth.

Material examined: Kerala, Thiruvananthapuram, Kombe, on leaves of Aporusa lindleyana (Wight) Baill. (Euphorbiaceae), 9 March 1996, V.B. Hosagoudar HCIO 44117, TBGT 549; Thiruvananthapuram, Peppara Wildlife Sanctuary, 26 February 1997, V.B. Hosagoudar HCIO 44198, TBGT 584; Thiruvananthapuram, Palode, TBGRI Campus, 18 December, 2001, H. Biju HCIO 46072, TBGT 1835; Kollam, Shankily forest, 23 November, 2004, V.B. Hosagoudar HCIO 46174, TBGT 1586; Kollam, Shankily forest, Vengulla, 23 November 2004, V.B. Hosagoudar HCIO 46011, TBGT 1775; Kollam, Kulathupuzha, Cheenikala, 5 December 2005, Jacob Thomas HCIO 47448 (p. p.), TBGT 2480 (p. p.); Pathanamthitta, Erumely, 29 December, 2005, V. Girish kumar \& P.J. Robin HCIO 46741, TBGT 2082; Kottayam, Ranni Division, Ponthanpuzha reserve forest, 22 December, 2006, P.J. Robin \& M. Harish HCIO 48325, TBGT 3046; Thiruvananthapuram, Peppara Wildlife Sanctuary, 3 March, 2007, Jacob Thomas \& al. HCIO 48075, TBGT 2858; Thiruvananthapuram, Kombe, March 11, 1996, V.B. Hosagoudar HCIO 44207, TBGT 579; Thiruvananthapuram, Kombe, 20 February, 1997, V.B. Hosagoudar HCIO 44285, TBGT 703; Alapuzha,Vandanam, 3 October, 2002, A. Manoj Kumar HCIO 44652, TBGT 934; Thiruvananthapuram, TBGRI campus, 2 March, 2005, G. R. Archana \& V. L. Sheeja HCIO 45958, TBGT 1722; 8 November, 2000, V.B. Hosagoudar HCIO 46102, TBGT 1865; TBGRI campus, 26 June, 2008, A. Sabeena \& M.C. Riju TBGT 4883; 10 April, 2008, A. Chandraprabha \& al. TBGT 5550; Arboratum, 23 May, 2008, Anil Kumar TBGT 5636; Kottayam, Ponthanpuzha, 29 December 2005 V. Gireesh Kumar \& P.J. Robin HCIO 46755, TBGT 2096; 29 December, 2005, V. Gireesh Kumar \& P.J. Robin HCIO 46804, TBGT 2145; Thiruvananthapuram, Palode, Chippanchira, 4 October, 2005, V. S. Sreelal
HCIO 46978, TBGT 2195; Pathanamthitta, Ranni forest division, 27 December 2005, P.J. Robin \& V. Girish Kumar HCIO 47335, TBGT 2373; Erumeli, 29 December 2005, V Gireesh Kumar \& P.J. Robin HCIO 47345, TBGT 2383; HCIO 47347, TBGT 2385; 27 December 2005, P.J. Robin \& V. Girish Kumar HCIO 47358, TBGT 2396; Ponthanpuzha, 29 December 2005 P.J. Robin \& V. Girish Kumar HCIO 47367, TBGT 2405; Thiruvananthapuram, Peppara, 5 February 1997, V.B. Hosagoudar HCIO 47412, TBGT 2450; Mylamood, 27 September 2006, V.B. Hosagoudar \& al. HCIO 47415, TBGT 2453; Neyyar Wildlife Sanctuary, 22 August 2007, Jacob Thomas HCIO 48147, TBGT 2883; Neyyar dam site, 18 November2007, Jacob Thomas \& Vimalkumar HCIO 48219, TBGT 2957; Thiruvananthapuram, TBGRI Campus, 28 June 2007, A. Sabeena \& al. HCIO 49133, TBGT 3388; Arboretum, 20 May 2008, Anilkumar HCIO 49365, 49367; TBGT 3610, 3612; Palode, Mylamoodu, 6 March 2008, V.B. Hosagoudar \& al. HCIO 49580, TBGT 3622; HCIO 49589, TBGT 3831; Wayanad, Begur, 15 February 2009, Jacob Thomas \& al. HCIO 49219, TBGT 3458; Periya, 17 September 2008, M.Harish \& P.J. Robin HCIO 49633, TBGT 3875; Padinharathara, 4 November 2009, A.Sabeena \& M. C. Riju TBGT 4294; Kottayam, Ponthanpuzha, Plachery, 12 October 2007, P.J. Robin HCIO 49993, TBGT 4145; Kollam, Achankovil, Amarakkathodu, on leaves of Aporusa sp., 4 December 2008, V.B. Hosagoudar \& al. HCIO 49485, TBGT 3727; Kottayam, Ponthanpuzha, 2 March 2008, Robin $\&$ al. HCIO 49504, TBGT 3746; Thiruvananthapuram, Peppara, 19 March 2009, Jacob Thomas HCIO 49511, TBGT 3753; HCIO 49519, TBGT 3761; Palghat, Silent valley National Park, Malakappara, 20 August 2007, M. Harish \& al. HCIO 49763, TBGT 3915; Kottayam, Ponthanpuzha, 22 November 2009, Robin \& al. TBGT 4242; Karnataka, South Canara, Kukke Subramanya, 15 November 2003, V.B. Hosagoudar \& al. HCIO 46084, TBGT 1847; South Canara, Mangalore, Hosmata, 11 November 2001, H. S. Shenoy HCIO 44286, TBGT 696; Kodagu, Hoddur, Mandrane, on leaves of Aporusa bourdillonii Stapf., 5 November 2009, C. Jagath Thimmaiah TBGT 5402; 5434. 
This species is often associated with Meliolaster aporusae Hosag. et al. but can be distinguished based on the morphology of appressoria and ascospores.

Distribution: Karnataka, Kerala

Asterina aporusae Hansf. var. cylindrica Kar \& Ghosh, Indian Phytopath. 39: 216, 1986.

Patches are on both surfaces of the leaves but vigorous growth on upper surface, forming black, scattered or aggregated, orbicular to semi-orbicular, dense, patches up to $5.5 \mathrm{~mm}$ in diameter. Mycelium superficial and loosely reticulate, composed of dark brown, septate, much branched, straight to substraight hyphae, 3-6 $\mu \mathrm{m}$ wide (cells mostly 18-36 $\mu \mathrm{m}$ long), giving rise to alternate branches (rarely one-side and at an acute angles just behind the septa). Appressoria alternatively arranged (rarely one side), unicellular, cylindrical, round at the apex, entire, straight or slightly bent, brown,12-19 $\times 4-6 \mu \mathrm{m}$. Thyriothecia many, scattered or in groups, seated in the mycelial colony like pin heads, orbicular, $216-331 \mu \mathrm{m}$ in diameter, sometimes 2-3 fruit bodies coalesce with each other and take an irregular shape, attached with free mycelium, dimidiate with radiate scutellum, stellately dehisced at first, subsequently developing in to an aperture, dark brown, convex, hymenium simple, margin fimbriate; asci many, oval to clavate, sessile, rounded tip, 8-spored, 23-28 $\times 10-11 \mu \mathrm{m}$, paraphysate; ascospores biseriate, ellipticoblong, uniseptate, upper cell slightly larger than the lower, end conic, constricted at the septum, brown, smooth walled, 23-28 $\times 10-11$ $\mu \mathrm{m}$.

West Bengal, Jalpaiguri, Baikunthapur forest, on leaves of Aporusa roxburghii Ball. (Euphorbiaceae), 24 February 1974, IMI 215554 (holotype).

Distribution: West Bengal

Asterina araliae Patil \& Pawar, Indian Phytopath. 42: 248, 1989.
Colonies epiphyllous, up to $3 \mathrm{~mm}$ in diameter. Hyphae reticulately branched, cells $12-15 \times 4-5 \mu \mathrm{m}$. Appressoria alternate, two celled, 11-13 $\mu \mathrm{m}$ long; stalk cells cuneate, 1-2 $\mu \mathrm{m}$ long; head cells irregularly 2-3 times lobate, 9-12 × $3 \mu \mathrm{m}$. Thyriothecia scattered, $150-195 \mu \mathrm{m}$ in diameter, stellately dehisced at the center; asci globose to subglobose, octosporous, $28-31 \times 25 \mu \mathrm{m}$; ascospores brown, conglobate, 1-septate, constricted at the septum, $25-28 \times 9 \mu \mathrm{m}$, wall smooth.

Material examined: Maharashtra, Sindhudurg, Amboli, on leaves of Brassaiopsis actinophila F. Muell (Araliaceae), 4 January 1984, A. B. Pawar HCIO 39413 (holotype).

Distribution: Maharashtra

Asterina ardisiae Hansf., Reinwardtia 3: 129, 1954; Hosag., Chandraprabha \& Agarwal, Asterinales of Kerala, p.38, 2011 . (Fig. 23)

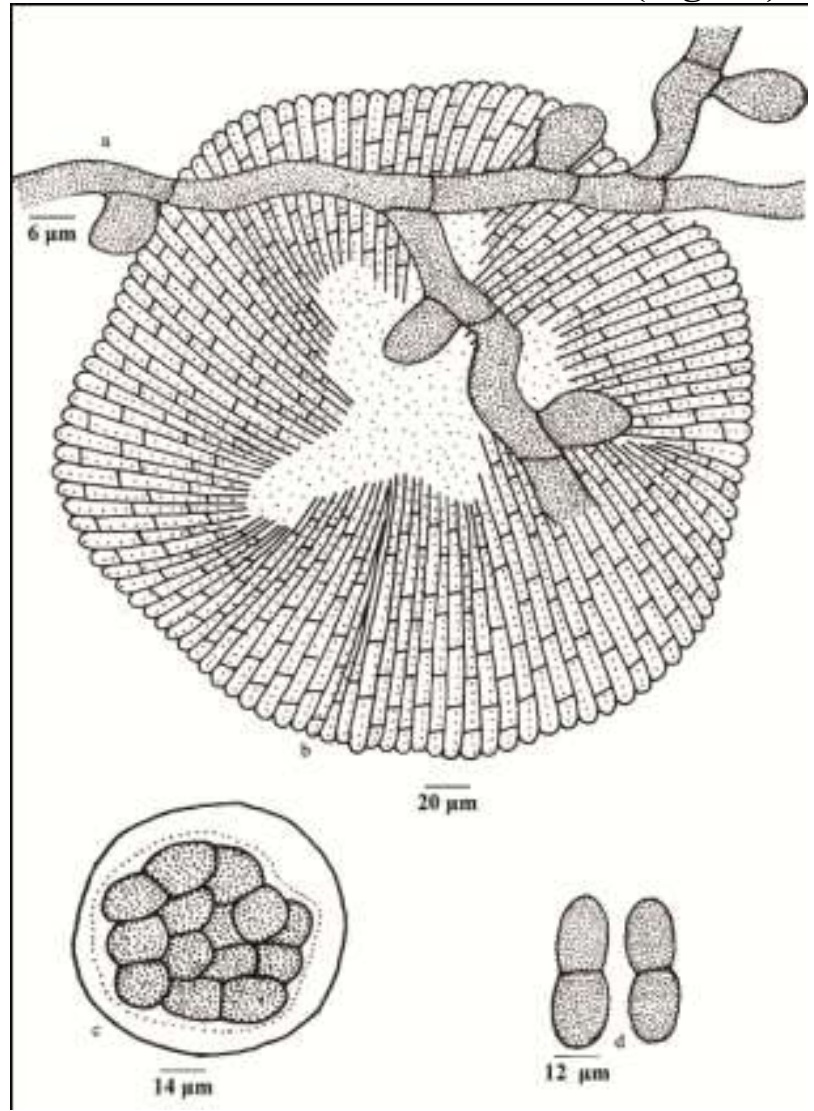

Figure 23 - Asterina ardisiae

Colonies epiphyllous, dense, crustose, up to $4 \mathrm{~mm}$ in diameter. Hyphae flexuous to crooked, branching irregular at acute to wide angles, loosely to closely reticulate, cells $15-24$ 
$\times$ 4-7 $\mu \mathrm{m}$. Appressoria unicellular, alternate to unilateral, scattered, ovate, globose, entire, 9-11 $\times 7-9 \mu \mathrm{m}$. Thyriothecia scattered to connate, orbicular, up to $200 \mu \mathrm{m}$ in diameter, stellately dehisced at the center, margin crenate to fimbriate; asci few, globose, octosporous, up to $35 \mu \mathrm{m}$ in diameter; ascospores conglobate, brown, uniseptate, slightly constricted at the septum, 20-25 × 10-13 $\mu \mathrm{m}$, wall smooth.

Material examined: Kerala, Idukki, Mannavan shola, on leaves of Ardisia solanacea Roxb. (Myrsinaceae), 23 September 1998, C.K. Biju HCIO 42962, TBGT 252.

This species was known on Ardisia colorata from Indonesia (Hansford, 1954).

Distribution: Kerala

Asterina ardisiicola Hosag. \& Chandra. ex Hosag. in Hosag., Chandra. \& Agarwal, Asterinales of Kerala, p.39, 2011.

Asterina ardisiicola Hosag. \& Chandra., Advances in Pollen Spore Research 27:156, 2009. (Fig. 24)

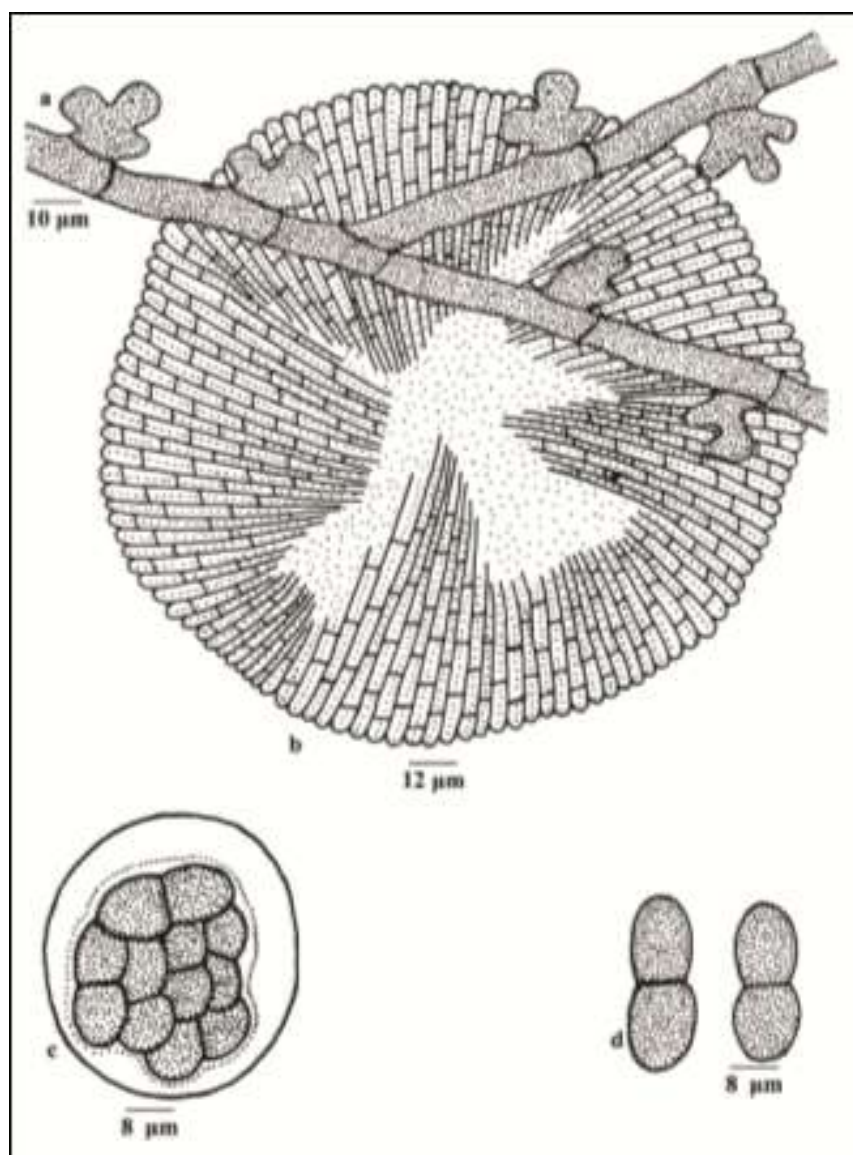

Figure 24 - Asterina ardisiicola
Colonies amphigenous, mostly epiphyllous, dense, crustose, up to $3 \mathrm{~mm}$ in diameter, confluent. Hyphae substraight, branching irregular at acute to wide angles, loosely reticulate, cells 11-24 $\times$ 4-7 $\mu \mathrm{m}$. Appressoria unicellular, alternate, unilateral, ovate, crenately to deeply lobate, broad based, 9-13 $\times$ 9-11 $\mu \mathrm{m}$. Thyriothecia scattered, orbicular, up to $110 \mu \mathrm{m}$ in diameter, stellately dehisced at the center, margin crenate; asci globose, octosporous, up to $30 \mu \mathrm{m}$ in diameter; ascospores conglobate, brown, uniseptate, constricted at the septum, 19-22 × 9-11 $\mu \mathrm{m}$, wall smooth.

Material examined: Kerala, Idukki, Eravikulam National Park, Munnar, on leaves of Ardisia sonchifolia Mez. (Myrsinaceae), 22 April 2008, A. Chandraprabha HCIO 48301 (holotype), TBGT 3022 (isotype).

Hosagoudar \& Chandraprabha (2009) proposed this species and was later validated by providing Latin (Hosagoudar et al., 2011).

Distribution: Kerala

Asterina arecacearum Hosag., Abraham \& C.K. Biju in Hosag., Abraham, C.K. Biju \& Hyde, Fungal Diversity 6: 70, 2001; Hosag., C.K. Biju \& Abraham, J. Mycopathol. Res. 40: 195, 2002; Hosag., Chandraprabha \& Agarwal, Asterinales of Kerala, p. 40, 2011.

(Figs 25-26)

Colonies amphigenous, thin to subdense, up to $10 \mathrm{~mm}$ in diameter, confluent. Hyphae substraight to crooked, branching irregularly at varying angles, loosely reticulate, cells 22-26 $\times$ 4-8 $\mu \mathrm{m}$. Appressoria few, scattered, unicellular, broad based, entire to sublobate, $9-15 \times 12-16 \mu \mathrm{m}$. Thyriothecia scattered, round to oval, up to $350 \mu \mathrm{m}$ in diameter, stellately dehisced at the center, margin crenate; asci saccate, bitunicate, 8spored, sessile, thickened at the apex, 70-100 $\times$ 50-75 $\mu \mathrm{m}$; ascospores ellipsoidal, brown, 2celled, strongly constricted at the septum, 36-49 $\times$ 14-19 $\mu \mathrm{m}$, wall smooth, surrounded by narrow mucilaginous sheath. 


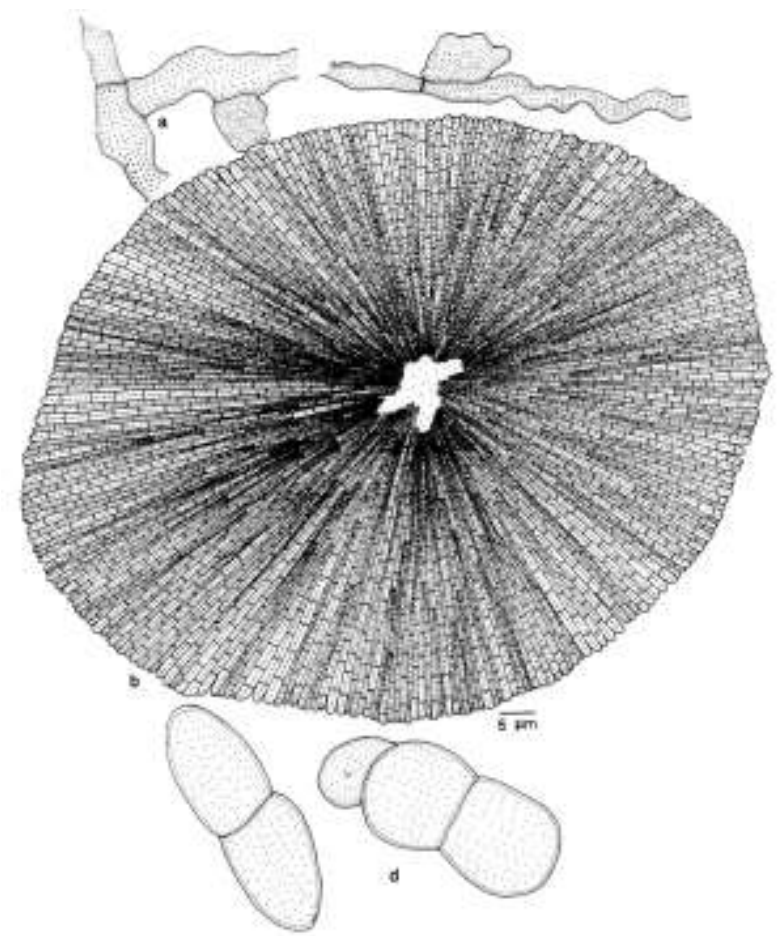

Figure 25 - Asterina arecacearum

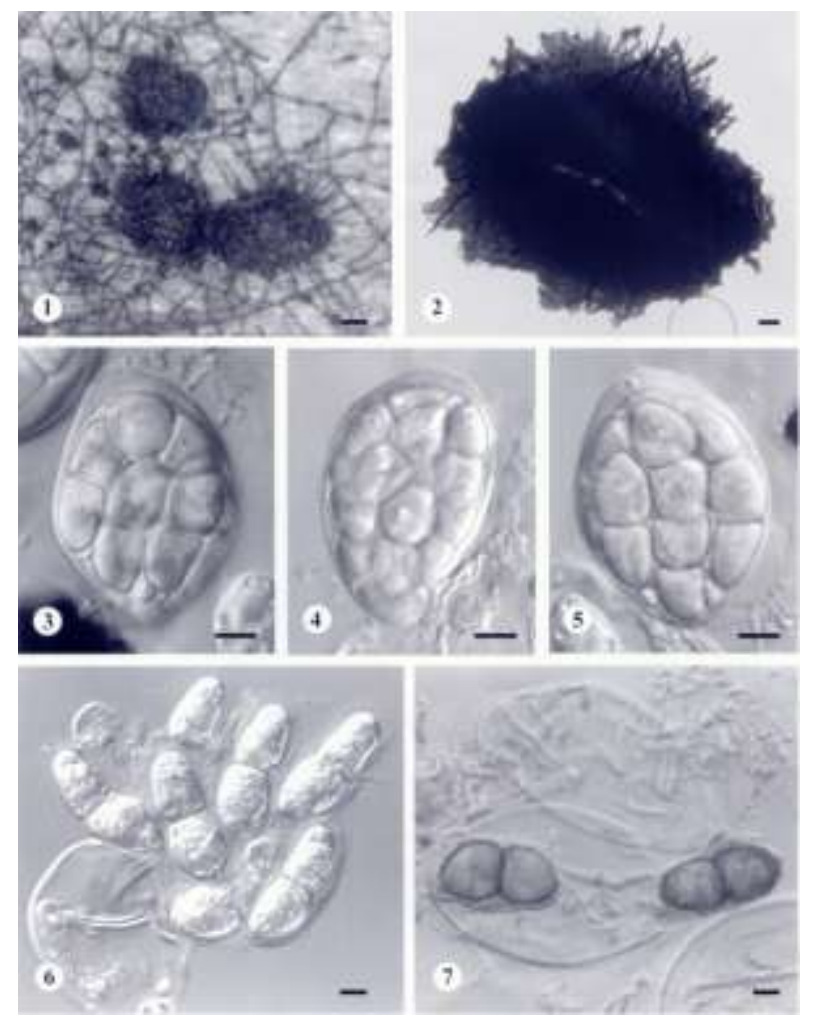

Figure 26 - Asterina arecacearum 1. Fungal colony, 2. Thyriothecium, 3-5. Asci, 6-7. Ascospores in ascus

Material examined: Kerala, Idukki, Munnar, on leaves of Calamus sp. (Arecaceae), 18 November 1998, C.K. Biju HCIO 43361 (holotype), TBGT 262 (isotype), $\mathrm{HKU}(\mathrm{M})$ 3353 (isotype).
Distribution: Kerala

Asterina argyreiae Hansf., Reinwardtia 3:130, 1954.

(Figs 27-28)

Colonies amphigenous, mostly epiphyllous, subdense, up to $1 \mathrm{~mm}$ in diameter. Hyphae substraight, flexuous to crooked, branching irregular at acute to wide angles, loosely reticulate to form a net, cells 18-27 × 3$5 \mu \mathrm{m}$. Appressoria alternate to unilateral, scattered, mostly perpendicular to the hyphae, globose, ovate, clavate, stipitate to broad based, lobate to deeply lobate, 5-7 $\times$ 5-8 $\mu \mathrm{m}$. Thyriothecia scattered to connate, orbicular, irregularly dehisced at the centre, up to $140 \mu \mathrm{m}$ in diameter, margin crenate to fimbriate, fringed hyphae small; asci many, globose, 8spored, 20-28 $\mu \mathrm{m}$ in diameter; ascospores conglobate, oblong to cylindrical,

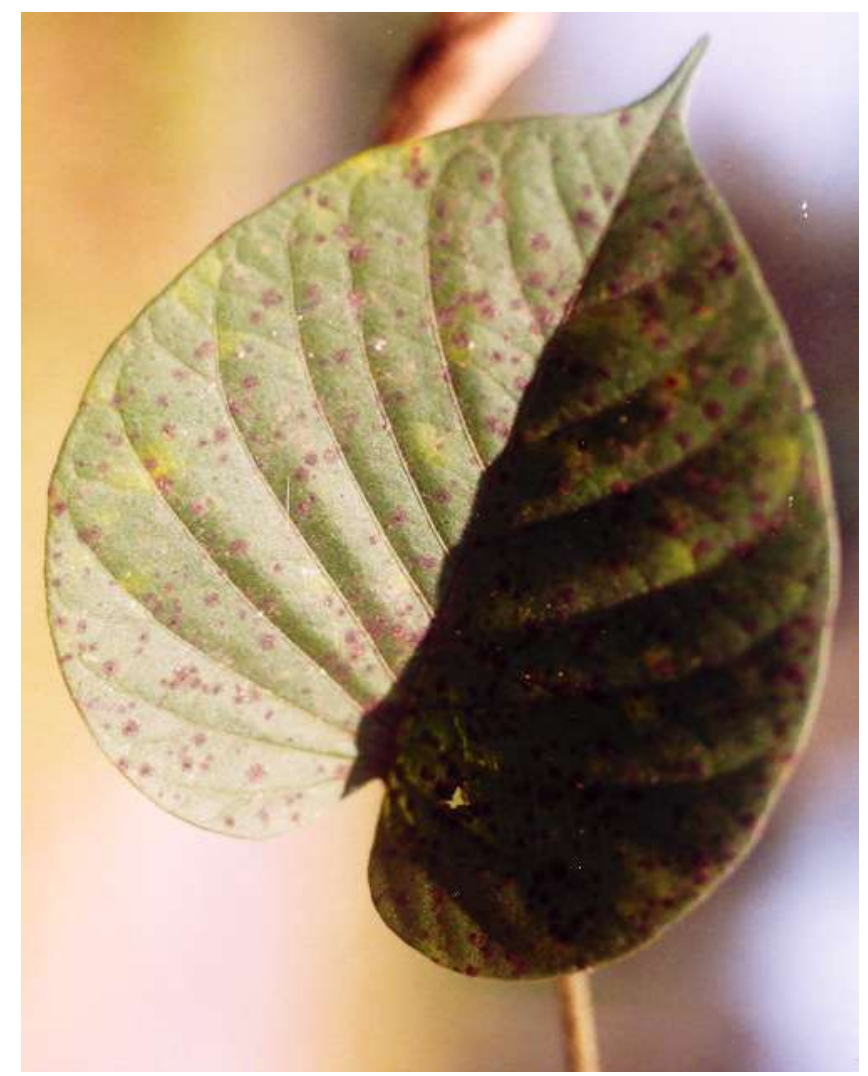

Figure 27 - Asterina argyreiae - Infected leaf

brown, uniseptate, constricted at the septum, $16-18 \times 6-8 \mu \mathrm{m}$.

\section{Material examined: Karnataka,} Kodagu, Hoddur, Tank Estate, on leaves of Argyreia sp. (Merremia sp.) (Convolvulaceae), 
25 November 2008, C. Jagath Thimmaiah TBGT 5705.

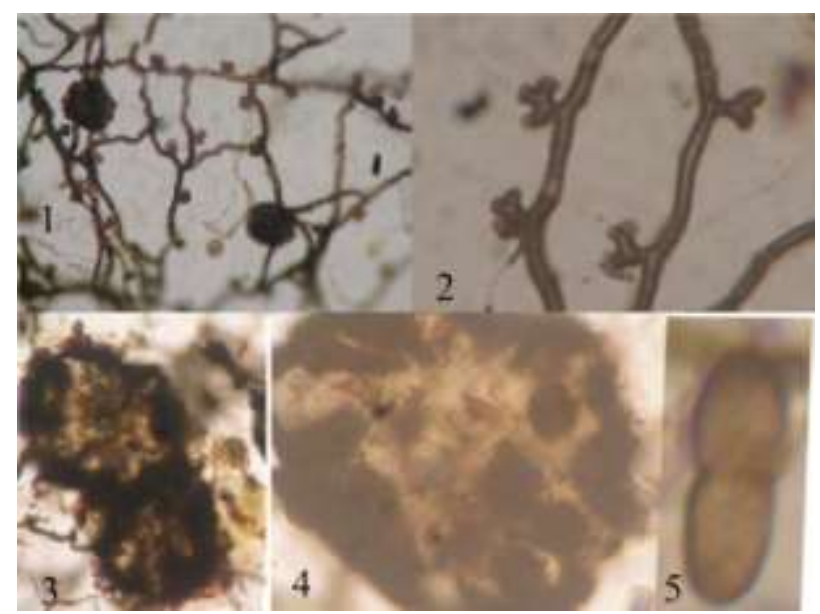

Figure 28 - Asterina argyreiae

1. Mycelium, 2. Appressoriate mycelium, 3.Thyriothecia, 4. Central portion of the thyriothecium dissolved by exposing the asci, 5 . Ascospore

This species was collected on Argyreia capitata from Java (Hansford, 1954).

Distribution: Karnataka

Asterina aristolochiae Hosag. \& JacobThomas, Taprobanica 3: 15, 2011.

(Figs 29-30)

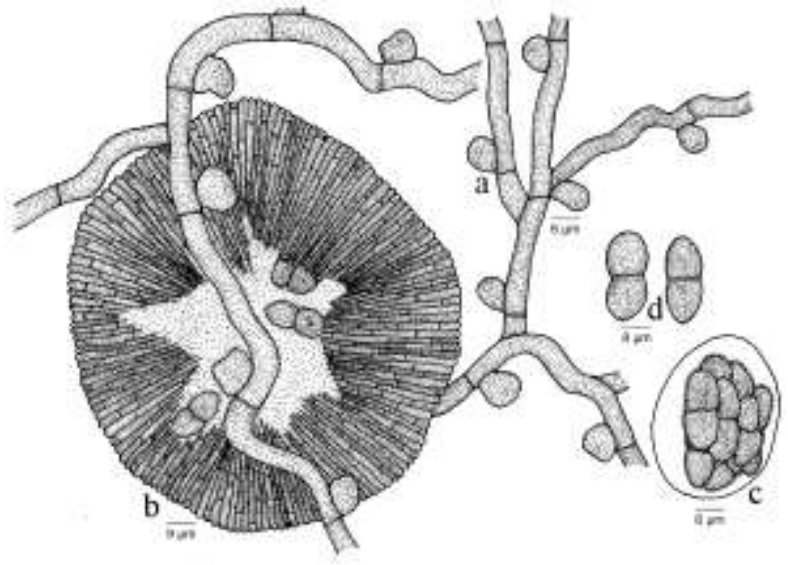

Figure 29- Asterina aristolochiae

Colonies amphigenous, mostly epiphyllous, thin, up to $3 \mathrm{~mm}$ in diameter, confluent and cover almost upper surface of the leaves. Hyphae pale brown, undulate, branching opposite to irregular at wide angles, loosely reticulate, cells 21-36 × 4-6 $\mu \mathrm{m}$. Appressoria alternate to unilateral, unicellular, ovate, subglobose, entire to sublobate, broad based, sessile, 4-12 × 7-12 $\mu \mathrm{m}$. Thyriothecia loosely scattered, orbicular, often connate, up to 100 $\mu \mathrm{m}$ in diameter, stellately dehisced at the centre, crenate to fimbriate at the margin, fringed hyphae flexuous; asci few to many, globose, octosporous, up to $43 \mu \mathrm{m}$ in diameter; ascospores oblong, conglobate, brown, uniseptate, constricted at the septum, 14-17 $\times 8$ $10 \mu \mathrm{m}$, wall echinulate.

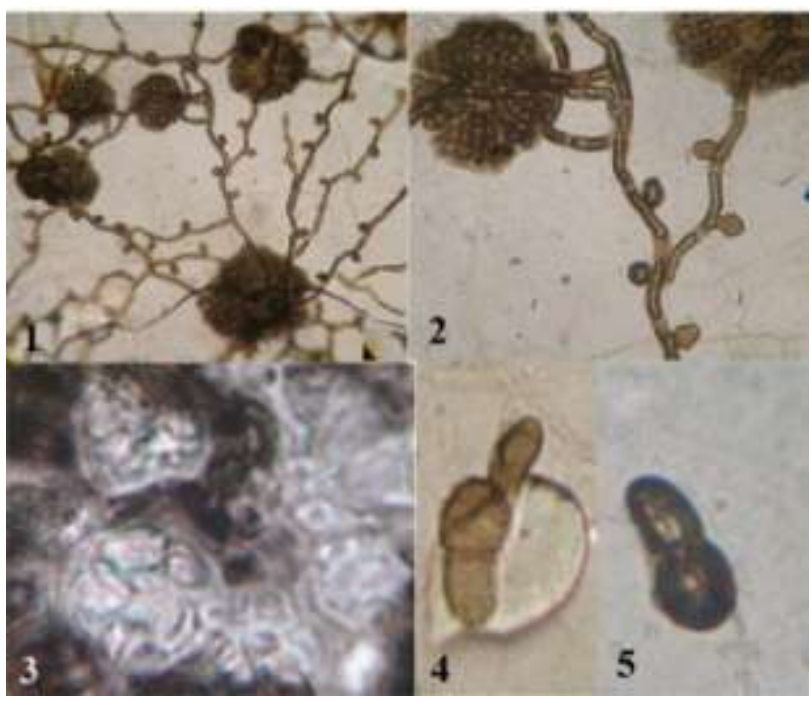

Figure 30 - Asterina aristolochiae

1. Mycelial colony with thyriothecia, 2. Appressoria, 3. Asci, 4-5. Ascospores

\section{Material examined: Kerala, Thiruvananthapuram, Peppara Wildlife Sanctuary, on leaves of Aristolochia tagala Cham. (Aristolochiaceae), 18 November 2007, Jacob Thomas \& Vimalkumar HCIO 48252 (holotype), TBGT 2991 (isotype).}

Asterina heterotropae Nakamura on Heterotropa hirsuticepala from Japan (Katumoto, 1975) and Asterina thotteae Hosag. \& Hanlin on Thottea spp. are known from India (Hosagoudar \& Hanlin, 1995). However, the present species differs from both in having unicellular appressoria.

\section{Distribution: Kerala}

Asterina asclepiadis Hosag. \& Goos, Mycotaxon 59: 152, 1996. (Fig. 31)

Colonies epiphyllous, subdense to dense, crustose, up to $3 \mathrm{~mm}$ in diameter, mostly 
confluent. Hyphae flexuous, branching opposite to irregular at acute to wide angles, loosely to closely reticulate, cells 15-19 × 3-4 $\mu \mathrm{m}$. Appressoria numerous, alternate, 2-celled, antrorse to retrorse, 12-16 $\mu \mathrm{m}$ long; stalk cells cylindrical, 3-10 $\mu \mathrm{m}$ long; head cells ovate, globose, entire to slightly angular, rounded to truncate at the apex, $6-10 \times 8-10 \mu \mathrm{m}$. Thyriothecia scattered, often 1-2 connate, orbicular, up to $124 \mu \mathrm{m}$ in diameter; margin crenate to fimbriate, dehisced stellately at the center; asci many, globose, 4-6 spored, 31-38 $\mu \mathrm{m}$ in diameter; ascospores conglobate, brown, 1-septate, deeply constricted at the septum, 18$22 \times 6-8 \mu \mathrm{m}$, wall smooth to tubercled at maturity.

Material examined: Tamil Nadu, Kanniyakumari, Veerapali Reserve forest, on leaves of Asclepias curassavica L. (Asclepiadaceae), 22 February 1994, V.B. Hosagoudar HCIO 41627 (holotype); Coimbatore, Valparai, Nirar dam, near Nooradi Settlement, 23 March 1990, V.B. Hosagoudar HCIO 30974.

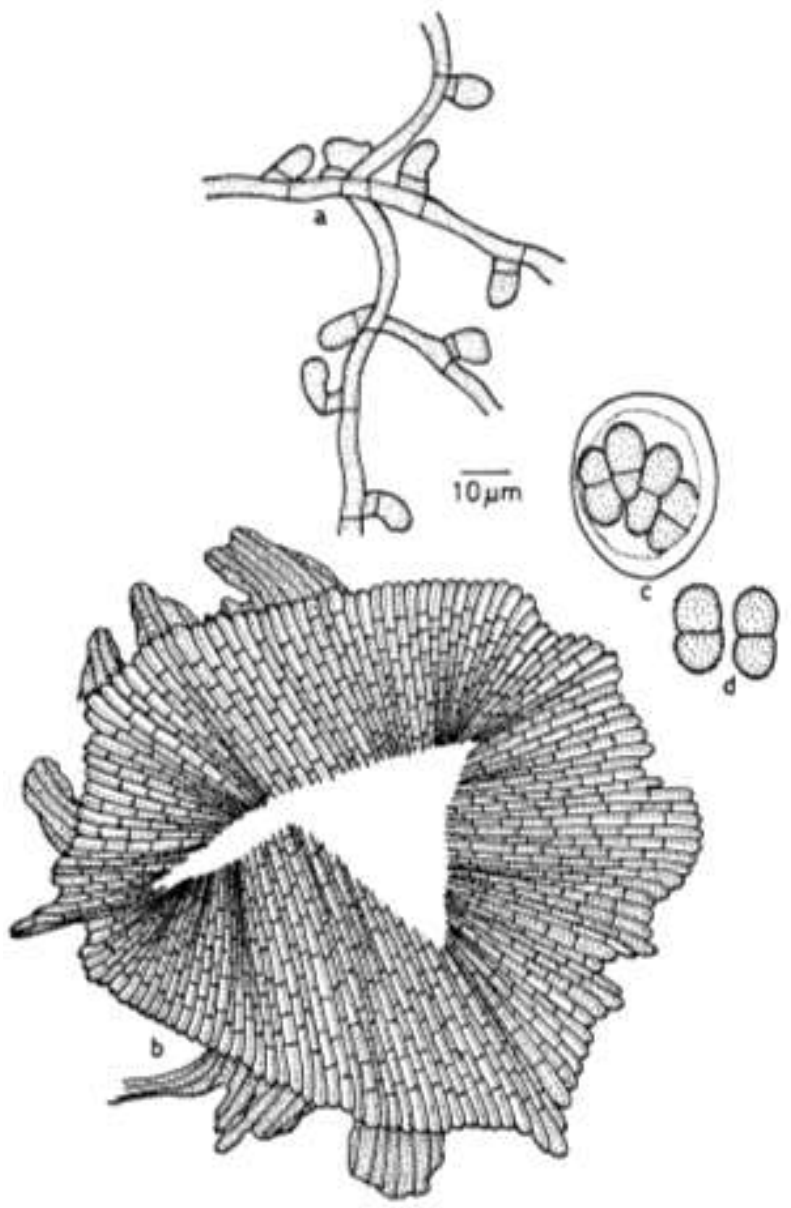

Figure 31 - Asterina asclepiadis
Numerous two celled appressoria distinguishes this species from other Asterina species known on the members of the family Asclepiadaceae.

Distribution: Tamil Nadu

Asterina atalantiae Hosag. \& Agarwal, Indian Phytopath. 56: 98, 2003; Hosag., Chandraprabha \& Agarwal, Asterinales of Kerala, p. 41, 2011. (Fig. 32)

Colonies hypophyllous, subdense, blackish brown, spreading, up to $10 \mathrm{~mm}$ in diameter, confluent. Hyphae straight to substraight, branching opposite, alternate to irregular at acute to wide angles, loosely to closely reticulate, cells $15-18 \times 4-5 \mu \mathrm{m}$. Appressoria alternate, unilateral to irregularly placed, unicellular, ovate, oblong, cylindrical, mostly entire, rarely truncate to slightly sublobate and often furcate, straight to curved, 9-13 $\times \quad 6-8 \mu \mathrm{m}$. Thyriothecia scattered, orbicular, up to $200 \mu \mathrm{m}$ in diameter, stellately dehisced at the centre, dehiscence extended up to margin, margin crenate to fimbriate, fringed hyphae very small; asci globose, octosporous, up to $30 \mu \mathrm{m}$ in diameter; ascospores oblong, brown, conglobate, uniseptate, constricted at the septum, 23-26 $\times 11-12 \mu \mathrm{m}$, wall smooth.

Material examined: Kerala, Thiruvananthapuram, Pandipathu, on leaves of Atalantia rotundifolia (Thw.) Tanaka (Rutaceae), 11 March 1997, V.B. Hosagoudar HCIO 44116 (holotype), TBGT 558 (isotype); A. wightii Tanaka, 21 March 2001, G. Rajkumar HCIO 45128, TBGT 1183; Palghat, Silent Valley National Park, on leaves of Atalantia sp., 27 February 2009, S.S. Shaji \& al. HCIO 49560, TBGT 3802; Pathanamthitta, Erumeli, 29 March 2009, Robin \& al. HCIO 49848, TBGT 4000.

This species is close to Asterina toddaliae Kar \& Ghosh known on Toddalia aculeata from Darjeeling, having unicellular appressoria. However, A. atalantiae differs from it in having only hypophyllous, blackish brown and spreading colonies; appressoria bent and never formed hook-like and ascospores are smaller. 


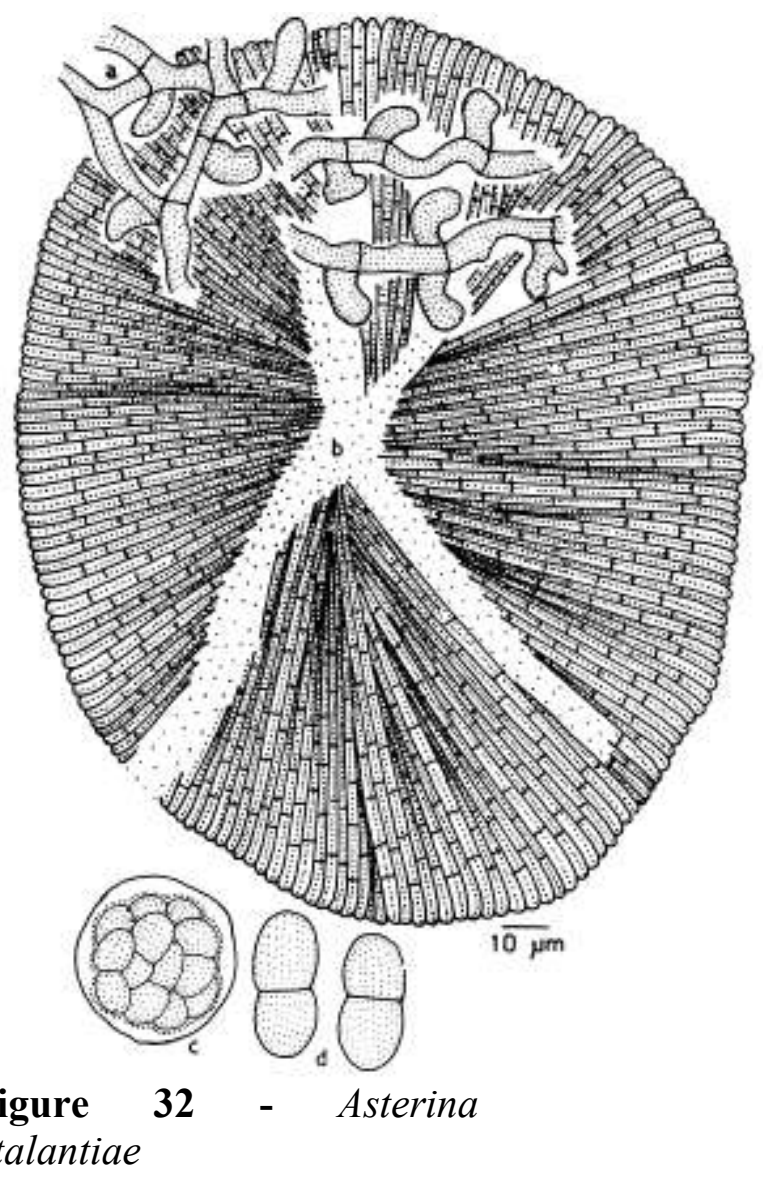

Distribution: Kerala

Asterina averrhoae Hosag., Kamar. \& K. P. Babu, Indian Phytopath. 55: 497, 2002; Hosag., Chandraprabha \& Agarwal, Asterinales of Kerala, p.43, 2011.

(Fig. 33)

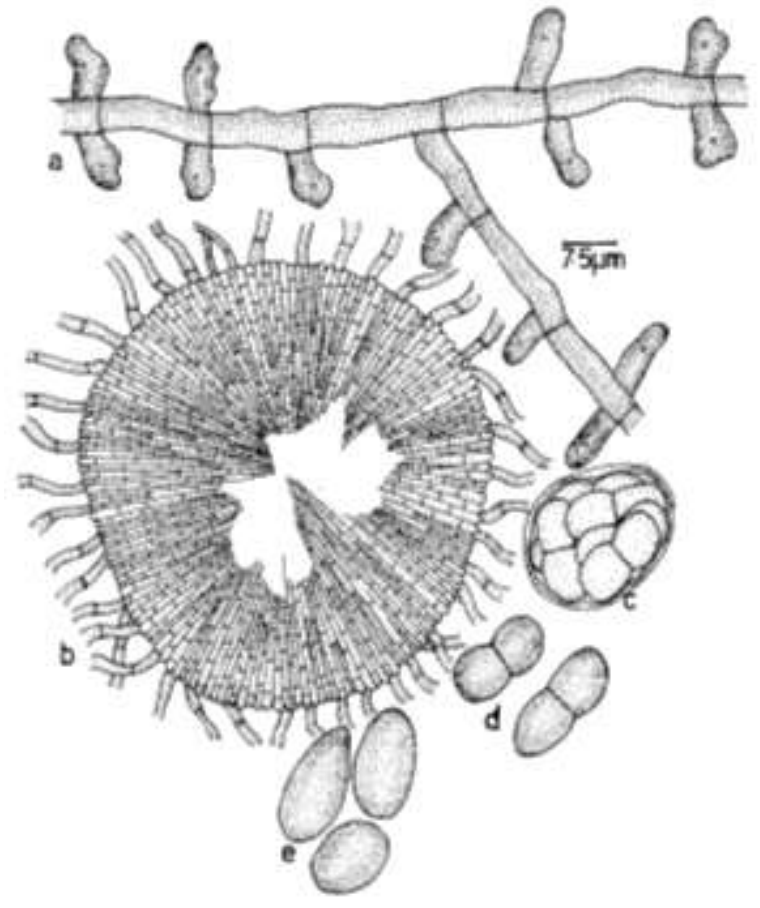

Figure 33 - Asterina averrhoae
Colonies mostly epiphyllous, dense, up to $3 \mathrm{~mm}$ in diameter, confluent. Hyphae straight to flexuous, branching opposite, alternate to irregular at acute angles, loosely reticulate, cells 11-16 × 4-6 $\mu \mathrm{m}$. Appressoria unicellular, alternate, about $30 \%$ opposite, ovate, oblong to rarely globose, entire, angular, sublobate to rarely furcate, $8-14 \times 4-6 \mu \mathrm{m}$. Thyriothecia few, scattered to connate, orbicular, up to 100 $\mu \mathrm{m}$ in diameter, margin crenate to fimbriate, fringed hyphae flexuous, stellately dehisced at the centre; asci few, globose, up to $30 \mu \mathrm{m}$ in diameter; ascospores brown, oblong, conglobate, 1-septate, constricted at the septum, $17-20 \times 8-10 \mu \mathrm{m}$, wall smooth to minutely echinulate. Pycnothyria numerous, similar to thyriothecia, smaller; pycnothyriospores oval, oblong, pyriform, 16-21 × 8-12 $\mu \mathrm{m}$, wall smooth.

Material examined: Kerala, Thiruvananthapuram, Palode, TBGRI Campus, on leaves of Averrhoa carambola L. (Averrhoaceae), 14 December 2000, K. M. Kamarudeen \& K. P. Babu HCIO 43713 (holotype), TBGT 367 (isotype).

This is the only species of the genus Asterina on the members of the family Averrhoaceae (Hosagoudar \& Abraham, 2000).

\section{Distribution: Kerala}

Asterina balakrishnanii Hosag. in Hosag., Balakr. \& Goos, Mycotaxon 59: 168, 1996; Hosag., Chandraprabha \& Agarwal, Asterinales of Kerala, p.44, 2011.

(Fig. 34)

Colonies epiphyllous, dense, crustose, up to $2 \mathrm{~mm}$ in diameter. Hyphae strongly appressed to the leaf surface, crooked, branching opposite to irregular at acute angles, loosely reticulate, cells 9-13 × 3-4 $\mu \mathrm{m}$. Appressoria alternate, sessile, deep brown, globose and angularly pointed towards the apex, 7-11 × 4-7 $\mu \mathrm{m}$. Thyriothecia closely scattered, frequently connate, orbicular to ovate, up to $120 \mu \mathrm{m}$ in diameter, dehisced stellately at the center, dehiscence extended up to margin, margin crenate; asci many, eight spored, ovate to globose, $30-32 \times 24-26 \mu \mathrm{m}$; 
ascospores conglobate, brown, 1-septate, slightly constricted at the septum, 18-22 ×9-10 $\mu \mathrm{m}$.

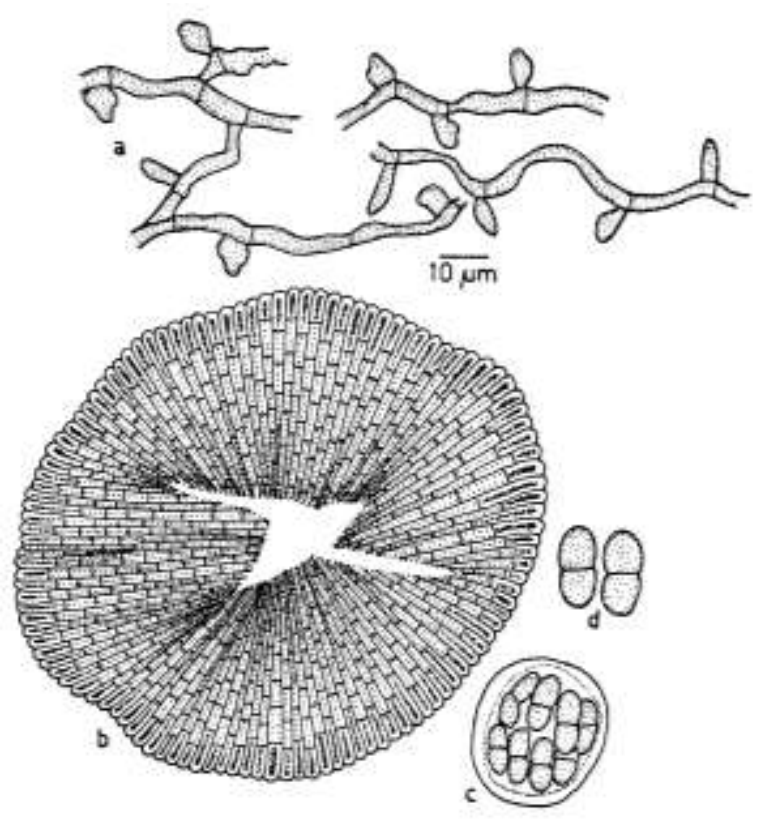

Figure 34- Asterina balakrishnanii

Material examined: Kerala, Idukki, Pamba, on leaves of Solanum torvum Sw. (Solanaceae), 10 October 1983; V.B. Hosagoudar HCIO 30953 (holotype).

This species is close to Asterina portoricensis Ryan known on Solanum sp. from Porto Rico (Ryan, 1924) but differs from it in having crooked mycelia and globose to angularly pointed appressoria.

Distribution: Kerala

Asterina balii Sydow, Ann. Mycol. 19: 308, 1921.

Colonies epiphyllous, up to $4 \mathrm{~mm}$ in diameter. Hyphae undulate, branched, closely reticulate, cells 3-4 $\mu \mathrm{m}$ broad. Appressoria scattered, unicellular, variously lobate, 6-8 $\times 8$ $10 \mu \mathrm{m}$. Thyriothecia closely scattered, rounded, orbicular, up to $130 \mu \mathrm{m}$ in diameter, margin fimbriate, fringed hyphae flexuous; asci globose to ovate-globose, octosporous, 30-40 $\times$ 8-10 $\mu \mathrm{m}$; ascospores conglobate, ellipsoidal, broadly rounded at both ends, 1 -septate, slightly constricted at the septum, $18-22 \times 8-10 \mu \mathrm{m}$.
Material examined: Orissa, Bhuvaneshwar, on leaves of Alangium lamarckii Thaites (Alangiaceae), October 1920, S.N. Bal.

\section{Distribution: Orissa}

Asterina banguiensis Yates, Philippine J. Sci. 13: 372, 1918; Kaul \& Nair, Acta Bot. Indica 16: 227, 1988.

Colonies epiphyllous, dense, sparse, confluent, orbicular to irregular, brown in colour. Mycelium irregular, reticulately branched and undulate, articulate, measuring 4$5 \mu \mathrm{m}$ wide, branching opposite. Appressoria sessile, alternate, hemispherical, one celled, and measure 10-12 $\mu \mathrm{m}$ long and $5 \mu \mathrm{m}$ wide. Thyriothecia flat, radiate, dense, stellately dehisced and measure $180-200 \mu \mathrm{m}$ in diameter; asci ovate, measure $35-40 \times 28-30 \mu \mathrm{m}$, aparaphysate; ascospores uniseptate with two cells of equal size, rounded at the ends, $20-28 \times$ 8-10 $\mu \mathrm{m}$.

Material examined: Tamil Nadu, Ootacamund, on leaves of Glycosmis pentaphylla (Retz.) DC. (Rutaceae), February 1977, L. N. Nair.

I had prepared the slide from the above said material but could not locate Asterina colonies. The identity of this material is to be confirmed by its subsequent collections.

Distribution: Tamil Nadu

Asterina betonicae Hosag. \& Goos, Mycotaxon 59: 153, 1996; Hosag., Chandraprabha \& Agarwal, Asterinales of Kerala, p.46, 2011 .

(Fig. 35)

Colonies epiphyllous, thin to subdense, up to $2 \mathrm{~mm}$ in diameter, rarely confluent. Hyphae straight to substraight, branching opposite to irregular at acute to wide angles, loosely reticulate, cells $12-25 \times 5-7 \mu \mathrm{m}$. Appressoria alternate, about $30 \%$ opposite, unicellular, ovate, mammiform, seated on broad base, sessile, entire, 6-10 × 4-6 $\mu \mathrm{m}$. Thyriothecia loosely scattered, orbicular, up to $220 \mu \mathrm{m}$ in diameter, margin crenate to fimbriate, fringed hyphae flexuous, dehisced 
stellately at the centre; asci many, octosporous, globose, 31-35 $\mu \mathrm{m}$ in diameter; ascospores brown, conglobate, 1 -septate, 15-19 × 9-10 $\mu \mathrm{m}$, wall smooth.

Material examined: Tamil Nadu, Tirunelveli, Kakachi forest, on leaves of Justicia betonica L. (Acanthaceae), 25 February 1994, V.B. Hosagoudar HCIO 41556 (holotype); Kerala, Wayanad, Pakshipathalam, 1 November 2007, A. Chandraprabha HCIO 48237, TBGT 2975.

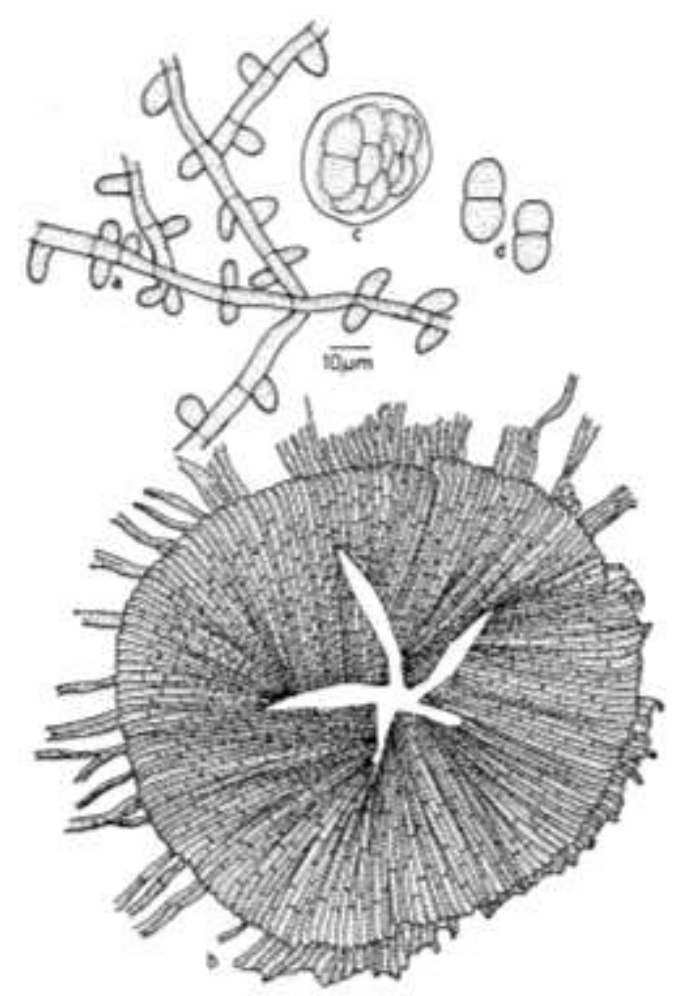

Figure 35 - Asterina betonicae

Alternate, opposite and entire head cells of the appressoria distinguishes this species from the other Asterina species reported on the members of the family Acanthaceae.

Distribution: Kerala, Tamil Nadu

Asterina blumeae Kar \& Ghosh, Indian Phytopath. 39: 214, 1986.

Colonies amphigenous, mostly epiphyllous, thin, up to $5 \mathrm{~mm}$ in diameter, confluent. Hyphae undulate, branching irregular to alternate at wide angles, loosely reticulate, cells $15-57 \times 3-7 \mu \mathrm{m}$. Appressoria alternate to unilateral, unicellular, straight to curved, deeply lobate, $5-10 \times 9-12 \mu \mathrm{m}$. Thyriothecia orbicular, up to $172 \mu \mathrm{m}$ in diameter, develop an aperture at the centre, margin subcrenate; asci many, round, 8-spored, up to $36 \mu \mathrm{m}$ in diameter; ascospores brown, fusiform-elliptic, uniseptate, constricted at the septum, rounded at both ends, $16-20 \times 7-9 \mu \mathrm{m}$, wall smooth.

Material examined: West Bengal, Darjeelling, Mirik, on leaves of Blumea sp. (Asteraceae), 26 November 1977, IMI 225670 (holotype).

Distribution: West Bengal

Asterina bottomleyae Doidge, Bothalia 4: 333, 1942; Hosag., Balakr. \& Goos, Mycotaxon 60: 170, $1996 . \quad$ (Fig. 36)

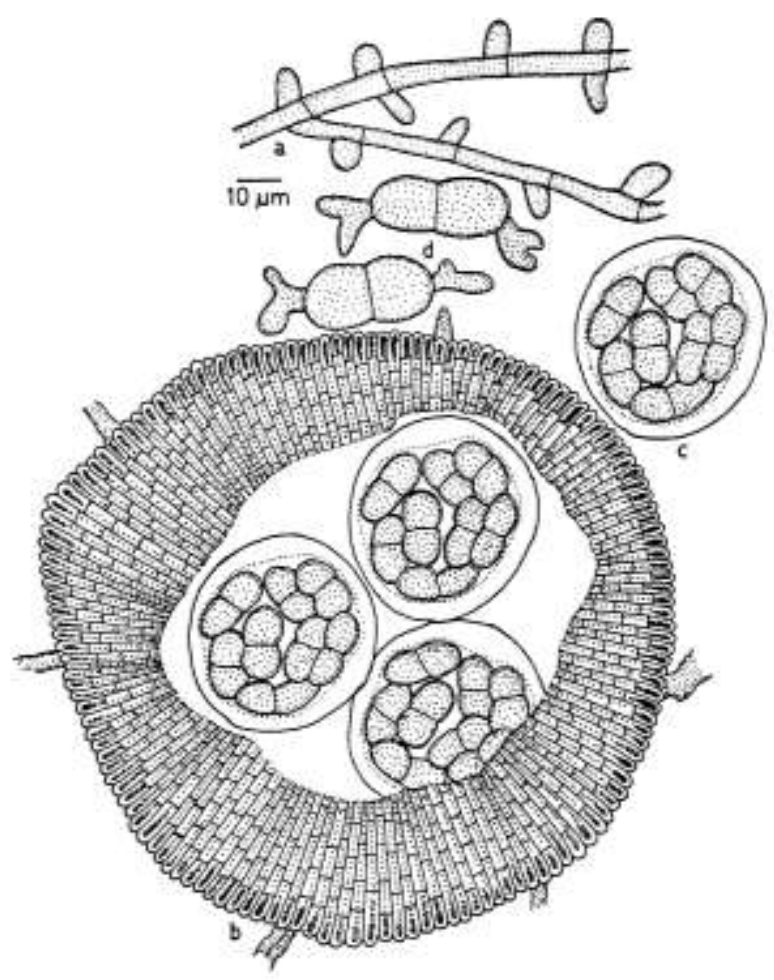

Figure 36- Asterina bottomleyae

Colonies foliicolous, amphigenous, mostly epiphyllous, thin to dense, up to $2 \mathrm{~mm}$ in diameter, confluent. Hyphae straight, branching opposite at acute angles, loosely reticulate, cells $12-22 \times 6-8 \mu \mathrm{m}$. Appressoria alternate and opposite (ca. 50\%), unicellular, conoid, broadly rounded at the apex, mostly straight and perpendicular to the bearing hyphae, 9-13 × 6-10 $\mu \mathrm{m}$. Thyriothecia scattered to loosely grouped, often connate, circular in outline, up to $180 \mu \mathrm{m}$ in diameter, the central 
portion dissolved and formed a wide opening, margin fimbriate, fringed hyphae tortuous and devoid of appressoria; asci globose to slightly ovate, eight spored, 31-53 $\mu \mathrm{m}$ in diameter; ascospores deep brown, conglobate, 1-septate, deeply constricted at the septum, lower cell slightly larger, 31-33 × 18-19 $\mu \mathrm{m}$, wall smooth.

Material examined: Tamil Nadu, Madurai, Kodaikanal, on leaves of Ilex walkeri Wight \& Gard. ex Thw. (Aquifoliaceae), 23 November 1991, K. Ravikumar HCIO 30954; Kodaikanal, Kuthuraiyar, on leaves of Ilex wightiana Wallich ex Wight, 4 August 2007, R. Nithyatharani TBGT 4412; 20 November 2007, R. Nithyatharani TBGT 4727; Periyakanal, 2 October 2008, V.B. Hosagoudar \& al. TBGT 4717.

Distribution: Tamil Nadu

Asterina cannonii Hosag. \& C.K. Biju, Indian Phytopath. 58: 194, 2005; Hosag., Chandraprabha \& Agarwal, Asterinales of Kerala, p. 46, 2011.

(Fig. 37)

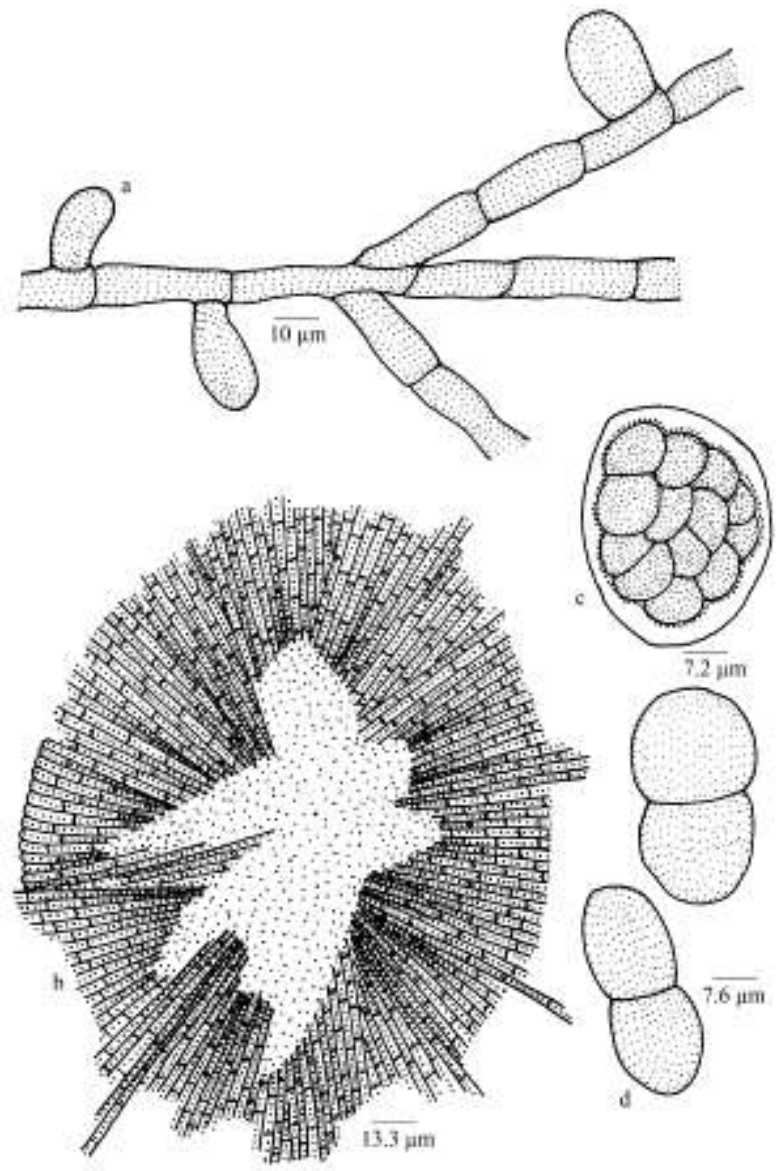

Figure 37 - Asterina cannonii
Colonies amphigenous, subdense, up to $3 \mathrm{~mm}$ in diameter, confluent. Hyphae straight to substraight, branching alternate to opposite at acute to wide angles, loosely to closely reticulate, cells 30-40 × 5-7 $\mu \mathrm{m}$. Appressoria alternate, unilateral, unicellular, oblong, ovate, clavate, cylindrical, entire, 19-24 × 8-10 $\mu \mathrm{m}$. Thyriothecia scattered, orbicular, up to $200 \mu \mathrm{m}$ in diameter, margin fimbriate, fringed hyphae small, stellately dehisced at the centre; asci few, globose, octosporous, up to $40 \mu \mathrm{m}$ in diameter; ascospores oblong, conglobate, brown, uniseptate, constricted at the septum, 35-38 $\times$ 16-18 $\mu \mathrm{m}$, wall smooth.

Material examined: Kerala, Idukki, Mannavan Shola, on leaves of Eurya japonica Thunb. (Theaceae), 16 September 1999, C.K. Biju HCIO 45143 (holotype), TBGT 1198 (isotype); on leaves of Eurya sp., 22 April 2008, A. Chandra Prabha HCIO 48823, TBGT 3199; 14 April 1997, C.K. Biju HCIO 47446, TBGT 2484.

Asterina theacearum Hosag. et al. known on this host from the same locality (Hosagoudar et al. 1997). A. cannonii differs from it in having robust hyphae, larger appressoria and ascospores. Colonies of $A$. theacearum were also present in the present collection.

Distribution: Kerala

Asterina cansjerae Ryan, Mem. Dept. Agric. India 15: 103, 1928.

(Fig. 38)

Colonies amphigenous, dense, up to 2 $\mathrm{mm}$ in diameter, confluent. Hyphae flexuous, branching irregular at acute to wide angles, loosely reticulate, $22-28 \times 3-5 \mu \mathrm{m}$. Appressoria alternate, scattered, sessile to stipitate, unicellular, slightly and mostly bilobate, 7-10 $\times$ 5-7 $\mu \mathrm{m}$. Thyriothecia scattered, orbicular, up to $160 \mu \mathrm{m}$ in diameter, stellately dehisced at the centre, margine margin distinctly radiate and crenate to fimbriate, fringed hyphae numerous; asci ovate to globose, up to $32 \mu \mathrm{m}$ in diameter; ascospores oblong to cylindrical, conglobate, uniseptate, constricted at the septum, 18-22 × 8$10 \mu \mathrm{m}$, wall smooth. Pycnothyria numerous, similar to thyriothecia, smaller; 
pycnothyriospores brown, pyriform, rounded at one end and attenuated at the other, $18-22 \times 8$ $10 \mu \mathrm{m}$.

Material examined: Karnataka, Dharwad, on leaves of Cansjera rheedi Gmel. (Opiliaceae), November 1918, L. S. Sedgwick HCIO 1966 (holotype).

Distribution: Karnataka

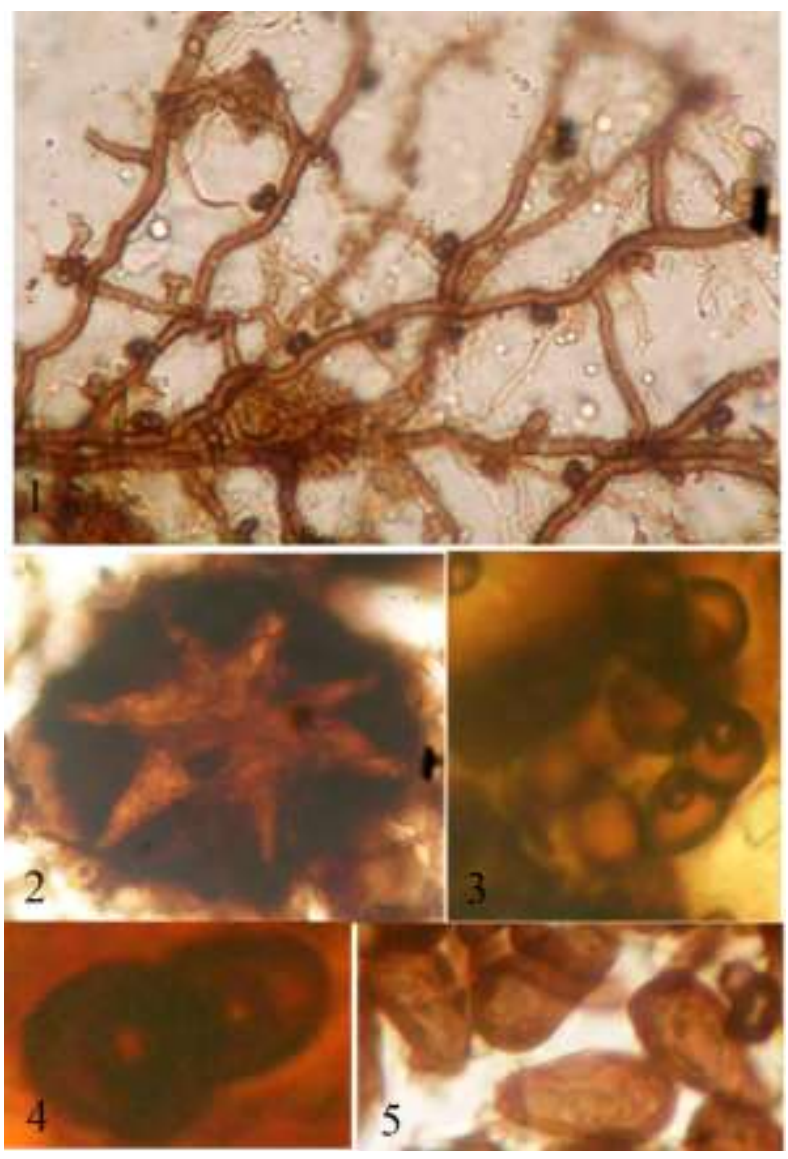

Figure 38 - Asterina cansjerae

1. Appressoriate branched mycelium, 2.Dehisced Thyriothecium, 3. Ascus, 4. Ascospore, 5. Pycnothyriospores

This species is known only from the present collection.

Distribution: Karnataka

Asterina cansjericola Hansf. \& Thirum., Farlowia 3: 304, 1948.

Colonies amphigenous, black, numerous, rather dense, rounded, usually discrete, 3-4 mm in diameter, smooth. Mycelium substraight to slightly sinuous, dark brown, branching opposite to irregular at acute to wide angles, loosely reticulate cells $25-40 \times 5-7 \mu \mathrm{m}$. Appressoria alternate or unilateral, in some colonies up to $5 \%$ opposite, conoid, usually slightly bend, dark brown, 12-16 × 6-8 $\mu \mathrm{m}$ thick at the base, slightly attenuate to the rounded apex, 4-5 $\mu \mathrm{m}$ thick; Thyriothecia densely scattered, numerous, circular convex black, up to $160 \mu \mathrm{m}$ diameter, or 2-3 connate into irregular compound ascomata; lower wall indistinct, hyaline, subradiate; upper wall subopaque, dark brown, radiate, hyphae 4-5 $\mu \mathrm{m}$ thick with cells 5-10 $\mu \mathrm{m}$ long; dehiscence by stellately fissures and the central parts falling away; margin usually stellately fimbriate, the fringing hyphae paler than the mycelium, tortuous-radiating, exappressoriate, up to $40 \mu \mathrm{m}$ long; asci few, 4-6, ripening in succession, globose to ellipsoid, 8-spored, aparaphysate, up to $50 \mu \mathrm{m}$ high by $40-45 \mu \mathrm{m}$ diameter; ascospores conglobate, dark brown, oblong with rounded ends, 1-septate, deeply constricted at the septum, $28-34 \times 14-17 \mu \mathrm{m}$, exospores finely, and closely echinulate. Pycnothyria similar to the thyriothecia; pycnothyriospores pyriform, dark brown, smooth, about $27 \times 18 \mu \mathrm{m}$, without hyaline band or prominent germ pores.

Material examined: Karnataka, Nandi hills, on leaves of Cansjera rheedii Gmel. (Opiliaceae), 18 November 1944, M. J. Thirumalachar

This species is known only from the description.

Distribution: Karnataka

Asterina cansjericola Hansf. \& Thirum. var. indica Hosag., Balakr. \& Goos, Mycotaxon 59: 170, 1996.

(Fig. 39)

Colonies amphigenous, predominantly epiphyllous, dense, crustose, up to $3 \mathrm{~mm}$ in diameter, confluent. Hyphae straight, cross hyphae rarely and slightly crooked, branching opposite to irregular at acute angles, loosely reticulate, cells $12-15 \times 3-5 \mu \mathrm{m}$. Appressoria predominantly alternate, about $15 \%$ opposite, unicellular, ovate to cylindrical with broadly rounded apices, 9-13 $\times 6-8 \mu \mathrm{m}$. Thyriothecia 
loosely grouped, circular to ovate, up to 190 $\mu \mathrm{m}$ in diameter, dehisce stellately at the center, later with a wide opening, margin crenate to fimbriate, fringed hyphae straight to flexuous; asci many, globose to slightly ovate, eight spored, $30-37 \times 24-31 \mu \mathrm{m}$; ascospores conglobate, dark-brown, 1-septate, strongly constricted at the septum, lower cell slightly larger, 24-26 × 12-13 $\mu \mathrm{m}$, wall smooth.

Material examined: Tamil Nadu, Nilgiris, Kotagiri, Jammunarai, on leaves of Cansjera rheedii Gmel. (Opiliaceae), 18 February 1991, V.B. Hosagoudar HCIO 30955; Kerala, Kannur, Mangattuparamba, Neeliyar Kottam, 21 January 2011, M. Reshma TBGT 4900; 14 February 2011, M. Reshma TBGT 5187; 26 December 2010, M. Reshma TBGT 5196.

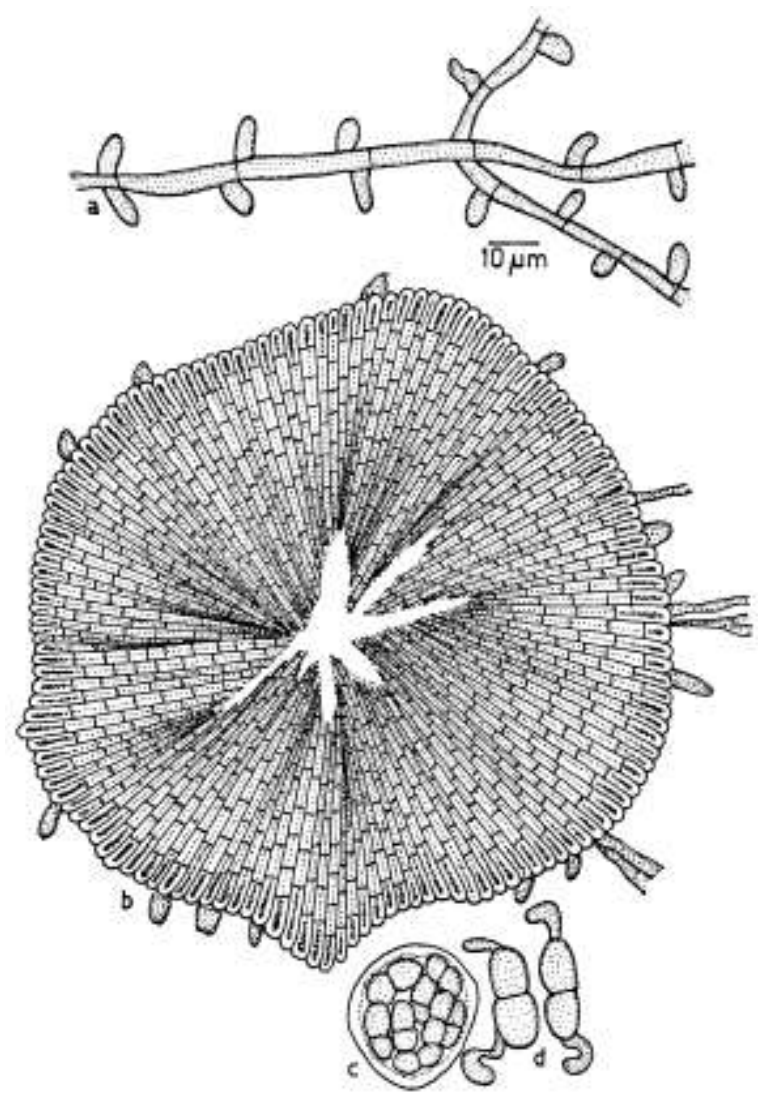

Figure 39 - Asterina cansjericola var. indica

This variety differs from the species in having smaller appressoria and ascospores.

This collection was associated with Meliola sp.

Distribution: Tamil Nadu
Asterina canthiigena Hosag., Archana \& Agarwal, Indian Phytopath. 60: 345, 2007.

(Fig. 40)

Colonies amphigenous, thin, up to $2 \mathrm{~mm}$ in diameter, confluent. Hyphae substraight to flexuous, branching alternate, opposite to irregular at acute to wide angles, loosely to closely reticulate, cells $8-29 \times 3-5 \mu \mathrm{m}$. Appressoria two celled, mostly alternate, often opposite to unilateral, antrorse to subantrorse, straight to curved, 9-19 $\mu \mathrm{m}$ long; stalk cells cuneate, 3-8 $\mu \mathrm{m}$ long; head cells oblong, ovate, angular to sublobate, 6-13 $\times$ 6-8 $\mu \mathrm{m}$. Thyriothecia scattered to grouped, orbicular, up to $176 \mu \mathrm{m}$ in diameter, stellately dehisced at the centre, margin crenate to fringed, fringed hyphae small; asci globose to slightly ovate, octosporous, up to $25 \mu \mathrm{m}$ in diameter; ascospores conglobate, uniseptate, constricted at the septum, 16-27 $\times 8-11 \mu \mathrm{m}$, wall smooth; pycnothyria similar to thyriothecia, smaller; pycnothyriospores pyriform, ovate, globose, $12-19 \times 8-11 \mu \mathrm{m}$.

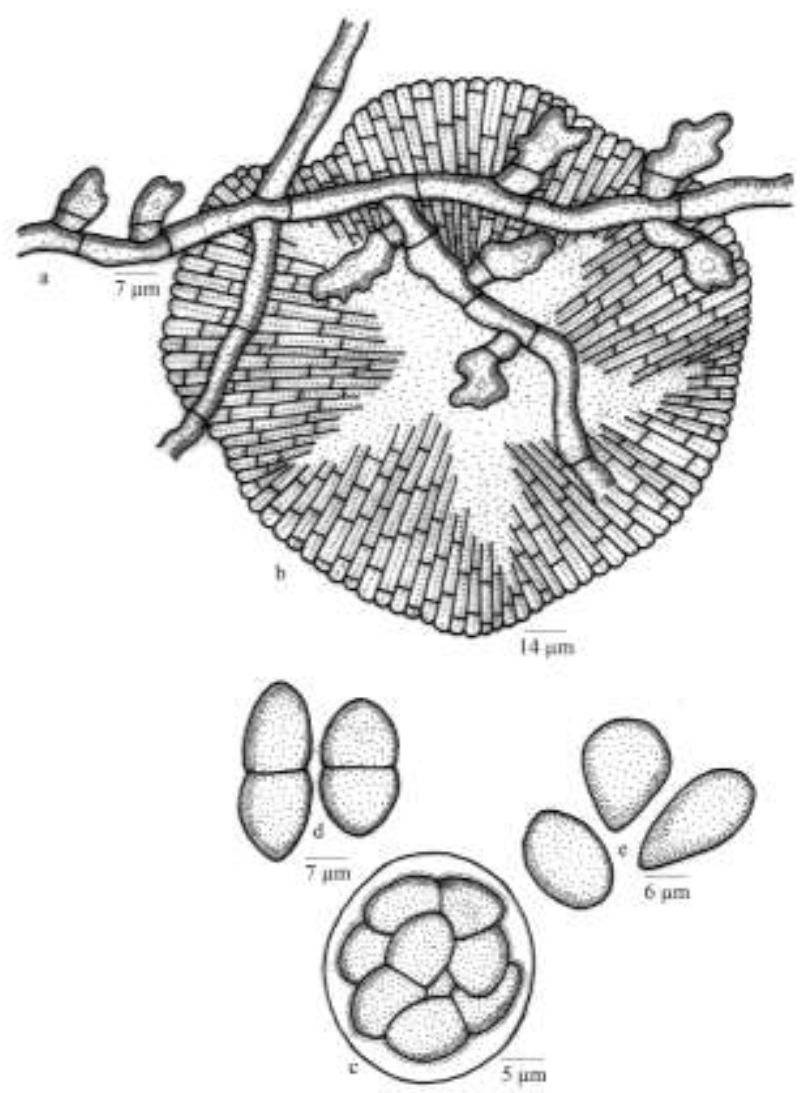

Figure 40 - Asterina canthiigena

Material examined: Karnataka, Coorg, Madikeri, Nishanemotta, on leaves of Canthium sp. (Rubiaceae), 11 November 2003, 
V.B. Hosagoudar \& al. HCIO 47319 (holotype), TBGT 2357 (isotype).

Asterina canthii Yates is known on Canthium sp. from Philippines (Yates, 1918a,b; Hosagoudar \& Abraham, 2000). However, A. canthiigena differs from it in having two celled in contrast to the unicellular appressoria.

Distribution: Karnataka

Asterina canthii-dicocci Hosag., Mycopathol. Res. 44: 6, 2006.

(Fig. 41)

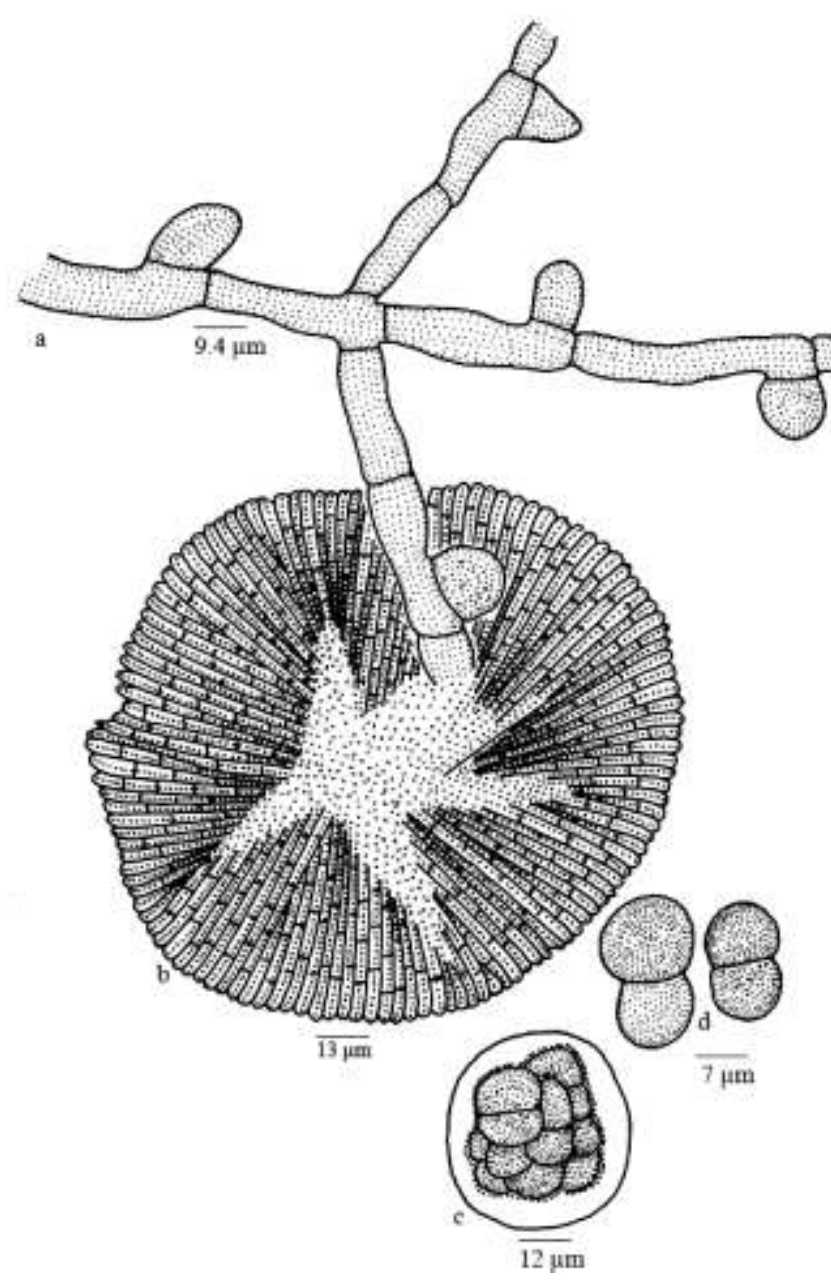

Figure 41 - Asterina canthii-dicocci

Colonies amphigenous, dense, up to $2 \mathrm{~mm}$ in diameter, rarely confluent. Hyphae straight, branching irregular at acute to wide angles, loosely to closely reticulate, cells $32-36 \times 5-7$ $\mu \mathrm{m}$. Appressoria alternate, unilateral, ovate, oblong, cylindrical, often attenuated at the apex, entire, 11-16 $\times$ 8-10 $\mu \mathrm{m}$. Thyriothecia scattered to connate, orbicular, up to $160 \mu \mathrm{m}$ in diameter, margin crenate to fimbriate, fringed hyphae straight, run parallel, often compact, thyriothecia stellately dehisced at the centre; asci globose, octosporous, up to $35 \mu \mathrm{m}$ in diameter; ascospores oblong, conglobate, uniseptate, constricted at the septum, 20-22 $\times$ 11-13 $\mu \mathrm{m}$, wall smooth.

Material examined: Karnataka, Kodagu (Coorg), Madikeri, Nishanemotta, on leaves of Canthium dicoccum (Gaertn.) Teijsm. J. \& Binn. (Rubiaceae), 11 November 2003, V.B. Hosagoudar \& al. HCIO 45762 (holotype), TBGT 1511 (isotype); Kerala, Thiruvananthapuram, Palode, TBGRI Campus, 31 December 2009, V.B. Hosagoudar TBGT 5039.

Asterina canthii-dicocci differs from $A$. canthii in absence of apposite appressoria and having larger ascospores. It differs from $A$. knysnae Doidge known on Canthium sp. from South Africa in having smaller ascospores against $(30-35 \times 16-20 \mu \mathrm{m})$. (Stevens \& Ryan, 1939; Doidge, 1942).

\section{Distribution: Karnataka}

Asterina capparidis Sydow \& Butler, Ann. Mycol. 9 390, 1911; Hosag., Balakr. \& Goos, Mycotaxon 60: 171, 1996.

(Fig. 42)

Colonies foliicolous, amphigenous, dense, mostly confluent. Hyphae flexuous, branching mostly opposite at wide angles, loosely reticulate, cells $18-28 \times 3-5 \mu \mathrm{m}$. Appressoria alternate, about 2\% opposite, unicellular to bicellular, mostly stalked but rarely sessile, straight to often curved, 12-22 $\mu \mathrm{m}$ long; basal cells cylindrical to cuneate, 3-10 $\mu \mathrm{m}$ long; apical cells ovate, globose, entire, angular to sublobate, $6-13 \times 6-10 \mu \mathrm{m}$. Thyriothecia scattered, round, up to $124 \mu \mathrm{m}$ in diameter, dehisce stellately at the center, the dehisced portion often dissolved, margin crenate to fimbriate, fringed hyphae small, tortuous; asci many, globose to ovate, eight spored, $30-38 \times 24-31 \mu \mathrm{m}$ in diameter; ascospores brown, ellipsoidal, conglobate, 1septate, slightly constricted at the septum, 17$19 \times 6-7 \mu \mathrm{m}$, both cells mostly equal, wall 
smooth. Pycnothyria many, like those of thyriothecia, smaller; pycnothyriospores deep brown, globose to pyriform, 18-21 × 10-12 $\mu \mathrm{m}$.

Material examined: Tamil Nadu, Coimbatore, Vellangiri hills, on leaves of Capparis zeylanica L., 20 September 1986, A. Diraviodoss HCIO 30956; Uttar Pradesh, Bahraich, on leaves of $C$. zeylanica L., M.A. Nomani TBGT 3503; Karnataka, Dharwad, Devikoppa, on leaves of Capparis horrida L. f., April 1919, Sedgwick HCIO 4421; Near Belgaum, on the way to Goa, Castle rock, $C$. spinosa L., 10 January 1984, C.R. Patil HCIO 40033.

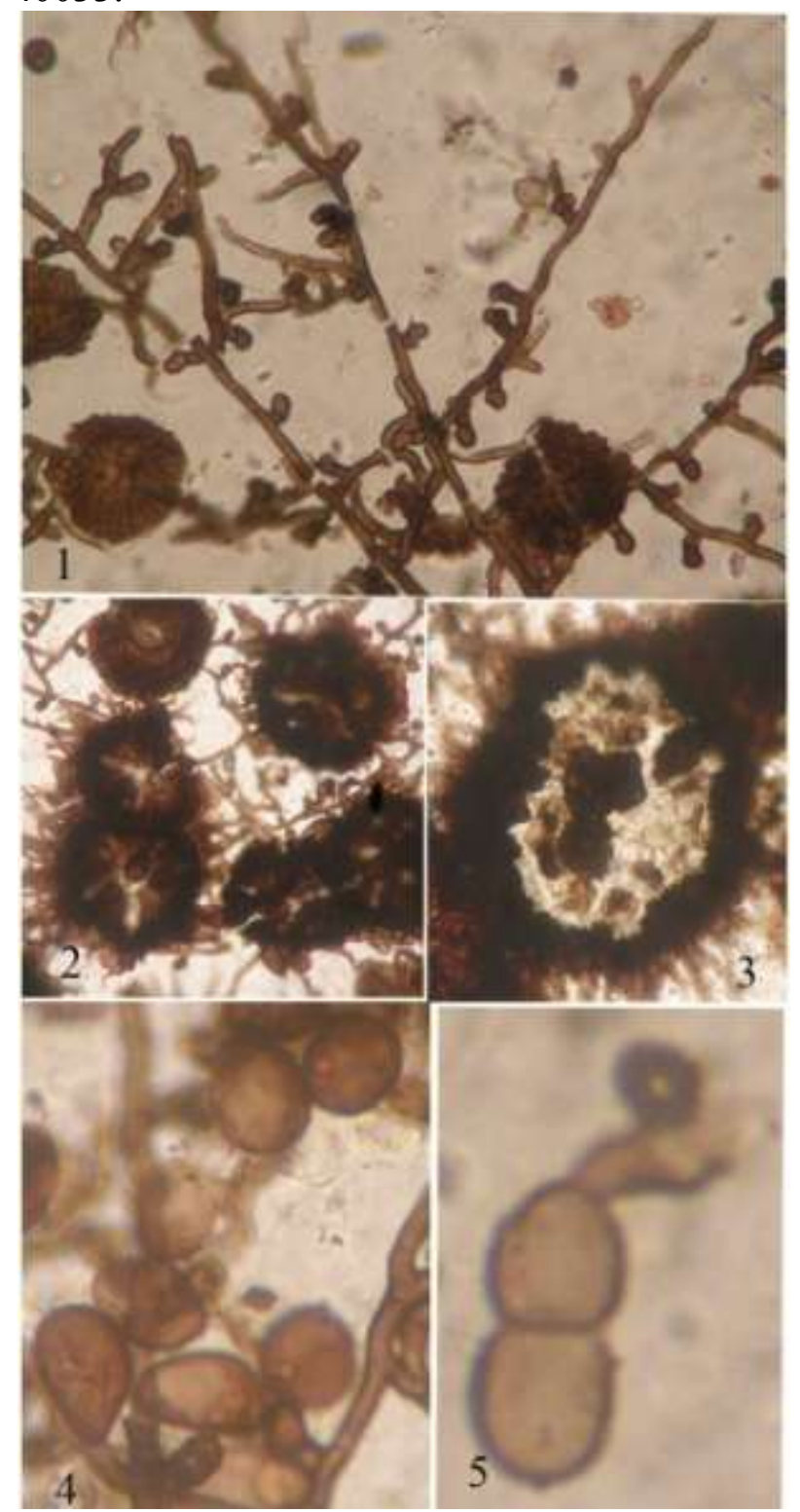

Figure 42 - Asterina capparidis

1. Appressoriate mycelium, 2. Stellately dehisced thyriothecia, 3 . Thyriothecium widely opened at the centre by exposing asci, 4 . Pycnothyriospores, 5. Germinating ascospore
This species was collected by E. J. Butler in 1903 on Capparis sp. from Madras (Sydow et al. 1911). However, the appressoria and pycnothyria were not described in the original description.

Distribution: Karnataka, Tamil Nadu, Uttar Pradesh

Asterina caseariae Hansf., Proc. Linn. Soc. London 156(2): 113, 1944.

Asterina caseariae Yamam., Sci. Rep. Hyogo Univ. Agric., Agric. Biol. Ser. 2: 35, 1956. (Fig. 43)

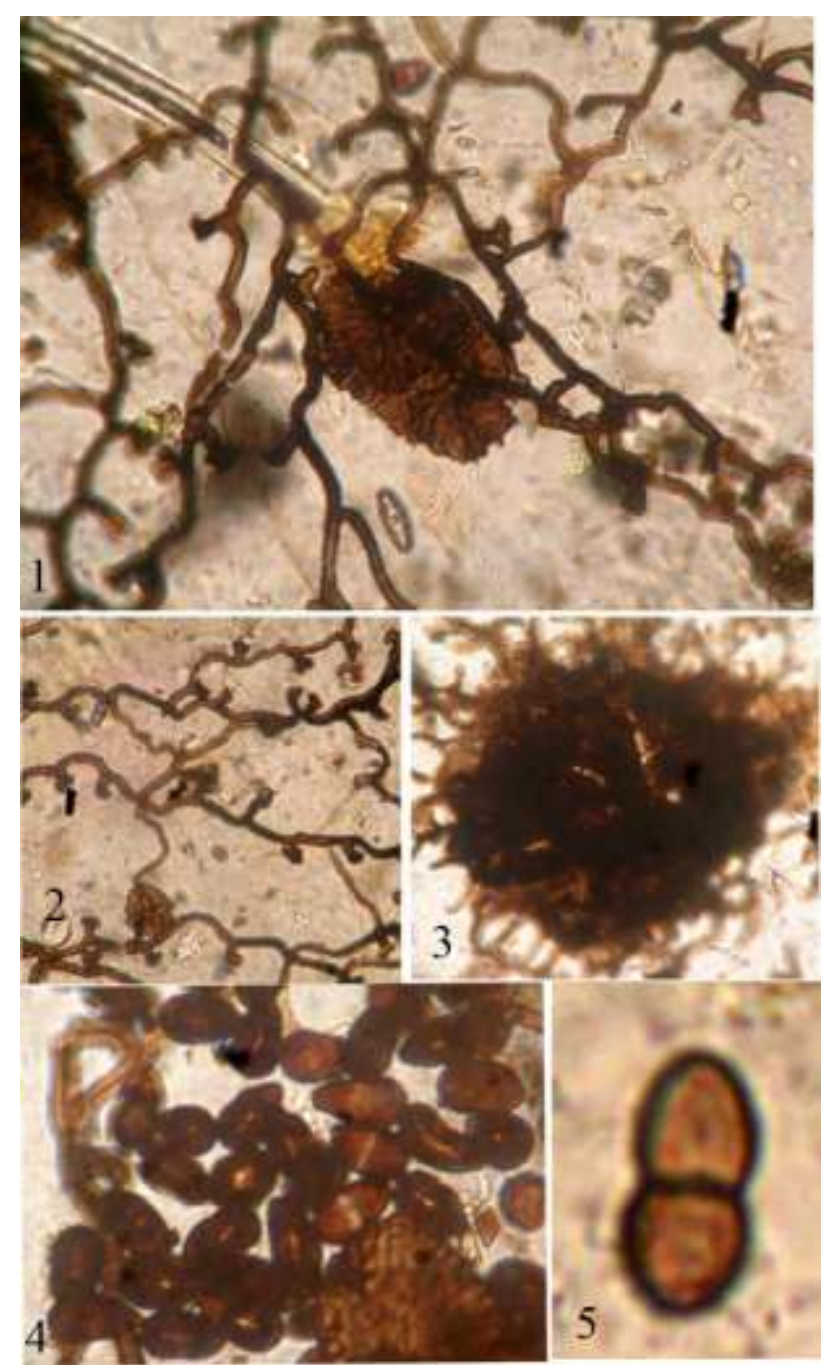

Figure 43 - Asterina caseariae

1-2. Appressoriate mycelium formed net, 3. Thyriothecium, 4. Pycnothyriospores with central hyaline band, 5. Ascospore

Colonies amphigenous, thin, up to $3 \mathrm{~mm}$ in diameter, confluent. Hyphae flexuous, branching irregular at acute to wide angles, loosely reticulate and form a loose mycelial net, 
cells 16-21 × 2-4 $\mu \mathrm{m}$. Appressoria scattered, alternate, two celled, 8-10 $\mu \mathrm{m}$ long; stalk cells cylindrical to cuneate, 2-4 $\mu \mathrm{m}$ long; head cells ovate, clavate, globose, straight to variously curved, hamate, irregularly sublobate to lobate, 5-8 $\times 6-8 \mu \mathrm{m}$. Thyriothecia scattered, orbicular, up to $110 \mu \mathrm{m}$ in diameter, stellately dehisced at the centre, margin crenate; asci few, globose, octosporous, up to $28 \mu \mathrm{m}$ in diameter; ascospores oblong, cylindrical, uniseptate, slightly constricted at the septum, upper cell globose, lower cell attenuated at the base, 18-20 $\times 8-10 \mu \mathrm{m}$. Pycnothyria numerous, similar to thyriothecia, smaller; pycnothyriospores pyriform, broadly rounded at the apex and narrowed towards the base, often slightly constricted in the middle, few spores possess hyaline band at the middle of the spores, 15-18 $\times 8-10 \mu \mathrm{m}$.

Material examined: Uttar Pradesh, Kataranya Ghat, on leaves of Casearia tomentosa Roxb. (Flacourtiaceae), M.A. Nomani TBGT 3505.

This species was known on Casearia engleri from Uganda (Hansford, 1944).

Distribution: Uttar Pradesh

Asterina caseariae-esculentae (Hosag. \& Goos) Hosag. \& Abraham, J. Econ. Taxon. Bot. 24: 568, 2000.

Asterina caseariicola Hosag. \& Goos, Mycotaxon 59: 153, 1996, (non Hansf., 1947).

Colonies epiphyllous, subdense, up to 5 $\mathrm{mm}$ in diameter, rarely confluent. Hyphae straight to substraight, branching alternate to irregular at wide angles, loosely to slightly closely reticulate, cells 21-25 $\times$ 3-5 $\mu \mathrm{m}$. Appressoria two celled, alternate, unilateral to about 5\% opposite, subantrorse to rarely recurved, 12-16 $\mu \mathrm{m}$ long; stalk cells cylindrical, 3-7 $\mu \mathrm{m}$ long; head cells ovate, long-ovate, globose, entire to sinuate, $9-11 \times 6-7 \mu \mathrm{m}$. Thyriothecia scattered, orbicular, up to $140 \mu \mathrm{m}$ in diameter; margin mostly crenate, rarely fringed, dehisce stellately at the center; asci many, globose to slightly ovate, octosporous, $37-41 \mu \mathrm{m}$ in diameter; ascospores conglobate, uniseptate, one cell slightly larger than the other, $18-22 \times 11-13 \mu \mathrm{m}$, wall smooth.

Material examined: Tamil Nadu, Nilgiris, Gudalur, Sri Madurai, on leaves of Cipadessa baccifera (Roth.) Miq. (Meliaceae), 25 January 1990, V.B. Hosagoudar HCIO 30957; Kerala, Thiruvananthapuram, TBGRI Campus, Palode, 4 December 1996, V.B. Hosagoudar HCIO 42412, TBGT 74; 17 January 2001, H. Biju HCIO 46111, TBGT 1874; river side, 10 October, 2001, M. Kamarudeen HCIO 44289, TBGT 649; 31 October 2003, H. Kamaleshkumar HCIO 46019, TBGT 1783; 25 January 2005, G. R. Archana \& al. HCIO 46015, TBGT 1779; 2 March 2005, G. R. Archana \& V. L. Sheeja HCIO 45926, TBGT 1688; 16 August 2005, G. R. Archana \& A. Sabeena HCIO 46705, TBGT 2046; 12 September 2007, G. R. Archana HCIO 48245, TBGT 2983; 25 January 2005, G.R. Archana \& al. HCIO 46017, TBGT 1781; 31 October 2005, Kamalesh Kumar HCIO 46019, TBGT 1783; 5 January 2001, H. Biju HCIO 46106, TBGT 1869; 17 January 2001, H. Biju HCIO 46111, TBGT 1874; 16 August 2005, G. R. Archana \& A. Sabeena HCIO 46705, TBGT 2046; 16 August 2005, G.R. Archana \& A. Sabeena HCIO 46710, TBGT 2051; 20 September 2005, M. Manu HCIO 46991, TBGT 2208; 6 July 2007, B. Bairavi HCIO 47537, TBGT 2559; 8 January 2008, A. Sabeena \& M.C. Riju TBGT 5265; 17 September 2008, K. Anil Kumar TBGT 5270; 19 February 2009, A. Sabeena TBGT 5296; Kollam, Palaruvi, 26 December 2000, G. Rajkumar HCIO 45135, TBGT 1190; 26 September 2007, A. Sabeena \& al. HCIO 48348, TBGT 3069; 10 April 2008, TBGT 5000; Palghat, Silent valley, Sairandhri, 2 August 2008, M.C. Riju \& al. TBGT 5229; 8 April 2008, A. Sabeena \& M.C. Riju TBGT 4789; Thenmala Ecotourism Adventure zone, 14 October 2002, A. Manojkumar HCIO 44668, TBGT 753; Thiruvananthapuram, Karakulam, 25 February 2003, G. Rajkumar HCIO 45133, TBGT 1188; Thiruvananthapuram, Peppara Wildlife Sanctuary, Bonoccord, 28 February 2008 Jacob Thomas \& al. HCIO 49053, TBGT 3308; Wayanad, Begur, 15 February 2009, Jacob Thomas \& al. HCIO 49207, TBGT 3446; HCIO 
49208, TBGT 3447; Thirunelli, 14 February 2009, Jacob Thomas \& al. HCIO 49211, TBGT 3450; Kottayam, Ponthanpuzha, 29 February 2008, Robin \& al. HCIO 49946, TBGT 4098; Wayanad, Padinharathara, 6 November 2009, A. Sabeena \& M.C. Riju TBGT 4273; Palghat, Silent Valley, Sairandhri, 13 December 2003, V.B. Hosagoudar \& al. HCIO 46038, TBGT 1801; Thiruvananthapuram, Palode, 3 June 2008, A. Chandraprabha HCIO 48822, TBGT 3198; Thrissur, Vazhachal Forests, 21 August 2007, Jacob Thomas \& al. HCIO 48187, TBGT 2923; on Meliaceae member, Thiruvananthapuram, Peppara Wild Life Sanctuary, Near Peppara Dam, 18 November 2007, Jacob Thomas \& Vimal Kumar HCIO 48249, TBGT 2988; Karnataka, Kodagu, Madikeri, Nishane motta, 11 November 2003, V.B. Hosagoudar \& al. HCIO 45649, TBGT 1395; Muddarmudi, Hakathur, 26 November 2008, V. B. Hosagoudar \& al. HCIO 49226, TBGT 3465; C. Jagath Thimmaiah TBGT 5274; Tamil Nadu, Kodaikanal, Samikanal, 31 December 2007, R. Nithyatharani TBGT 4351; 5 August 2007, R. Nithyatharani TBGT 4415; 15 November 2007, R. Nithyatharani TBGT 4723; Kuthuraiyar, 22 October 2007, R. Nithyatharani TBGT 4413.

This is the only species known on this host and is common in the Southern Western Ghats.

Distribution: Karnataka, Kerala, Tamil Nadu.

Asterina cassiicola V.B. Hosagoudar \& G.R. Archana, sp. nov. (Fig. 44).

MycoBank 801001

Etymology - Named after the host genus

Colonies epiphyllous, thin, crustose, up to $3 \mathrm{~mm}$ in diameter, confluent. Hyphae substraight to flexuous, branching opposite, alternate to irregular at ac+ute to wide angles, loosely to closely reticulate, cells $12-24$ $\times 3-5 \mu \mathrm{m}$. Appressoria opposite, alternate to unilateral, antrorse to subantrorse, sessile, ovate, oblong, sublobate, angular, 3-10 × 4-8 $\mu \mathrm{m}$. Thyriothecia scattered to grouped, orbicular, up to $150 \mu \mathrm{m}$ in diameter, stellately dehisced at the centre, margin fimbriate, fringed hyphae flexuous; asci globose to ovate, octosporous, $20-24 \times 16-20 \mu \mathrm{m}$; ascospores conglobate, uniseptate, slightly constricted at the septum, 14-21 $\times$ 9-13 $\mu \mathrm{m}$, wall smooth. Pycnothyria orbicular, smaller than thyriothecia; pycnothyriospores unicellular, pyriform, ovate, $12-16 \times 6-8 \mu \mathrm{m}$.

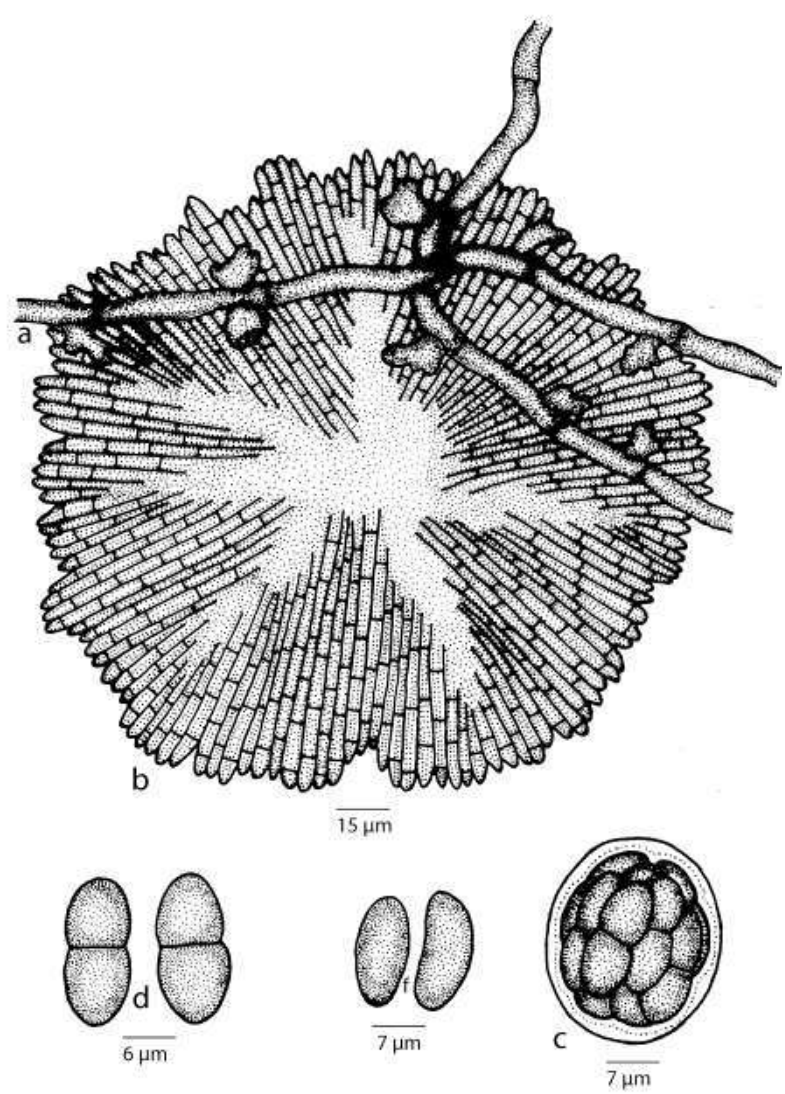

Figure 44 - Asterina cassiicola

Material examined - Kerala, Thiruvananthapuram, Palode, Karimancode, on leaves of Cassia fistula $L$. (Caessalpiniaceae), 12 September 2009, G.R. Archana TBGT 3143 (holotype) (as A. cassiae), $\mathrm{HCIO} 48422$ (isotype) (as $A$. cassiae); HCIO 48424, TBGT 3145 .

This species differs from Asterina ciferriana Petrak and Asterina contigua Sydow on Caesalpinia crista and Dialium heudeloti from San Domingo and Sierra Leone, respectively, in having single celled, alternate to opposite and angular to sublobate appressoria (Hosagoudar \& Abraham 2000).

Distribution - Kerala 
Asterina chukrasiae Hosag. in Hosag., H. Biju \& Appaiah, J. Mycopathol. Res. 44: 40, 2006; Hosag., Chandraprabha \& Agarwal, Asterinales of Kerala, p.47, 2011. (Fig. 45)

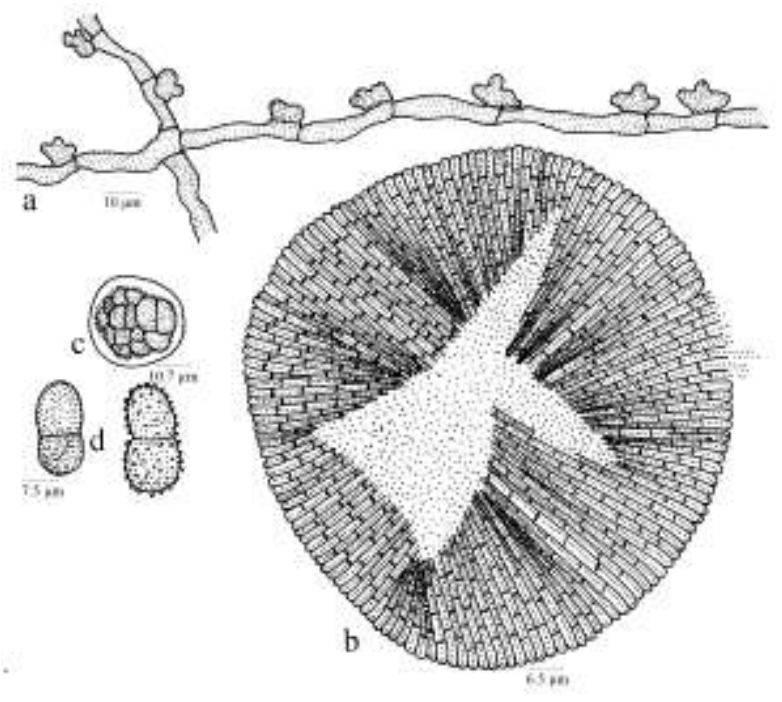

Figure 45 - Asterina chukrasiae

Colonies epiphyllous, thin to subdense, up to $2 \mathrm{~mm}$ in diameter, rarely confluent. Hyphae substraight, branching irregular at acute to wide angles, loosely reticulate, cells 19-23 $\times$ 3-5 $\mu \mathrm{m}$. Appressoria alternate to unilateral, minutely stipitate to mostly broad based, globose, 2-3-times sublobate to lobate, 4-6 × 6$7 \mu \mathrm{m}$. Thyriothecia loosely aggregated to closely aggregated, orbicular, up to $100 \mu \mathrm{m}$ in diameter, margin crenate, stellately dehisced at the centre; asci globose, octosporous, up to 30 $\mu \mathrm{m}$ in diameter; ascospores oblong, conglobate, uniseptate, constricted at the septum, brown, 20-24 × 11-13 $\mu \mathrm{m}$, wall smooth to tubercled.

Material examined: Kerala, Palghat, Silent Valley, Sairandhri, on leaves of Chukrasia tabularis A. Juss. (Meliaceae), 13 December 2003, V.B. Hosagoudar \& al. HCIO 45760 (holotype), TBGT 1509 (isotype); Karnataka Kodagu, Hoddur, C. fabularis Akane, 17 October.2009, Jagath Thimmaiah TBGT 5430; Devarakadu, on leaves of $C$. tabularis A. Juss., 4 December 2009, Jagat Thimmaiah TBGT 5469.

Based on the alternate appressoria, $A$. chukrasiae is similar to A. turraeae Hansf. known on Turraea floribunda from Uganda (Hansford, 1944, Hosagoudar \& Abraham,
2000). However, differs from it in having 2-3times sublobate to lobate appressoria.

\section{Distribution: Kerala}

Asterina cinnamomi Sydow, Ann. Mycol. 21: 103, 1923; Kaul \& Nair, Acta Bot. Indica 16: 228, 1988; Hosag. \& Abraham, J. Econ. Taxon. Bot. 4: 570, 2000; Hosag., Zoos' Print J. 18: 1283, 2003; Hosag., Chandraprabha \& Agarwal, Asterinales of Kerala, p.48, 2011. (Fig. 46)

Colonies amphigenous, dense, crustose, up to $4 \mathrm{~mm}$ in diameter, confluent. Hyphae crooked, branching irregular at acute to wide angles, loosely to closely reticulate, cells 18-24 $\times$ 4-7 $\mu \mathrm{m}$. Appressoria two celled, alternate to unilateral, subantrorse, spreading, entire, 11-18 $\mu \mathrm{m}$ long; stalk cells cylindrical to cuneate, 4-9 $\mu \mathrm{m}$ long; head cells ovate, globose, entire, 9-11 $\times 7-9 \mu \mathrm{m}$. Thyriothecia scattered, orbicular, up to $110 \mu \mathrm{m}$ in diameter, stellately dehisced at the center, margin crenate; asci few to many, globose, octosporous, up to $40 \mu \mathrm{m}$ in diameter; ascospores brown, conglobate, 1-septate, constricted at the septum, $20-24 \times 9-11 \mu \mathrm{m}$, wall smooth.

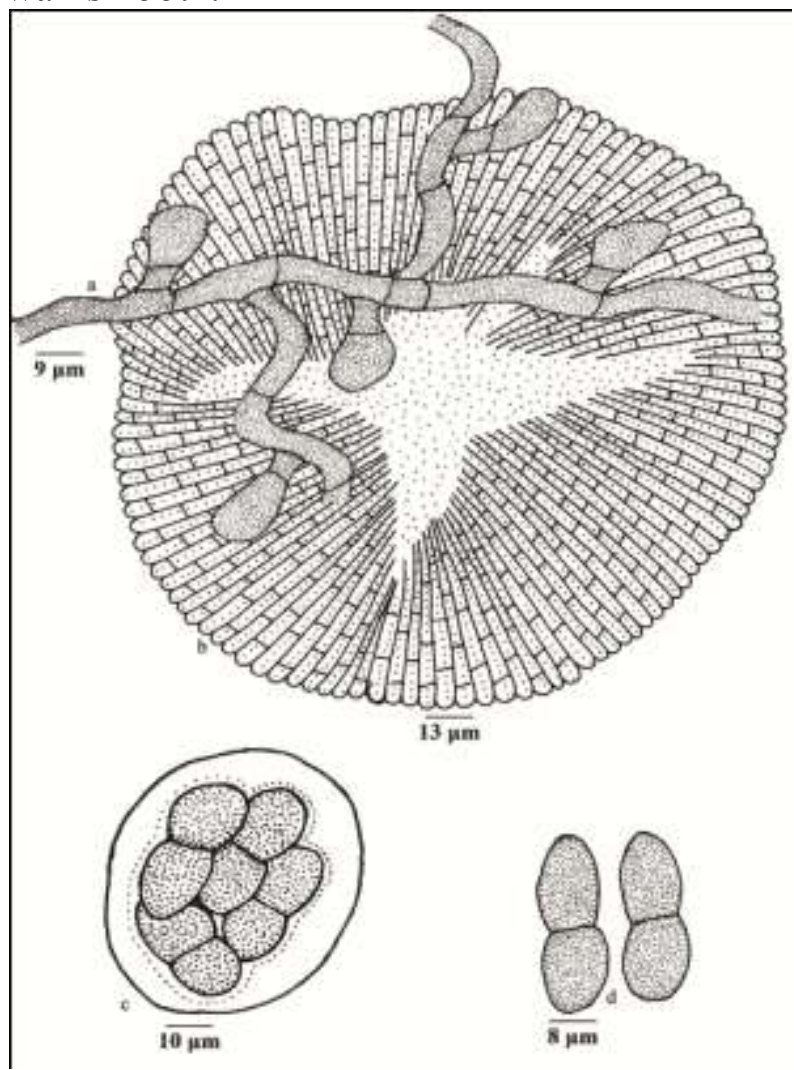

Figure 46 - Asterina cinnamomi 
Material examined: Kerala, Idukki, Periyar Reserve, on leaves of Cinnamomum sp. (Lauraceae), 16 December 2004, V.B. Hosagoudar \& al. HCIO 46789, TBGT 2130.

This species was first reported from Philippines (Sydow, 1923) and later from Kaul \& Nair (1988) on Cinnamomum zeylanicum Blume from Ooty, Tamil Nadu.

Distribution: Kerala, Tamil Nadu

Asterina cinnamomicola Hansf., Proc. Linn. Soc. London, 1944-45: 202, 1946; Hosag. \& Abraham, J. Econ. Taxon. Bot. 4: 570, 2000; Hosag., Chandraprabha \& Agarwal, Asterinales of Kerala, p. 49, 2011. (Fig. 47)

Colonies amphigenous, dense, crustose, up to $4 \mathrm{~mm}$ in diameter, confluent. Hyphae crooked, branching irregular at acute to wide angles, loosely to closely reticulate, cells $15-21$ $\times 3-4 \mu \mathrm{m}$. Appressoria unicellular, alternate, ampulliform, proliferated at the apex, entire, 9$18 \times 3-7 \mu \mathrm{m}$. Thyriothecia scattered, orbicular, up to $92 \mu \mathrm{m}$ in diameter, stellately dehisced at the center, margin crenate; asci few to many, globose, octosporous, up to $35 \mu \mathrm{m}$ in diameter; ascospores brown, conglobate, 1-septate, constricted at the septum, $18-22 \times 9-11 \mu \mathrm{m}$, wall smooth.

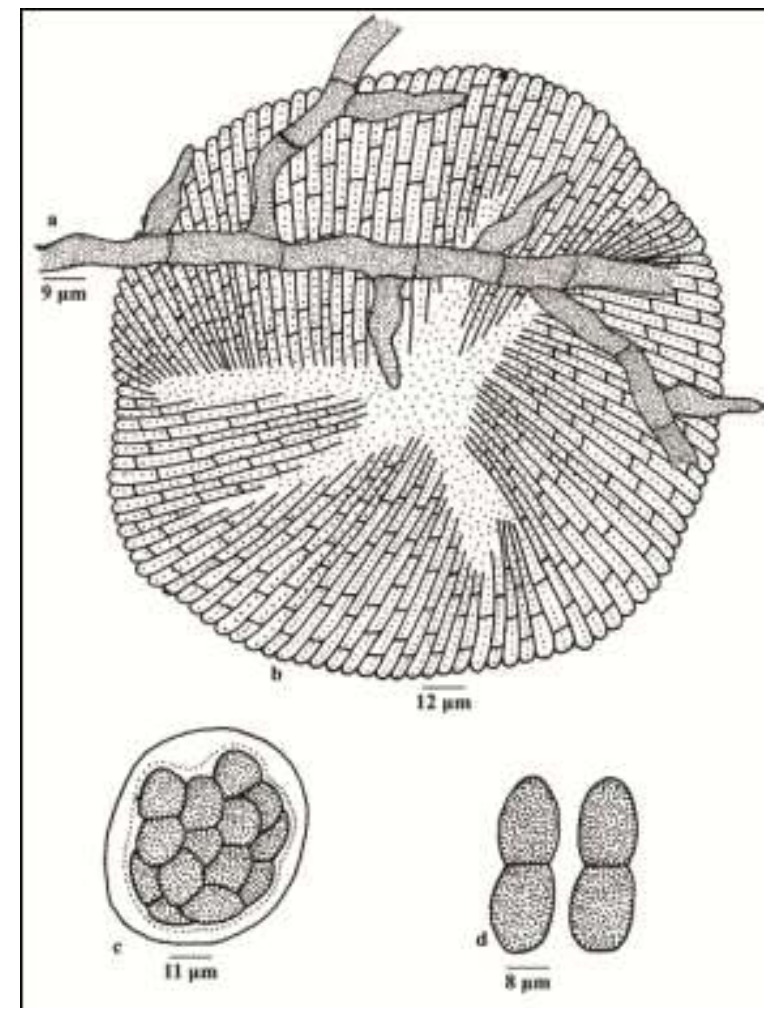

Figure 47 - Asterina cinnamomicola
Material examined: Kerala, Idukki, Periyar Tiger Reserve, on leaves of Cinnamomum sp. (Lauraceae), 16 December 2004, V. B. Hosagoudar \& al. HCIO 46789, TBGT 2130; Mannavan Shola, 16 September 1999, V.B. Hosagoudar HCIO 46789, TBGT 3486; Periyar, near Project Tiger Reserve, 16 December 2004, V.B. Hosagoudar \& al. HCIO 47676, TBGT 2698; Tamil Nadu, Kodaikanal, Near Poombarai shola, Cinnamomum wightii Meissner, 17 July 2006, R. Nithyatharani HCIO 47692, TBGT 2714; Bear shola West, 22 July 2006, R. Nithyatharani HCIO 47706, TBGT 2728.

Asterina cinnamomicola Hansf. differs from Asterina cinnamomi Sydow in having unicellular, proliferated appressoria ( Sydow, 1923).

Distribution: Kerala, Tamil Nadu

Asterina cipadessae Yates, Philippine J. Sci. 12: 371, 1917; Hosag., Balakr. \& Goos, Mycotaxon 60: 172, 1996; Hosag. \& Abraham, J. Econ. Taxon. Bot. 4: 574, 2000; Hosag., Zoos' Print J. 18: 1283, 2003; 21: 2326, 2006; Hosag., H. Biju \& Appaiah, J. Mycopathol. Res. 44: 6, 2006. Parasterina cipadessae (Yates) Mendoza, Philippine J. Sci. 49: 446, 1932; Hosag., Chandraprabha \& Agarwal, Asterinales of Kerala, p.51, 2011. (Fig. 48)

Colonies epiphyllous, dense, up to 2 $\mathrm{mm}$ in diameter, confluent. Hyphae straight, flexuous to crooked, branching mostly opposite at acute angles loosely to closely reticulate, cells 12-34 × 4-6 $\mu \mathrm{m}$. Appressoria alternate and opposite, sessile, entire to mostly lobate, $9-13 \times$ 7-10 $\mu \mathrm{m}$. Thyriothecia scattered to grouped, often connate, orbicular, up to $202 \mu \mathrm{m}$ in diameter, dehisce stellately at the center, margin crenate, rarely slightly fimbriate; asci many, ovate to globose, eight spored, 30-44 $\times$ 30-35 $\mu \mathrm{m}$; ascospores conglobate, deep brown, 1-septate, slightly constricted at the septum, 24$28 \times 12-15 \mu \mathrm{m}$, upper cell ovate and lower cell globose, wall smooth. Pycnothyria many, similar to the thyriothecia, smaller; pycnothyriospores brown, ovoid to pyriform, $12-16 \times 4-7 \mu \mathrm{m}$. 


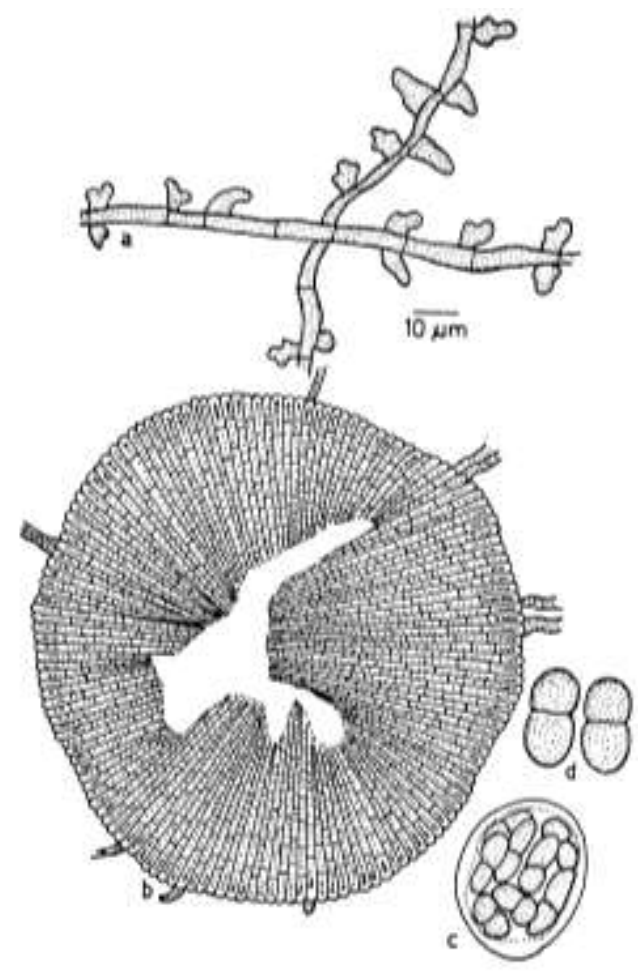

Figure 48 - Asterina cipadessae

Material examined: Tamil Nadu, Nilgiris, Gudalur, Sri Madurai, on leaves of Cipadessa baccifera (Roth.) Miq. (Meliaceae), 25 January 1990, V.B. Hosagoudar HCIO 30957; Kerala, Thiruvananthapuram, Palode, TBGRI Campus, 4 December 1996, V.B. Hosagoudar HCIO 42412, TBGT 74; Thiruvananthapuram, Palode, TBGRI Campus, 17 January 2001, H. Biju HCIO 46111, TBGT 1874; TBGRI river side, 10 October 2001, M. Kamarudeen HCIO 44289, TBGT 649; Kollam, Thenmala Ecotourism Adventure zone, 14 October 2002, A. Manojkumar HCIO 44668, TBGT 753; Thiruvananthapuram, Karakulam, 25 February 2003, G. Rajkumar HCIO 45133, TBGT 1188; Thiruvananthapuram, Palode, TBGRI Campus, 31 October 2003, H. Kamaleshkumar HCIO 46019, TBGT 1783; Karnataka, Kodagu, Madikeri, Nishane motta, 11 November 2003, V.B. Hosagoudar \& al. HCIO 45649, TBGT 1395; Kerala, Palghat, Silent Valley, Sairandhri, 13 December 2003, V.B. Hosagoudar \& al. HCIO 46038, TBGT 1801; Palode, TBGRI Campus, 25 January 2005, G.R. Archana \& al. HCIO 46015, TBGT 1779; 2 March, 2005, G.R. Archana \& V.L. Sheeja HCIO 45926, TBGT 1688; 16 August 2005, G.R. Archana \& A. Sabeena HCIO 46705, TBGT 2046; 12 September 2007, G. R. Archana HCIO 48245, TBGT 2983;
Thiruvananthapuram, Palode, 3 June 2008, A. Chandra Prabha HCIO 48822, TBGT 3198; TBGRI Campus, 25 January 2005, G.R. Archana \& al. HCIO 46017, TBGT 1781; 31 October 2005, Kamalesh Kumar HCIO 46019, TBGT 1783; 5 January 2001, H. Biju HCIO 46106, TBGT 1869; 17 January 2001 H. Biju HCIO 46111, TBGT 1874; 16 August 2005, G.. R. Archana \& A. Sabeena HCIO 46705, TBGT 2046; HCIO 46710, TBGT 2051; 20September 2005, M. Manu HCIO 46991, TBGT 2208; 6 July 2007, B. Bairavi HCIO 47537, TBGT 2559; Thrissur, Vazhachal Forests, 21 August 2007, J. Thomas \& al. HCIO 48187, TBGT 2923; Thiruvananthapuram, Peppara Sanctuary, Near Peppara Dam, Meliaceae, 18 November 2007, Jacob Thomas \& Vimal Kumar HCIO 48249, TBGT 2988; Thiruvananthapuram, Palode, TBGRI Campus, 26 September 2007, A. Sabeena \& al. HCIO 48348, TBGT 3069; Thiruvananthapuram, Peppara Sanctuary, Bonoccord, 6 March 2008, Athirumala, Peppara Sanctuary, 28 February 2008 J. Thomas \& al. HCIO 49053, TBGT 3308; Wayanad, Begur, 15 February 2009, J. Thomas \& al HCIO 49207, TBGT 3446; HCIO 49208, TBGT 3447; Wayanad, Thirunelli, 14 February 2009, J. Thomas \& al HCIO 49211, TBGT 3450; Thirunelli, 14 February 2009, J. Thomas \& al HCIO 49221, TBGT 3460; Karnataka, Coorg, Muddarmudi, Hakathur, 26 November, 2008, V.B. Hosagoudar \& al HCIO 49226, TBGT 3465; Kerala, Kottayam, Ponthanpuzha, 29 February 2008, Robin \& al HCIO 49946, TBGT 4098; Wayanad, Padinharathara, 6 November 2009, A. Sabeena \& M.C. Riju TBGT 4273;Tamil Nadu, Kodaikanal, Samikanal, 31 December 2007, R. Nithya Tharani TBGT 4351; Kuthuraiyar, 22 October.2007, R. Nithya Tharani TBGT 4413; Samikanal. 5 August 2007, R. Nithya Tharani TBGT 4415; S. Kanal, 15 November 2007, R. Nithyatharani TBGT 4723; Kerala, Palode, TBGRI campus 10 April 2008, TBGT 5000; Palghat, Silent Valley, Sairandhri, 2 August 2008, M.C. Riju \& al TBGT 5229; Palode, TBGRI campus, 8 January 2008, A. Sabeena \& M.C. Riju TBGT 5265; 17 February 2008, K. Anil Kumar TBGT 5270; 19 February 2009, A. Sabeena TBGT 5296; Karnataka, Kodagu, Hakathur, 26 November 2008, C. Jagath Thimmaiah TBGT 5274. 
This is the only species known on this host and is common in the Southern Western Ghats.

Nadu.

Distribution: Kerala, Karnataka, Tamil

Asterina cissi Hughes, Mycol. Pap. 48: 10, 1952; Hosag., C.K. Biju \& Abraham, J. Mycopathol. Res. 40: 195, 2002; Indian Phytopath. 57: 114, 2004; Hosag., Chandraprabha \& Agarwal, Asterinales of Kerala, p. 52, 2011. (Fig. 49)

Colonies epiphyllous, subdense, up to 3 $\mathrm{mm}$ in diameter, confluent. Hyphae straight to flexuous, branching irregular at acute angles, loosely reticulate, cells $22-26 \times 4-6 \mu \mathrm{m}$. Appressoria unicellular, alternate to unilateral, ovate to globose, entire, angular to 2-3- times sublobate to lobate, $10-15 \times 10-13 \mu \mathrm{m}$. Thyriothecia predominantly scattered, rarely connate, orbicular, up to $125 \mu \mathrm{m}$ in diameter, margin crenate to fimbriate, fringed hyphae substraight, stellately dehisced at the centre; asci globose, octosporous, up to $40 \mu \mathrm{m}$ in diameter; ascospores conglobate, brown, 1septate, constricted at the septum, 20-23 × 10$13 \mu \mathrm{m}$, wall smooth.

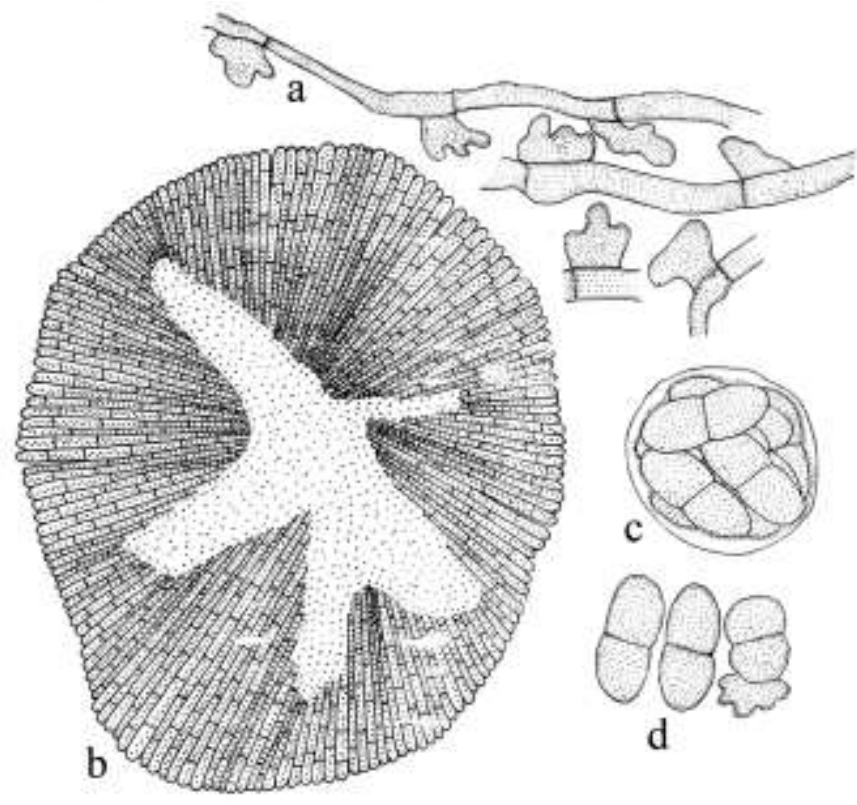

Figure 49 - Asterina cissi

Material examined: Kerala, Idukki, Rajmala hills, on leaves of Cissus sp. (Vitis sp.) (Vitaceae), 25 September 1998, C.K. Biju HCIO 42941, TBGT 213.
The present collection perfectly matches with the assigned species except the smooth walled ascospores.

Distribution: Kerala

Asterina clausenicola Doidge, Trans. Royal Soc. South Africa 8: 263, 1920; Hosag., Indian J. Forestry 18: 274, 1995; Hosag., Chandraprabha \& Agarwal, Asterinales of Kerala, p. 52, 2011.

(Fig. 50)

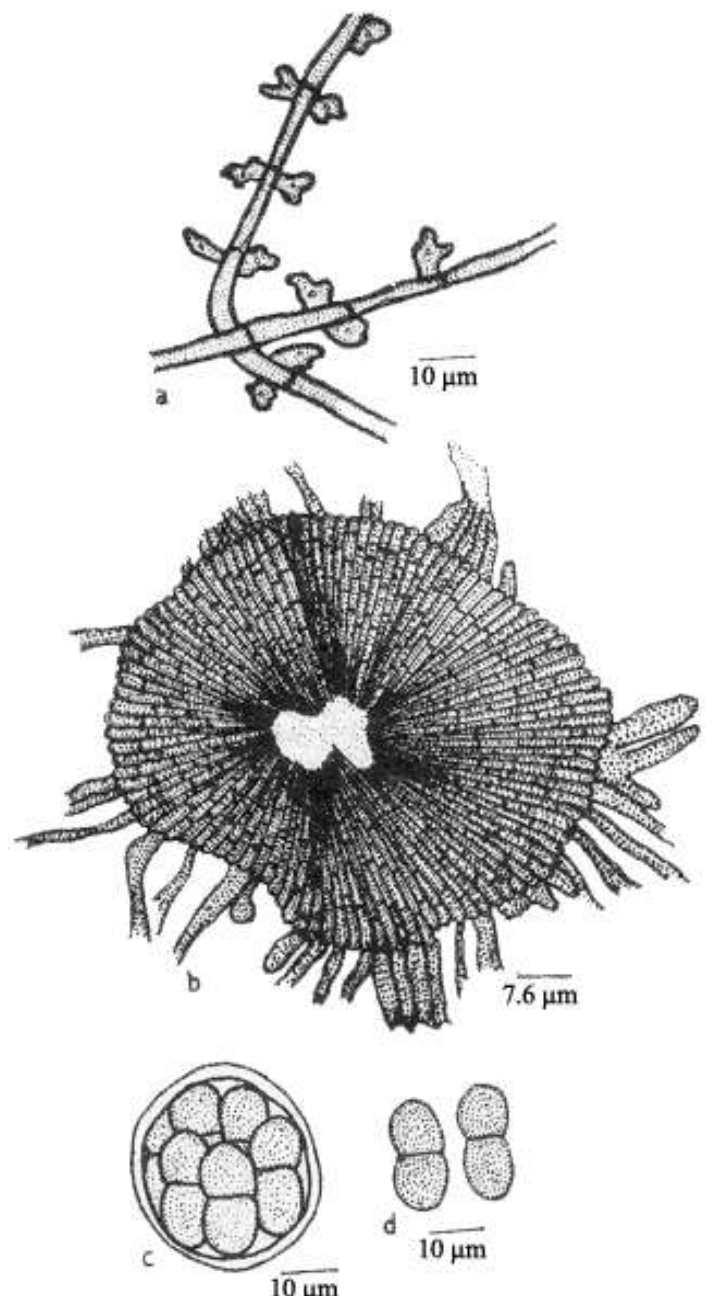

Figure 50 - Asterina clausenicola

Colonies epiphyllous, dense to subdense, crustose, up to $3 \mathrm{~mm}$ in diameter, rarely confluent. Hyphae substraight to flexuous, branching mostly opposite at acute angles, loosely reticulate, cells $18-31 \times 3-5 \mu \mathrm{m}$. Appressoria alternate and about $12 \%$ opposite, straight to curved, oblong to globose, unicellular, entire and bluntly conoid towards the apex, uni-to multilobate, 6-13 $\times 4-8 \mu \mathrm{m}$. Thyriothecia scattered, rarely 2-3 connate, roughly circular in outline, up to $125 \mu \mathrm{m}$ in diameter, margin crenate to fimbriate, fringed hyphae flexuous, stellately dehisce at the 
center; asci globose, rarely ovate or oblong, hexasporous, $30-38 \mu \mathrm{m}$ in diameter; ascospores brown, conglobate, oblong, 1-septate, strongly constricted at the septum, rounded at both ends, $18-22 \times 10-12.5 \mu \mathrm{m}$, wall smooth.

Material examined: Tamil Nadu, Coimbatore, Anamalai, Near Sholayar dam, on leaves of Melicope lunu-ankenda (Gaertn.) T. Hartley (Euodia lunu-ankenda (Gaertn.) Merr. (Rutaceae), 28 February 1993, V.B. Hosagoudar HCIO; Kerala, Wyanad, Periya, 8 January 2001, M. Kamarudeen HCIO 44550, TBGT 836; Kottayam, Ponthanpuzha, 12 October 2007, P.J. Robin HCIO 48409, TBGT 3130 .

This species was recorded on Clausena anisata from South Africa (Doidge, 1942).

Distribution: Kerala, Tamil Nadu.

Asterina claviflori Kar \& Maity, Trans. Brit. Mycol. Soc. 54: 441, 1970; Hosag., Chandraprabha \& Agarwal, Asterinales of Kerala, p.54, 2011. (Fig. 51)

Colonies epiphyllous, dense, up to 2 $\mathrm{mm}$ in diameter, confluent. Hyphae flexuous, branching alternate to irregular at acute to wide angles, loosely reticulate, cells 25-32 × 4-8 $\mu \mathrm{m}$. Appressoria alternate to unilateral, unicellular, ovate, oblong, cylindrical, antrorse to retrorse, straight to curved, entire, 9-18 $\times 6-8 \mu \mathrm{m}$. Thyriothecia scattered, rarely connate, orbicular, up to $250 \mu \mathrm{m}$ in diameter, margin fimbriate, fringed hyphae flexuous, stellately dehisced at the centre; asci few to many, ovate to globose, octosporous, $30-45 \mu \mathrm{m}$ in diameter; ascospores oblong, brown, conglobate, uniseptate, constricted, 14-18 × 11-13 $\mu \mathrm{m}$, wall smooth to slightly verrucose.

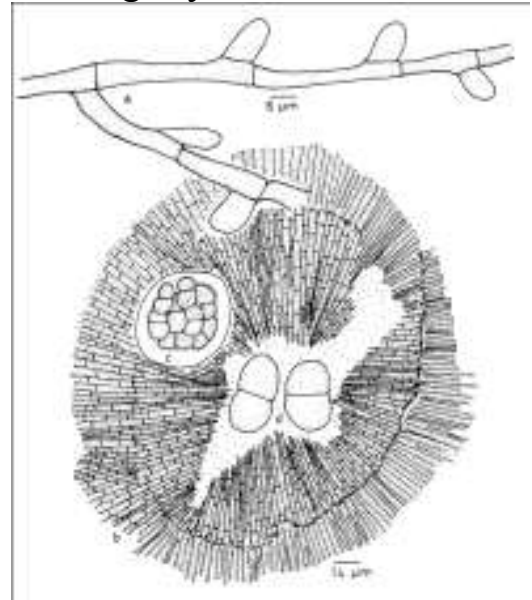

Figure 51 - Asterina claviflori
Material examined: Kerala, Thiruvananthapuram, Palode, TBGRI Campus, on leaves of Syzygium mundagam (Bourd.) Chitra (Myrtaceae), 10 January 2001, H. Biju HCIO 46076, TBGT 1839; S. jambolana (Lam.) DC., TBGRI Campus, Medicinal plant garden, 17 January 2001, H. Biju HCIO 47084, TBGT 2301; 12 March 2008, A. Chandra Prabha HCIO 48893, TBGT 3269; 17 January 2001, H. Biju HCIO 47084,TBGT 2301; TBGRI campus, 10 January 2001, H. Biju HCIO 46076, TBGT 1839; TBGRI Campus, on leaves of Syzygium cumini (L.) Skeels, 12 March 2008, A. Chandra prabha HCIO 48893, TBGT 3269; Wayanad, Chunkathara, 8 December 2009, Sam P. Mathew TBGT 4645; Karnataka, Kodagu, Vanachalu, 24 November, 2008, C. Jagath Thimmaiah TBGT 5322; Madikeri, 15 January 2009, C. Jagath Thimmaiah TBGT 5396; Kerala, Chengannur, Karakkad, on leaves of Syzygium zeylanicum (L.) DC., 2 March 2008, M. Harish TBGT 4635; Karnataka, Kodagu, Madikeri, FMC Campus, 26 November 2008, C. Jagath Thimmaiah TBGT 5373; Tamil Nadu, Marthandam, on leaves of Syzygium sp., 9 February 2003, G. Rajkumar HCIO 45088, TBGT 1143; Kerala, Palghat, Silent Valley National Park, Sairandri, 13 June 2007, Jacob Thomas \& al. HCIO 48844, TBGT 3220; Karnataka, Coorg, Madikari, 25 November 2008, V.B. Hosagoudar \& al. HCIO 49149, TBGT 3404: 24 November 2008, V.B. Hosagoudar \& al. HCIO 49157, TBGT 3412; Madikari, FMC College, 26 November 2008, V.B. Hosagoudar \& al. HCIO 49160, TBGT 3415; Vanachalu, 24 November 2008, V.B. Hosagoudar \& al. HCIO 49161, TBGT 3416; Kodagu, Vanachalu, 24 November 2008, C. Jagath Thimmaiah TBGT 5332; Kerala, Thiruvananthapuram, Palode, TBGRI Campus, Arboretum, 7 August 2008, K. Anilkumar HCIO 49347, TBGT 3592; HCIO 49350, TBGT 3595; May 13, 2008, K. Anilkumar HCIO 49353, TBGT 3598; Idukki, Munnar, Mattupetti, 15 March 2007, Robin \& al. HCIO 49501, TBGT 3743; Palghat, Silent Valley National Park, 1 March 2009, S.S. Shaji \& al. HCIO 49558, TBGT 3800; 3 March 2009, S.S. Shaji \& al. HCIO 49576, TBGT 3818; Pathanamthitta, Sabarimala forest division, Nilakal, 29 March 2009, P.J. Robin \& al. HCIO 
49655, TBGT 3897; 27 April 2007, M. Harish \& al. HCIO 49756, TBGT 3908; Wayanad, Periya, 6 December 2006, M. Harish \& al. HCIO 49759, TBGT 3911; 6 December 2006, Gireesh \& al. HCIO 50030, TBGT 4182; 15 September 2008, P.J. Robin \& al. HCIO 49808, TBGT 3960; Thirunelli, 13 February 2009, Jacob Thomas \& al. HCIO 49782, TBGT 3934; Puthuserrykadavu, 14 March 2007, M.C. Riju HCIO 49971, TBGT 4223; Gurukulam Botanical Garden, Periya, 5 November 2009, M.C. Riju \& A. Sabeena TBGT 4664; Palghat, Silent valley, Sairandhri, 13 December 2003, V.B. Hosagoudar \& al. HCIO 50009, TBGT 4161; Pathanamthitta, Kochu Pamba, 18 January 2007, Girish Kumar \& al. TBGT 4823; Wayanad, Gurukulam Botanic Garden, 5 November 2009, A. Sabeena \& M.C. Riju TBGT 4983; Kozhikode, Malabar Botanic Garden, 4 November 2009, A. Sabeena \& M.C. Riju TBGT 4985; Palghat, Silent valley National park, Pulippara, 13 February 2007, M.C. Riju \& al. TBGT 5019; Valakadu, 4 March 2008, Robin \& al. TBGT 5100; Thiruvananthapuram, Palode, TBGRI campus, 30 August 2008, A. Sabeena \& M.C. Riju TBGT 5262; Tamil Nadu, Kodaikanal, Samikanal, 21 October 2008, R. Nithyatharani TBGT 4371; Kuthuraiyar, 4 May 2008, R. Nithyatharani TBGT 4382; 30 June 2008, R. Nithyatharani TBGT 4384; Koruthodu, 25 March 2009, TBGT 4518; Talavakanal, 31 December 2007, R. Nithyatharani TBGT 4718: Kutharaiyar, 27 November 2007, R. Nithyatharani TBGT 4722; Samy Kanal, 2 June 2008, R. Nithytharani TBGT 4775.

About 30 species of the genus Asterina are known on the members of the family Myrtaceae. The unicellular appressoria matches with the assigned species. However, revision of this on Myrtaceae is needed.

Distribution: Karnataka, Kerala, Tamil Nadu

Asterina clematidis Hansf., Proc. Linn. Soc. New South Wales 79(3-4): 107, 1954. (Fig. 52)

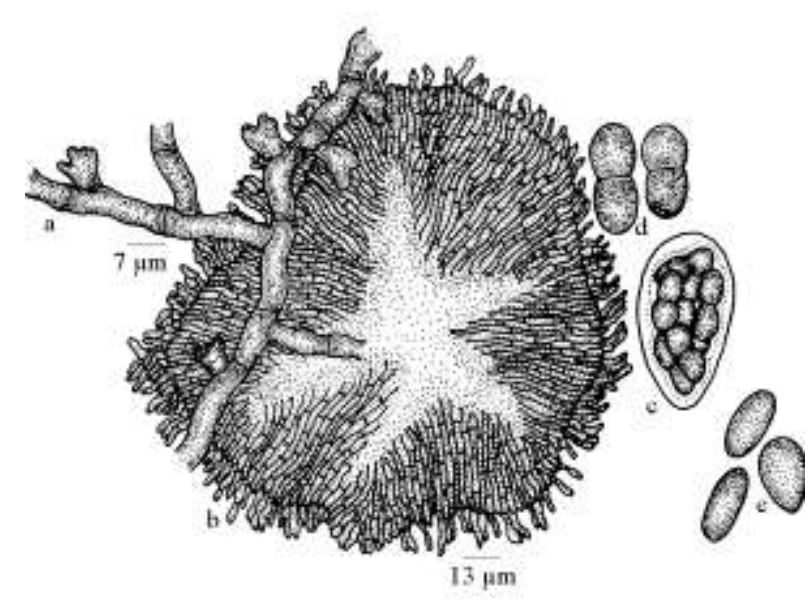

Figure 52 - Asterina clematidis

Colonies amphigenous, thin, up to 2 $\mathrm{mm}$ in diameter, confluent. Hyphae flexuous, branching alternate to irregular at acute to wide angles, cells 14-32 × 2-4 $\mu \mathrm{m}$. Appressoria alternate to unilateral, unicellular, sessile, ovate, oblong, globose, angular, sublobate, 4-11 $\times 4-8 \mu \mathrm{m}$. Thyriothecia scattered, orbicular, up to $150 \mu \mathrm{m}$ in diameter, stellately dehisced at the centre, margin crenate to fringed, fringed hyphae flexuous; asci ovate, globose, octosporous, up to $22 \mu \mathrm{m}$ in diameter; ascospores conglobate, uniseptate, 16-18 $\times 8$-10 $\mu \mathrm{m}$. pycnothyriospores scattered in the colony, ovate, pyriform, $9-13 \times 4-7 \mu \mathrm{m}$.

Material examined: Tamil Nadu, Kodaikanal, Periyakanal, on leaves of Clematis sp. (Ranunculaceae), 15 February 2005, R. Nithyatharani TBGT 5745.

This species was reported on Clematis glycinoides from Australia (Hansford, 1954a).

Asterina clusiacearum V.B. Hosagoudar \& C. Jagath Thimmaiah, sp. nov. (Fig. 53)

MycoBank 801003

Etymology: Named after the host genus

Colonies epiphyllous, thin to dense, up to $3 \mathrm{~mm}$ in diameter, confluent. Hyphae straight to substraight, branching irregular at acute angles, loosely reticulate, cells 11-15 × 3-4 $\mu \mathrm{m}$. Appressoria alternate, about $20 \%$ opposite, antrorse, subantrorse, straight to curved, 12-16 $\mu \mathrm{m}$ long; stalk cells cylindrical to cuneate, 5-6 $\mu \mathrm{m}$ long; head cells ovate, oblong to 
cylindrical, sinuately lobate to irregularly sublobate, 7-10 × 3-6 $\mu \mathrm{m}$. Thyriothecia few, scattered to connate, orbicular, stellately dehisced at the centre, up to $100 \mu \mathrm{m}$ in diameter, margine mostly fimbriate, fringed hyphae solitary, substraight to flexuous; asci few, globose, up to $20 \mu \mathrm{m}$ in diameter; ascospores conglobate, brown, uniseptate, deeply constricted at the septum, 19-25 × 10-12 $\mu \mathrm{m}$, wall smooth. Pycnothyria numerous, similar to thyriothecia, smaller; pycnothyriospores brown, globose, oval, pyriform, $15-18 \times 8-10 \mu \mathrm{m}$.

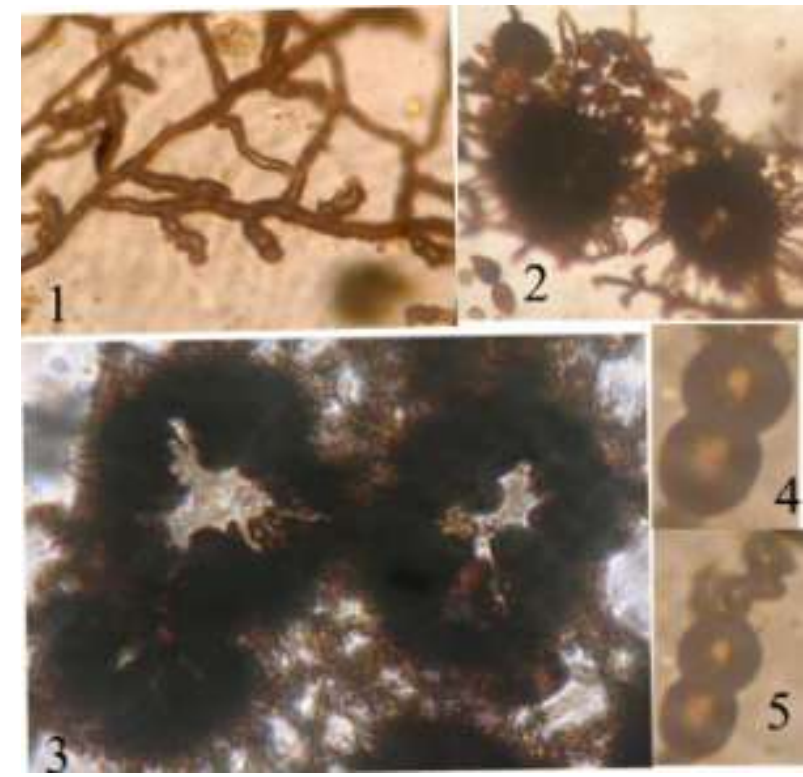

Figure 53 - Asterina clusiacearum

1.Appressoriate mycelium, 2-3. Thyriothecia, 4-5. Germinating ascospores

Material examined: Karnataka, Kodagu, Hoddur, Mandrane, on leaves of Garcinia gummigutta (L.) Robs. (Clusiaceae), 22 February 2009, C. Jagath Thimmaiah TBGT 5702 (holotype).

Asterina garciniae Hansf., A. morellae Hosag. et al. and A. garciniicola Ouyang \& Song are known on this host genus from China and India (Hosagoudar \& Abraham, 2000; Ouyang et al., 1995). However, Asterina clusiacearum differs from these in having two celled appressoria with irregularly lobate head cells.

This species was associated with Meliola garciniae and Lembosia garciniae
Asterina combreti Sydow, Engl. Bot. Jahrb. 45: 264, 1910; Hosag. \& Abraham, Indian Phytopath. 51: 389, 1998; Hosag. \& Appaiah, J. Mycopathol. Res. 43:172, 2005; Hosag., Chandraprabha \& Agarwal, Asterinales of Kerala, p.55, 2011 .

(Fig. 54)

Colonies epiphyllous, dense, crustose to velvety, up to5 $\mathrm{mm}$ in diameter, confluent. Hyphae flexuous to rarely crooked, branching irregular at acute to wide angles, loosely reticulate, cells 9-13 × 1.5-2 $\mu \mathrm{m}$. Appressoria alternate, two celled, distantly placed, mostly perpendicular to the hyphae, 6-20 $\mu \mathrm{m}$ long; stalk cells cylindrical to cuneate, $3-12 \mu \mathrm{m}$ long; head cells oval, globose, irregularly angular to sublobate, straight to uncinate, 4-7 $\times$ 7-9 $\mu \mathrm{m}$. Thyriothecia scattered, orbicular, up to $100 \mu \mathrm{m}$ in diameter, stellately dehisced at the centre, margin mostly crenate, rarely fimbriate, fringed hyphae flexuous; asci globose, octosporous, 24$30 \mu \mathrm{m}$ in diameter; ascospores brown, conglobate, 1-septate, constricted at the septum, 11-13 $\times$ 6-7 $\mu \mathrm{m}$, wall smooth. Pycnothyria smaller and similar to thyriothecia; pycnothyriospores brown, oval to ellipsoidal, straight to curved, slightly constricted in the middle, often hyaline transverse band visible in the middle, $17-20 \times 8-10 \mu \mathrm{m}$.

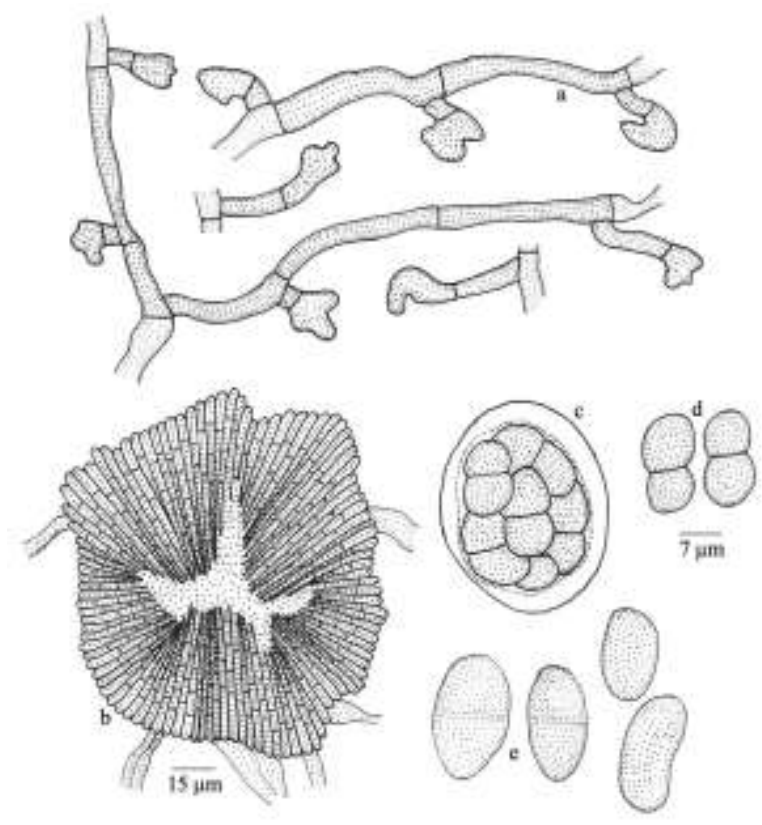

Figure 54 - Asterina combreti 
Material examined: Kerala, Thiruvananthapuram, Peppara Wildlife Sanctuary, on leaves of Calycopteris floribunda (Roxb.) Poiret (Combretaceae), 3 February 1997, V.B. Hosagoudar HCIO 42535, TBGT 172; Kollam, Shendhurney dam site, 20 October 2005, V.B. Hosagoudar HCIO 46964, TBGT 2181; HCIO 46965, TBGT 2182; Thiruvananthapuram, Palode Forest, 27 September 2006, V.B. Hosagoudar \& al. HCIO 47426, TBGT 2464; Neyyar Wild Life Sanctuary, 21 August 2007, Jacob Thomas HCIO 48149, TBGT 2885; Kottayam, Ponthenpuzha Reserve Forest, 3 March 2008, P.J. Robin HCIO 49531, TBGT 3773; Palghat, Silent Valley National Park, 23 July 2009, Jayakumar \& al. HCIO 49858, TBGT 4010; Kottayam, Mukkada, 3 March 2008, P.J. Robin HCIO 49879, TBGT 4031; Kollam, Chozhiakodu, 3 January 2011, V.B. Hosagoudar \& al. TBGT 5081; Kollam, Madathara, on leaves of Calycopteris sp., 7 July 2001, M. Kamarudeen HCIO 44291, TBGT 651; Thiruvananthapuram, TBGRI campus, on leaves of Terminalia arjuna (Roxb. ex DC.) Wight \& Arn., 20 November 2000, T. Sabu HCIO 44019, TBGT 412; Alapuzha, Vandanam, 3 October 2002, A. Manoj Kumar HCIO 44654, TBGT 936; Thiruvananthapuram, Palode, TBGRI Campus, on leaves of $T$. cuneata Roth. \{(T. arjuna (Rox. ex DC.) Wight $\&$ Arn.) $\}$ (Combretaceae), 20 November 2000, T. Sabu HCIO 44019, TBGT 412; Alapuzha, Vandanam, 3 October 2002, A. Manoj Kumar HCIO 44654, TBGT 936; Kollam, Kuzhathupuzha, on leaves of $T$. paniculata Roth, 26 December 2000, G. Rajkumar HCIO 45275, TBGT 1313; Kottayam, Vazhoor, T.P. Puram, 30 December 2005, V.Gireesh Kumar \& P.J. Robin HCIO 46809, TBGT 2150; Thiruvananthapuram, Peppara Wildlife Sanctuary, Kottoor forest, 16 February 2008, Jacob Thomas HCIO 48877, TBGT 3253; Amarakathodu, 12 October 2007, V.B. Hosagoudar \& al. HCIO 49994, TBGT 4146; Kollam, Chozhiakode, 3 January 2011, V.B. Hosagoudar \& al. TBGT 4849; 4851; Thiruvananthapuram, Palode, TBGRI campus, 12 November 2008, K. Anil Kumar TBGT 5142; 17 September 2008, K. Anil Kumar TBGT 5247; Kottayam, Thazhathuvadakara, on leaves of Terminalia sp., 29 December 2005, V.
Gireesh Kumar \& P.J. Robin HCIO 46744, TBGT 2085; HCIO 46959, TBGT 2176; Alapuzha,Vandanam, 8 December 2002, A. Manoj Kumar HCIO 44728, TBGT 1009; Kottayam, Vazhoor, T.P. Puram, 30 December, 2005, P.J. Robin HCIO 46960, TBGT 2177; Thiruvananthapuram, Nanniyode, 14 December 2005, V.B. Hosagoudar \& al. HCIO 47022, TBGT 2239; Thiruvananthapuram, TBGRI campus, 12 October 2006, A. Sabeena \& M.C. Riju HCIO 47552, TBGT 2574.

These collections showed variation in species description having longer appressoria and ascospores. Doidge (1942) stated that this is a highly variable species varying even on the same leaf. This species was associated with Asteridiella combreti (Stev.) Hansf. var. leonensis Hansf.

\section{Distribution: Kerala}

Asterina combreti Sydow var. cylindrica Kar \& Ghosh, Indian Phytopath. 39: 218, 1986.

Patches are on both surface of the leaves but vigorous growth on the upper surface, forming black, scattered, round, widely effused thin patches, up to $6 \mathrm{~mm}$ in diameter, sometimes the patches coalesce and cover almost all the leaf surface; mycelium superficial, densely reticulate, composed of dark brown, septate, much branched, wavy hyphae, 4.32-7.56 $\mu \mathrm{m}$ wide, (cells mostly 14.4$39.6 \mu \mathrm{m}$ long) giving rise to alternate or one sided (rarely opposite or irregular) appressoria at an acute to wide angle just behind the septa; appressoria are one sided or alternately arranged, unicellular, cylindrical, rounded at end, entire, straight or bent, brown, 10.08-15.2 $\times 6.84-7.56 \mu \mathrm{m}$; thyriothecia many, scattered or in groups, seated in the centre of the mycelial colony like pin heads, orbicular, 253-468 $\mu \mathrm{m}$ diameter, sometimes 2-3 fruit bodies coalesce with each other and take an irregular shape, attached with free mycelium, dimidiate with radiate scutellum, stellately dehisce at first, subsequently developing in to an aperture, dark brown, convex, hymenium simple, margin fimbriate; asci many, oval to clavate, sessile, rounded tip, 8-spored, 50.4-68.4 × 19.8-27 $\mu \mathrm{m}$, 
paraphysate; ascospores are biseriate, ellipticoblong, uniseptate, upper cell slightly larger than the lower, upper end round, lower end conic, constricted at each septum, dark brown, smooth-walled, 19.8-25.2 × 10.08-11.16 $\mu \mathrm{m}$.

West Bengal, Darjeeling, Sukha forest, on leaves of Combretum sp. (Combretaceae), 5 March 1976, IMI 215557 (holotype).

Distribution: West Bengal

Asterina congesta Cooke, Grevillea 8: 95, 1879; Hansf. \& Thirum., Farlowia 3: 305, 1948; Hosag., Balakr. \& Goos, Mycotaxon 59: 172, 1996; Hosag. Krishnan \& Abraham, New Botanist 24: 28, 1997; Hosag., Chandraprabha \& Agarwal, Asterinales of Kerala, p.57, 2011. (Figs 55a-c)

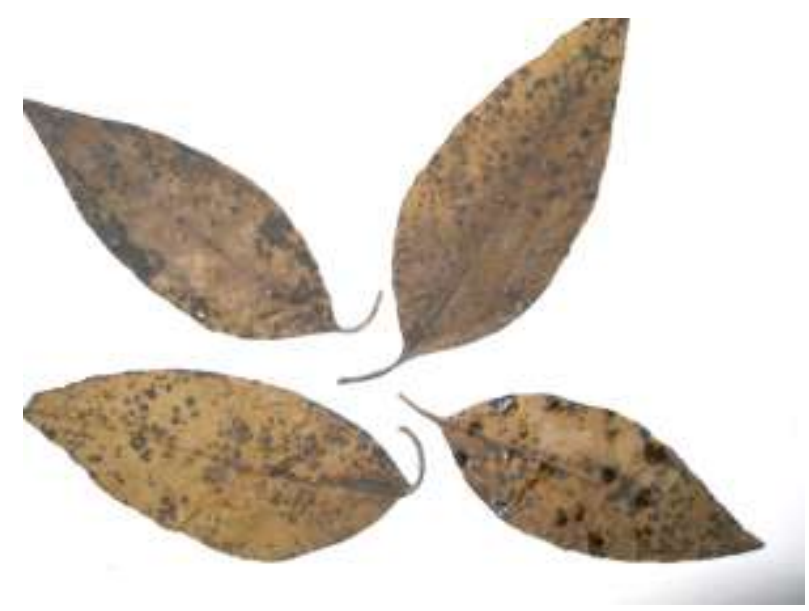

Figure 55a - Infected leaves of Santalum

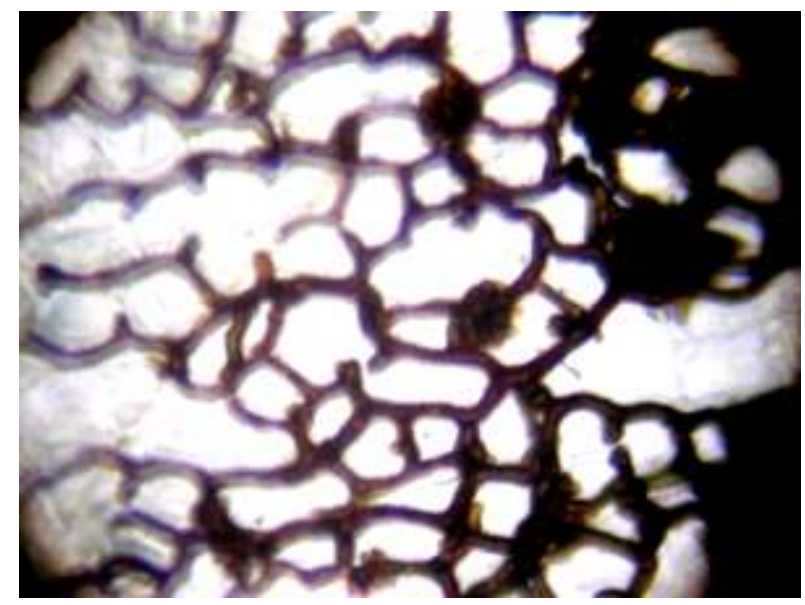

Figure 55b- Asterina congesta - mycelial net
Colonies initially epiphyllous, later on both surfaces of the leaves, caulicolous, often on tender stems, form a coating of black mat and are confluent. Hyphae straight to crooked, cells $10-15 \times 4-6 \mu \mathrm{m}$. Appressoria alternate to unilateral, unicellular, ovate to cylindrical, straight to curved, entire to sinuately lobate, 5$10 \times 4-5 \mu \mathrm{m}$. Thyriothecia scattered, loosely aggregated, often coalesced, up to $130 \mu \mathrm{m}$ in diameter; asci many, aparaphysate, globose, octosporous, bitunicate, $35-45 \times 28-40 \mu \mathrm{m}$; ascospores oblong, conglobate, uniseptate, slightly constricted at the septum, 13-26 × 9-11 $\mu \mathrm{m}$, wall smooth. Pycnothyria scattered to connate, orbicular, up to $100 \mu \mathrm{m}$ in diameter, crenate to fimbriate at the margin, stellately dehisce at the centre; Pycnothyriospores pyriform to obpyriform, cinnamon brown, 17$20 \times 8-10 \mu \mathrm{m}$, wall smooth, often with a single hyaline band at the middle.

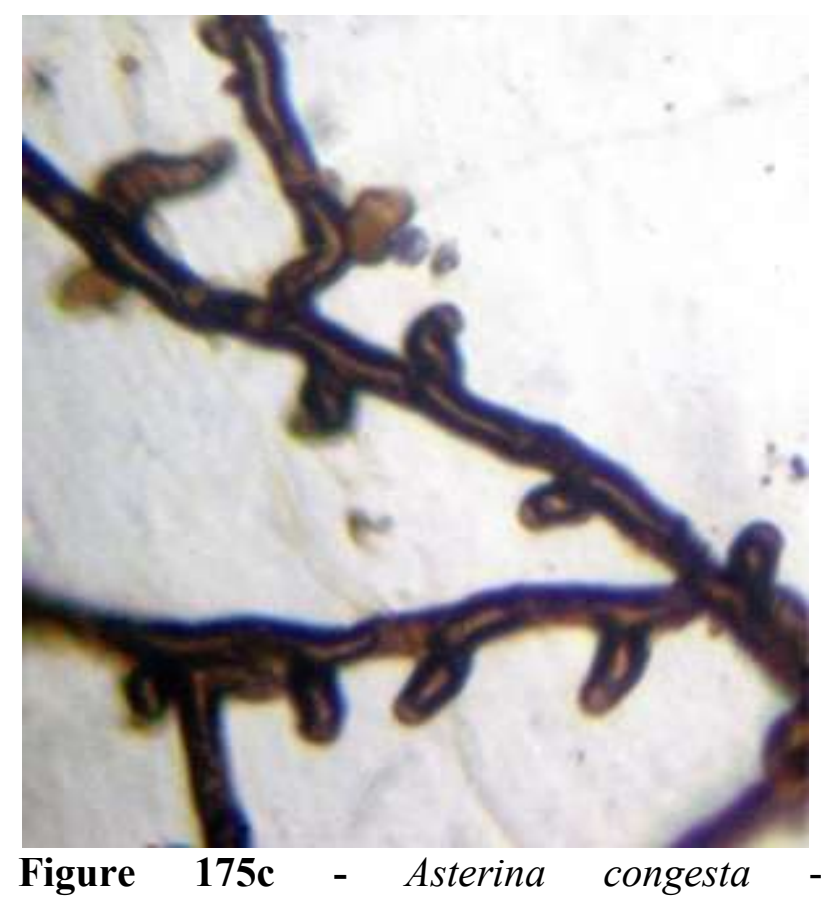

Appressoriate mycelium

Material examined: Kerala, Palghat, Ramagirikotta, on leaves of Santalum sp. (Santalaceae), 16 July 2002, A.Manoj Kumar \& H. Biju HCIO 44521, TBGT 807; Trissur, Chalakudy, Pariyaram, on leaves of Santalum album L., 22 October 2002, A.Manoj Kumar HCIO 44685, TBGT 966; Idukki, Kumili, 23 May 2003, P.A. Jose HCIO 45076, TBGT 1131; Thiruvananthapuram, Pangode, 14 
January 2003, G. Rajkumar HCIO 45089, TBGT 1144; Palode, TBGRI campus, 16 October, 2001, H. Biju HCIO 46075, TBGT 1838; 2 November, 2000, H. Biju HCIO 46100, TBGT 1863; Kollam, Tenmala, forest Nursery, 13 January 2005, V.B. Hosagoudar \& al. HCIO 45951, TBGT 1715; HCIO 45953, TBGT 1717; TBGRI Campus, Palode, 6 October, 2005, V.S. Sreelal HCIO 46969, TBGT 2186; 22 September 2005, M. Manu HCIO 46997, TBGT 2214; 22 October 2007, Mathew Dan HCIO 48239, TBGT 2977; Arboretum, 31 October 2007, K. Anil Kumar HCIO 48312, TBGT 3033; 11 June 2007, A. Sabeena \& Bijeesh HCIO 48360, TBGT 3081; 25 February 2009, A. Sabeena TBGT 4696; 21 August 2008, K. Anil Kumar TBGT 5132; 30 August 2008, A. Sabeena \& M.C. Riju TBGT 5263; Kozhikode, Malabar Botanic Garden, 16 November 2006, A. Sabeena \& al. HCIO 47405, TBGT 2443; HCIO 47593, TBGT 2615; 10 April 2008, A. Sabeena \& M.C. Riju TBGT 4795; 24 December 2007, Bijeesh HCIO 48384, TBGT 3105; 3 November 2009, M.C. Riju \& A. Sabeena TBGT 4749; Thekkadi, Periyar Tiger Reserve, Sakunthalakadu, 23 March 2006, H. Biju \& V. Gireesh Kumar HCIO 47621, TBGT 2643; Thiruvananthapuram, Govt. Botanic Garden, 3 October 2006, A. Sabeena \& G.R. Archana HCIO 47420, TBGT 2458; 12 June 2009, M.C. Riju \& A. Sabeena TBGT 4747; Wyanad, Thirunelli, 30 October 2007, A. Chandraprabha HCIO 48302, TBGT 3023; Thiruvalla, Kuttapuzha, Mar Thoma College Campus, 11 December 2007, Jacob Thomas HCIO 48309, TBGT 3030; Kannur, Mangattu Paramba, Neeliyar Kottam, 21 January 2011, M. Reshma TBGT 4899; 21 January 2011, M. Reshma TBGT 5195; 14 February 2011, M. Reshma TBGT 5184; Payyanur, Edat, 21 January 2011, M. Soumya TBGT 4946; Wayanad, Tiruneli, on leaves of Santalum sp., 15 September 2008, Robin \& al. HCIO 49805, TBGT 3957; Pulpally, 20 September 2008, Gireesh \& al HCIO 50013, TBGT 4165; Karnataka, Chitradurga, Jogimatti state forest, 17 December 2005, N.M. Ganesh babu HCIO 46818, TBGT 2159; Kodagu, Hoddur, 29 December 2008, C. Jagath Thimmaiah TBGT 5397.
This fungus is very common throughout the Western Ghats of Peninsular India. Hansford \& Thirumalachar (1948) reported this species from Karnataka. Further, this is the first species of the genus Asterina known from India (Cooke, 1884)

Distribution: Kerala, Karnataka.

Asterina crebra H. Sydow, Ann. Mycol. 11: $327,1913$.

(Fig. 56)

Colonies amphigenous, thin to subdense, up to $2 \mathrm{~mm}$ in diameter. Hyphae substraight to flexuous, branching irregular at acute to wide angles, loosely reticulate and form a net, cells 12-16 × 3-5 $\mu \mathrm{m}$. Appressoria unicellular, alternate to irregular, antrorse to retrorse, straight to curved, ovate, oblong, pyriform, entire, $8-13 \times 4-6 \mu \mathrm{m}$. Thyriothecia scattered to connate, orbicular, up to $150 \mu \mathrm{m}$ in diameter, stellately dehisced at the centre, margin mostly fimbriate, fringed hyphae small, compact; asci globose to slightly ovate, 8spored, up to $30 \mu \mathrm{m}$ in diameter; ascospores conglobate, brown, uniseptate, constricted at the septum, 20-24 × 9-11 $\mu \mathrm{m}$, wall smooth. Pycnothyria similar to thyriothecia, smaller; pycnothyriospores ovate, oblong, pyriform, 19$22 \times 11-13 \mu \mathrm{m}$.

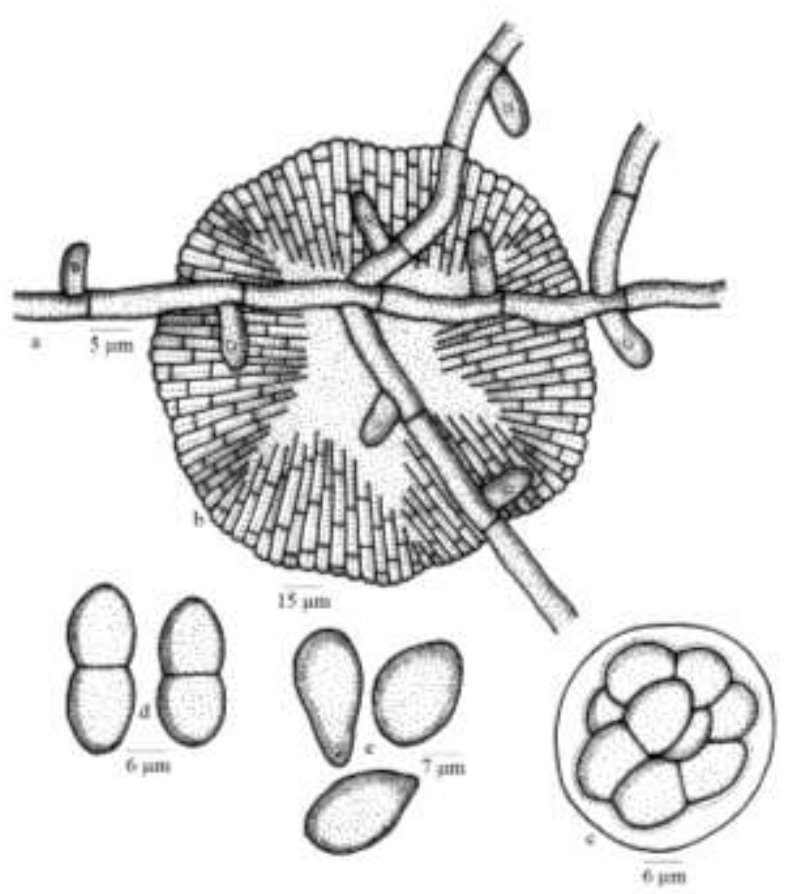

Figure 56 - Asterina crebra 
Material examined: Karnataka, Chitradurga, Jogimatti State forest, on leaves of Opilia amentacea Roxb. (Opiliaceae), 12 March 2005, N. M. Ganesh Babu HCIO 5990, TBGT 1754; 17 December 2005, N.M. Ganesh Babu HCIO 46994, TBGT 2211.

Distribution: Karnataka.

Asterina cryptocariicola Hosag., C.K. Biju \& Abraham, Indian Phytopath. 54: 137, 2001; J. Mycopathol. Res. 40:195, 2002; Hosag., Chandraprabha \& Agarwal, Asterinales of Kerala, p.59, 2011. (Fig. 57)

Colonies amphigenous, dense, up to 4 $\mathrm{mm}$ in diameter. Hyphae substraight to flexuous, branching mostly opposite at wide angles, loosely reticulate, cells 19-26 × 3-4 $\mu \mathrm{m}$. Appressoria scattered, alternate, unicellular, globose to ovoid, entire, 4-7 $\times$ 4-6 $\mu \mathrm{m}$. Thyriothecia scattered, orbicular, up to $100 \mu \mathrm{m}$ in diameter, stellately dehisced at the center, margin crenate; asci not seen; ascospores oblong, conglobate, brown, uniseptate, constricted at the septum, $12-16 \times 6-8 \mu \mathrm{m}$. Wall smooth.

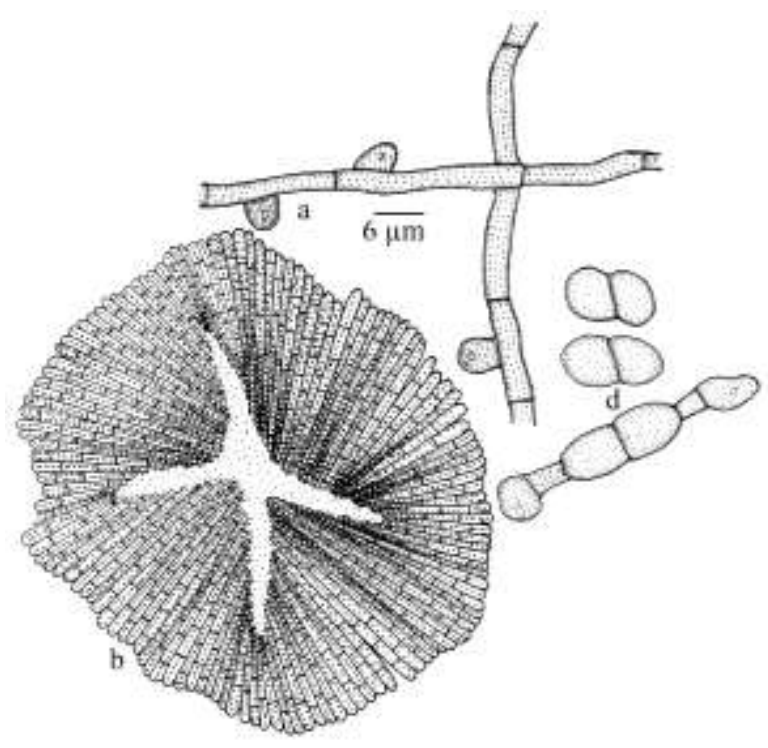

Figure 57 - Asterina cryptocariicola

Material examined: Kerala, Idukki, Rajamala, on leaves of Cryptocarya wightiana Thwaites ( $C$. bourdillonii Gamble) (Lauraceae), 25 October 1998, C.K. Biju HCIO 42961 (holotype), TBGT 236 (isotype); Kottayam,
Ponthanpuzha, Cryptocarya sp., 7 November 2007, Robin \& al. HCIO 49949, TBGT 4101; Wayanad, Perya, on leaves of Litsea floribunda (Blume) Gamble, 16 February 2009, Jacob Thomas \& al. HCIO 49224, TBGT 3463; Kannur, Aaralam Wildlife Sanctuary, 28 February 2007, Jacob Thomas \& al. HCIO 48845, TBGT 3221; Palghat, Silent valley National Park, 23 July 2009, Jayakumar \& al. HCIO 50050, TBGT 4202; Thiruvananthapuram, Peppara wild life sanctuary, on leaves of Litsea sp., 5 January, 2008, Jacob Thomas HCIO 50040, TBGT 4192; Tamil Nadu, Kodaikanal, Shembaganoor, 8 June 2006, R. Nithyatharani HCIO 47090, TBGT 2307; Pambar shola, on leaves of Litsea wightiana (Nees) Hook.f., 22 July 2006, R. Nithyatharani HCIO 47088, TBGT 2305.

Asterina cryptocaryae Cooke is known on this host genus. After examining the holotype, Stevens \& Ryan transferred it to the genus Prillieuxina because of the lack of appressoria. Asterina woodiana Doidge is known on this host genus from South Africa. The present species differs from it in having only unicellular appressoria, smaller thyriothecia and ascospores.

Distribution: Kerala, Tamil Nadu

Asterina cynanchi Hosag. \& Shiburaj, Zoos' Print J., 17: 943, 2002; Hosag., Chandraprabha \& Agarwal, Asterinales of Kerala, p.59, 2011. (Fig. 58)

Colonies amphigenous, mostly hypophyllous, dense, crustose to velvety, up to $4 \mathrm{~mm}$ in diameter, rarely confluent. Hyphae substraight, flexuous to crooked, branching irregular at acute to wide angles, form an irregular net, loosely to closely reticulate, cells 16-19 × 3-5 $\mu \mathrm{m}$. Appressoria many, alternate, unilateral, straight to curved, 11-15 $\mu \mathrm{m}$ long; stalk cells cylindrical to cuneate, often gibbous, 1.5-5 $\mu \mathrm{m}$ long; head cells ovate to globose, entire, stellately to irregularly sublobate to deeply lobate, $8-10 \times 6-11 \mu \mathrm{m}$. Thyriothecia scattered, loosely grouped to rarely connate, orbicular, up to $140 \mu \mathrm{m}$ in diameter, stellately dehisced at the centre, margin fringed, fringed 
hyphae flexuous, exappressoriate; asci ovate to globose, octosporous, $28-35 \mu \mathrm{m}$ in diameter; ascospores conglobate, brown, 1-septate, constricted at the septum, 14-16 × 7-9 $\mu \mathrm{m}$, wall smooth. Pycnothyria few, orbicular, similar to thyriothecia, smaller; pycnothyriospores pyriform, broadly rounded at one end, attenuated and truncate at the other side, 14-16 $\times 9-11 \mu \mathrm{m}$.

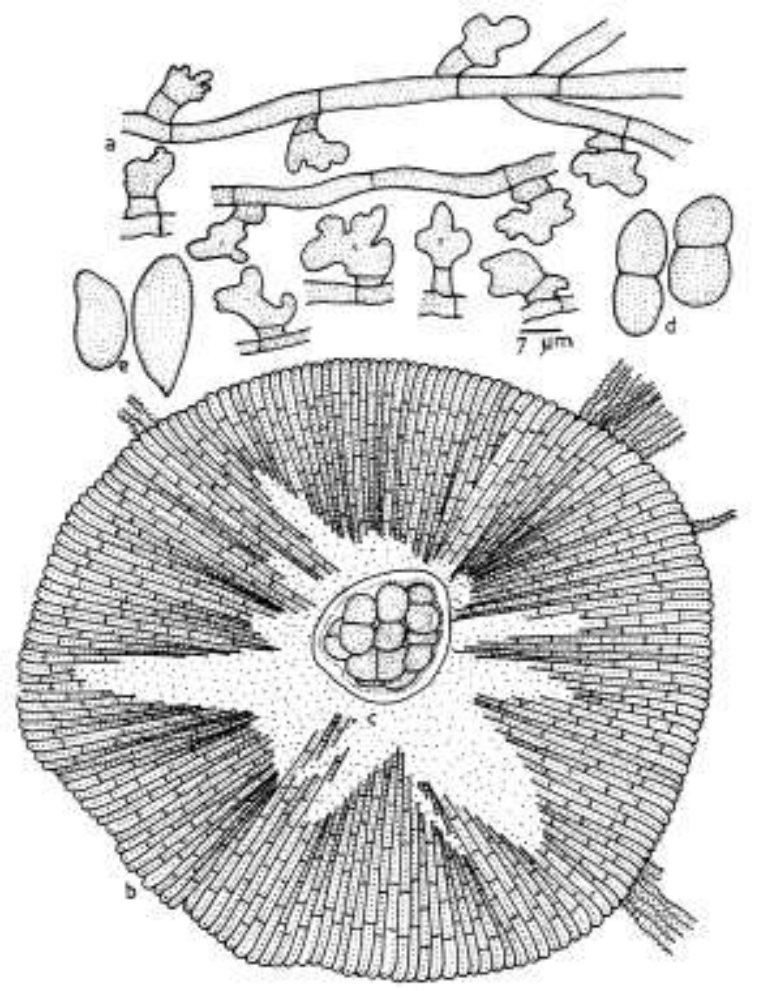

Figure 58 - Asterina cynanchi

Material examined: Kerala, Idukki, Munnar, Gravel Banks, on leaves of Cyananchum callialatum Buch. Ham. ex Wight \& Arn. (Asclepiadaceae), 18 December 2001, S. Shiburaj HCIO 44294 (holotype), TBGT 619 (isotype).

Having numerous, two celled and only alternate appressoria, the present species can be compared with Asterina concinna Sydow known on Macroscepis sp. from Venezuela. However, the species differs from it in having gibbous basal cells and sublobate to deeply lobate head cells of appressoria in contrast to entire to sublobate head cells.

Distribution: Kerala.
Asterina cynanchicola V.B. Hosagoudar \& G.R. Archana, sp.nov. (Fig. 59)

MycoBank 801042

Etymology: Named after the host genus

Colonies epiphyllous, thin to subdense, up to $2 \mathrm{~mm}$ in diameter, confluent. Hyphae substraight, flexuous to undulate, branching opposite, alternate to irregular at acute to wide angles, loosely to closely reticulate, cells 11-24 $\times 3-5 \mu \mathrm{m}$. Appressoria alternate to unilateral, straight to slightly curved, 11-21 $\mu \mathrm{m}$ long; stalk cells cylindrical to cuneate, 3-9 $\mu \mathrm{m}$ long; head cells ovate, mammiform, entire to angular, antrorse to subantrorse, $6-11 \times 4-8 \mu \mathrm{m}$. Thyriothecia scattered to grouped, orbicular, up to $200 \mu \mathrm{m}$ in diameter, stellately dehisced at the centre, margin fimbriate, fringed hyphae small, flexuous; asci few to many, globose, ovate, octosporous, $24-29 \times 16-22 \mu \mathrm{m}$; ascospores conglobate, uniseptate, slightly constricted at the septum, $17-22 \times 8-11 \mu \mathrm{m}$, wall smooth. Pycnothyria similar to thyriothecia, orbicular, smaller; pycnothyriospores unicellular, ovate, $12-16 \times 6-13 \mu \mathrm{m}$.

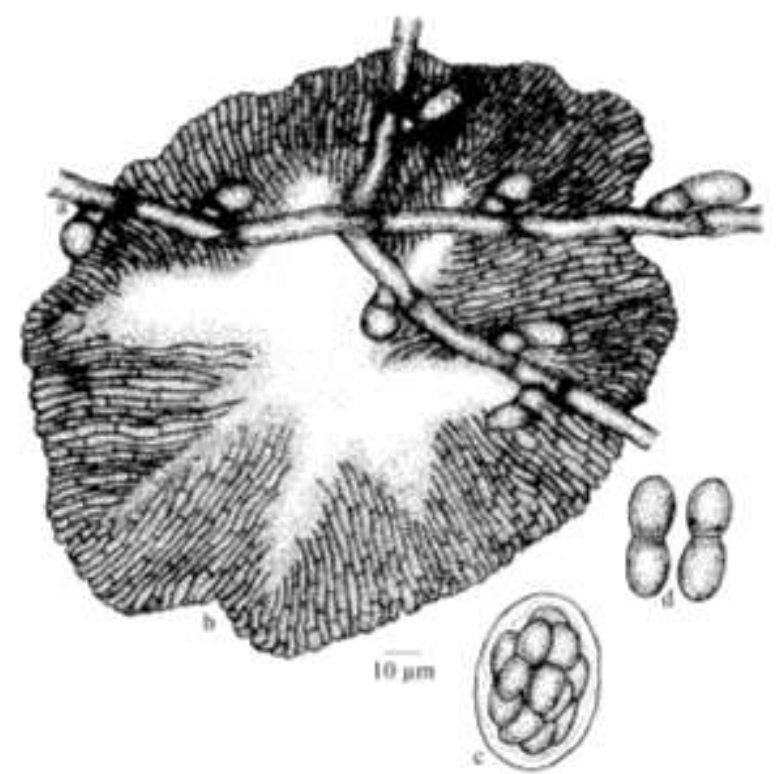

Figure 59 - Asterina cynanchicola

Material examined: Kerala, Idukki, Mannavan Shola, on leaves of Cynanchum alatum Wight \& Arn. (Ascleipidaceae), 11 May 1999, C.K.Biju TBGT 4815 (holotype).

This species differs from Asterina cynanchi Hosag. \& Shiburaj in having an entire margin of the head cells of appressoria (Hosag., 2002). 
Distribution: Kerala.

Asterina dallasica Petrak, Sydowia 8:14, 1954; Hosag., Riju \& Uma Maheswari, Indian J. Sci. \& Techn. 1:1, 2008; Hosag., Chandraprabha \& Agarwal, Asterinales of Kerala, p. 60, 2011. (Figs 60a, b)

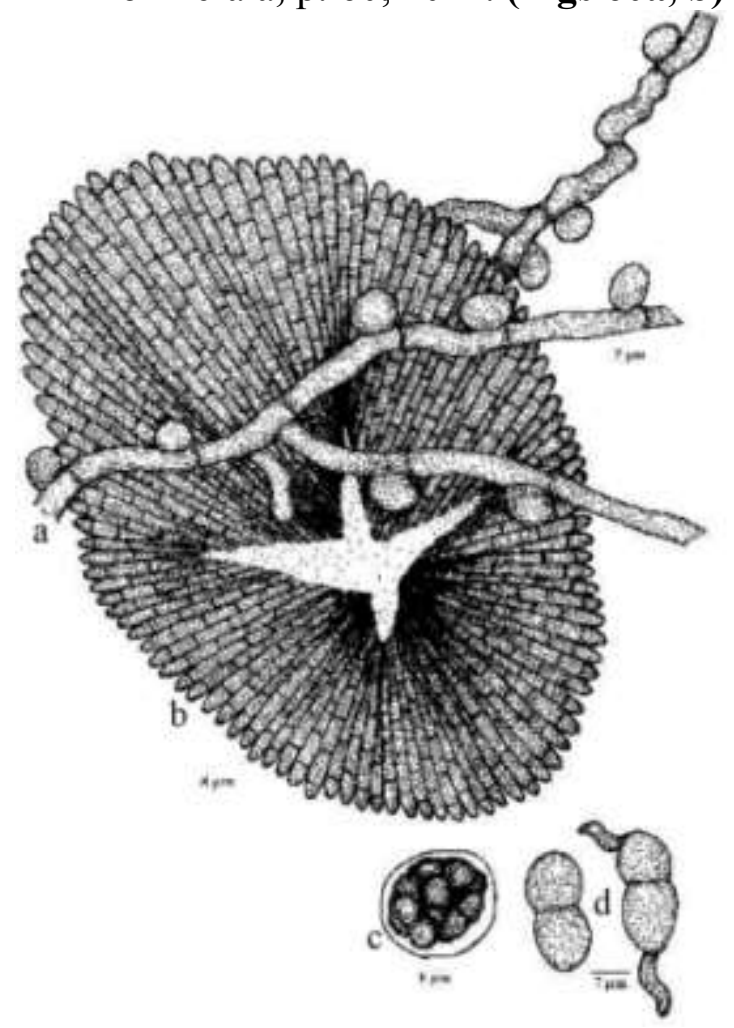

Figure 60a - Asterina dallasica

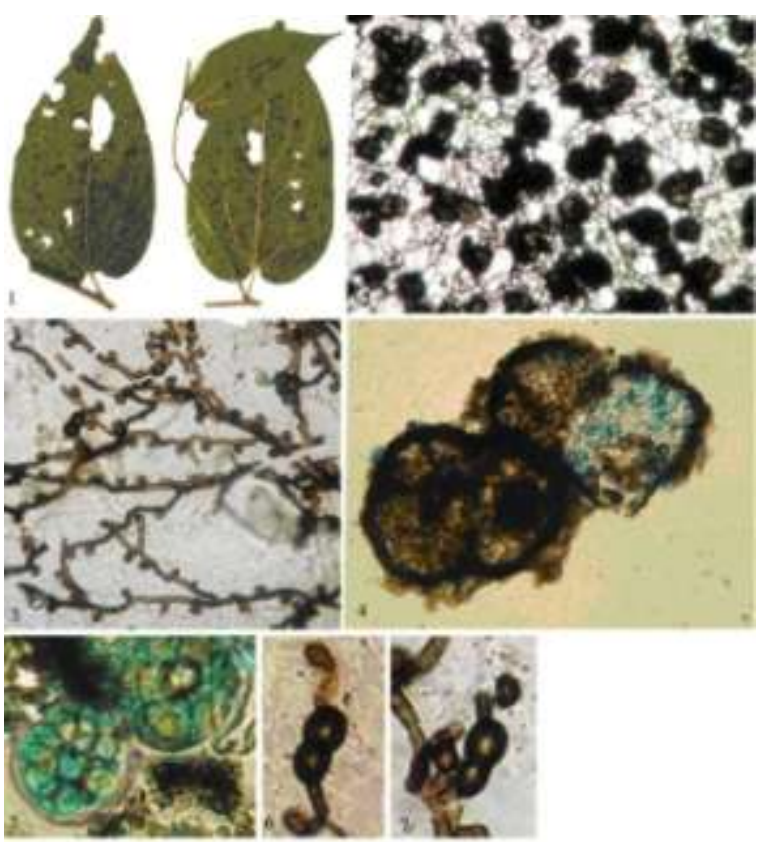

Figure 60b - Asterina dallasica

1. Infected leaves, 2. Mycelial colony with thyriothecia, 3. Appressoriate mycelium, 4. Thyriothecia with exposed asci, 6-7. Germinating ascospores.
Colonies epiphyllous, scattered, up to 3 $\mathrm{mm}$ in diameter. Hyphae straight, flexuous to crooked, branching irregular at acute to wide angles, loosely reticulate, cells 11-26 × 6$11 \mu \mathrm{m}$. Appressoria scattered, unicellular, alternate, unilateral, about $2 \%$ opposite, antrorse to subantrorse, globose, mammiform, mostly entire, rarely angular to crenately lobate, 6-11 $\times 6-11 \mu \mathrm{m}$. Thyriothecia closely scattered, orbicular, up to $115 \mu \mathrm{m}$ in diameter, stellately dehisced at the centre, margin fimbriate; asci globose, octosporous, up to $26 \mu \mathrm{m}$ in diameter; ascospores brown, conglobate, uniseptate, constricted at the septum, $17-22 \times 6-11 \mu \mathrm{m}$, wall smooth.

Material examined: Kerala, Wyanad, Manandavady, on leaves of Trema orientalis (L.) Blume (Ulmaceae), 9 November 2007, M. C. Riju HCIO 48324, TBGT 3045; Thiruvananthapuram, Peppara Wildlife Sanctuary, Bonoccord, 6 March 2008, Jacob Thomas HCIO 48875, TBGT 3251.

\section{Distribution: Kerala.}

Asterina celtidicola Henn., A. dallasica Petrak and $A$. sponiae Racib. are known on Celtis, Trema and Sponia species, respectively. A. dallasica matches well with that of assigned species. This species was known on Trema species from Borneo Islands (Petrak, 1954; Hosagoudar \& Abraham, 2000) and it reveals an affinity between the fungal flora of Wyanad and Borneo Islands.

Asterina deightonii Sydow, Ann. Mycol. 36: 172, 1938; Hosag., C.K. Biju, Abraham \& Agarwal, Indian Phytopath. 55: 497, 2002; Hosag., Zoos' Print J. 21: 2326, 2006; Hosag., Chandraprabha \& Agarwal, Asterinales of Kerala, p. 62, 2011. (Figs 61a, b)

Colonies amphigenous, mostly epiphyllous, thin to subdense, up to $2 \mathrm{~mm}$ in diameter, rarely confluent. Hyphae substraight to flexuous, branching irregular at acute angles, loosely reticulate, cells 17-21 $\times$ 4-5 $\mu \mathrm{m}$. Appressoria unicellular, many, alternate, about $1 \%$ opposite, globose to ovate, entire, rarely 


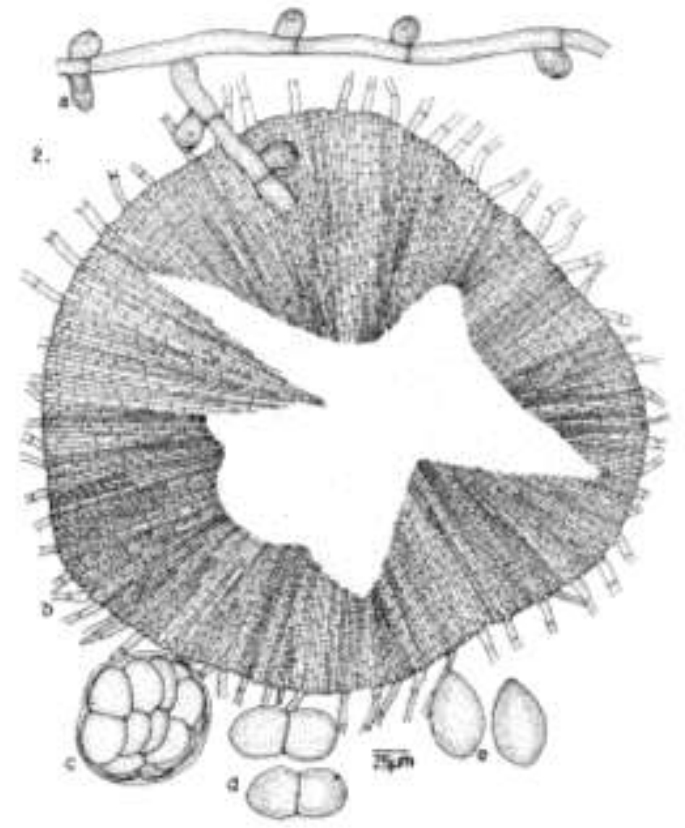

Figure 61a - Asterina deightonii
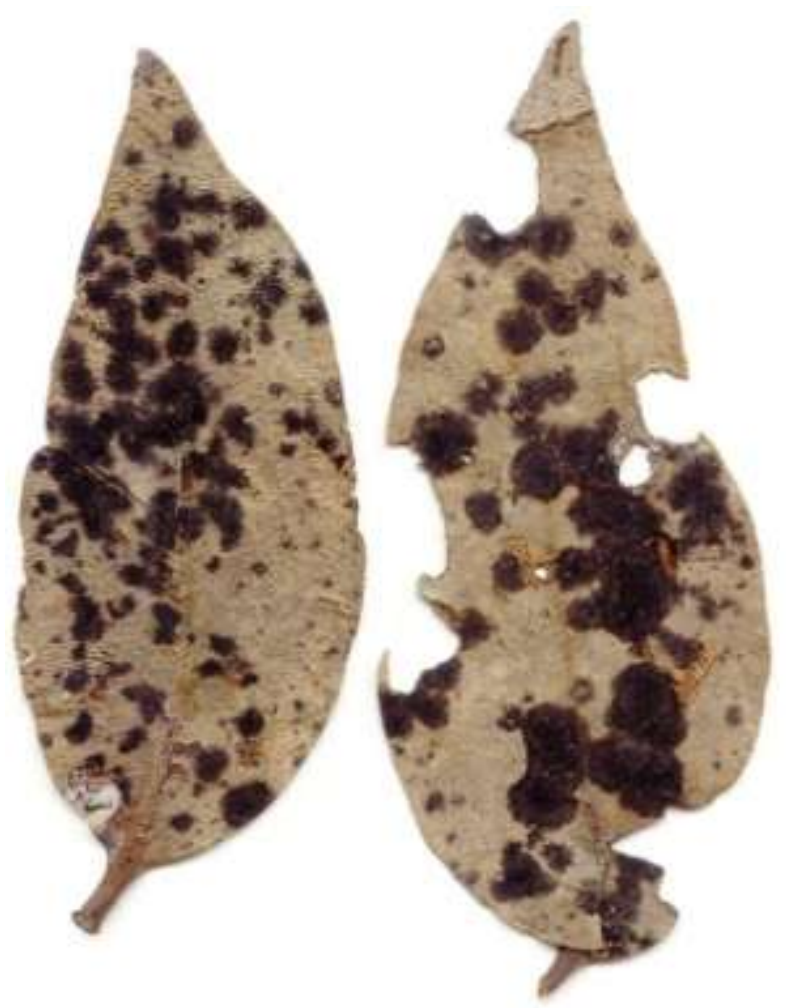

Figure 61b - Asterina deightonii-Infected leaves

slightly angular, 6-10 $\times 5-7 \mu \mathrm{m}$. Thyriothecia scattered, often loosely grouped, orbicular, up to $145 \mu \mathrm{m}$ in diameter, margin crenate to fimbriate, fringed hyphae flexuous, stellately dehisced at the centre; asci few to many, globose, octosporous, up to $40 \mu \mathrm{m}$ in diameter; ascospores brown, oblong, conglobate, uniseptate, constricted, $21-23 \times 11-13 \mu \mathrm{m}$; wall glabrous to minutely echinulate. Pycnothyria similar to thyriothecia, smaller; Pycnothyriospores few, globose to pyriform, brown, $16-18 \times 12-18 \mu \mathrm{m}$, wall smooth.

Material examined: Kerala, Idukki, Munnar, near Mannavan shola, on leaves of Dendrophthoe sp. (Loranthus sp.) (Loranthaceae), Parasitic on Tea plants, 15 January 1999, C.K. Biju HCIO 43710, TBGT 368; Thiruvananthapuram, Palode, TBGRI Campus, 16 August 2001, S. Shibu HCIO 44144, TBGT 547; TBGRI quarters, 15 December 2001, C.K. Biju HCIO 44295, TBGT 612; Idukki, Munnar, Gravel banks, 18 December 2001, S. Shiburaj HCIO 44296, TBGT 697; Idukki, Munnar, Mannavan shola, on leaves of Taxillus sp., 11 May 1999, C.K. Biju HCIO 45196, TBGT 1232; Thiruvananthapuram, Palode, TBGRI Campus, on leaves of Helixanthera sp., 10 January 2003, G. Rajkumar HCIO 45236 (p. p.), TBGT 1273 (p. p.); Thiruvananthapuram, Palode, TBGRI campus, on leaves of Loranthus falcatus L.f., 8 March 2001, H. Biju HCIO 46117, TBGT 1880; Kozhikode, Malabar Botanic Garden, Loranthus sp., 16 December 2006, A. Sabeena $\&$ al. HCIO 47603, TBGT 2625; Tamil Nadu, Kodaikanal, Mathikettan shola, Taxillus sp., 22 July 2006, Nityatharani HCIO 47695, TBGT 2717; Idukki, Chinnar Wildlife Sanctuary, on leaves of Dendrophthoe falcata (L.f.) Ettingsh (parasitic on Pterocarpus sp.), 23 April 2008, A. Chandraprabha HCIO 48421, TBGT 3142; Thiruvananthapuram, Palode, TBGRI campus, 31 March, 2009, A. Sabeena \& M.C. Riju TBGT 4867; Kerala, Kollam, Achenkovil, Amarakkathodu, Loranthus sp., 4 December 2008, V.B. Hosagoudar \& al. HCIO 49021, TBGT 3276; Palghat, Silent valley National Park, Walakkad, Onnampuzha, 2 August 2008, Jacob Thomas \& al. HCIO 49041, TBGT 3296; Wayanad,Thirunelli, 12 February 2009, Gireesh Kumar \& al. HCIO 49244, TBGT 3483; Kottayam, Plachery, 25 November 2007, Robin \& al. HCIO 49777, TBGT 3229; Palghat, Silent Valley National Park, 2 March 2009, Jayakumar \& al. HCIO 49850, TBGT 4002; Thiruvananthapuram, Palode, TBGRI campus, 18 November 2009, A. Sabeena TBGT 4298; 8 January 2010, A. Sabeena TBGT 4300; 
Pathanamthitta, Sabarigiri project area, 10 January 2007, M.C. Riju \& al. TBGT 5067; Kollam, Chozhiakodu, 3 January 2011, V.B. Hosagoudar \& al. TBGT 5068; 5095; Tamil Nadu, Kodaikanal, Kukkal Shola, Kuthirayar, 21 February 2007, R. Nithyatharani TBGT 4581; Talavakanal, 16 February 2008, R. Nithyatharani TBGT 4354; Samy Kanal, 2 June 2008, R. Nithyatharani TBGT 4714; Periyakanal, 4 January 2010, R. Nithyatharani TBGT 5252; TBGT 5254; Karnataka, Kodagu, Hoddur, 22 February 2009, C. Jagath Thimmaiah TBGT 5388.

This species was known on Loranthus leonensis from Sierra Leone, collected by F. C. Deighton no. 1378 (Sydow, 1938). Hughes (1952) proposed Asterina aburiensis and stated that the same fungus is also represented in two of Mr. F. C. Deighton's collections from Sierra Leone.

\section{Tamil Nadu}

Distribution: Karnataka, Kerala,

Asterina delicatula Sydow \& Bal, Ann. Mycol. 19:308, 1921. (Fig. 62)

Colonies epiphyllous, dense, scattered, up to $3 \mathrm{~mm}$ in diameter, confluent. Hyphae straight, substraight to flexuous, branching alternate to opposite at acute to wide angles, loosely reticulate, cells $18-25 \times 3-5 \mu \mathrm{m}$. Appressoria alternate, more scattered, two celled, straight to curved, $12-16 \mu \mathrm{m}$ long; stalk cells cylindrical to cuneate, often gibbous at the base, 2-4 $\mu \mathrm{m}$ long; head cells straight to curved, ovate to oblong, deeply and irregularly lobate, 10-12 × 7-9 $\mu \mathrm{m}$. Thyriothecia scattered, orbicular, up to $100 \mu \mathrm{m}$ in diameter, margin crenate to fimbriate ; asci few, orbicular, up to $32 \mu \mathrm{m}$ in diameter; ascospores oblong to cylindrical, conglobate, uniseptate, constricted at the septum, $18-22 \times 8-11 \mu \mathrm{m}$.

Material examined: West Bengal, Kolkata, Hoogly, on leaves of Aegle marmelos (L.) Correa (Rutaceae), 10 September 1919, P. Pal and S.N. Bal HCIO 4436 (holotype). host genus.

This is the only species known on this

Distribution: West Bengal

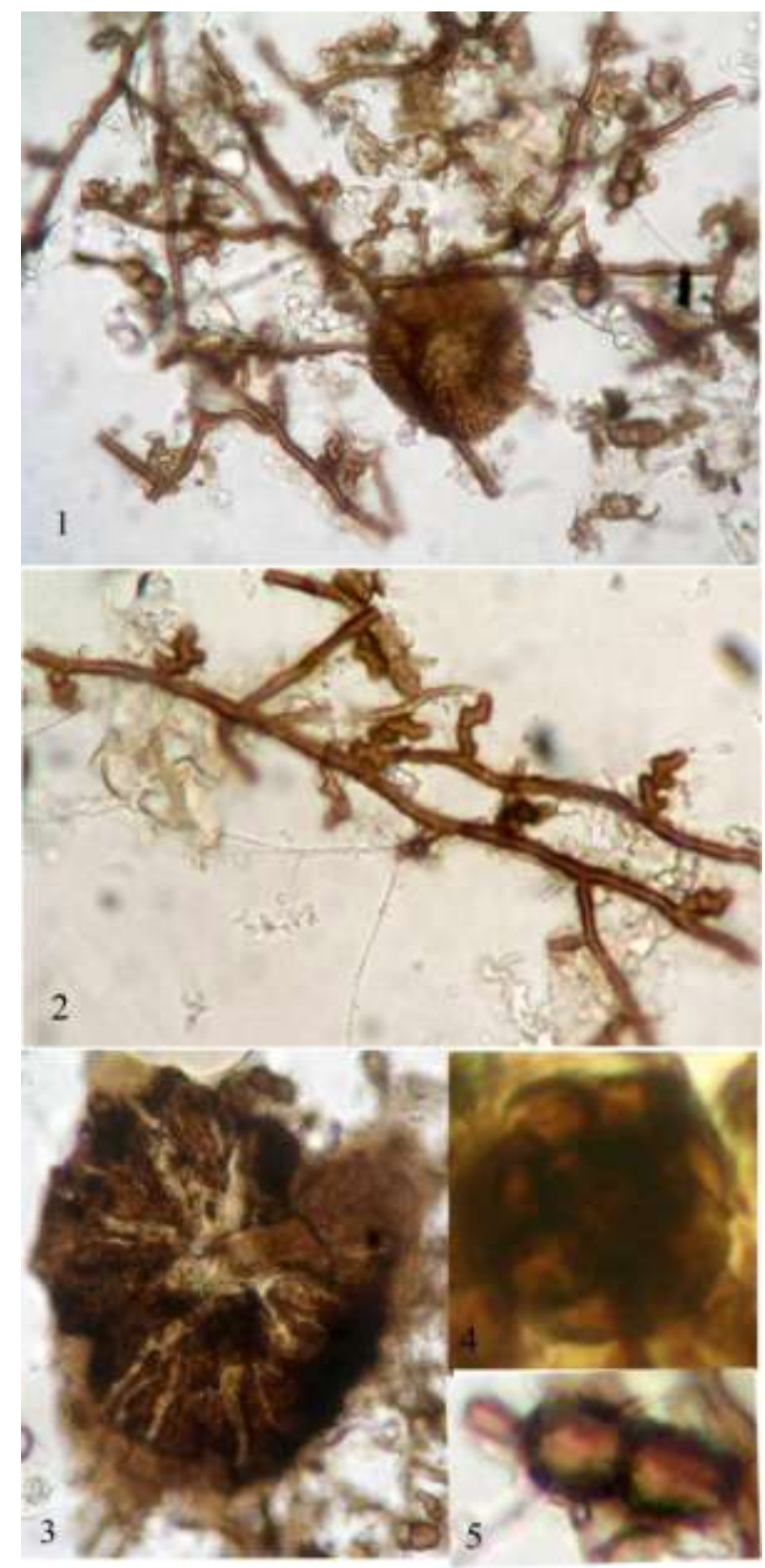

Figure 62 - Asterina delicatula

1. Mycelium with thyriothecia, 2.Two celled appressoriate mycelium, 3. Thyriothecium, 4. Ascus, 5.Ascospore

Asterina dichapetali Hansf. \& Thirum., Farlowia 3: 305. 1948.

Colonies epiphyllous, thin, black, smooth, rounded, up to $5 \mathrm{~mm}$. diameter, often confluent over the leaf. Mycelium closely reticulate, dark brown, crooked, cells 4-5 $\mu \mathrm{m}$ thick, the cells mostly $15-20 \mu \mathrm{m}$ long, branching opposite or irregular. Appressoria continuous, opposite to unilateral at wide angles, usually straight, cylindrical, with rounded apex, 5-10 $\times$ 4-5 $\mu \mathrm{m}$. Thyriothecia 
closely scattered, often 2-3 connate; rounded when single, 120-130 $\mu$ m diameter, black; basal wall of radiating pale grey or olivaceous hyphae forming a membrane; upper wall opaque, black brown, of closely compacted dark brown hyphae, 4-5 $\mu$ m thick, the cells 3-8 $\mu \mathrm{m}$ long; margin more or less fimbriate and the fringed hyphae tortuous-radiating, exappressoriate, up to $30 \mu \mathrm{m}$ long, loose, Dehiscence by 3-5 stellate fractures at the centre extending to the margin, forming broadly triangular segments becoming more or less erect around the exposed layer of asci; asci 6-8, globose, sessile, thin walled, 20-30 $\mu \mathrm{m}$ diameter, 4-8 spored, aparaphysate; ascospores opaque, dark brown, oblong with broadly rounded ends, 1-septate, constricted at the septum, 20-24 × 10-12 $\mu \mathrm{m}$, the upper cell slightly larger than the lower; wall closely echinulate. Pycnothyria similar to the thyriothecia; pycnothyriospores ovate to pyriform, chestnut-brown, smooth, 17-23 × 12$15 \mu \mathrm{m}$, with 2-3 equatorial germ pores, but no hyaline band.

Karnataka, Balehonnur, on leaves of Dichapetalum gelaniodes (Roxb.) Engl. (Dichapetalaceae), 29 April 1945, M. J. Thirumalachar 900 .

\section{Distribution: Karnataka.}

Asterina diospyri Hosag. \& C.K. Pradeep in Hosag., C.K. Biju, Abraham \& C.K. Pradeep, J. Econ. Taxon. Bot. 25: 279, 2001; Hosag., Chandraprabha \& Agarwal, Asterinales of Kerala, p.64, 2011 .

(Fig. 63)

Colonies amphigenous, mostly hypophyllous, dense, up to $5 \mathrm{~mm}$ in diameter, confluent. Hyphae straight to substraight, branching opposite to irregular at acute angles, closely reticulate, cells 17-24 $\times$ 6-7 $\mu \mathrm{m}$. Appressoria alternate, about 20\% opposite, unicellular, cylindrical to ampulliform, subantrorse, entire, broadly rounded at the apex, 11-14 $\times 5-7 \mu \mathrm{m}$. Thyriothecia scattered to grouped, orbicular, up to $56 \mu \mathrm{m}$ in diameter, stellately dehisced and later widely opened at the center, margin crenate; asci few, globose, octosporous, 20-28 $\mu \mathrm{m}$ in diameter; ascospores oblong, brown, uniseptate, constricted at the septum, 24-29 × 12-15 $\mu \mathrm{m}$, lower cell slightly smaller, wall smooth. Pycnothyriospores many, pyriform, brown, 17-21 × 14-16 $\mu \mathrm{m}$, wall smooth, Pycnothyriospores on germination produce appressoria.

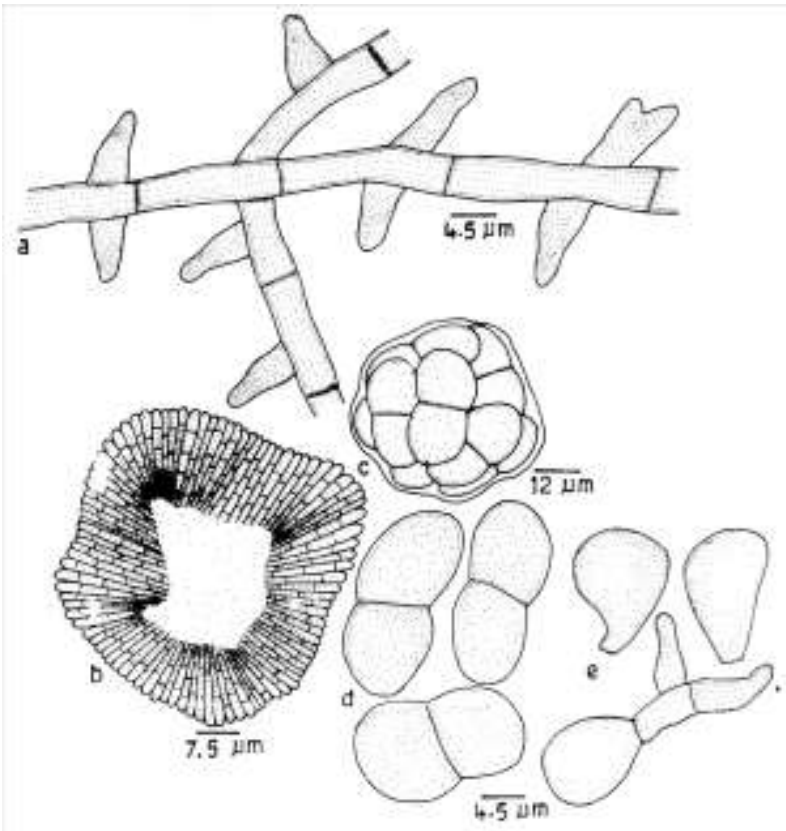

Figure 63 - Asterina diospyri

Material examined: Kerala, Thiruvananthapuram, Kallar, on leaves of Diospyros sp. (Ebenaceae), 5 October 1998, C.K. Pradeep HCIO 42939 (holotype), TBGT 210 (isotype).

Asterina clasterosporium Hughes and Asterina mabae Patil \& Pawar are known on Maba warnecke and M. nigrescense from Gold Coast and India, respectively (Hughes, 1953; Patil \& Pawar, 1989). The present new species differs from the former in having cylindrical to ampulliform appressoria and in absence of hyphomycetous conidia. It also differs from the later species in having unicellular, entire and $20 \%$ opposite appressoria.

Distribution: Kerala.

Asterina diplocarpa Cooke, Grevillea 10: 129, 1882; Hosag., Zoos' Print J. 21: 2304, 2006; Hosag., Chandraprabha \& Agarwal, Asterinales of Kerala, p. 64, 2011.

Asterina similes Cooke, Grevillea 10: 130, 1882. 
Seynesia balansae Speg. var. africana Sacc., Hedwigia 38: 133, 1899.

Asterina balansae (Speg.) Theiss. var. africana (Sacc.) Theiss., Die Gattung Asterina, p. 88, 1913. (Figs 64-65)

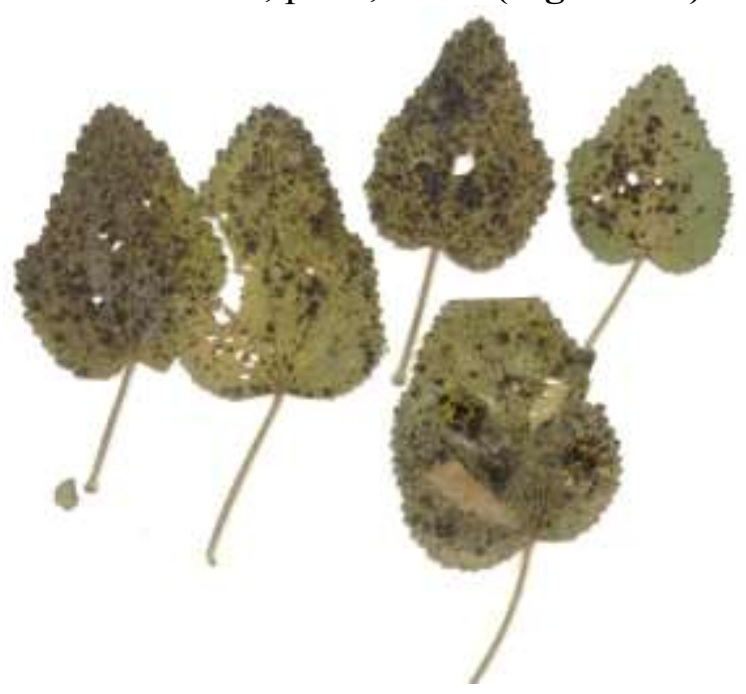

Figure 64 Asterina diplocarpa-Infected leaves

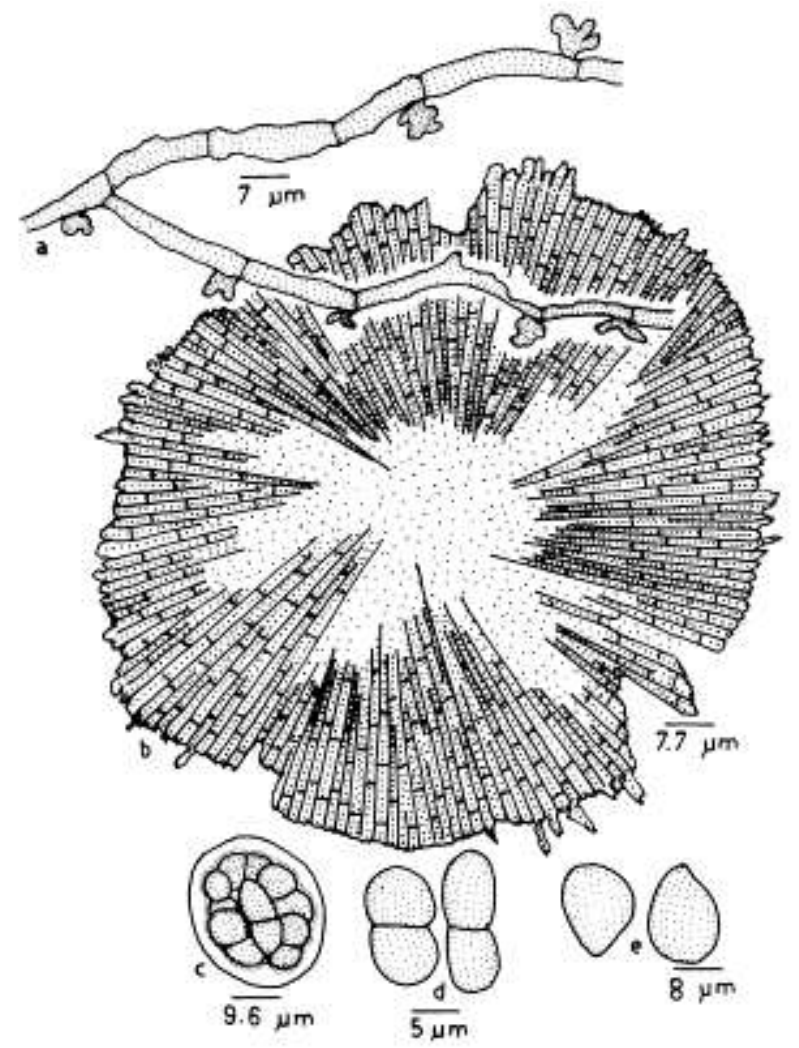

Figure 65 - Asterina diplocarpa

Colonies amphigenous, mostly epiphyllous, subdense, up to $3 \mathrm{~mm}$ in diameter, confluent. Hyphae crooked, branching irregular at acute to wide angles, loosely to closely reticulate, cells 18-22 × 3-4 $\mu \mathrm{m}$. Appressoria scattered, alternate to unilateral, antrorse to retrorse, unicellular, broad based to slightly stipitate, ovate to globose, angular, sublobate to deeply lobate, bisect, mostly irregularly trilobate, 6-10 $\times$ 7-9 $\mu \mathrm{m}$. Thyriothecia scattered to grouped, orbicular, up to $120 \mu \mathrm{m}$ in diameter, margin crenate, stellately dehisced at the centre; asci globose, octosporous, 30-32 $\mu \mathrm{m}$ in diameter; ascospores brown, conglobate, uniseptate, constricted at the septum, 14-16 $\times 7$ $9 \mu \mathrm{m}$, wall slightly crenulate. Pycnothyria many, similar to thyriothecia, smaller; pycnothyriospores pyriform, brown, 11-16 $\times 9$ $12 \mu \mathrm{m}$.

Material examined: Kerala, Thiruvananthapuram, Palode, Kushavoor, on leaves of Sida cordata (Burm. f.) Borssum (Malvaceae), Forest area, November 16 2002, A. Manojkumar HCIO 44689, TBGT 970; Kottayam, Wagamon, 5 December 2002, A. Manojkumar HCIO 44717, TBGT 998; Kollam, Venkolla, Sankily forest, on leaves of Sida glutinosa auct non. Cav., 23 November 2004, V.B. Hosagoudar HCIO 46159, TBGT 1571; HCIO 46207, TBGT 1619; HCIO 46299, TBGT 1945; Pathanamthitta, Moozhiar forest, Anathodu dam site, on leaves of Abutilon sp. (Malvaceae), 15 December 2004, V.B. Hosagoudar \& al. HCIO 46340, TBGT 1986.

This species was known from South Africa (Doidge, 1942).

Distribution: Kerala.

Asterina diplopoda Sydow, Ann. Mycol. 25: 56, 1927; Hansf., Proc. Linn. Soc. London 160: 144, 1949; Hosag., C.K. Biju \& Abraham, J. Mycopathol. Res. 40: 195, 2002; Hosag., Chandraprabha \& Agarwal, Asterinales of Kerala, p. 65, 2011.

(Fig. 66)

Colonies epiphyllous, dense, up to 3 $\mathrm{mm}$ in diameter, confluent. Hyphae crooked, branching irregular at acute to wide angles, loosely reticulate, cells 13-24 $\times$ 4-7 $\mu \mathrm{m}$. Appressoria two celled, alternate, rarely opposite, spreading, subantrorse, 11-15 $\mu \mathrm{m}$ long; stalk cells cylindrical to cuneate, $4-7 \mu \mathrm{m}$ long; head cells ovate, cylindrical, entire to sublobate, $9-11 \times 7-9 \mu \mathrm{m}$. Thyriothecia scattered, orbicular, up to $100 \mu \mathrm{m}$ in diameter, 
dehisce stellately at the center, margin crenate; asci many, globose, octosporous, up to $35 \mu \mathrm{m}$ in diameter; ascospores brown, conglobate, uniseptate, $18-24 \times 9-11 \mu \mathrm{m}$, wall smooth.

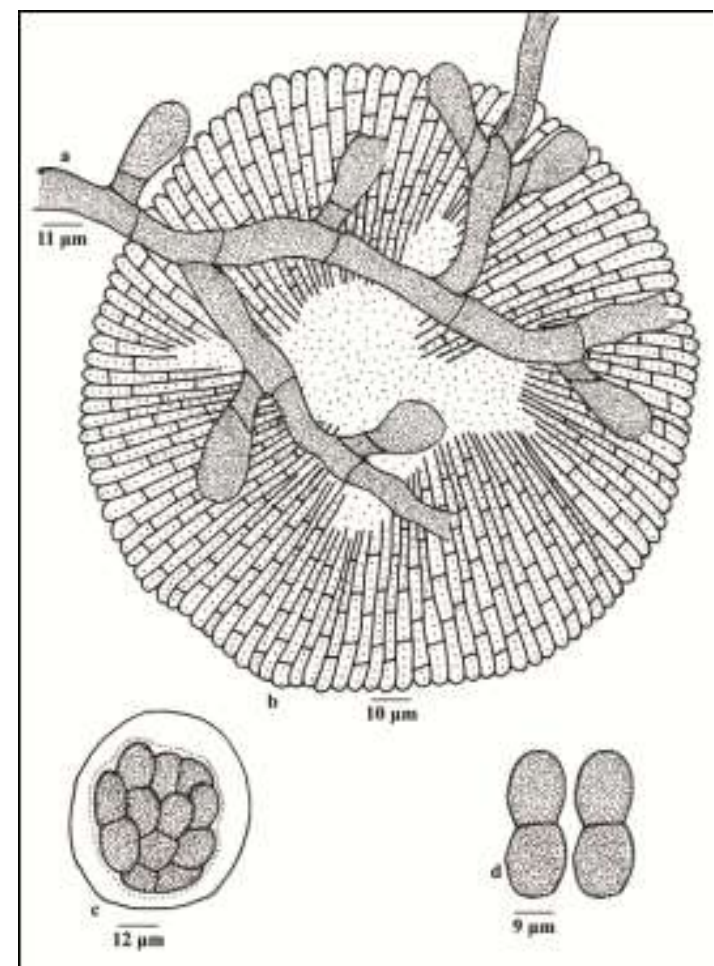

Figure 66 - Asterina diplopoda

Material examined: Kerala, Idukki, Mannavan Shola, on leaves of Solanum ferox L. (Solanaceae), 23 September 1998, C.K. Biju HCIO 42960, TBGT 253.

Asterina diplopoda differs from Asterina balakrishnanii Hosag. in having two celled appressoria.

Distribution: Kerala

Asterina disciferae Hosag. in Hosag., Balakr. \& Goos, Mycotaxon 59: 172, 1996.

(Fig. 67)

Colonies foliicolous, epiphyllous, dense, crustose, up to $2 \mathrm{~mm}$ in diameter. Hyphae carbonaceous black, flexuous to crooked, branching opposite to irregular at acute angles, loosely to closely reticulate, cells 24-38 × 6-8 $\mu \mathrm{m}$. Appressoria carbonaceous black, stipitate, two celled, antrorse to spreading, 12-16 $\mu \mathrm{m}$ long; stalk cells cylindrical to cuneate, 3-7 $\mu \mathrm{m}$ long; head cells globose, ovate, entire, 9-13 $\times$ 9-12 $\mu \mathrm{m}$.
Thyriothecia scattered, rarely connate, orbicular, up to $250 \mu \mathrm{m}$ in diameter, dehisce stellately at the center, dehiscence extending up to margin, margin crenate to fimbriate, fringed hyphae exappressoriate, tortuous; asci eight spored, initially globose, slightly ovate at maturity, 60-72 $\mu \mathrm{m}$ in diameter; ascospores cinnamon brown, conglobate, oblong, 1-septate, deeply constricted at the septum, 34-41 × 15-19 $\mu \mathrm{m}$, lower cell slightly rounded and upper cell ovate, wall smooth.
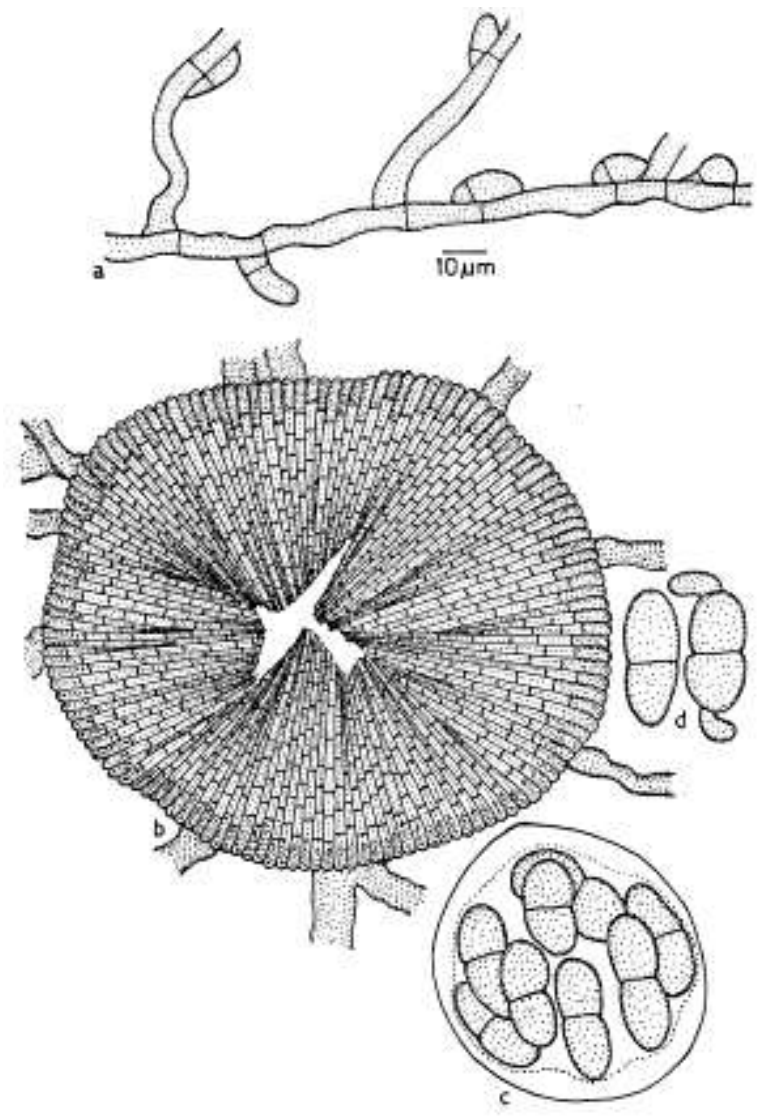

Figure 67 - Asterina disciferae

Material examined: Tamil Nadu, Coimbatore, Anamalai, near Nirar dam, Nooradi settlement, on leaves of Eugenia discifera Gamble (Myrtaceae), 26 December 1990, V.B. Hosagoudar HCIO 30959 (holotype).

This species is close to Asterina bukobensis Hansf. and A. pemphidioides Cooke (Hansford, 1946; Doidge, 1942) in having 2celled appressoria, and ascospores up to $40 \mu \mathrm{m}$ long. It differs from the former species in having smaller but normal two-celled appressoria with entire head cells, and from the 
latter species in having dense epiphyllous colonies with sinuous to crooked hyphae. It also differs from Asterina jambolana Kar \& Maity in having epiphyllous colonies and larger ascospores.

However, the genus Asterina on Myrtaceae is to be revised.

Distribution: Tamil Nadu.

Asterina dissiliens (Sydow) Doidge, Bothalia 4: 287, 1942; Hosag. \& Goos, Mycotaxon 52: 467, 1994; Hosag., Balakr. \& Goos, Mycotaxon 60: 174, 1996.

Asterinella dissiliens Sydow, Ann. Mycol. 22: 425, 1924.

Parasterina reticulata Doidge, Bothalia 1: 200, 1924 \{(non Asterina reticulata (Kalch. \& Cooke) Doidge)\}.

(Fig. 68)

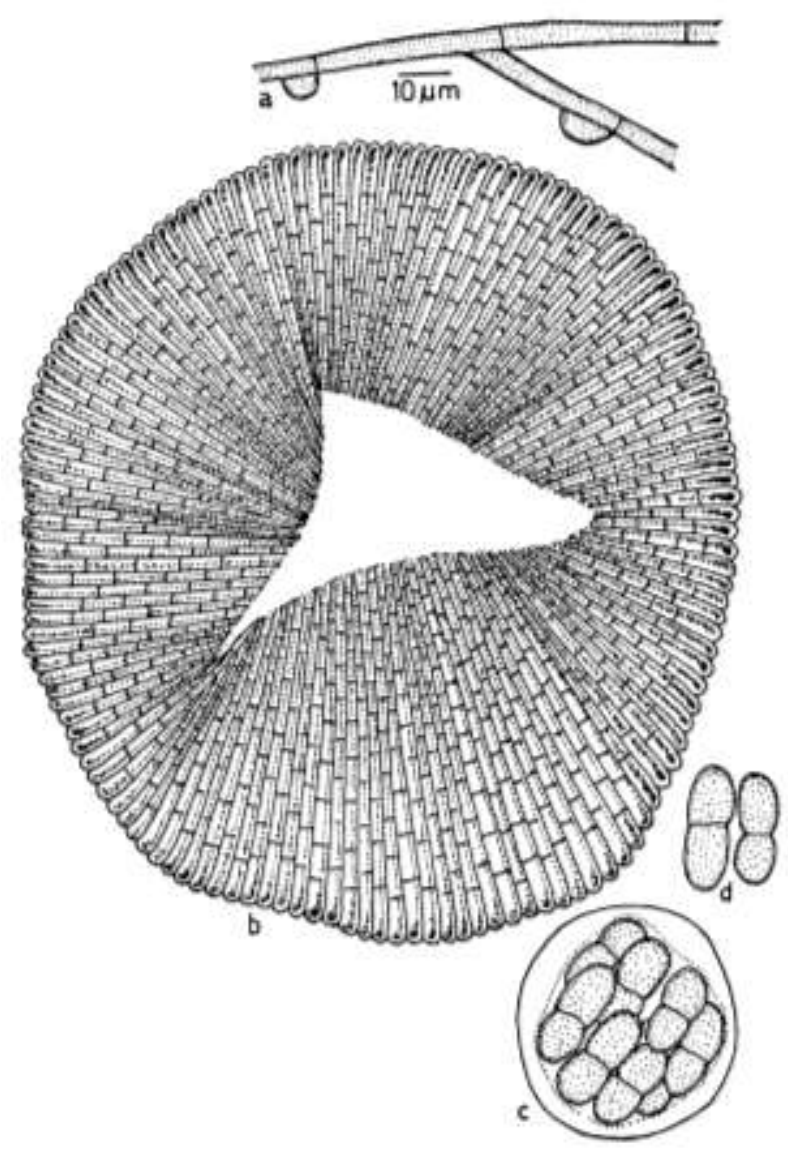

Figure 68 - Asterina dissiliens

Colonies amphigenous, mostly epiphyllous, dense, orbicular, up to $5 \mathrm{~mm}$ in diameter, rarely confluent. Hyphae straight to flexuous, branching alternate to opposite, loosely to closely reticulate, cells $12-22 \times 3-5$ $\mu \mathrm{m}$. Appressoria very few, mammiform, entire to sublobate, 4-7 $\times 6-10 \mu \mathrm{m}$. Thyriothecia numerous, centrally situated in the colonies, loosely to closely grouped, carbonaceous black, circular, up to $265 \mu \mathrm{m}$ in diameter, dehisce stellately at the center, margin crenate to fimbriate, fringed hyphae numerous, tortuous; asci many, eight spored, initially globose, slightly ovoid at maturity, 43-47 × 21-28 $\mu \mathrm{m}$; ascospores deep brown, conglobate, 1- septate, deeply constricted at the septum, 24-28 $\times 8-10$ $\mu \mathrm{m}$.

Material examined: Tamil Nadu, Salem, Yercaud, Kaka sholai, on leaves of Pleurostylia opposita (Wall.) Alston (Celastraceae), February 1992, A. A. Ansari HCIO 40848; Madurai, Pachakoomachi Hills, on leaves of Maytenus ovata (Wallich ex Wight \& Arn.) Loes. (Celastraceae), 28 January 1991, V. Lakshmanan HCIO 30960; Maharashtra, Pune, Bhimasankar wildlife sanctuary, Rai forest, 11 February 2001, K. Ravikumar HCIO 44705, TBGT 986; Mahabaleswar, on leaves of Gymnosporia rothiana (Walp) Lawson (Celastraceae), 1 January 2009, V.B. Hosagoudar \& al. HCIO 49446, TBGT 3691; HCIO 49448, TBGT 3693; HCIO 49450, TBGT 3695.

Careful observation is needed to locate the appressoria.

Distribution: Tamil Nadu, Maharashtra

Asterina dhivaharanii Hosag. \& Nithyatharani, J. Sci. Environ. Technnov. 4: 46, 2010.

(Fig. 69)

Colonies epiphyllous, thin, up to $2 \mathrm{~mm}$ in diameter, confluent. Hyphae substraight to flexuous, branching opposite to alternate at acute to wide angle, loosely reticulate, cells 10$12 \times \quad 8-12 \mu \mathrm{m}$. Appressoria alternate, unicellular, entire, pyriform, globose, cylindrical, stellately lobate, often bifid, 10-12 $\times 8-12 \mu \mathrm{m}$. Thyriothecia scattered to connate, orbicular, up to $96 \mu \mathrm{m}$ in diameter, dehisced stellately at the center, margin crenate; asci many, globose, eight spored, 32-35 $\mu \mathrm{m}$ in 
diameter; ascospores conglobate, 1-septate, slightly constricted at the septum, 16-19 $\times 7-9$ $\mu \mathrm{m}$, wall smooth.

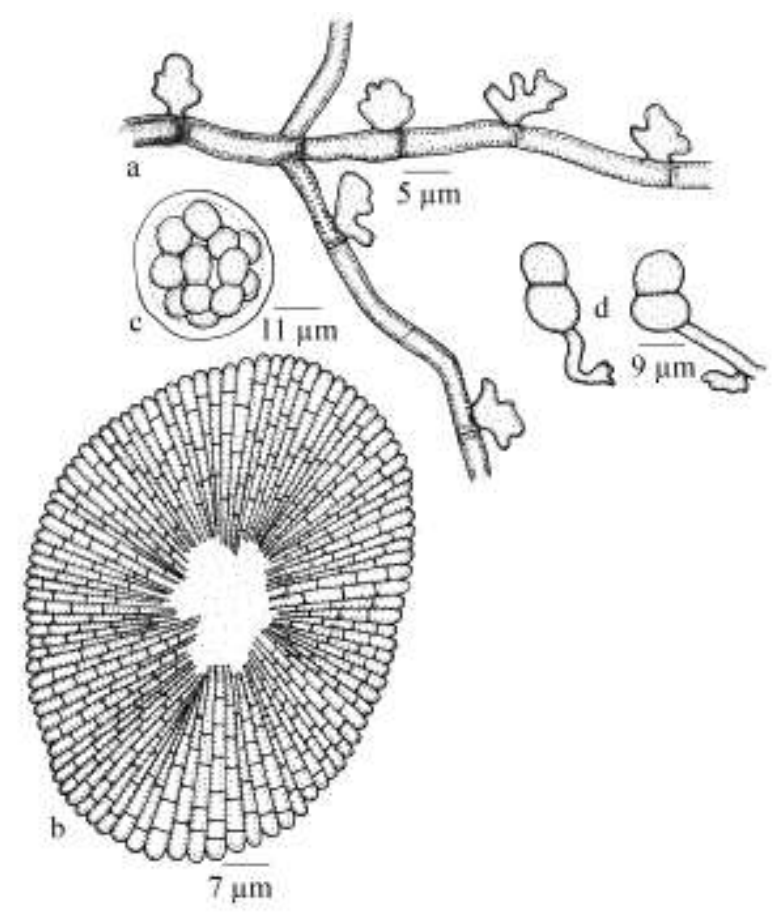

Figure 69 - Asterina dhivaharanii

Material examined: Tamil Nadu, Kodaikanal, Kukkal shola forest, on leaves of Impatiens viscida Wight (Balsaminaceae), 6 August 2007, R. Nithyatharani TBGT 4261 (holotype); Periya Kanal, 19 November 2007, R. Nithyatharani TBGT 4479; 6 February, 2008, R. Nithytharani TBGT 4756; Samykanal, Kodaikanal, 2 June 2008, R. Nithytharani TBGT 4753; Kuthirayar, Kukkal Shola, Kodaikanal, 30 June 2008, R. Nithytharani TBGT 4770; 3 March 2008, R. Nithytharani TBGT 4772.

This is the only species of the genus Asterina known here on the members of the family Balsaminaceae (Doidge, 1942; Hosagoudar \& Abraham, 2000; Stevens \& Ryan, 1939). However, Asterina balsamina Peck. in herb. is Dimeriella balsamicola (Peck) Pett. (Chen, 1974).

Distribution: Tamil Nadu

Asterina drimycarpi Kar \& Maity, Trans. Brit. Mycol. Soc. 54: 435, 1970.

On both surface of the leaves, but very few on the lower surface, forming black, scattered, orbicular, thin patches, up to $5 \mathrm{~mm}$ in diameter, sometimes 2-3 patches coalesce producing an irregular shape; mycelium superficial and loosely reticulate, composed of dark brown, septate, branched, straight hyphae, 5-6.6 $\mu \mathrm{m}$ wide, (cells mostly 14-36 $\mu \mathrm{m}$ long) giving rise to alternate branches (rarely one sided) at an acute angle and appressoria at an acute to wide angle just behind the septa. Appressoria alternately arranged, unicellular, cylindrical, rounded at the end, entire, straight, brown, 13-18 × 4.9-6.6 $\mu \mathrm{m}$; Thyriothecia many, scattered or in groups, seated in the mycelial colony like pin heads, orbicular, 231-412 $\mu \mathrm{m}$ in diameter, sometimes 2-3 fruit bodies coalesce with each other and take an irregular shape, attached with free mycelium, dimidiate with radiate scutellum, stellately dehisce at first, subsequently developing into widely opened aperture, dark brown, convex, hymenium simple, margin fimbriate; asci many, arranged towards the periphery of the fruit body, oval to clavate, sessile, tip round, 8-spored, 49.5-67.6 $\times$ 21.5-24.8 $\mu \mathrm{m}$, paraphysate; ascospores biseriate, elliptic-oblong, uniseptate, upper cell slightly larger than the lower, upper end round, lower end conic, constricted at the septum, dark brown, smooth-walled, 24.7-27.2 × 9.9-13.2 $\mu \mathrm{m}$.

West Bengal, Darjeeling, Tung (2286 $\mathrm{m})$, on leaves of Drimycarpus racemosus Hook. f. (Anacardiaceae), 12 May 1967, IMI 134491 (holotype).

Distribution: West Bengal

Asterina elaegni (Sydow) Sydow \& Petrak, Ann. Mycol. 29: 225, 1931; Hansf. \& Thrium., Farlowia 3: 306, 1948.

(Fig. 70)

Colonies epiphyllous, thin to subdense, up to $3 \mathrm{~mm}$ in diameter, rarely confluent. Hyphae straight to substraight, branching alternate to irregular at acute to wide angles, loosely reticulate, cells 25-42 $\times 3-4 \mu \mathrm{m}$. Appressoria scattered, alternate to unilateral, distantly placed, subglobose, entire, broad based, 5-7 $\times 5-10 \mu \mathrm{m}$. Thyriothecia scattered to connate, orbicular, up to $350 \mu \mathrm{m}$ in diameter, margin crenate to fimbriate, stellately dehisced at the centre; asci numerous, octosporous, oval, 
globose, 42-62 × 12-30 $\mu \mathrm{m}$; ascospores, conglobate, 1-septate, constricted at the septum, 20-22 × 9-11 $\mu \mathrm{m}$, wall smooth.

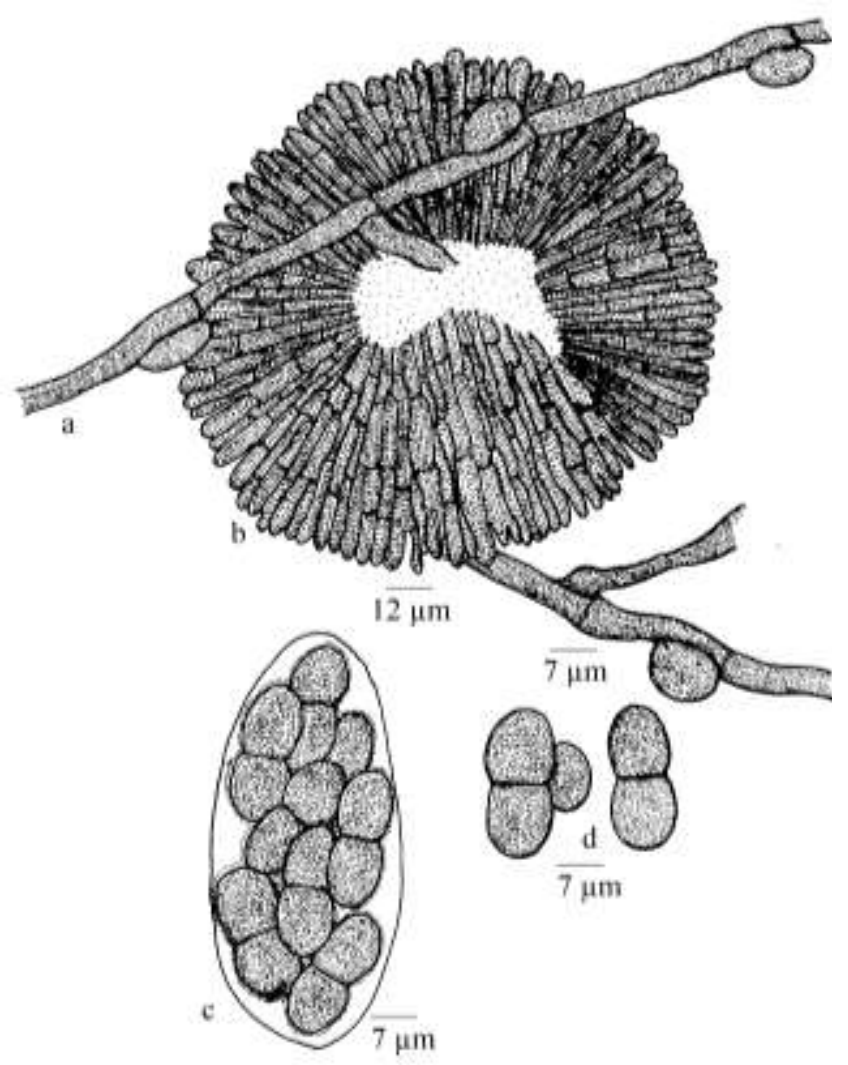

Figure 70 - Asterina elaegni

Material examined: Tamil Nadu, Kodaikanal, Periyakanal, on leaves of Elaegnus kologa Schlecht. (Elaegnaceae), 6 January 2010, TBGT 5739.

This species was collected from Karnataka on August 1948, M.J. Thirumalachar (Hansford \& Thirumalachar 1948).

Distribution: Karnataka, Tamil Nadu.

Asterina elaeocarpicola Hansf., Reinwardia 3: 131, 1954; Hosag. \& Goos, Mycotaxon 59: 154, 1996; Hosag., Zoos' Print J. 18: 1282, 2003; 21: 2327, 2006; Hosag., H. Biju \& Appaiah, J. Mycopathol. Res. 44: 7, 2006; Hosag. J. Appl. \& Nat. Sci. 1(1); 29, 2009; Hosag., Chandraprabha \& Agarwal, Asterinales of Kerala, p. 67, 2011.

(Fig. 71)
Colonies amphigenous, mostly hypophyllous, subdense, up to $3 \mathrm{~mm}$ in

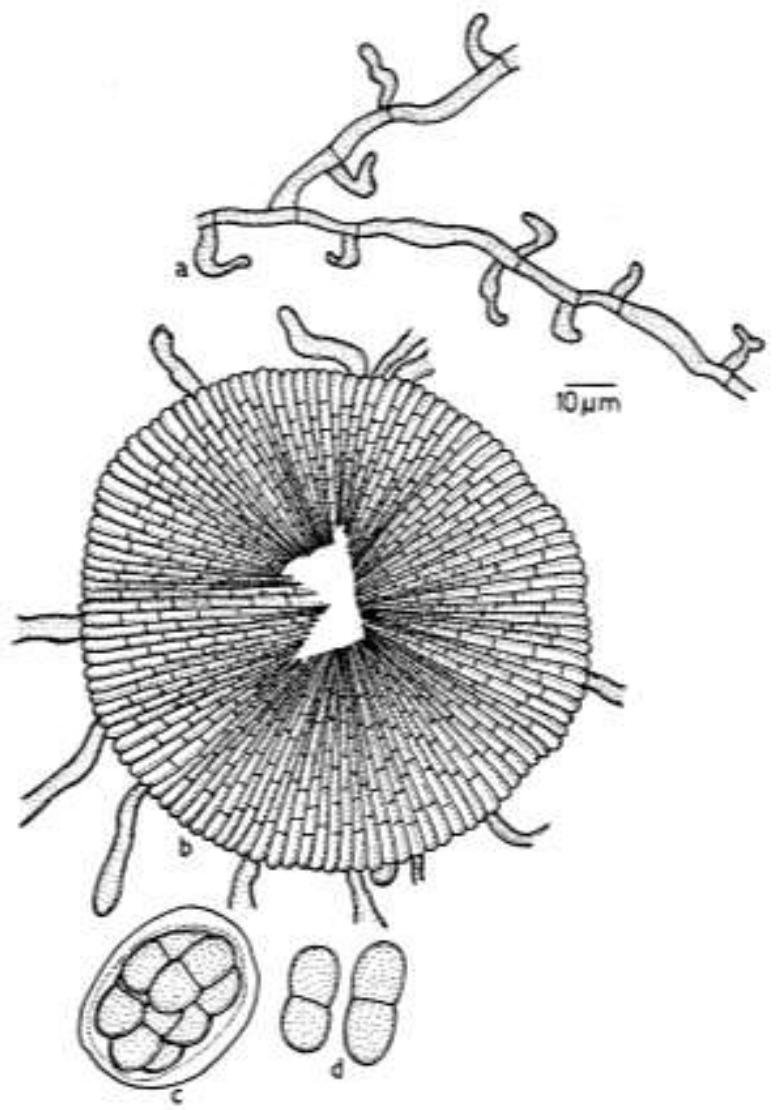

Figure 71 - Asterina elaeocarpicola

diameter, confluent and cover the entire lower surface of the leaves. Hyphae sinuous to crooked, branching irregular at acute angles, loosely reticulate, cells $15-19 \times 3-5 \mu \mathrm{m}$. Appressoria unicellular, mostly alternate, rarely opposite, ovate, ampulliform, straight, flexuous, uncinate irregularly, rarely forked, 9-19 × 3-5 $\mu \mathrm{m}$. Thyriothecia closely scattered and often connate, orbicular, up to $186 \mu \mathrm{m}$ in diameter, margin crenate, rarely fimbriate, dehisce stellately at the center; asci many, octosporous, globose, $40-44 \mu \mathrm{m}$ in diameter; ascospores conglobate, brown, 1- septate, 24-28 × 9-13 $\mu \mathrm{m}$.

Material examined: Tamil Nadu, Tirunelveli, Kakachi forest, on leaves of Elaeocarpus munronii (Wight) Masters (Elaeocarpaceae), 21 February 1994, V.B. Hosagoudar HCIO 41630; Kerala, Pathanamthitta, Mozhiar Forest, Pachakanam, 15 December 2004, V.B. Hosagoudar \& al. HCIO 46045, TBGT 1808; Pathanamthitta, Mozhiar Forest, 14 December 2004, V.B. Hosagoudar \& al. HCIO 46047, TBGT 1810; 
Pathanamthitta, Mozhiar Forest, Kakki Dam, 14 December 2004, V.B. Hosagoudar \& al. HCIO 46288, TBGT 1934; Palghat, Silent valley National Park, Cheriya Walakkad, 2 August 2008, Jacob Thomas \& al. HCIO 49234, TBGT 3473. Karnataka, Coorg, Talacauveri, Bramhagiri, MPCA, on leaves of Elaeocarpus sp., 13 November 2003, V.B. Hosagoudar \& al. HCIO 45614, TBGT 1357; Madikeri, Jodupal, 12 November 2003, V.B. Hosagoudar \& al. HCIO 45679, TBGT 1426; Coorg, 24 November 2008, V.B. Hosagoudar \& al. HCIO 49147, TBGT 3402.

This species was described by Hansford (1954) from Indonesia. It differs from other species known on the members of the family Elaeocarpaceae in having ovate ampulliform, flexuous, uncinate and forked appressoria.

Distribution: Kerala, Karnataka, Tamil Nadu.

Asterina elaeocarpi Sydow var. ovalis Kar \& Maity, Indian Phytopath. 39: 218, 1986; Hosag., Balakr. \& Goos, Mycotaxon 60: 175, 1996; Hosag., J. Appl. \& Nat. Sci. 1(1): 29, 2009; Hosag., Chandraprabha \& Agarwal, Asterinales of Kerala, p. 68, 2011. (Figs 72-73)

Colonies epiphyllous, thin to subdense, up to $2 \mathrm{~mm}$ in diameter, confluent and cover the entire upper surface of the leaves. Hyphae straight to substraight, branching alternate to opposite at acute to wide angles, loosely reticulate, cells 8-13 × 3-4 $\mu \mathrm{m}$. Appressoria alternate, opposite to subopposite, ovate to oblong, long, elongated, unicellular, entire, 4$24 \times 4-5 \mu \mathrm{m}$. Thyriothecia scattered to connate, orbicular, up to $160 \mu \mathrm{m}$ in diameter, stellately dehisced at the centre, crenate to fimbriate at the margin, fringed hyphae flexuous; asci few to many, globose to ovate, octosporous, 35-45 $\mu \mathrm{m}$ in diameter; ascospores oblong, conglobate, deep brown, uniseptate, constricted at the septum, 22-24 × 9-13 $\mu \mathrm{m}$, wall coarsely echinulate.

Material examined: Tamil Nadu, Coimbatore, Anamalai, Valparai, Karingundru, on leaves of Elaeocarpus tuberculatus Roxb.
(Elaeocarpaceae), 26 December 1990, V.B. Hosagoudar HCIO 30961; Kerala, Kollam, Shendurney, on leaves of Elaeocarpus sp., 14 January 2003, V.B. Hosagoudar HCIO 44854, TBGT 41; Thiruvananthapuram, Athirumala, 26 March 1996, V.B. Hosagoudar HCIO 42176, TBGT 48; Attayar, 19 March 1997, V.B. Hosagoudar HCIO 44077, TBGT 485; Wyanad, Periya, 6 February 2002, M. Kamarudeen, HCIO 44297, 622; Wyanad,Tirunelly, 20 May 2002, S. Shiburaj HCIO 44638, TBGT 920; Wyanad, Periya, 27 December 2002, M. Kamarudeen \& P.A. Jose HCIO 44787, TBGT 1024; Idukki, Thekkadi, Periyar Tiger Reserve, Cheveloda, 21 March 2001, H. Biju \& V. Gireesh HCIO 47621, TBGT 2648; Wayanad, Periya, 6 February 2002, M. Kamarudeen HCIO44297, TBGT 622; Thirunelli, 20 May 2002, S. Shiburaj HCIO 44638, TBGT 920; Palghat, SilentValley, Sairandhri, 13 December

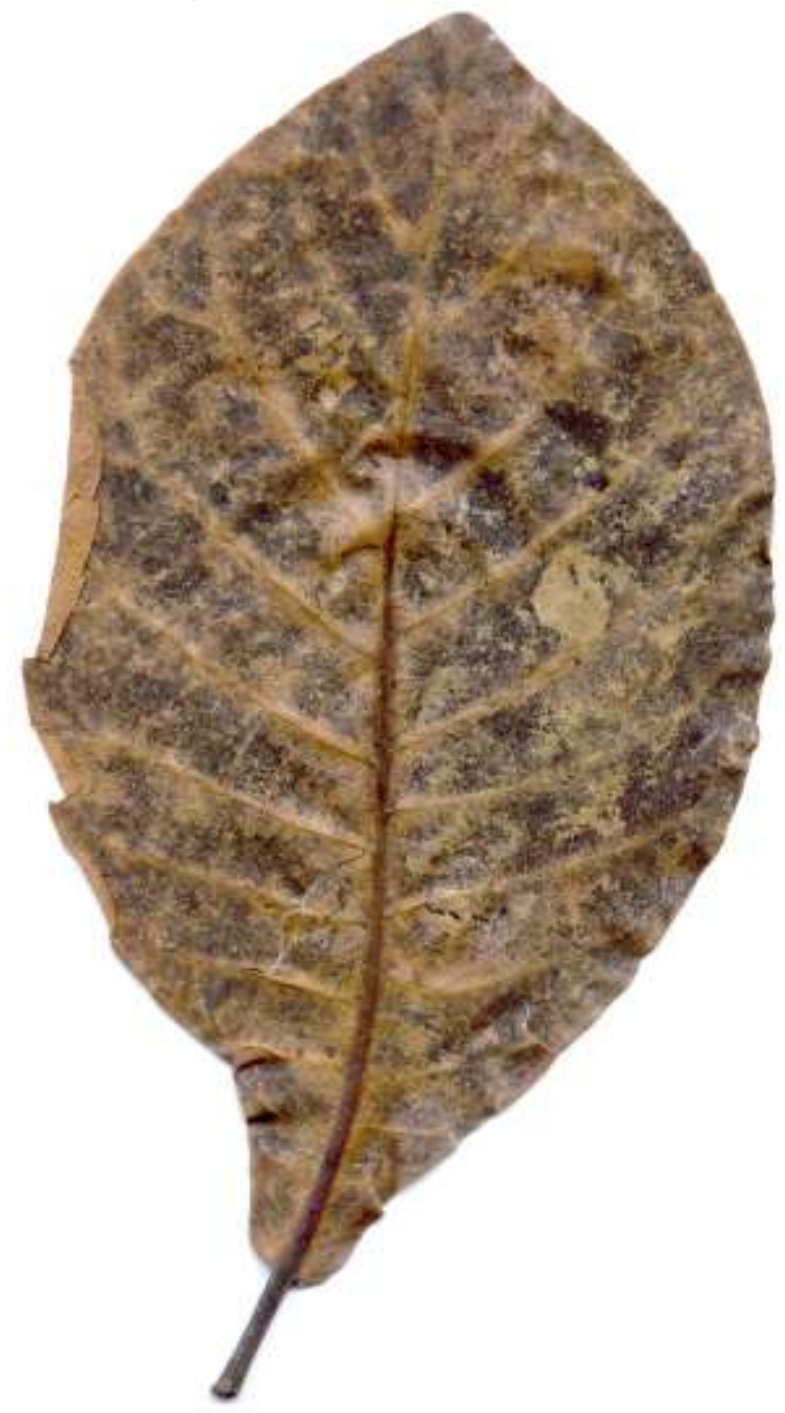

Figure 72 - Asterina elaeocarpi var. ovalis 
2003, V.B. Hosagoudar \& al. HCIO 45772, TBGT 1521; Pathanamthitta, Mozhiar forest, 14 December 2004, V.B. Hosagoudar \& al. HCIO 46232, TBGT 1644; Kakki Dam, 14 December 2004, V.B. Hosagoudar HCIO 45986, TBGT 1750; Palghat, Silent valley, Sairandhri, 13 December 2003, V.B. Hosagoudar \& al. HCIO 46062, TBGT 1825; Thekkadi, Periyar Tiger Reserve, Cheveloda, 21 March 2006, H.Biju \& V.Gireesh Kumar HCIO 47626, TBGT 2648; Wayanad, Mylattumala, 6 December 2006, M. Harish \& al. HCIO 48035, TBGT 2818;Pathanamthitta, Thiruvalla, 11 June 2007, Jacob Thomas HCIO 48263, TBGT 3002; Thrissur, Vazhachal, 21 August 2007, Jacob Thomas \& al. HCIO 48265, TBGT 3004; Idukki, Kuttikanam, 17 March 2007, Harish \& al. HCIO 48323, TBGT 3044; Thiruvananthapuram, Palode, TBGRI Campus, 15 November 2003, A. Sabeena \& al. HCIO 48377, TBGT 3098; 6 March 2008, A.

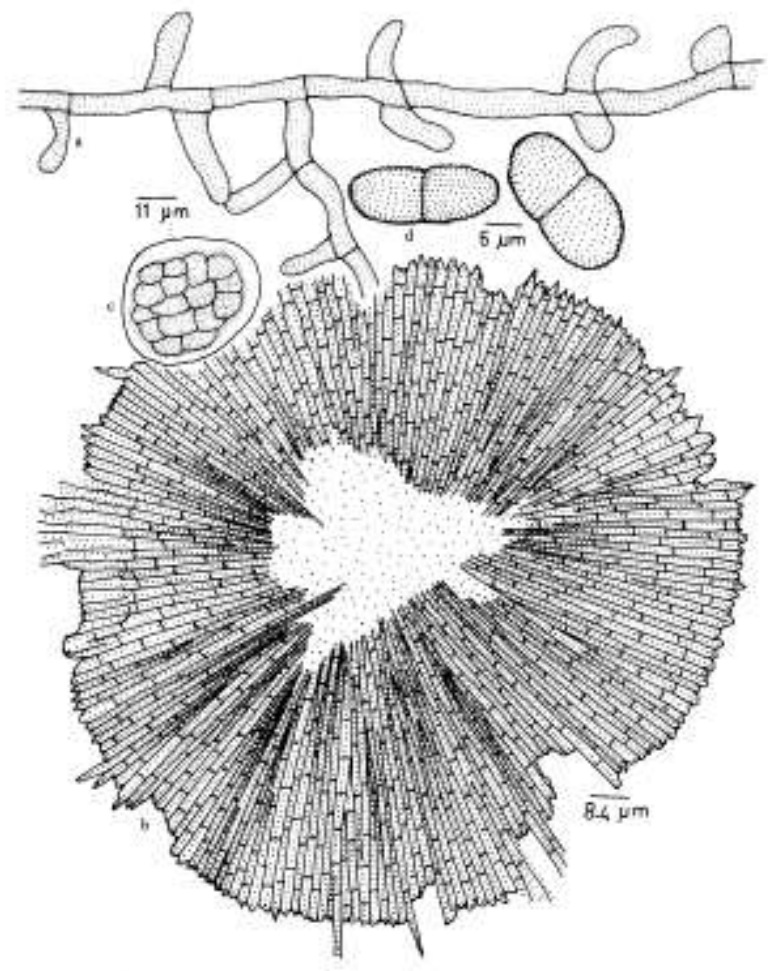

Figure 73 - Asterina elaeocarpi var. ovalis

Sabeena \& al. HCIO 48387, TBGT 3108; Kottayam, Ponthenpuzha Reserve Forest, 4 March 2008, P.J. Robin HCIO 48808, TBGT 3184; HCIO 48818, TBGT 3194; Wayanad, Periya, 16 September 2008, Harish et al. HCIO 49245, TBGT 3484; Pathanamthitta, Erumeli, 25 March 2009, P.J. Robin \& al. HCIO 49404, TBGT 3649; Kottayam, Kandankayam, 25 March 2009, P.J. Robin \& al. HCIO 49650,
TBGT 3892; Sabarimala forest division, Nilakal, 29 March 2009, P.J. Robin \& al. HCIO 49656, TBGT 3898; Wayanad,Periya, 16 February 2009, Gireesh \& al. HCIO 49815, TBGT 3967; Palghat, Silent Vally National Park, 2 March 2009, P.P. Rajesh Kumar \& al. HCIO 49828, TBGT 3980; 2 August 2008, P.P. Rajesh Kumar \& al. HCIO 49830, TBGT 3982; 30 July 2009, Jayakumar \& al. HCIO 49859, TBGT 4011; 28 July 2009, Jayakumar \& al. HCIO 49867, TBGT 4019; 2 March 2009, V.B. Hosagoudar TBGT 4536; Thiruvananthapuram, Palode, TBGRI campus, 16 August 2010, A. Sabeena TBGT 5003; Kottayam, Thalappally, 25 March 2009, P.J. Robin \& al. TBGT 5120; Palghat, Silent valley, Valakkad, 6 August 2008, M.C. Riju \& al. TBGT 239; Karnataka, Jodupal, Madikeri, Coorg, 12 November 2003, V.B. Hosagoudar \& al. HCIO 45670, TBGT 1417; South Canara, Kukke Subramanya, 15 November2003, V.B. Hosagoudar \& al. HCIO 45718, TBGT 1467; Coorg, Madikeri, Jodupal, 12 November 2003, V.B. Hosagoudar \& al. HCIO 45750, TBGT1499; HCIO 45779, TBGT 1528; Kodagu, Galibeedu, 24 November 2008, C. Jagath Thimmaiah TBGT 5329; Kodagu, Jodupal, 12 November, 2003, V.B. Hosagoudar $\&$ al. HCIO 45750, TBGT 1499; HCIO 45670, TBGT 1417; November 12, 2003, V.B. Hosagoudar \& al. HCIO 45750, TBGT 499; South Canara, Kukke Subramanya, 15 November 2003, V.B. Hosagoudar \& al. HCIO 45784, TBGT 1534.

This species is very specific and infects this host plant throughout Western Ghats region.

Distribution: Kerala, Karnataka, Tamil Nadu.

Asterina elatostematis Hosag. \& Goos, Mycotaxon 59: 156, 1996; Hosag., Chandraprabha \& Agarwal, Asterinales of Kerala, p. 69, 2011.

(Fig. 74)

Colonies epiphyllous, scattered, dense, minute, crustose, up to $1 \mathrm{~mm}$ in diameter. Hyphae flexuous, branching alternate to irregular at acute angles, loosely reticulate, cells 9-25 $\times 3-5 \mu \mathrm{m}$. Appressoria alternate to unilateral, unicellular, mammiform, non- 
stipitate, crenately lobate, 6-10 $\times$ 9-10 $\mu \mathrm{m}$. Thyriothecia aggregated, orbicular, up to 124 $\mu \mathrm{m}$ in diameter, margin crenate to fimbriate, dehisce stellately at the center; asci many, globose, octosporous, 24-26 × 18-20 $\mu \mathrm{m}$; ascospores conglobate, 1-septate, initially hyaline but turn brown at maturity, 12-16 $\times 5-7$ $\mu \mathrm{m}$, wall smooth.

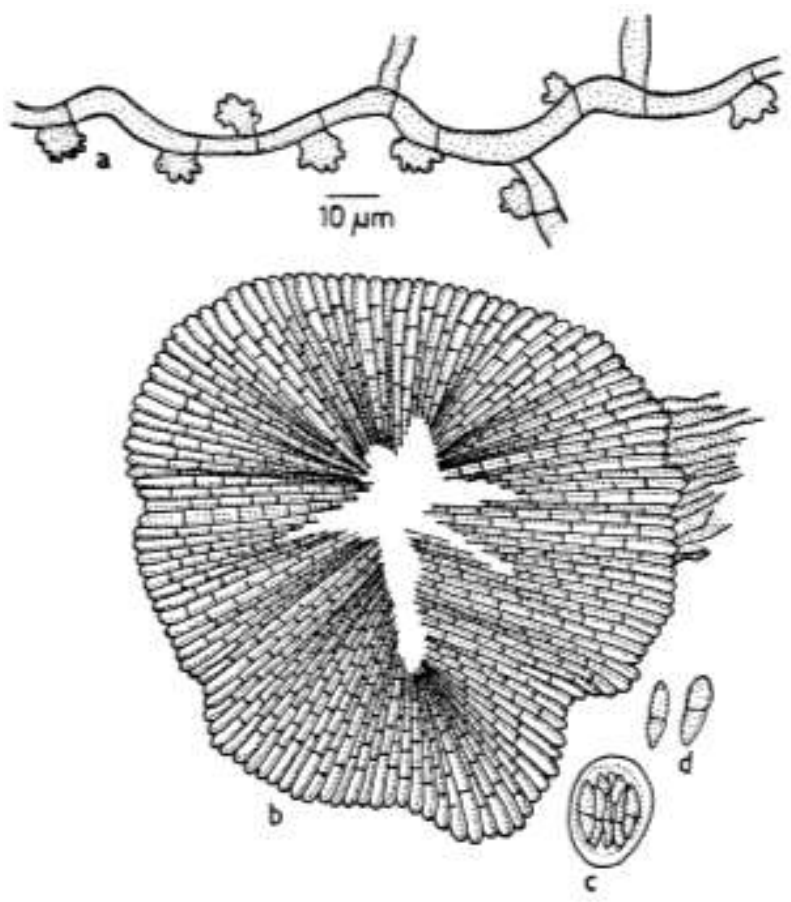

Figure 74 - Asterina elatostematis

Material examined: Tamil Nadu, Tirunelveli, Manjoli forest, on leaves of Elatostema lineolatum Wight (Urticaceae), 24 February 1994, V.B. Hosagoudar HCIO 41619 (holotype).

Minute and dense colonies, unicellular and crenately lobate appressoria distinguishes this species from other Asterina species reported on the members of the family Urticaceae.

Distribution: Kerala.

Asterina emciciana Hosag., Robin \& Archana, Sydowia 61: 246, 2009. (Fig. 75)

Colonies amphigenous, mostly epiphyllous, thin, up to $3 \mathrm{~mm}$ in diameter. Hyphae straight to flexuous, branching opposite to alternate at acute angles, loosely reticulate, cells $22-34 \times 5-7 \mu \mathrm{m}$. Appressoria alternate, unilateral, about $3 \%$ opposite, $12-18 \mu \mathrm{m}$ long; basal cells cylindrical to cuneate, 5-7 $\mu \mathrm{m}$ long; head cells ovate, globose, entire, angular to sublobate, $5-10 \times 5-7 \mu \mathrm{m}$. Thyriothecia scattered, orbicular, up to $101 \mu \mathrm{m}$ in diameter, dehisce stellately at the center, margin crenate to fimbriate; asci globose, octosporous, up to 41 $\mu \mathrm{m}$ in diameter; ascospores brown, ellipsoidal, uniseptate, constricted at the septum, 19-36 × 7$10 \mu \mathrm{m}$, wall smooth.

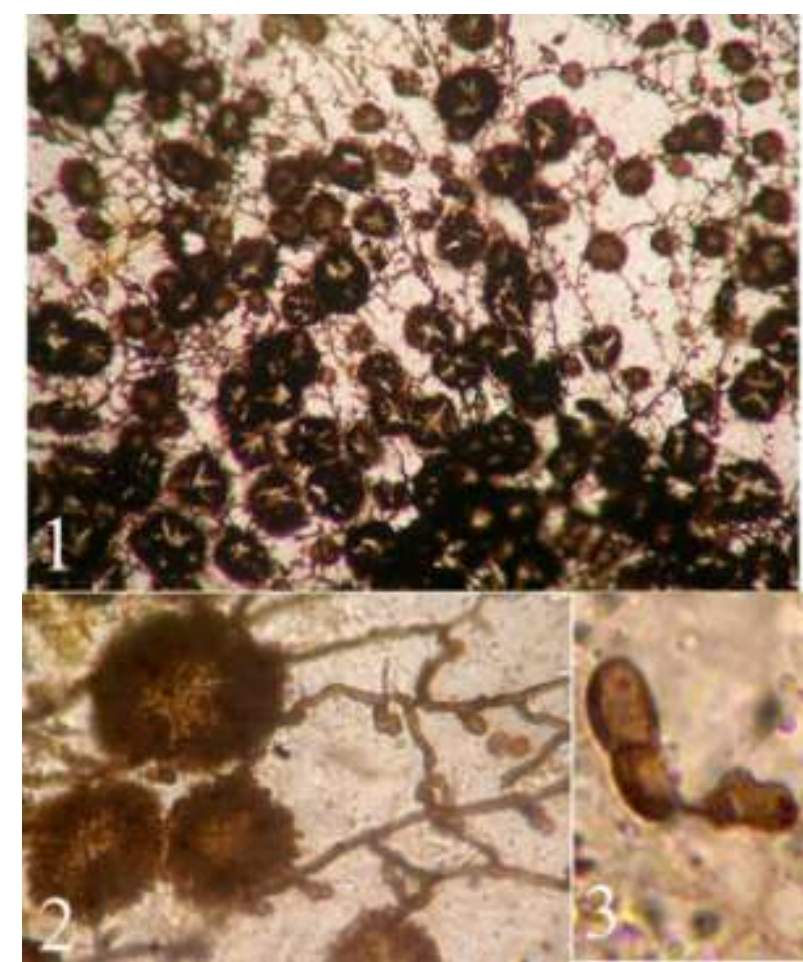

Figure 75 - Asterina emciciana

1. Colony with dehisced thyriothecia, 2.Thyriothecia and two celled appressoriate mycelium, 3. Germinating ascospore

Material examined: Tamil Nadu, Chennai, in the Campus of Madras Christian College, on leaves of Maerua apetala (Spreng.) M. Jacobs (Capparaceae), 24 December 2007, V. B. Hosagoudar et al. HCIO 48441 (holotype), TBGT 3162 (isotype).

Asterina capparis Sydow, Sydow \& Butler (capparidis) was collected on Capparis sp. from Chennai by E. J. Butler during the year 1903 (Sydow et al., 1911, Hosagoudar \& Abraham, 2000). The present species differs from it in having 3\% opposite appressoria and distinctly longer ascospores.

Distribution: Tamil Nadu 
Asterina enicostematis Hosag. \& Chandra., Indian J. Sci. \& Techn. 2(6):15, 2009 (enicostematis); Hosag., Chandraprabha \& Agarwal, Asterinales of Kerala, p. 70, 2011.

(Figs 76-77)

Colonies amphigenous, dense, up to 3 $\mathrm{mm}$ in diameter. Hyphae crooked, branching opposite at acute to wide angles, loosely to closely reticulate, cells 18-33 $\times$ 4-7 $\mu \mathrm{m}$. Appressoria unicellular, alternate, ovate, mammiform, sessile, 7-13 $\times$ 4-9 $\mu \mathrm{m}$. Thyriothecia scattered, orbicular, up to $132 \mu \mathrm{m}$ in diameter, dehisce stellately at the center, margin crenate to fimbriate; asci globose, octosporous, up to $40 \mu \mathrm{m}$ in diameter; ascospores conglobate, uniseptate, constricted at the septum, 11-20× 7-9 $\mu \mathrm{m}$, wall smooth. Pycnothyria similar to thyriothecia, orbicular, up to $110 \mu \mathrm{m}$ in diameter, margin crenate to
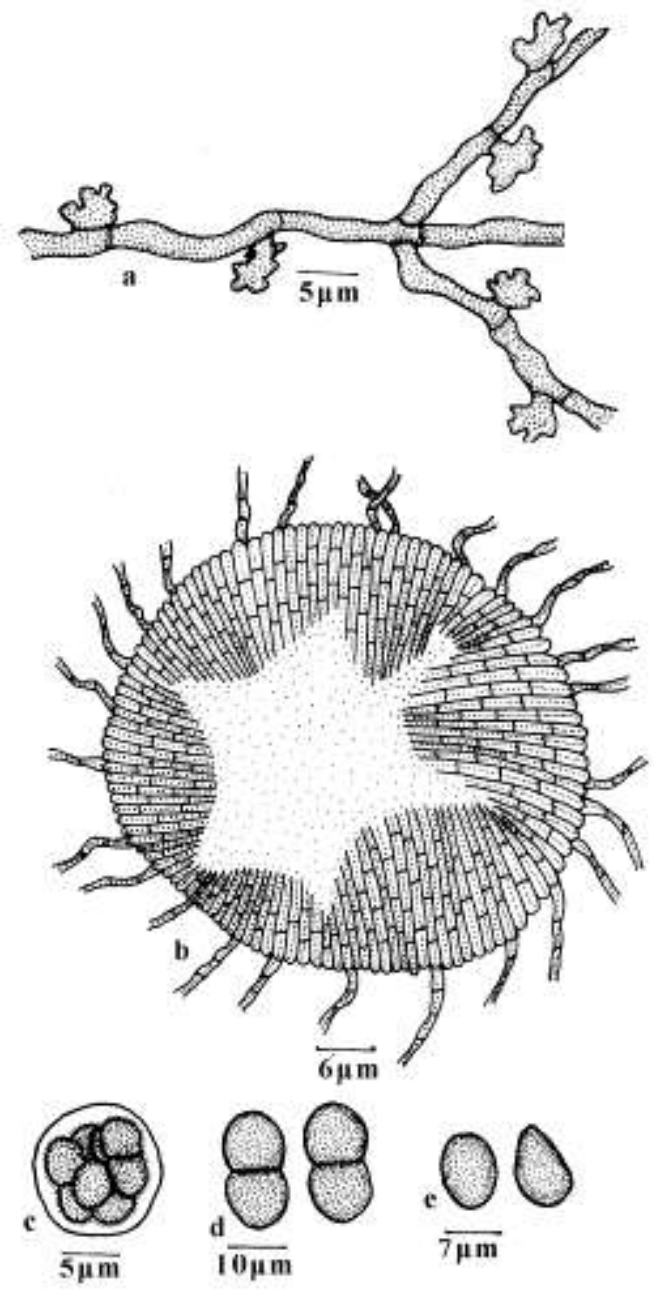

Figure 76 - Asterina enicostematis

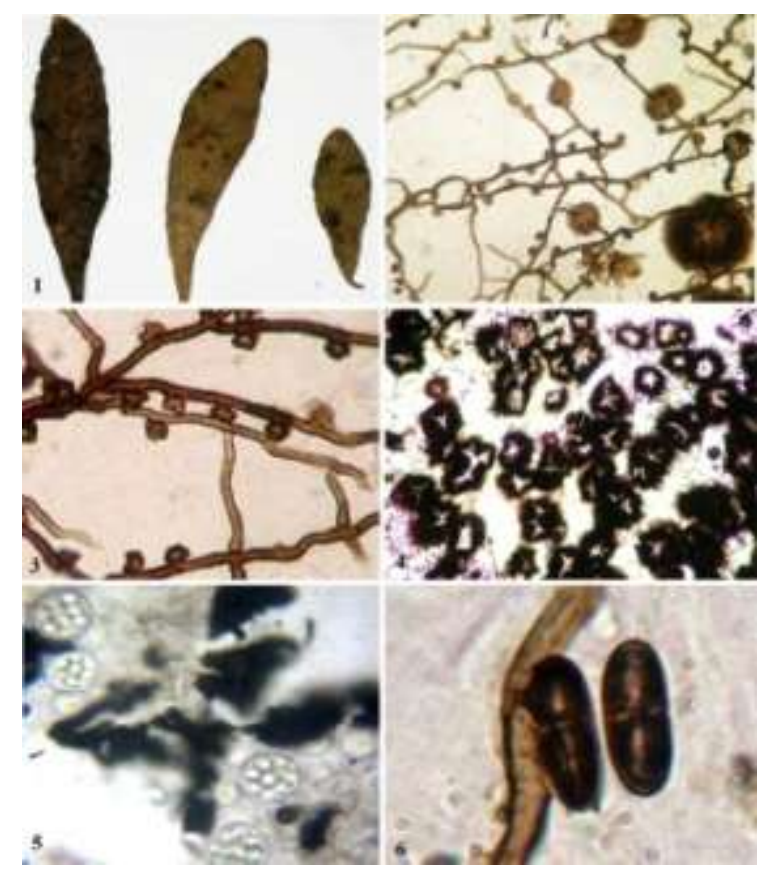

Figure 77 - Asterina enicostematis

1. Infected leaf, 2. Fungal colony with thyriothecia, 3. Appressoriate Mycelium, 4. Dehisced thyriothecia, 5. Asci, 6. Ascospores

fimbriate; pycnothyriospores ovate, pyriform, brown, 9-18 × 4-13 $\mu \mathrm{m}$, wall smooth.

Material examined: Kerala, Wayanad, upper peak of Pakshipathalam, on the leaves of Enicostema axillare (Lam.) A. Raynal. (Gentianaceae), 30 October 2007, A. Chandraprabha HCIO 48242 (holotype), TBGT 2980 (isotype).

Lembosia microtheca Theiss. is known on Goeppertia sp. of the family Gentianaceae (Stevens \& Ryan, 1939) and is the first report of genus Asterina on the members of the family Gentianaceae (Hosagoudar \& Abraham, 2000).

Distribution: Kerala.

Asterina erysiphoides Kalch. \& Cooke, Grevillea 9: 32, 1880 emend. Doidge, Trans. Roy. Soc. South Africa 8: 256, 1920; Hansf. \& Thirum., Farlowia 3: 306, 1948; Hosag., Balakr. \& Goos, Mycotaxon 59: 175, 1996; Hosag., H. Biju \& Appaiah, J. Mycopathol. Res. 44: 7, 2006; Hosag., Chandraprabha \& Agarwal, Asterinales of Kerala, p. 72, 2011.

(Fig. 78) 


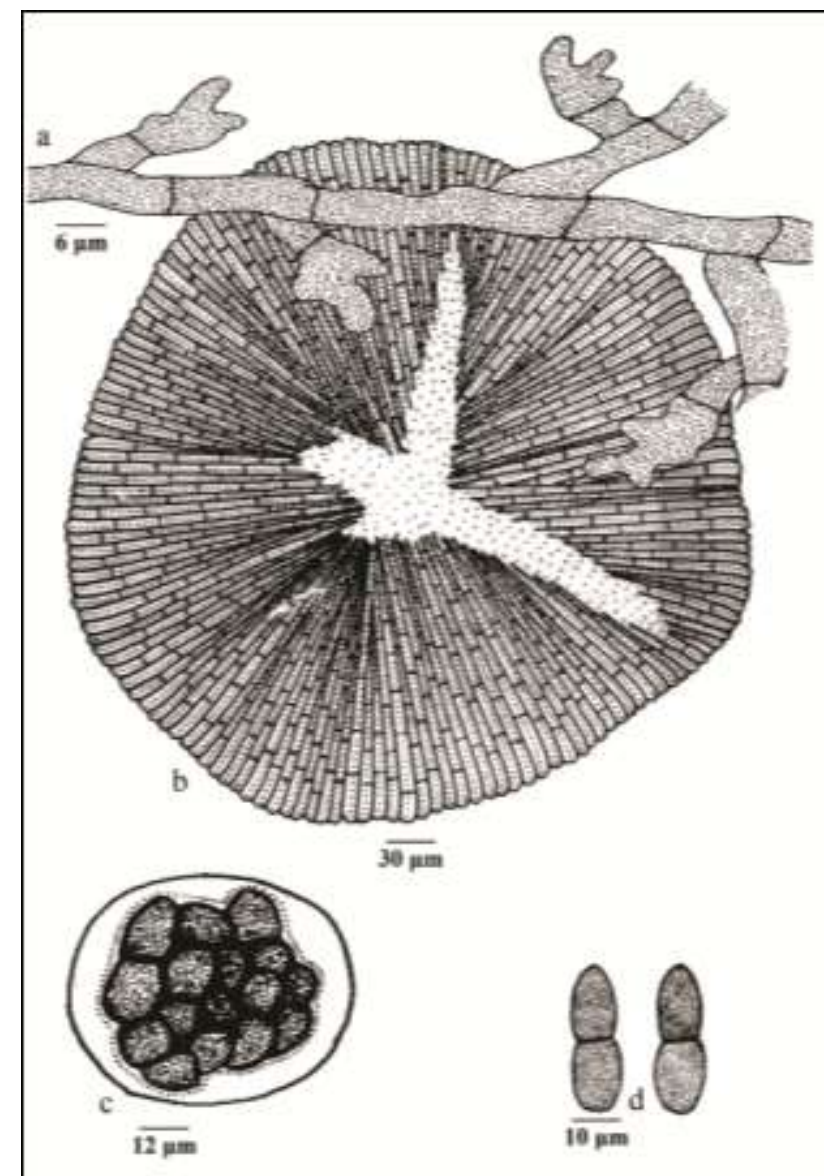

Figure 78 - Asterina erysiphoides

Colonies epiphyllous, dense, up to 2 $\mathrm{mm}$ in diameter, rarely confluent. Hyphae flexuous to crooked, branching mostly opposite at acute angles, loosely to closely reticulate, cells 18-25 × 2-5 $\mu \mathrm{m}$. Appressoria opposite and alternate, antrorse to reflexed, straight to variously curved, 15-20 long; stalk cells cylindrical to cuneate, $4-13 \mu \mathrm{m}$ long; head cells straight to curved, entire to lobate, $6-11 \times 7-10$ $\mu \mathrm{m}$. Thyriothecia numerous, scattered, often confluent, initially closed, stellately dehisced at center at maturity, margin crenate to fimbriate; asci numerous, ovoid to globose, octosporous, 30-33 × 27-30 $\mu \mathrm{m}$; ascospores initially hyaline, brown at maturity, conglobate, oblong, rounded at both ends, 1-septate, constricted at septum, $18-22 \times 9-13 \mu \mathrm{m}$.,

Material examined: Tamil Nadu, Nilgiris, Gudalur, Sri Madurai, on leaves of Jasminum retchiei Clarke (Oleaceae), 25 January 1990, V.B. Hosagoudar HCIO 30962; Karnataka, Nandi hills, Jasminum rigidum Zenker, 16 November 1994, M. J. Thirumalachar; Kerala, Wayanad, Banasuran mala, Jasminum cordifolium Wallich ex G.
Don, 16 April 1999, C.K. Biju HCIO 45111, TBGT 1166; Thiruvananthapuram, Ponmudi, 7 March 2003, G. Rajkumar \& P. A. Jose HCIO 45138, TBGT 1193; Thiruvananthapuram, Palode, TBGRI Campus, Central nursery, 5 September 2005, G. R. Archana \& A. Sabeena HCIO 46731, TBGT 2072; Nedumangad, 30 October 2005, Mahanteshagowda V. Patil HCIO 46707, TBGT 2048; Thiruvananthapuram, Palode, TBGRI Campus, on leaves of $J$. angustifolium (L.) Willd., 16 July 2005, A. Sabeena \& al. HCIO 46971, TBGT 2188; Jasminum sp., Wayanad, Thirunelli, 15 May, 1999, C.K. Biju HCIO 45082, TBGT 1137; Thiruvananthapuram, Palode, Kusavoor, 22 June 2002, A. Manojkumar HCIO 44602, TBGT 889; Nedumangad, 8 July 2003, Mahanteshagouda V. Patil HCIO 45100, TBGT 1155; Pathanamthitta, Uppupara, Pachakanam, 15 December 2004, V.B. Hosagoudar HCIO 45994, TBGT 1758; HCIO 45996, TBGT 1760; Thiruvananthapuram, Palode, Jawahar colony, 21 December 2005, V. Gireesh \& P.J. Robin HCIO 46773, TBGT 2114; Thiruvananthapuram, TBGRI Campus, 16 August 2005, G.R. Archana \& A. Sabeena HCIO 46704, TBGT 2045; Kottayam,Thazhathuvadakara, 27 December 2005, V. Gireesh Kumar \& P.J. Robin HCIO 46751, TBGT 2092; Nedumangad, 7 July 2005, P. V. Mahanteshagouda HCIO 46810, TBGT 2151; Palode, TBGRI, Central nursery, 16 August 2008, A. Sabeena \& al. HCIO 46961, TBGT 2178; Karimancode, 25 December 2005, G.R. Archana HCIO 46963, TBGT 2180; Palode, TBGRI, Central nursery, 18 August 2008, A. Sabeena \& al. TBGT 2183; HCIO 46970, TBGT 2187; Palghat, Shoranoor, 16 September 2005, K. Nandini HCIO 47608, TBGT 2630; Wayanad,Periya, Kunkichira, 6 December 2006, M. Harish \& al. HCIO 48055, TBGT 2838; Karnataka, Kodagu, Madikeri, Nishane motta, 11 November 2003, V.B. Hosagoudar \& al. HCIO 45631, TBGT 1375; HCIO 45634, TBGT 1378; 12 November 2003, V.B. Hosagoudar \& al. HCIO 45630, TBGT 1373; HCIO 45780, TBGT 1529; HCIO 45645, TBGT 1391; HCIO 45795, TBGT 1544; HCIO 45796, TBGT 1545; HCIO 45797, TBGT 1546; 11 November 2003, V.B. Hosagoudar \& al. HCIO 46354, TBGT 2000; 14 November 2003, 
V.B. Hosagoudar \& al. HCIO 47066, TBGT 2283; Kodagu, Vanachalu, 24 November 2008, V.B. Hosagoudar \& al. HCIO 49146, TBGT 3401; 24 November 2008, V.B. Hosagoudar \& al. HCIO 49165, TBGT 3420; Madikari, 26 November 2008, V.B. Hosagoudar \& al. HCIO 49152, TBGT 3407; Galibeedu, 24 November 2008, V.B. Hosagoudar \& al. HCIO 49154, TBGT 3409; Madikari, 25 November 2008, V.B. Hosagoudar \& al. HCIO 49168, TBGT 3423; Tamil Nadu, Kodaikanal,Three star Shola, 19 July 2006, V. Bhairavi HCIO 47099, TBGT 2316.

In most of the collections, the colonies were associated with the colonies of Meliola gemellipoda Doidge and Meliola jasminii Hansf. \& Stev.

This species is common in the Southern Western Ghats.

Distribution: Kerala, Karnataka, Tamil Nadu.

Asterina erysiphoides Kalch. \& Cooke var. indica Hosag., Balakr. \& Goos, Mycotaxon 59: 175, 1996. (Fig. 79)

Colonies epiphyllous, dense, carbonaceous black, up to $5 \mathrm{~mm}$ in diameter, confluent and cover the entire lower surface of the leaves. Hyphae light brown, straight, flexuous to crooked, branching alternate, opposite to irregular at wide angles, loosely reticulate, cells $12-16 \times 3-5 \mu \mathrm{m}$. Appressoriate alternate and opposite (30\%), straight to curved, 2-celled, 8-16 $\mu \mathrm{m}$ long; stalk cells cylindrical to cuneate, 3-7 $\mu \mathrm{m}$ long; head cells ovate, globose, hamate, entire to variously lobate and in various shapes, 5-15.5 $\times$ 5-7 $\mu \mathrm{m}$. Thyriothecia scattered to loosely grouped, orbicular, up to $125 \mu \mathrm{m}$ in diameter, dehisce stellately at the center, margin crenate to fimbriate, fringed hyphae tortuous; asci many, globose to slightly ovate, eight spored, 27-31 $\times$ 24-26 $\mu \mathrm{m}$; ascospores conglobate, cinnamon brown, 1-septate, constricted at the septum, 18$19 \times 12-13 \mu \mathrm{m}$, lower cell globose, upper cell ovate, wall smooth. Pycnothyria similar to thyriothecia; pycnothyriospores brown, globose to pyriform, $15-21 \times 12-13 \mu \mathrm{m}$.

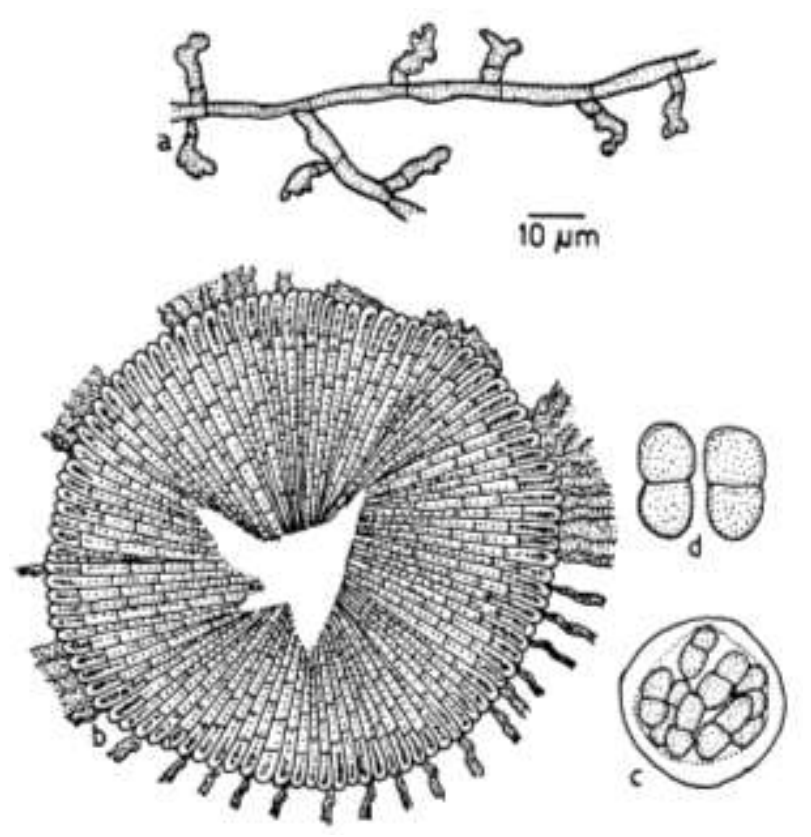

Figure 79 - Asterina erysiphoides var. indica

Material examined: On leaves of Jasminum cordifolium Wallich ex G. Don (Oleaceae), Sri Madurai, Gudalur, Nilgiris, Tamil Nadu, 25 January 1990, V.B. Hosagoudar HCIO 30962.

This variety differs from the species in having only hypophyllous colonies and more opposite appressoria.

Distribution: Tamil Nadu.

Asterina erythropalicola Hosag. \& Goos, Mycotaxon 59: 156, 1996; Hosag., Chandraprabha \& Agarwal, Asterinales of Kerala, p. 73, 2011. (Fig. 80)

Colonies epiphyllous, dense, crustose, up to $2 \mathrm{~mm}$ in diameter, confluent. Hyphae straight to substraight, branching mostly opposite at acute to wide angles, loosely reticulate, cells 21-28 × 6-8 $\mu \mathrm{m}$. Appressoria alternate, unilateral, about $30 \%$ opposite, subantrorse to perpendicular to the hyphae, mostly straight, 2-celled, 12-22 $\mu \mathrm{m}$ long; stalk cells cylindrical, 3-10 $\mu \mathrm{m}$ long; head cells ovate, globose, entire, rarely truncate, 9-13 × 6$8 \mu \mathrm{m}$. Thyriothecia closely scattered, orbicular, up to $217 \mu \mathrm{m}$ in diameter, margin fringed, rarely crenate, fringed hyphae flexuous, with appressoria, thyriothecia dehisce stellately at the center and widely opened; asci many, globose, octosporous, 40-46 $\mu \mathrm{m}$ in diameter; 
ascospores conglobate, 1 -septate, constricted at the septum, both cells unequal, 24-28 $\times 12-16$ $\mu \mathrm{m}$, wall smooth. Pycnothyria attached or mixed with thyriothecia, slightly smaller; pycnothyriospores oval to pyriform 18-20 × 12$14 \mu \mathrm{m}$.

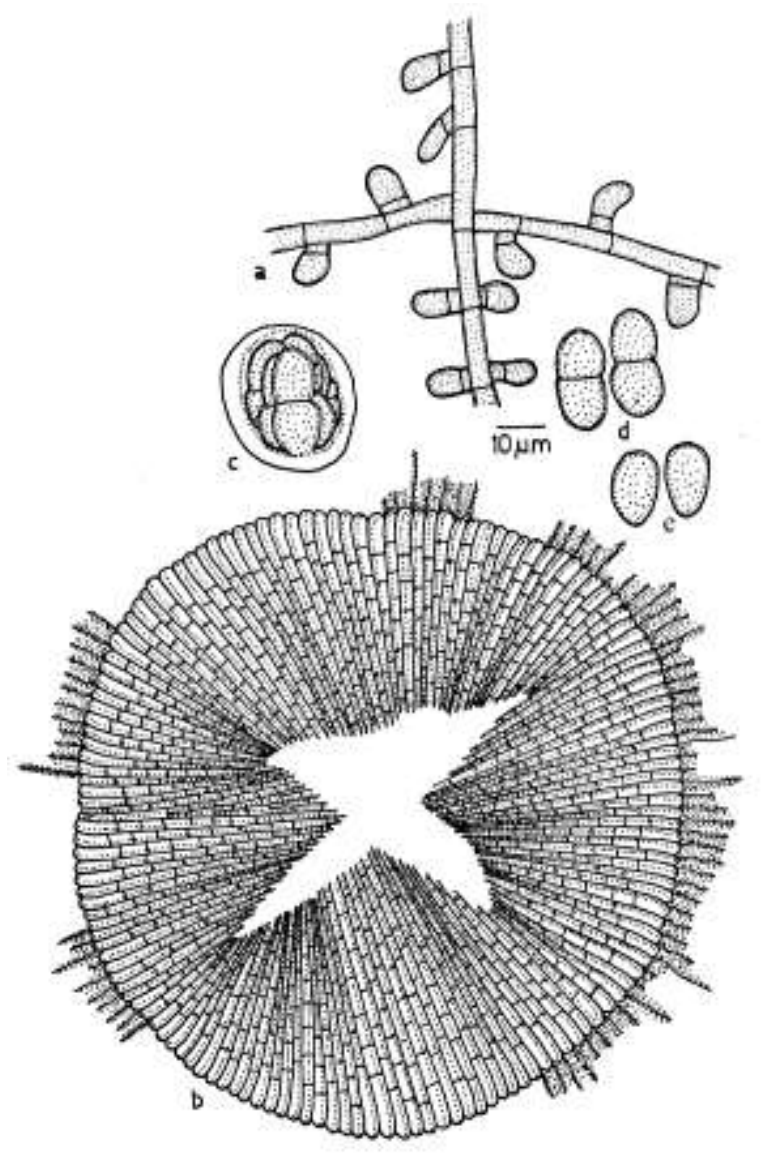

Figure 80 - Asterina erythropalicola

Material examined: Tamil Nadu, Kanniyakumari, Veerapuli Reserve forest, on leaves of Erythropalum populifolium (Arn.) Masters (Erythropalaceae), 22 February 1994, V.B. Hosagoudar HCIO 41642; Kerala, Thiruvananthapuram, Athirumala, 26 March 1996, V.B. Hosagoudar HCIO 42177, TBGT 49; HCIO 44006, TBGT 416; Wyanad, Periya, Kunkichira, 6 December 2006, M. Harish \& al. HCIO 48037, TBGT 2820; Kottayam, Ponthanpuzha reserve forest, 10 February 2007, P.J. Robin \& al. HCIO 48156, TBGT 2892; Palghat, Silent valley, Sairandhri, 13 December 2003, V.B. Hosagoudar \& al. HCIO 46063, TBGT 1826; Silent Valley National Park, 30 July 2009, Jayakumar \& al. HCIO 49863, TBGT 4015; Kottayam, Ponthenpuzha Reserve Forest, 3 March 2008, P.J. Robin HCIO 48819, TBGT 3195; Thiruvananthapuram, Peppara
Wildlife Sanctuary, Athirumala, 28 February 2008, HCIO 48879, TBGT 355.

This species differs from Asterina erythropali Hansf. in having epiphyllous colonies and appressoria with entire head cells (Hansford, 1954).

Distribution: Kerala, Tamil Nadu.

Asterina escharoides Sydow, Abh. K. K. Zool. Bot. Ges. 7: 101, 1913; Hosag., Indian J. Forestry 18: 274, 1995; Hosag., H. Biju \& Appaiah, J. Mycopathol. Res. 44: 7, 2006; Hosag., Chandraprabha \& Agarwal, Asterinales of Kerala, p. 74, 2011.

\section{(Figs 80-81)}

Colonies epiphyllous, dense, crustose, spreading, up to $2 \mathrm{~mm}$ in diameter, confluent. Hyphae substraight to crooked, branching alternate to irregular at acute to wide angles, loosely reticulate and form a mycelial net, cells 24-31 $\times$ 3-5 $\mu \mathrm{m}$. Appressoria few, distantly placed, two celled, alternate, scattered, mostly straight, rarely curved, 12-25 $\mu \mathrm{m}$ long; stalk cells cylindrical, 5-7 $\mu \mathrm{m}$ long; head cells globose, clavate, entire to sublobate, $6-13 \times 6$ $10 \mu \mathrm{m}$. Thyriothecia mostly scattered, often
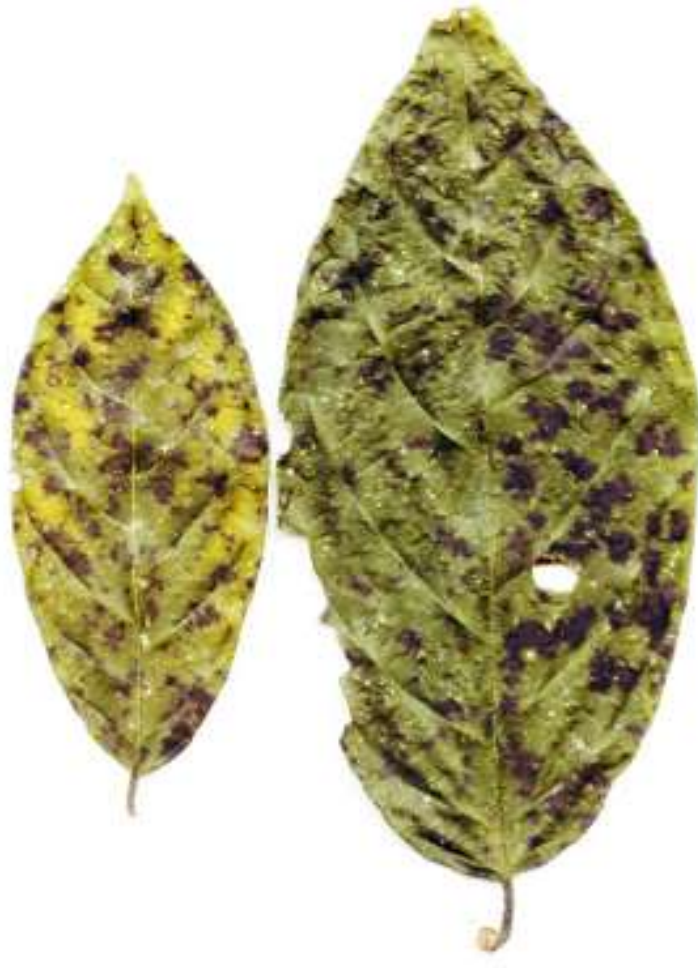

Figure 81 - Asterina escharoides - Infected leaves 


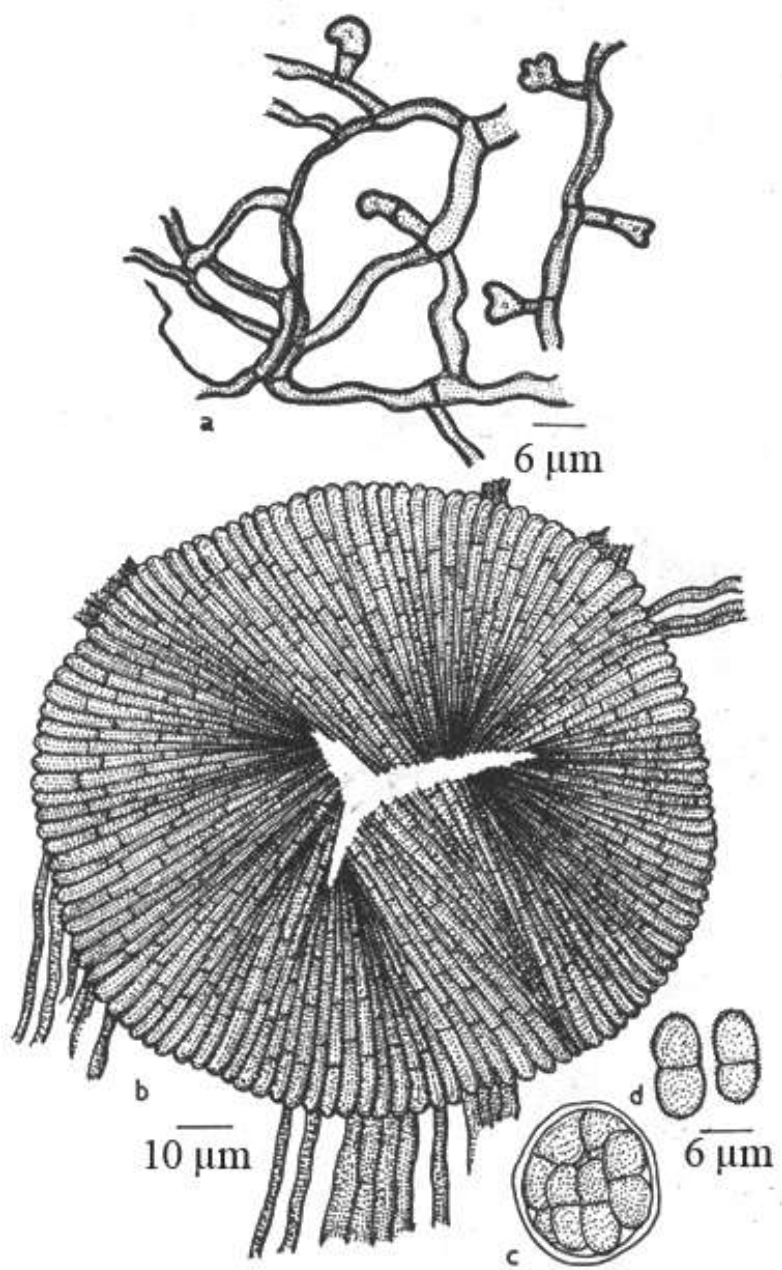

Figure 82 - Asterina escharoides

connate, circular, margin crenate to fimbriate, fringed hyphae loosely to compactly arranged, rupturing stellately at the center; asci globose to slightly clavate, octosporous, 27-41 × 21-31 $\mu \mathrm{m}$; ascospores brown, conglobate, oblong, 1septate, strongly constricted at the septum, rounded at both ends, one cell larger, $18-22 \times$ 8-10 $\mu \mathrm{m}$, wall minutely verrucose.

Material examined: Kerala, Thiruvananthapuram, Palode, TBGRI Campus, on leaves of Quisqualis indica L. (Combretaceae), 17 January 2001, H. Biju HCIO 46077, TBGT 1840; 10 April 2008, A. Sabeena \& M.C. Riju TBGT 4782; 21 November 2002, P. A. Jose HCIO 44669, TBGT 950; Karimancode, 21 January 2005, G. R. Archana \& al. HCIO 46226, TBGT 1638; Nedumangad, Kallara, 8 November 2005, A. Sabeena HCIO 46815, TBGT 2156; HCIO 46820, TBGT 2159; TBGRI Campus, 11 November 2005, A. Sabeena \& G. R. Archana HCIO 47006, TBGT 2223; Ponmudy, 20
February 2007, G.R. Archana \& al. TBGT 4544; near Peppara Dam, 18 November 2007, Jacob Thomas \& Vimalkumar HCIO 48223, TBGT 2959; Pangode, 23 January 2001, S. Shiburaj HCIO 44074, TBGT 486; Palode, TBGRI Campus, 14 December 2001, G. Rajkumar HCIO 45085, TBGT 1140; 11 November 2005, G.R. Archana \& A. Sabeena HCIO 47003, TBGT 2220; HCIO 47006, TBGT 2223; Kozhicode, Malabar Botanic garden, 16 November 2006, A. Sabeena \& al. HCIO 47392, TBGT 2430; HCIO 47392, TBGT 2430; HCIO 47567, TBGT 2589; 3 November, 2009, A. Sabeena \& M.C. Riju TBGT 4551; Pathanamthitta, Thiruvalla, Kavumbhagam, 21 September 2007, Jacob Thomas HCIO 48222, TBGT 2958; 21 September 2007, Jacob Thomas 48306, TBGT 3027; Karnataka, Kodagu, Jodupal, 12 November 2003, V.B. Hosagoudar \& al. HCIO 45648, TBGT 1394; HCIO 45669, TBGT 1416; HCIO 45648, TBGT 1394; 45669, TBGT 1416.

This species was recorded from Philippines (Stevens \& Ryan, 1939).

Distribution: Karnataka, Kerala.

Asterina euonymi Hosag. \& Goos, Mycotaxon 52: 467, 1994. (Fig. 83)

Colonies amphigenous, caulicolous, ramicolous, dense, velvety, widely confluent. Hyphae straight to substraight, rarely crooked, branching alternate to irregular at acute angles, loosely to closely reticulate, cells 21 $25 \times 4-7 \mu \mathrm{m}$. Appressoria continuous, mammiform, obovoid to cylindrical, rounded at the apex, entire to rarely sublobate, $6-37 \times$ 5-7 $\mu \mathrm{m}$. Thyriothecia scattered to loosely grouped, round, margin crenate to rarely fringed, stellately dehisced at the center, often upper portion dissolved and widely opened, up to $110 \mu \mathrm{m}$ in diameter; asci many, globose, octosporous, $55-75 \mu \mathrm{m}$ in diameter; ascospores conglobate, 1-septate, deeply constricted at the septum, upper cell ovate, lower cell globose, 31-34 × 14-16 $\mu \mathrm{m}$, wall verrucose. Pycnothyria few, similar to thyriothecia, slightly smaller; pycnothyriospores brown, globose, pyriform, often slightly beaked, 18-25 × 14-16 $\mu \mathrm{m}$. 


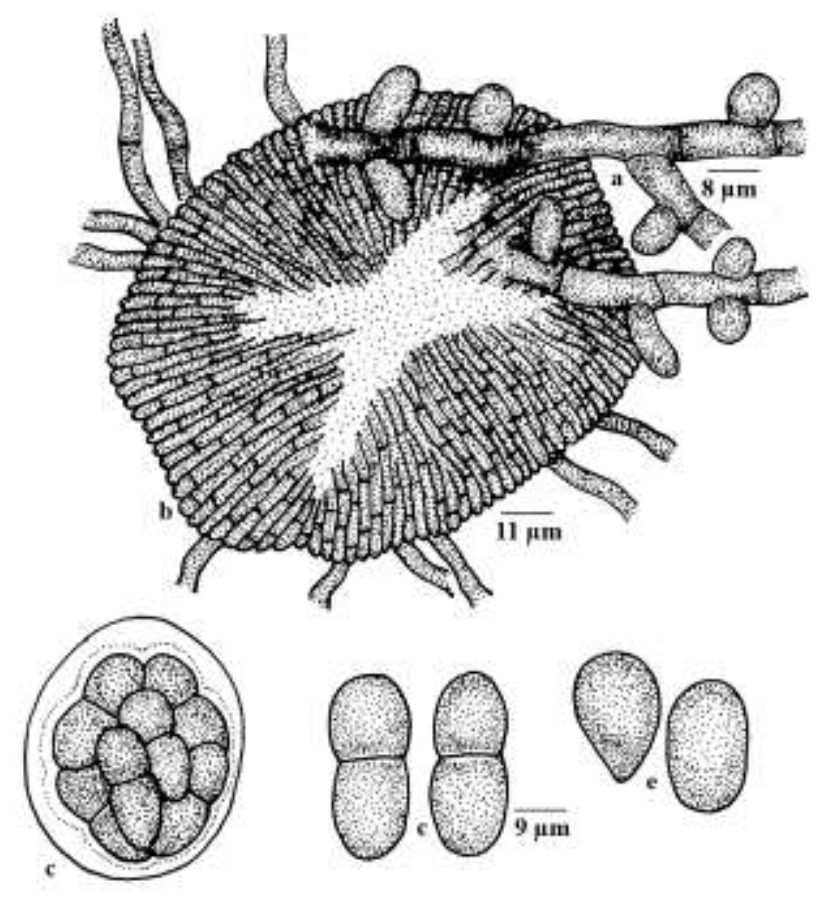

Figure 83 - Asterina euonymi

Material examined: Tamil Nadu, Kamarajar dist., Seithur hills, on leaves of Euonymous crenulatus Wall. ex Wight \& Arn. (Celastraceae), 12 November 1992, V.B. Hosagoudar HCIO 40849 (holotype).

This species differs from Asterina dissiliens (Sydow) Doidge in having numerous appressoria, scattered thyriothecia and larger ascospores.

Distribution: Tamil Nadu.

Asterina eupatorii Kar \& Ghosh, Indian Phytopath. 39: 214, 1986.

Colonies epiphyllous, scattered, up to $3.5 \mathrm{~mm}$ in diameter. Hyphae undulate, branching alternate, irregular to opposite, closely reticulate, cells $18-33 \times 4-6 \mu \mathrm{m}$. Appressoria alternate to unilateral, unicellular, straight to bent, 2-5 deeply lobed, 4-10 $\times 5-8$ $\mu \mathrm{m}$. Thyriothecia scattered to gregarious at the centre of the colony, orbicular, 81-205 $\mu \mathrm{m}$ in diameter, stellately dehisce at the centre; asci many, globose to oval, 8-spored, 16-30 $\mu \mathrm{m}$ in diameter, paraphysate; ascospores brown, elliptic-oblong, uniseptate, constricted at the septum, $12-15 \times 4-8 \mu \mathrm{m}$, wall smooth.
West Bengal, Darjelling, Mirik, on leaves of Eupatorium glandulosum Kunth. (Asteraceae), 15 July 1979, IMI 237718 (holotype).

Distribution: West Bengal.

Asterina flacourtiacearum Hosag. \& Ravikumar in Hosag., Balakr. \& Goos, Mycotaxon 59: 176, 1996.

(Fig. 84)

Colonies amphigenous, mostly epiphyllous, up to $2 \mathrm{~mm}$ in diameter, frequently confluent. Hyphae straight, branching alternate to irregular at acute angles, loosely reticulate, cells 24-31 $\times$ 5-7 $\mu \mathrm{m}$. Appressoria scattered, distantly placed, alternate, mostly two celled, occasionally several celled, 9-30 $\mu \mathrm{m}$ long; stalk cells mostly unicellular, rarely up to 3-celled, mostly cylindrical to cuneate, rarely irregularly curved, 3-22 $\mu \mathrm{m}$ long; head cells obpyriform, frequently 2-3 lobate, rarely entire to angular, 6-10 $\times$ 9-13 $\mu \mathrm{m}$. Thyriothecia orbicular, carbonaceous black, closely grouped to scattered, up to $190 \mu \mathrm{m}$ in diameter, dehiscing stellately at the center, margin crenate to fimbriate, fringed hyphae tortuous; asci many, globose, eight spored, 31-41 $\mu \mathrm{m}$ in diameter; ascospores cinnamon brown, conglobate, 1septate, deeply constricted at the septum, upper cell slightly larger, $24-28 \times 12-16 \mu \mathrm{m}$, wall smooth. Pycnothyria similar to thyriothecia, up to $90 \mu \mathrm{m}$ in diameter; pycnothyriospores ovate to globose, slightly papillate at one end, cinnamon brown, 15-22 × 15-19 $\mu \mathrm{m}$.

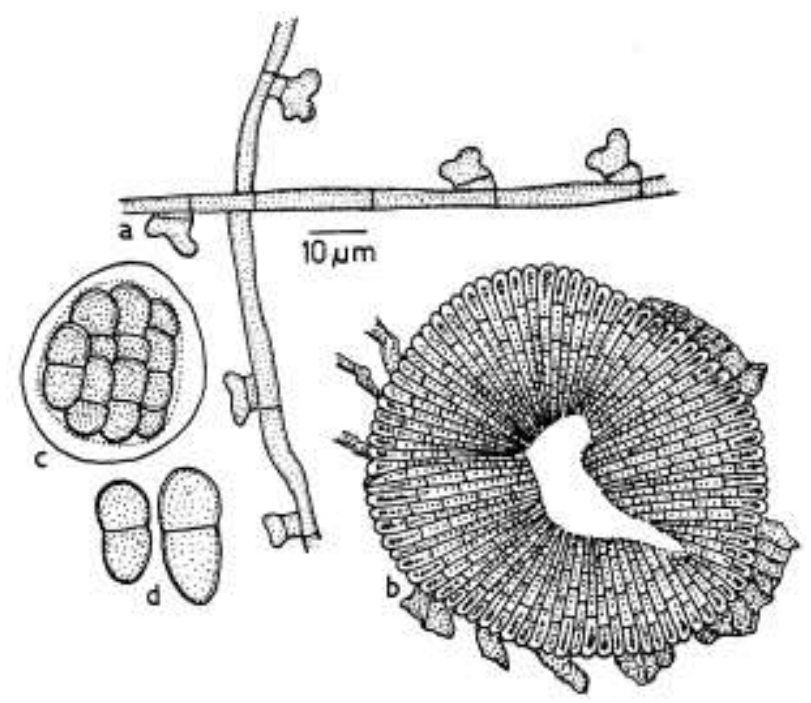

Figure 84 - Asterina flacourtiacearum 
Material examined: Tamil Nadu, Madurai, Kodaikanal, Pamba Ravane forest, on leaves of Scolopia crenata (Wgiht \& Arn.) D. Clox. (Flacourtiaceae) 18 October 1991, K. Ravikumar HCIO 30964 (holotype); Karnataka, Coorg, Madikari, member of Flacourtiaceae, 26 November 2008, V.B. Hosagoudar \& al. HCIO 49959, TBGT 4111.

Of the Asterina species reported on the members of Flacourtiaceae, this species is close to Asterina uncinata Doidge (Hansford, 1944, Doidge, 1942, Stevens \& Ryan, 1939) in having one to several celled appressoria but differs from it in having regularly lobate head cells and mostly single celled stalk cells of the appressoria.

Distribution: Karnataka, Tamil Nadu.

Asterina gamsii Hosag. \& C.K. Biju in Hosag., Indian Phytopath. 58: 195, 2005; Hosag. J. Appl. \& Nat. Sci. 1(1): 27, 2009; Hosag., Chandraprabha \& Agarwal, Asterinales of Kerala, p. 77, 2011.

(Figs 85-86)

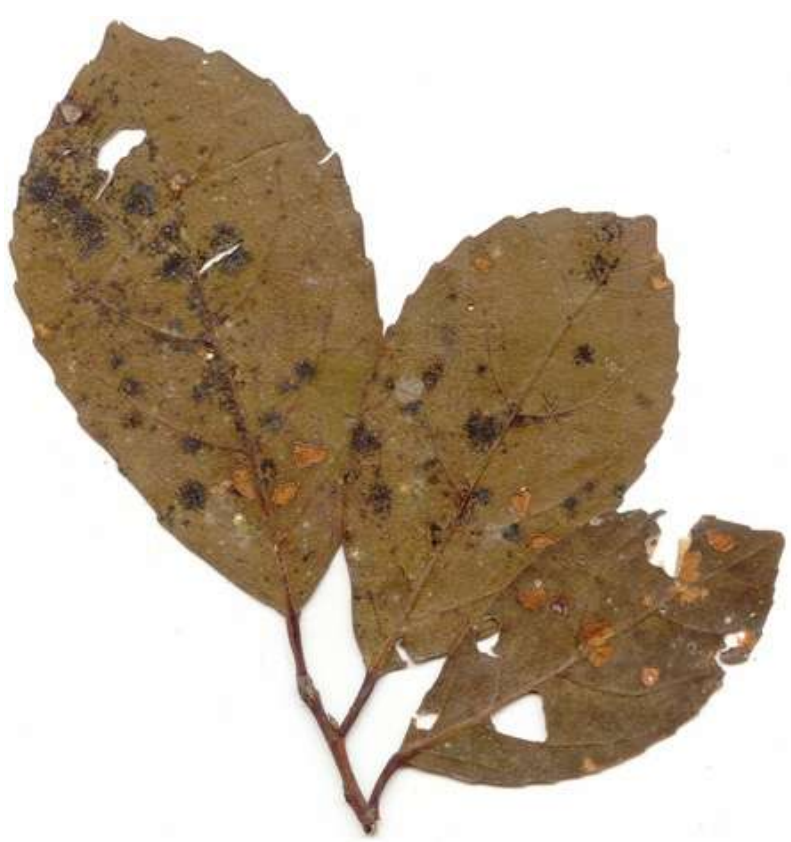

Figure 85 - Asterina gamsii

Colonies epiphyllous, dense, velvety, up to $3 \mathrm{~mm}$ in diameter and cover an entire upper portion of the leaves. Hyphae straight to substraight, branching irregular at acute angles, loosely to closely reticulate, cells $16-23 \times 4-7$ $\mu \mathrm{m}$. Appressoria alternate, unilateral and about
$20 \%$ opposite to subopposite, mostly straight, subantrorse to rarely retrorse, ovate to cylindrical, entire, rounded at the apex, 8-13 $\times$ 6-8 $\mu \mathrm{m}$. Thyriothecia closely scattered, orbicular, up to $300 \mu \mathrm{m}$ in diameter, stellately dehisced at the centre, crenate to fimbriate at the margin, fringed hyphae flexuous; asci few to many, globose, octosporous, up to $38 \mu \mathrm{m}$ in diameter; ascospores oblong, brown, uniseptate, constricted at the septum, $32-36 \times 11-18 \mu \mathrm{m}$, wall smooth. Pycnothyria similar to thyriothecia, smaller; pycnothyriospores pyriform, apiculate, brown, $22-26 \times 16-18 \mu \mathrm{m}$.

Material examined: Kerala, Wyanad, Banasuran mala, on leaves of Elaeocarpus variabilis Zmarzty (Elaeocarpus tectorius (Lour.) Poir.) (Elaeocarpaceae), 19 November 2000, C.K. Biju HCIO 45166 (holoype), TBGT 1221 (isotype); Palghat, Silent valley, Sairandhri, 13 December 2003, V.B. Hosagoudar \& al. HCIO 45753, TBGT 1502; Chempatty, 14 December 2003, V.B. Hosagoudar \& al. HCIO 46137, TBGT 1900; Pathanamthitta, Mozhiyar forest, 14 December 2004, V.B. Hosagoudar \& al. HCIO 46098, TBGT 1861; Tamil Nadu, Kodaikanal, Mathikettan sholai, Elaeocarpus variablilis Zmarzty, 9 March 2007, R. Nithyatharani TBGT 4352; Kodaikanal, Kuthereyar, on leaves of Elaeocarpus glandulosus Sensu auct., 7 March 2007, R. Nithyatharani TBGT 5105; Thiruvananthapuram, Palode, TBGRI Campus, Arboretum, Elaeocarpus sp., 23 January 2008, Anilkumar HCIO 49427, TBGT 3672.

There are five species, namely Asterina borneensis Hansf., A. elaeocarpi Sydow, $A$. elaeocarpi Sydow var. ovalis Kar \& Ghosh, $A$. elaeocarpicola Hansf. and $A$. elaeocarpikobenmochi Yamam., known on the members of the family Elaeocarpaceae (Hosagoudar \& Abraham, 2000). Asterina gamsii differs from A. elaeocarpicola and A. borneensis in having octosporous asci and straight appressoria (Hansford, 1954,). It differs from A. elaeocarpi in having opposite appressoria and larger ascospores. It also differs from $A$. elaeocarpi var. ovalis in having ovate appressoria in contrast to longer and cylindrical ones (Kar \& Ghosh, 1986, Hosagoudar, 2009). In $A$. elaeocarpi-kobanmochi appressoria are 
predominantly opposite and oblong and ascospores are smaller (Yamamoto, 1957).

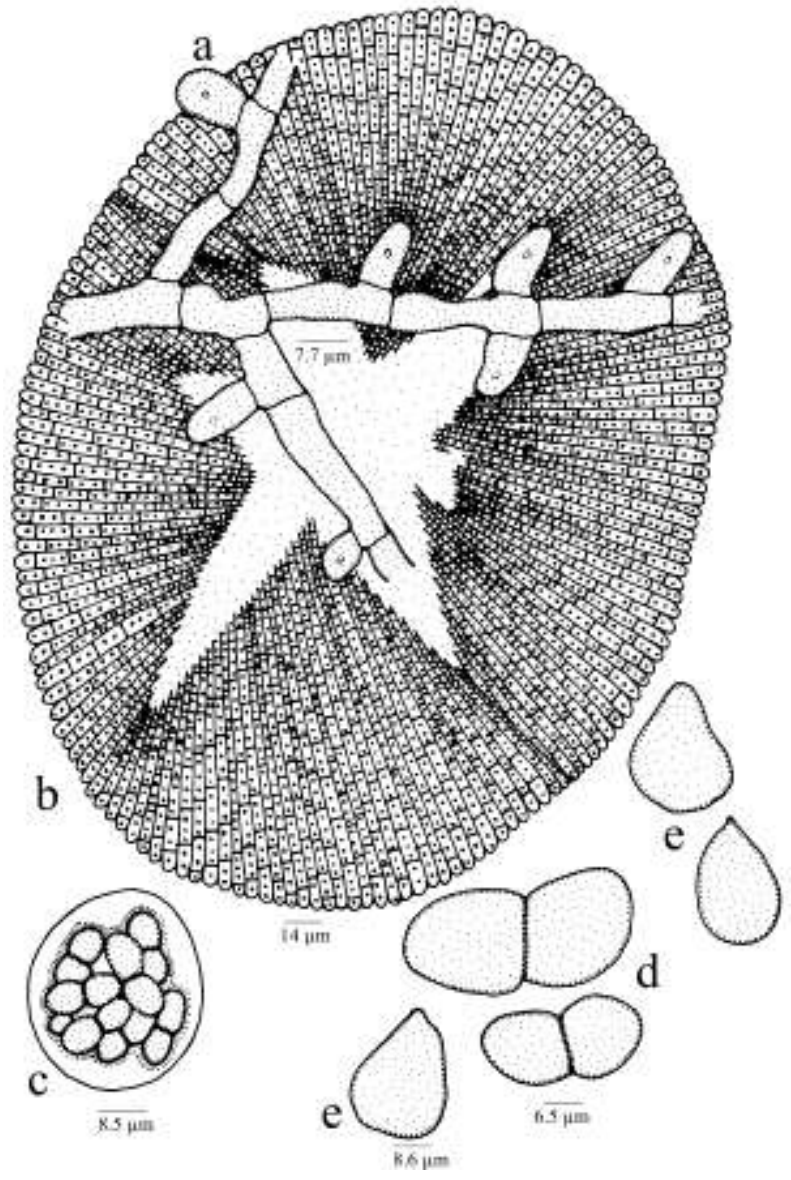

Figure 86 - Asterina gamsii

Distribution: Kerala, Tamil Nadu

Asterina garciniae Hansf., Proc. Linn. Soc. London 157: 204, 1946; Hosag. \& Archana, Zoos' Print J. 22: 2870, 2007; Hosag., Chandraprabha \& Agarwal, Asterinales of Kerala, p. 79, 2011. (Fig. 87)

Colonies epiphyllous, subdense, up to $2 \mathrm{~mm}$ in diameter, confluent. Hyphae straight to substraight, branching opposite, alternate, irregular at acute to wide angles, loosely to closely reticulate, cells 9-32 $\times$ 4-7 $\mu \mathrm{m}$. Appressoria ovate, alternate, unilateral, mostly sessile, 6-13 × 8-13 $\mu \mathrm{m}$., rarely 2 -celled, 11-16 $\mu \mathrm{m}$ long; stalk cells cylindrical to cuneate, 3-5 $\mu \mathrm{m}$ long; head cells globose, ovate, entire to angular, $8-13 \times 8-10 \mu \mathrm{m}$. Thyriothecia scattered to grouped, orbicular, up to $343 \mu \mathrm{m}$ in diameter, margin fimbriate, fringed hyphae flexuous, dehiscing stellately at the centre; asci globose, octosporous, up to $40 \mu \mathrm{m}$ in diameter; ascospores conglobate, brown, uniseptate, constricted at the septum, 24-27x 10-14 $\mu \mathrm{m}$, smooth walled.

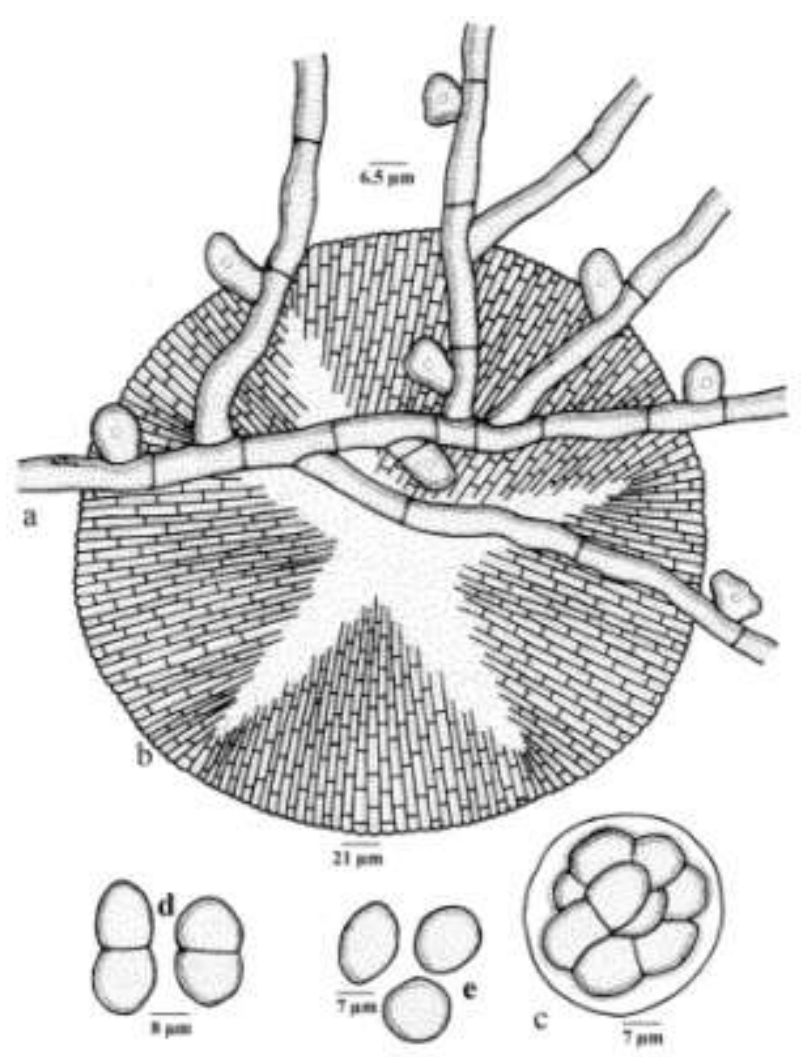

Figure 87 - Asterina garciniae

\section{Material examined: Kerala,} Thiruvananthapuram, Ponmudi, on leaves of Garcinia sp. (Clusiaceae), Chemunji hill ca. 1040 m, MSL, 22 November 2004, E. S. Santhosh Kumar TBGT 1977; 10 October 2008, A. Chandra Prabha HCIO 48894, TBGT 3270; Chemunji, on leaves of Garcinia travancorica Bedd., 3 January 2001, G. Rajkumar HCIO 49457, TBGT 3699; Kottayam, Ponthanpuzha, Garcinia sp., 26 March 2009, P.J. Robin \& al. HCIO 50033, TBGT 4185.

This species differs from $A$. morellae Hosag. et al. known on this host genus in having entire head cells (Hosagoudar et al., 2001).

\section{Distribution: Kerala}

Asterina garciniicola Ouyang \& Song in Ouyang, Song \& Hu, Acta Mycol. Sinica 14: 244, 1995; Hosag., Jacob- 
Thomas \& Agarwal, Taprobanica 3: 42, 2011. (Figs 88-89)

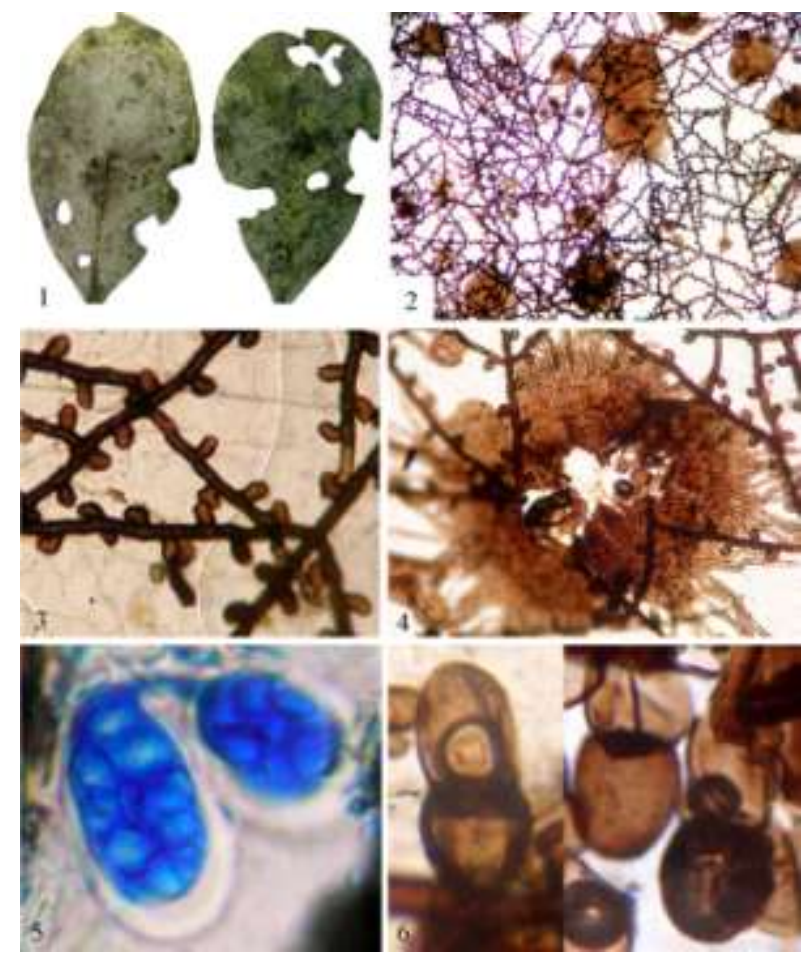

Figure 88 - Asterina garciniicola

1. Infected leaves, 2.Colony, 3.Appressoriate mycelium, 4. Thyriothecium, 5. Asci, 6. Ascospores

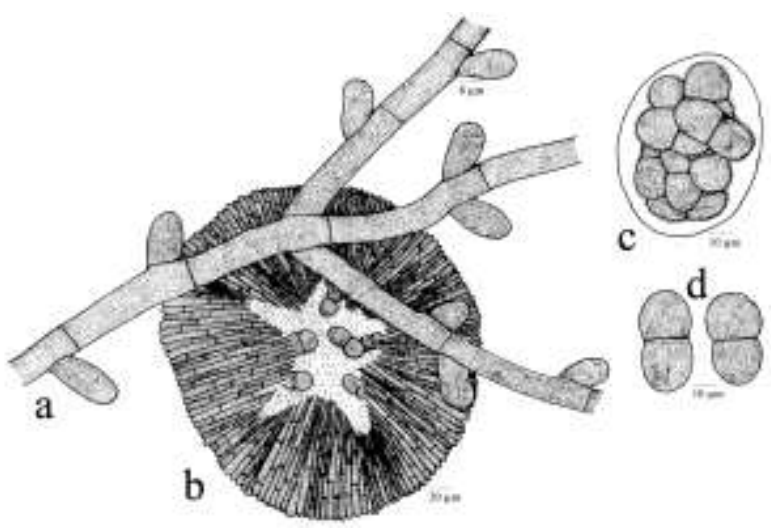

Figure 89 - Asterina garciniicola

Colonies epiphyllous, dense, velvety, up to $5 \mathrm{~mm}$ in diameter, rarely confluent. Hyphae straight to substraight, branching opposite at acute to wide angles, loosely to closely reticulate, cells $14-29 \times 7-10 \mu \mathrm{m}$. Appressoria alternate to $2 \%$ opposite, subopposite, unicellular, globose to cylindrical, straight to curved, antrorse to spreading, entire, 9-15 × 7$10 \mu \mathrm{m}$. Thyriothecia scattered, orbicular, often connate, up to $270 \mu \mathrm{m}$ in diameter, stellately dehisced at the centre, margin crenate to fimbriate, fringed hyphae flexuous; asci globose to ovate, octosporous, up to $65 \mu \mathrm{m}$ in diameter; ascospores conglobate, uniseptate, strongly constricted at the septum, 31-36 × 14$17 \mu \mathrm{m}$.

Material examined: Kerala, Thiruvananthapuram, Peppara Wildlife Sanctuary, Pandipathal, on leaves of Garcinia sp. (Clusiaceae), 29 February 2008, Jacob Thomas \& al. TBGT 3223, HCIO 48847.

However, our collection differs from the species type in having about $2 \%$ opposite appressoria and few are curved and closely appressed to mycelium. This species was reported on G. multiflora from China.

\section{Distribution: Kerala}

Asterina girardiniae Hosag. \& C.K. Biju in Hosag., Indian Phytopath. 58: 196, 2005; Hosag., Chandraprabha \& Agarwal, Asterinales of Kerala, p. 79, 2011. (Fig. 90)

Colonies epiphyllous, thin, up to $2 \mathrm{~mm}$ in diameter, rarely confluent. Hyphae flexuous, branching alternate to irregular at acute to wide angles, loosely reticulate, cells $24-28 \times 3-4 \mu \mathrm{m}$. Appressoria alternate, about $10 \%$ opposite, antrorse, subantrorse to retrorse, straight to curved, mostly 2-cells, rarely basal cell septate, 9-16(-24) $\mu \mathrm{m}$ long; stalk cells cylindrical to cuneate, mostly straight, flexuous when it is septate, 3-7(-16) $\mu \mathrm{m}$ long; head cells ovate, oblong to globose, entire, angular, rarely sublobate to lobate, broadly rounded to truncate at the apex, 6-7 $\times$ 4-7 $\mu \mathrm{m}$. Thyriothecia scattered to connate, orbicular, up to $112 \mu \mathrm{m}$ in diameter, crenate at the margin, stellately dehisced at the centre; asci globose, octosporous, up to $30 \mu \mathrm{m}$ in diameter; ascospores conglobate, oblong, uniseptate, constricted at the septum, pale brown to deep brown, 16-18 × 7-9 $\mu \mathrm{m}$, wall smooth.

Material examined: Kerala, Idukki, Munnar, Bendhar, on leaves of Girardinia diversifolia (Link) Fries (Urticaceae), 23 January 2003, A. Manojkumar \& H. Biju HCIO 
44865 (holotype), TBGT 1093 (isotype); Mannavan shola, 5 January 1999, C.K. Biju HCIO 45280, TBGT 1318; Tamil Nadu, Boothkanal, Kodaikanal, 12 November 2007, K. Thiyagesan TBGT 5751.

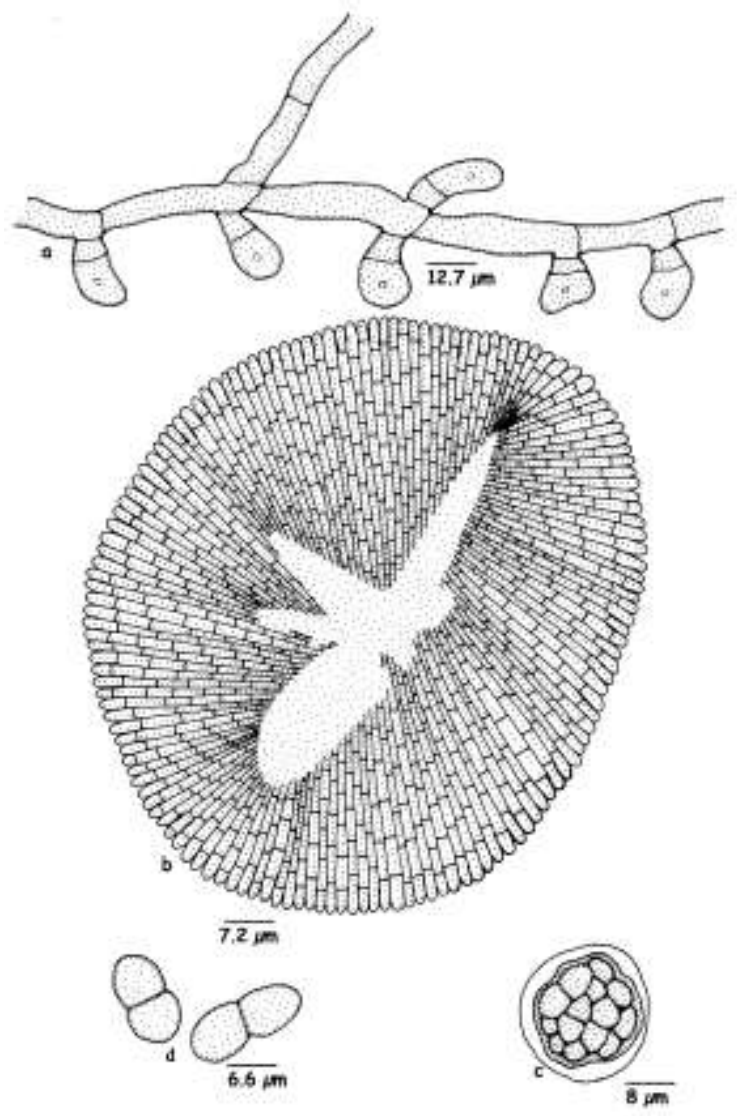

Figure 90 - Asterina girardiniae

This species differs from other Asterina species known on the members of the family Urticaceae in having two celled and 10\% opposite appressoria. This forms the first report of the genus Asterina on the genus Girardinia (Hosagoudar \& Abraham, 2000).

Distribution: Kerala, Tamil Nadu

Asterina glycosmidigena Hosag. \& JacobThomas, J. Appl. Nat. Sci. 2: 102, 2010. (Fig. 91)

Colonies epiphyllous, thin, up to $2 \mathrm{~mm}$ in diameter, confluent. Hyphae pale brown, straight to slightly crooked, branching irregular at acute to wide angles, loosely reticulate and form a loose mycelial net, cells $12-19 \times 2-5 \mu \mathrm{m}$.
Appressoria sessile, mostly alternate, about 2\% opposite, unicellular, ovate, subglobose, irregularly sublobate, entire, 4-10 × 4-7 $\mu \mathrm{m}$. Thyriothecia scattered, orbicular, often connate, up to $110 \mu \mathrm{m}$ in diameter, stellately dehisced at the centre, margin crenate; asci globose, octosporous, 50-60 $\mu \mathrm{m}$ in diameter; ascospores oblong, conglobate, brown, uniseptate, strongly constricted at the septum, 14-19 $\times 7-10 \mu \mathrm{m}$, wall smooth.
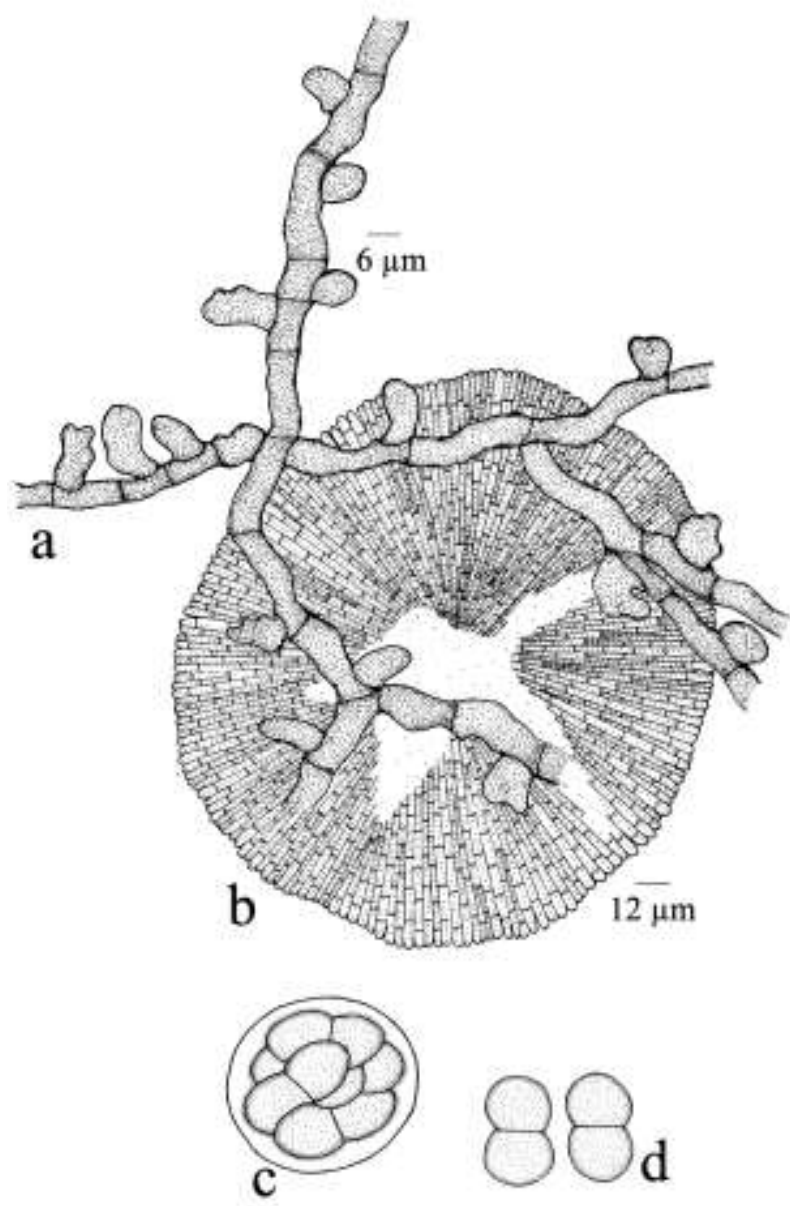

Figure 91 - Asterina glycosmidigena

Material examined: Kerala, Wayanad, on leaves of Glycosmis pentaphylla (Retz.) DC. (Rutaceae), Jacob Thomas \& al. TBGT 3669 (holotype), HCIO 49424 (isotype); Karnataka, Hoddur, Devarakadu, on leaves of Glycosmis sp., 4 December 2009, C. Jagath Thimmaiah TBGT 5477.

The present species differs from Asterina glycosimidis Hosag. \& Rajkumar and A.banguiensis Yates known on the host genus in having hemispherical, broad based, 
irregularly sublobate to lobate appressoria (Hosagoudar, 2005; Yates, 1918a,b).

These colonies were mixed with the colonies of Meliola sp.

Distribution: Karnataka, Kerala

Asterina glycosmidis Hosag. \& Rajkumar in Hosag., Indian Phytopath. 58: 194, 2005; Hosag., Chandraprabha \& Agarwal, Asterinales of Kerala, p. 80, 2011. (Fig. 92)

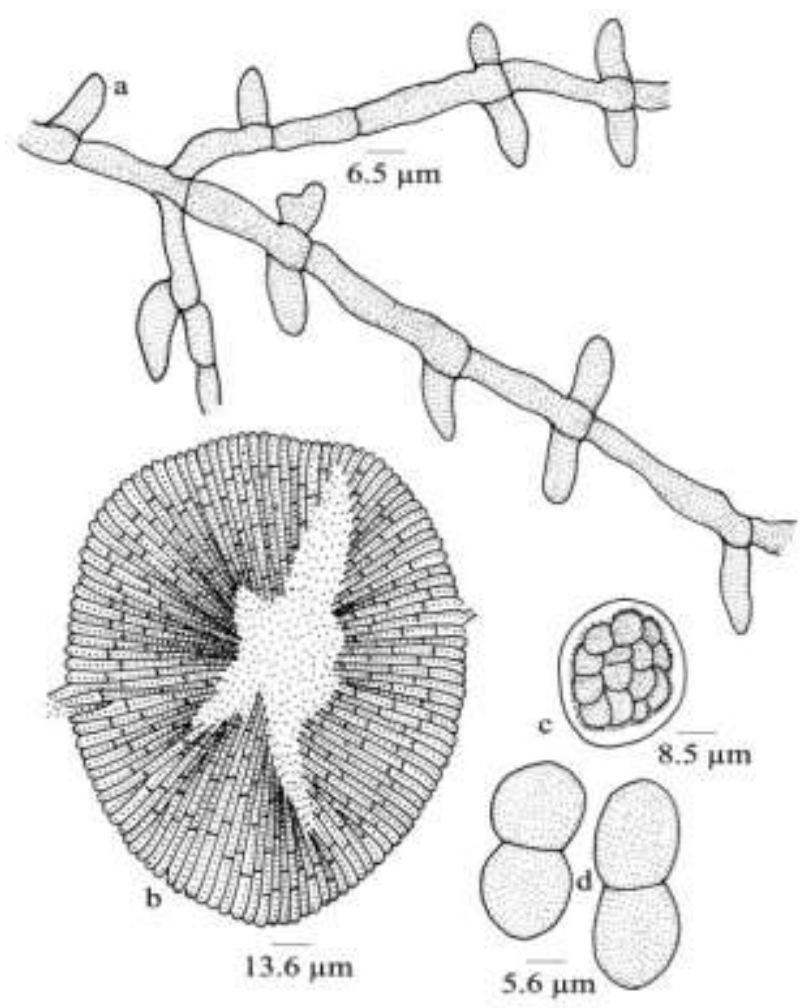

Figure 92 - Asterina glycosmidis

Colonies mostly epiphyllous, subdense to dense, thinly velvety, up to $2 \mathrm{~mm}$ in diameter, confluent. Hyphae straight, substraight to flexuous, branching opposite to irregular at acute to wide angles, loosely to closely reticulate, cells 20-26 × 4-6 $\mu \mathrm{m}$. Appressoria unicellular, alternate, unilateral, about $40 \%$ opposite, ovate, conoid, oblong, often cylindrical, entire to rarely angular to sublobate, 6-13 $\times 4-6 \mu \mathrm{m}$. Thyriothecia scattered to closely grouped, orbicular, up to $150 \mu \mathrm{m}$ in diameter, margin crenate to fimbriate, fringed hyphae small, stellately dehisced at the centre; asci few, globose, octosporous, up to $30 \mu \mathrm{m}$ in diameter; ascospores conglobate, brown, uniseptate, deeply constricted at the septum, $25-28 \times 14-18$ $\mu \mathrm{m}$, wall smooth.

Material examined: Kerala, Wayanad, on leaves of Glycosmis sp. (Rutaceae), 6 March 2001, G. Rajkumar HCIO 45174 (holotype), TBGT 1335 (isotype).

Asterina banguiensis Yates is known on this host genus from Philippines (Yates, 1918a,b; Hosagoudar \& Abraham, 2000). Asterina glycosmidis differs from it in having opposite, alternate and unilateral appressoria, differ in the shape of the appressoria, and possessing larger ascospores.

Distribution: Kerala.

Asterina glyptopetali Hosag. \& C.K. Biju, Indian Phytopath. 58: 198, 2005; Hosag., Chandraprabha \& Agarwal, Asterinales of Kerala, p. 81, 2011. (Fig. 93)

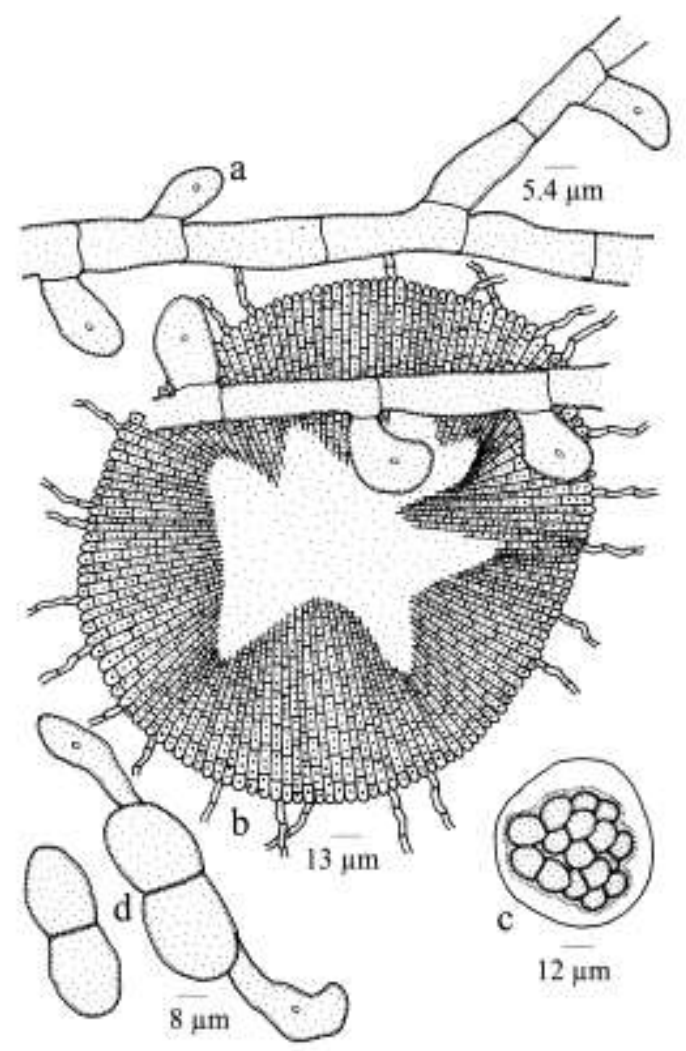

Figure 93 - Asterina glyptopetali

Colonies amphigenous, dense, velvety, up to $2 \mathrm{~mm}$ in diameter, rarely confluent. Hyphae straight to substraight, branching alternate, opposite to irregular at acute to wide angles, loosely to closely reticulate, cells 11-24 $\times 7-9 \mu \mathrm{m}$. Appressoria unicellular, alternate, 
clavate, cylindrical, straight to slightly curved, entire, $17-21 \times 8-10 \mu \mathrm{m}$. Thyriothecia scattered to connate, orbicular, up to $170 \mu \mathrm{m}$ in diameter, stellately dehisced at the centre, margin fimbriate, fringed hyphae flexuous; asci globose, octosporous, up to $50 \mu \mathrm{m}$ in diameter; ascospores conglobate, uniseptate, constricted at the septum, brown, $36-40 \times 18-22 \mu \mathrm{m}$, wall smooth.

Material examined: Kerala, Idukki, Munnar, Mannavan Shola, on leaves of Glyptopetalum zeylanicum Thw. (Celastraceae), 6 January 1999, C.K. Biju HCIO 45278 (holotype), TBGT 1316 (isotype).

Based on the unicellular appressoria and having straight hyphae, this taxon can be compared with $A$. euonymi Hosag. \& Goos known on Euonymus crenulatus from Western Ghats of Southern India. However, $A$. glyptopetali differs from it in having only cylindrical to clavate and entire appressoria and have larger ascospores.

\section{Distribution: Kerala}

Asterina gomphandrae Hosag. \& C.K. Biju in Hosag., Zoos' Print J. 17: 835, 2002; Hosag., Chandraprabha \& Agarwal, Asterinales of Kerala, p. 82, 2011. (Fig. 94)

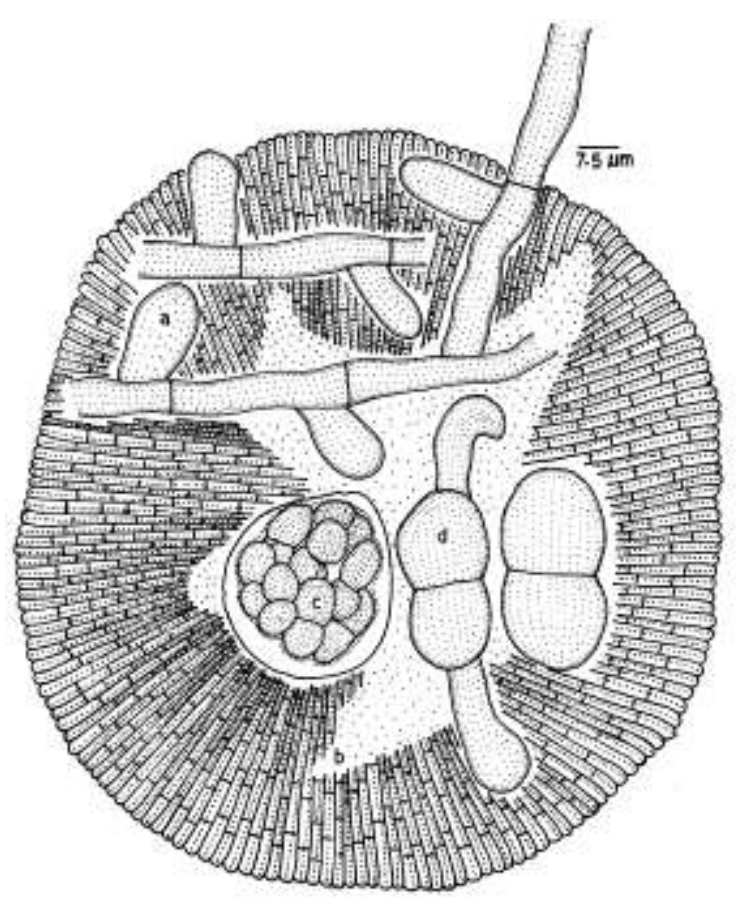

Figure 94 - Asterina gomphandrae
Colonies epiphyllous, dense, up to 2 $\mathrm{mm}$ in diameter, confluent. Hyphae substraight to flexuous, branching alternate at acute to wide angles, loosely reticulate, cells 11-21 × 4-7 $\mu \mathrm{m}$. Appressoria alternate, unicellular, cylindrical, clavate, straight to curved, entire, 9-13 $\times 7-9$ $\mu \mathrm{m}$. Thyriothecia scattered, connate, orbicular, up to $110 \mu \mathrm{m}$ in diameter, stellately dehisced at the center, margin crenate to fimbriate; asci few, globose, octosporous, up to $30 \mu \mathrm{m}$ in diameter; ascospores oblong, conglobate, brown, uniseptate, constricted at the septum, 20-26 × 9-11 $\mu \mathrm{m}$, wall smooth.

Material examined: Kerala, Idukki, Mannavan shola, on leaves of Gomphandra sp. (Icacinaceae), 6 January 1999, C.K. Biju HCIO 43963 (holotype), TBGT 400 (isotype).

Asterina mappiae Petrak \& Cifferi and A. natsiati Kar \& Maity were known on the members of the family Icacinaceae (Petrak \& Cifferi, 1930; Kar \& Maity, 1970; Hosagoudar \& Abraham, 2000). However, A. gomphandrae differs from both in having unicellular, oblong to cylindrical, entire appressoria and larger ascospores.

Distribution: Kerala.

Asterina goosii Hosag. \& Balakr. in Hosag., Balakr. \& Goos, Mycotaxon 59: 178, 1996; Hosag., Chandraprabha \& Agarwal, Asterinales of Kerala, p. 83, 2011.

(Fig. 95)

Colonies epiphyllous, very rarely few colonies hypophyllous, dense, up to $3 \mathrm{~mm}$ in diameter, confluent. Hyphae brown, straight to substraight, branching alternate to irregular at acute angles, loosely reticulate, cells 21-25 $\times 3$ $5 \mu \mathrm{m}$. Appressoria brown, alternate, about 1\% opposite, straight, antrorse, 2-celled, 9-13 $\mu \mathrm{m}$ long; stalk cells cuneate, 3-4 $\mu \mathrm{m}$ long; head cells globose, irregularly sublobate to lobate, 6$10 \times 9-10 \mu \mathrm{m}$. Thyriothecia round to ovate, scattered to connate, up to $186 \mu \mathrm{m}$ in diameter, dehiscing stellately at the center, margin fimbriate; fringed hyphae pale, flexuous, nonappressoriate, up to $62 \mu \mathrm{m}$ long; asci many, eight spored, globose, $27-35 \mu \mathrm{m}$ in diameter; ascospores conglobate, initially hyaline, dark 
brown at maturity, oblong, rounded at both ends, 1-septate, deeply constricted at the septum, 21-25 × 9-13 $\mu \mathrm{m}$, wall smooth.
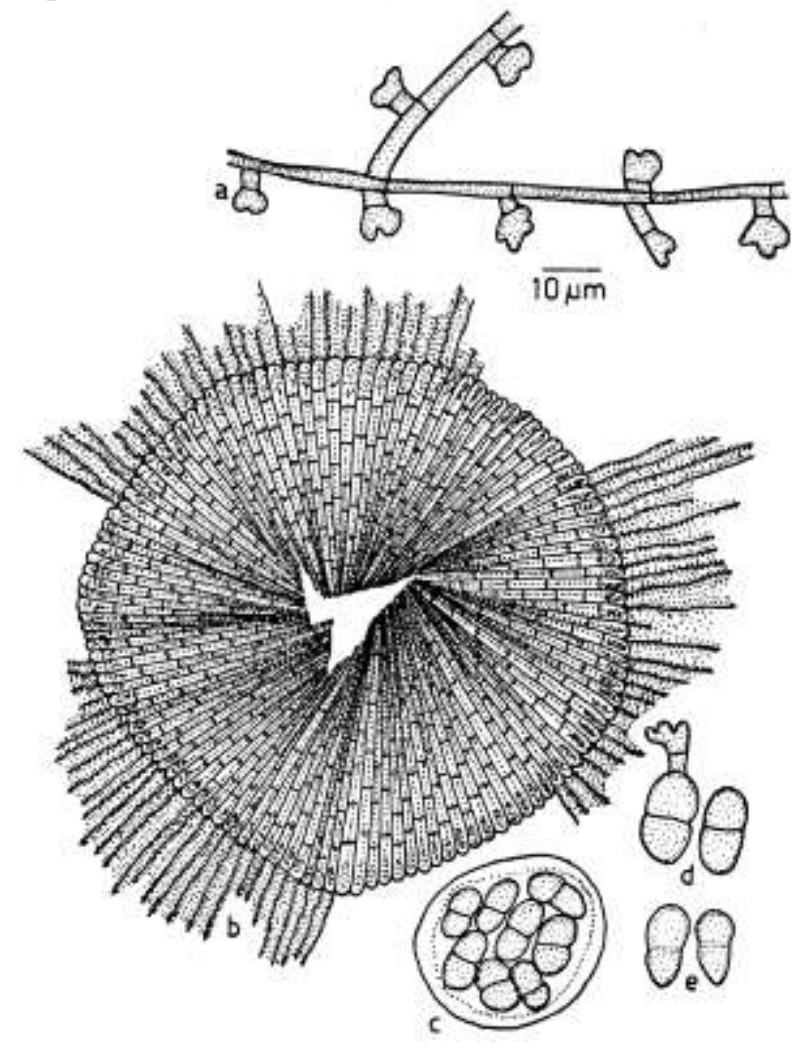

Figure 95 - Asterina goosii

Material examined: Tamil Nadu, Kodaikanal, on leaves of Mahonia leschenaultii (Wallich ex Wight \& Arn.) Takeda ex Gamble (Berberidaceae), 10 March 1986, V.B. Hosagoudar HCIO 30965; Poomparai shola, 19 July 2006, R. Nithyatharani HCIO 47104, TBGT 2321; Gundan shola West, 21 July 2006, R. Nithyatharani HCIO 47722, TBGT 2744; Bombay shola, 13 July 2006, Bairavi \& al. HCIO 47585, TBGT 2607; Then Shola, 8 March 2007, V.B. Hosagoudar \& al. HCIO 48064, TBGT 2847; Periyakanal, 5 January 2010, R. Nithyatharani TBGT 5107; Kerala, Idukki, Mannavan Shola, 23 September 1998, C.K. Biju HCIO 42938, TBGT 215.

Asterina mahoniae Keibl. known on this host genus from China. Based on the endemic status of the host and host specificity of the genus, it has been accommodated in a separate species (Hosagoudar and Abraham, 2000).

Distribution: Kerala, Tamil Nadu

Asterina gopalakrishnanii Nair \& Kaul, Curr. Sci. 56: 1071, 1987.
Colonies amphigenous, scattered to aggregated, dense to subdense. Mycelium superficial, reticulate, substraight, cells 20-25 $\times$ 5-7 $\mu \mathrm{m}$. Appressoria sessile, alternate, hemispherical, one celled, 5-7 $\mu \mathrm{m}$ broad. Thyriothecia carbonaceous, ostiolate, 226-240 $\mu \mathrm{m}$ in diameter, stellately dehisced; asci ovate, aparaphysate, $\quad 50-65 \mu \mathrm{m} \times 25-50 \mu \mathrm{m}$; ascospores two celled, $12-16 \times 30-45 \mu \mathrm{m}$, thick walled, spiny, brown with two dark brown bands in the middle of each cell.

Maharashtra, Mahabaleshwar, on leaves of Syzygium cumini (L.) Skeels (Myrtaceae), February 1979.

Distribution: Maharashtra

Asterina granulosa (Hansf.) Hosag., Balakr. \& Goos, Mycotaxon 59: 179, 1996; Hosag., Chandraprabha \& Agarwal, Asterinales of Kerala, p. 85, 2011. Asterina grewiae Cooke var. granulosa Hansf., Proc. Linn. Soc. London 156: 114, 1944. (Fig. 96)

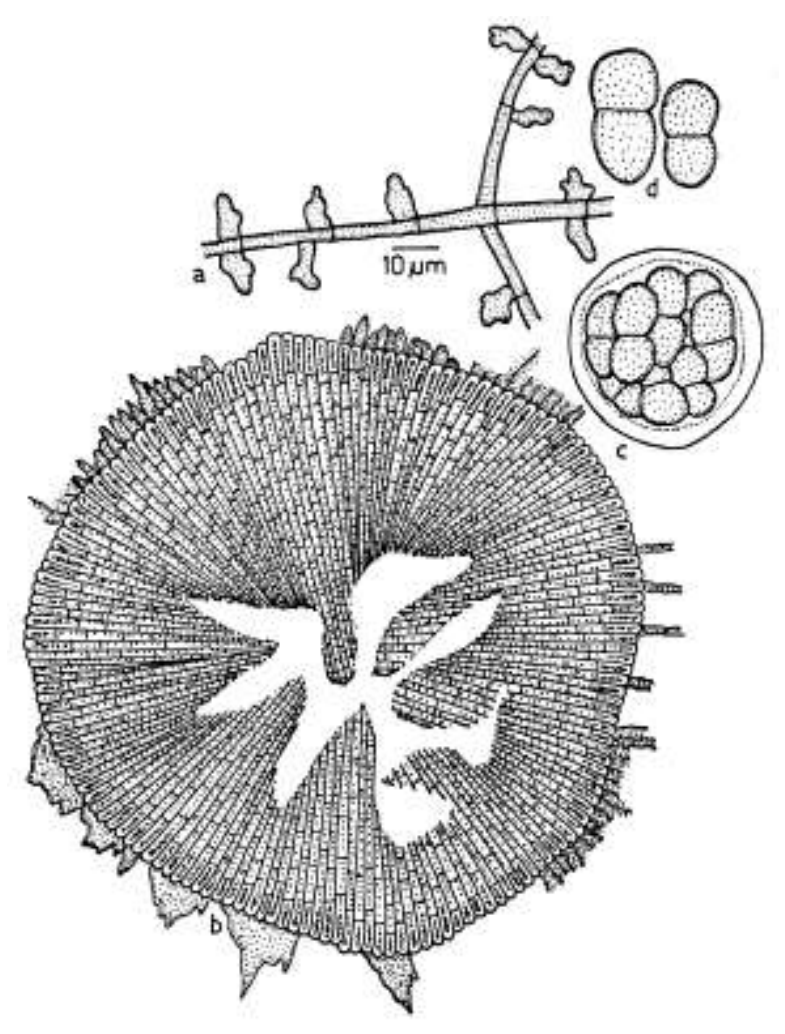

Figure 96 - Asterina granulosa

Colonies amphigenous, thin, up to 5 $\mathrm{mm}$ in diameter, confluent and covering most 
of the leaf surface. Hyphae straight to flexuous, branching mostly opposite at wide angles, loosely reticulate, cells $12-22 \times 3-4 \mu \mathrm{m}$. Appressoria alternate and opposite, straight to curved, cylindrical, ovate, entire to sublobate, rounded to truncate at the apex, 8-14 $\times 6-8 \mu \mathrm{m}$. Thyriothecia scattered, round, up to $140 \mu \mathrm{m}$ in diameter, dehisce stellately at the center, margin crenate to fimbriate, fringed hyphae brown, flexuous; asci many, globose, eight spored, 42-45 $\mu \mathrm{m}$ in diameter; ascospores conglobate, deep brown, 1-septate, both cells almost equal in size, $27-31 \times 12-16 \mu \mathrm{m}$, wall smooth. Few pycnothyriospores were observed, deep brown, pyriform, slightly beaked, 24-26 $\times$ 15-16 $\mu \mathrm{m}$.

Material examined: Tamil Nadu, Nilgiris, Kotagiri, Kolithorai, on leaves of Scolopia crenata (Wight \& Arn.) Clos. (Flacourtiaceae), 10 October 1991, V.B. Hosagoudar HCIO 30966; Kerala, Kollam, Shankily forest, 23 November 2004, V.B. Hosagoudar \& al. HCIO 46267, TBGT 1913; Thiruvananthapuram, Palode, TBGRI Campus, on leaves of Scolopia sp., 15 November 2007, A. Sabeena HCIO 48405, TBGT 3126.

Hansford (1944) described Asterina grewiae Cooke var. granulosa Hansf. on Scolopia sp. from Uganda. Doidge (1942) discussed the identity of the host of Asterina grewiae Cooke. Since this taxon has distinct characters, Hosag. et al. (1996) gave a species status.

Distribution: Kerala, Tamil Nadu.

Asterina gymnemae Hosag. \& Jacob-Thomas, J. Appl. Nat. Sci. 2: 102, 2010.(Figs 9798)

Colonies epiphyllous, subdense, up to 1 $\mathrm{mm}$ in diameter, confluent. Hyphae substraight to undulate, branching opposite to irregular at acute to wide angles, loosely to closely reticulate, cells 19-34 $\times 2-5 \mu \mathrm{m}$. Appressoria alternate, two celled, straight to curved, antrorse, 12-14 $\mu \mathrm{m}$ long; stalk cells cylindrical to cuneate, straight to crooked, 2-5 $\mu \mathrm{m}$ long; head cells ovate, globose, oblong, angular, sublobate to variously lobate, 4-7 $\times$
4-10 $\mu \mathrm{m}$. Thyriothecia scattered, orbicular, up to $150 \mu \mathrm{m}$ in diameter, stellately dehisced at the centre, margin crenate; asci few to many, globose,

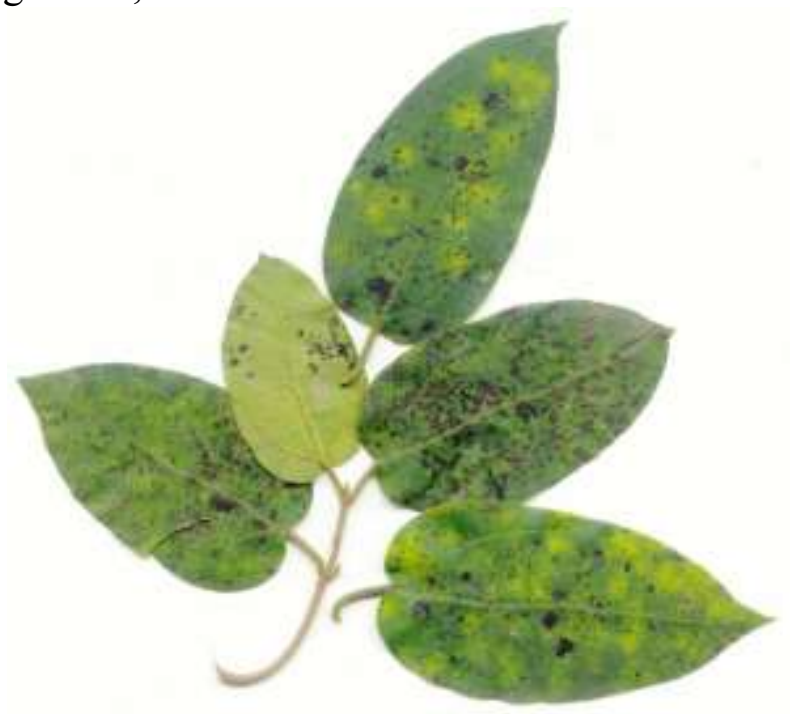

Figure 97 - Asterina gymnemae - Infected leaves

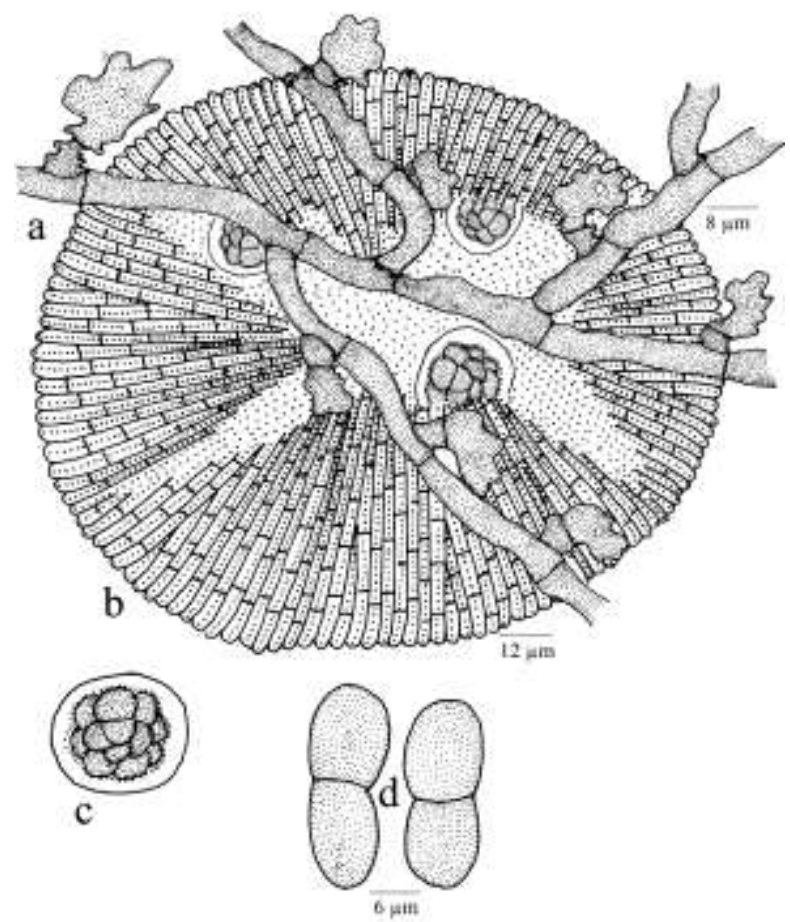

Figure 98 - Asterina gymnemae

octosporous, 40-60 $\mu \mathrm{m}$ in diameter; ascospores conglobate, uniseptate, hyaline, constricted at the septum, 16-19 $\times$ 7-10 $\mu \mathrm{m}$, wall smooth.

Material examined: Kerala, Wayanad, Thirunelli, on leaves of Gymnema sylvestre R.Br. (Asclepiadaceae), 14 February 2009, Jacob Thomas \& al. TBGT 3667a (holotype), HCIO 49422a (isotype); Periya, on 
the leaves of a member of Asclepiadaceae, 8 March 2008, Robin \& al. HCIO 49803, TBGT 3955; Tamil Nadu, Kodaikanal, Periya Kanal, on leaves of Gymnema sp., 7 January 2010, R. Nithyatharani TBGT 4355.

There are six species of the genus Asterina known on the members of the family Asclepiadaceae, namely, Asterina asclepiads Hosag. \& Goos (1996), A.coccina Sydow (1930), A. cynanchi Hosag. \& Shiburaj (Hosagoudar, 2002), A. leonensis Sydow (1938), and A. paraffinis Speg. (Theissen, 1913). Based on the character of angular to sublobate head cells and evenly placed appressoria, the present species is closer to $A$. cynanchi, $A$. leonensis and $A$. paraffinis. However, the present species differs from $A$. paraffinis in having narrow ascospores and from $A$. cynanchi in having longer ascospores. It also differs from $A$. leonensis in having only alternate appressoria.

Distribution: Kerala, Tamil Nadu.

Asterina gymnosporiae Castellani, Neuovo G. Bot. Ital. 49: 11, 1942; Thite \& Kulkarni, Indian Phytopath. 29: 169, 1976.

The infection in the form of a thick, black mass on the upper surface of the leaves and no definite colonies are formed. Hyphae Straight to sub straight, branched, branching opposite to irregular, closely interwoven, reticulate, septate, cells mostly 25-35 $\times 5-7$ $\mu \mathrm{m}$. Appressoria in whorls of five to six at each septum, spreading, 12-35 $\mu \mathrm{m}$ long; stalk cells cylindrical, 3-8 $\mu \mathrm{m}$ long; head cells ovate, entire, rounded at apex, 10-15 $\times 8-10$ $\mu \mathrm{m}$. Thyriothecia scattered with free mycelium in groups of four to five closely attached to each other, dimidiate radiate, circular, 300-450 $\mu \mathrm{m}$ in diameter; ascospores are irregularly arranged in the oval ascus, they are elliptical to oblong, one septate, $30-40 \times 15-20 \mu \mathrm{m}$.

Maharashtra, Amboli and Amba Ghat, Radhanagari, on the leaves of Gymnosporia puberula Laws. (Celastraceae), October 1969, A. N. Thite HCIO 31680.
Asterina gymnosporiae E. Castel was first described by Castellani (1942) from Italy growing on gymnosporia species. If the appressoria are in whorls, it has more affinity towards Ishwaramyces than Asterina.

Distribution: Maharashtra

Asterina hakgalensis Hansf., Proc. Linn. Soc. London 158: 45, 1947; Hosag., Jacob Thomas \& Robin, Indian J. Sci. Techn. 2: 2, 2009; Hosag., Chandraprabha \& Agarwal, Asterinales of Kerala, p. 86, 2011. (Figs 99-100)

Colonies epiphyllous, dense, crustose, orbicular, up to $3 \mathrm{~mm}$ in diameter, rarely confluent. Hyphae substraight to crooked, branching alternate to irregular at acute angles, loosely to closely reticulate, cells $14-20 \times 4-5$ $\mu \mathrm{m}$. Appressoria moderate to sparse, mostly present in the peripheral hyphae, globose, mammiform, broad based, entire, 6-7 $\mu \mathrm{m}$ high and 7-9 $\mu \mathrm{m}$ broad. Thyriothecia scattered to grouped, discrete to often connate, orbicular, up to $215 \mu \mathrm{m}$ in diameter, margin fringed, fringed hyphae join with the fringed hyphae of the other thyriothecia, carbonaceous black and stellately or irregularly splitted at the centre, radiating cells visible only towards the margin of the thyriothecia; asci few to many, ovate to globose, octosporous, $30-35 \mu \mathrm{m}$ in diameter; ascospores conglobate, oblong, brown, 1septate, slightly constricted at the septum, 20$23 \times 10-12 \mu \mathrm{m}$, wall smooth.

Material examined: Kerala, Idukki, Munnar, Sylvan valley hill, on leaves of Rhododendron arboreum J. E. Smith ssp. nilagiricum (Zenk.) Tagg. (Ericaceae), 25 April 2002, S. Shiburaj HCIO 44554, TBGT 840; Pettimudi, 22 April 2008, A. Chandra Prabha HCIO 48303, TBGT 3024; HCIO 48304, TBGT 3024;Idukki, Munnar, Mannavan shola, on leaves of Rhododendron sp., 6 January 1999, C.K. Biju HCIO 47395, TBGT 2433; 11 May 1999, C.K. Biju HCIO 45282, TBGT 1320; S. Shiburaj HCIO 44644, TBGT 926; Rajamala, 20 January 2003, H. Biju \& A. Manojkumar HCIO 44827, TBGT 1064; Meghalaya, Shillong, Mawphlang, 21 January 2007, Jacob Thomas \& P.J. Robin HCIO 48038, TBGT 2821; 20 January 2007, P.J. Robin \& Jacob 
Thomas HCIO 48052, TBGT 235; Tamil Nadu, Kodaikanal, Mathikettan shola, 18 July 2006, B. Bairavi HCIO 47033, TBGT 2250; 18 July 2006, R. Nithyatharani

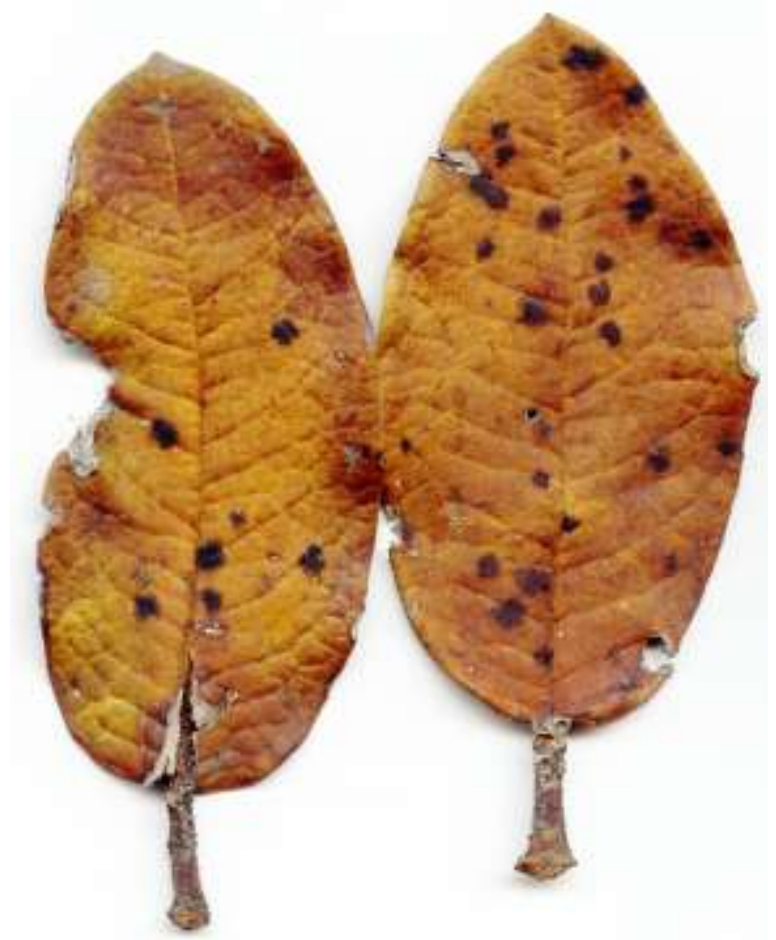

Figure 99 - Asterina hakgalensis-Infected leaves

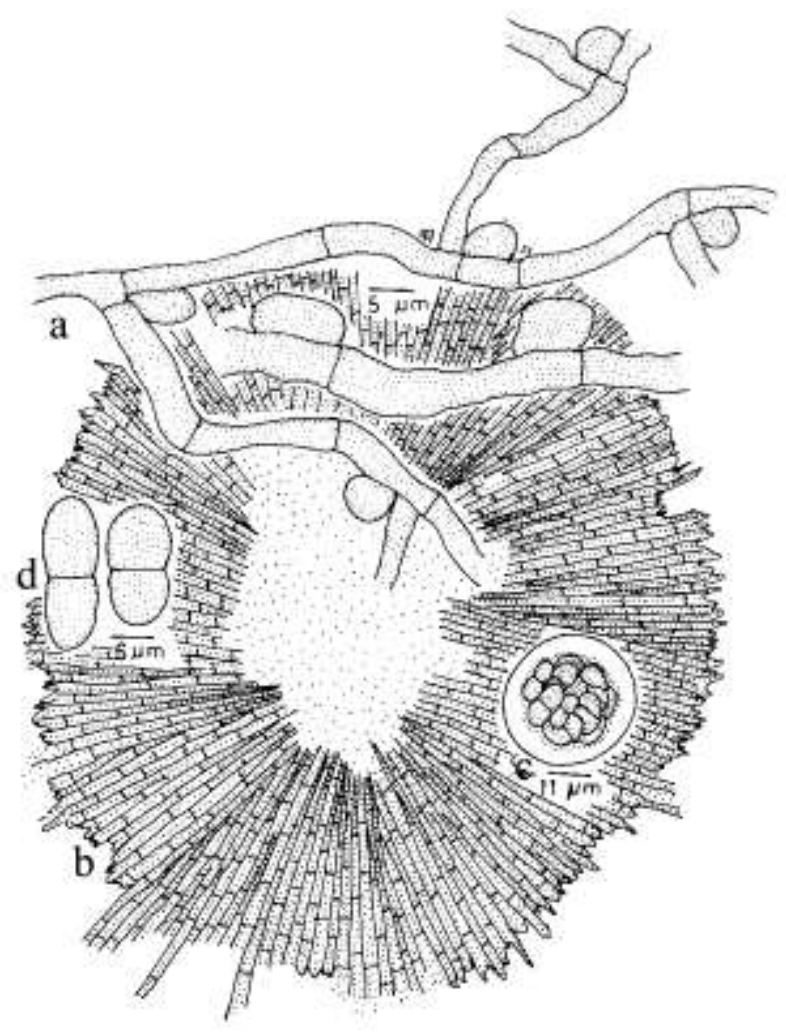

Figure 100 - Asterina hakgalensis

HCIO 47036,TBGT 2253; Bear shola, 22 July 2006, R. Nithyatharani HCIO 47045, TBGT
2262; Bombay shola, 13 June 2006, Bairavi \& al. HCIO 47585, TBGT 2607; Gundan shola West, 22 July 2006, R. Nithyatharani HCIO 47687, TBGT 2709; Bear shola West, 22 July 2006, R. Nithyatharani HCIO 47705, TBGT 2727; Gundan shola West, 21 July 2006, R. Nithyatharani HCIO 47724, TBGT 2746; Gundan shola West, 30 July 2006, B. Bhairavi HCIO 48440, TBGT 3161; Kerala, Thiruvananthapuram, Palode, 14 December 2007, A. Chandraprabha HCIO 48304, TBGT 3025; Meghalaya, Shillong, Mawphlang, on leaves of Rhododendron arboreum Smith (Ericaceae), 21 January 2007, Jacob Thomas \& P.J. Robin HCIO 48038, TBGT 2821.

This species was known on Rhododendron arboreum var. arboreum from Sri Lanka (Hansford, 1947).

This is the only species known on this host genus.

Distribution: Kerala, Tamil Nadu, Meghalaya.

Asterina helicteris Ouyang \& $\mathrm{Hu}$ in Yousheng, Song \& Hu, Acta Mycol. Sinica 15: 88, 1996; Hosag., Chandraprabha \& Agarwal, Asterinales of Kerala, p. 87, 2011.

Asterostomella helicteridis Hosag., Balakr. \& Goos, Mycotaxon 58: 491, 1996.

(Fig. 101)

Colonies amphigenous, dense, crustose to velvety, up to $2 \mathrm{~mm}$ in diameter, confluent. Hyphae flexuous to crooked, branching irregular at acute to wide angles, loosely reticulate, cells 15-28 $\times 2-4 \mu \mathrm{m}$. Appressoria scattered, alternate, unicellular, stipitate to sessile, clavate, globose, hamate, straight to curved, angular, slightly to deeply 3-5-lobate, straight to deeply curved, $8-10 \times 9-11 \mu \mathrm{m}$. Thyriothecia scattered, loosely grouped to rarely connate, orbicular, up to $150 \mu \mathrm{m}$ in diameter, stellately dehisced at the centre, crenate at the margin; asci few to many, globose, octosporous, bitunicate, up to $30 \mu \mathrm{m}$ in diameter; ascospores oblong, conglobate, brown, uniseptate, constricted at the septa, 16$18 \times 8-10 \mu \mathrm{m}$, wall smooth. Pycnothyria many, 
similar but smaller than the thyriothecia; pycnothyriospores oblong, pyriform, brown, $14-18 \times 6-11 \mu \mathrm{m}$.

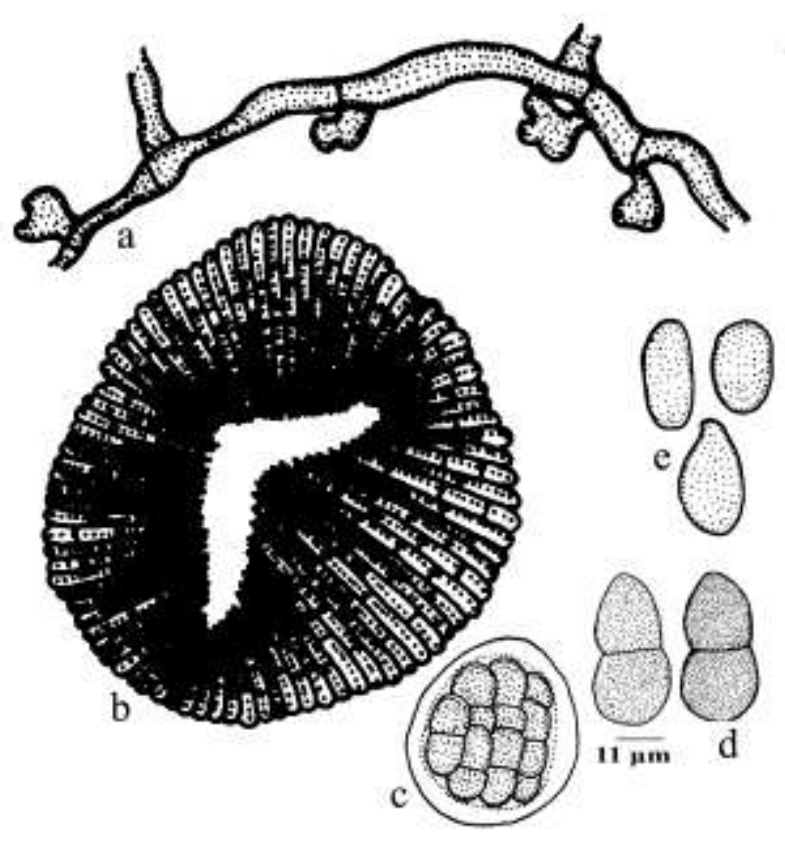

Figure 101 - Asterina helicteridis

\begin{abstract}
Material examined: Kerala, Pathanamthitta, Erumeli, on leaves of Helicteres isora L. (Sterculiaceae), 29 December 2005, P.J. Robin \& V. Gireesh HCIO 47332, TBGT 2370; Thiruvananthapuram, Peppara Wildlife Sanctuary, 18 November 2007, Jacob Thomas \& Vimal HCIO 48267, TBGT 3006; Palode, TBGRI campus, 16 October 2008, K. Anil Kumar TBGT 5140; Kollam, Arippa, 27 October 2006, V.B. Hosagoudar \& al. TBGT 4674; Choliakodu, 27 October 2006, V.B. Hosagoudar \& al. TBGT 4680. Anamorph: Tamil Nadu, Gudalur, Sri Madurai, on leaves of Helicteres isora Miq. (Urticaeae), 25 January 1990, V.B. Hosagoudar HCIO 30979; Kerala, Thiruvananthapuram, Peppara, 2 February 1997 V.B. Hosagoudar TBGT 137a, HCIO 42502.
\end{abstract}

Asterina leptalea Sydow has been reported on this host (Sydow \& Petrak, 1931) but $A$. helicteridis differs from it in the morphology and measurement.

Distribution: Kerala, Tamil Nadu
Asterina henianii Verma, Tripathi \& Chaudhary, Indian Phytopath. 52:377, 1999.

Infection spots amphigenous, forming brownish black, circular, scattered or aggregated, dense, hard, widely effused patches up to $8 \mu \mathrm{m}$ in diameter, coalescing to cover almost whole leaf surface but it retains the original shape. Mycelium superficial, loosely reticulate, composed of dark brown, septate, branched, wavy hyphae, individual cells measuring 4.5-8.7 $\mu \mathrm{m}$ in diameter, branching irregular. Appressoria mostly bicellular, sometimes unicellular, measuring 12.2-20.5 $\times$ 5.6- $9.3 \mu \mathrm{m}$. dark brown, straight to bent, lower cell of appressoria smaller, cylindrical to cuneate, head cell capitates, spherical to hemispherical. Thyriothecia many, scattered or grouped together, present in the mycelial colonies like a pin heads, rounded about 112.0$157.4 \mu \mathrm{m}$, in diameter, dimidiate with radiate scutellum stellately dehiscing at first, subsequently developing in to an aperture, convex, dark brown, hymenium, simple, margin fimbriate; asci many, rounded to oval, sessile, 8 -spored, measuring 37.5-56.3 × 61.2-67.5 $\mu \mathrm{m}$; ascospores crowded, elliptical, oblong, uniseptate, constricted at the septum, rounded at ends, bluish brown to brownish yellow, smooth walled, measuring 8.1-16.2 $\times 24-37.5 \mu \mathrm{m}$.

Uttar Pradesh, Mathrjganj, on leaves of Syzygium henianum (Myrtaceae), March 1997, R. C. Verma HCIO 42574.

Distribution: Utter Pradesh

Asterina hibisci (Doidge) Hosag. in Hosag., C.K. Biju \& Abraham, J. Econ. Taxon. Bot. 28: 175, 2004; Hosag., Zoos' Print J. 21: 2327, 2006; Hosag., Chandraprabha \& Agarwal, Asterinales of Kerala, p. 88, 2011.

Asterina diplocarpa Cooke var. hibisci Doidge, Botahalia 4: 331, 1942.

(Fig. 102)

Colonies mostly epiphyllous, thin to subdense, up to $5 \mathrm{~mm}$ in diameter, confluent and thinly cover an entire upper surface of the leaves. Hyphae substraight to undulate, 
branching alternate to opposite at acute angles, loosely reticulate, $20-32 \times 2-4 \mu \mathrm{m}$. Appressoria unicellular, alternate, scattered, straight to rarely curved, ovate, globose, entire to sublobate, $9-12 \times 4-9 \mu \mathrm{m}$.. Thyriothecia scattered, orbicular, up to $110 \mu \mathrm{m}$ in diameter, stellately dehisced at the center, margin crenate; asci few to many, mostly globose, octosporous, 25-35 $\mu \mathrm{m}$ in diameter; ascospores brown, conglobate, uniseptate, constricted at the septum, 20-23 × 9-12 $\mu \mathrm{m}$., wall verrucose. Pycnothyria few to many, similar but smaller to thyriothecia; pycnothyriospores unicellular, globose to pyriform, brown, 16-18 $\times 13-15$, wall smooth.

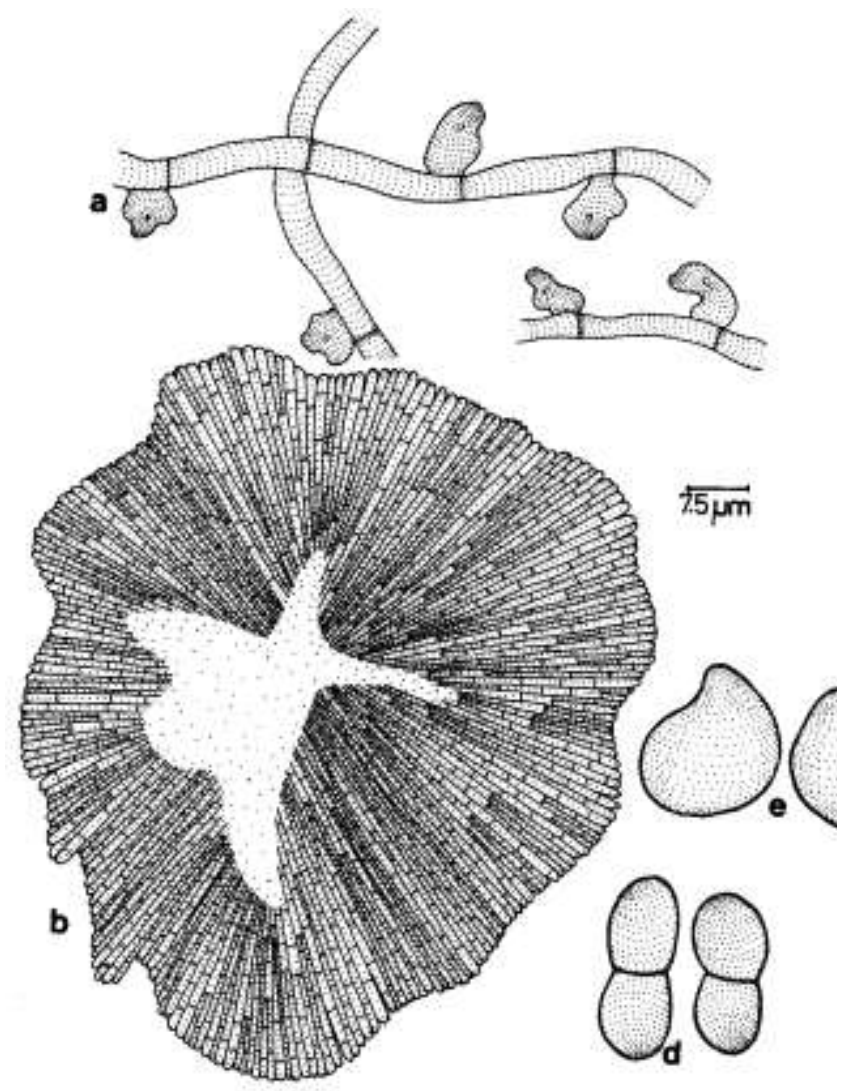

Figure 102 - Asterina hibisci

Material examined: Kerala, Thiruvananthapuram, Palode, TBGRI campus, on leaves of Hibiscus sp. (Malvaceae), 17 October 2000, V.B. Hosagoudar HCIO 43812, TBGT 357; 18 February 2001, V.B. Hosagoudar HCIO 43997, TBGT 413; Jawahar colony, near TBGRI, 21 December 2005, V. Gireesh \& P.J. Robin HCIO 46750, TBGT 2091; Wayanad, $16^{\text {th }}$ mile, Padinharathara, Hibiscus rosa-sinensis L., 25 September 2008, M.C. Riju TBGT 4496, 4498; Padinharathara, 4
November 2009, A. Sabeena \& M.C. Riju TBGT 4644.

Doidge (1942) distinguished and placed this fungus by giving the status of variety. However, loosely reticulate mycelia, morphologically different and less number of appressoria and distinctly larger ascospores justifies it species status.

\section{Distribution: Kerala.}

Asterina homaligena V.B. Hosagoudar and C. Jagath Thimmaiah, sp. nov. (Fig. 103)

MycoBank 801043

Etymology: Named after the host genus

Colonies amphigenous, mostly epiphyllous, subdense, up to $4 \mathrm{~mm}$ in diameter. Hyphae substraight to flexuous, branching irregular at wide angles, loosely reticulate, cells 16-31 $\times$ 4-7 $\mu \mathrm{m}$. Appressoria alternate to opposite, few unilateral, antrorse to retrorse, straight to flexuous, two celled, 17-22 $\mu \mathrm{m}$ long; stalk cells cylindrical, 4-7 $\mu \mathrm{m}$ long; head cells ovate, ampulliform, cylindrical, flexuous, straight to curved, hamate, margin entire to flexuous, $10-12 \times 5-9 \mu \mathrm{m}$. Thyriothecia scattered to loosely grouped, orbicular, up to $100 \mu \mathrm{m}$ in diameter; stellately dehisced at the centre, often the central portion dissolved to form wide opening by exposing the asci, margin crenate to fimbriate, fringed hyphae small; asci few, globose, 8-spored, up to $25 \mu \mathrm{m}$ in diameter; ascospores brown, conglobate, uniseptate, deeply constricted at the septum, 24$28 \times 15-18 \mu \mathrm{m}$, wall smooth. Pycnothyria numerous, similar to thyriothecia, orbicular, smaller; pycnothyriospores numerous, ovate, oblong, pyriform, $18-27 \times 14-18 \mu \mathrm{m}$.

Material examined: Karantaka, Kodagu, Hoddur, River side, on leaves of Homalium zeylanica (Gardner) Benth. (Flacourtiaceae), 22 August 2009, C. Jagath Thimmai TBGT 5696 (holotype).

Asterina homalii Sydow on homalium alnifolium from Sierra Leone and $A$. homaliicola Hughes on Homalium dolichophyllum from Gold coast are known (Sydow, 1939; Hughes, 1952). However, the 
new species differs from both in having two celled appressoria.

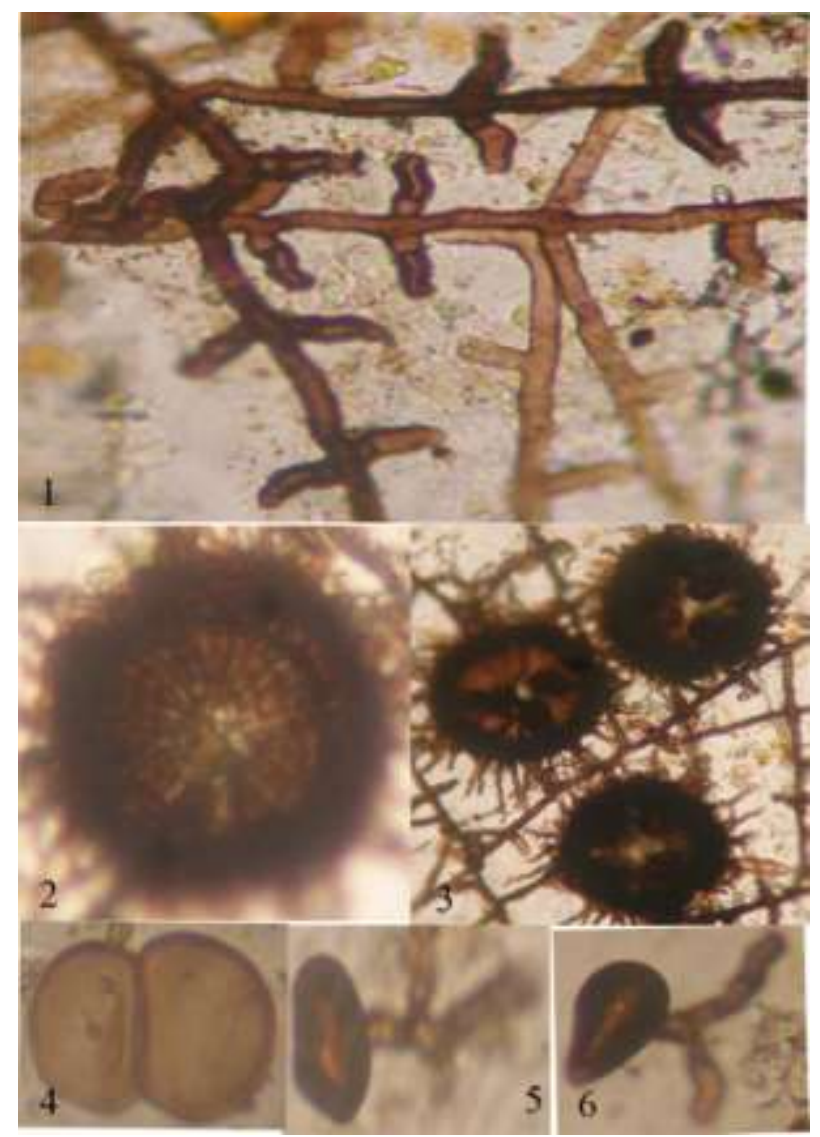

Figure 103 - Asterina homaligena

1. Appressoriate mycelium, 2-3. Thyriothecia, 4. Ascospore, 5-6. Germinated pycnothyriospores

Distribution: Karnataka

Asterina homonoiae Hosag. \& Sabeena, Bull. Basic Appl. Plant Biol. 1: 49, 2011. (Fig. 104)

Colonies mostly hypophyllous, thin to subdense, up to $3 \mathrm{~mm}$ in diameter, confluent. Hyphae crooked, branching opposite to unilateral at acute to wide angles, loosely reticulate, cells 15-37 $\times 5-7 \mu \mathrm{m}$. Appressoria straight to curved, unicellular, alternate to unilateral, ovate, oblong, entire to sublobate, 7$12 \times 5-12 \mu \mathrm{m}$. Thyriothecia scattered, orbicular, up to $230 \mu \mathrm{m}$ in diameter, margin crenate, stellately dehisced at the centre; asci few, globose, up to $30 \mu \mathrm{m}$ in diameter; ascospores conglobate, 1-septate, constricted at the septum, $17-20 \times 8-10 \mu \mathrm{m}$, wall smooth.
Material examined: Kerala, Kottayam, Koruthodu, on leaves of Homonoia riparia Lour. (Euphorbiaceae), 25 March 2009, V. Gireesh Kumar \& al. TBGT 5096 (holotype p.p.). Part of the collection has been deposited in HCIO, New Delhi.

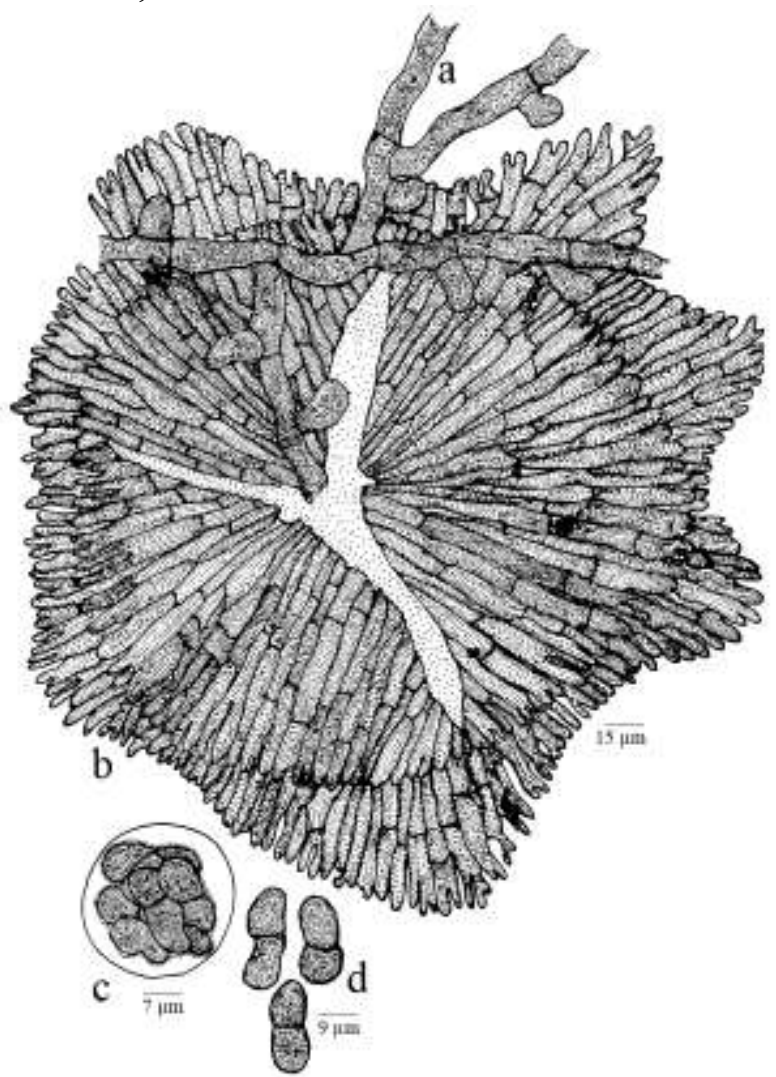

Figure 104 - Asterina homonoiae

On members of the family Euphorbiaceae, the genus Phyllanthus harbours Asterina phyllanthi-beddomei Hosag. et al., A. phyllanthigena Hosag. and A. phyllanthicola Singh; Glochidion harbours A. lobulifera Sydow and $A$. lobulifera Sydow var. indica Hosag. \& Chandra.; Mallotus harbours $A$. malloticola Hosag. et al. and Aporusa harbours $A$. aporusae Hansf. and $A$. aporusae Hansf. var. cylindrica Kar \& Maity (Hosagoudar et al., 2011). This is the first report of the genus Asterina on this host genus.

Distribution: Kerala

Asterina hopeae Hosag. \& Kamar., in Hosag., Zoos' Print J. 17: 815, 2002; Hosag. \& Appaiah, J. Mycopathol. Res. 43:170, 2005; Hosag., H. Biju \& Appaiah, J. Mycopathol. Res. 43:203, 2005; Hosag., 
Chandraprabha \& Agarwal, Asterinales of Kerala, p. 89, 2011. (Fig. 105)

\section{(Fig. 105)}

Colonies epiphyllous, subdense, up to 3 $\mathrm{mm}$ in diameter, confluent. Hyphae straight to substraight, branching alternate to irregular at acute angles, loosely reticulate, cells 16-24 × 3$4 \mu \mathrm{m}$. Appressoria alternate to unilateral, mostly conoid, clavate, often ovate, attenuated and broadly rounded at the apex, entire, 6-10 $\times$ 4-7 $\mu \mathrm{m}$. Thyriothecia scattered, rarely connate, orbicular, up to $176 \mu \mathrm{m}$ in diameter, stellately dehisced at the centre, margin fimbriate, fringed hyphae long, flexuous; asci many, globose to ovate, octosporous, up to $45 \mu \mathrm{m}$ in diameter; ascospores oblong, conglobate, brown, 1septate, constricted at the septum, 24-28 $\times 11$ $13 \mu \mathrm{m}$, wall smooth, tubercled in matured spores.

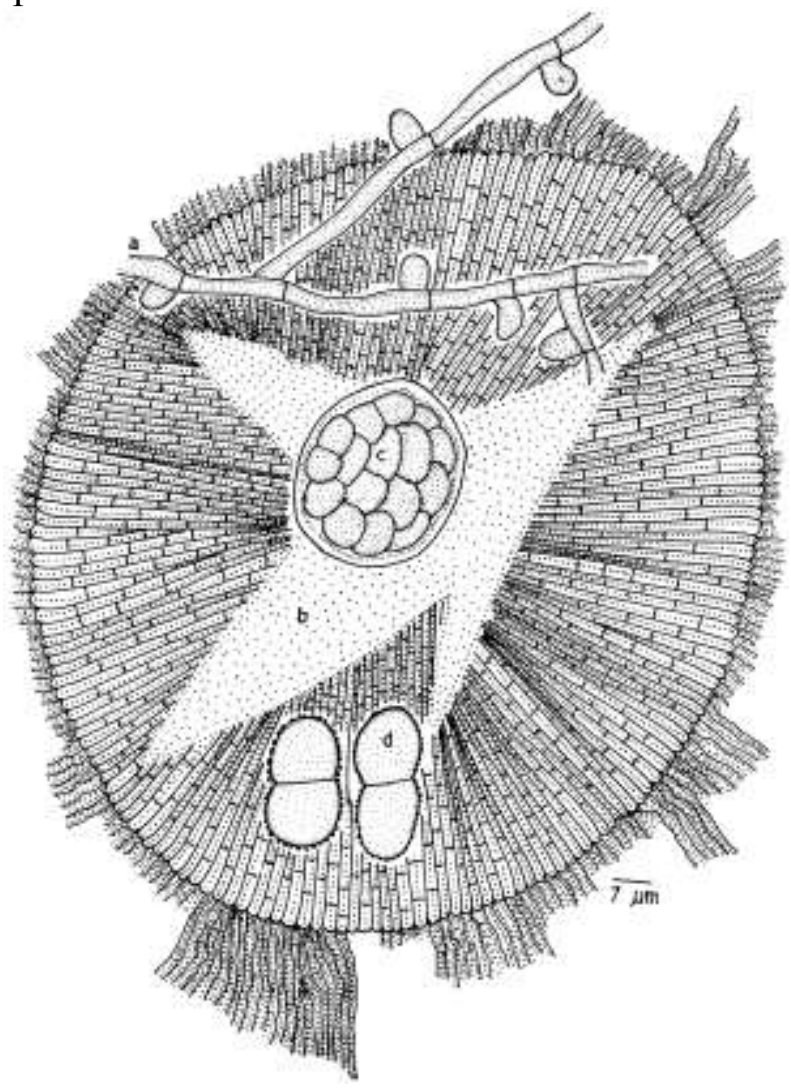

Figure 105 - Asterina hopeae

Material examined: Kerala, Thiruvananthapuram, Palode, in the campus of Tropical Botanic Garden and Research Institute, on leaves of Hopea ponga (Dennst.) Moberly (Dipterocarpaceae), 10 January 2001, M. Kamarudeen HCIO 43995 (holotype), TBGT 414 (isotype); 15 November 2003, V.B. Hosagoudar \& al. HCIO 45724, TBGT 1473;
Palghat, Silent valley, Neelakal forest, 30 March 2009, M.C. Riju TBGT 4534; Thiruvananthapuram, Peppara Wildlife Sanctuary, near Peppara Dam, 31 March 2007, Jacob Thomas HCIO 48846, TBGT 3222.

So far six species of the genus Asterina are known on the host genera Anisoptera, parashorea and Shorea of the family Dipterocarpaceae (Hosagoudar \& Abraham, 2000). Of these, Asterina shoreana Sacc. does not represent external mycelium, while, $A$. camarinensis H. Sydow \& P. Sydow and $A$. anisopterae H. Sydow \& P. Sydow have nodulose appressoria in the mycelium (Saccardo, 1924). Hence, these are the doubtful species of the genus Asterina. Asterina hopeae differs from $A$. anisoptericola Hansf. in having unicellular appressoria and from $A$. rizalensis Hansf. in absence of $90 \%$ opposite appressoria (Hansford, 1949). A. plurispora Ryan, known from India, differs from the present species in having crook-shaped appressoria.

\section{Distribution: Kerala.}

Asterina hopiicola Hosag. \& Abraham, Mycol. Res. 102: 184, 1998; Hosag.,C.K. Biju \& Abraham, J. Econ. Taxon. Bot. 25: 305, 2001; Hosag., Zoos' Print J. 18: 1282, 2003; Hosag. \& Appaiah, J. Mycopathol. Res. 43:170, 2005; Hosag., Chandraprabha \& Agarwal, Asterinales of Kerala, p. 91, 2011.

(Fig. 106)

Colonies epiphyllous, subdense to dense, up to $3 \mathrm{~mm}$ diameter, widely confluent and covering most of the leaf area. Hyphae straight, branching alternate to irregular at acute angles, loosely reticulate, cells 19-24 × 3.5-5 $\mu \mathrm{m}$. Appressoria unicellular, alternate, scattered, ovate, attenuated at the apex, straight to curved, mostly entire, rarely angular, 7-10 $\times$ 6-8 $\mu \mathrm{m}$. Thyriothecia scattered, orbicular, rounded, carbonaceous black, up to $252 \mu \mathrm{m}$ diameter, stellately dehisced at the centre, margin fimbriate, fringed hyphae numerous, straight to flexuous; asci initially globose, slightly ovate at maturity, 8-spored, 48-60 × 28$36 \mu \mathrm{m}$; ascospores brown, conglobate, 1- 
septate, deeply constricted at the septum, 21-24 $\times$ 11-13 $\mu \mathrm{m}$, wall smooth.

$\begin{array}{crr}\text { Material } & \text { examined: } & \text { Kerala, } \\ \text { Thiruvananthapuram, } & \text { Neyyar } & \text { Wildlife }\end{array}$ Sanctuary, Meenmutty, in the forest near Kombe, on leaves of Hopea parviflora Bedd. (Dipterocarpaceae), 9 March 1996, V.B. Hosagoudar HCIO 42142 (holotype), TBGT 004 (isotype).
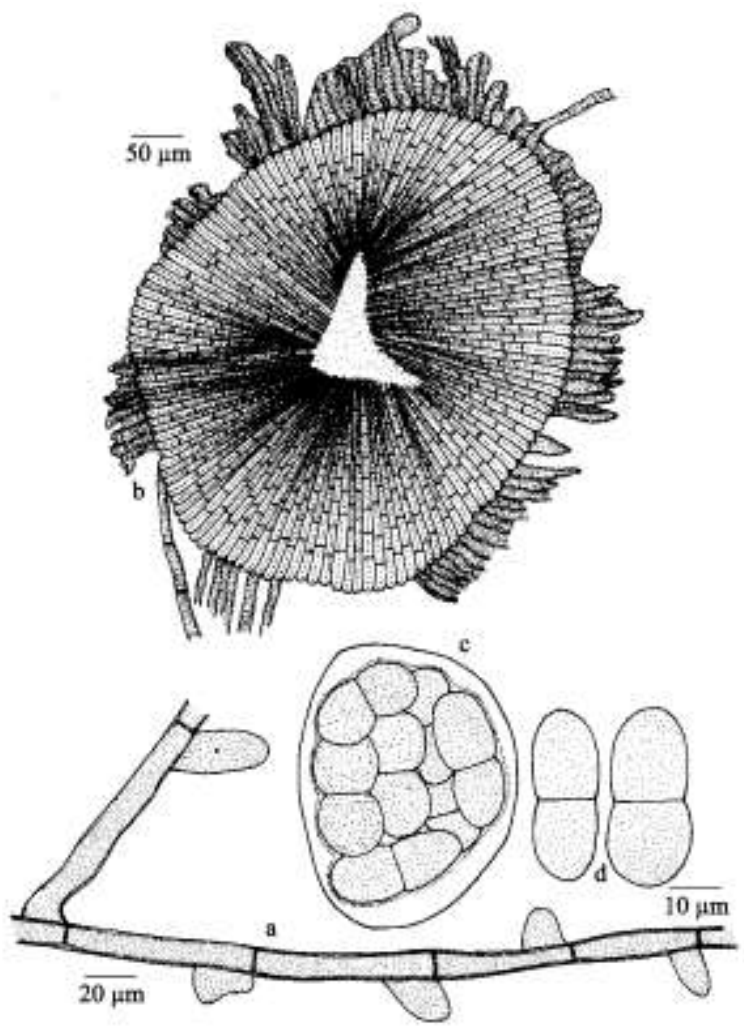

Figure 106 - Asterina hopiicola

Asterina anisopterae Sydow \& Sydow, A. camarinensis Sydow \& Sydow and $A$. shoreana Sacc. are known from the Philippines on Anisoptera and Parashorea (Dipterocarpaceae). Sydow \& Sydow (1917) considered $A$. shoreana a synonym of $A$. camarinensis. Asterina hopiicola differs from A. anisopterae and A. camarinensis in having ovate and attenuated appressoria; the ascospores are shorter (21-24 $\mu \mathrm{m}$ long, against $32-38 \mu \mathrm{m}$ and $38-43 \mu \mathrm{m}$, respectively). Further, occurrence of the genera Anisoptera and Parashorea in India is doubtful (Santapau \& Henry, 1984).

Distribution: Kerala.
Asterina hugoniae Hosag., Sabeena \& Riju, J. Threatened Taxa 3(6): 1880, 2011.

(Fig. 107)

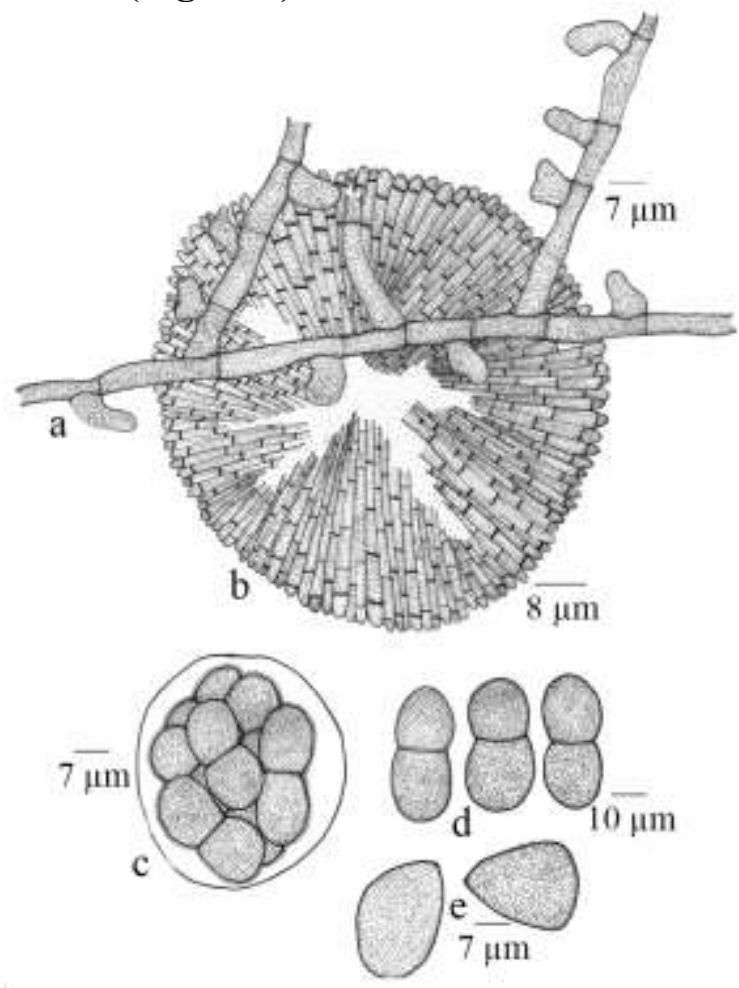

Figure 107 - Asterina hugoniae

Colonies amphigenous, mostly epiphyllous, subdense to dense, up to $2 \mathrm{~mm}$ in diameter, confluent. Hyphae substraight to flexuous, branching opposite to irregular at acute to wide angles, loosely to closely reticulate, cells $12-27 \times 3-5 \mu \mathrm{m}$. Appressoria one celled, alternate to unilateral, ovate, entire to mostly sublobate, $5-12 \times 5-10 \mu \mathrm{m}$. Thyriothecia scattered, orbicular, stellately dehisced at the centre, up to $300 \mu \mathrm{m}$ in diameter, margin rarely fimbriate; asci ovate to globose, octosporous, $20-37 \mu \mathrm{m}$ in diameter; ascospores conglobate, 1 -septate, constricted at the septum, $22-27 \times 10-15 \mu \mathrm{m}$, wall slightly verrucose. Pycnothyria numerous, scattered, orbicular, up to $100 \mu \mathrm{m}$ in diameter, stellately dehisced at the centre, margin crenate to fimbriate; pycnothyriospores unicellular, globose, ovate, $15-22 \times 12-20 \mu \mathrm{m}$, wall smooth.

Material examined: Kerala, Kozhikode, Malabar Botanic Garden, on leaves of Hugonia mystax L. (Linaceae), 3 November 2009, A. Sabeena \& M.C. Riju TBGT 4249 (holotype); TBGT 4250, 4260. Part of the 
collection has been deposited in HCIO, New Delhi.

This species differs from Asterina hapala Sydow reported on Hugonia platysepala from Uganda (Sydow, 1938) in having larger thyriothecia (300 $\mu \mathrm{m}$ against $100-140 \mu \mathrm{m})$, larger ascospores $(22-27 \times 10-15 \mu \mathrm{m}$ versus 17 $20 \times 7-8 \mu \mathrm{m})$ and larger pycnothyriospores $(15$ $22 \times 12-20$ against $14-17 \times 8-9 \mu \mathrm{m})$.

Distribution: Kerala

Asterina hydnocarpi Hosag. \& Abraham, Indian Phytopath. 51: 389, 1998; Hosag.,C.K. Biju \& Abraham, J. Econ. Taxon. Bot. 25: 305, 2001; Hosag., Zoos' Print J. 18: 1283, 2003 Hosag., Chandraprabha \& Agarwal, Asterinales of Kerala, p. 92, 2011. (Fig. 108)

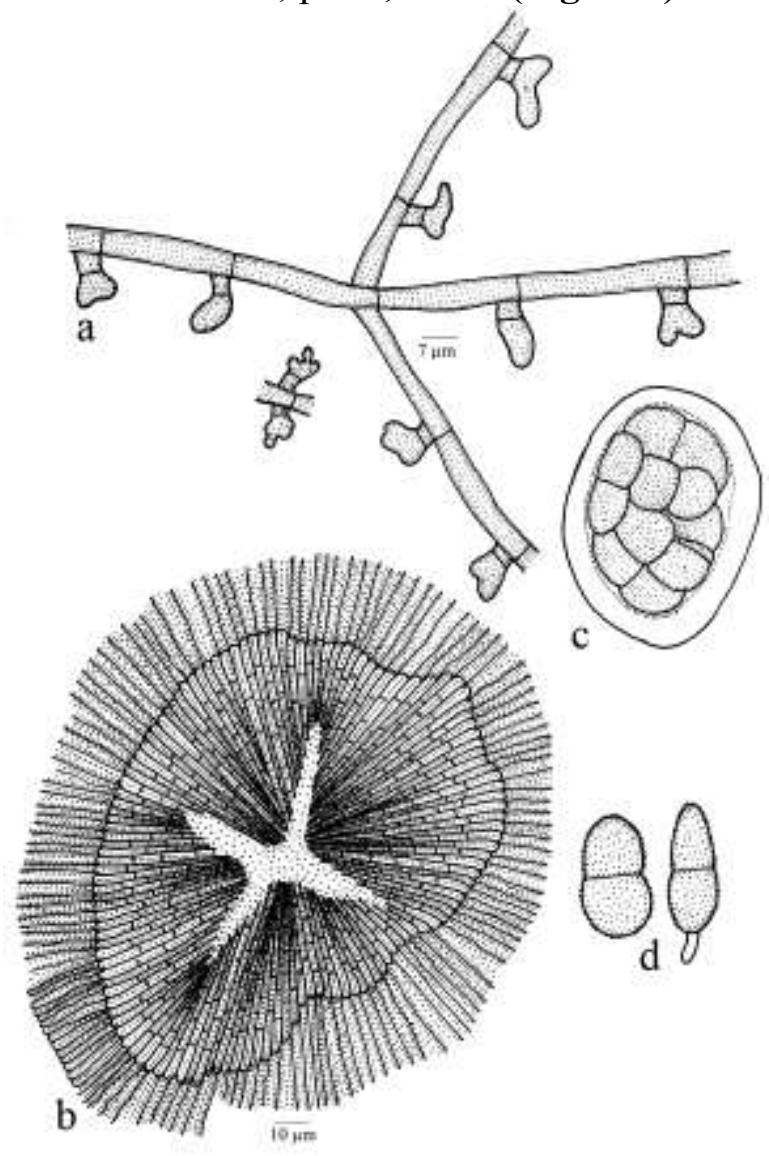

Figure 108 - Asterina hydnocarpi

Colonies hypophyllous, subdense to dense, up to $3 \mathrm{~mm}$ in diameter, confluent. Hyphae straight to substraight, branching mostly opposite at wide angles, loosely reticulate, cells 12-16 $\times$ 4-6 $\mu \mathrm{m}$. Appressoria alternate, about $3 \%$ opposite, 2 -celled, straight, curved to uncinate, $8-13 \mu \mathrm{m}$ long; stalk cells cylindrical to cuneate, $1.5-5 \mu \mathrm{m}$ long; head cells ovate, globose, hamate, straight to curved, entire, bifid to 3-4 times sublobate, 6-8 × 3-10 $\mu \mathrm{m}$. Thyriothecia scattered to loosely grouped, orbicular, up to $112 \mu \mathrm{m}$ in diameter, stellately dehisced at the centre, margin mostly fimbriate, fringed hyphae run parallel, elongated; asci few, globose, octosporous, up to $50 \mu \mathrm{m}$ in diameter; ascospores brown, conglobate, 1septate, constricted at the septum, 20-24 × 9-11 $\mu \mathrm{m}$, wall tubercled.

\section{Material examined: Kerala,} Thiruvananthapuram, Neyyar Wildlife Sanctuary, Kombe, on leaves of Hydnocarpus macrocarpa (Bedd.) Warb. (Flacourtiaceae), 19 February 1997, V.B. Hosagoudar HCIO 42536 (holotype), TBGT 178 (isotype); Karnataka, Kodagu, Hoddur, on leaves of Hydnocarpus sp., 29 September 2009, C. Jagath Thimmaiah TBGT 5424.

Twelve species of the genus Asterina are known on the members of the family Flacourtiaceae. The present species differs from all in having only 3\% opposite, 2-celled appressoria; entire, bifid to 3-4 times sublobate head cells of the appressoria distinguishes this species.

Distribution: Karnataka, Kerala.

Asterina hydrocotyles Hosag. \& C.K. Biju, Indian Phytopathol. 58: 198, 2005; Hosag., Chandraprabha \& Agarwal, Asterinales of Kerala, p. 93, 2011.

(Fig. 109)

Colonies epiphyllous, dense, minute, up to $1 \mathrm{~mm}$ in diameter, rarely confluent. Hyphae substraight, often parallel and compact, branching alternate to irregular at acute to wide angles, loosely to closely reticulate, cells $16-20$ $\times$ 3-5 $\mu \mathrm{m}$. Appressoria scattered, alternate, unilateral, unicellular, oval, globose, slightly stipitate to broad based, irregularly sublobate to deeply lobate, 7-9 $\times 9-11 \mu \mathrm{m}$. Thyriothecia scattered to loosely grouped, orbicular, up to $120 \mu \mathrm{m}$ in diameter; margin crenate, stellately dehisced at the centre; asci globose, octosporous, up to $35 \mu \mathrm{m}$ in diameter; ascospores oblong, conglobate, brown, 
uniseptate, deeply constricted, 35-38 $\times$ 15-18 $\mu \mathrm{m}$, wall smooth. Pycnothyria few, similar to thyriothecia, smaller; pycnothyriospores ovate, oblong, brown, $18-20 \times 8-10 \mu \mathrm{m}$, few spores with hyaline band at the centre.

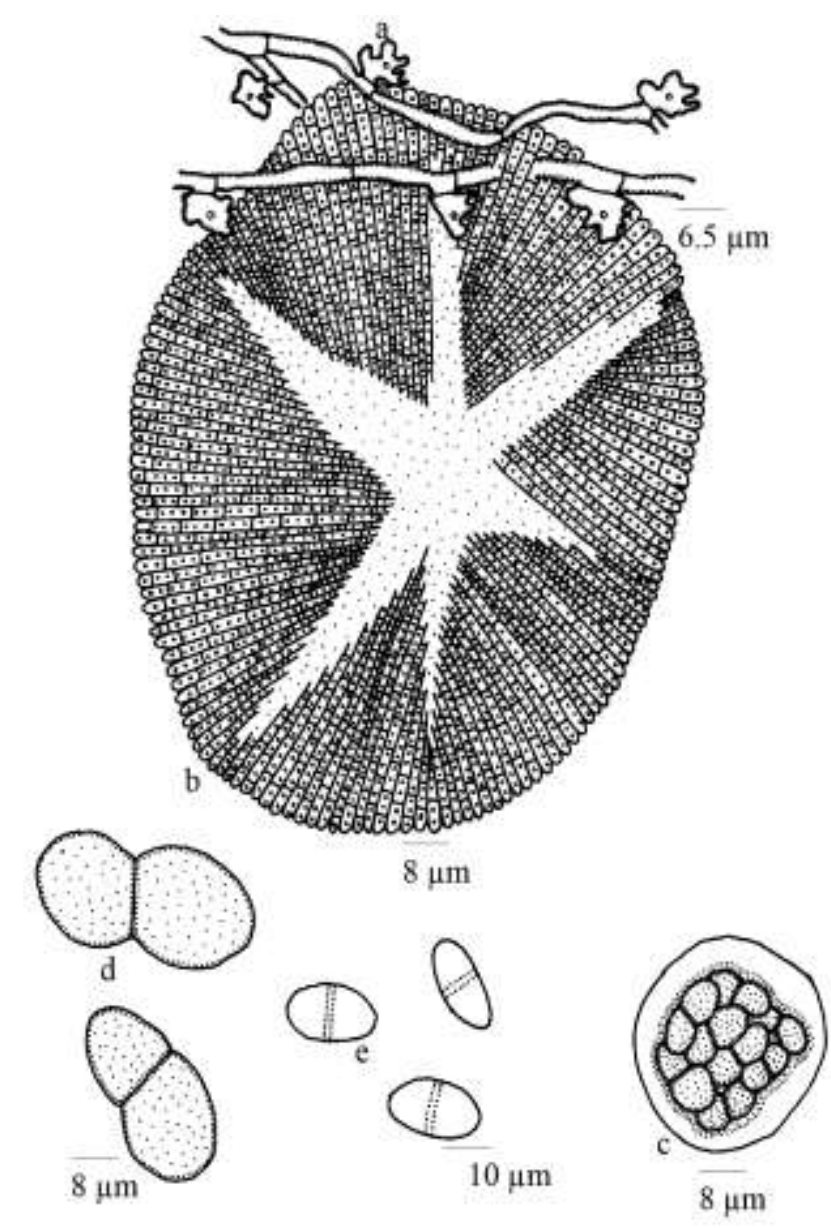

Figure 109 - Asterina hydrocotyles

Material examined: Kerala, Idukki, Munnar, Mannavan Shola, on leaves of Hydrocotyle sp. (Apiaceae), 11 May 1999, C.K. Biju HCIO 45284 (holotype), TBGT 1310 (isotype); TBGT 1322; Tamil Nadu, Kodaikanal, Periya Kanal, 15 February 2008, R. Nithyatharani TBGT 4720; on leaves of Hydrocotyle javanica Thumb., 2 March 2008, R. Nithyatharani TBGT 4355.

Asterina saniculae Doidge and $A$. saniculicola Hansf. are known on the host genus Sanicula from Java and South Africa (Doidge, 1942; Hansford, 1954). The present taxon is similar to $A$. saniculicola in having unicellular appressoria but has distinctly larger ascospores $(35-38 \times 15-18 \mu \mathrm{m}$ in contrast to $13-16 \times 6-7 \mu \mathrm{m})$.
Distribution: Kerala, Tamil Nadu

Asterina hyptidicola Hosag. in Hosag., H. Biju \& Appaiah, J. Mycopathol. Res. 44: 7, 2006. (Fig. 110)

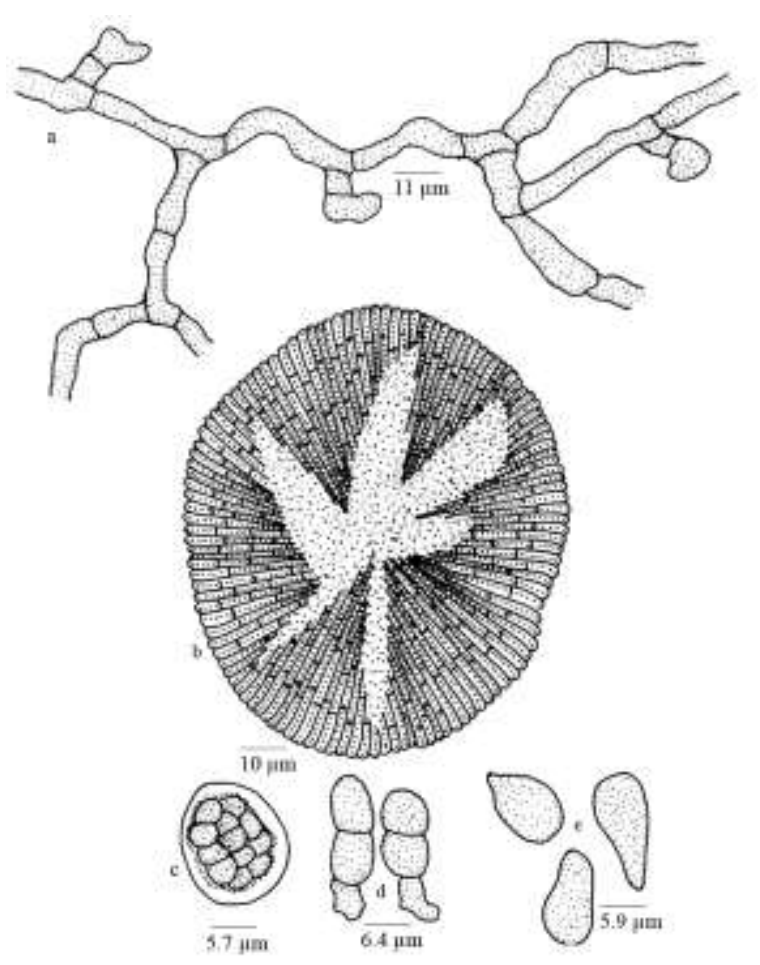

Figure 110 - Asterina hyptidicola

Colonies epiphyllous, minute, dense, up to $1 \mathrm{~mm}$ in diameter, confluent. Hyphae flexuous, branching irregular at acute to wide angles, loosely reticulate, form loose net, cells 19-43 $\times 3-5 \mu \mathrm{m}$. Appressoria very much distantly placed, bicellular, straight to curved, 9-16 $\mu \mathrm{m}$ long; stalk cells cylindrical to cuneate, 3-5 $\mu \mathrm{m}$ long; head cells ovate, globose, curved, entire, sublobate to deeply lobate, $6-12 \times 8-10$ $\mu \mathrm{m}$. Thyriothecia scattered to connate, orbicular, up to $100 \mu \mathrm{m}$ in diameter, stellately dehisced at the centre, margin crenate; asci globose, octosporous, up to $30 \mu \mathrm{m}$ in diameter; ascospores conglobate, oblong, brown, uniseptate, $12-16 \times 6-8 \mu \mathrm{m}$. Pycnothyria mixed with thyriothecia, similar to thyriothecia but smaller; pycnothyriospores oval, pyriform, unicellular, brown, 13-16 × 7-9 $\mu \mathrm{m}$.

Material examined: Karnataka, Coorg, Madikeri, Nishanemotta, on leaves of Hyptis sauveolense (L.) Poit. (Lamiaceae), 11 November 2003, V.B. Hosagoudar HCIO 45656 (holotype), TBGT 1402 (isotype); Jodupal, Madikeri, Coorg, on leaves of Hyptis 
sp., 12 November 2003, V.B. Hosagoudar \& al. HCIO 45800, TBGT $1549\{$ (as Asterina hyptidis (Rehm) Hosag. \& Abraham)\}.

Asterina acanthopoda Speg. var. hyptidis Rehm was known on Hyptis sp. but was made synonymous to Asterina carbonacea Cooke (Stevens \& Ryan, 1939). Later, Hosagoudar \& Abraham (2000) gave a species status to it. However, Asterina hyptidis (Rehm) Hosag. \& Abraham differs from it in having distinctly smaller thyriothecia, asci and ascospores.

Distribution: Karnataka.

Asterina indica Sydow in Sydow, Sydow \& Butler, Ann. Mycol. 9: 390, 1911; Patil \& Thite, J. Shivaji Univ. 17: 152, 1977; Hosag., Zoos' Print J. 18: 1285, 2003; 21: 2327, 2006; Hosag., H. Biju \& Appaiah, J. Mycopathol. Res. 44: 8, 2006; Hosag., Jacob Thomas \& Robin, Indian J. Sci. Techn. 2: 2, 2009; Hosag., Chandraprabha \& Agarwal, Asterinales of Kerala, p. 94, 2011. (Fig. 111)

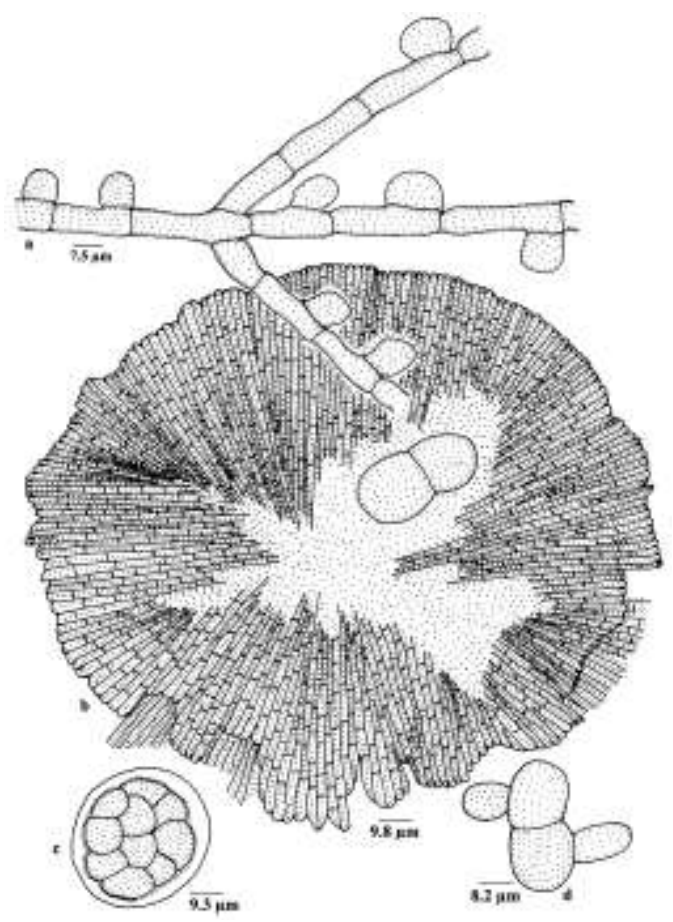

Figure 111 - Asterina indica

Colonies epiphyllous, thin, up to $2 \mathrm{~mm}$ in diameter. Hyphae straight to substraight, branching opposite to alternate at acute to wide angles, loosely reticulate, cells 31-38 $\times 12 \mu \mathrm{m}$. Appressoria 95\% alternate and 5\% opposite, unicellular, slightly antrorse, 14-22 × 7-10 $\mu \mathrm{m}$. Thyriothecia scattered, orbicular, up to $72 \mu \mathrm{m}$ in diameter, stellately dehisce at the centre; ascospores dark brown, 1-septate, constricted at the septum, $38-43 \times 14-19 \mu \mathrm{m}$.

\section{Material examined: Karnataka,} Kodagu, Talacauvery, Bramhagiri, MPCA, on leaves of Symplocos sp. (Symplocaceae), 13 November 2003, V.B. Hosagoudar \& al. HCIO 45689, TBGT 1436; TBGT 1447; HCIO 45711, TBGT 1460; HCIO 45717, TBGT 1466; HCIO 45736, TBGT 1485; HCIO 45738, TBGT 1487; Nishane motta, 14 November 2003, V.B. Hosagoudar \& al. HCIO 45662, TBGT 1408; Kerala, Idukki, Munnar, Mannavan Shola, on leaves of Symplocos cochinchinensis ssp. laurina (Retz.) Nooteb. (Symplocaceae), 23 September 1990, C.K. Biju HCIO 45210, TBGT 1246; Thiruvananthapuram, Neyyar Wild Life Sanctuary, Pongalapara, 26 March 1996, V.B. Hosagoudar HCIO 45240, TBGT 1278; Thiruvananthapuram, Agasthyamala (MPCA), 29 June 2001, M. Kamarudeen HCIO 44298, TBGT 607; Meghalaya, Shillong, Mawphlang, on leaves of Symplocos theacifolia Ham. (Symplocaceae), 21 January 2007, Jacob Thomas \& P.J. Robin HCIO 48053, TBGT 2836; Kerala, Thiruvananthapuram, Chemunji, on leaves of Symplocos sp., 11 March 1997, V.B. Hosagoudar HCIO 45118, TBGT 1173; Idukki, Munnar, Mannavan Shola, 5 January 1999, C.K. Biju HCIO 45113, TBGT 1162; Thiruvananthapuram, Palode, TBGRI Campus, 17 February 2000, C.K. Biju HCIO 45160, TBGT 1215; Kollam, Shendhurney Wildlife Sanctuary, 14 June 2003, V.B. Hosagoudar \& al. HCIO 45079, TBGT 1128; Pathanamthitta, Pachakanam, 15 December 2004, V.B. Hosagoudar HCIO 46255, TBGT 1667; Idukki, Thekkadi, Periyar Tiger Reserve, 21 March 2006, H. Biju \& V. Gireesh Kumar HCIO 4728, TBGT 2650.

Asterina grammocarpa Sydow is known on the host genus Symplocos. Asterina indica differs from it in having unicellular appressoria.

Distribution: Kerala, Meghalaya, Tamil Nadu, Karnataka.

Asterina ixorae Ryan, Mycologia 16: 182, 1924; Thite \& Kulkarni, Indian Phytopath. 29: 171, 1976. 
Colonies amphiphyllous, thin, 2-4 mm in diameter. Hyphae undulate to substraight, septate, cells mostly $20-25 \times 6-7 \mu \mathrm{m}$, branching opposite to irregular at acute to wide angles, loosely interwoven reticulate. Appressoria in whorls of four to five at each septum; stalk cells cylindrical, 2-5 $\mu \mathrm{m}$ long; head cells ovate, entire, 11-14 $\mu \mathrm{m}$ in diameter. Thyriothecia in groups of few, flat, radiate, dimidiate, 100-200 $\mu \mathrm{m}$ in diameter; asci globose to subglobose, bitunicate, eight spored, $40-50 \mu \mathrm{m}$ in diameter; ascospores oblong, obtuse, one septate, cells unequal, slightly constricted at the septum, 30$35 \times 13-16 \mu \mathrm{m}$.

Maharashtra, Panhala, on leaves of Ixora lanceolariae Colebr. (Rubiaceae), December 1968, A. N. Thite HCIO 31681.

Whirled appressoria is the character of the genus Ishwaramyces.

Distribution: Maharashtra.

Asterina jambolana Kar \& Maity, Trans. Brit. Mycol. Soc. 54: 438, 1970; Hosag., Balakr. \& Goos, Mycotaxon 59: 180, 1996; Hosag. \& Abraham, J. Econ. Taxon. Bot. 4: 576, 2000; Hosag.,C.K. Biju\& Abraham, J. Econ. Taxon. Bot. 25: 306, 2001; J. Mycopathol. Res. 40:195, 2002; Hosag., Zoos' Print J. 18: 1283, 2003; Hosag., Zoos' Print J. 21: 2327, 2006; Hosag., Chandraprabha \& Agarwal, Asterinales of Kerala, p. 96, 2011. (Figs 112-113)

Colonies amphigenous, mostly epiphyllous, dense, crustose to velvety, up to 2 $\mathrm{mm}$ in diameter, confluent. Hyphae substraight to flexuous, branching irregular at acute angles, loosely to closely reticulate, cells 25-32 × 4-7 $\mu \mathrm{m}$. Appressoria scattered, alternate, unilateral, mostly closely antrorse and appressed to the hyphae, often, curved, subantrorse to spreading, 16-20 $\mu \mathrm{m}$ long; stalk cells cylindrical to cuneate, 6-7 $\mu \mathrm{m}$ long; head cells ovate to globose, straight to slightly curved, entire, 9-13 $\times 8-10 \mu \mathrm{m}$. Thyriothecia scattered to connate and often fused, orbicular, up to $300 \mu \mathrm{m}$ in diameter, stellately dehisced at the centre, margin crenate to fimbriate, fringed hyphae short; asci globose, octosporous, up to $50 \mu \mathrm{m}$ in diameter; ascospores oblong, conglobate, brown, uniseptate, slightly constricted at the septum, 32-34 × 13-16 $\mu \mathrm{m}$.

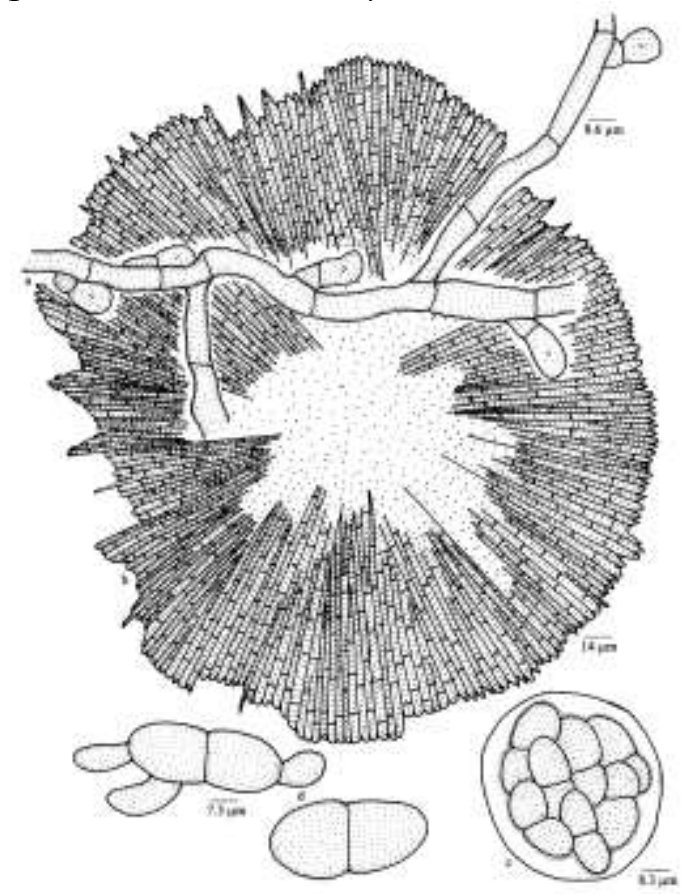

Figure 1118 - Asterina jambolana

Material examined: Tamil Nadu, Coimbatore, Anamalai, Manjaparai, on leaves of Syzygium cumini (L.) Skeels (Myrtaceae), 26 March 1990, V.B. Hosagoudar HCIO 30967; Kerala, Thiruvananthapuram, Palode, TBGRI Campus, 25 January 2005, G. R. Archana \& V. L. Sheeja HCIO 45929, TBGT

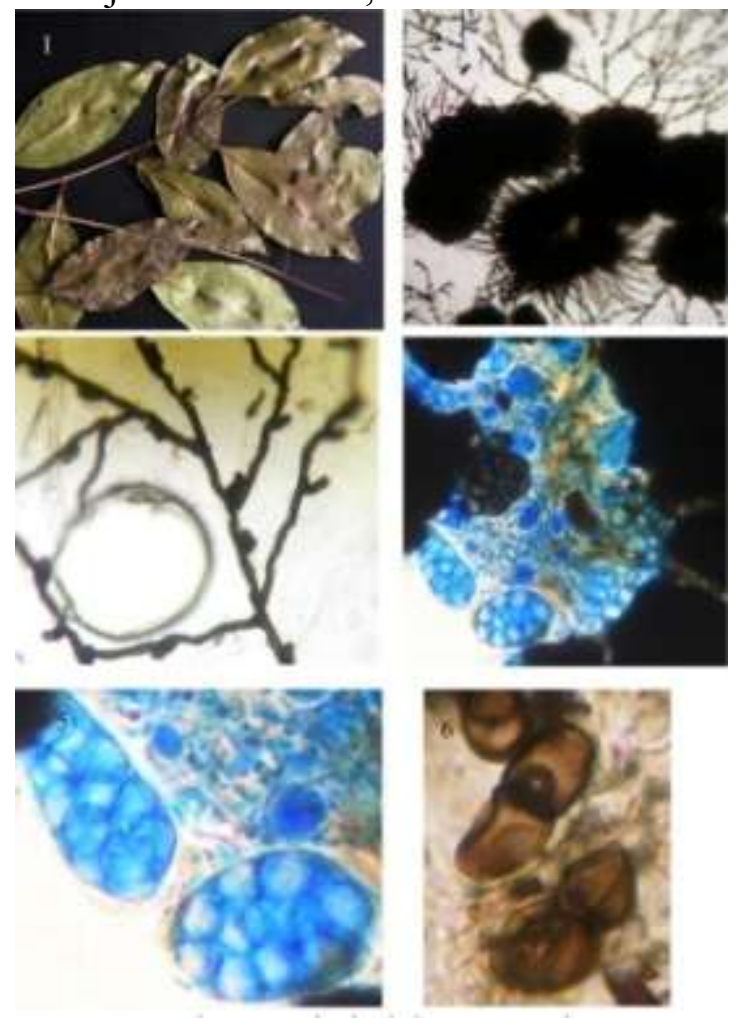

Figure 113 - Asterina jambolana 
1. Infected leaf, 2. Fungal colony with thyriothecia, 3.Appressoriate mycelium, 4. Dehisced thyriothecia with asci, 5. Asci, 6. Ascospores

1691; 6 October 2005, V.S. Sreelal HCIO 47059, TBGT 2276; Karnataka, Kodagu, Talacauveri, 25 November 2008, V.B. Hosagoudar \& al. HCIO 49174, TBGT 3429; Vanachalu, 24 November 2008, C. Jagath Thimmaiah TBGT 5322; Kodagu, Talacauveri, on leaves of Syzygium mundagam (Bourd.) Chithra, 25 November 2008, C. Jagath Thimmaiah TBGT 5359; Kerala, Pathanamthitta, Erumeli, 20 September 2007, P.J. Robin HCIO 49800, TBGT 3952; Iduki, Nedumkandam, 18 March 2007, Robin \& al. HCIO 49939, TBGT 4091; Kottayam, Ponthanpuzha reserve forest, 5 March 2008, P.J. Robin HCIO 50010, TBGT 4162; Kozhikode, Malabar Botanic Garden, 3 November 2009, A. Sabeena \& M.C. Riju TBGT 4629; Tamil Nadu, Kodaikanal, Mathikettan shola, on leaves of Syzygium densiflorum Wallich ex Wight \& Arn., 18 July 2006, G. Sangeetha HCIO 47100, TBGT 2317; 10 February 2008, A. Chandra Prabha HCIO 48892, TBGT 3268; Kerala, Munnar, Mannavan shola, on leaves of Syzygium sp., 11 May 1999, C.K. Biju HCIO 43810, TBGT 372; Tamil Nadu, Kodaikanal, Kuthuraiyar, 3 February 2008, R. Nithyatharani TBGT 4337; Kerala, Thiruvananthapuram, Palode, TBGRI campus, 28 February 2007, A. Sabeena \& M.C. Riju TBGT 4997; Palghat, Silent valley national park, 13 February 2007, M.C. Riju \& al. TBGT 5072; Silent valley, Poochipara, 8 March 2010, P.J. Robin \& al. TBGT 5162; TBGT 5164; 4 August 2008, M.C. Riju \& al. TBGT 5225; Thiruvananthapuram, Palode, TBGRI Campus, 16 March 2009, A. Sabeena TBGT 5301; Idukki, Munnar, Mannavan shola, 16 September 1999, C.K.Biju HCIO 43815, TBGT 377; Karnataka, Kodagu, Hoddur, 29 November 2009, C. Jagath Thimmaiah TBGT 5461; Alapuzha, Vandanam sacred grove, 3 October 2002, A. Manojkumar HCIO 44653, TBGT 935; Uttara Pradesh, Maharganj, on leaves of Syzygium heyneanum (Duthie) Wall. Ex Gamble (?henianum), M.A. Nomani TBGT 3520; Maharganj, S. jambolanum (Lam.) DC., M.A. Nomani TBGT 3519.

Kar \& Maity (1970) described this species from West Bengal. This is one of the most common species throughout the Southern Western Ghats.

Distribution: Karnataka, Kerala, Tamil Nadu, Uttar Pradesh, West Bengal.

Asterina jasmini Hansf. var. indica Hosag., Indian Phytopathol. 58: 199, 2005; Hosag., Zoos' Print J. 21: 2327, 2006; Hosag., Chandraprabha \& Agarwal, Asterinales of Kerala, p. 97, 2011.

(Fig. 114)

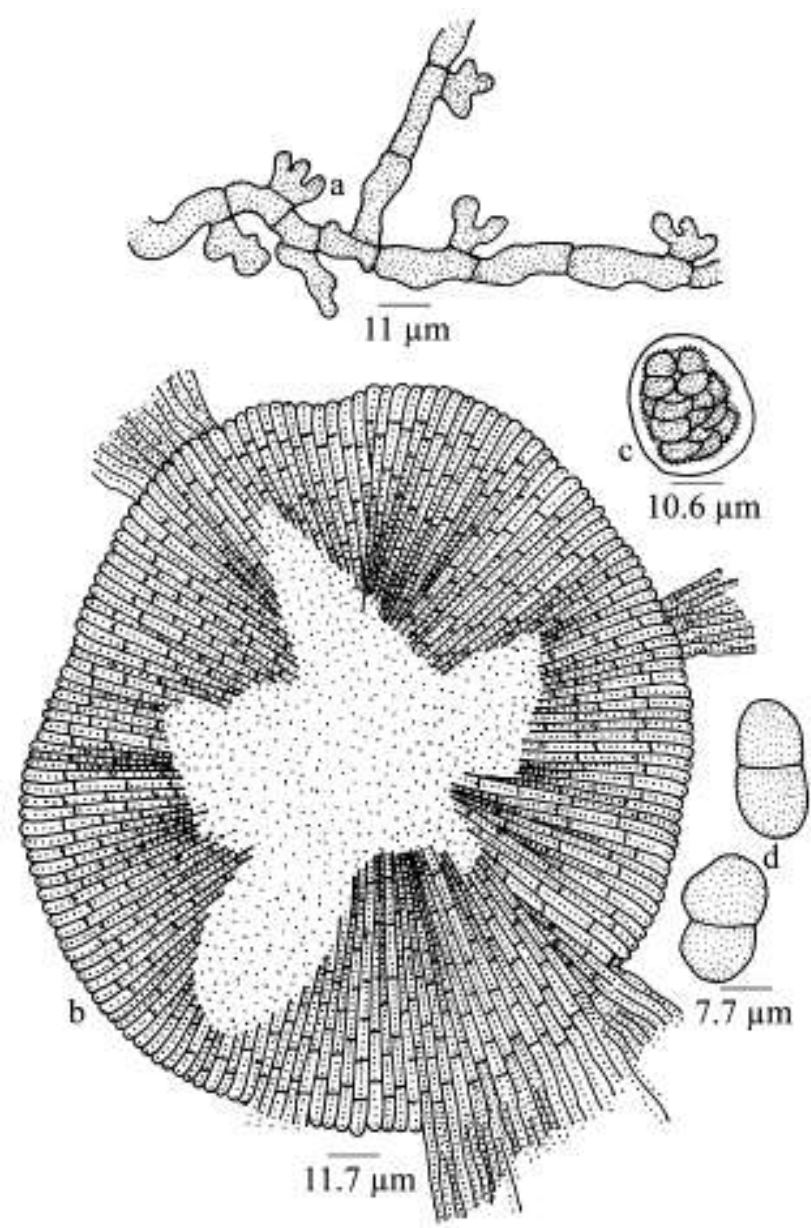

Figure 114 - Asterina jasmini var. indica

Colonies amphigenous, dense, crustose, up to $1 \mathrm{~mm}$ in diameter, confluent. Hyphae substraight to flexuous, branching irregular at acute to wide angles, loosely to closely reticulate, cells $24-32 \times 4-6 \mu \mathrm{m}$. Appressoria alternate to unilateral, globose, stipitate to broad based, 2-4 times slightly to deeply lobate, 6-8 $\times 9-13 \mu \mathrm{m}$, often some portion of the mycelium is devoid of appressoria. Thyriothecia scattered to loosely grouped, orbicular, up to $200 \mu \mathrm{m}$ in diameter, stellately 
dehisced to dissolved at the centre, margin fringed, fringed hyphae long, elongated; asci few, globose, octosporous, up to $35 \mu \mathrm{m}$ in diameter; ascospores conglobate, brown, oblong, uniseptate, constricted at the septum, $20-24 \times 10-12 \mu \mathrm{m}$.

Material examined: Kerala, Idukki, Munnar, Silent valley, on leaves of Jasminum bignoniacearum Wall. ex G. Don (Oleaceae), 29 August 2003, S. Shiburaj HCIO 45224 (holotype), TBGT 1261 (isotype); Karnataka, Madikeri, Field Marshal Cariappa college campus, on leaves of Jasminum sp., 23 November 2009, C. Jagath Thimmaiah TBGT 5449.

Asterina jasmini Hansf. is distinct from other Asterina species known on Oleaceae in having unicellular appressoria (Hansford, 1948; Hosagoudar \& Abraham, 2000). The variety indica differs from the var. jasmini in having smaller ascospores.

Distribution: Karnataka, Kerala.

Asterina jasminicola Yates, Philippine J. Sci. 13: 373, 1918; Maity, Indian J. Mycol. Res. 16: 24, 1978.

Colonies mostly amphigenous, form leaf spots, scattered to coalesced, $2-6 \mathrm{~mm}$ in diameter. Hyphae tortuous, cells 4-5 $\mu \mathrm{m}$ long. Appressoria numerous, scattered, 2-celled, alternate, stalk cells 5.5-6.5 $\times$ 4-4.5 $\mu \mathrm{m}$; upper cell irregularly angulose to lobate, $8-10 \mu \mathrm{m}$ in diameter. Thyriothecia irregularly dehisced; asci globose, octosporous, 22-26 $\mu \mathrm{m}$ diameter; ascospores ellipsoidal-oblong, 1-septate, brown, $17-19 \times 8-10 \mu \mathrm{m}$.

Material examined: West Bengal, Howrah, Panchla, on leaves of Jasminum pubescens Willd. (Oleaceae), June 1977, M. K. Maity IMI 224595, RPM 11.

Distribution: West Bangal.

Asterina kannurensis V.B. Hosagoudar, G.R. Archana, K.M. Khaleel and P. Ramya sp. nov. (Fig. 115)

MycoBank 801004

Etymology: Specific epithet based on the collection locality

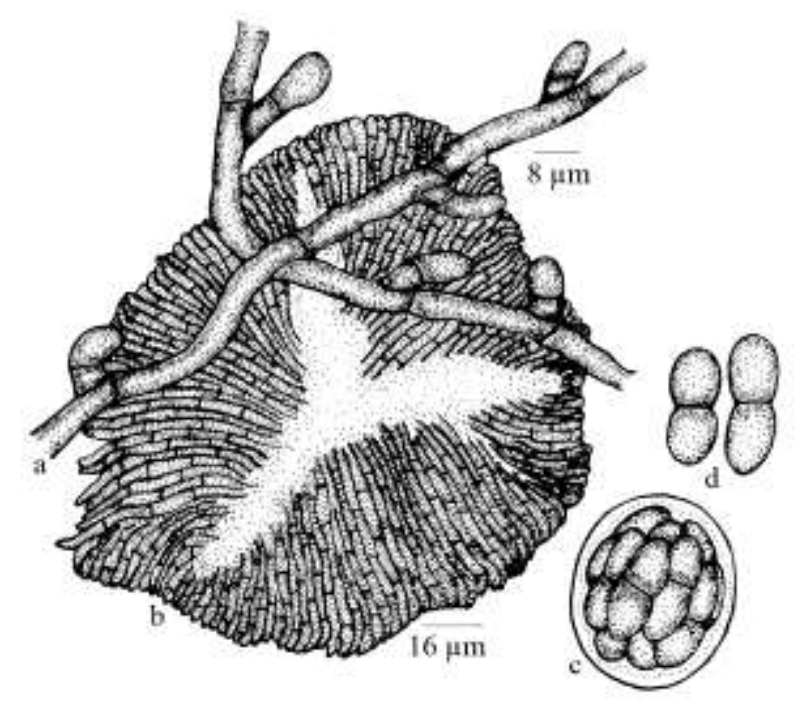

Figure 115 - Asterina kannurensis

Colonies epiphyllous, thin, up to $3 \mathrm{~mm}$ in diameter, confluent. Hyphae flexuous, branching opposite to irregular at acute to wide angles, loosely to closely reticulate, cells 11-30 $\times 2-4 \mu \mathrm{m}$. Appressoria alternate, unilateral to $2 \%$ opposite, antrorse to subantrorse, $7-15 \mu \mathrm{m}$ long; stalk cells cylindrical to cuneate, 3-7 $\mu \mathrm{m}$ long; head cells ovate, oblong, entire to angular, 4-8 $\times$ 4-7 $\mu \mathrm{m}$. Thyriothecia scattered to grouped, orbicular, up to $125 \mu \mathrm{m}$ in diameter, stellately dehisced at the centre, margin fimbriate, fringed hyphae flexuous; asci globose to ovate, octosporous, $20-26 \times 16-22$ $\mu \mathrm{m}$; ascospores conglobate, uniseptate, slightly constricted at the septum, $14-20 \times 6-12 \mu \mathrm{m}$, wall smooth.

Material examined: Kerala, Kannur, Payyannur, Edat, on leaves of Clerodendrum viscosum Vent. (Verbenaceae), 21 January 2011, P. Ramya TBGT 4935 (holotype). Part of the collection has been deposited in HCIO, New Delhi.

Asterin pusilla Sydow \& Sydow was described on Premna nauseosa from Philippines (Sydow \& Sydow, 1915) and Hosagoudar \& Sabeena (2007) on Premna corymbosa from Malabar Botanic Garden, Kozhikode, Kerala, India. However, Asterina kannurensis differs from it in having two celled appressoria.

\section{Distribution: Kerala}

Asterina knemae-attenuatae Hosag., Abraham \& T. S. Nayar, Mycotaxon 67: 487, 
1998; Hosag., Zoos' Print J. 18: 1283, 2003; Hosag., Zoos' Print J. 21: 2328, 2006; Hosag., Chandraprabha \& Agarwal, Asterinales of Kerala, p. 97, 2011. (Fig. 116)

Colonies epiphyllous, dense, crustose, up to $5 \mathrm{~mm}$ in diameter. Hyphae straight to rarely crooked, branching irregular at acute to wide angles, loosely reticulate, cells $25-30 \times 4$ $6 \mu \mathrm{m}$. Appressoria alternate, about 1\% opposite, distantly placed, unicellular, broad based to stipitate, mammiform to cylindrical, entire to 1-4 times sublobate, 7.5-20 × 10-12.5 $\mu \mathrm{m}$. Thyriothecia scattered to 1-3 connate, orbicular to slightly ovate, $150-250 \mu \mathrm{m}$ in diameter, margin crenate, irregularly opened at the centre; asci very few, globose to slightly ovate, bitunicate, eight spored, 54-58 $\times$ 45-50 $\mu \mathrm{m}$; ascospores conglobate, brown, 2-celled, slightly constricted at the septum, 25-27 × 11$13 \mu \mathrm{m}$, wall glabrous.

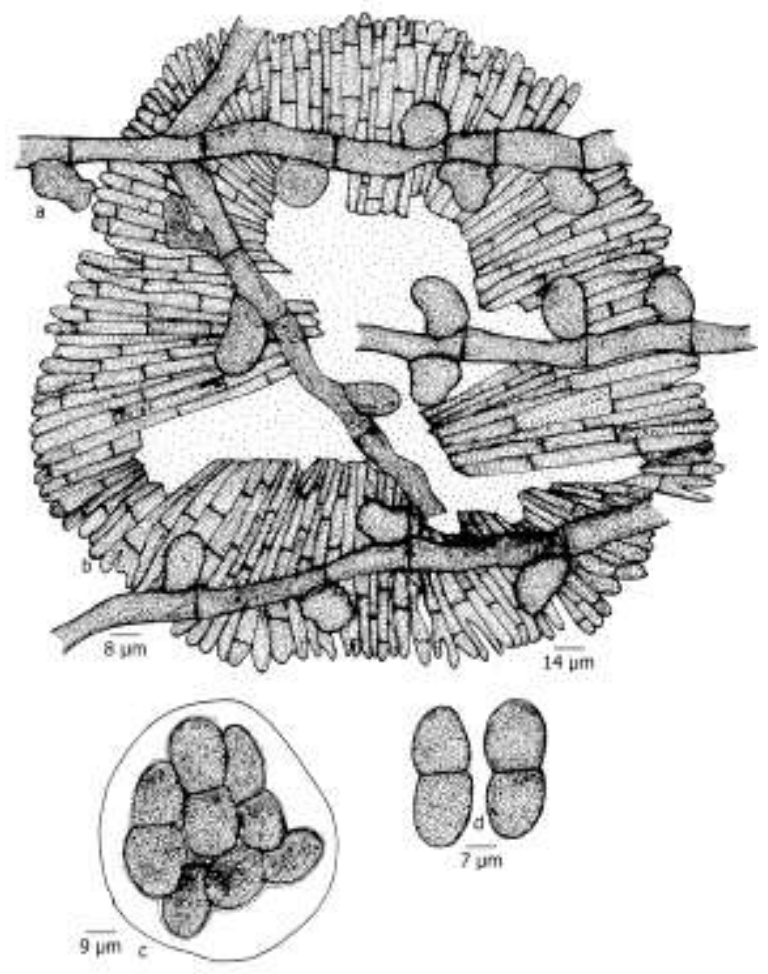

Figure 116 - Asterina knemae-attenuatae

Material examined: Kerala, Thiruvananthapuram, Palode, TBGRI Campus, on leaves of Knema attenuata (Wallich ex Hook. f. \& Thomson) Warb. (Myristicaceae), 14 February 1997, T. S. Nayar HCIO 42462 (holoype), TBGT 483 (isotype); Attayar, 19
March 1997, V.B. Hosagoudar HCIO 44145, TBGT 513; Peppara Wildlife Sanctuary, 10 February 1997, V.B. Hosagoudar HCIO 44299, TBGT 719; Kollam, Sankily, 10 March 2010, V.B. Hosagoudar \& al. TBGT 4607, 4609, 4611.

Asterina coelocaryi Hansf., A. horsfieldiae Hansf. and $A$. pycnanthi Hansf. are known or the members of the family Myristicaceae. Based on the morphology of the appressoria and epiphyllous colonies, the present species can be compared with Asterina horsfieldiae Hansf. However, the present species differs from it in having only $1 \%$ opposite appressoria, only crenate margins of the thyriothecia and smaller and smooth-walled ascospores (Hansford, 1954). Further, the present host is endemic to the Western Ghats of the Peninsular India while, Horsfieldia is not represented in India (Santapau \& Henry, 1984; Nayar et al., 2006).

Distribution: Kerala.

Asterina kodajadriensis V.B. Hosagoudar, A. Sabeena \& K. Ravikumar, sp. nov. (Figs 117-118)

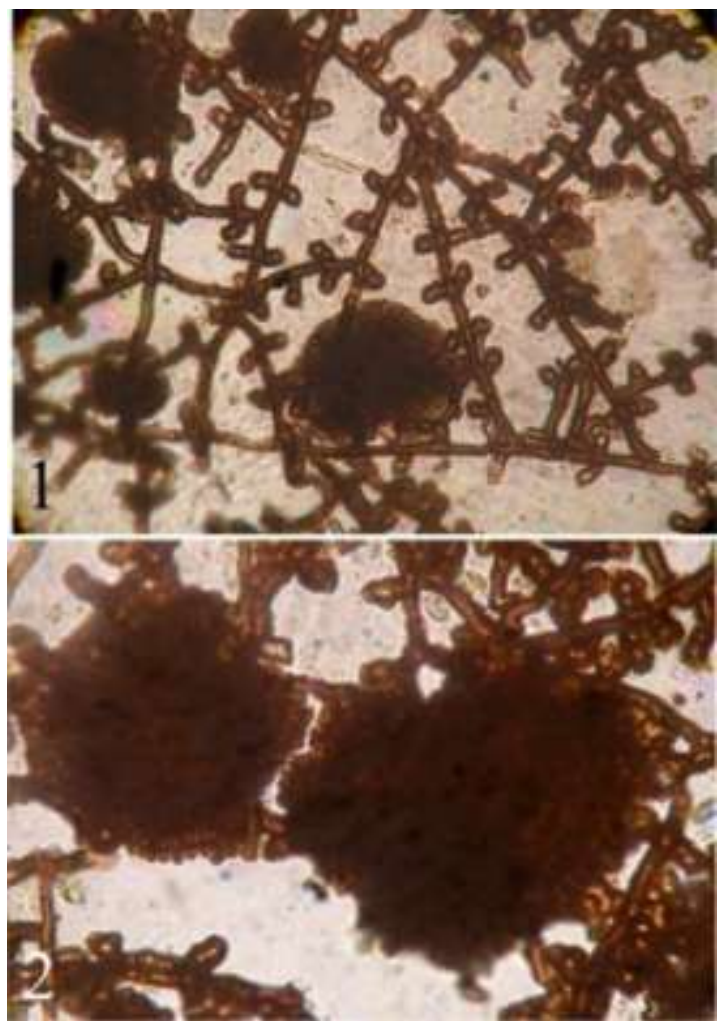

Figure 117 - Asterina kodajadriensis 1. Appressoriate mycelium, 2. Thyriothecia 
MycoBank 801005

Etymology: Named after the collection locality

Colonies mostly epiphyllous, subdense to dense, velvety, up to $4 \mathrm{~mm}$ in diameter, confluent. Hyphae straight to substraight, branching alternate to unilateral at acute to wide angles, loosely to closely reticulate, cells $15-37 \times 3-5 \mu \mathrm{m}$. Appressoria rarely produced in clusters, opposite to unilateral, antrorse to subantrorse, 7-12 $\mu \mathrm{m}$ long; stalk cells cylindrical to cuneate, $2-5 \mu \mathrm{m}$ long; head cells ovate, sublobate to lobate, 5-10 $\times 5-10 \mu \mathrm{m}$. Thyriothecia, scattered, orbicular, up to $162 \mu \mathrm{m}$ in diameter, stellately dehisced at the centre; asci few, octosporous, globose, 25-32 $\mu \mathrm{m}$ in diameter; ascospores conglobate, 1-septate, constricted at the septum, 20-25 $\times 11-13 \mu \mathrm{m}$, wall smooth; Pycnothyria similar to thyriothecia; pycnothyriospores ovate to globose, $12-20 \times 12-15 \mu \mathrm{m}$.

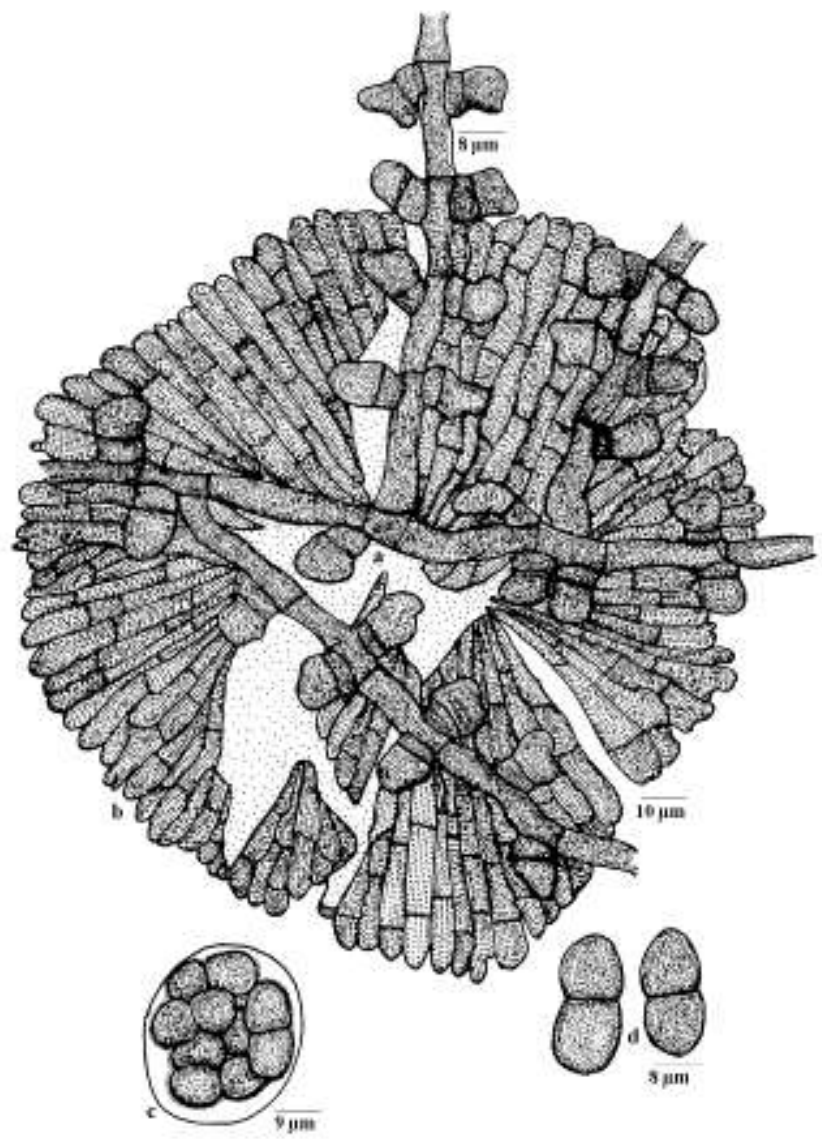

Figure 1119 - Asterina kodajadriensis
Material examined: Karnataka, Uduppi, Kodajadri Hills, on leaves of Capparis clegghornii Dunn ex Gamble (Capparaceae), 10 January 2012, K. Ravikumar HCIO 44287(holotype as A. capparidis), TBGT 717 (isotype as $A$. capparidis).

Typically opposite appressoria distinguishes this species from rest of the Asterina species known on the members of Capparaceae. This species shows an affinity with Ishwaramyces having rarely clustered appressoria.

Distribution: Karnataka

Asterina kukkalensis Hosag., Dhivaharan \& Riju, J. Sci. Environ. Techn. 4:164, 2010. (Figs 119-120)

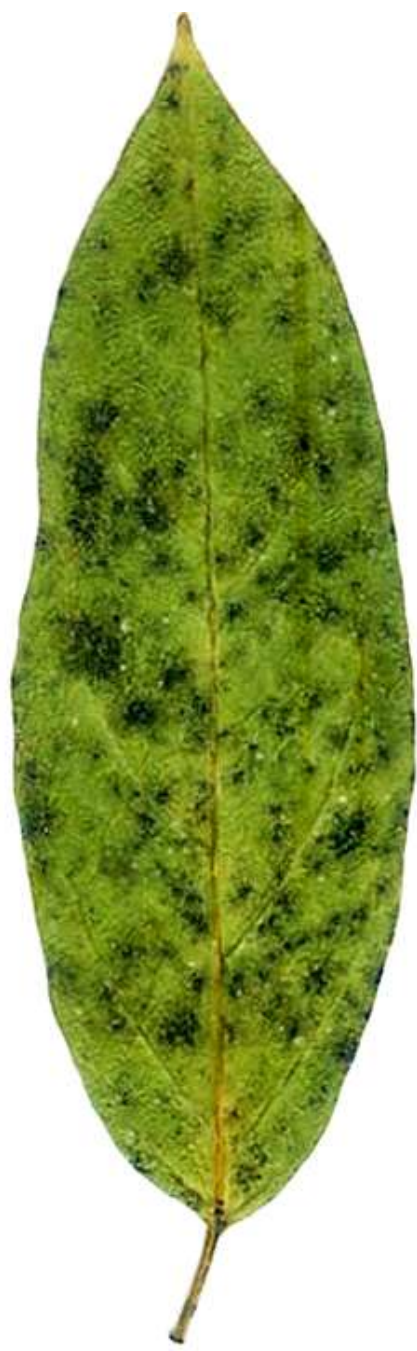

Figure 119 - Asterina kukkalensis - Infected leaves 


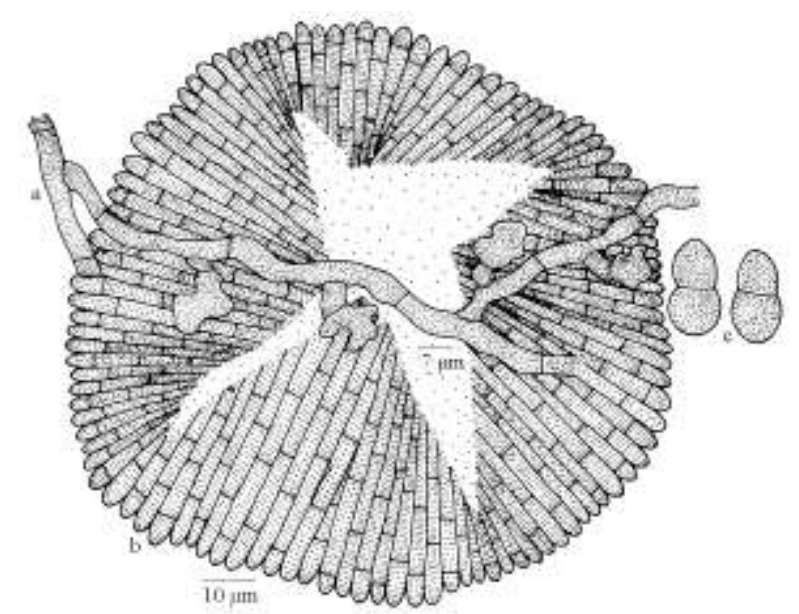

Figure 120 -20. Asterina kukkalensis

Colonies epiphyllous, subdense to dense, scattered, 2-5 mm in diameter, confluent and cover entire upper surface of the leaves. Hyphae substraight to flexuous, branching alternate to opposite at acute to wide angles, loosely reticulate, cells 25-35 $\times$ 5-7 $\mu \mathrm{m}$. Appressoria two celled, alternate, about 2\% opposite, straight to curved, 12-15 $\mu \mathrm{m}$ long; stalk cells cylindrical to cuneate, 5-7 $\mu \mathrm{m}$ long; head cells ovate, elongated to cylindrical, globose, bifid, sublobate to deeply lobate, 7-10 $\times 10-12 \mu \mathrm{m}$. Thyriothecia scattered to grouped, orbicular, up to $137 \mu \mathrm{m}$ in diameter, stellately dehisced at the centre, margin crenate to fimbriate, fringed hyphae solitary, flexuous and devoid of appressoria; asci globose, octosporous, up to $40 \mu \mathrm{m}$ in diameter; ascospores conglobate, 1-septate, constricted at the septum, $17-20 \times 7-8 \mu \mathrm{m}$.

Material examined: Tamil Nadu, Kodaikanal, Kukkal shola forest, Periyakanal, on leaves of Premna sp. (Verbenaceae), 11 November 2007, V. Dhivaharan \& al. TBGT 4447 (holotype). Part of the collection has been deposited in HCIO as isotype.

Asterina pusilla is known on this host genus from Phillipines and India (Sydow, 1913; Hosagoudar \& Sabeena, 2007). However Asterina kukkalensis differs from it in having two celled appressoria.

Distribution: Tamil Nadu

Asterina lanneae Hosag. \& Manoj. in Hosag., Zoos' Print J. 18: 1037, 2003; Hosag.,
Zoos' Print J. 21: 2328, 2006' Hosag., Chandraprabha \& Agarwal, Asterinales of Kerala, p. 99, 2011. (Fig. 121)

Colonies epiphyllous, dense, crustose, up to $5 \mathrm{~mm}$ in diameter, confluent. Hyphae straight to substraight, branching mostly opposite at acute angles, loosely to closely reticulate, cells $14-18 \times 3-5 \mu \mathrm{m}$. Appressoria opposite, about $10 \%$ solitary or unilateral, ovate, conoid, entire to variously lobed, 8-10 $\times$ 4-7 $\mu \mathrm{m}$. Thyriothecia scattered to grouped, often connate, orbicular, up to $200 \mu \mathrm{m}$ in diameter, margin fimbriate to crenate, fringed hyphae few, flexuous, stellately dehisced at the centre; asci globose, octosporous, $30-40 \mu \mathrm{m}$ in diameter; ascospores conglobate, brown, uniseptate, $19-21 \times 9-11 \mu \mathrm{m}$, wall punctate.

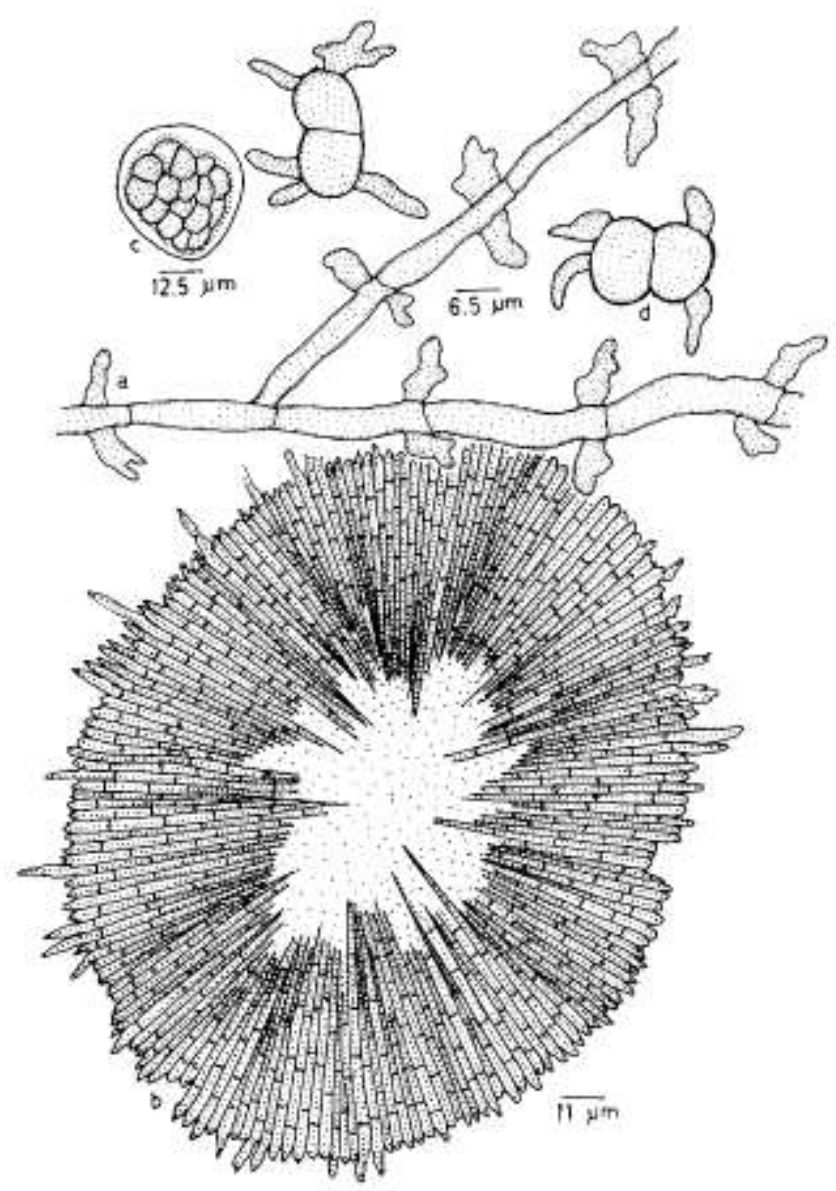

Figure 121 - Asterina lanneae

Material examined: Kerala, Pathanamthitta, Ranni, Placherry forest, on leaves of Lannea coromandelica (Houtt.) Merr. (Anacardiaceae), 4 October 2002, A. Manojkumar HCIO 44616 (holotype), TBGT 
898 (isotype); Konni, 4 December 2002, A. Manojkumar HCIO 44719, TBGT 1000.

Asterina anacardii (Ryan) Hosag. \& Abraham, A. drimycarpi Kar \& Maity and $A$. nothopegiae Ryan are known on the members of Anacardiaceae (Hosagoudar \& Abraham, 2000). Opposite and conoid appressoria of Asterina lanneae is similar to A. nothopegiae Ryan known on Nothopegia species from India (Ryan, 1928, Hosagoudar et al. 1996). However, this species differs from it in having all opposite but $10 \%$ solitary appressoria, shorter ascospores with punctate wall.

\section{Distribution: Kerala}

Asterina lawsoniae Henn. \& Nyn., Monsumia 1:159, 1899; Patil \& Thite, J. Shivaji Univ. 17: 152, 1977; Hosag., Chandraprabha \& Agarwal, Asterinales of Kerala, p. 102, 2011.

Asterina lawsoniae Gawande, D.K. Agarwal \& Hosag., Indian Phytopath. 57 (3): 287, 2004. (Fig. 122)

Colonies amphigenous, caulicolous, cover the softer portion of the stem and the total coverage of the black colonies on the plant can be noticed even from a distance during winter. Hyphae flexuous, branching irregular at acute to wide angles, loosely to closely reticulate, cells 9-16 $\times$ 3-5 $\mu \mathrm{m}$. Appressoria alternate, scattered, sessile, unicellular, ovate, mostly globose, rarely entire, mostly 1-3-sublobate to lobate, 4-8 $\times$ 4-7 $\mu \mathrm{m}$. Thyriothecia scattered, orbicular, up to $120 \mu \mathrm{m}$ in diameter, margin crenate, stellately dehisced at the centre; asci few to many, octosporous, ovate to globose, 30$36 \mu \mathrm{m}$ in diameter; ascospores conglobate, brown, uniseptate, constricted at the septum, $18-22 \times 8-10 \mu \mathrm{m}$, wall smooth. Pycnothyria similar to thyriothecia, mixed with thyriothecia, orbicular, smaller; pycnothyriospores pyriform, brown, unicellular, 14-17 × 7-9 $\mu \mathrm{m}$.

Material examined: Karnataka, Coorg, Madikeri, Jodupal, on leaves of Lawsonia inermis L. (Lythraceae), 21 November 2003, V.B. Hosagoudar \& al. HCIO 45807, TBGT 1556; Kerala, Thiruvananthapuram, Peppara, 4 February 1997, V.B. Hosagoudar HCIO 42539,
TBGT 153; Palode, 19 December, 2001, K.Vijayakumar HCIO 44300, TBGT 655; Plavara, June 19, 2002, A. Manoj Kumar HCIO 44603, TBGT 890; Ernakulam, Kolenchery, 11 September 2000, P.N. Krishnan HCIO 44073, TBGT 487; Pathanamthitta, Konni, 4 December 2002, A. Manoj Kumar 44720, TBGT 1001; Idukki, Munnar, 28 December 2004, P.V. Mahanteshgowda HCIO 46182, TBGT 1594; Thiruvananthapuram, Palode, Karimancode, 21 January 2005, Archana \& al. HCIO 46224, TBGT 1636; June

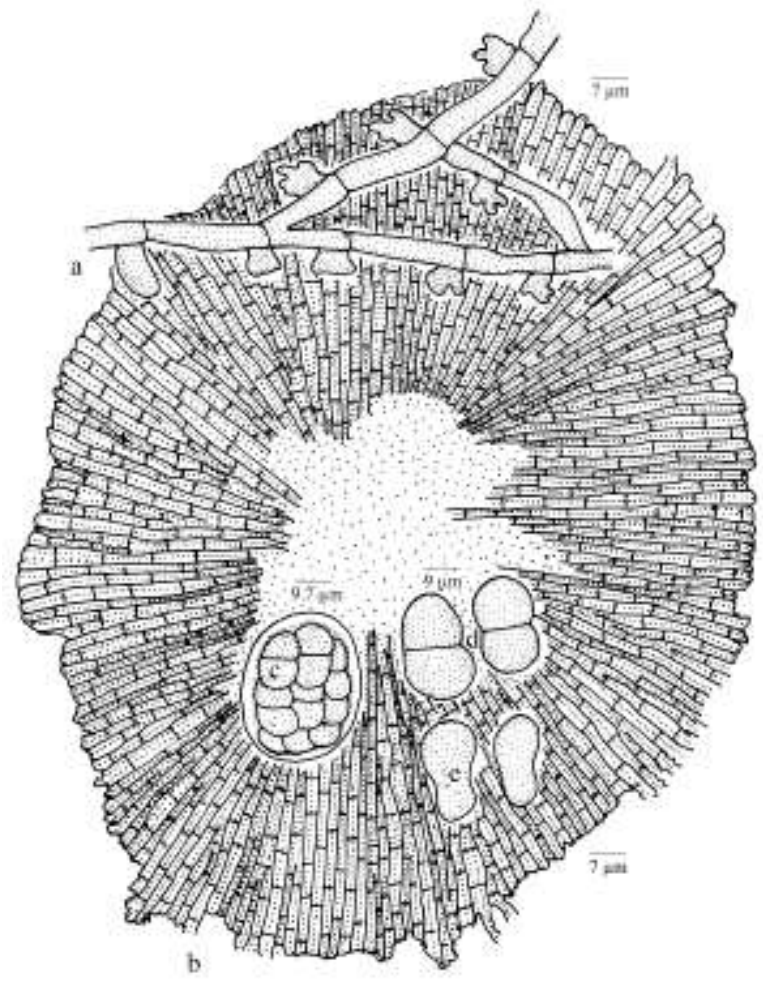

Figure 122 - Asterina lawsoniae

3, 2005, G.R. Archana HCIO 46367, TBGT 2013; Palode, TBGRI Campus, 25 January 2005, G.R. Archana \& V.L. Sheeja HCIO 45925, TBGT 1687; 8 November 2000, H. Biju 46101, TBGT 1864; HCIO 46370, TBGT 2016; August 22, 2005, G.R. Archana HCIO 46728, TBGT 2069; TBGRI Campus, 6 October 2005, V.S. Sreelal HCIO 46737, TBGT 2078; Jawahar colony, 21 December 2005, V.Gireesh Kumar \& al. HCIO 46747, TBGT 2088; Palode, Karimancode, 25 September 2005, Archana G.R HCIO 46972, TBGT 2189;TBGRI Campus, 20 September 2005, G.K. Anish Kumar HCIO 46998, TBGT 2215; Thiruvananthapuram, Nedumangad, Chullimanoor, 3 July 2006, Nasaruddeen 
47615, TBGT 2637; TBGRI Campus, 20 October 2006, V.B. Hosagoudar HCIO 47646, TBGT 2668; Bharathannoor, 21 January 2007, S. Divya HCIO 48030, TBGT 2813; Neyyar Wild Life Sanctuary, 24 August 2007, Jacob Thomas 48144, TBGT 2880; Palode, 14 December 2007, A. Chandraprabha HCIO 48304, TBGT 3025; TBGRI Campus, 19 October 2008, A. Sabeena HCIO 49125, TBGT 3380; 15 May 2008, V. Gireeshkumar 49345, TBGT 3590; 26 June 2008, A. Sabeena TBGT 4886; TBGRI campus, 6 September 2008, K. Anil Kumar TBGT 5279; Kottayam,Thazhathuvadakara, 27 December 2005, V.Gireesh Kumar \& P.J. Robin HCIO 46784, TBGT 2125; Kozhikode, Malabar Botanic Garden, 16 November 2006, Sabeena, Archana \& Riju 47391, TBGT 2429; Palghat, Shoranoor, 16 September 2005, K.Nandini 47610, TBGT 2632; Muvattupuzha, Pambakuda, 14 March 2007, Robin \& al. 48320, TBGT 3041; Kozhikode, Malabar Botanic Garden, 3 November 2009, A.Sabeena \& M. C. Riju TBGT 4320; Kannur, Mangattu Paramba, Neeliyar Kottam, 21 January 2011, M.P. Libina TBGT 4914; TBGT 4916

The fungus is always associated with the host plant throughout the winter season. Common in the Southern Western Ghats.

\section{Distribution: Karnataka, Kerala, Tamil Nadu, Maharashtra.}

Asterina laxiuscula Sydow, Phillipine J. Sci. 8: 276, 1912; M. S. Patil \& Pawar, Indian Phytopathol. 42: 248, 1989.

Mycelium epiphyllous, slightly to moderately developed, thin, scattered, effuse, cells $4.5 \mu \mathrm{m}$ broad, branched, distantly septate. Appressoria alternate, unicellular, broadly cylindrical, obtuse at the tip, 6-10 $\times 4-6 \mu \mathrm{m}$; thyriothecia loosely scattered to rarely aggregated, orbicular, stellately dehisced at the centre, $150-220 \mu \mathrm{m}$ in diam., slightly to moderately fimbriate at the margin; asci ovate, 55-75 $\times 40-50 \mu \mathrm{m}$ in diam., octosporous, spores, conglobate, oblong, rounded at both ends, uniseptate, constricted at the septum, pale brown, 32-40 × 15-18 $\mu \mathrm{m}$ wall smooth.
Maharashtra, Sindhudurgh, Amboli, on leaves of Xanotis tomentosum (Roxb.) Rafin. (Sapotaceae), 1 January 1977, M. S. Patil HCIO 40460.

This specis was known on Sideroxylon anguistifolia and $S$. ferugineum from Philppines. Patil and Pawar have reported it on Xanotis tomentosum. Since we did not get the material the description is based on Saccardo (1924).

Distribution: Maharashtra.

Asterina lepianthis (Hosag., Balakr. \& Goos) Hosag. in Hosag.,C.K. Biju, Abraham \& Agarwal, Indian Phytopath. 55: 498, 2002 (lepianthedis); Hosag., Chandraprabha \& Agarwal, Asterinales of Kerala, p. 103, 2011. (Fig. 123) Anamorph: Asterostomella lepianthedis Hosag., Balakr. \& Goos, Mycotaxon 58: 492, 1996.

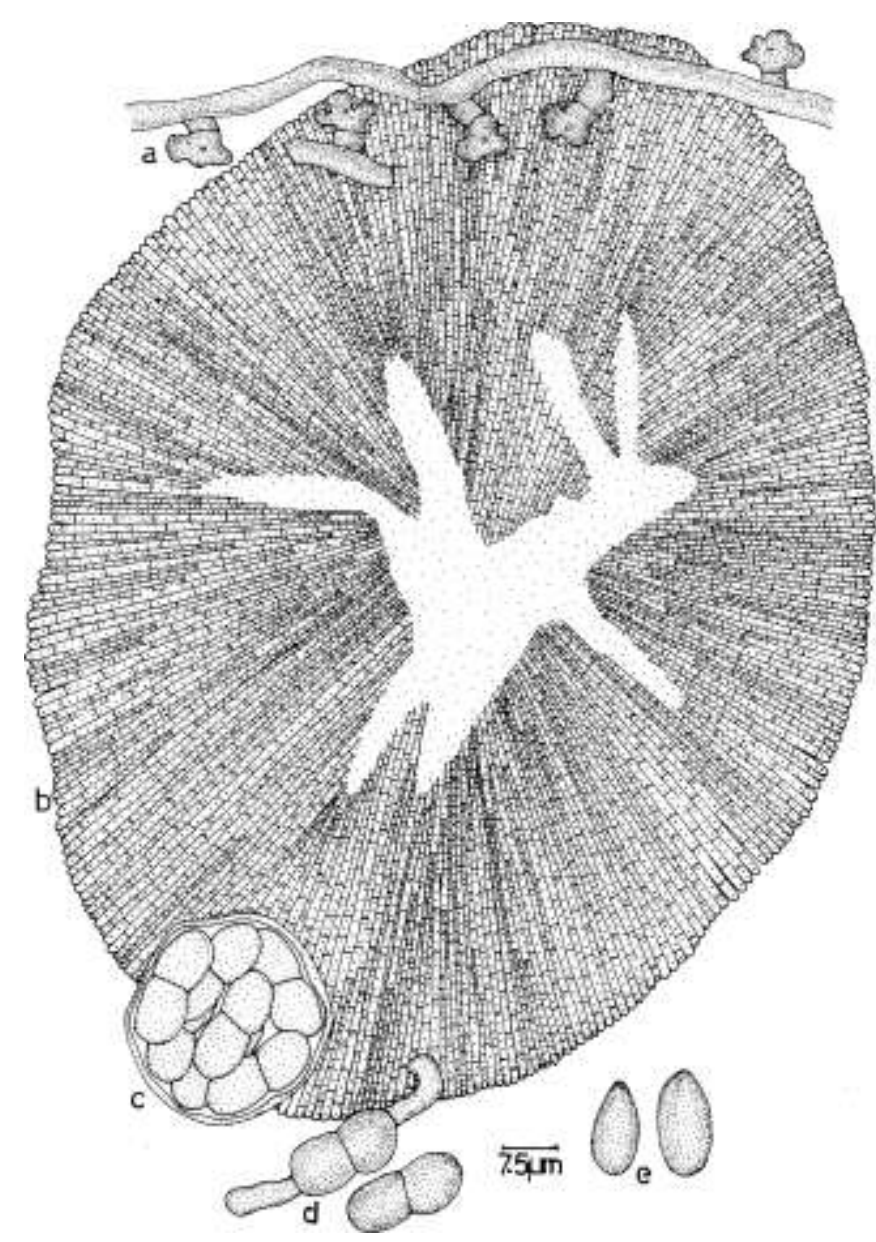

Figure 123. Asterina lepianthedis 
Colonies amphigenous, mostly epiphyllous, thin to dense, up to $1 \mathrm{~mm}$ in diameter, confluent. Hyphae st4raight, flexuous to crooked, branching irregular at acute angles, loosely reticulate, cells $12-33 \times 3-5 \mu \mathrm{m}$. Appressoria scattered, alternate to unilateral, straight to curved, two-celled, 9-13 $\mu \mathrm{m}$ long; basal cells cuneate to cylindrical, 3-7 $\mu \mathrm{m}$. Pycnothyria scattered, orbicular, center, margin crenate; Pycnothyriospores unicellular, globose to ellipsoidal, brown, 9-15 × 6-10 $\mu \mathrm{m}$.

Material examined: Tamil Nadu, Nilgiris, Gudalur, Benne Forest, on leaves of Lepianthes umbellata, (L.) Rafin. [Hackeria subpeltata (Willd.) Kunth] (Piperaceae), 24 January 1990, V.B. Hosagoudar HCIO 30980; Karnataka, Coorg, Madikeri, Jodupal, 12 November 2003, V.B. Hosagoudar \& al. HCIO 45686, TBGT 1433; Jodupal, Madikeri, Coorg, Karnataka, November 12, 2003,V.B. Hosagoudar \& al. HCIO 45802, TBGT 1551; Kerala, Palghat, Silent valley, Sairandhri, 13 December 2003, V.B. Hosagoudar \& al. HCIO 45698, TBGT 1445; Wyanad, 8 December 2000, M. Kamarudeen HCIO 43714, TBGT 364; Sairandhri, Silent valley, Kerala, December 13, 2003,V.B. Hosagoudar \& al. HCIO 45804, TBGT 1553; Sairandhri, Silent valley, Kerala, December 12, 2004, V.B. Hosagoudar \& al. HCIO 46089, TBGT 1852.

Repeated collections from Nilgiris and Anamalai (Coimbatore dist.) of Tamil Nadu did not show any ascigerous states.

This species was associated with Meliola sp.

Distribution: Tamil Nadu, Karnataka, Kerala

Asterina leucadis Hosag. \& Robin, Bioscience Discovery 2: 264, 2011. (Fig. 124)

Colonies epiphyllous, thin, up to $2 \mathrm{~mm}$ in diameter, confluent. Hyphae substraight to undulate, branching alternate to opposite, irregular at acute to wide angles, loosely reticulate, form a net, cells $17-22 \times 5-7 \mu \mathrm{m}$. Appressoria two celled, alternate to unilateral, antrorse to subantrorse, $17-19 \mu \mathrm{m}$ long; stalk cells cylindrical to cuneate, 5-7 $\mu \mathrm{m}$ long; head cells globose, ovate, clavate, irregularly sublobate to 2-4-times lobate, 10-12 × 7-10 $\mu \mathrm{m}$. Thyriothecia scattered, orbicular, up to $144 \mu \mathrm{m}$ in diameter, stellately dehisced at the center, margin fimbriate, rarely crenate, fringed hyphae straight to flexuous; asci not seen; ascospores conglobate, brown, 1-septumte, constricted at the septum, 17-22 $\times 7-10 \mu \mathrm{m}$, wall smooth.

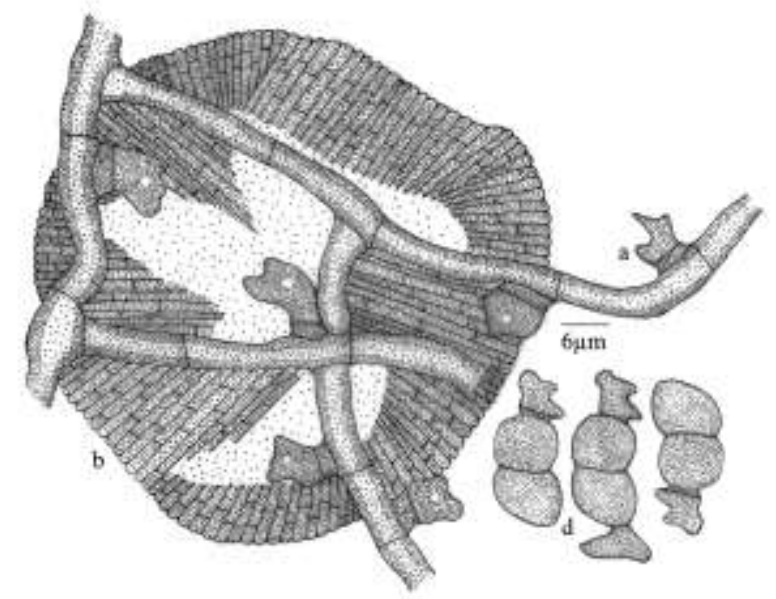

Figure 124 - Asterina leucadis

Material examined: Karnataka, Coorg, on leaves of Leucas sp. (Lamiaceae), 27 November 2007, V.B. Hosagoudar \& P.J. Robin 4655 (holotype). Part of the collection has been deposited in HCIO, New Delhi.

There are five species of the genus Asterina known on the members of the family Lamiaceae (Hosagoudar \& Abraham, 2000). Based on the nature of two celled appressoria, A.lucadis can be compaired with $A$. geniospori and $A$. pogostemonis. It differs from $A$. geniospori in having substraight to undulate mycelium in contrast to strongly crooked. It also differs from $A$. pogostemonis in having scattered but good number of appressoria.

Asterina ligustricola Hosag. \& Kamar. in Hosag., J. Econ. Taxon. Bot. 28: 187, 2004; Hosag., Chandraprabha \& Agarwal, Asterinales of Kerala, p. 106, 2011.

Asterostomella ligustri Hosag., Balakr. \& Goos, Mycotaxon 58: 493, 1996.

(Fig. 125)

Colonies amphigenous, mostly epiphyllous, subdense to dense, up to $3 \mathrm{~mm}$ in 
diameter, confluent. Hyphae substraight to flexuous, branching irregular at acute angles, loosely reticulate, cells 11-15 $\times 3-4 \mu \mathrm{m}$. Appressoria alternate to unilateral, scattered, unicellular, sessile to stipitate, irregularly and stellately sublobate to lobate, globose, 4-8 $\times 6$ $10 \mu \mathrm{m}$. Pycnothyria numerous, scattered, orbicular, up to $50 \mu \mathrm{m}$ in diameter, margin fimbriate to crenate, stellately dehisced at the center; pycnothyriospores ellipsoidal, taper towards both ends, brown, 19-21 $\times$ 9-10 $\mu \mathrm{m}$. Thyriothecia scattered, orbicular, up to $130 \mu \mathrm{m}$ in diameter, margin crenate to fimbriate, fringed hyphae tortuous, thyriothecia stellately dehisced at the center; asci globose, octosporous, 30-35 $\mu \mathrm{m}$ in diameter; ascospores conglobate, brown, uniseptate, constricted at the septum, $16-19 \times 9-10 \mu \mathrm{m}$, wall minutely echinulate.

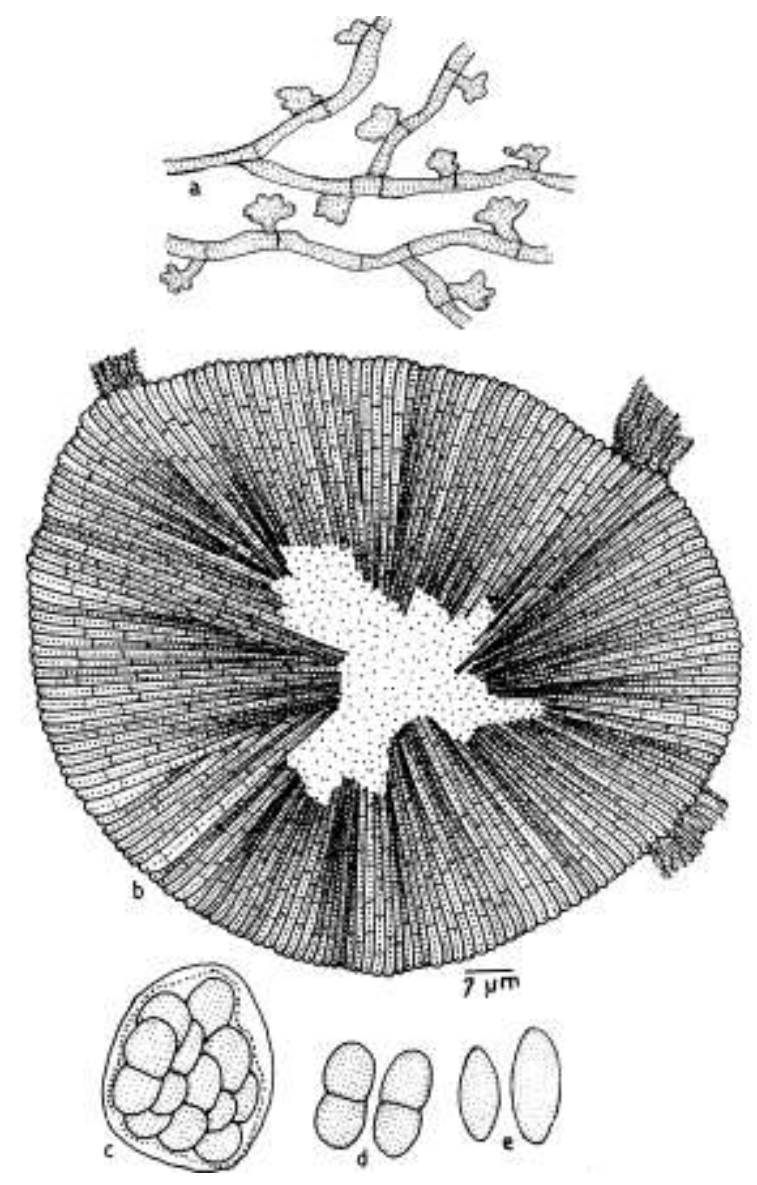

Figure125 - Asterina ligustricola

Material examined: Kerala, Wyanad, on leaves of Ligustrum travencoricum Gamble (Oleaceae), 24 May 2001, M. Kamarudeen HCIO 44129 (holotype), TBGT 528 (isotype); Wyanad, Periya, on leaves of Ligustrum walkeri auct., 7 March 2001, G. Rajkumar \&
P.A. Jose HCIO 44867, TBGT 1095; Pathanamthitta, Mozhiar forest, Anathodu Dam site, on leaves of Ligustrum sp., 15 December 2004, V.B. Hosagoudar \& al. HCIO 46078, TBGT 1841; 19 December 2004, V.B. Hosagoudar \& al. HCIO 46188, TBGT 1600; HCIO 46090, TBGT 1853; HCIO 46329, TBGT 1975; Pathanamthitta, Pachakanam, 15 December 2004, V.B. Hosagoudar \& al. HCIO 46283, TBGT 1929.

This species differs from Asterina ligustri P. Henn in having unicellular appressoria and is the teleomorph of Asterostomella ligustri Hosag. et al. (Katumoto, 1975; Hosag. et al., 1996). It was parasitised by Zygosporium sp.

Distribution: Kerala.

Asterina litseae Yates, Philippine J. Sci. 13: 373, 1918; Hosag., Balakr. \& Goos, Mycotaxon 59: 180, 1996. (Fig. 126)

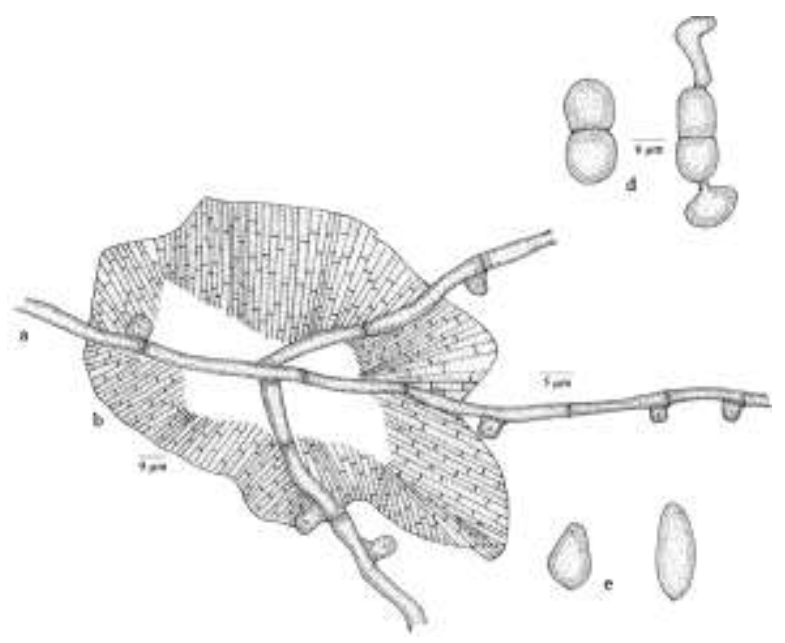

Figure 126 - Asterina litseae

Colonies amphigenous, mostly epiphyllous, thin to subdense, up to $5 \mathrm{~mm}$ in diameter, rarely confluent. Hyphae straight to substraight, branching opposite to irregular at wide angels, loosely reticulate, cells 12-22 × 3$5 \mu \mathrm{m}$. Appressoria alternate, about $40 \%$ opposite, unicellular, straight to curved, conoid to ampulliform, broadly rounded at the apex, 6$13 \times 5-7 \mu \mathrm{m}$. Thyriothecia closely scattered, orbicular, up to $125 \mu \mathrm{m}$ in diameter, dehiscing stellately at the center, dehiscence extending up to the margin, the dehisced portion often dissolved and the inner deep yellow content 
exposed, margin fimbriate, fringed hyphae many, crooked; asci many, globose, eight spored, 18-25 $\mu \mathrm{m}$ in diameter; ascospores conglobate, deep brown, 1-septumte, deeply constricted at the septum, $18-19 \times 8-10 \mu \mathrm{m}$, upper cell ovate, lower cell globose, wall smooth.

Material examined: Tamil Nadu, Nilgiris, Kotagiris, Kolithorai, on leaves of Litsea deccanensis Gamble (Lauraceae), 16 February 1991, V.B. Hosagoudar HCIO 30968; Kodaikanal, Vattakanal, 22 July 2006, G. Sangeeta HCIO 47097 TBGT 2314; Uttar Pradesh, Maharajganj, on leaves of Litsea chinensis Lam. (Lauraceae), M.A. Nomani TBGT 3512.

This species was reported from Philippines (Hansford, 1949).

Distribution: Tamil Nadu, Uttar Pradesh

Asterina litseae-ligustrinae Hosag., Balakr. \& Goos, Mycotaxon 59: 180, 1996.

(Fig. 127)

Colonies hypophyllous, dense, crustose, up to $5 \mathrm{~mm}$ in diameter, rar3ely confluent. Hyphae straight to substraight in few places crooked, branching opposite to irregular at acute to wide angles, loosely reticulate, cells $15-22 \times 3-5 \mu \mathrm{m}$. Appressoria alternate, about $15 \%$ opposite, straight to curved, unicellular, conoid, entire to variously sublobate, 9-13 × 6$10 \mu \mathrm{m}$. Thyriothecia scattered to loosely grouped, round to ovate, up to $110 \mu \mathrm{m}$ in diameter, stellately fissured at the center, inner content deep yellow, margin crenate to fimbriate, fringed hyphae long and tortuous; asci many, globose, eight spored, 24-26 $\mu \mathrm{m}$ in diameter; ascospores conglobate, 1-septumte, constricted at the septum, $18-19 \times 6-10 \mu \mathrm{m}$, upper cell slightly ovate, lower cell globose, wall echinulate.

Material examined: Tamil Nadu, Nilgiris, Kotagiri, Kolithoirai, on leaves of Litsea ligustrina (Nees) Hook. f. (Lauraceae), 6 February 1991, V.B. Hosagoudar HCIO 30969 (holotype).
This species is similar to Asterina litseae Yates in having yellow contents in the thyriothecia (Hansford, 1949) but differs from it in having hypophyllous colonies, substraight to crooked hyphae, $15 \%$ opposite, and entire to variously sublobate appressoria and echinulate ascospores.

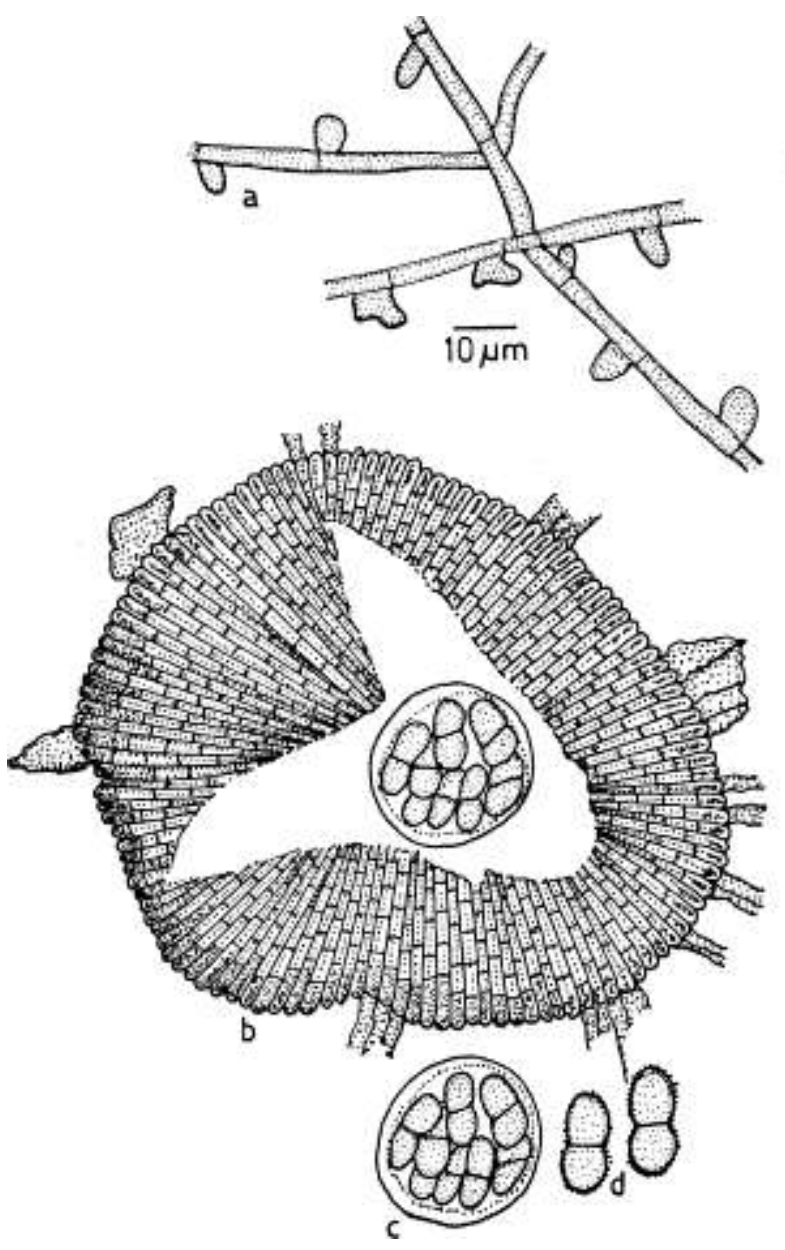

Figure 127 - Asterina litseae-ligustrinae

Distribution: Tamil Nadu

Asterina lobeliacearum Hosag.,C.K. Biju in Hosag.,C.K. Biju \& Abraham, J. Mycopathol. Res. 40:195, 2002; Indian Phytopath. 57: 115, 2004; Hosag., Chandraprabha \& Agarwal, Asterinales of Kerala, p. 107, 2011.

\section{(Figs 128-129)}

Colonies epiphyllous, minute, dense, black, up to $1 \mathrm{~mm}$ in diameter. Hyphae straight to flexuous, branching irregular at wide angles, loosely reticulate, cells $12-20 \times 2-3 \mu \mathrm{m}$. Appressoria scattered, alternate, unicellular, globose to cylindrical, angular to 2-3 times 
sublobate, 7-13 $\times 5-8 \mu \mathrm{m}$. Thyriothecia mostly scattered, rarely connate, orbicular, up to 120 $\mu \mathrm{m}$ in diameter, stellately dehisced at the centre, margin crenate; asci globose, octosporous, up to $40 \mu \mathrm{m}$ in diameter, stellately dehisced at the center, margin crenate; asci globose, octosporous, up to $40 \mu \mathrm{m}$ in diameter; ascospores oblong, brown, 1-septumte, constricted at the septum, $15-17 \times 7-8 \mu \mathrm{m}$, wall smooth.

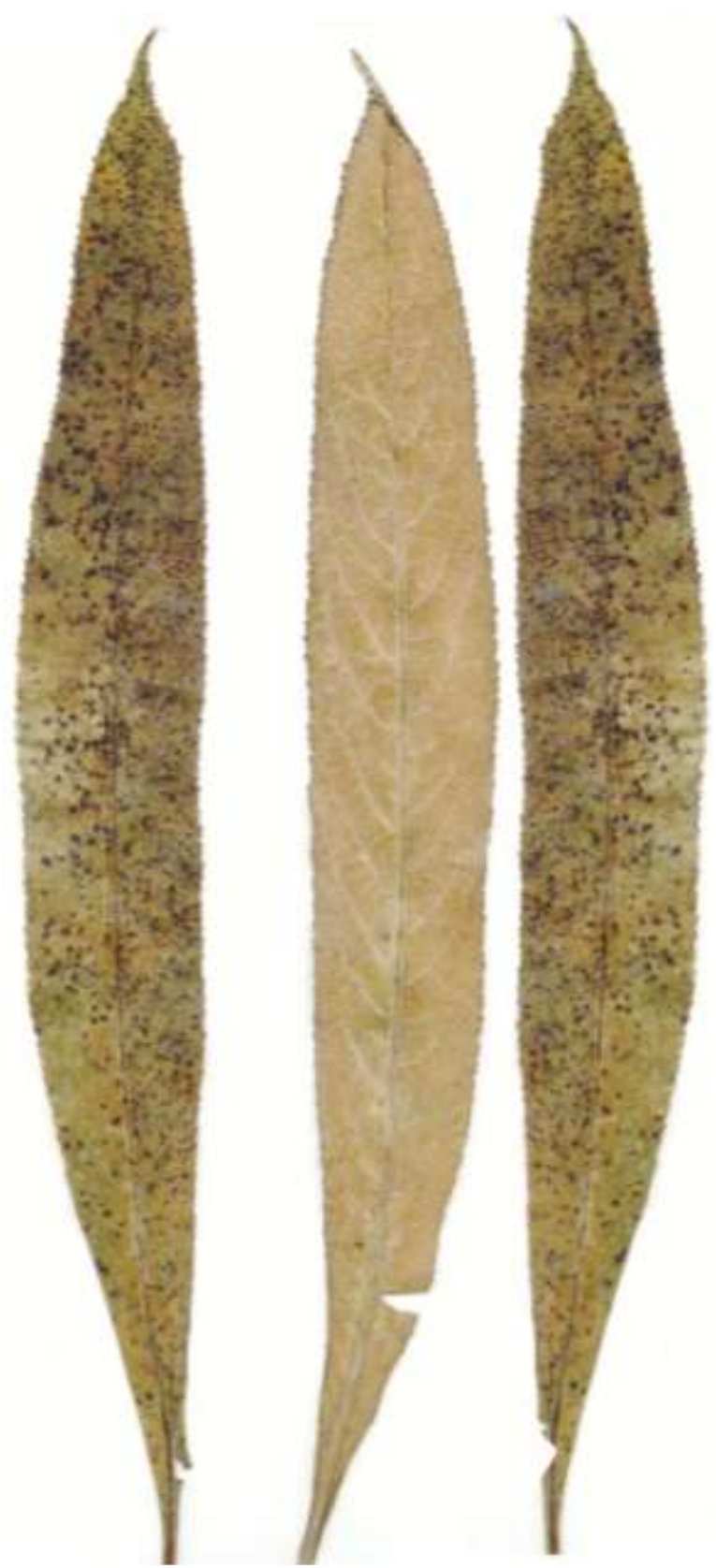

Figure 128 - Asterina lobeliacearum

Material examined: Kerala, Idukki, Mannavan shola, on leaves of Lobelia nicotinifolia Roth ex Schultes (Lobeliaceae), 29 September 1998, C.K. Biju HCIO 42936 (holotype), TBGT 217 (isotype); 6 January
1999, C.K. Biju HCIO 43814, TBGT 393; Munnar,Vagapara, 22 January 2003, A. Manojkumar \& H. Biju HCIO 44855, TBGT 42; Mannavanshola, 6 January 1999, C.K.Biju HCIO 43814, TBGT 293; Munnar, Mattupatti, 17 March 2007, Jacob Thomas \& al. HCIO 48160, TBGT 2896.

Asterina lobeliae Stev. \& Ryan and $A$. xumensis Doidge are known on the host genus Lobelia from Porto Rico and South Africa (Diodge, 1942; Stevens \& Ryan, 1939). A. lobeliacearum differs from the former species in having smaller thyriothecia, larger asci and ascospores. It also differs from the latter species in having only alternate and unicellular appressoria, and larger ascospores.

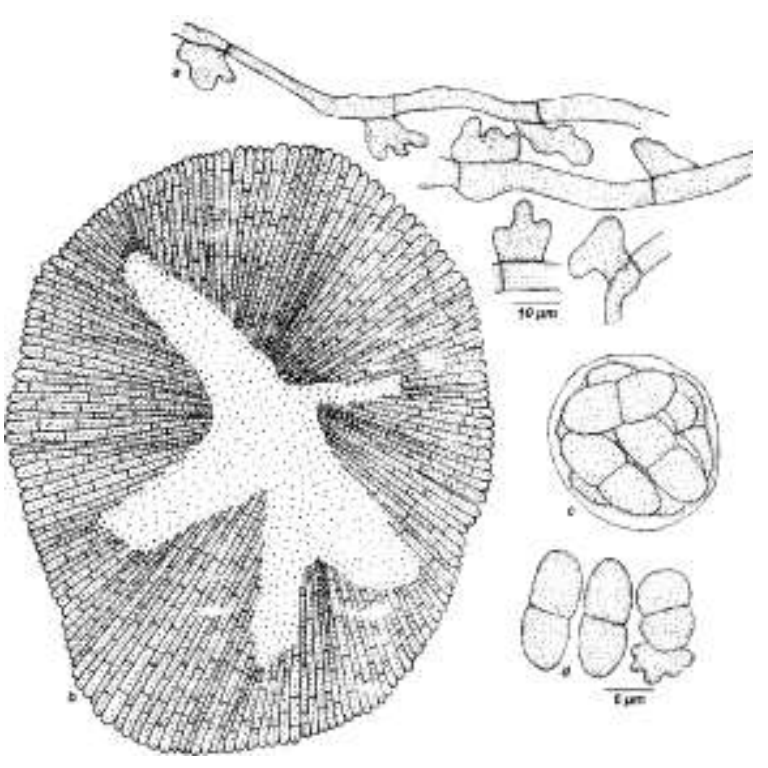

Figure 129 - Asterina lobeliacearum

Distribution: Kerala.

Asterina lobulifera Sydow in Sydow \& Sydow, Philippine J. Sci. 9: 181, 1914; Hosag.,C.K. Biju\& Abraham, J. Mycophathol. Res. 40:195, 2002; J. Econ. Taxon. Bot. 28: 175, 2004; Hosag., Chandraprabha \& Agarwal, Asterinales of Kerala, p. 108, 2011.

(Fig. 130)

Colonies epiphyllous, subdense to dense, up to $3 \mathrm{~mm}$ in diameter, rarely confluent. Hyphae straight to flexuous, branching irregular 
at acute angles, loosely reticulate, cells $16-32 \times$ 4-5 $\mu \mathrm{m}$. Appressoria two celled, alternate, antrorse, 8-15 $\mu \mathrm{m}$ long; stalk cells cylindrical to cuneate, 5-8 $\mu \mathrm{m}$ long; head cells ovate, globose, entire, sublobate to lobate, 5-8 6-11 $\mu \mathrm{m}$. Thyriothecia scattered to loosely grouped, often connate, orbicular, up to $144 \mu \mathrm{m}$ in diameter, stellately dehisce at the center, margin crenate to fimbriate, fringed hyphae straight to flexuous; asci not seen; ascospores conglobate, brown, 1-septumte, strongly constricted at the septum, 16-21 $\times 9-10 \mu \mathrm{m}$, wall smooth. Pycnothyria smaller and similar to thyriothecia; Pycnothyriospores brown, obpyriform, truncate at the base, 16-18 $\times 9-10$ $\mu \mathrm{m}$, wall smooth.

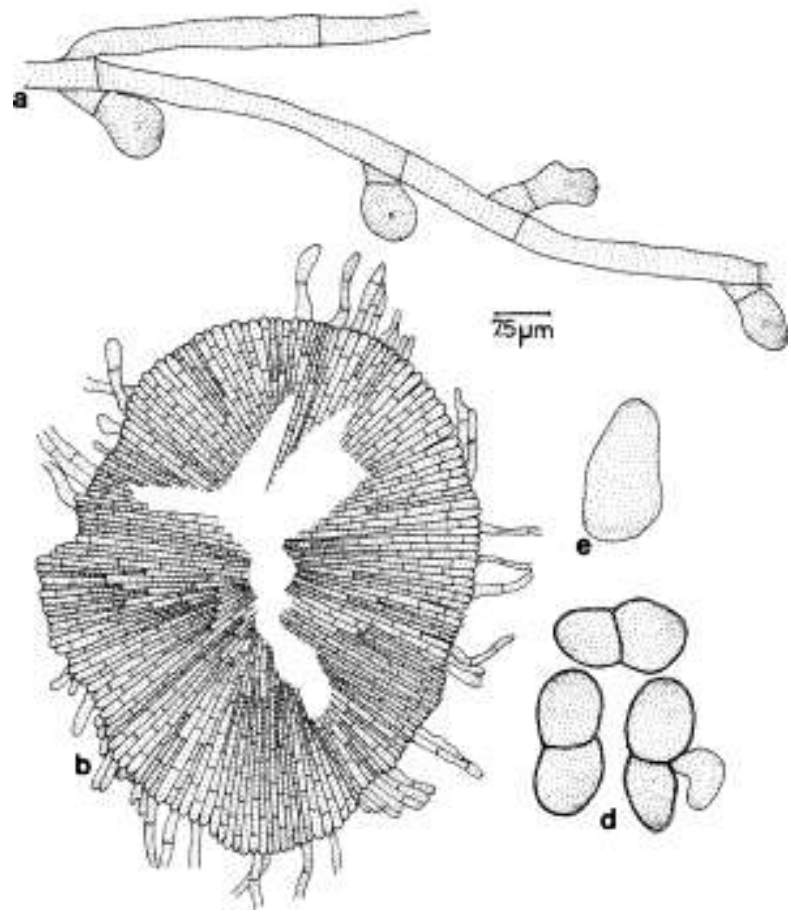

Figure 130 - Asterina lobulifera

Material examined: Kerala, Idukki, Munnar, Mannavan shola, on leaves of Glochidion sp. (Euphorbiaceae), 5 January 1998, C.K. Biju HCIO 43362, TBGT 265.

This species was known from Philippines, Taiwan and Japan (Sydow \& Sydow, 1914; Yamamoto, 1956; Katumoto, 1991) and is reported here for the first time from India (Bilgrami et al., 1991; Sarbhoy et al., 1996).

Distribution: Kerala.
Asterina lobulifera Sydow var. indica Hosag. \& Chandra., Indian J. Sci. Techn. 2: 15, 2009; Hosag., Chandraprabha \& Agarwal, Asterinales of Kerala, p. 109, 2011. (Figs 131-132)

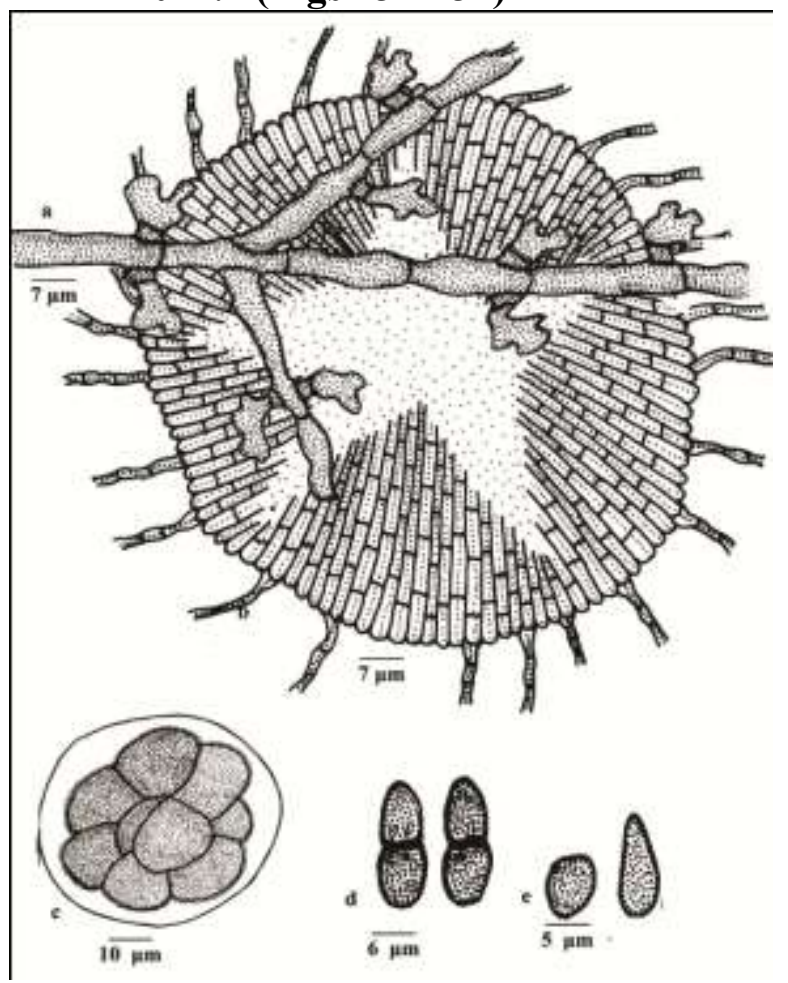

Figure 131 - Asterina lobulifera var. indica

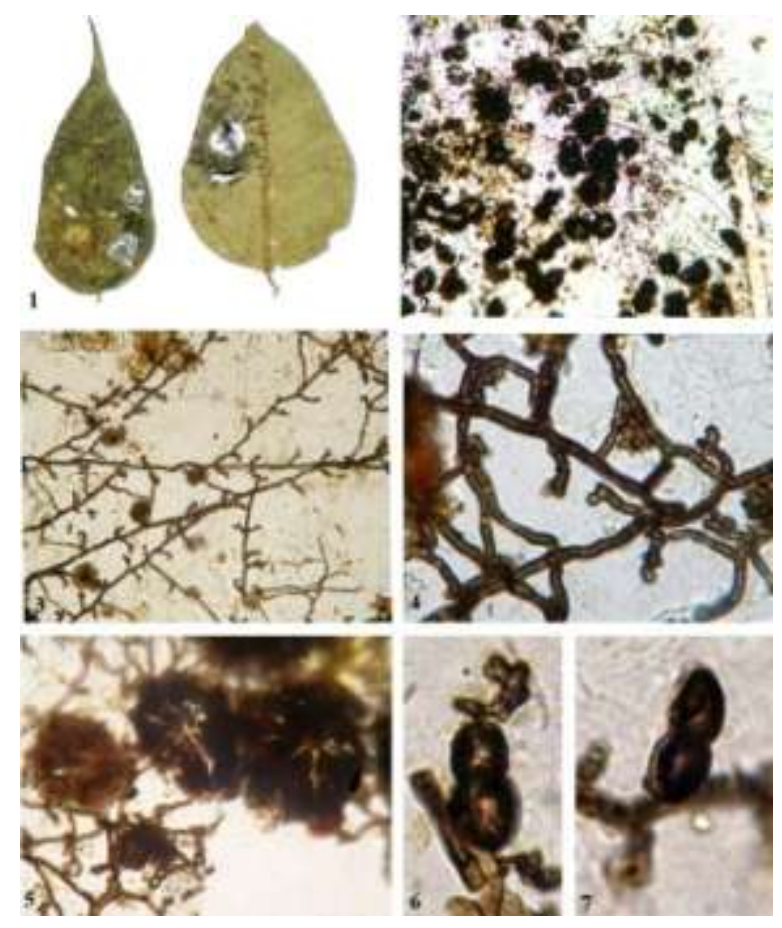

Figure 132 - Asterina lobulifera var. indica

1. Infected leaf, 2. Fungal colony with thyriothecia, 3. Appressoriate mycelium,4. Two celled appressoriate mycelium, 5. Dehisced thyriothecia, 6. Germinating ascospore, 6. Ascospore 
Colonies amphigenous, dense, up to $2 \mathrm{~mm}$ in diameter. Hyphae flexuous to crooked, branching opposite to irregular at acute to wide angles, loosely to closely reticulate, cells $15-26$ $\times$ 4-7 $\mu \mathrm{m}$. Appressoria 2-celled, alternate, opposite $(5-10 \%)$ subantrorse straight to curved, 11-15 $\mu \mathrm{m}$ long; stalk cells cylindrical to cuneate, 4-7 $\mu \mathrm{m}$ long; head cells ovate, globose, sublobate to lobate, $7-11 \times 4-7 \mu \mathrm{m}$. Thyriothecia scattered two grouped at the centre of the colonies, orbicular, up to $121 \mu \mathrm{m}$ in diameter, margin crenate to fimbriate, stellately dehisced at the center; ascospores oblong, conglobate, uniseptate, constricted at the septum, 13-22 $\times$ 7-9 $\mu \mathrm{m}$, wall smooth. Pycnothyria smaller, similar to thyriothecia; pycnothyriospores ovate, pyriform, brown, 11$22 \times 7-11 \mu \mathrm{m}$, wall smooth.

Material examined: Kerala, Wyanad, Periya, Upper peak, on the leaves of Glochidion sp. (Euphorbiaceae), 29 October 2007, A. Chandra Prabha HCIO 48236 (holotype), TBGT 2974 (isotype).

Asterina cassiae Sydow and $A$. lobulifera Sydow are known on the genus Glochidion from Philippines (Sydow \& Sydow, 1914; Hosagoudar \& Abraham, 2000). $A$. lobulifera differs from $A$. cassiae in having two celled appressoria. However, A. lobulifera Sydow var. indica differs from the var. lobulifera in having $5-10 \%$ opposite appressoria (Saccardo, 1924; Katumoto, 1991).

Distribution: Kerala.

Asterina loeseneriellae Hosag. \& Goos, Mycotaxon, 59: 157, 1996. (Fig. 133)

Colonies amphigenous, mostly hypophyllous, dense, velvety, up to $5 \mathrm{~mm}$ in diameter, confluent. Hyphae straight to substraight, branching opposite to irregular at acute angles, loosely reticulate, cells 15-22 × 6$8 \mu \mathrm{m}$. Appressoria opposite, alternate $(20 \%)$, continuous, ovoid, cylindrical, ampulliform, antrorse to subantrorse, mostly straight, attenuated and rounded at the apex, 12-19 $\times 6-8$ $\mu \mathrm{m}$. Thyriothecia scattered, round, up to $78 \mu \mathrm{m}$ in diameter; margin crenate, widely opened to dehiscing stellately at the center; asci very few
(2-4), globose, octosporous, $37-47 \mu \mathrm{m}$ in diameter; ascospores conglobate, 1-septumte, deeply constricted at the septum initially hyaline and later turn deep brown, upper cell smaller than lower, 30-35 × 15-19 $\mu \mathrm{m}$, wall smooth. Pycnothyria few, similar to thyriothecia; pycnothyriospores dark brown, pyriform, $18-22 \times 15-19 \mu \mathrm{m}$, wall smooth.

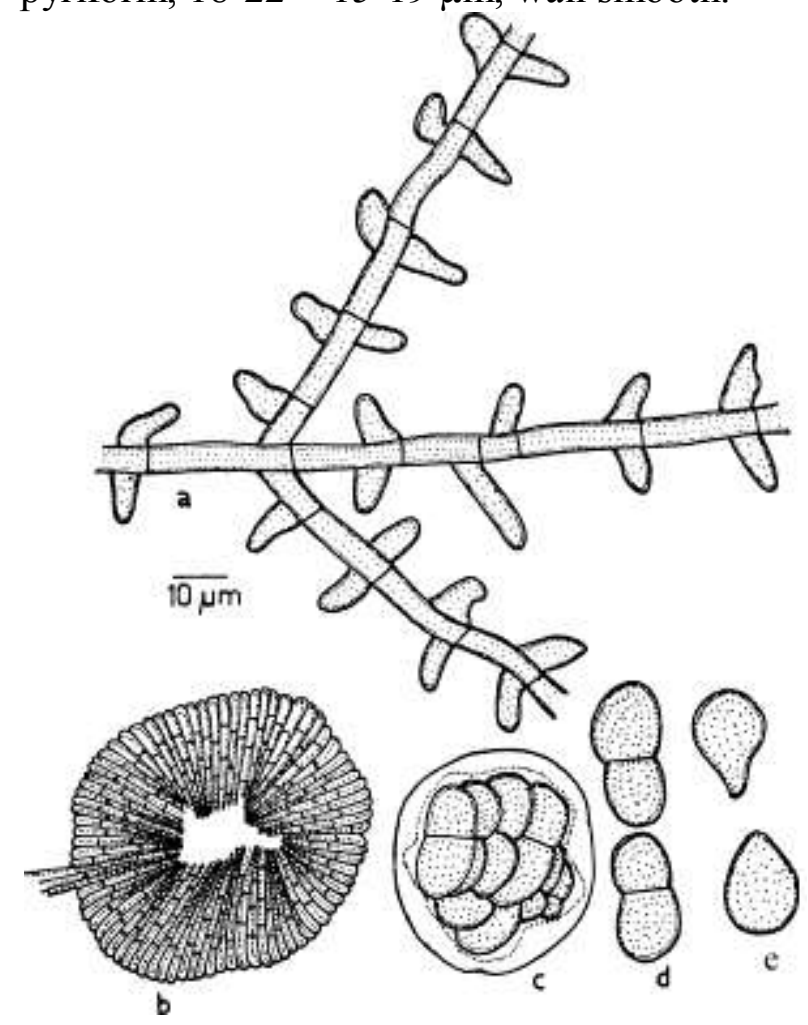

Figure 133 - Asterina loeseneriellae

Material examined: Tamil Nadu, Coimbatore, Anamalai, Topslip, on leaves of Loeseneriella obtusifolia (Roxb.) A. C. Smith (Celastraceae), 12 March 1994, V.B. Hosagoudar HCIO 41562 (holotype).

Asterina hippocrateae Ryan and $A$. salaciae Allesch. are known on the members of the family Hippocrataceae. This species differs from the former in having smaller thyriothecia and larger ascospores and is distinguished from the latter in having both smaller thyriothecia and ascospores.

Distribution: Tamil Nadu.

Asterina loranthigena Hosag., Agarwal, H. Biju \& Archana, Indian Phytopathol. 59: 525, 2006; Hosag., Chandraprabha \& Agarwal, Asterinales of Kerala, p. 111, 2011. (Figs 134-135) 
Colonies amphigenous, dense, velvety, up to $2 \mathrm{~mm}$ in diam., confluent. Hyphae flexuous to crooked, branching irregular and form a net, cells 22-26 × 4-7 $\mu \mathrm{m}$. Appressoria
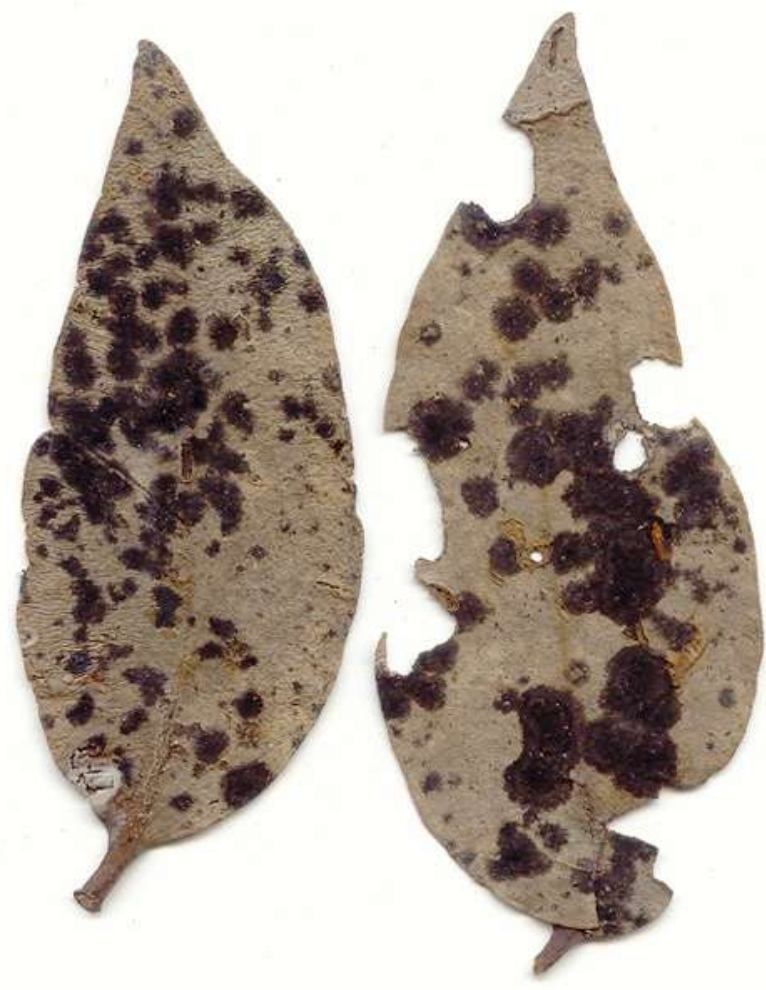

Figure 134. Asterina loranthigena

places randomly, often some portion of the Hyphae devoid of appressoria, alternate to unilateral, oblong, clavate, straight to mostly curved to uncinate, entire, $12-21 \times 6-8 \mu \mathrm{m}$. Thyriothecia scattered to connate, orbicular, up to $200 \mu \mathrm{m}$ in diam., margin crenate to fimbriate, fringed hyphae flexuous, stellately dehisced at the centre; asci few, globose, up to $35 \mu \mathrm{m}$ in diam.; ascospores oblong, conglobate, brown, uniseptate, constricted at the septum, 27-30 $\times 14-16 \mu \mathrm{m}$, wall echinulate at maturity. Pycnothyria similar to thyriothecia, smaller; pycnothyriospores pyriform, brown, 22-24 $\times$ 14-16 $\mu \mathrm{m}$.

Material examined: Kerala, Kollam, Shankily forest, on leaves of Dendrophthoe sp. (Loranthus sp.) (Loranthaceae), 23 November 2004, V.B. Hosagoudar HCIO 46160 (holotype), TBGT 1572 (isotype).

There are five species of the genus Asterina reported on the members of the family Loranthaceae (Hosagoudar \& Abraham, 2000).

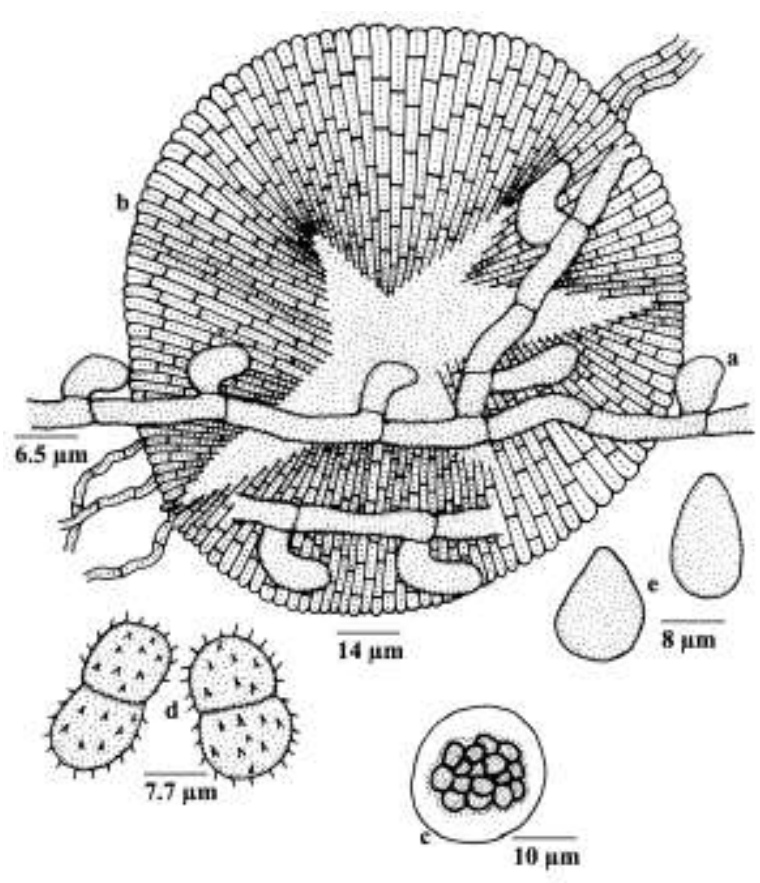

Figure 135 - Asterina loranthigena

Of these, Asterina loranthigena is similar to Asterina loranthicola Sydow known on Loranthus sp. from Africa (Doidge, 1942; Stevens \& Ryan, 1939) in having uncinate appressoria. However, Asterina loranthigena differs from it in having only unicellular and longer appressoria $(12-21 \times 6-8 \mu \mathrm{m}$ against 7 $11 \times 5-6 \mu \mathrm{m})$ and longer ascospores $(27-30 \times$ 14-16 $\mu \mathrm{m}$ against $20-21 \times 8-14 \mu \mathrm{m})$ with echinulate wall.

\section{Distribution: Kerala.}

Asterina lycianthedis Hosag. \& Abraham, Indian Phytopath. 50: 216, 1997; Hosag., C.K. Biju \& Abraham, J. Econ. Taxon. Bot. 25: 305, 2001; Hosag., Chandraprabha \& Agarwal, Asterinales of Kerala, p. 112, 2011.

(Fig. 136)

Colonies subdense to dense, up to 2 $\mathrm{mm}$ in diameter. Hyphae flexuous to crooked, branching mostly opposite at acute angles, loosely to closely reticulate, cells $14-22 \times 4-5$ $\mu \mathrm{m}$. Appressoria unicellular, alternate, unilateral, about $20 \%$ opposite, ovate, globose, mammiform, entire, 7-10 $\times$ 6-8 $\mu \mathrm{m}$. Thyriothecia scattered, 2-3 connate, orbicular, up to $185 \mu \mathrm{m}$ in diameter, dehisce stellately at the center, margin crenate; asci many, globose to ovate, octosporous, $40-55 \times 35-45 \mu \mathrm{m}$; 
ascospores brown, conglobate, 1-spptate, 24-26 $\times$ 11-13 $\mu \mathrm{m}$, upper cell smaller, wall smooth.
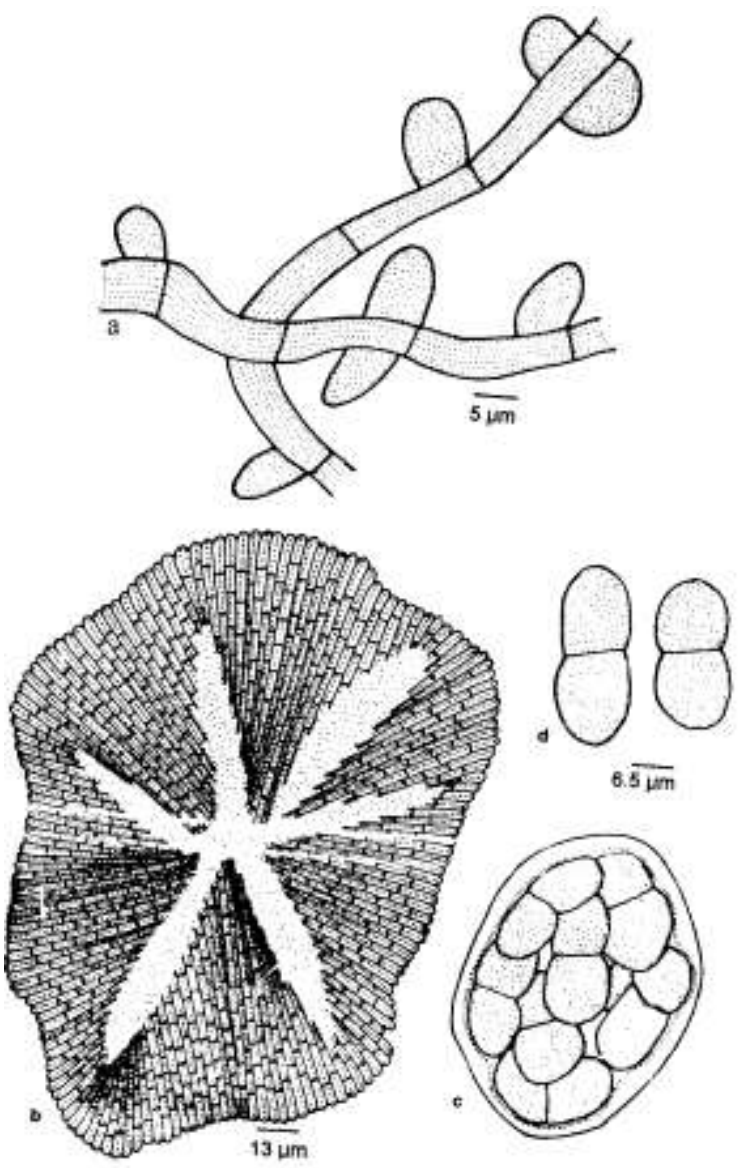

Figure 136 - Asterina lycianthedis

\section{Material examined: Kerala,}

Thiruvananthapuram, Agastiar hill, on leaves of Lycianthes laevis (Dunnal) Bitter (Solanaceae), 26 March 1996, V.B. Hosagoudar HCIO 42290 (holotype), TBGT 75 (isotype).

Seven taxa of the genus Asterina are known on the members of the family Solanaceae (Stevens and Ryan, 1939; Hansford, 1949; Hosagoudar and Goos, 1996). The present new species differs from all in having $20 \%$ opposite appressoria.

Distribution: Kerala.

Asterina mabae M. S. Patil \& Pawar, Indian Phytopathol. 42: 249, 1989.

Colonies amphigenous, up to $7 \mathrm{~mm}$ in diameter. Hyphae reticulate, cells 15-22 × 3-4 $\mu \mathrm{m}$. Appressoria alternate, 2-celled, 13-16 $\mu \mathrm{m}$ long; stalk cells cylindrical, $2-4 \mu \mathrm{m}$ long; head cells 1-3 lobate, lobes irregular, 9-13 $\times 3 \mu \mathrm{m}$. Thyriothecia gregarious, orbicular, 150-200 $\mu \mathrm{m}$ in diameter, stellately dehisced at the center; asci globose, octosporous, 27-31 $\mu \mathrm{m}$ in diameter; ascospores ovate-elliptic, brown, 1septumte, constricted, rounded at both ends, 19$22 \times 10 \mu \mathrm{m}$, wall smooth.

Maharashtra, Kolhapur, Amba, on leaves of Maba nigrescens Dalz. (Ebenaceae), 11 January 1984, A. B. Pawar HCIO 39415.

\section{Distribution: Maharashtra.}

Asterina madikeriensis Hosag. J. Mycopathol. Res. 44: 9, 2006. (Figs 137-138)

Colonies epiphyllous, dense, minute to $3 \mathrm{~mm}$ in diameter, confluent. Hyphae straight, branching alternate to opposite at acute wide angles, loosely to closely reticulate, cells 17-24 $\times 4-6 \mu \mathrm{m}$. Appressoria opposite, rarely solitary or attenuate, ovate, entire, attenuated to broadly rounded at the apex, $8-10 \times 6-8 \mu \mathrm{m}$. Thyriothecia orbicular, scattered, up to $170 \mu \mathrm{m}$ in diam., stellately dehisced at the centre, margin fimbriate to crenate, fringed hyphae straight to flexuous, compact; asci globose, octosporous, up to $30 \mu \mathrm{m}$ in diam.; ascospores oblong, conglobate, brown, uniseptumte, constricted at the septum, $22-25 \times 8-11 \mu \mathrm{m}$, wall tubercled.

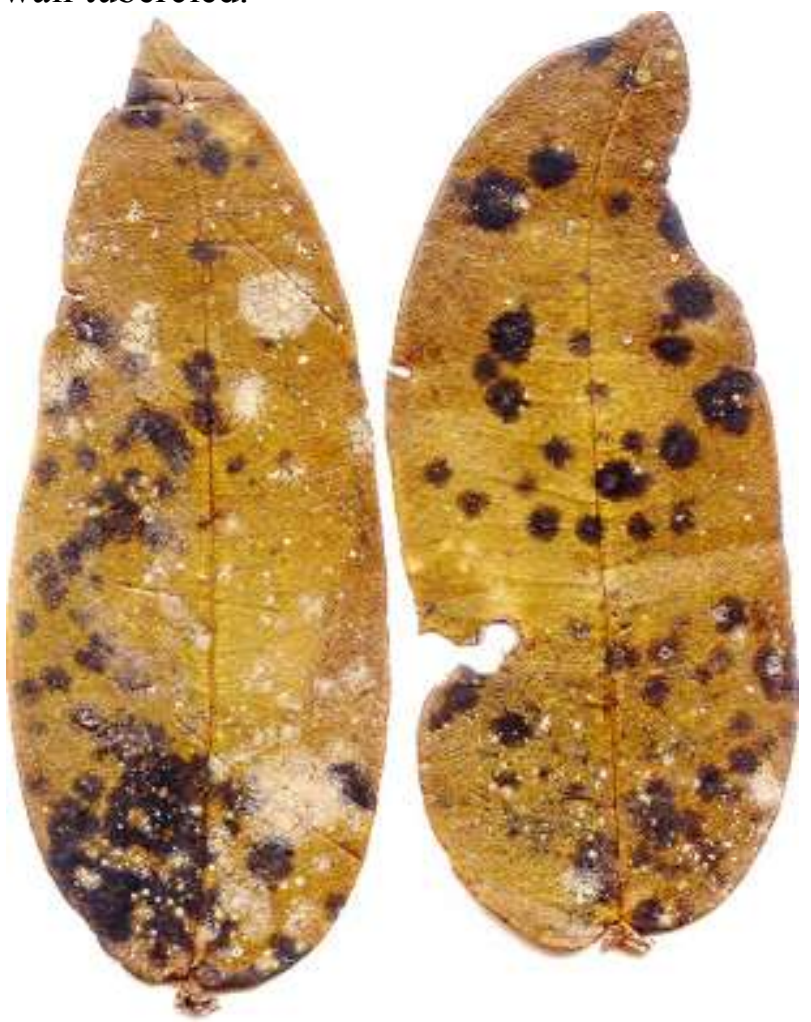

Figure 137 - Asterina madikeriensis 
Material examined: Karnataka, Kodagu (Coorg), Madikeri, Nishanemotta, on leaves of Memecylon sp. (Melastomataceae), 12 November 2003, V.B. Hosagoudar \& al. TBGT 1455 (holotype).

Asterina memecylonis Ryan is the only species known on the genus Memecylon from Karwar, Karnataka (Ryan, 1928; Hosagoudar \& Abraham, 2000). It was relocated from Kerala (Hosagoudar, 2003). However, $A$. madikeriensis differs from it in having regularly opposite and densely placed appressoria.

The colonies were associated with Meliola memecyli Syd. var. microspora Hosag. et al.
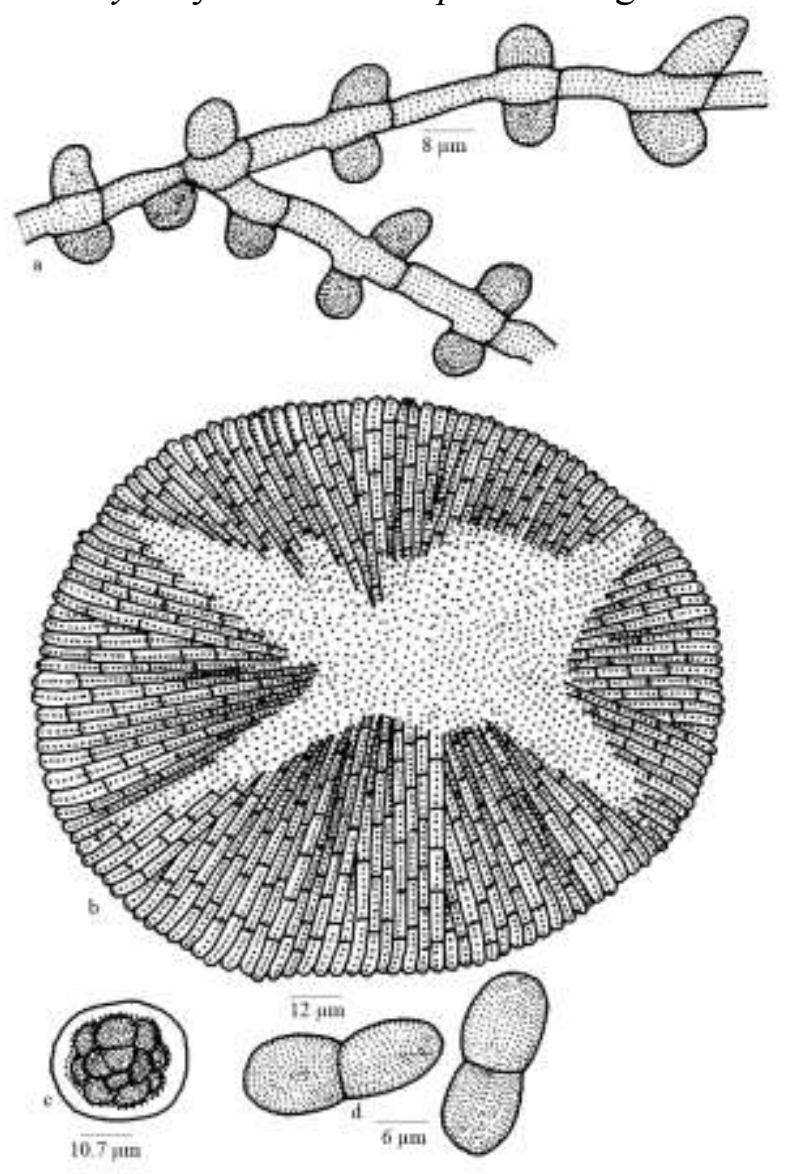

Figure 138 - Asterina madikeriensis

Distribution: Karnataka.

Asterina malloticola Hosag., Kamar. \& Rajkumar in Hosag. \& Agarwal, Indian Phytopath. 56: 99, 2003; Hosag. \& Appaiah, J. Mycopathol. Res. 43:168, 2005; Hosag., Chandraprabha \&
Agarwal, Asterinales of Kerala, p. 113, 2011. (Fig. 139)

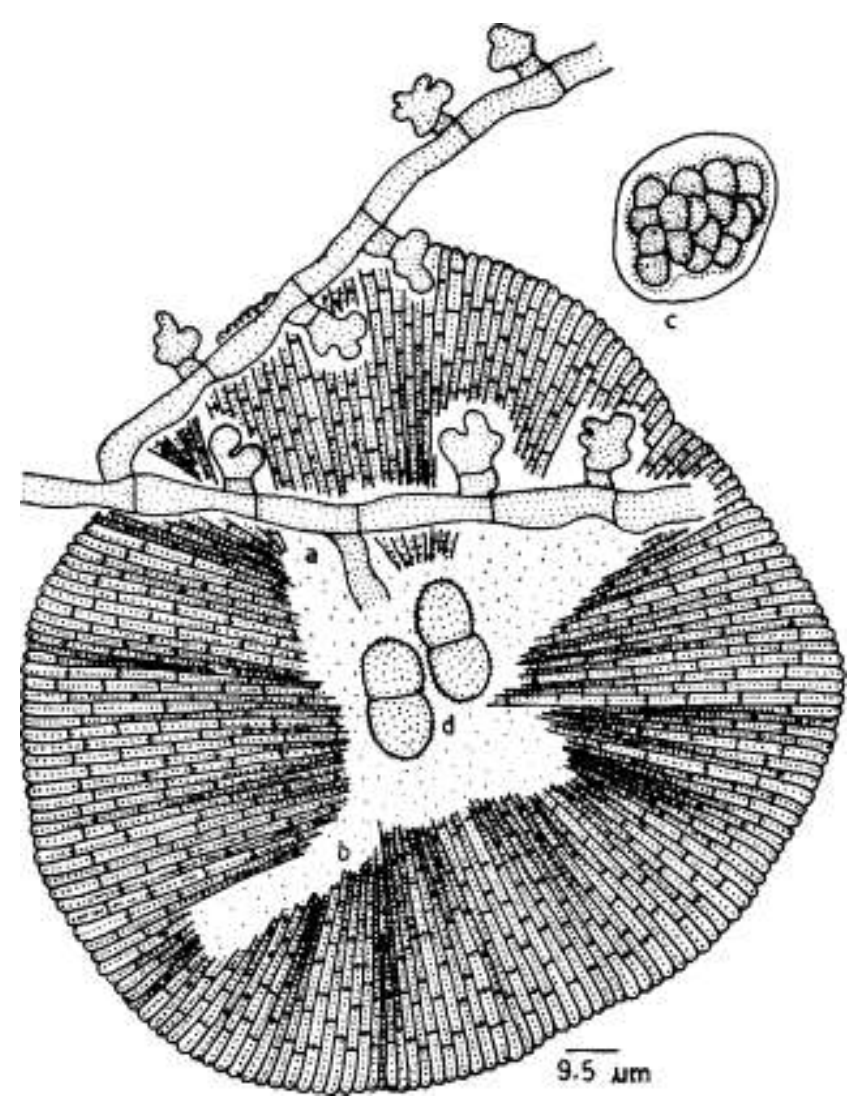

Figure 139 - Asterina malloticola

Colonies epiphyllous, subdense, up to 3 $\mathrm{mm}$ in diameter, confluent. Hyphae straight to flexuous, branching alternate, opposite to irregular at acute angles, loosely to closely reticulate, cells $19-23 \times 4-5 \mu \mathrm{m}$. Appressoria alternate, about $1 \%$ opposite, two celled, antrorse to subantrorse, $8-13 \mu \mathrm{m}$ long; stalk cells cylindrical to cuneate, $1.5-5 \mu \mathrm{m}$ long; head cells globose, stellately to irregularly lobate, 6-8 $\times$ 9-12 $\mu \mathrm{m}$. Thyriothecia scattered, orbicular, up to $130 \mu \mathrm{m}$ in diameter, stellately dehisced at the centre, margin crenate to fimbriate, fringed hyphae very small; asci globose, octosporous, up to $30 \mu \mathrm{m}$ in diameter; ascospores oblong, conglobate, brown, uniseptate, constricted at the septum, 19-23 $\times$ 11-13 $\mu \mathrm{m}$, wall minutely echinulate.

Material examined: Kerala, Kollam, Ariyankavu, Kulirkadu, on leaves of Mallotus philippensis (Lam.) Muell.-Arg. (Euphorbiaceae), 20 February 2001, M. Kamarudeen \& G. Rajkumar HCIO 44118 (holotype), TBGT 559 (isotype). 
Asterina malloti Sawada is known on the host genus Mallotus from Taiwan and India (Sawada, 1959; Thite \& Kulkarni, 1976). However, Asterina malloticola differs from it in having $1 \%$ opposite appressoria with stellately lobate head cells in contrast to entire ones.

Distribution: Kerala.

Asterina mallotigena V.B. Hosagoudar \& A. Sabeena, sp. nov. (Fig. 140)

MycoBank 101006

Etymology: Named after the host genus

Colonies epiphyllous, thin to subdense, crustose, up to $2 \mathrm{~mm}$ in diameter, confluent. Hyphae substraight to flexuous, branching irregular at acute angles, loosely to closely reticulate, cells 12-20 × 2-5 $\mu \mathrm{m}$. Appressoria alternate, subopposite to opposite, unicellular, narrowly ovate, oblong, tubular, entire, 7-15 $\times$ 2-7 $\mu \mathrm{m}$. Thyriothecia scattered, orbicular, up to $140 \mu \mathrm{m}$ in diameter, stellately dehisced at the centre; asci globose, octosporous, up to $28 \mu \mathrm{m}$ in diameter; ascospores conglobate, 1-septate, constricted at the septa, $17-25 \times 10-12 \mu \mathrm{m}$, wall smooth.

Material examined: Kerala, Kottayam, Ponthanpuzha, on leaves of Malotus sp. (Euphorbiaceae), 1 March 2008, P.J. Robin TBGT 5738 (holotype).

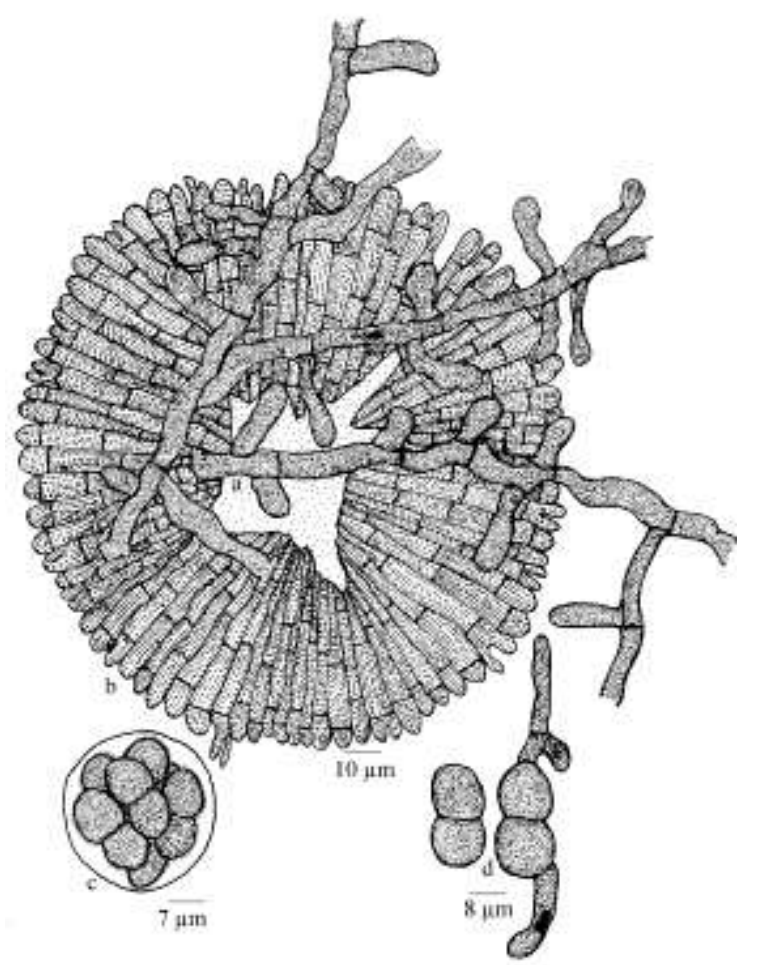

Figure 140 - Asterina mallotigena
Asterina malloti Sawada and A.malloticola Hosag. et al. are known on this host from Thaiwan and India (Sawada 1959; Hosag et al. 2003). However, the present species differs from both in having unicellular appressoria.

Distribution: Kerala

Asterina melicopecola Hosag. \& Abraham, Indian Phytopath. 50: 216, 1997; Hosag., C.K. Biju \& Abraham, J. Econ. Taxon. Bot. 25: 305, 2001; Hosag., Zoos' Print J. 18: 1284, 2003; 21: 2328, 2006; Hosag., H. Biju \& Appaiah, J. Mycopathol. Res. 44: 9, 2006; Hosag., Chandraprabha \& Agarwal, Asterinales of Kerala, p. 115, 2011.

(Fig. 141)

Colonies amphigenous, mostly epiphyllous, dense, up to $1 \mathrm{~mm}$ in diameter, confluent. Hyphae straight, branching opposite at acute angles, loosely reticulate, cells 19-24 $\times$ 3.5-5 $\mu \mathrm{m}$. Appressoria opposite, about 20\% alternate, unicellular, ovate, globose, clavate, pyriform, irregularly sublobate to lobate, 9-12.5 $\times 6-7.5 \mu \mathrm{m}$. Thyriothecia orbicular, loosely grouped in the center of the colony, scattered to connate, up to $150 \mu \mathrm{m}$ in diameter, stellately dehisce at the center, splitting up to margin, crenate to fimbriate, fringed hyphae small, profusely branched; asci globose, rounded, octosporous, up to $42 \mu \mathrm{m}$ in diameter; ascospores conglobate, brown, 1-septate, deeply constricted at the septum, upper cell globose, lower cell slightly ovate, 31-34 × 12$13.5 \mu \mathrm{m}$.

$\begin{array}{crr}\text { Material } & \text { examined: } & \text { Kerala, } \\ \text { Thiruvananthapuram, } & \text { Neyyar } & \text { Wildlife }\end{array}$ Sanctuary, Agastiyar hill, on leaves of Melicope lunuankanda (Gaertn.) T. G. Hartley (Rutaceae), 26 March 1996, V.B. Hosagoudar HCIO 42291; Wyanad, Periya, 10 January 2002, M. Kamarudeen HCIO 44301, TBGT 588; Wyanad, Banasuran mala, on leaves of Euodia luna-ankenda (Gaertner) Merr. (Rutaceae), 16 April 1999, C.K. Biju HCIO 45147, TBGT 1202; 19 November 1999, C.K. Biju HCIO 45149, TBGT 1204; Perya, 16 February 2009, Jacob Thomas \& al HCIO 49223, TBGT 3462; 15 February 2009, Jacob Thomas \& al. HCIO 49785, TBGT 3937; Palghat, Silent Valley National Park, 27 April 
2007, M. Harish \& al. HCIO 49760, TBGT 3912; Kottayam, Ponthanpuzha, 22 November 2007, P.J. Robin \& al. HCIO TBGT 5213; Karnataka, Nishane motta, 11 November 2003, V.B. Hosagoudar \& al. HCIO 45607, TBGT 1350; Madikeri, Field Marshal Cariappa college campus, 23 November 2009, C. Jagath Thimmaiah TBGT 5445.

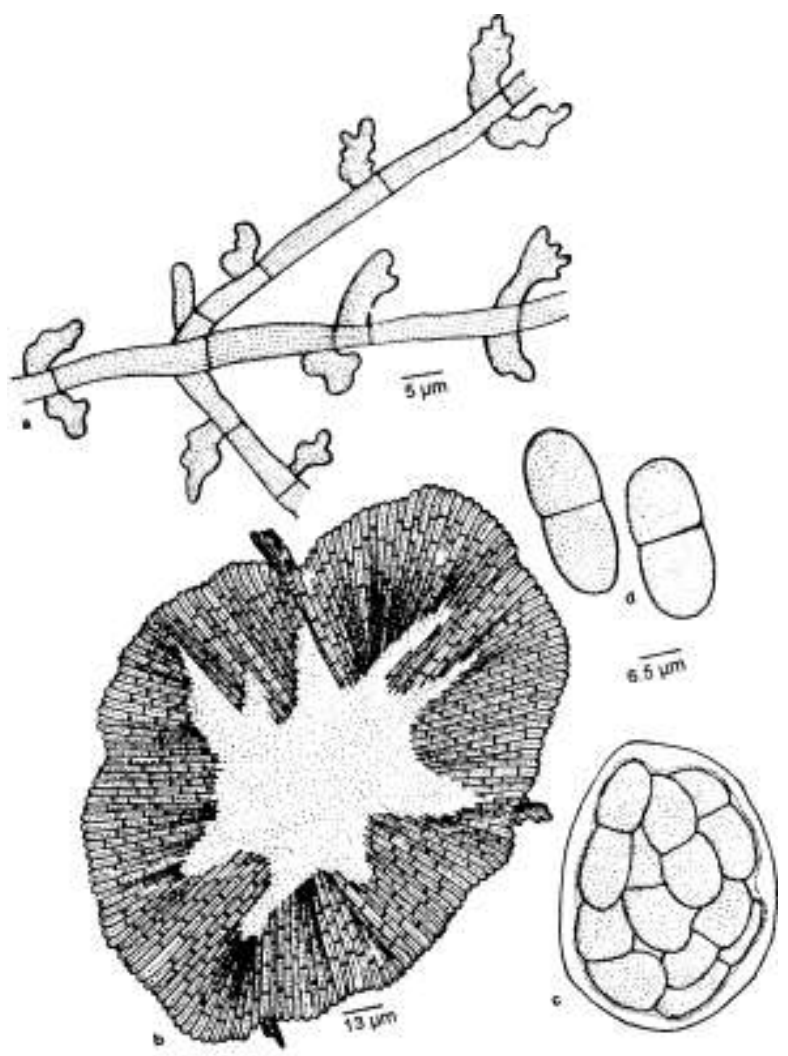

Figure 141 - Asterina melicopecola

Asterina clausenicola Doidge, $A$. evodiae Hansf. and $A$. evodiicola Yamam. are known of the host genus Evodia (Doidge, 1942; Hansford, 1954; Hosagoudar, 1995; Yamamoto, 1956). The present species differs from all these in having only $20 \%$ alternate and irregularly sublobate to lobate appressoria.

This species was mixed with Amazonia sp.

Distribution: Kerala, Karnataka.

Asterina meliosmae-simplicifoliae Hosag., C.K. Biju \& Abraham, J. Mycopathol. Res. 40:195, 2002; Hosag., Chandraprabha \& Agarwal, Asterinales of Kerala, p. 115, 2011.

(Fig. 142)
Colonies amphigenous, mostly hypophyllous, dense, up to $4 \mathrm{~mm}$ in diameter, confluent. Hyphae flexuous, branching opposite to irregular at acute angles, loosely reticulate, cells 11-26 × 4-7 $\mu \mathrm{m}$. Appressoria scattered, alternate, unilateral, unicellular, oblong, cylindrical, straight to curved, entire, sublobate, 10-13 × 7-9 $\mu \mathrm{m}$. Thyriothecia scattered, orbicular, up to $113 \mu \mathrm{m}$ in diameter, dehisce stellately at the center, margin crenate to fimbriate; asci many, octosporous, globose, up to $38 \mu \mathrm{m}$ in diameter; ascospores oblong, conglobate, brown, 1-septate , slightly constricted at the septum, 23-25 × 10-13 $\mu \mathrm{m}$. Pycnothyria similar to thyriothecia, smaller; pycnothyriospores globose, pyriform, brown, $15-22 \times 11-13 \mu \mathrm{m}$, wall smooth.

Material examined: Kerala, Idukki, Mannavan shola, on leaves of Meliosma simplicifolia (Roxb.) Walp. (Sabiaceae), 24 September 1998, C.K. Biju HCIO 42957 (holotype), TBGT 254 (isotype); Karnataka, Karnataka, Hoddur, Kodagu, 2 December, 2009, C. Jagath Thimmaiah TBGT 5465.

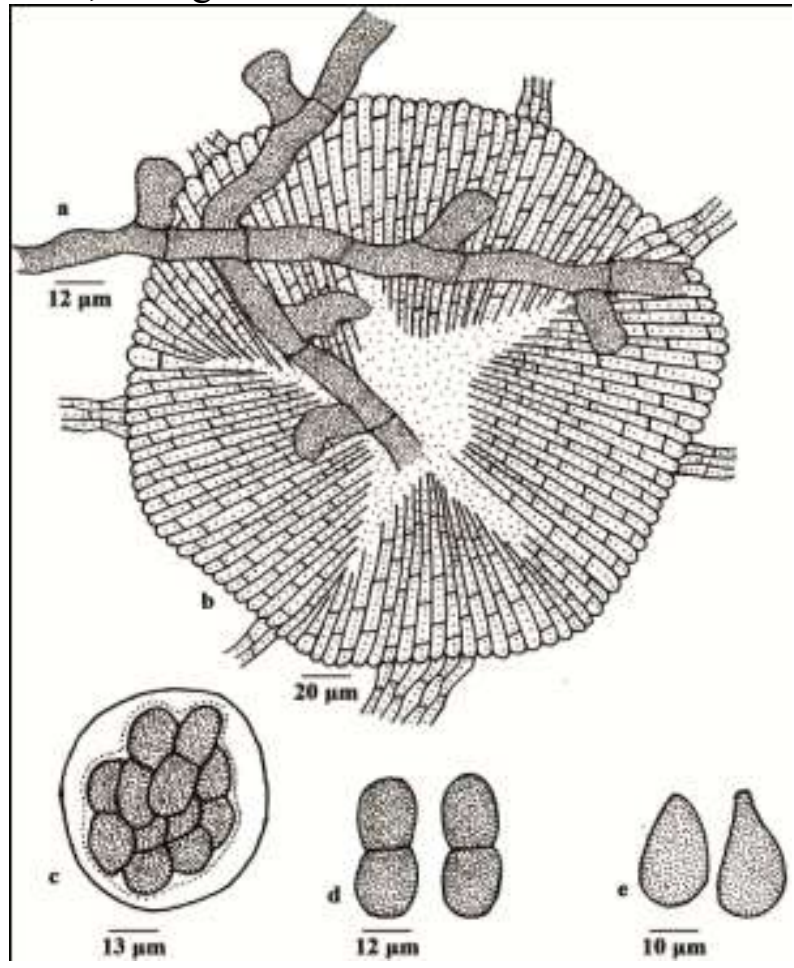

Figure 142 - Asterina meliosmae-simplicifoliae

Asterina meliosmae- simplicifoliae is close to Asterina meliosmaticola Petrak \& Cifferi, in having cylindrical appressoria. However, Asterina meliosmae- simplicifoliae differs from it in having straight, entire to 
lobate appressoria. It can also be compared with Asterina sabiacearum Hosag. \& Goos known from the Western Ghats of Peninsular India. However, Asterina meliosmae-simplicifoliae differs from it in having cylindrical appressoria in contrast to mammiform.

Distribution: Karnataka, Kerala.

Asterina memecylonis Ryan, Mem. Dept. Agric. India 15: 105, 1921; Hosag., Zoos' Print J. 19: 1386, 2004; Hosag., H. Biju \& Appaiah, J. Mycopathol. Res. 43:204, 2005; Hosag., Zoos' Print J. 21: 2328, 2006; Hosag., Chandraprabha \& Agarwal, Asterinales of Kerala, p. 116, 2011. (Fig. 143)

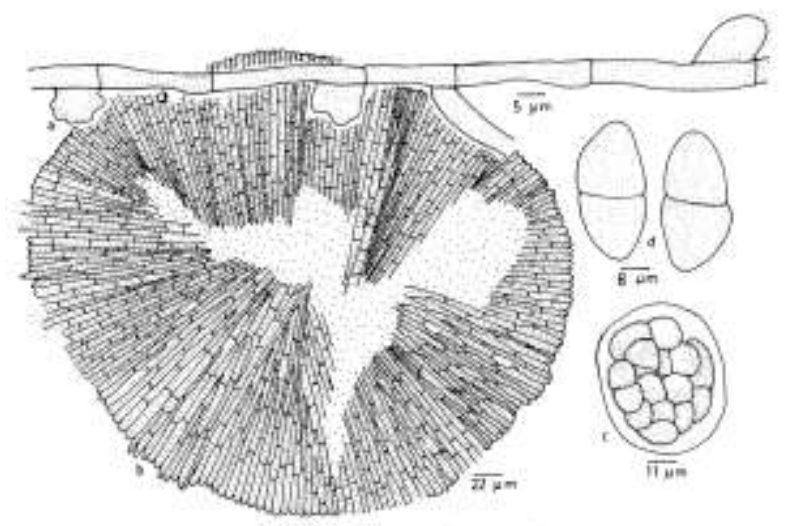

Figure 143 - Asterina memecylonis

Colonies amphigenous, subdense to dense, up to $2 \mathrm{~mm}$ in diameter, confluent. Hyphae straight, branching alternate, opposite to irregular at acute angles, loosely to closely reticulate, cells 28-32 × 4-7 $\mu \mathrm{m}$. Appressoria scattered, alternate, unicellular, broad based, mammiform, globose, entire, angular, crenately lobate to slightly lobate, $11-13 \times 11-15 \mu \mathrm{m}$. Thyriothecia scattered, orbicular, up to $441 \mu \mathrm{m}$ in diameter; crenate at margin, irregularly dehisce at the centre; asci few, globose to ovate, octosporous, 56-62 $\mu \mathrm{m}$ in diameter; ascospores brown, conglobate, uniseptate , constricted at the septum, taper at both the ends, 38-42 × 14$16 \mu \mathrm{m}$.

Material examined: Kerala, Thiruvananthapuram, Palode, TBGRI campus, on leaves of Memecylon edule Roxb. (Melastomataceae), 12 May 2009, A. Sabeena \& M.C. Riju TBGT 5309; on leaves of Memecylon sylvaticum Thwaites, 24 April
2002, H. Biju HCIO 44585, TBGT 872; Memecylon sp., 15 November 2003, V.B. Hosagoudar \& al. HCIO 45684, TBGT 1431; Wyanadu, Pulpally, 20 September 2008, M. Harish \& P.J. Robin HCIO 49629, TBGT 3871; TBGRI campus, September 19, 2008, K. Anilkumar TBGT 4519; Palghat, Silent valley National park, 3 March 2009, V.B. Hosagoudar TBGT 4517; 4 August 2008, M.C. Riju \& al. TBGT 5033; Kannur, Neeliyar Kottam, Mangattu Paramba, 26 December 2010, Libina TBGT 5189; Karnataka, Coorg, Hakathoor, 26 November 2008, V.B. Hosagoudar \& al. HCIO 49919, TBGT 4071; Hoddur,Devarakadu, 4 December2009, Jagat Thimmaiah TBGT 5468; Maharashtra, Satara, Kas lake, 2 January 2009, V.B. Hosagoudar \& al. TBGT 5091.

The present collection matches well with the species assigned to it except larger thyriothecia.

This species was known from Karwar, Karnataka (Ryan, 1928).

\section{Distribution: Kerala, Karnataka, Maharashtra}

Asterina mezonevronis V.B. Hosagoudar and C. Jagath Thimmaiah, sp. nov.

(Fig. 144)

MycoBank 101008

Etymology: Named after the host genus

Colonies epiphyllous, dense, up to 4 $\mathrm{mm}$ in diameter. Hyphae straight to substraight, branching alternate to opposite at acute to subacute angles, loosely reticulate, cells 13-22 $\times$ 4-6 $\mu \mathrm{m}$. Appressoria about $80 \%$ opposite, subopposite to alternate, rarely two borne from the same place from a cell, antrorse, subantrorse to retrorse, straight to slightly curved, 9-15 $\mu \mathrm{m}$ long; stalk cells cylindrical, 2$4 \mu \mathrm{m}$ long; head cells ovate, cylindrical, entire, lobate, angular, 7-12 × 4-7 $\mu \mathrm{m}$. Thyriothecia scattered, orbicular, irregularly dehisced at the centre, up to $130 \mu \mathrm{m}$ in diam., margin mostly crenate; asci few, globose, up to $25 \mu \mathrm{m}$ in diam.; ascospores few, brown, conglobate, uniseptate, constricted at the septum, 23-26 $\times$ $1013 \mu \mathrm{m}$, wall ciliated. Pycnothyria numerous, 
similar but smaller than the thyriothecia; pycnothyriospores unicellular, globose, ovate, pyriform, often apiculate, $16-20 \times 12-14 \mu \mathrm{m}$.

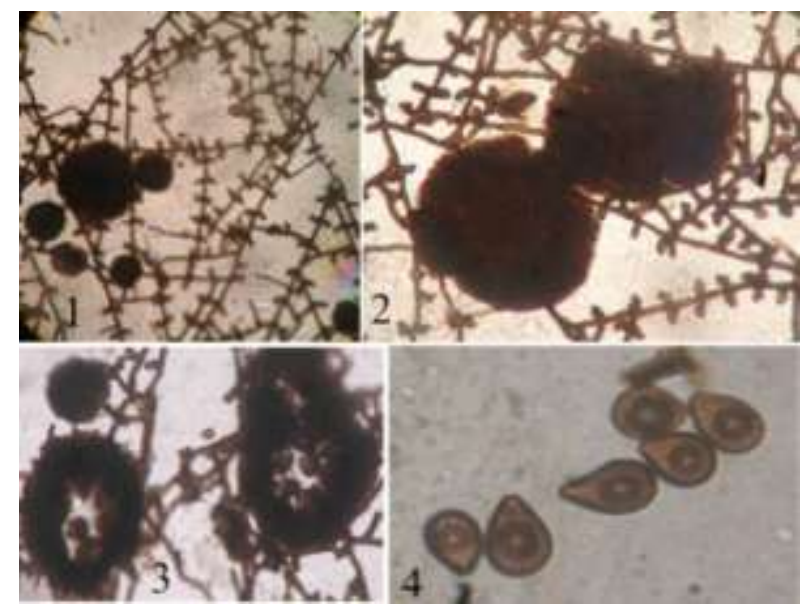

Figure 144 - Asterina mezonevronis

1. Mycelial colony, 2.Thyriothecia, 3.Stellately dehisced pycnothyria, 4. Pycnothyriospores

Material examined: Karnataka, Kodagu, Madikeri, Bharatiya Vidya Bhavan Kendriya Vidyalaya, on leaves of Mezonevron cucullatum (Roxb.) Wight \& Arn. (Caesalpiniaceae), 21 November 2009, C. Jagath Thimmaiah TBGT 5744.

Opposite appressoria (80\%) with entire to sublobate head cells distinguishes this species from rest known on the members of Caesalpiniaceae (Hosagoudar \& Abraham, 2000). Ascospores were few. Further, there were aerial whip like hyphae but could not confirm the intercalary appressoria to place it in Beemamyces.

Distriburion: Karnataka

Asterina micheliifolia Hosag. \& Riju, J. Threatened Taxa 3: 1942, 2011.

(Figs 145-146)

Colonies hypophyllous, thin, up to 2 $\mathrm{mm}$ in diameter, confluent. Hyphae flexuous to crooked, branching irregular at acute to wide angles, forming closely reticulated rings, cells 12-40 $\times$ 3-5 $\mu \mathrm{m}$. Appressoria scattered, unicellular, opposite, alternate, unilateral, antrorse to retrorse, globose to cylindrical, entire, 5-18 $\times 5-8 \mu \mathrm{m}$. Pycnothyria scattered, orbicular, up to $58 \mu \mathrm{m}$ in diameter, stellately dehisced and widely opened at the centre; pycnothyriospores globose, clavate, $15-20 \mu \mathrm{m}$, wall smooth. Thyriothecia scattered, orbicular, up to $85 \mu \mathrm{m}$ in diameter, stellately dehisced and widely opened at the centre by exposing asci; asci globose to ovate, $37-45 \mu \mathrm{m}$ in diameter; ascospores brown, uniseptate, constricted at the septum, $22-25 \times 10-13 \mu \mathrm{m}$, wall smooth.

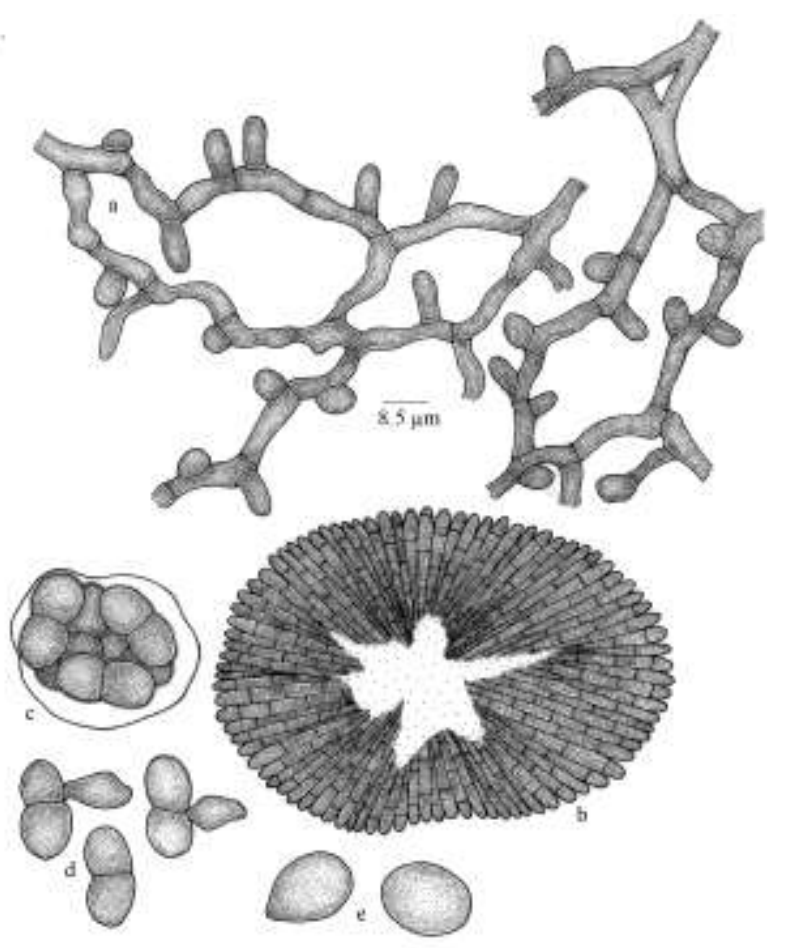

Figure 145. Asterina micheliifolia

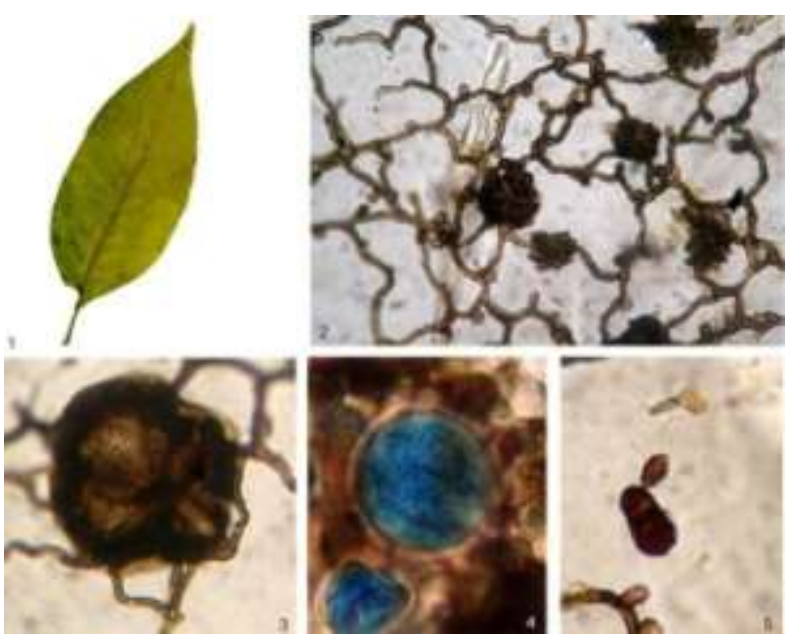

Figure 146 - Asterina micheliifolia

1. Infected leaf, 2. Colony with thyriothecia, 3. Thyriothecium, 4. Asci. 5. Germinating ascospore

Material examined: Kerala, Wayanad, Padinharathara, Chennalode, on leaves of Michelia chempaka L. (Magnoliaceae), 20 September 2008, M. C. Riju HCIO 49111 
(holotype), TBGT 3366a (isotype); HCIO 49112a, TBGT 3367a; HCIO 49113a, TBGT 3368 a; HCIO 49114a, TBGT 3369a; HCIO 49115a, TBGT 3370a.

This species differs from Asterina micheliae Hansf. in having typically thyriothecium like fruiting bodies and differs from $A$. micheligena in having straight mycelium and larger ascospores.

Distribution: Kerala.

Asterina micheliigena Hosag. \& Riju, J. Threatened Taxa 3: 1944, 2011.

(Figs 147-148)

Colonies epiphyllous, dense, up to 3 $\mathrm{mm}$ in diameter, confluent and often trait along the major veins of the upper surface of the leaves. Hyphae substraight to flexuous, branching opposite, alternate to irregular at acute to wide angles, loosely to closely reticulate, cells 9-24 × 4-6 $\mu \mathrm{m}$. Appressoria scattered, unicellular, opposite, alternate, unilateral, globose, entire, mammiform, 4-7 $\times$ 4-9 $\mu \mathrm{m}$. Pycnothyria scattered, orbicular, up to $75 \mu \mathrm{m}$ in diameter, stellately dehisced and widely opened at the centre;

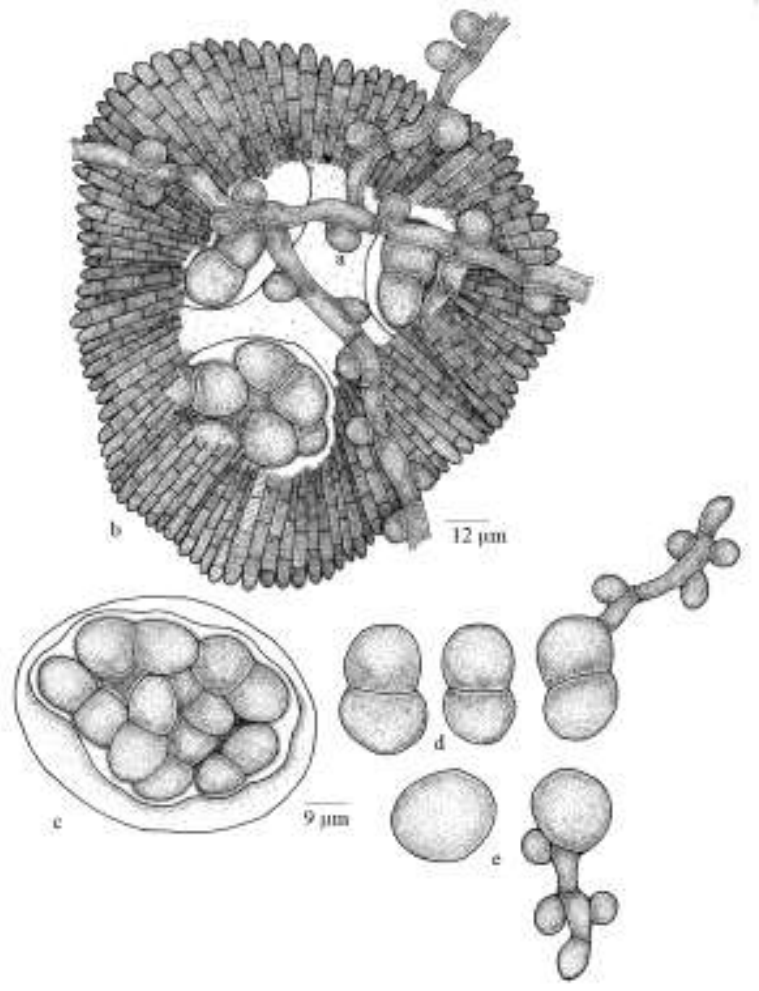

Figure 147. Asterina micheliigena pycnothyriospores globose to slightly ovate, 17-25 $\mu \mathrm{m}$ in diameter, wall smooth. Thyriothecia scattered, orbicular, up to $188 \mu \mathrm{m}$ in diameter, stellately dehisced and widely opened at the centre by exposing asci; asci globose to ovate, up to $63 \mu \mathrm{m}$ in diameter; ascospores brown, uniseptate, constricted at the septum, 25-33 × 15-18 $\mu \mathrm{m}$, wall smooth.

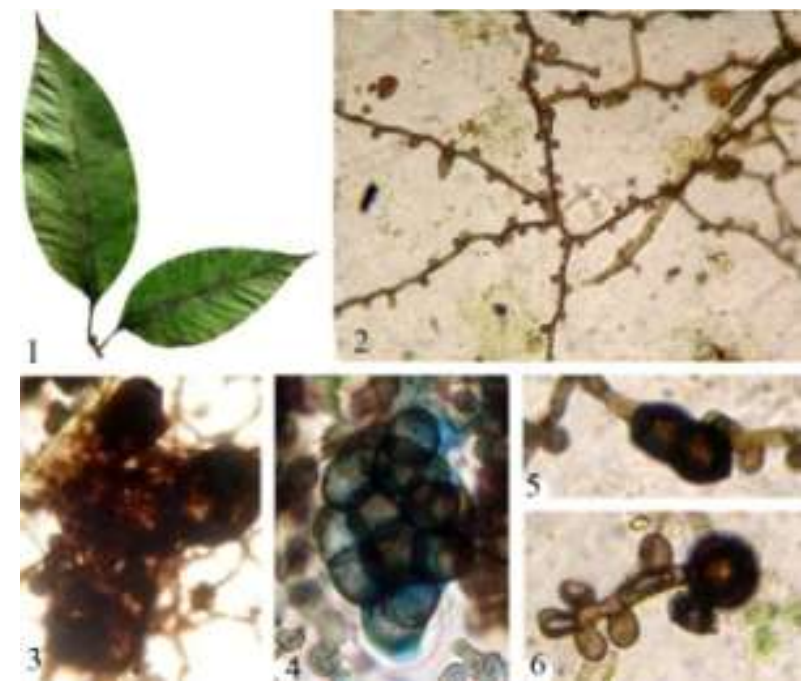

Figure 148 - Asterina micheliigena

1. Infected leaves, 2. Colony with appressoriate mycelium, 3. Thyriothecia, 4. Asci, 5. Germinating ascospore, 6. Germinating pycnothyriospore

Material examined: Kerala, Wayanad, Padinharathara, Chennalode, on leaves of Michelia chempaka L. (Magnoliaceae), 20 September 2008, M. C. Riju HCIO 49111b (holotype), TBGT 3366b (isotype); HCIO 49112b, TBGT 3367b; HCIO 49113b, TBGT 3368b; HCIO 49114b, TBGT 3369b; HCIO 49115b, TBGT 3370b.

Distribution: Kerala.

Asterina microtropidicola Hosag. \& C.K. Biju in Hosag.,C.K. Biju, Abraham \& Agarwal, Indian Phytopath. 55: 499, 2002; Hosag., Zoos' Print J. 21: 2328, 2006; Hosag., Chandraprabha \& Agarwal, Asterinales of Kerala, p. 118, 2011. (Fig. 149)

Colonies amphigenous, dense, velvety, up to $5 \mathrm{~mm}$ in diameter, rarely confluent. Hyphae straight, rarely substraight to slightly 
flexuous, branching irregular at acute angles, loosely to closely reticulate, cells $12-20 \times 3-5$ $\mu \mathrm{m}$. Appressoria unicellular, alternate, about $30 \%$ opposite, straight to slightly curved, conoid, attenuated and broadly rounded at the apex, entire, $11-20 \times 6-8 \mu \mathrm{m}$. Thyriothecia closely scattered, often connate, orbicular, up to $125 \mu \mathrm{m}$ in diameter, mostly crenate at the margin, stellately dehisced and widely opened at the centre; asci many, octosporous, globose, up to $40 \mu \mathrm{m}$ in diameter; ascospores oblong, conglobate, uniseptate, deeply constricted at the septum, 30-34 × 14-16 $\mu \mathrm{m}$, wall smooth.

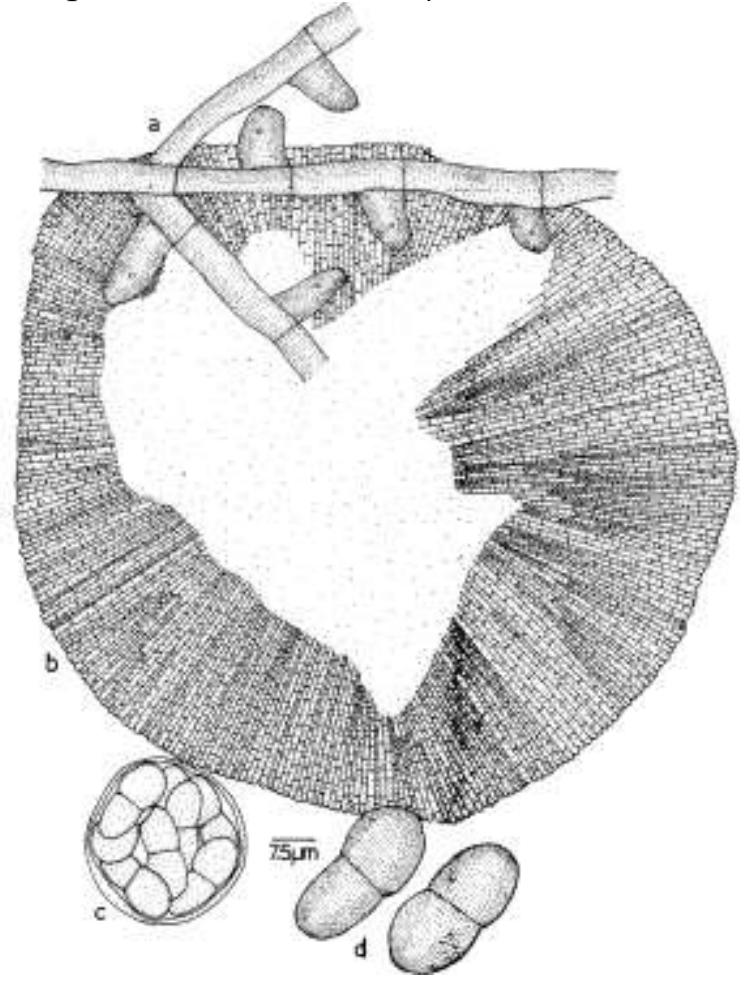

Figure 149 - Asterina microtropidicola

Material examined: Kerala, Wayanad, Thirunelli shola forest, on leaves of Microtropis latifolia Wight \& Lawson (Celastraceae), 12 August 1999, C.K. Biju HCIO 43712 (holotype), TBGT 370 (isotype); Palghat, Silent valley, Sairandhri, on leaves of Pleurostylia sp. (Celastraceae), 13 December 2003. V.B. Hosagoudar \& al. HCIO 46379, TBGT 2025.

Asterina microtropidis Hosag. et al. is known on Microtropis ovalifolia from the Western Ghats of peninsular India (Hosagoudar et al. 1996). However, Asterina microtropidicola differs from it in having alternate and opposite, conoid and straight appressoria.

Distribution: Kerala.

Asterina microtropidis Hosag., Balakr. \& Goos, Mycotaxon 59:181, 1996; Hosag. \& Abraham, J. Econ. Taxon. Bot. 4: 564, 2000; Hosag., Zoos' Print J. 18: 1282, 2003; Hosag., Chandraprabha \& Agarwal, Asterinales of Kerala, p. 119, 2011.

(Fig. 150)

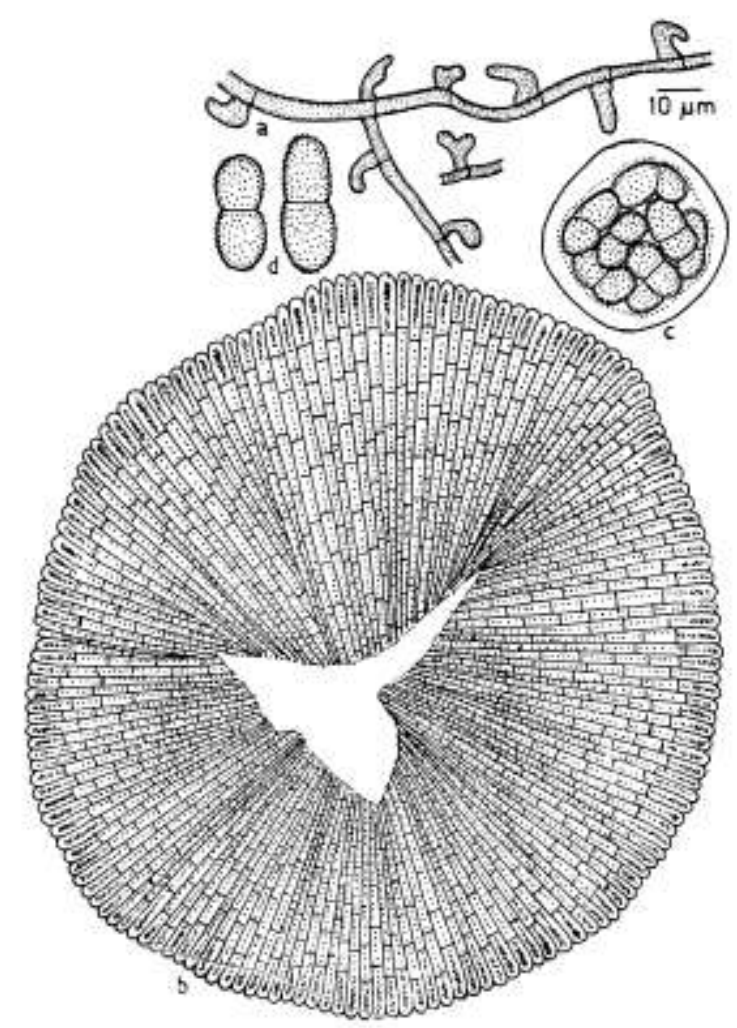

Figure 150 - Asterina microtropidis

Colonies amphigenous, dense, velvety, up to $2 \mathrm{~mm}$ in diameter, confluent. Hyphae brown, septate, substraight to crooked, branching opposite to irregular at acute to wide angles, closely reticulate, cells 21-28 × 4-7 $\mu \mathrm{m}$. Appressoria unicellular, alternate to unilateral, cylindrical, straight to predominantly and variously curved, broadly rounded at the apex, $12-16 \times 3-5 \mu \mathrm{m}$. Thyriothecia scattered to grouped, orbicular, up to $172 \mu \mathrm{m}$ in diameter, dehiscing stellately at the center, margin crenate; asci many globose to ovate, eight spored, 55-62 $\times$ 43-47 $\mu \mathrm{m}$; ascospores conglobate, initially hyaline, dark brown at maturity, 1-septate, deeply constricted at the 
septum, upper cell slightly larger than the lower $24-28 \times 12-15 \mu \mathrm{m}$, wall echinulate.

Material examined: Kerala, Idukki, Painavu, on leaves of Microtropis ovalifolia Wight (Celastraceae), 24 October 1984, V.B. Hosagoudar HCIO 30970 (holotype).

This species is close to Asterina dissiliens (Sydow) Doidge but differs from it in having cylindrical and longer appressoria, smaller thyriothecia, and larger asci and ascospores. It also differs from $A$. dissiliens (Sydow) Doidge var. senegalensis Doidge in having cylindrical appressoria and larger ascospores.

Distribution: Kerala.

Asterina miliusae Hosag. \& C.K. Biju in Hosag., C.K. Biju \& Abraham, J. Econ. Taxon. Bot. 28: 177, 2004; Hosag., Chandraprabha \& Agarwal, Asterinales of Kerala, p. 120, 2011.

(Fig. 151)

Colonies epiphyllous, dense, up to 3 $\mathrm{mm}$ in diameter, rarely confluent. Hyphae straight, branching irregular at acute to wide angles, loosely to closely reticulate, cells $17-26$ $\times$ 5-7 $\mu \mathrm{m}$. Appressoria alternate, about 30\% opposite, unicellular, ovate, taper and broadly rounded towards apex, entire to rarely slightly lobate, 9-16 × 6-8 $\mu \mathrm{m}$. Thyriothecia scattered but mostly connate, orbicular, up to $200 \mu \mathrm{m}$ in diameter, stellately dehisced at the center, margin fimbriate, fringed hyphae crooked, compact; asci globose, octosporous, up to 40 $\mu \mathrm{m}$ in diameter; ascospores conglobate, two celled, uniseptate, constricted at the septum, brown at maturity, $24-32 \times 12-16 \mu \mathrm{m}$, wall tubercled to slightly echinulate.

Material examined: Kerala, Idukki, Rajamala, on leaves of Miliusa sp. (Annonaceae), 14 September 1999, C.K. Biju HCIO 43456 (holotype), TBGT 273 (isotype).

Based on the morphology and measurements the present collection is close to Asterina clemensiae Petrak known on Polyalthia sp. from Philippines (Sydow \&
Petrak, 1931). However, Asterina miliusae differs from it in having $30 \%$ opposite and longer appressoria.

Distribution: Kerala.

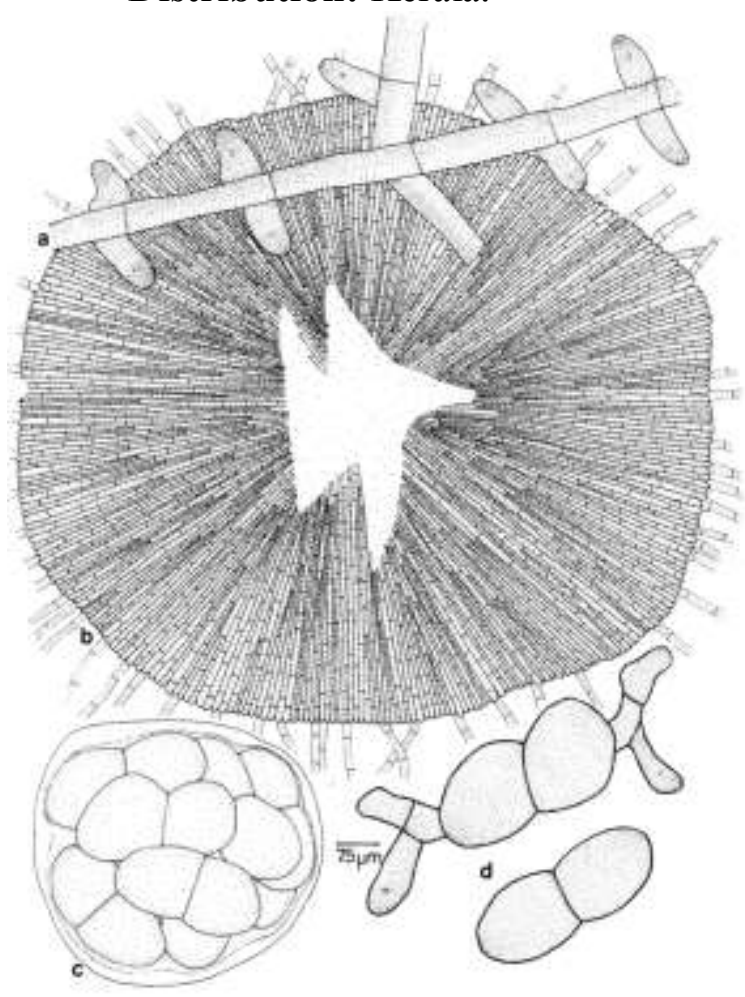

Figure 151 - Asterina miliusae

Asterina millettiae Hosag., Archana, Kamar. \& Jose in Hosag., Chandraprabha \& Agarwal, Asterinales of Kerala, p. 121, 2011. (Fig. 152)

Colonies hypophyllous, crustose, dense, up to $4 \mathrm{~mm}$ in diameter, confluent. Hyphae crooked, branching irregular at acute angles, closely reticulate, cells $11-20 \times 3-5 \mu \mathrm{m}$. Appressoria unicellular, alternate, about $1 \%$ opposite, ovate, globose, entire, angular, 7-9 $\times$ 4-7 $\mu \mathrm{m}$. Thyriothecia scattered, orbicular, up to $100 \mu \mathrm{m}$ in diameter, stellately dehisced at the center, margin crenate to rarely fimbriate; asci few, globose, octosporous, up to $33 \mu \mathrm{m}$ in diameter; ascospores brown, conglobate, 1septate, constricted at the septum, 18-22 × 9-11 $\mu \mathrm{m}$, wall smooth.

Material examined: Kerala, Idukki, on leaves of Millettia rubiginosa Wight \& Arn. (Fabaceae), 6 September 2001, M. Kamarudeen \& P. A. Jose HCIO 47434 (holotype), TBGT 2472 (isotype); Thiruvananthapuram, Peppara 
Wildlife Sanctuary, on leaves of Millettia sp., Pandipathal, Athirumala, 29 February 2008, Jacob Thomas \& al. HCIO 48835 (holotype), TBGT 3211(isotype).

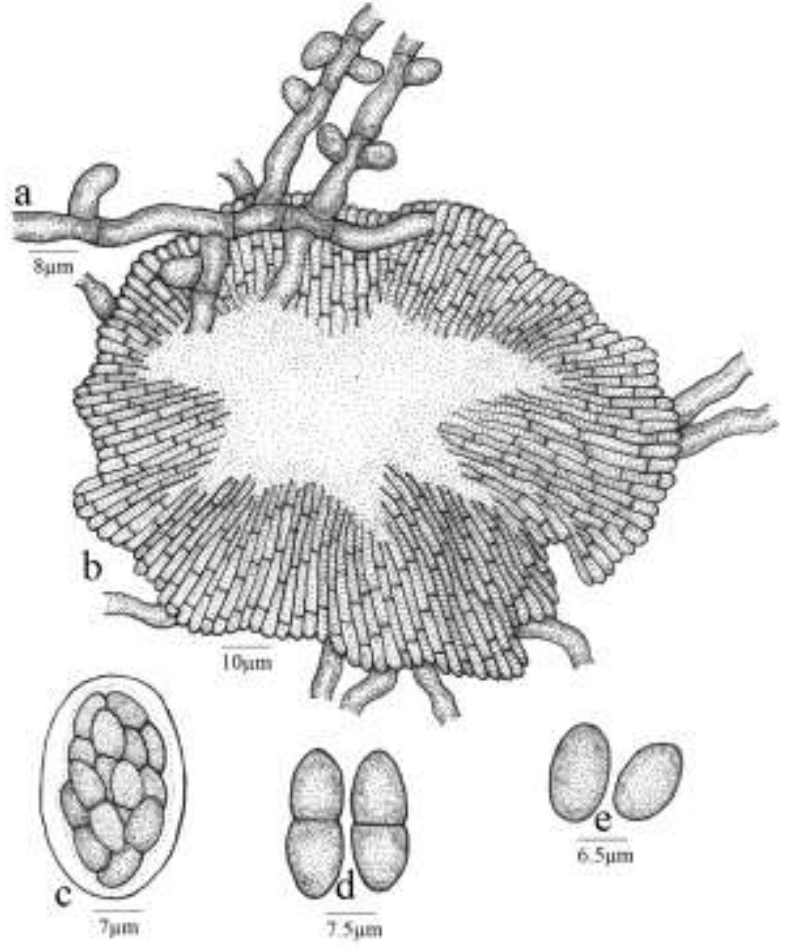

Figure 152 - Asterina millettiae

This is the only species known on this host genus (Hosagoudar \& Abraham, 2000). Based on the host specificity, it has been accommodated into a new species.

Distribution: Kerala.

Asterina mimusopsidicola Hosag., Sabeena \& Agarwal, Indian Phytopath. 62: 229, 2009; Hosag., Chandraprabha \& Agarwal, Asterinales of Kerala, p. 122, 2011.

(Figs 153-154)

Colonies mostly hypophyllous, subdense, up to $3 \mathrm{~mm}$ in diameter, confluent. Hyphae flexuous, branching opposite, alternate to unilateral at acute angles, loosely to closely reticulate, cells 22-35x4-6 $\mu \mathrm{m}$. Appressoria alternate, unilateral, antrorse, subantrorse to closely appressed to the hyphae, two celled,1122 long; stalk cells cylindrical to cuneate,4-9 long; head cell ovoid, oblong, cylindrical to linear, entire, 6-13 $\times 4-9 \mu \mathrm{m}$. Thyriothecia scattered to connate, orbicular, up to $500 \mu \mathrm{m}$ in diameter, stellately dehisced or dissolved at the centre; margin crenate to fimbriate, fringed hyphae few to numerous, straight to flexuous, compact to loosely bound, devoid of appressoria; asci globose, octosporous, up to 55 $\mu \mathrm{m}$ in diam.; ascospores conglobate, brown, uniseptate, constricted at the septum, 20-22 $\times$ 11-13 $\mu \mathrm{m}$, wall smooth.

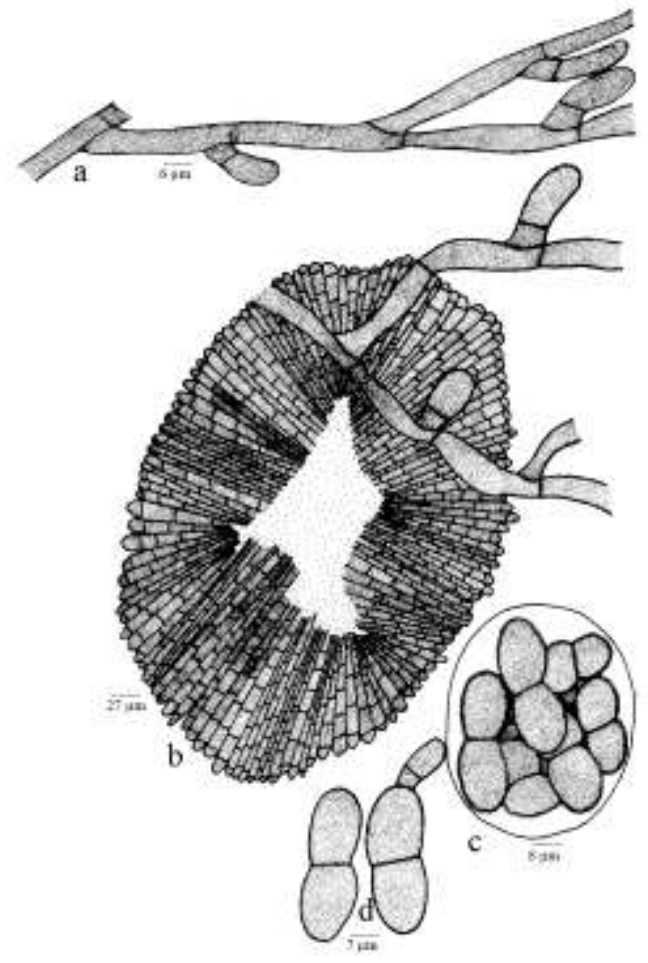

Figure 153 - Asterina mimusopsidicola

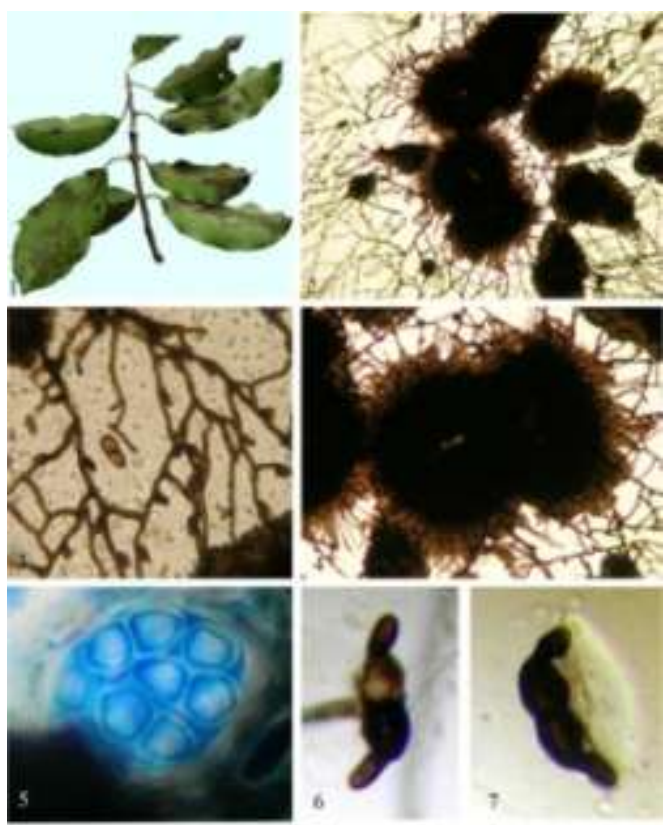

Figure 154 - Asterina mimusopsidicola

Infected leaves, 2. Colony with thyriothecia, 3. Closely appressed appressoria, 4. Thyriothecia, 5. Ascus, $6 \& 7$. Germinating ascospores 
Material examined: Kerala, Thiruvananthapuram, Palode, TBGRI Campus, on leaves of Mimusops elengi L. (Sapotaceae), 11 June 2007, A. Sabeena HCIO 48271 (holotype), TBGT 3020 (isotype); HCIO 48272, TBGT 3021.

Asterina mimusopsidis Hansf. is known on Mimusopsis welwitsctus from Congo Belge (Hansford, 1957). However, strictly hypophyllous colonies and two celled appressoria make the present species different from it.

Distribution: Kerala.

Asterina morellae Hosag.,C.K. Biju \& Abraham, Indian Phytopath. 54: 137, 2001; Hosag., Zoos' Print J. 21: 2328, 2006; Hosag., Chandraprabha \& Agarwal, Asterinales of Kerala, p. 123, 2011.

(Fig. 155)

Colonies amphigenous, mostly epiphyllous, dense, spreading, up to $5 \mathrm{~mm}$ in diameter, confluent. Hyphae straight to flexuous, branching opposite to irregular at acute angles, loosely to closely reticulate, cells $12-30 \times 2-4 \mu \mathrm{m}$. Appressoria alternate to unilateral, unicellular, mammiform, broad based, entire, angular, 2-3 times sublobate to lobate, $6-8 \times 5-8 \mu \mathrm{m}$. Thyriothecia scattered, orbicular, up to $175 \mu \mathrm{m}$ in diameter, stellately dehisced at the center, margin crenate; asci many, globose, octosporous, 22-28 $\mu \mathrm{m}$ in diameter; ascospores oblong, conglobate, brown, uniseptate, constricted, 21-23 × 11-13 $\mu \mathrm{m}$, wall echinulate. Pycnothyria similar to thyriothecia, smaller; Pycnothyriospores oval to oblong, brown, unicellular, 15-18 × 7-10 $\mu \mathrm{m}$, wall smooth.

Material examined: Kerala, Thiruvananthapuram, in the forest near Bonacaud, on leaves of Garcinia morella (Gaertn.) Descr. (Clusiaceae), 11 March 1997, V.B. Hosagoudar HCIO 42953 (holotype), TBGT 240 (isotype); Chemunji, 3 January 2001, G. Rajkumar HCIO 44860, TBGT 1088; 19 May 1999, C.K. Biju HCIO 45209, TBGT 1245; Bonacaud, 8 March 2003, G. Rajkumar HCIO 45125, TBGT 1180.

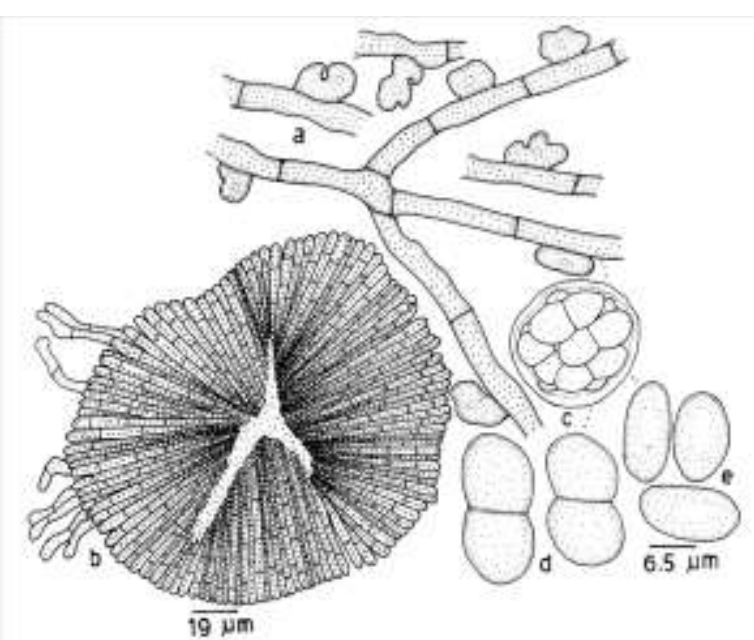

Figure 155 -Asterina morellae

Asterina garciniae Hansf. and $A$. garciniicola Ouyang \& Song are known on the host genus Garcinia from India and China. The present collection is close to the former species in having epiphyllous colonies, broad based appressoria. However, the new species differs from it in having sublobate to lobate appressoria and strongly echinulate ascospores.

Distribution: Kerala.

\section{Asterina munnarensis nom. nov.}

Asterina lauracearum Hosag. \& C.K. Biju, Indian Phytopath. 57: 114, 2004; Hosag., C.K. Biju \& Abraham, J. Mycopathol. Res. 40:195, 2002 (non. Song et al. 2003); Hosag., Chandraprabha \& Agarwal, Asterinales of Kerala, p. 101, 2011.

(Fig. 156)

MycoBank 101024

Colonies hypophyllous, thin to subdense, spreading, up to $10 \mathrm{~mm}$ in diameter, confluent. Hyphae straight to substraight, branching opposite to irregular at wide angles, loosely reticulate, cells $12-20 \times 2-3 \mu \mathrm{m}$. Appressoria alternate, not numerous, globose, straight to curved, entire, angular to 2-3 times sublobate, $8-12 \times 7-10 \mu \mathrm{m}$. Thyriothecia scattered, rarely connate, orbicular, up to $65 \mu \mathrm{m}$ in diameter, stellately dehisced at the centre and the central upper portion dissolved by exposing golden yellow content, margin crenate, rarely fimbriate; asci globose, octosporous, up to 30 $\mu \mathrm{m}$ in diameter; ascospores conglobate, oblong, 
brown, 1-septate, deeply constricted, 17-20 $\times$ 7-8 $\mu \mathrm{m}$, wall tuberculate.

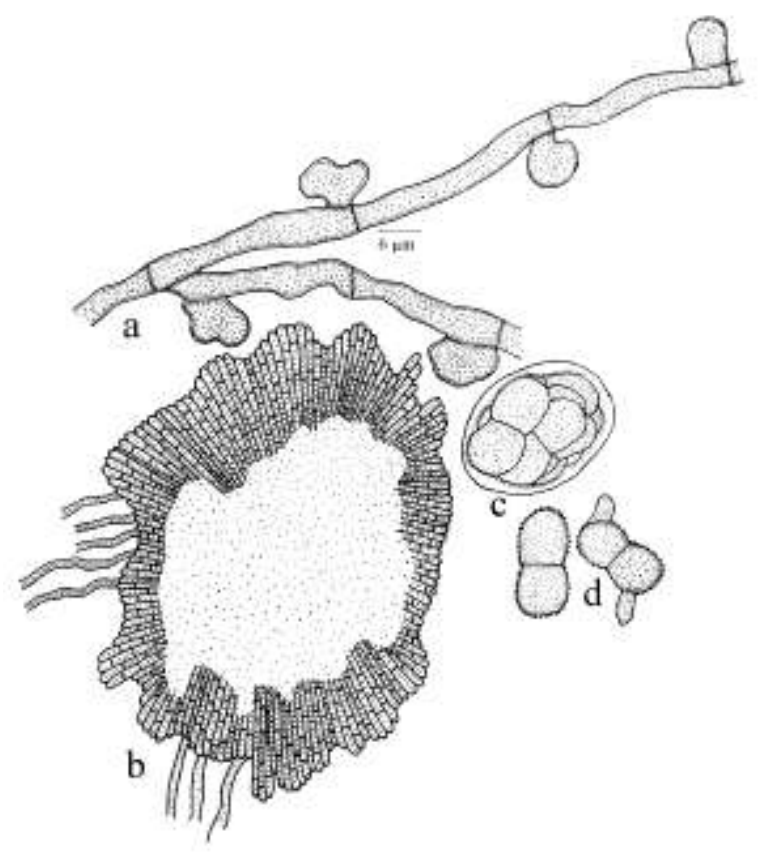

Figure 156 - Asterina munnarensis

Material examined: Kerala, Idukki, Mannavan shola, on leaves of Cinnamomum sp. (Lauraceae), 24 September 1998, C.K. Biju HCIO 42937 (holotype), TBGT 216 (isotype).

Asterina cinnamomi Sydow and $A$. cinnamomicola Hansf. are known on the host genus Cinnamomum from Philippines and Sri Lanka. The present species differs from the former in having smaller thyriothecia and ascospores. It differs from the latter species in having only alternate appressoria, smaller ascospores with tubercled wall in contrast to echinulate.

Distribution: Kerala.

Asterina murrayae Hansf. Proc. Linn. Soc. London 158: 45, 1947; Hosag. \& Abraham, J. Econ. Taxon. Bot. 4: 581, 2000; Hosag. Zoos' Print J. 19: 1387, 2004; Hosag., Chandraprabha \& Agarwal, Asterinales of Kerala, p. 125, 2011. (Fig. 157)

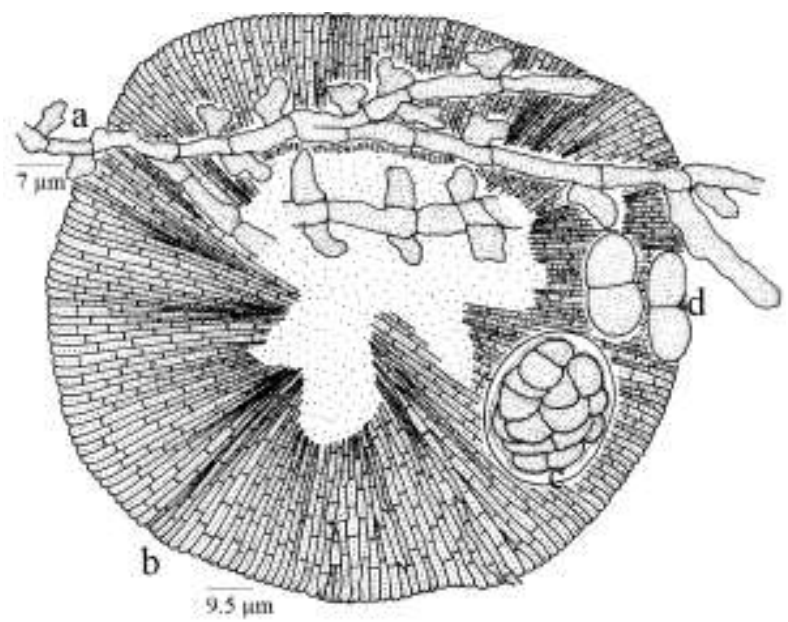

Figure 157 - Asterina murrayae

Colonies amphigenous, mostly epiphyllous, dense, up to $2 \mathrm{~mm}$ in diameter, confluent. Hyphae straight, substraight to crooked, branching alternate to irregular at acute angles, loosely to closely reticulate, cells 12-16 × 4-7 $\mu \mathrm{m}$. Appressoria alternate, about $40 \%$ opposite, unicellular, globose, ovate, oblong, cylindrical, entire, sinuately lobate to irregularly and sharply lobate, 5-13 × 4-7 $\mu \mathrm{m}$. Thyriothecia scattered to loosely grouped, orbicular, up to $175 \mu \mathrm{m}$ in diameter, stellately dehisced at the centre, margin mostly crenate to rarely fimbriate and the fringed hyphae very small; asci few, ovate to globose, octosporous, 28-35 $\mu \mathrm{m}$ in diameter; ascospores oblong, conglobate, brown, uniseptate, slightly constricted at the septum, $20-23 \times 8-10 \mu \mathrm{m}$.

Material examined: Kerala, Thiruvananthapuram, Palode, in the Medicinal plant garden, TBGRI, on leaves of Murraya koenigii (Rutaceae), 17 January 2001, H. Biju HCIO 44512, TBGT 798.

This species was known on the same host from Sri Lanka (Hansford, 1947).

Distribution: Kerala.

Asterina murrayicola V.B. Hosagoudar \& A. Sabeena, sp. nov.

MycoBank 101009

(Fig. 158)

Etymology: Named after the host genus 


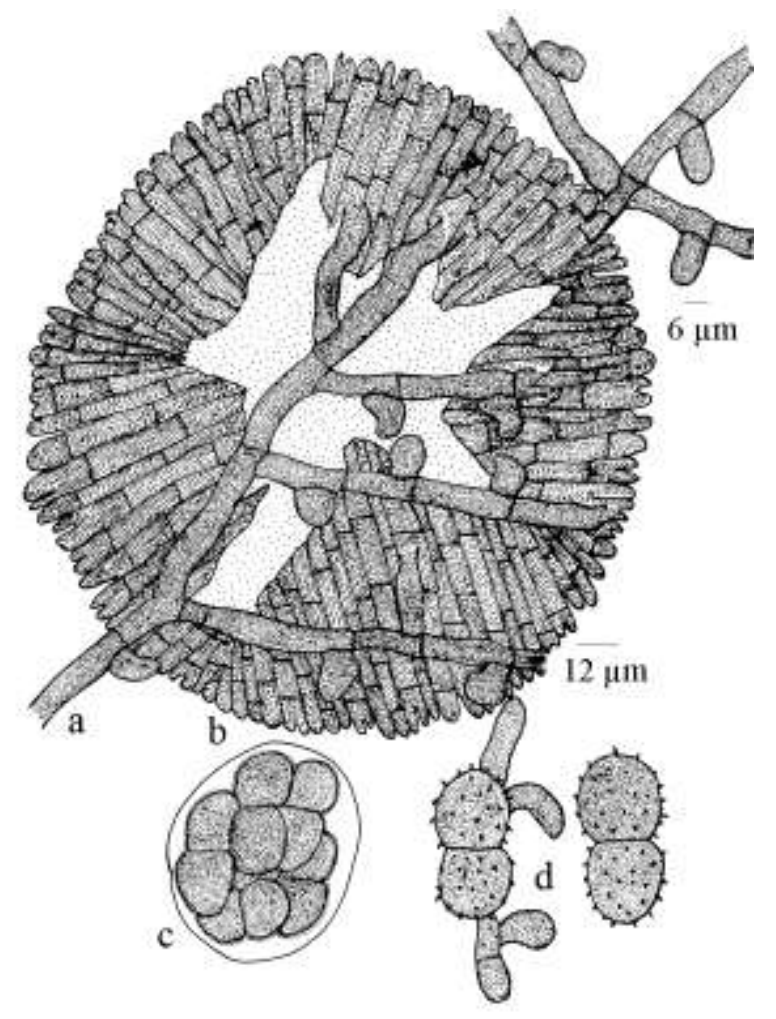

Figure 158 - Asterina murrayicola

Colonies amphigenous, subdense, crustose, up to $3 \mathrm{~mm}$ in diameter, confluent. Hyphae straight to substraight, branching opposite to unilateral at acute to wide angles, loosely reticulate, cells 20-25 $\times$ 4-5 $\mu \mathrm{m}$. Appressoria alternate to unilateral, unicellular, ovate, subglobose, entire, 7-12 × 5-10 $\mu \mathrm{m}$. Thyriothecia scattered, orbicular, up to $230 \mu \mathrm{m}$ in diameter, margin crenate to fimbriate, fringed hyphae flexuous, stellately dehisced at the centre; asci globose, octosporous, $25-30 \mu \mathrm{m}$ in diam.; ascospores, conglobate, 1-septate, constricted at the septum, $30-32 \times 12-15 \mu \mathrm{m}$, wall echinulate.

\section{Material examined: Kerala,} Pathanamthitta, Moozhiyar forest, on leaves of Murraya exotica (Rutaceae), 17 January 2007, M. Harish \& M.C. Riju TBGT 5740 (holotype).

Asterina murrayae Hansf. known on this host genus from Srilanka and India (Hansford, 1947; Hosag. \& Abraham, 2000). However, the present species differs from it in having ovate and entire appressoria in contrast to variously angular appressoria.

Distribution: Kerala
Asterina myristicae Hosag. \& Sabeena, J. Threatened Taxa 3: 2144, 1911.

(Fig. 159)

Colonies hypophyllous, thin, crustose, up to $3 \mathrm{~mm}$ in diameter, confluent. Hyphae substraight, flexuous to slightly crooked, branching opposite, alternate to irregular at acute to wide angles, loosely to closely reticulate, cells 12-29 × 3-5 $\mu \mathrm{m}$. Appressoria alternate, opposite, unilateral, antrorse, subantrorse to retrorse, 1-3 celled, straight, curved, flexuous to crooked, 9-35 $\mu \mathrm{m}$ long; stalk cells unicellular to 1-2 septate, straight, flexuous to crooked, 3-27 $\mu \mathrm{m}$ long; head cells ovate, oblong, straight to curved, entire to sublobate, $4-11 \times 3-8 \mu \mathrm{m}$. Thyriothecia scattered to grouped, orbicular to slightly ovate, up to $160 \mu \mathrm{m}$ in diameter, margin crenate, stellately dehisced at the centre; asci many, globose to ovate, octosporous, 19-40 $\times 16-25$ $\mu \mathrm{m}$; ascospores brown, oblong, conglobate, uniseptate , 16-21 × 8-10 $\mu \mathrm{m}$, wall smooth. Pycnothyriospores brown, pyriform, unicellular, $12-15 \times 6-10 \mu \mathrm{m}$.

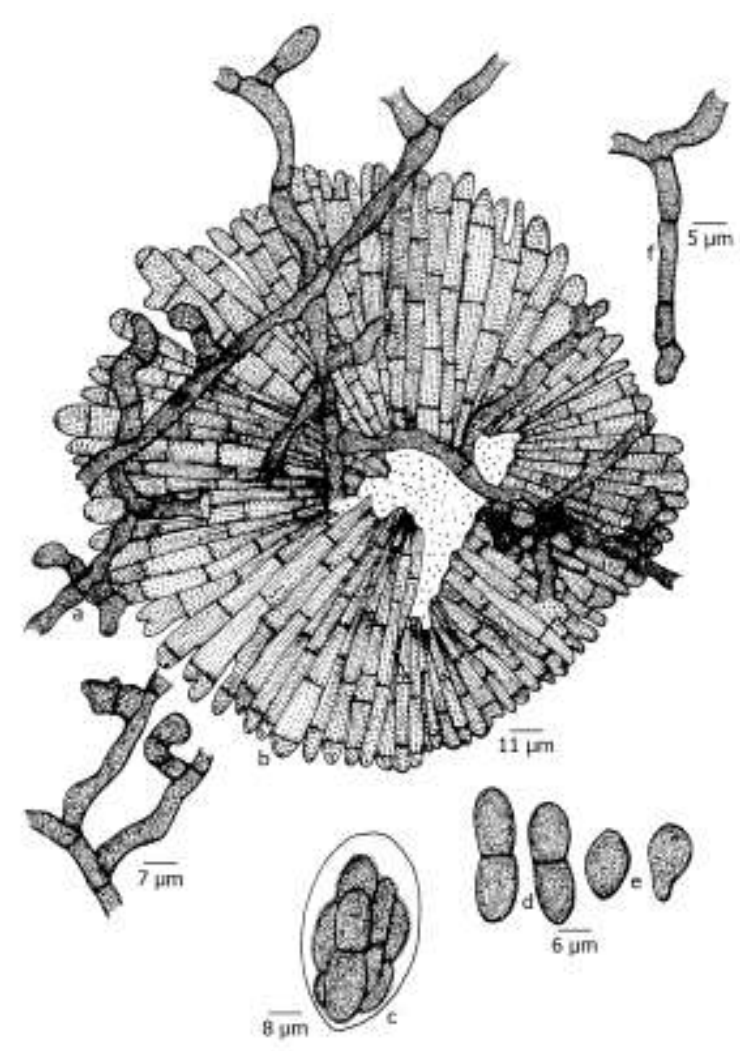

Figure 159 - Asterina myristicae

Material examined: Kerala, Thiruvananthapuram, Palode, TBGRI Campus, Arboretum, on leaves of Myristica sp. 
(Myristicaceae), 7 January 2008, K. Anilkumar TBGT 4614 (holotype). Part of the collection has been deposited in HCIO, New Delhi; on leaves of M. malabarica Lam., 16 May 2008, K. Anil Kumar TBGT 4610; 15 October 2008, K. Anil Kumar TBGT 4612; 10 April 2008, A. Sabeena \& M.C. Riju TBGT 4981, 4977; on leaves of Myristica sp., 19 September 2008, K. Anil Kumar TBGT 5245.

This species stands distinct from all other Asterina species known on the members of the Myristicaceae in having 1-3-celled appressoria.

\section{Distribution: Kerala}

Asterina myristicacearum Hosag. \& Sabeena, J. Threatened Taxa 3: 2144, 1911.

(Fig. 160)

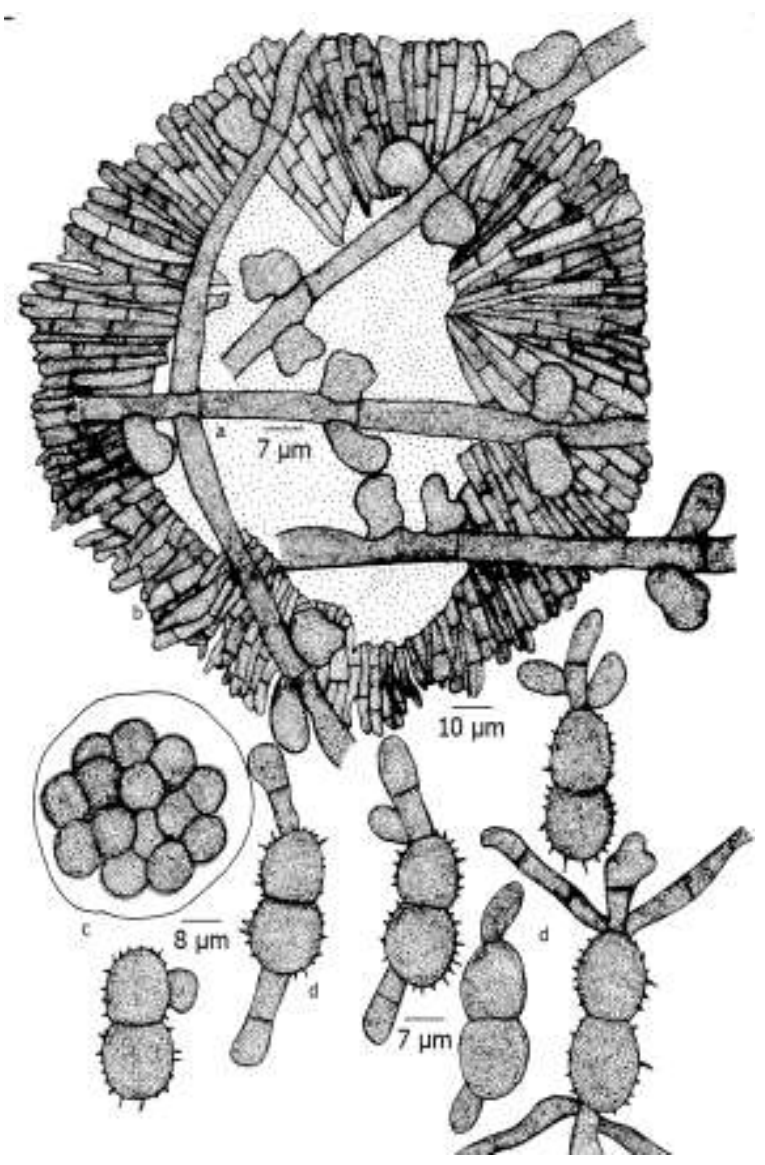

Figure 160 - Asterina myristicacearum

Colonies epiphyllous, subdense, up to 3 $\mathrm{mm}$ in diameter. Hyphae straight to substraight, branching opposite to unilateral at acute to wide angles, loosely reticulate, cells 17-47 × 2-5 $\mu \mathrm{m}$. Appressoria often crowded, alternate, opposite to subopposite, unicellular, often broad based, ovate, globose, entire, angular to sublobate, 7-
$15 \times 7-10 \mu \mathrm{m}$. Thyriothecia scattered to connate, ovate, up to $170 \mu \mathrm{m}$ in diam., margin crenate, stellately dehisced at the centre or the central portion dissolved by exposing the asci; asci globose, octosporous, 37-50 $\mu \mathrm{m}$ in diam.; ascospores conglobate, brown, uniseptate, constricted at the septum, $25-32 \times 12-17 \mu \mathrm{m}$, wall echinulate.

Material examined: Kerala, Thiruvananthapuram, Palode, TBGRI Campus, on leaves of Myristica malabarica Lam. (Myristicaceae), 30 April 2008, A. Sabeena \& M. C. Riju TBGT 553 (holotype), HCIO 44140 (isotype); 25 February 2009, A. Sabeena \& M.C. Riju TBGT 4608; April 30, 2008, A. Sabeena \& M.C. Riju TBGT 4979.

Crowded appressoria on this host distinguishes from Asterina knemae attenuatae Hosag. et al. (Hosagoudar \& Abraham, 2000). By adding these three taxa, the number of Asterina species known on Myristicaceae in the world is raised to eight and the key is provided here to facilitate their rapid identification.

\section{Distribution: Kerala}

Asterina myrsines Kar \& Matiy, Trans. Brit. Mycol. Soc. 54: 438, 1970.

Confined to the upper surface of the leaves, forming black, scattered, round thin patches, up to $4 \mathrm{~mm}$ diam. Sometimes all the patches coalesce and cover almost all the leaf surface; Mycelium superficial, densely reticulate, composed of dark brown, septate , branched straight hyphae, $6.6 \mu \mathrm{m}$ wide, (cells mostly 19-33 $\mu \mathrm{m}$ long) giving rise to opposite branches and appressoria at an acute angle. Appressoria usually opposite, rarely solitary, unicellular, entire, rounded at the apex, brown, straight or bent, 6.6-11.5 $\times 6.6 \mu \mathrm{m}$; Thyriothecia many, scattered or aggregated, orbicular, 150-280.5 $\mu \mathrm{m}$ diam., attached with free mycelium, stellately dehiscing at first, subsequently developing in to an aperture, dimidiate with radiate scutellum, dark brown, convex, hymenium simple, margin subcrenate; Asci many, rounded to oval, arranged towards the periphery of the fruit body, sessile, 8spored, 42.9-62.7 × 39.6-46.2 $\mu \mathrm{m}$, paraphysate; 
Ascospores crowded, oblong-elliptical, uniseptate, constricted at the septum, rounded at the ends, dark brown, smooth-walled, guttulate, 26.4.7-33 × 12-13.2 $\mu \mathrm{m}$.

Material examined: West Bengal, Darjelling, on leaves of Myrsine semiserrata Wall. var. subsinosa, Birch hill (2134 m.), 23 July 1966, IMI 134487( holotype).

Distribution: West Bengal.

Asterina mysorensis Hansf. Farlowia 3: 307, 1948.

Colonies amphigenous, black orbicular smooth, 2-3 $\mathrm{mm}$ in diam., or confluent dense in the centre. Mycelium of crooked dark brown hyphae 6-8 $\mu \mathrm{m}$ thick, the cells mostly 20-30 $\mu \mathrm{m}$ long, branching opposite or irregular at 45$90^{\circ}$, closely reticulate. Appressoria opposite or alternate, continuous, bluntly conoid to cylindrical with obtuse rounded apex straight or variously bent, $10-15 \times 5-8 \mu \mathrm{m}$. Thyriothecia densely scattered, but usually discrete, black, circular, convex, up to $130 \mu \mathrm{m}$ diam.; lower wall indistinct; upper wall of subopaque dark brown radiating hyphae 6-7 $\mu \mathrm{m}$ thick, the cells 6-10 $\mu \mathrm{m}$ long, margin not or only slightly fimbriate; the centre dehiscent by irregularly stellate fissures and soon falling away to expose the asci. Asci aparaphysate, subglobose, 8spored, sessile, about $30 \mu \mathrm{m}$ diam., Spores conglobate, oblong with rounded ends, subopaque dark brown, 1-septate, constricted, 26-32 $\times 14-17 \mu \mathrm{m}$, the surface finely and closely verruculose.

Karnataka, Nandi hills, on leaves of Ficus sp. (Moraceae), 18 October 1944, M. J. Thirumalachar.

Distribution: Karnataka.

Asterina naraveliae Hosag., C.K. Biju \& Agarwal, Indian Phytopath. 55: 499, 2002; Hosag., Chandraprabha \& Agarwal, Asterinales of Kerala, p. 126, 2011. (Fig. 161)

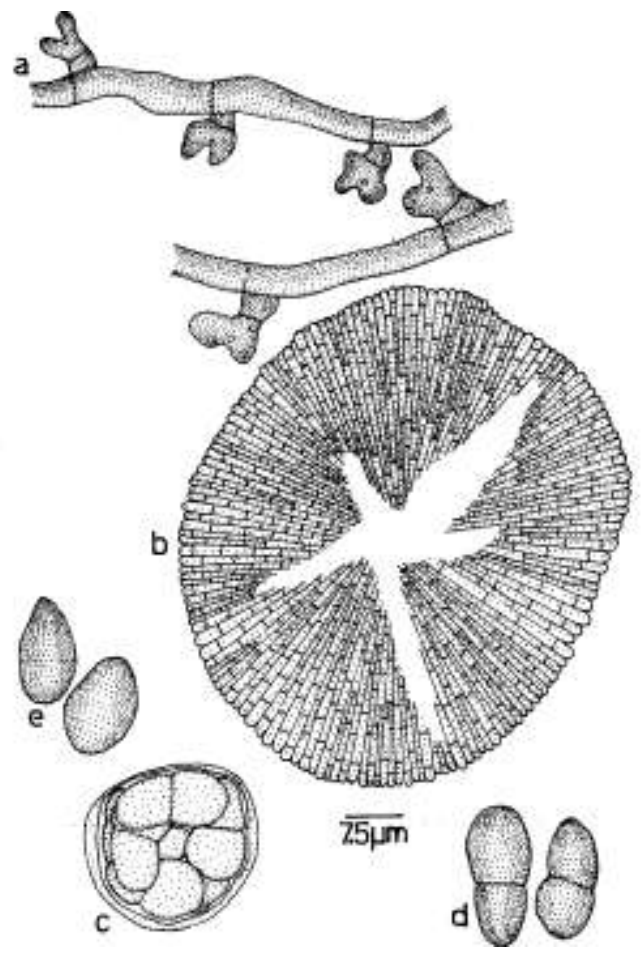

Figure 161 - Asterina naraveliae

Colonies amphigenous, thin to subdense, ad $2 \mathrm{~mm}$ in diameter, rarely confluent. Hyphae flexuous to crooked, branching irregular at acute angles, loosely reticulate, cells 16-20 × 3-4 $\mu \mathrm{m}$. Appressoria two celled, very much scattered, antrorse, 9-15 $\mu \mathrm{m}$ long; stalk cells cylindrical to cuneate, 1.5$5 \mu \mathrm{m}$ long; head cells ovate, globose, mostly bilobate, rarely 3-4-times lobate, $8-10 \times 6-10$ $\mu \mathrm{m}$. Thyriothecia scattered, orbicular, up to 65 $\mu \mathrm{m}$ in diameter, stellately dehisced at the centre, crenate at the margin; asci few to many, globose, octosporous, up to $28 \mu \mathrm{m}$ in diameter; ascospores oblong, brownish, conglobate, 1septate, upper cell slightly larger, 14-16 × 6-8 $\mu \mathrm{m}$, wall smooth. Pycnothyria not seen; Pycnothyriospores ovate, pyriform, sometimes pale hyaline band present at the middle, 14-16 $\times 7-9 \mu \mathrm{m}$.

Material examined: Kerala, Wyanad, Banasuran mala, on leaves of Naravelia zeylanica (L.) DC. (Ranunculaceae), 19 November 1999, C.K. Biju HCIO 43711 (holotype), $\quad$ TBGT 369 (isotype); Thiruvananthapuram, TBGRI Campus, 10 April 2008, A. Sabeena \& M. C. Riju HCIO 49130, TBGT 3385; Wayanadu, Puthuserry kadavu, 14 March 2007, M.C. Riju HCIO 49968, TBGT 4120, 27 December 2007, M.C. Riju HCIO 50006, TBGT 4158; Periya, Gurukulam 
Botanical Garden, 5 November 2009, M.C. Riju \& A. Sabeena TBGT 4657; HCIO TBGT 5008; Thyriode, 6 November 2009, A. Sabeena \& M.C. Riju TBGT 5055; Karnataka, Kodagu, Hoddur, 1 November 2009, C. Jagath Thimmaiah TBGT 5395, 5433.

Asterina clematidis Hansf. is known on Clematis glycinoides from Australia (Hansford, 1954). Asterina naraveliae differs from it in having sparsely arranged, alternate appressoria having deeply lobate head cells.

Distribution: Karnataka, Kerala.

Asterina natsiati Kar \& Maity, Trans. Brit. Mycol. Soc. 54: 438, 1970.

On the lower surface of the leaves, forming black, scattered, orbicular, superficial, thick patches, up to $5 \mathrm{~mm}$ diam.; Mycelium superficial, densely reticulate, composed of brown, septate, much branched, wavy hyphae, 3.3-5 $\mu \mathrm{m}$ wide, (cells mostly 13.2-33 $\mu \mathrm{m}$ long) giving rise to opposite or irregular branches and appressoria at an acute to wide angle. Appressoria one sided or alternately arranged, unicellular, 2-4 deeply lobed, brown, straight or bent, 6.6-9.9 × 3.3-8.2 $\mu \mathrm{m}$; Thyriothecia many, scattered, superficial, orbicular, 108-181.5 $\mu \mathrm{m}$ diam., attached with free mycelium, dimidiate with radiate scutellum, stellately dehiscing at first, subsequently developing in to an aperture, dark brown, convex, hymenium simple, margin subcrenate; Asci many, arranged towards the periphery of the fruit body, round to obovate, sessile, 8-spored, 23.1-33 $\times 19.8-23 \mu \mathrm{m}$, paraphysate; Ascospores crowded, fusiformelliptical, uniseptate, constricted at the septum, upper cell slightly conic, brown, smoothwalled, $16.5-19 \times 6.6-7.4 \mu \mathrm{m}$.

Material examined: West Bengal, Jalpaiguri, Rajabhatkhawa forest, on living leaves of Natsiatum hypericum Ham. (Icacinaceae) October 28, 1967, IMI 134493( holotype).

Distribution: West Bengal.

Asterina neolitsiicola Hosag., C.K. Biju \& Abraham, Indian Phytopath. 54: 138,
2001; J. Mycopathol. Res. 40:195, 2002; Hosag., Zoos' Print J. 21: 2328, 2006; Hosag., Chandraprabha \& Agarwal, Asterinales of Kerala, p. 127, 2011. (Figs 162-163)

Colonies hypophyllous, thin to subdense, up to $10 \mathrm{~mm}$ in diameter, rarely confluent. Hyphae flexuous to crooked, branching opposite to irregular at acute angles, loosely reticulate, cells $12-18 \times 3-5 \mu \mathrm{m}$. Appressoria numerous, ovate, globose, mammiform, broad based, entire, angular to sublobate, $7-13 \times 7-8 \mu \mathrm{m}$. Thyriothecia many, scattered, orbicular, up to $75 \mu \mathrm{m}$ in diameter, stellately dehisced at the center, margin fimbriate, fringed hyphae flexuous, exappressoriate; asci globose, octosporous, 12$18 \mu \mathrm{m}$ in diameter; ascospores oblong, conglobate, brown, 1-septate, strongly constricted at the septum, 17-25 $\times 7-10 \mu \mathrm{m}$, wall verruculose.

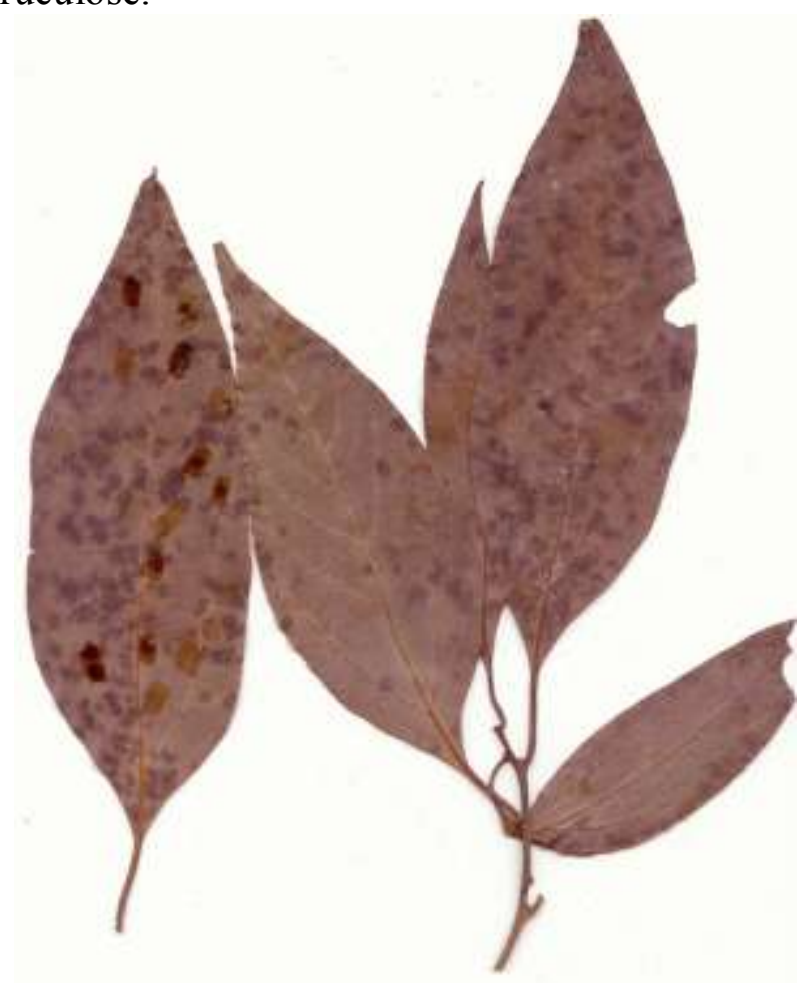

Figure 162 - Asterina neolitsiicola - Infected leaves

Material examined: Kerala, Idukki, Rajamal a, on leaves of Neolitsea sp. (Lauraceae), 15 September 1998, C.K. Biju HCIO 42952 (holotype), TBGT 241 (isotype); Munnar, Mannavan Shola, 29 September 1998, C.K. Biju HCIO 45276, TBGT 1314; Tamil 
Nadu, Kodaikanal, Pambar shola, 22 July 2006, B. Bairavi HCIO 47034, TBGT2251; July 19, 2006,G. Sangeeta HCIO 47294, TBGT 2332; July 19, 2006, R. Nithyarani HCIO 47704, TBGT 2726; Poomparai shola, Neolitsea scrobiculata (Meissner) Gamble, 19 July 2006, R. Nithyatharani HCIO 47039, TBGT 2256; Pambar shola, 22 July 2006, R. Nithyatharani HCIO 47043, TBGT 2260.

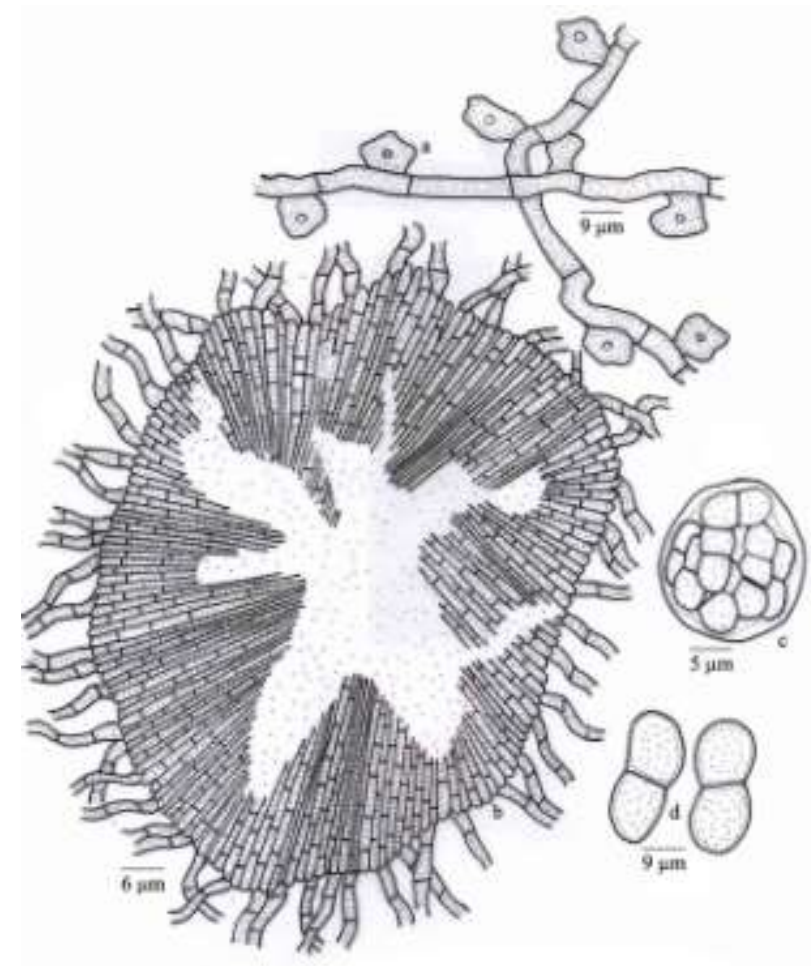

Figure 163 - Asterina neolitsiicola

Asterina neolitseae Yates is the only species known on the host genus Neolitsea from Philippines. However, the new species differs from it in having hypophyllous colonies, unicellular appressoria and smaller ascospores.

Distribution: Kerala, Tamil Nadu

Asterina nothopegiae Ryan, Mem. Dept. Agric. India 15: 104, 1928; Patil \& Thite, J. Shivaji Univ. 17: 152, 1977; Hosag., Balakr. \& Goos, Mycotaxon 59: 182, 1996; Hosag.,C.K. Biju \& Abraham, J. Econ. Taxon. Bot. 25: 305, 2001; Hosag., Zoos' Print J. 18: 1280, 2003; Hosag., Zoos' Print J. 21: 2328, 2006; Hosag., Chandraprabha \& Agarwal, Asterinales of Kerala, p. 128, 2011.

(Fig. 164)
Colonies amphigenous, mostly epiphyllous, thin, up to $5 \mathrm{~mm}$ in diameter, confluent. Hyphae straight, branching opposite at wide angles loosely reticulate, cells 9-22 $\times 3$ $5 \mu \mathrm{m}$;. Appressoria alternate and about $40 \%$ opposite, unicellular, ovate, globose to conoid, entire to deeply and irregularly shallowly to deeply lobate, $6-13 \times 6-8 \mu \mathrm{m}$; Thyriothecia scattered, round to slightly ovate, up to $155 \mu \mathrm{m}$ in diameter, dehiscing stellately at the center, margin crenate to fimbriate, fringed hyphae flexuous; asci many, globose to ovate, eight spored, $30-32 \times 21-25 \mu \mathrm{m}$.

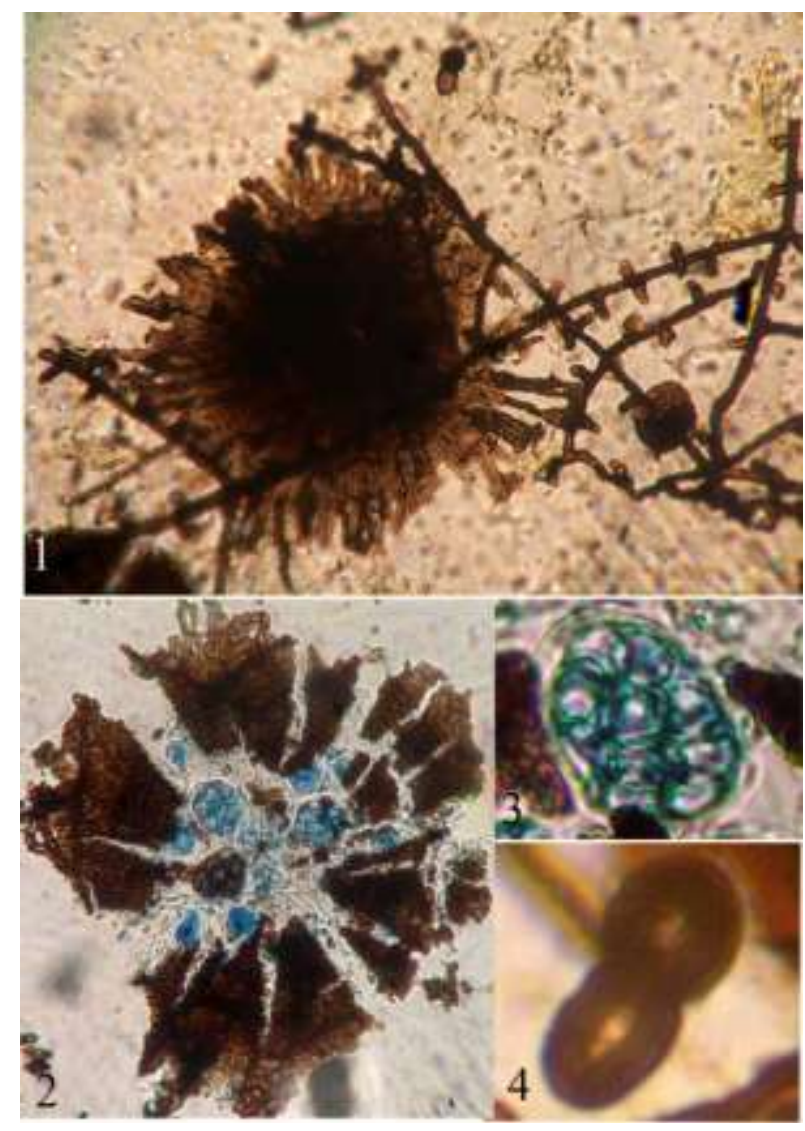

Figure 164 - Asterina nothopegiae

1. Appressoriate mycelium with Thyriothecium, 2. Dehisced thyriothedium with asci, 3. Ascus, 4. Ascospore

Material examined: Tamil Nadu, Nilgiris, Kotagiri, Kolithorai, on leaves of Nothopegia colebrookiana (Wight) Blume (Anacardiaceae), 16 February 1991, V.B. Hosagoudar HCIO 30971; Thiruvananthapuram, Agasthyar hill, Pongalapara, on leaves of $N$. travancorica Bedd. ex Hook. f., 26 March 1996, V.B. Hosagoudar HCIO 42178, TBGT 50; Attayar, 
on leaves of $N$. aureo-fulva Bedd. ex Hook. f., 18 March 1997, V.B. Hosagoudar HCIO 42494, TBGT 113; Karnataka, Kodagu, Hoddur, Mandrane, on leaves of Nothopegia racemosa (Dalz.) Ramam., 8 January 2009, C. Jagath Thimmaiah TBGT 5391; Tamil Nadu, Kuttalam, on leaves of Nothopegia sp., 27 December 2000, G. Rajkumar HCIO 45168, TBGT 1223; Kerala, Palghat, Silent Valley National Park, 1 August 2008, P.P. Rajesh Kumar \& al. HCIO 49802, TBGT 3954; Karnataka, Hoddur, Devarakadu, 2 December 2009, C. Jagat Thimmaiah TBGT 5464.

This is the only species of Asterina known on the host genus Nothopegia. The host plants are endemic to Southern Western Ghats.

Distribution: Karnataka, Kerala, Tamil Nadu.

Asterina olacicola Hansf., Proc. Linn. Soc. London 158: 46, 1947; Patil \& Thite, J. Shivaji Univ. 18: 220, 1998; Hosag., Chandraprabha \& Agarwal, Asterinales of Kerala, p. 129, 2011.

(Figs 165-166)

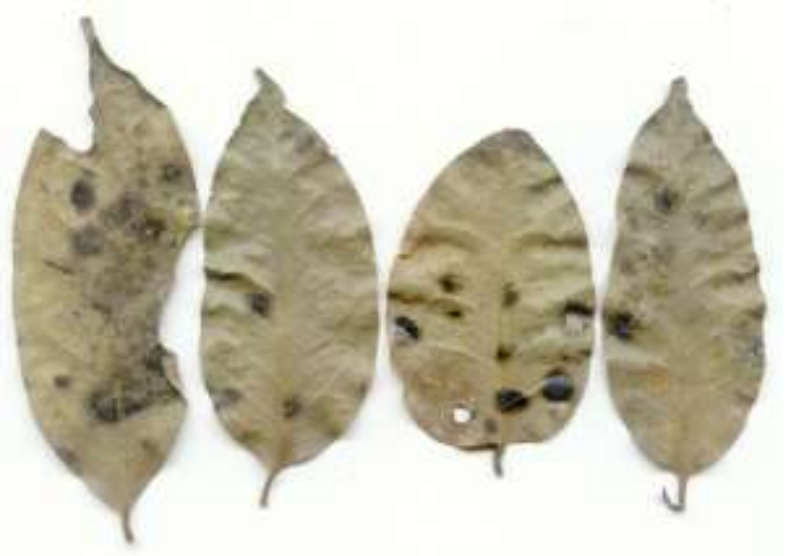

Figure 165 - Asterina olacicola - Infected leaves

Colonies amphigenous, mostly epiphyllous, dense, crustose to velvety, up to 5 $\mathrm{mm}$ in diameter. Hyphae straight to substraight, branching mostly opposite at acute angles, loosely to closely reticulate, cells $19-23 \times 8-10$ $\mu \mathrm{m}$. Appressoria alternate, about 3\% opposite, unilateral, ovate, broad, straight to slightly curved, entire, $11-13 \times 6-7 \mu \mathrm{m}$. Thyriothecia densely scattered, orbicular, up to $160 \mu \mathrm{m}$ in diam., stellately dehisced at the centre, margin crenate to slightly fimbriate, fringed hyphae small; asci globose, octosporous, up to $40 \mu \mathrm{m}$ in diam.; ascospores oblong, conglobate, brown, uniseptate, strongly constricted at the septa, 27$36 \times 14-19 \mu \mathrm{m}$, wall tubercled. Pycnothyria mixed with thyriothecia, similar but smaller than thyriothecia; pycnothyriospores pyriform, deep brown, 19-23 $\times 15-17 \mu \mathrm{m}$, wall smooth.

\section{Material examined: Kerala,}

Thiruvananthapuram, Ponmudi, on leaves of Olax scandens Roxb. (Olacaceae), 25 January 2005, N. Mohanan \& P. A. Jose HCIO 46369, TBGT 2015.

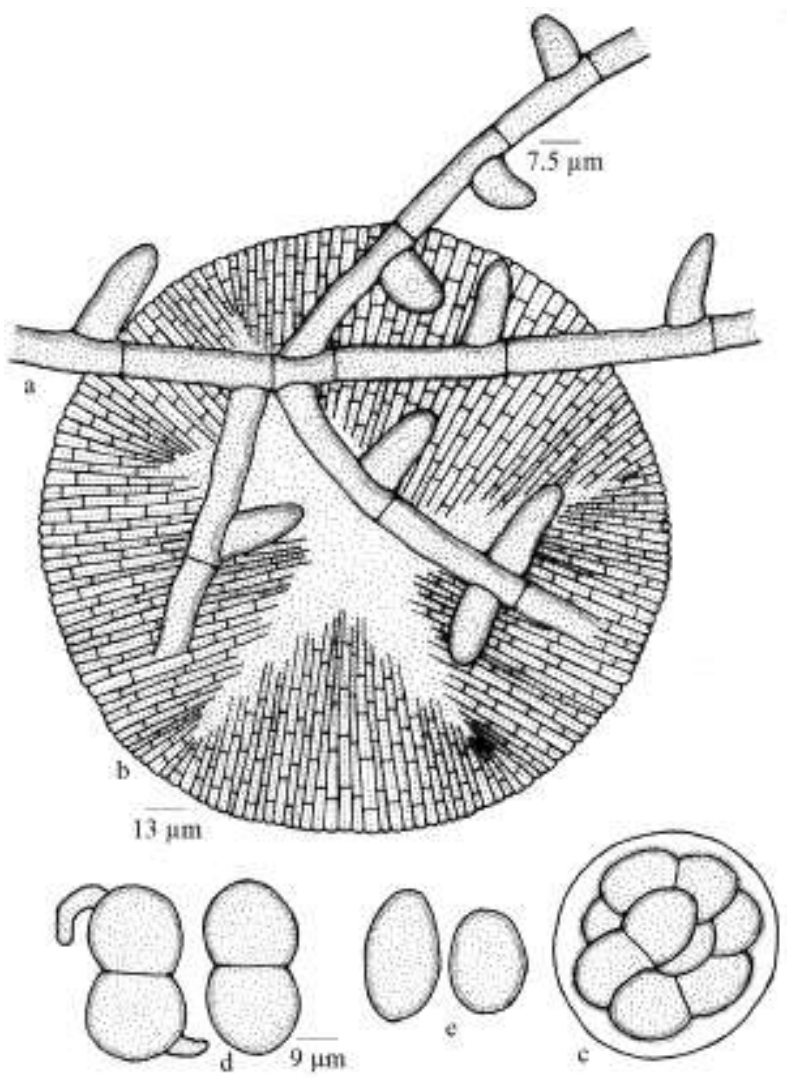

Figure 166 - Asterina olacicola

This species was collected by M. J. Thirumalachar on February 19, 1944 from Nandi Hills, Karnataka on Olax wightiana (Hansford, 1947). Subsequently, Patil \& Thite (1978) have just listed it from Maharashtra and has extended its distribution in the Western Ghats up to Kerala state.

Maharashtra

Distribution: Karnataka, Kerala,

Asterina oreocnidecola Hosag., Balakr. \& Goos, Mycotaxon 59: 183, 1996; Hosag. \& H. Biju, J. Mycopathol. Res. 44: 42, 2006; Hosag., Chandraprabha \& 
Agarwal, Asterinales of Kerala, p. 130, 2011.

(Fig. 167)

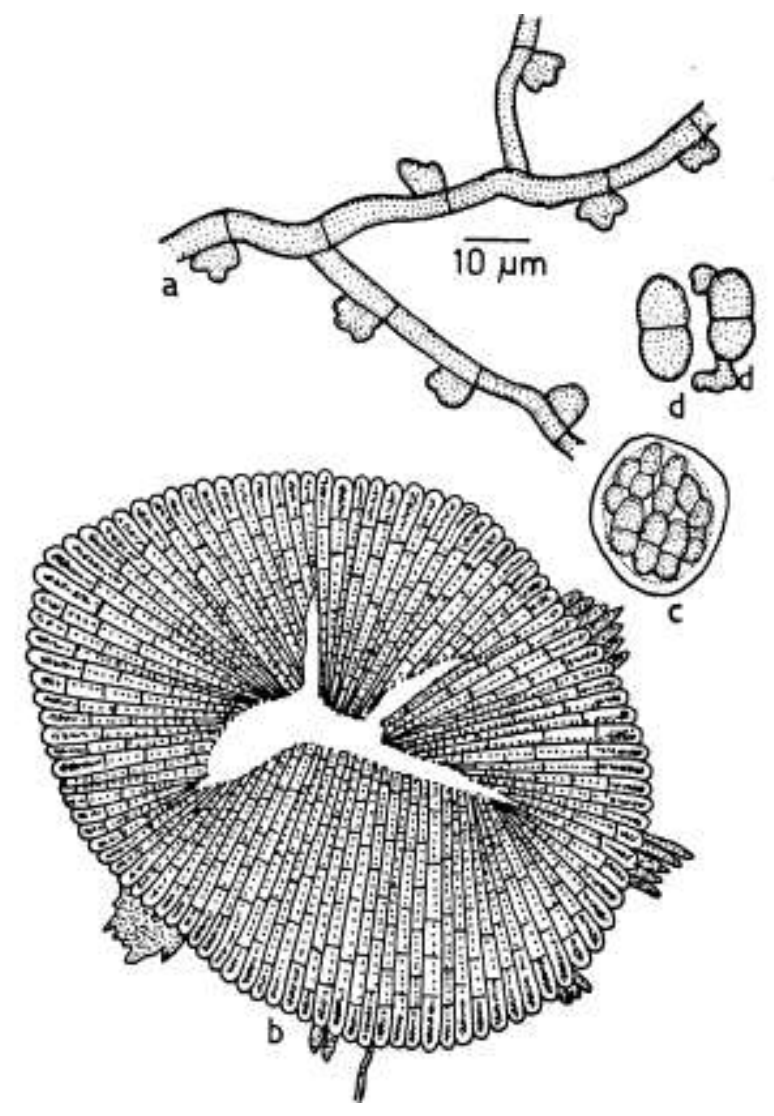

Figure 167 - Asterina oreocnidecola

Colonies amphigenous mostly epiphyllous, rarely hypophyllous, up to $3 \mathrm{~mm}$ in diameter, confluent, very thin, sometimes difficult to trace. Hyphae brown, straight to undulate, branching alternate to opposite at wide angles, loosely reticulate, cells 9-16 × 3-5 $\mu \mathrm{m}$. Appressoria pale brown, unicellular, alternate to unilateral, globose, stellately sublobate, rarely entire, 4-6 × 7-10 $\mu \mathrm{m}$. Thyriothecia scattered, mostly rounded, up to $100 \mu \mathrm{m}$ in diameter, dehiscing stellately at the center, margin crenate to fimbriate, fringed hyphae yellow, slightly flexuous; asci many, globose to ovate, eight spored, 27-28 $\times$ 21-25 $\mu \mathrm{m}$; ascospores brown, conglobate, 1-septate, $18-19 \times 9-11 \mu \mathrm{m}$, one cell slightly larger, wall smooth.

Material examined: Tamil Nadu, Coimbatore, Anamalai, Near Manjaparai, on leaves of Oreocnide integrefolia (Gaud. ex Wedd.) Miq. (Urticaceae), 23 March 1990, V.B. Hosagoudar HCIO 30972; Kerala, Palghat, Silent valley, Sairandhri,on leaves of
Oreocnide sp., 13 December 2003, V.B. Hosagoudar \& al. HCIO 45914, TBGT 1676; HCIO 46280, TBGT 1626; HCIO 45767, TBGT 1516; 14 December 2003, V.B. Hosagoudar HCIO 45916, TBGT 1678; Chemmpatti, 14 December 2003, V.B. Hosagoudar \& al. HCIO 47729, TBGT 2751; Pathanamthitta, Gavi, 16 December, 2004, V.B. Hosagoudar \& al. HCIO 46292, TBGT 1938; Moozhiyar forest, 14 December 2004, V.B. Hosagoudar \& al. HCIO 46300, TBGT 1946; TBGT 1938; Kottayam, Ponthanpuzha, on leaves of Urticaceae member, 7 November 2007, P.J. Robin \& al. HCIO 49951, TBGT 4103; Thrissur, Vazhachal, Mallakkapara, on leaves of Oreocnide integrifolia (Gaudich) Miq. (Villebrunea integrifolia Gaudich.) (Urticaceae), 18 October 2007, Jacob Thomas HCIO 48305, TBGT 3026; Thiruvananthapuram, Peppara Wildlife Sanctuary, Athirumala, 28 February 2008, Jacob Thomas HCIO 48878, TBGT 3254, HCIO 48880, TBGT 3256; Pathanamthitta, Anathodu, on leaves of Villebrunea sp., 18 January 2007, M.C. Riju \& al. TBGT 4971; Palakkad, Silent Valley National Park, on leaves of Villebrunea sp., 16. February 2007, Riju. M.C \& V. Gireesh Kumar TBGT 4972.

Asterina fleuryae Doidge, A. phaleriae Mandoza, A. phenacis Sydow and $A$. pipturi Sydow have been reported on the genera Fleurya, Phaleria, Phenax and Pipturus of the family Urticaceae. The present species is distinct from all these in having very thin epiphyllous colonies, alternate to unilateral and stellately lobate appressoria.

Distribution: Kerala, Tamil Nadu.

Asterina oreocnidegena Hosag. in Hosag., H. Biju \& Appaiah, Mycopathol. Res. 44: 42, 2006; Hosag., Chandraprabha \& Agarwal, Asterinales of Kerala, p. 131, 2011. (Fig. 168)

Colonies epiphyllous, thin to subdense, up to $3 \mathrm{~mm}$ in diameter. Hyphae straight, branching mostly opposite at acute angles, loosely reticulate, cells 19-28 × 4-7 $\mu \mathrm{m}$. Appressoria mostly opposite, about 3\% alternate to solitary, unicellular, ovate, conoid, 
broadly rounded at the apex, entire, 9-12 × 4-7 $\mu \mathrm{m}$. Thyriothecia scattered to connate, orbicular, up to $180 \mu \mathrm{m}$ in diameter, stellately dehisced at the centre, margin crenate to fimbriate, fringed hyphae compact; asci globose, octosporous, $30-40 \mu \mathrm{m}$ in diam.; ascospores conglobate, brown, uniseptate, constricted at the septa, $25-30 \times 14-16 \mu \mathrm{m}$, wall smooth.

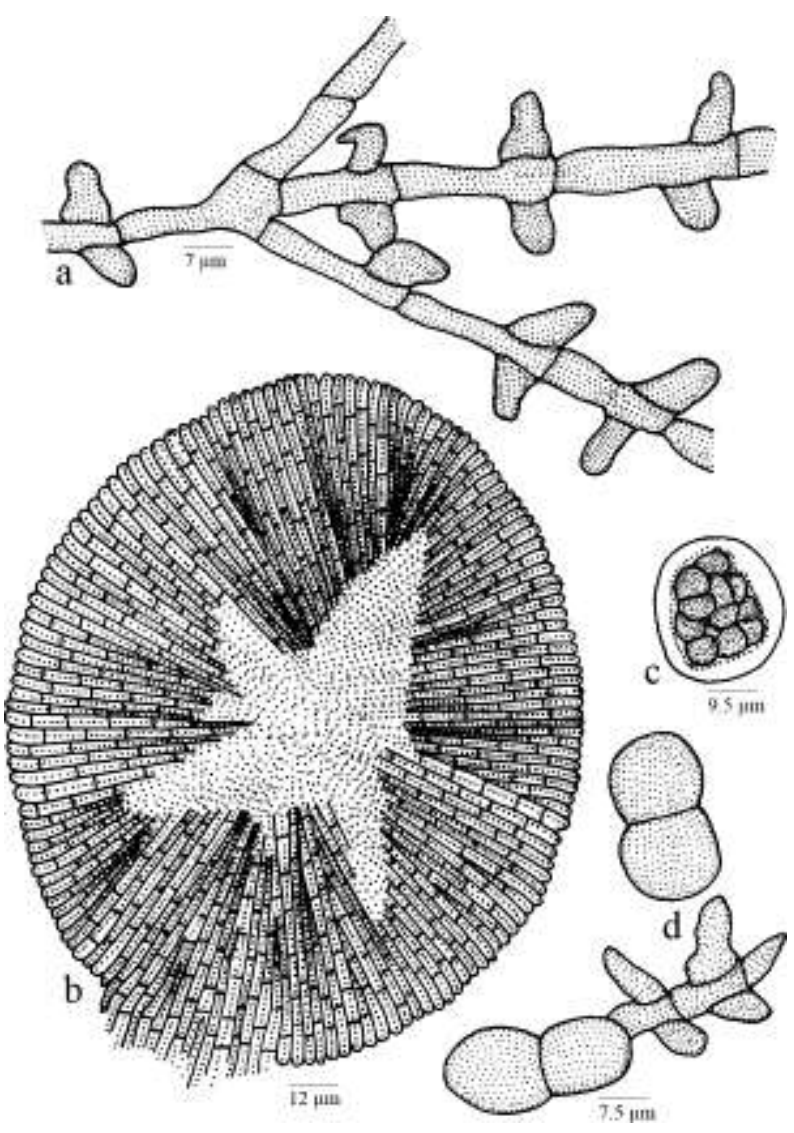

Figure 168 - Asterina oreocnidegena

Material examined: Kerala, Silent Valley, Palghat, Sairandhri, on leaves of Oreocnide integrifolia (Gaud. ex Wedd.) Miq. (Urticaceae), 13 December 2003, V.B. Hosagoudar \& al. HCIO 45771 (holotype), TBGT 1520 (isotype).

Asterina oreocnidecola Hosag. et al. is known on the same host from the Western Ghats (Hosagoudar et al. 1996a,b,c). However, $A$. oreocnidegena differs from it in having opposite appressoria and larger ascospores.

Distribution: Kerala.
Asterina palaquii Hosag. \& Goos, Mycotaxon 59: 158, 1996. (Fig. 169)

Colonies epiphyllous, dense, crustose, up to $3 \mathrm{~mm}$ in diameter. Hyphae straight to substraight, branching irregularly at acute angles, loosely reticulate, cells 24-28 $\times 3-5 \mu \mathrm{m}$. Appressoria mostly alternate, about 20\% opposite, mammiform, seated on broad base, entire to rarely truncate, $6-8 \times 5-6 \mu \mathrm{m}$. Thyriothecia scattered, orbicular, up to $200 \mu \mathrm{m}$ in diameter, margin mostly crenate, dehiscing stellately at the center; asci many, mostly globose to slightly ovate, octosporous, 46-62 $\times$ 37-47 $\mu \mathrm{m}$; ascospores conglobate, brown, 1septate, deeply constricted at the septum, 24-28 $\times$ 11-13 $\mu \mathrm{m}$, wall smooth.

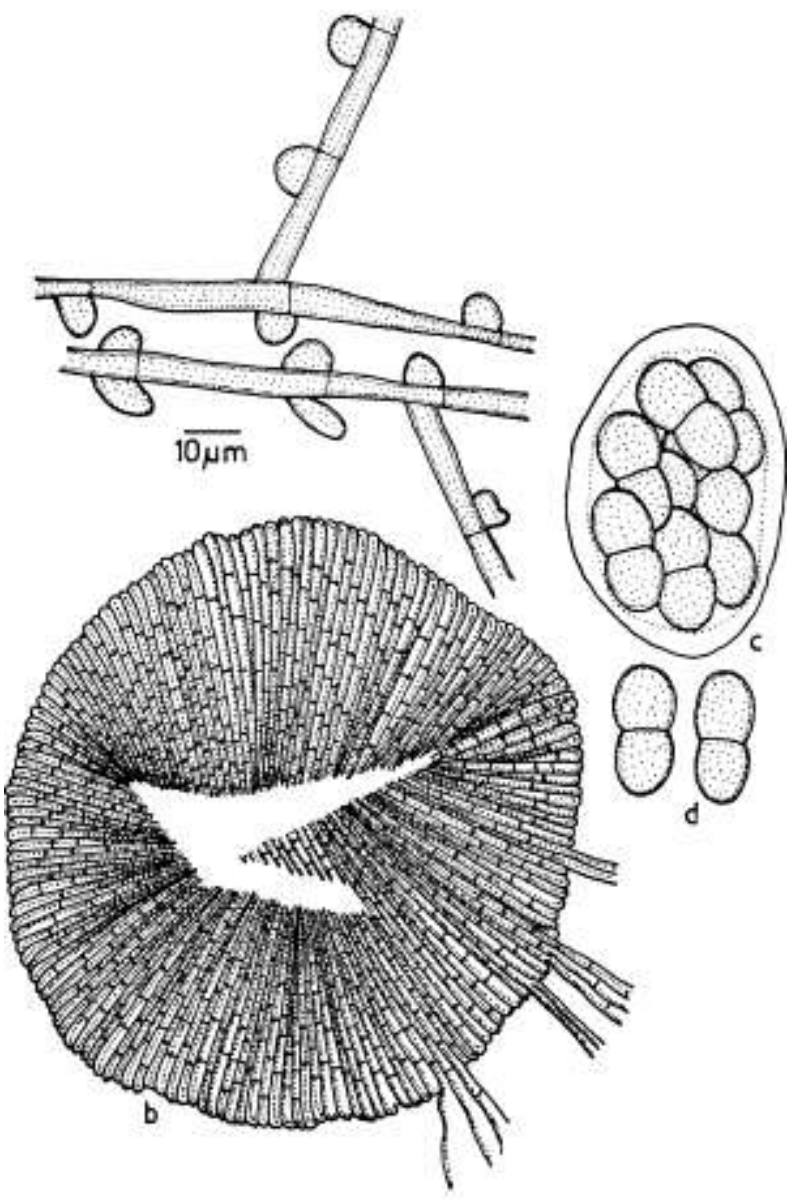

Figure 169 - Asterina palaquii

Material examined: Tamil Nadu, Tirunelveli, Kudrevetti, on leaves of Palaquium ellipticum (Dalz.) Baillon (Sapotaceae), 25 February 1994, V.B. Hosagoudar HCIO 41612 (holotype). 
Broad based and 20\% opposite appressoria, crenate margin of the thyriothecia and narrow ascospores distinguishes this species from the similar Asterina opaca Sydow (Doidge, 1942).

Distribution: Tamil Nadu.

Asterina parsonsiae Hosag. in Hosag., H. Biju \& Appaiah, J. Mycopathol. Res. 44: 9, 2006. (Fig. 170)

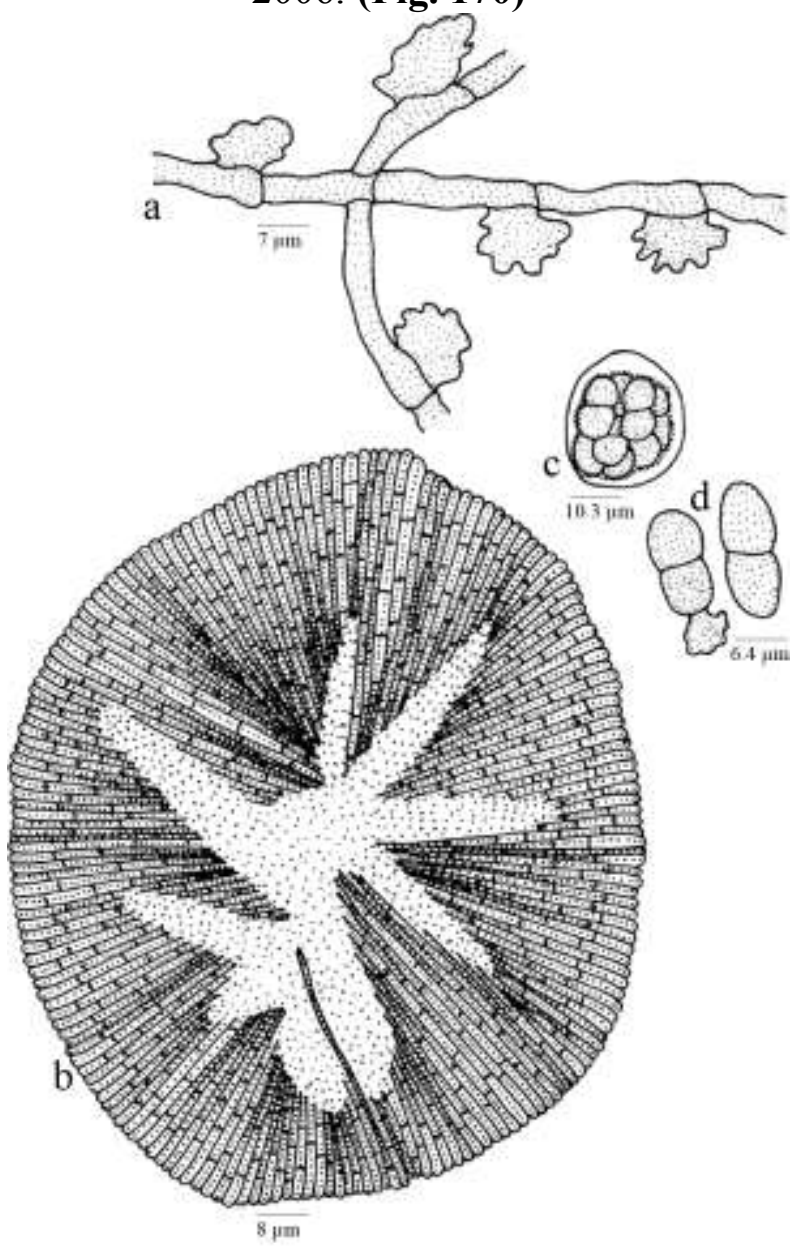

Figure 170 - Asterina parsonsiae

Colonies amphigenous, mostly hypophyllous, subdense to dense, up to $5 \mathrm{~mm}$ in diameter, confluent. Hyphae undulate, branching irregular at acute to wide angles, loosely reticulate and form a loose net, cells 16$24 \times 3-5 \mu \mathrm{m}$. Appressoria alternate to unilateral, mammiform, broad based, crenately to irregularly lobate, $7-9 \times 11-14 \mu \mathrm{m}$. Thyriothecia scattered to connate, orbicular, up to $130 \mu \mathrm{m}$ in diameter, stellately dehisced at the centre, margin crenate; asci globose, octosporous, up to $30 \mu \mathrm{m}$ in diameter; ascospores oblong, conglobate, uniseptate, slightly constricted, brown, 14-18 $\times 7-9 \mu \mathrm{m}$, wall smooth.

Material examined: Karnataka, Coorg, Madikeri, Talacauvery, Brahmagiri, Medicinal Plant Conservation Area, on leaves of Parsonsia alboflavescens (Dennst.) Mabberley (Apocynaceae), 13 November 2003, V.B. Hosagoudar HCIO 45657 (holotype), TBGT 1403 (isotype).

Based on the morphology and measurements of the ascospores and appressoria, Asterina parsonsiae is similar to $A$. aganosmae Sydow but differs from it having mammiform and crenately lobate appressoria (Sydow \& Petrak, 1931).

Distribution: Karnataka.

Asterina pavoniae Werd., Rep. Spec. Nor. Res. Ves. 9: 50, 1923; Hosag. \& Goos, Mycotaxon 60: 160, 1996.

(Fig. 171)

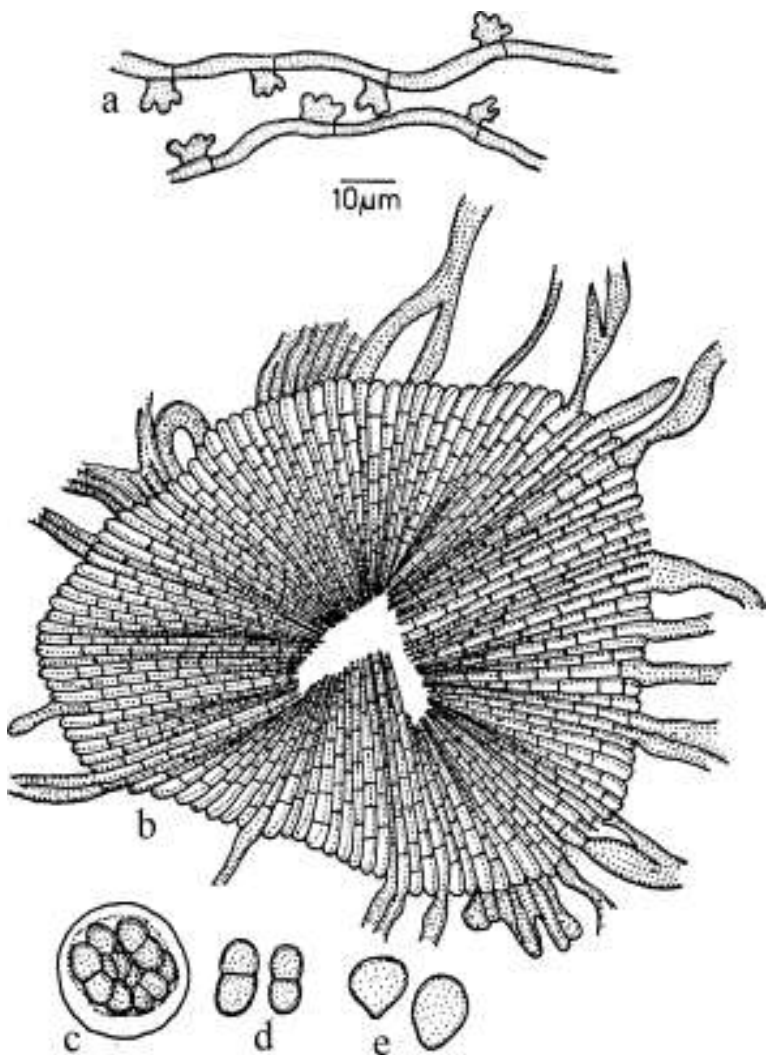

Figure 171 - Asterina pavoniae

Colonies epiphyllous, minute, subdense, crustose and covering the entire upper surface of the leaves. Hyphae flexuous, branching irregular at acute angles, closely reticulate, cells 
15-22 × 3-4 $\mu \mathrm{m}$. Appressoria alternate to unilateral, sessile to slightly stipitate, unicellular, mammiform to clavate, 1-4 lobed, $6-10 \times 6-10 \mu \mathrm{m}$. Thyriothecia scattered to connate, orbicular, up to $110 \mu \mathrm{m}$ in diameter, margin fringed, fringed hyphae flexuous to crooked, thyriothecia dehisced stellately at the center; asci many, globose, octosporous, up to $35 \mu \mathrm{m}$ in diameter; ascospores deep brown, conglobate, 1-septate, deeply constricted at the septum, $14-16 \times 6-10 \mu \mathrm{m}$, both cells identical in size in some spores while, the lower cell is larger in others. Pycnothyria many, orbicular, dehiscing stellately at the center, up to $62 \mu \mathrm{m}$ in diameter; pycnothyriospores deep brown, ovoid to pyriform, $12-16 \times 9-13 \mu \mathrm{m}$.

Material examined: Tamil Nadu, Kanniyakumari, Veerapali reserve forest, on leaves of Sida rhombifolia L. (Malvaceae), 22 February 1994, V.B. Hosagoudar HCIO 41626.

Werdermann (1923) described this species based on the collected on Pavoniasp. from Natal. Doidge (1942) determined the host to be Sida hislopii. This species stands distinct from the other Asterina species in having mostly 3-lobed appressoria.

Distribution: Tamil Nadu.

Asterina perpusilla Sydow, Ann. Mycol. 14: 366, 1916.

Anamorph: Asterostomella alangii Hosag. \& Mohanan, Indian J. Forestry 19: 371, 1996. (Fig. 172)

Colonies epiphyllous, thin, up to $2 \mathrm{~mm}$ in diameter, confluent. Hyphae substraight, branching opposite, alternate to irregular, at acute to wide angles, loosely to closely reticulate, cells 11-24 × 2-3 $\mu \mathrm{m}$. Appressoria sessile, unilateral, alternate, angular, broad based, $4-10 \times 4-10 \mu \mathrm{m}$. Thyriothecia scattered to grouped, orbicular, stellately dehisced at the centre, up to $160 \mu \mathrm{m}$ in diameter; ascospores conglobate, uniseptate, constricted at the septum, 19-26 $\times$ 9-11 $\mu \mathrm{m}$. Pycnothyria scattered, rarely connate, orbicular, up to $78 \mu \mathrm{m}$ in diameter, margin crenate, rarely fringed, stellately dehisced at the center; pycnothyriospores numerous, cinnamon brown, pyriform, $21-25 \times 12-16 \mu \mathrm{m}$.

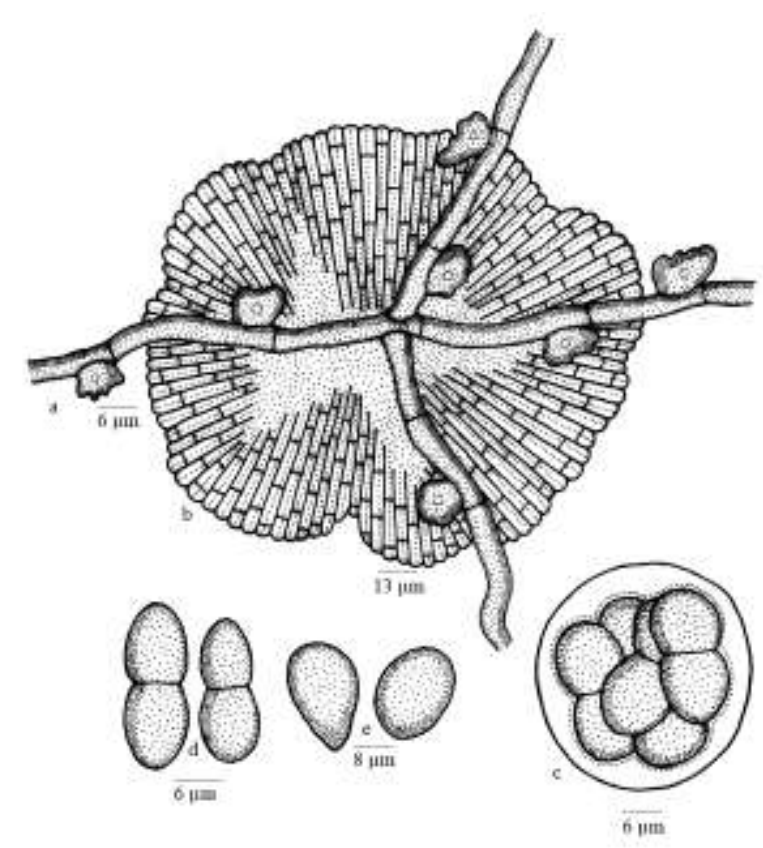

Figure 172 - Asterina perpusilla

Material examined: Andhra Pradesh, East Godavari, Valamuru forest, Maredumilli, on leaves of Alangium salvifolium (L.F) Wans (Alangiaceae), 23 February 1994, M. Mohanan AMH 7864; Karnataka, South Canara, Kukke Subramanya, 15 November 2003, V.B. Hosagoudar \& al. HCIO 46779, TBGT 2120; Kerala, Thiruvananthapuram, Palode, TBGRI Campus, 26 September 2008, A. Sabeena \& M.C. Riju HCIO 49127, TBGT 3382; HCIO 49128, TBGT 3383; 2 November 2008, A. Sabeena \& M.C. Riju HCIO 49129, TBGT 3384; 5 October 2009, A. Sabeena TBGT 4599; 16 June 2010, A. Sabeena TBGT 4316; Kozhikode, Malabar Botanic Garden, 3 November 2009, A. Sabeena \& M.C. Riju TBGT 4624; TBGT 4669; Wayanad, Padinharathara, Alangium sp., 4 November 2009, A. Sabeena \& M.C. Riju TBGT 4299; Anamorphic state: on leaves of Alangium salviifolium (L. f.) Wangerin (Alangiaceae), TBGRI Campus, Palode, Thiruvananthapuram, 16 December 2000, M. Kamarudeen HCIO 44013, TBGT 419; 8 February 2001, H. Biju HCIO 46069, TBGT 1832; Karakulam, Thiruvananthapuram, February 26, 2003, G. Rajkumar HCIO 45130, TBGT 1185; Malabar Botanic Garden, Kozhikode, 24 December 2007, Bijeesh TBGT 3127, HCIO 48406. 


\section{Distribution: Andhra Pradesh,} Karnataka, Kerala.

Asterina phlogacanthi Kar \& Ghosh, Indian Phytopathol. 39: 211, 1986.

Patches are on both surface of the leaves, forming black, scattered, superficial, thin patches, up to $9.5 \mathrm{~mm}$ in diam., sometimes the patches coalesce and take an irregular shape; mycelium superficial, densely reticulate, composed of brown, septate much branched wavy hyphae, 3.45-5.73 $\mu \mathrm{m}$ wide, (cells mostly 11.46-38.2 $\mu \mathrm{m}$ long) giving rise to irregular branches and appressoria at an acute to wide angle; appressoria are few, one sided or scattered, unicellular, 2-4 deeply lobed, brown, straight or bent, 8.4-12.22 × 5.35-8.4 $\mu \mathrm{m}$; thyriothecia many, scattered, superficial, orbicular, 76.4-164.26 $\mu \mathrm{m}$ diam., attached with free mycelium, dimidiate with radiate scutellum, stellately dehiscing at first, subsequently developing in to an aperture, dark brown, convex, hymenium simple, margin subcrenate; asci many, round to obovate, sessile, 8-spored, 24.93-42.02 $\mu \mathrm{m}$, paraphysate; ascospores crowded, oblong-elliptical, uniseptate, constricted at the septum, rounded at ends, dark brown, smooth-walled, 16.04-17.95 $\times 7.25-8.02 \mu \mathrm{m}$.

Material examined: West Bengal, Darjeeling, Sukna forest (500-1000 ft.), on leaves of Phlogacanthus curviflorus Nees (Acantthaceae), 22 May 1976, IMI 215562 (holotype).

Distribution: West Bengal.

Asterina phyllanthicola Singh, Trans. Brit. Mycol. Soc. 74:204, 1980.

Colonies epiphyllous, dark brown to black, irregularly confluent, often covering the entire leaf surface. Mycelium of sinuous to flexuous Hyphae, light brown, septate, smooth, 2-4.5 $\mu \mathrm{m}$ in diam., (cells 7.5-25 $\mu \mathrm{m}$ long), branching mostly alternate, rarely opposite, loosely reticulate; appressoria predominantly alternate, antrorse, straight to slightly curved, 2celled, 11-18 $\mu \mathrm{m}$ long; stalk cell cylindrical, 4$7.5 \times 2-4 \mu \mathrm{m}$, head cell cylindrical to ovate, straight, often slightly curved, smooth, entire, comparatively thick walled, 6.5-11.5 × 3-5 $\mu \mathrm{m}$. Thyriothecia numerous, scattered uniformly, dark brown to black, globose to sub globose, smooth,90-160 $\mu \mathrm{m}$ diam., lower wall distinct, subhyaline to pale olivaceous brown, plate like; upper wall with radiating Hyphae, opaque dark brown, 3-5 $\mu \mathrm{m}$ thick (cells 5-12 $\mu \mathrm{m}$ long), splitting star-like, margin fimbriate; fimbriating hyphae few, 30-60 $\mu \mathrm{m}$ long, exappressoriate. Asci numerous, bitunicate, subglobose to pyriform, 8- spored, sessile, hyaline to subhyaline, aparaphysate, 20.5-30.5 $\mu \mathrm{m}$ diam. Spores hyaline when young becoming light brown towards maturity, conglobate, oblongclavate, more or less rounded at both ends, one septate, constricted at the septum, smooth, 12$18 \times 6-10 \mu \mathrm{m}$, cells ovate to subglobose and slightly unequal in size. Pycnothyria not obtained.

Material examined: Uttara Pradesh, Telkonia range, on leaves of Phyllanthus reticulates Pior (Euphorbaceae), February 1976, S. Singh 393, IMI 200116 (holotype).

Distribution: Utter Pradesh.

Asterina phyllanthi-beddomei Hosag. \& JacobThomas, Taprobanica 3: 16, 2011. (Fig. 173)

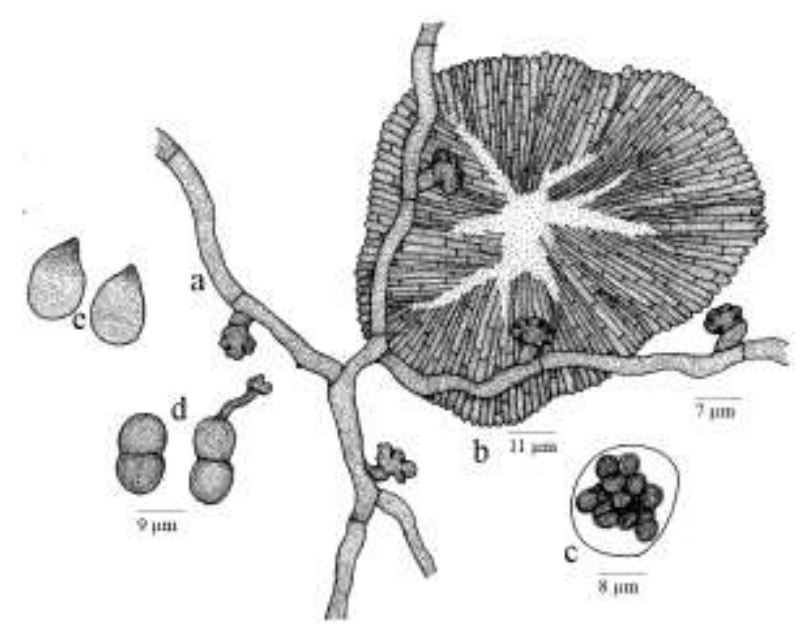

Figure 173 - Asterina phyllanthi-beddomei

Colonies epiphyllous, subdense, up to 1 $\mathrm{mm}$ in diameter, confluent. Hyphae flexuous to crooked, branching alternate to irregular at 
wide angles, loosely reticulate, cells $28-43 \times 3$ $5 \mu \mathrm{m}$. Appressoria alternate to unilateral, two celled, subantrorse to spreading, straight to curved, 9-15 $\mu \mathrm{m}$ long; stalk cells cylindrical to cuneate, 2-5 $\mu \mathrm{m}$ long; head cells ovate to mostly globose, 3-5-times stellately lobate, 7$10 \times 9-12 \mu \mathrm{m}$. Thyriothecia scattered, orbicular, up to $140 \mu \mathrm{m}$ in diameter, stellately dehisced at the centre, margin crenate; asci many, globose, octosporous, up to $38 \mu \mathrm{m}$ in diam.; ascospores, oblong, conglobate, brown, uniseptate, constricted at the septum, $16-24 \times 7-10 \mu \mathrm{m}$, wall smooth.

\section{Material examined: Kerala, Thiruvananthapuram, Peppara Wildlife Sanctuary, Athirumala, on leaves of Phyllanthus beddomi (Gamble) M. Mohanan (Euporbiaceae), 27 February 2008, Jacob Thomas HCIO 48869 (holotype), TBGT 3245 (isotype).}

Two species of the genus Asterina, namely, $A$. phyllanthicola Singh and $A$. phyllanthigena Hosag. are known on the host genus Phyllanthus (Singh, 1980; Hosagoudar, 2004). However, the present new species differs from both in having typically lobate head cells of the appressoria.

Distribution: Kerala.

Asterina phyllanthigena Hosag., Zoos' Print J. 19: 1522, 2004; Hosag., Chandraprabha \& Agarwal, Asterinales of Kerala, p. 132, 2011. (Fig. 174)

Colonies epiphyllous, thin to subdense, up to $1 \mathrm{~mm}$ in diameter, confluent. Hyphae flexuous, form a loose net of hyphae, cells 16$20 \times 3-4 \mu \mathrm{m}$. Appressoria few, scattered, twocelled, straight to curved, 9-11 $\mu \mathrm{m}$ long; stalk cells cylindrical to cuneate, 2-3 $\mu \mathrm{m}$ long; head cells ovate to oblong, slightly attenuated and broadly rounded at the apex, entire, 4-6 $\times$ 4-5 $\mu \mathrm{m}$. Thyriothecia scattered, orbicular, up to 80 $\mu \mathrm{m}$ in diameter, stellately dehisced at the centre, margin crenate; asci few, globose, octosporous, up to $30 \mu \mathrm{m}$ in diameter; ascospores oblong, conglobate, brown, uniseptate, slightly constricted, 12-14 × 6-8 $\mu \mathrm{m}$, wall smooth. Pycnothyria similar to thyriothecia, smaller; pycnothyriospores oblong, pyriform, often apiculate, brown, 9-18 $\times 8-10 \mu \mathrm{m}$, wall smooth.
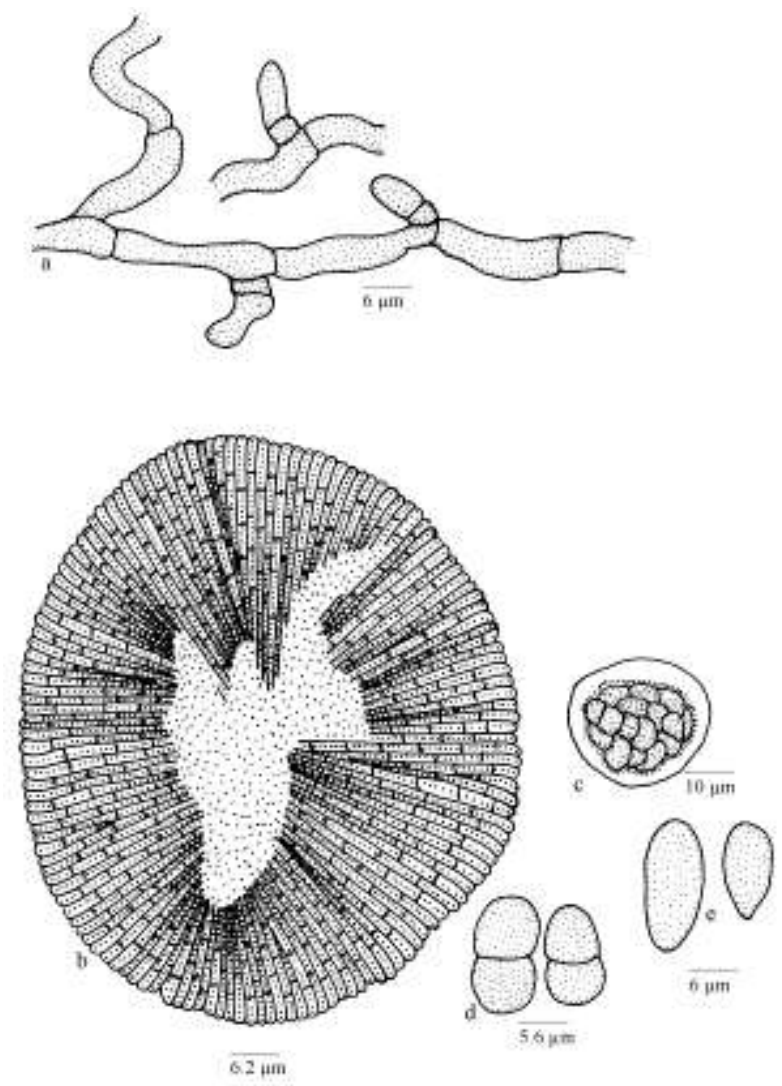

Figure 174 - Asterina phyllanthigena

Material examined: Kerala, Kollam, Sankili forest, on leaves of Phyllanthus sp. (Euphorbiaceae), 27 December 2001, V.B. Hosagoudar HCIO 42888 (holotype), TBGT 206 (isotype).

Asterina phyllanthicola Singh is known on the genus Phyllanthus from the Himalayan region of Uttar Pradesh (Hosagoudar \& Abraham, 2000; Singh, 1980). However, A. phyllanthigena differs from it in having loosely net forming hyphae, remotely placed shorter appressoria and smaller ascospores.

Distribution: Kerala.

Asterina physalidis V.B. Hosagoudar \& G.R. Archana, sp. nov. (Fig. 175)

MycoBank 101011

Etymology: Named after the host genus

Colonies amphigenous, subdense, up to $5 \mathrm{~mm}$ in diameter, confluent. Hyphae straight to 
slightly flexuous, branching opposite at acute to wide angles, loosely to closely reticulate, cells 9-21 $\times$ 4-6 $\mu \mathrm{m}$. Appressoria alternate to unilateral, antrorse to subantrorse, sessile ovate, globose, $6-10 \times 6-8 \mu \mathrm{m}$. Thyriothecia scattered to grouped, orbicular, up to $200 \mu \mathrm{m}$ in diam., stellately dehisced at the centre, margin crenate to fimbriate, fringed hyphae flexuous, exappressoriate; asci globose, octosporous, up to $58 \mu \mathrm{m}$ in diam.; ascospores conglobate, uniseptate, slightly constricted at the septum, 19-24 × 12-15 $\mu \mathrm{m}$, wall coarsely echinulate. Pycnothyria similar to thyriothecia, orbicular, smaller; pycnothyriospores unicellular, pyriform, ovate, $8-11 \times 4-6.5 \mu \mathrm{m}$.

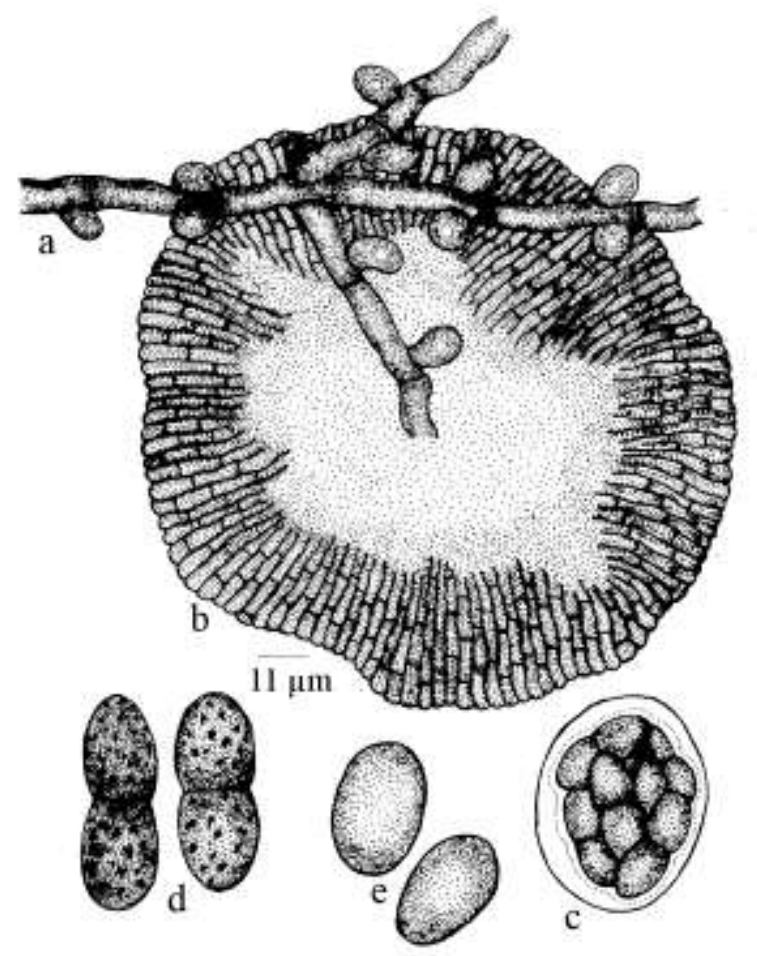

Figure 175 - Asterina physalidis

Material examined: Kerala, Thiruvananthapuram, Palode Forest, on leaves of Physalis sp. (Solanaceae), 14 May 2009, G.R. Archana TBGT 4812 (holotype).

This species differs from $A$. balakrishnanii Hosag. known on the leaves of Solanum torvum from Kerala in having alternate and opposite appressoria.

Distribution: Kerala.
Asterina piperina Sydow, Ann. Mycol. 15: 243, 1917; M. S. Patil \& Pawar, Indian Phytopathol. 42: 251, 1989.

Asterina piperis Yates, Philippine J. Sci. 13: 374, 1918. (Fig. 176)
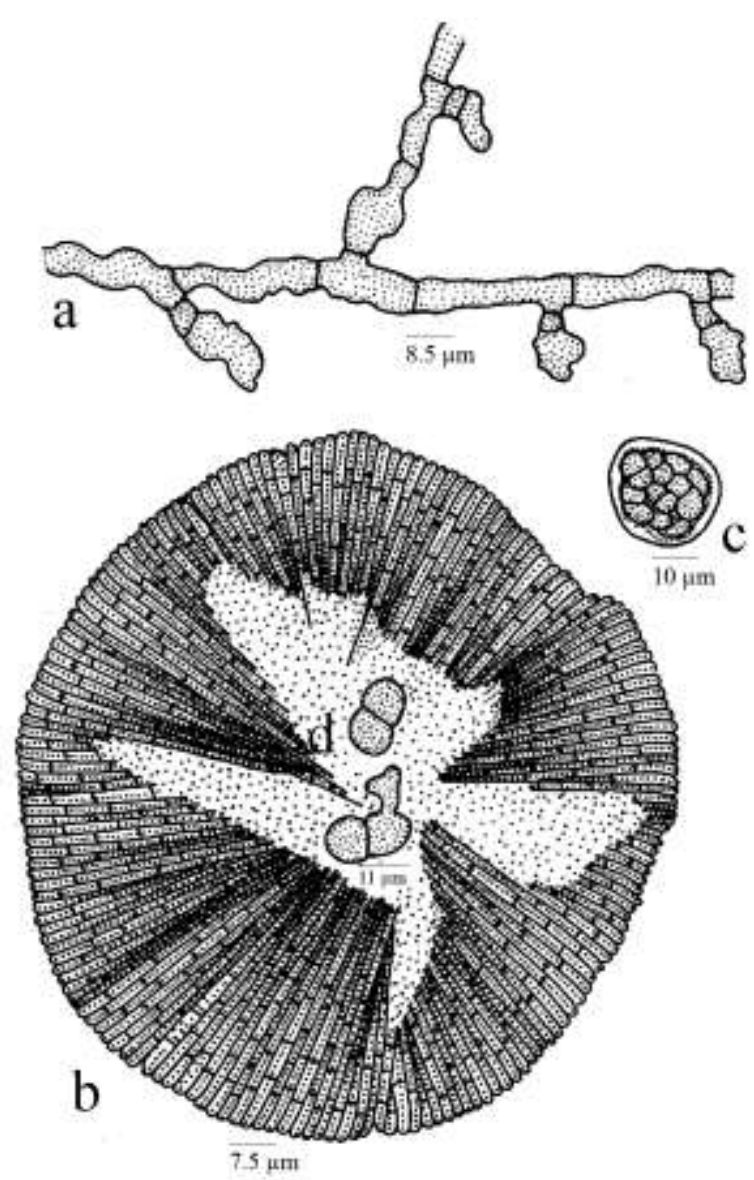

Figure 176 - Asterina piperina

Colonies amphigenous, thin to dense, up to $1 \mathrm{~mm}$ in diameter, rarely confluent. Hyphae crooked, branching irregular at acute to wide angles, loosely to closely reticulate, cells 19-24 $\mu \mathrm{m}$. Appressoria scattered, alternate to unilateral, very closely antrorse, antrorse, subantrorse to retrorse, straight to curved, 12$20 \mu \mathrm{m}$ long; stalk cells cylindrical to cuneate, 3-7 $\mu \mathrm{m}$ long; head cells ovate, globose, oblong, straight to curved, entire, angular, crenately lobate to deeply lobate, $9-13 \times 9-11 \mu \mathrm{m}$. Thyriothecia scattered to rarely connate, orbicular, up to $120 \mu \mathrm{m}$ in diameter, stellately dehisced at the centre, margin crenate to slightly fimbriate, fringed hyphae very small; asci globose, octosporous, up to $28 \mu \mathrm{m}$ in diameter; ascospores conglobate, brown, uniseptate, constricted, $14-21 \times 6-11 \mu \mathrm{m}$, wall smooth. 
Material examined: Karnataka, Coorg, Madikeri, Jodupal, on leaves of Piper sp. (Piperaceae), 12 November 2003, V.B. Hosagoudar HCIO 45685, TBGT 1432; Thiruvananthapuram, Palode, TBGRI Campus, 13 August 2009, V.B. Hosagoudar \& al. HCIO 49623, TBGT 3865; Wayanad, Periya, 17 September 2008, M. Harish \& P.J. Robin HCIO 49636, TBGT 3878.

Asterina elachista Sydow, A. piperina Sydow and $A$. piperis Yates are known on the host genus Piper (Sydow \& Sydow, 1917; Sydow, 1938; Yates, 1918a,b; Hosagoudar \& Abraham, 2000). The former species known from Uganda and the latter two species are known from Philippines. A. piperis Yates is identical with that of $A$. piperina in having two celled appressoria. The present collection matches well with the assigned species except having slightly longer appressoria and larger ascospores.

Distribution: Karnataka, Kerala.

Asterina pittospori Hansf., Reinwardtia 3(1): 135, 1954. (Fig. 177)

Colonies hypophyllous, thin, up to 2 $\mathrm{mm}$ in diameter, confluent. Hyphae crooked, branching irregular at acute to wide angles, loosely to closely reticulate, cells $30-37 \times 4-5$ $\mu \mathrm{m}$. Appressoria alternate to unilateral, unicellular, narrowly ovate, clavate, globose to cylindrical, entire to often truncate at the apex, 7-15 $\times$ 7-12 $\mu \mathrm{m}$. Thyriothecia scattered, orbicular, up to $190 \mu \mathrm{m}$ in diameter, stellately dehisced at the centre, margin crenate to fimbriate, fringed hyphae flexuous; asci globose, octosporous, up to $37 \mu \mathrm{m}$ in diameter; ascospores conglobate, 1-septate, constricted at the septum, 20-27 × 10-15 $\mu \mathrm{m}$, wall smooth.

Material examined: Kerala, Idukki, Kulamavu forest, on leaves of Pittosporum dasycaulon Miq. (Pittosporaceae), 17 December 2009, P.A. Jose TBGT 5741.

There are eight taxa of Asterina known on this host genus (Hosagoudar \& Abraham 2000). Based on the ascospore measurement and nature of appressoria, the present collection has been assigned to $A$. pittospori Hansf. known on Pittosporum ferrugineum. This species was earlier known from Java (Hansford, 1954).

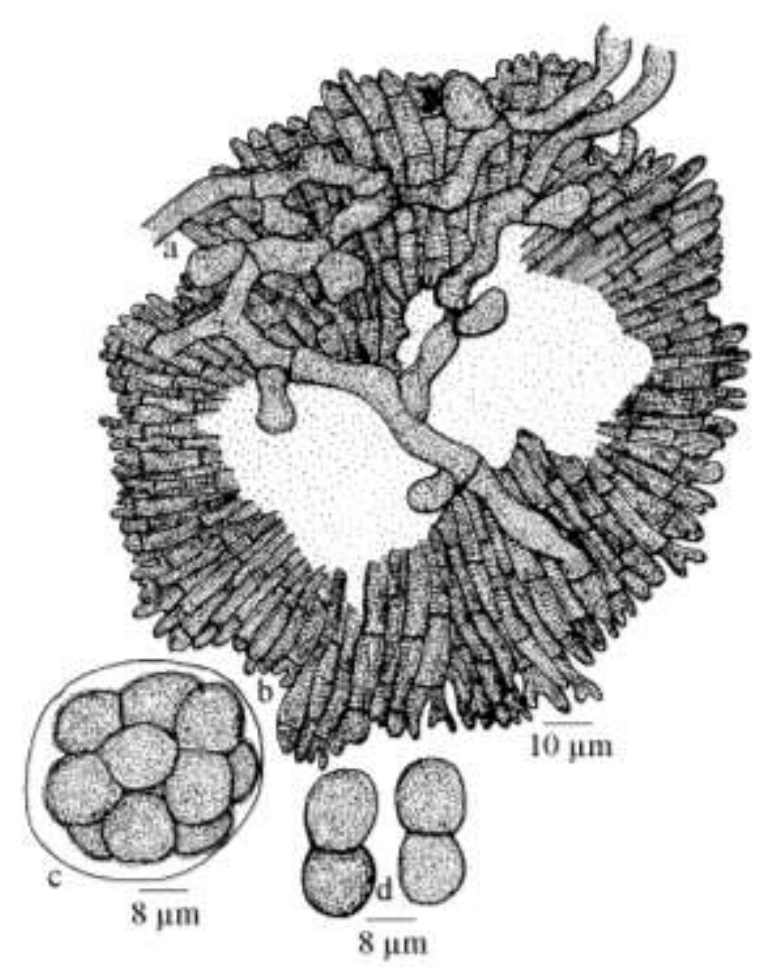

Figure 177 - Asterina pittospori

Distribution: Kerala.

Asterina plectranthi Hosag., Manojkumar \& H. Biju in Hosag., Indian Phytopath. 58: 200, 2005; Hosag., Chandraprabha \& Agarwal, Asterinales of Kerala, p. 134, 2011. (Figs 178-179)

Colonies epiphyllous, dense, up to $1 \mathrm{~mm}$ in diameter, rarely confluent. Hyphae flexuous to rarely crooked, branched irregular at acute to wide angles, form a loose circular mycelial net, cells 16-21 × 4-6 $\mu \mathrm{m}$. Appressoria two celled, straight to curved, antrorse, retrorse to spreading, 9-11 $\mu \mathrm{m}$ long; stalk cells cylindrical to cuneate, 3-5 $\mu \mathrm{m}$ long; head cells ovate, globose, entire, angular to mostly irregularly to palmately lobate, 4-6 × 6-8 $\mu \mathrm{m}$. Thyriothecia scattered, orbicular, up to $120 \mu \mathrm{m}$ in diam., stellately dehisced at the centre, margin crenate to fimbriate, fringed, hyphae small; asci globose, octosporous, up to $30 \mu \mathrm{m}$ in diam.; ascospores oblong, brown, conglobate, one septate, slightly constricted, 14-16 × 6-8 $\mu \mathrm{m}$, wall smooth. Pycnothyria similar to 
thyriothecia, smaller; pycnothyriospores unicellular, brown, oval to pyriform, often hyaline band present at the centre, 17-21 $\times 9-11$ $\mu \mathrm{m}$, wall smooth.

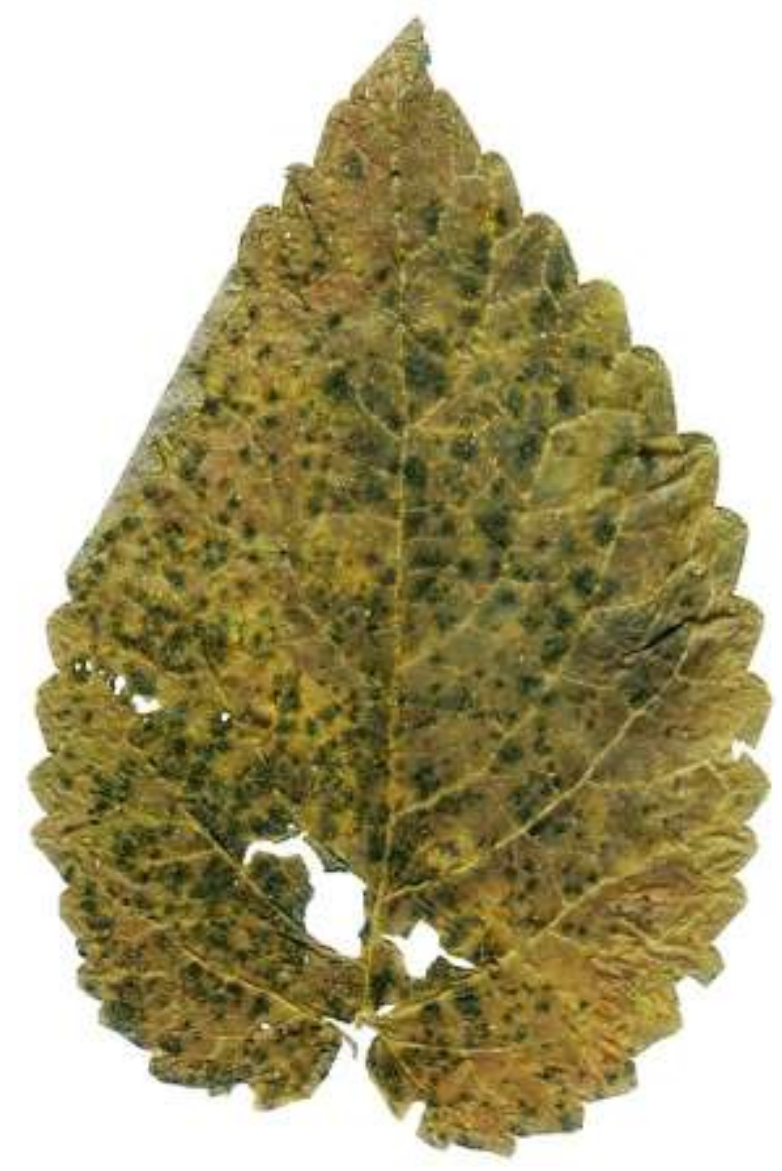

Figure 178. Asterina plectranthi - Infected leaf

Material examined: Kerala, Idukki, Munnar, Rajamala, on leaves of Plectranthus sp. (Lamiaceae), 20 January 2003, A. Manoj Kumar \& H. Biju HCIO 45179 (holotype), TBGT 1340 (isotype); Munnar, Mannavan Shola, 11 May 1999, C.K. Biju HCIO 45184, TBGT 1345; Tamil Nadu, Kodaikanal, Periya Kanal, 11 November 2007, R. Nithyatharani TBGT 4719; Kuthareyar, 19 November 2007, R. Nithyatharani TBGT 4721; 18 August 2008, R. Nithyatharani TBGT 4729; 7 February 2008, R. Nithyatharani TBGT 4731.

There are five species of the genus Asterina known on the members of the family Lamiaceae (Hosagoudar \& Abraham, 2000). Based on the two celled appressoria and ascospore measurements, the present taxon is similar to A. geniospori Hansf. but differs from it in having entire to deeply lobate head cells in contrast to lobate ones.

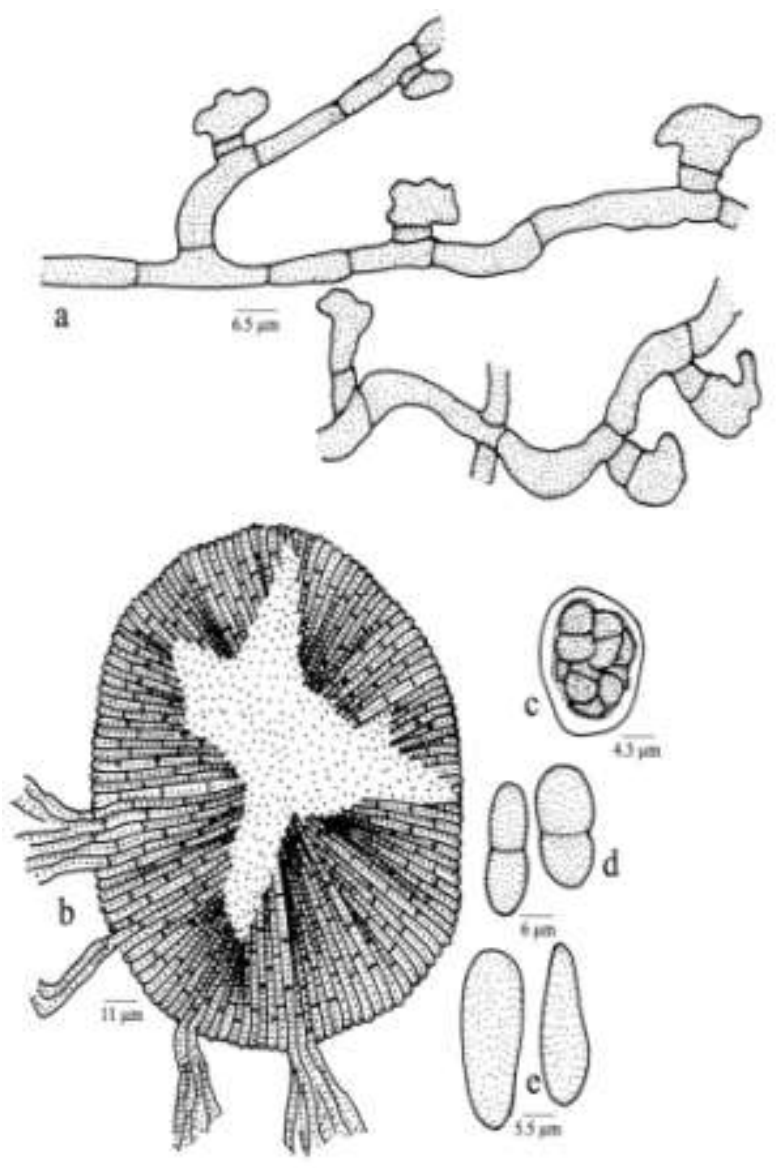

Figure 179 - Asterina plectranthi

Distribution: Kerala, Tamil Nadu

Asterina plurisporus Ryan, Mem. Dept. Agric. Ind. Bot. 15:104, 1928.

Colonies hypophyllous, form large irregular black patches, $2-10 \mathrm{~mm}$ in diam. Hyphae brown, flexuous, up to $6 \mu \mathrm{m}$ broad; appressoria crook-shaped, one cells, 12-14 $\times$ 24-36 $\mu \mathrm{m}$. Thyriothecia round, ostiolate (?), margin light brown, radiate, 264-848 $\mu \mathrm{m}$ in diam.; asci ovate to globose, 80-84 $\times$ 84-104 $\mu \mathrm{m}$; ascospores brown, 2-celled, 24- $\times$ 40-42 $\mu \mathrm{m}$.

Karnataka, Karwar, North Canara, Siddapur, on leaves of Shorea talura Roxb. (Dipterocarpaceae), October 1919, L.J. Sedwick HCIO 1975a (holotype).

Distribution: Karnataka 
Asterina pogostemonis Petrak, Sydowia 12: 476, 1958; Hosag. \& Abraham, J. Econ. Taxon. Bot. 4: 570, 2000; Hosag.,C.K. Biju\& Abraham, J. Mycopathol. Res. 40:195, 2002; Hosag.,C.K. Biju \& Abraham, J. Econ. Taxon. Bot. 28: 179, 2004; Hosag., Zoos' Print J. 21: 2328, 2006; Hosag., Chandraprabha \& Agarwal, Asterinales of Kerala, p. 135, 2011. (Fig. 180)

Colonies amphigenous, mostly epiphyllous, dense, up to $2 \mathrm{~mm}$ in diameter, rarely confluent. Hyphae flexuous to crooked, branching irregular at acute angles, 3-4 hyphal strands run together to form compact mycelial strip, loosely to closely reticulate, cells $17-21 \times$ 3-4 $\mu \mathrm{m}$. Appressoria few, two celled, alternate, straight to rarely curved, antrorse to subantrorse or perpendicular to the hyphae, 11-13 $\mu \mathrm{m}$ long; stalk cells cylindrical, 3-5 $\mu \mathrm{m}$ long; head cells ovate, oblong, clavate hamate, straight to curved, angular, sublobate to deeply lobate, 6$10 \times 6-13 \mu \mathrm{m}$. Thyriothecia scattered, loosely grouped to connate, orbicular, up to $110 \mu \mathrm{m}$ in diameter, stellately dehisced at the center, margin crenate; asci not seen; ascospores conglobate, brown, 1-septate, constricted, 19-24 $\times 8-10 \mu \mathrm{m}$, wall smooth to punctate.

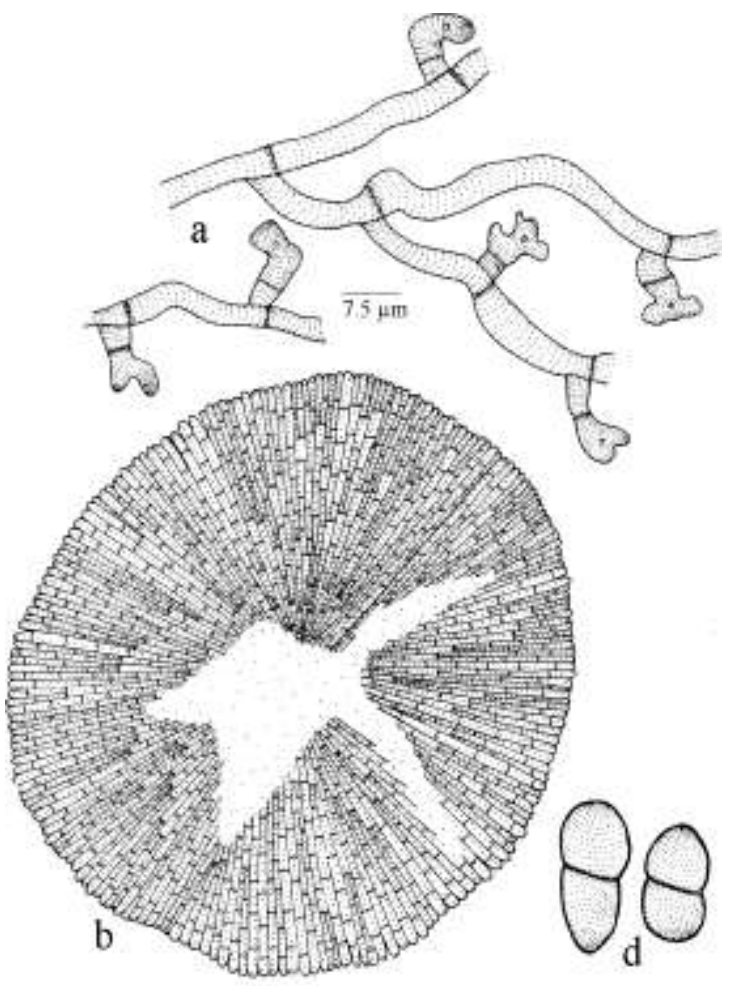

Figure 180 - Asterina pogostemonis
Material examined: Kerala, Idukki, Rajamala, on leaves of Sentellaria violacea Heyne ex Benth. (Lamiaceae), 7 January 1999, C.K. Biju HCIO 43001, TBGT 401; Tamil Nadu, Kodaikanal, Periya Kanal, Pogostemon bengalensis (Burm.f.) Kuntze (Lamiaceae), 15 February 2008, R. Nithyatharani TBGT 4385; Kerala, Idukki, Rajamala, on leaves of Pogostemon sp. (Lamiaceae), 14 September 1999, C.K. Biju HCIO 43612, TBGT 288.

This species was known on Pogostemon velatus from Philippines (Petrak, 1958) and is reported here for the first time from India. Ascospores in the present collection are slightly larger.

Distribution: Kerala, Tamil Nadu

Asterina polygalae Hosag., Dhivaharan \& Nithyatharani, J. Sci. Environ. Technov. 4:46, 2010. (Fig. 181)

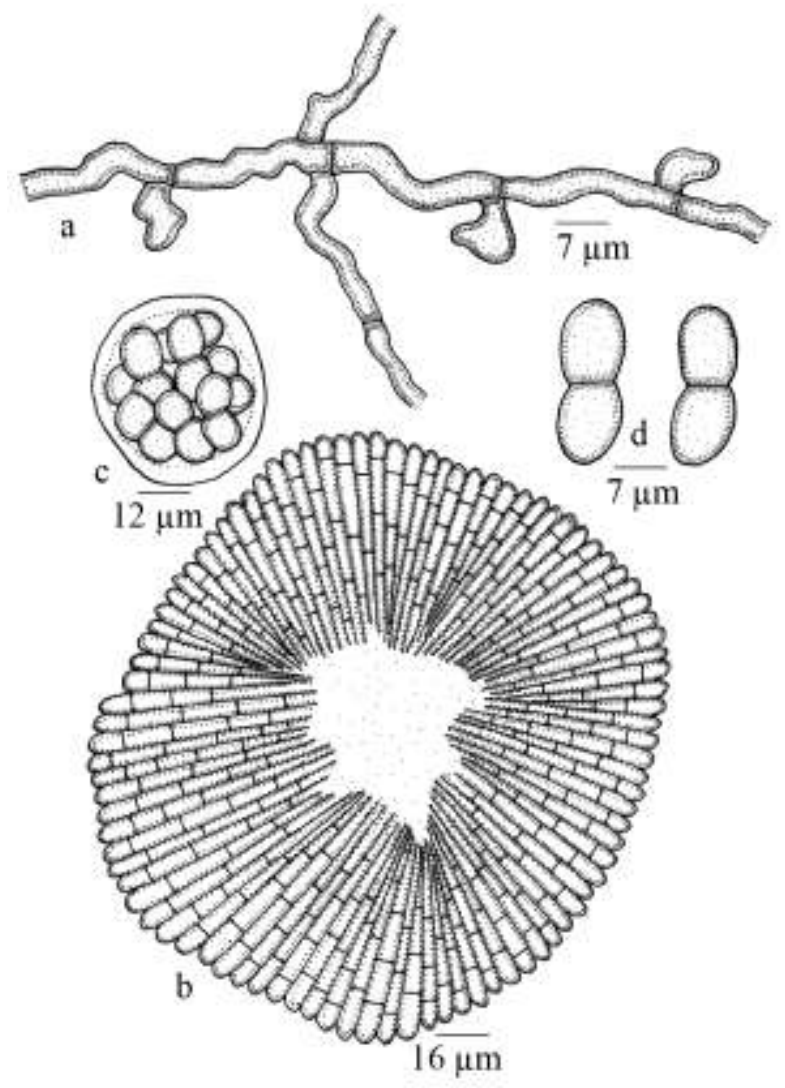

Figure 181. Asterina polygalae

Colonies amphigenous, subdense, up to $2 \mathrm{~mm}$ in diameter, confluent. Hyphae crooked, branching opposite at wide angle, loosely reticulate, cells $17-20 \times 6-7 \mu \mathrm{m}$. Appressoria alternate, unilateral, pyriform, globose, ovate, 
angular to sublobate, $7-12 \times 7-10 \mu \mathrm{m}$. Thyriothecia grouped, orbicular, up to $165 \mu \mathrm{m}$ in diameter, dehiscing stellately at the center, margin fimbriate; asci many, globose, eight spored, 30-35 $\mu \mathrm{m}$ in diameter; ascospores conglobate, 1-septate, slightly constricted at the septum, $20-22 \times 7-10 \mu \mathrm{m}$, wall smooth.

Material examined: Tamil Nadu, Kodaikanal, Kukkal shola forest, on leaves of Polygala arillata Buch.-Ham. ex D. Don (Polygalaceae), 2 November 2007, R. Nithyatharani TBGT 4262a (holotype); Kodaikanal, Kuthuraiyar, 21 September 2007, R. Nithya tharani TBGT 4416; 2 September 2008, R. Nithyatharani TBGT 4444; 26 December 2007, R. Nithyatharani TBGT 4458; Kodaikanal,Thalavakanal, 8 February 2008, R. Nithya tharani TBGT 4465.

\section{Asterina bredmeyerae Rehm on} Bredmeyera sp. and A. purchella Petrak on Seridaca sp. are known on the members of the family Polygalaceae (Hosagoudar \& Abraham, 2000). However, A. polygalae differs from the former species in having crooked hyphae with angular to sublobate cells of appressoria (Theissen, 1913a,b); it also differs from $A$. purchella Petrak in having smaller thyriothecia, asci and ascospores (Stevens \& Ryan, 1939).

Distribution: Tamil Nadu.

Asterina pongalaparensis Hosag., C.K. Biju \& Abraham, Indian Phytopath. 54: 138, 2001; Hosag., Chandraprabha \& Agarwal, Asterinales of Kerala, p. 136, 2011. (Fig. 182)

Colonies amphigenous, subdense to dense, up to $4 \mathrm{~mm}$ in diameter, rarely confluent. Hyphae undulate to crooked, branching alternate to unilateral at acute angles, loosely to closely reticulate, cells 17-23 $\times$ 4-6 $\mu \mathrm{m}$. Appressoria alternate, unilateral, two celled, straight to variously curved, smooth to variously bulged, entire to lobate, 5-10 $\mu \mathrm{m}$ long; head cells clavate, ovate, cylindrical, hamate, straight to curved, 3-7 times sublobate to lobate, $7-13 \times 11-13 \mu \mathrm{m}$. Thyriothecia scattered, orbicular, up to $150 \mu \mathrm{m}$ in diameter, stellately dehisced at the center, margin fringed, fringed hyphae flexuous, exappressoriate; asci globose, octosporous, 22-33 $\mu \mathrm{m}$ in diameter; ascospores oblong, cylindrical, brown, uniseptate, strongly constricted at the septum, 20-25 × 10-13 $\mu \mathrm{m}$, wall echinulate.

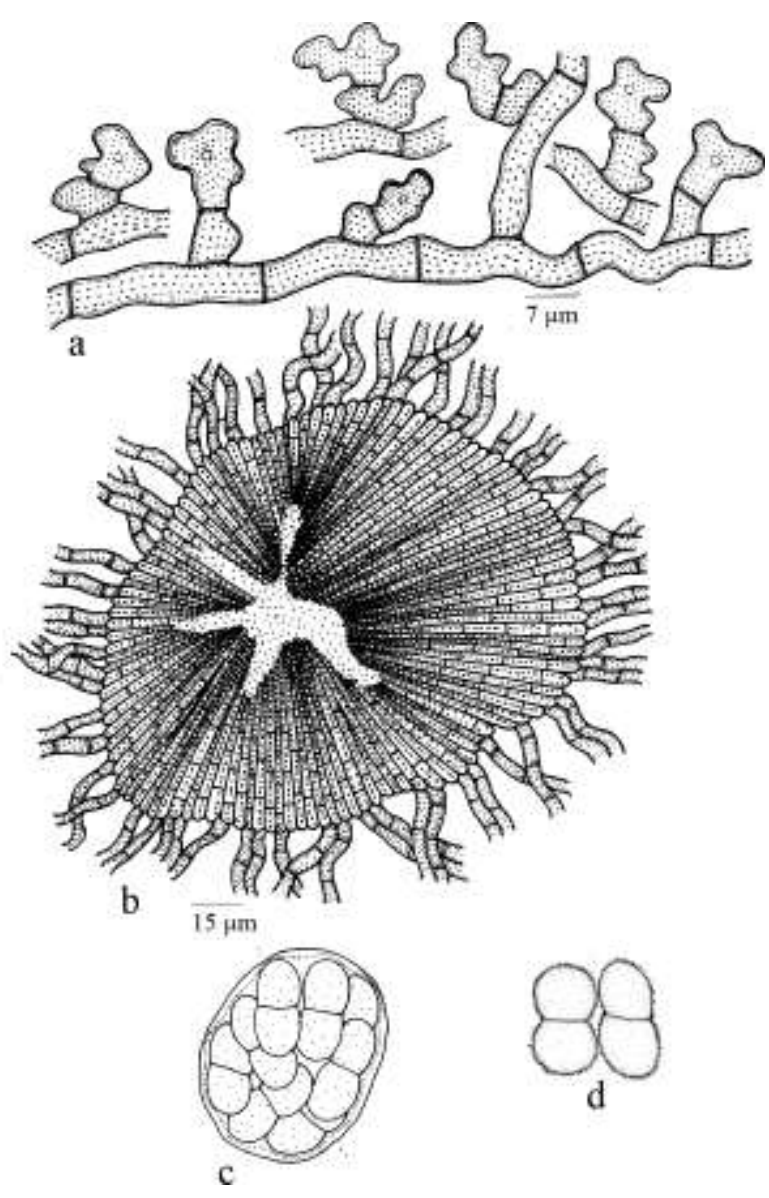

Figure 182 - Asterina pongalaparensis

Material examined: Kerala, Thiruvananthapuram, Chemunji, on leaves of Jasminum sp. (Oleaceae), 11 March 1997, V.B. Hosagoudar HCIO 44554, TBGT 846; Thiruvananthapuram, Ponmudi, 29 November 2005, V.B. Hosagoudar HCIO 47675, TBGT 2701; Thiruvananthapuram, Palode, on leaves of J. sambac (L.) Aiton, 12 March 2008, A. Chandra Prabha HCIO 48295, TBGT 3014; 10 October 2008, A. Chandra Prabha HCIO 48891, TBGT 3267.

There are six taxa of the genus Asterina known on the members of the family Oleaceae (Yates, 1918a; Doidge, 1942; Hansford, 1945, 1948; Yamamoto, 1956; Hosagoudar \& Goos, 1996). Asterina spissa Sydow known on this host genus is a doubutful species since it does not have appressoria (Sydow et al., 1911). 
However, Asterina pongalaparensis is similar to Asterina jasmini- trinervis Yamam. but differs from it in having dense colonies, irregularly bulged and gibbous stalk cells.

Distribution: Kerala.

Asterina prataparajii Hosag. \& Jacob-Thomas, J. Appl. Nat. Sci. 2: 93, 2010.

(Fig. 183)

Colonies hypophyllous, subdense, up to $3 \mathrm{~mm}$ in diameter, mostly confluent. Hyphae flexuous, branching opposite to alternate at acute angles, loosely to closely reticulate, cells 17-22x 3-4 $\mu \mathrm{m}$. Appressoria numerous, alternate, 2-celled, antrorse to subantrorse, 12$16 \mu \mathrm{m}$ long; stalk cells cylindrical, 3-10 $\mu \mathrm{m}$ long; head cells ovate, globose, lobate, , 6-10 $\times$ 8-10 $\mu \mathrm{m}$. Thyriothecia scattered, often 1-2 connate, orbicular, up to $108 \mu \mathrm{m}$ in diameter; margin crenate to fimbriate, dehiscing stellately at the center; asci many, globose, octosporous, 31-38 $\mu \mathrm{m}$ in diameter; ascospores conglobate, brown, 1-septate, deeply constricted at the septum, 14-19 × 10-12 $\mu \mathrm{m}$, wall smooth.

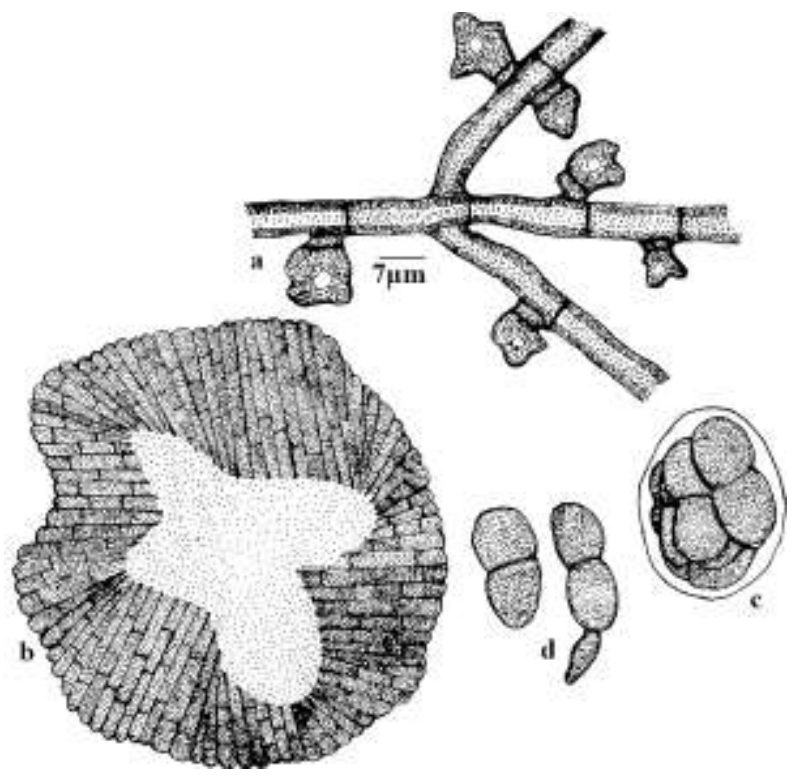

Figure 183 - Asterina prataparajii

Material examined: Maharashtra, Mahabeleswar, on leaves of Tylophora dalzelli (Burm.f.) Merr. (Asclepiadaceae), 1 January 2009, V.B. Hosagoudar \& al. TBGT 3689 (holotype), HCIO 49444 (isotype).
There are eight species of the genus Asterina known on the members of the family Asclepiadaceae, namely Asterina asclepiadis Hosag. \& Goos (Hosag. \& Goos, 1996), A. concinna Sydow (Sydow, 1930), A. cynanchi Hosag. \& Shiburaj (Hosag., 2002), A. heterostemmatis Yamam. (Yamamoto, 1956), A. leonensis Sydow (Sydow, 1938), $A$. peraffinis Speg. (Theissen, 1913), A. toxocarpi Hosag. \& C.K. Biju (Hosagoudar, 2005), A. travancorensis Sydow \& Sydow (Sydow \& Sydow, 1915), and A. tylophorae-indicae Hosag. et al. (Hosag., 2006). All these species having two celled appressoria except Asterina tylophorae-indicae. However, the present species differs from it in having typically lobate appressoria.

\section{Distribution: Maharashtra}

Asterina psychotriicola Hosag. \& Archana, Indian J. Sci. \& Technov. 2(6):1, 2009. (Fig. 184)

Colonies epiphyllous, subdense to dense, up to $3 \mathrm{~mm}$ in diameter, often confluent. Hyphae flexuous, branching irregular at acute to wide angles, form a loose net, cells 9-35 $\times 3$ $5 \mu \mathrm{m}$. Appressoria ovate, clavate, slightly stipitate, unicellular, attenuated and broadly rounded at the apex, entire, 8-13 × 6-10 $\mu \mathrm{m}$. Thyriothecia scattered, orbicular, up to $400 \mu \mathrm{m}$ in diameter, stellately dehisced at the centre, margin mostly crenate; asci globose, octosporous, up to $30 \mu \mathrm{m}$ in diameter; ascospores oblong, brown, conglobate, uniseptate, constricted at the septum, 20-24 ×9$13 \mu \mathrm{m}$, wall strongly tubercled.

Material examined: Karnataka, South Canara, Mangalore, Kukke Subramanya, on leaves of Psychotria sp. (Rubiaceae), 15 November 2003, V.B. Hosagoudar \& al. HCIO 46689 (holotype), TBGT 2030 (isotype).

Asterina psychotriae Ryan is known on this host genus from Puerto Rico (Stevens \& Ryan, 1939). A. psychotiicola differs from it in having longer appressoria and ascospores.

Distribution: Karnataka. 


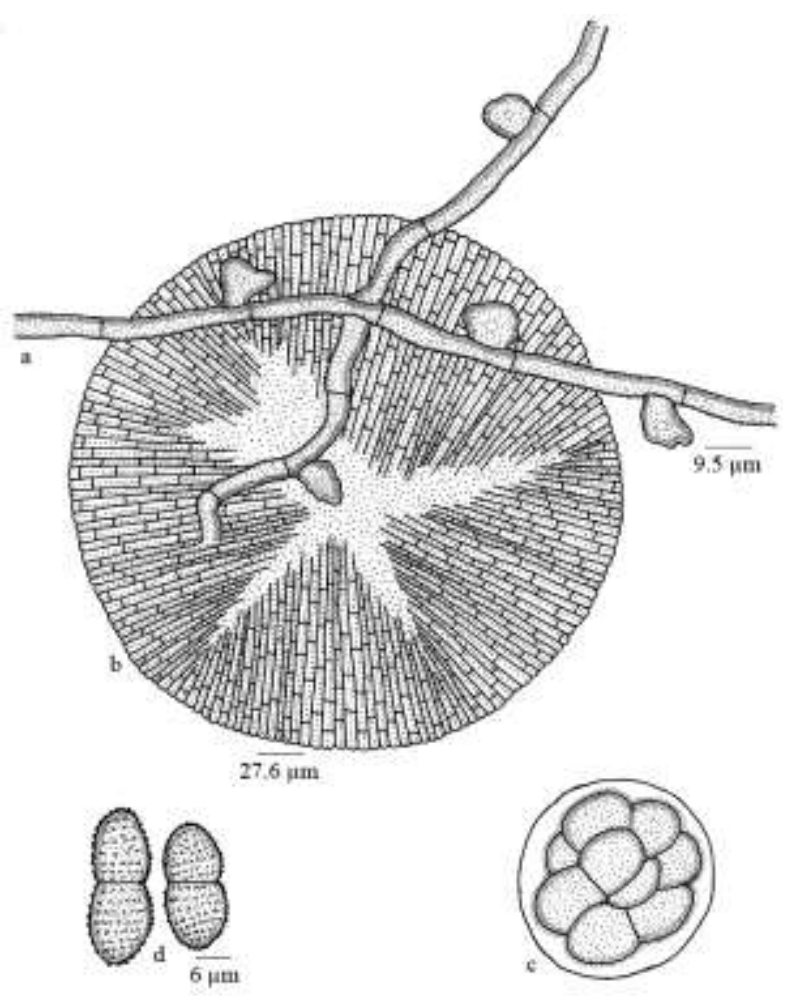

Figure 184 - Asterina psychotriicola

Asterina pusilla Sydow \& Sydow, Sydow Philippine J. Sci. 8: 488, 1913; Hosag. \& Sabeena, Zoos' Print J. 22: 2786, 2007; Hosag., Chandraprabha \& Agarwal, Asterinales of Kerala, p. 137, 2011. (Fig. 185)

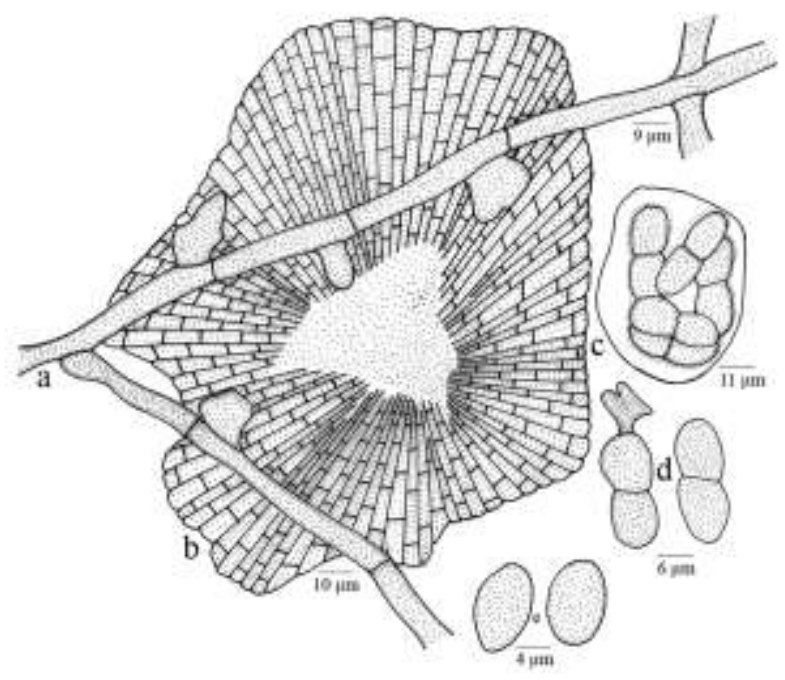

Figure 185 - Asterina pusilla

Colonies epiphyllous, thin, dense, crustose, up to $2 \mathrm{~mm}$ in diameter, confluent. Hyphae straight to undulate, branching alternate, opposite to irregular at acute to wide angles, loosely reticulate, cells 20-48 $\times 3-5 \mu \mathrm{m}$. Appressoria alternate, scattered, unicellular, antrorse, subantrorse to retrorse, sublobate to lobate, mostly broad based, $8-11 \times 6-11 \mu \mathrm{m}$. Thyriothecia closely scattered, orbicular, up to $136 \mu \mathrm{m}$ in diameter, stellately dehisced at the centre, margin fimbriate, fringed hyphae long, crooked; asci globose, up to $34 \mu \mathrm{m}$ in diameter; ascospores brown, conglobate, uniseptate, constricted at the septum, 16-22 $\times 6-10 \mu \mathrm{m}$, wall smooth. Pycnothyria numerous; pycnothyriospores brown, ovate, globose, pyriform, $10-15 \times 10-14 \mu \mathrm{m}$.

Material examined: Kerala, Kozhikode, Malabar Botanic Garden, Kerala, on leaves of Premna corymbosa Rottl. \& Willd. (Verbenaceae), 16 October 2006, A. Sabeena \& al. HCIO 47492, TBGT 2530; on leaves of $P$. serratifolia L., 3 September 2009, A. Sabeena \& M.C. Riju TBGT 4552; Thiruvananthapuram, TBGRI Campus, 26 September 2008, A. Sabeena \& M. C. Riju HCIO 49127, TBGT 3382; HCIO 49129, TBGT 3383; 5 September 2008, A. Sabeena \& M.C. Riju HCIO 49522, TBGT 3764; 17 September 2008, K. Anil Kumar TBGT 5134; 9 February 2009, K. Anil Kumar TBGT 5269; Kannur, Payyanur, Edat, on leaves of $P$. latifolia Roxb., 21 January 2011, P. Ramya TBGT 4926; Thiruvananthapuram, TBGRI Campus, Palode, Medicinal Plants Garden, on leaves of Premna sp., 15 May 2007, G.R. Archana \& al. HCIO 49344, TBGT 3589; Karnataka, Kodagu, Hoddur, 11 January 2009, C. Jagath Thimmaiah TBGT 5394.

This species was known on Premna nauseosa from Philippines (Sydow \& Sydow, 1913; Hosagoudar \& Abraham, 2000). Perhaps, this is the first collection after its type collection.

Distribution: Karnataka, Kerala.

Asterina rhabdodendri Sydow var. levibus M. S. Patil \& Pawar, Indian Phytopathol. 42:251, 1989.

Colonies hypophyllous, circular to irregular in out line, black crust-like, 3-10 mm in diam.; brown; hyphae reticulately branched, 
cells cylindrical, 12-16 $\times 4 \mu \mathrm{m}$; appressoriate; appressoria alternate, sparse, 2-celled, cylindrical to ovate, $11-16 \times 5 \mu \mathrm{m}$, stalk cell short, cylindrical, $2-4 \mu \mathrm{m}$ in length, head cells 9-12 $\times 4 \mu \mathrm{m}$, globose, brown; scutellum composed of radially arranged cells, dehisce by fissures, 100-125 $\mu \mathrm{m}$ in diam.; asci globose, bitunicate, 8-spored, pseudoparahysate, 32-48 $\mu \mathrm{m}$ in diam.; ascospores oblong-ovate, 1septate, constricted at the septa, rounded at both the ends, brown, smooth, poystichous, 21-25 $\times$ $10 \mu \mathrm{m}$. Conidial state not observed.

Maharshtra, Kolhapur, Patgaon, on leaves of Acronychia laurifolia (Rutaceae), 19 October 1984, A. B. Pawar HCIO 39419.

Distribution: Maharashtra

Asterina rhamni Kar \& Ghosh, Indian Phytopathol. 39: 206, 1986.

Patches continued to the upper surface of the leaves, forming black, scattered, round, thin patches, up to $3 \mathrm{~mm}$ in diam. Sometimes the patches coalesce producing an irregular shape; mycelium superficial, densely reticulate, composed of dark brown, septate much branched, straight to wavy hyphae, 3.6-8 $\mu \mathrm{m}$ wide, (cells mostly $12-56 \mu \mathrm{m}$ long) giving rise to opposite, one sided or irregular branches and appressoria at an acute to wide angle; appressoria are one sided, alternate or irregularly arranged, unicellular, 2-4 lobed, brown, straight or bent, 6-11.2 $\mu \mathrm{m}$; thyriothecia many, scattered or in groups, present in the centre of the mycelial colony like pin heads, round, $60-180 \mu \mathrm{m}$ diam., sometimes coalescing with each other and taking an irregular shape, attached with free mycelium, dimidiate with radiate scutellum, stellately dehiscing at first, subsequently developing in to an aperture, brown, convex, margin subcrenate, hymenium simple; asci many, round, sessile, 8-spored, 20$48 \mu \mathrm{m}$, paraphysate; ascospores crowded, fusiform-elliptical, uniseptate, constricted at the septum, upper cell slightly conic, brown, smooth-walled, 15.6-17.2 × 8-9.2 $\mu \mathrm{m}$.

Material examined: West Bengal, Kalijhora (1, $000 \mathrm{ft}$.), Darjeeling, on the living leaves of Rhamnus sp. (Rhamnaceae), 15 September 1977, IMI 225664 (holotype).
Distribution: West Bengal.

Asterina rhodomyrti Hosag., H. Biju \& Manoj. in Hosag., Zoos' Print J. 21: 2335, 2006; Hosag., Chandraprabha \& Agarwal, Asterinales of Kerala, p. 138, 2011. (Fig. 186)

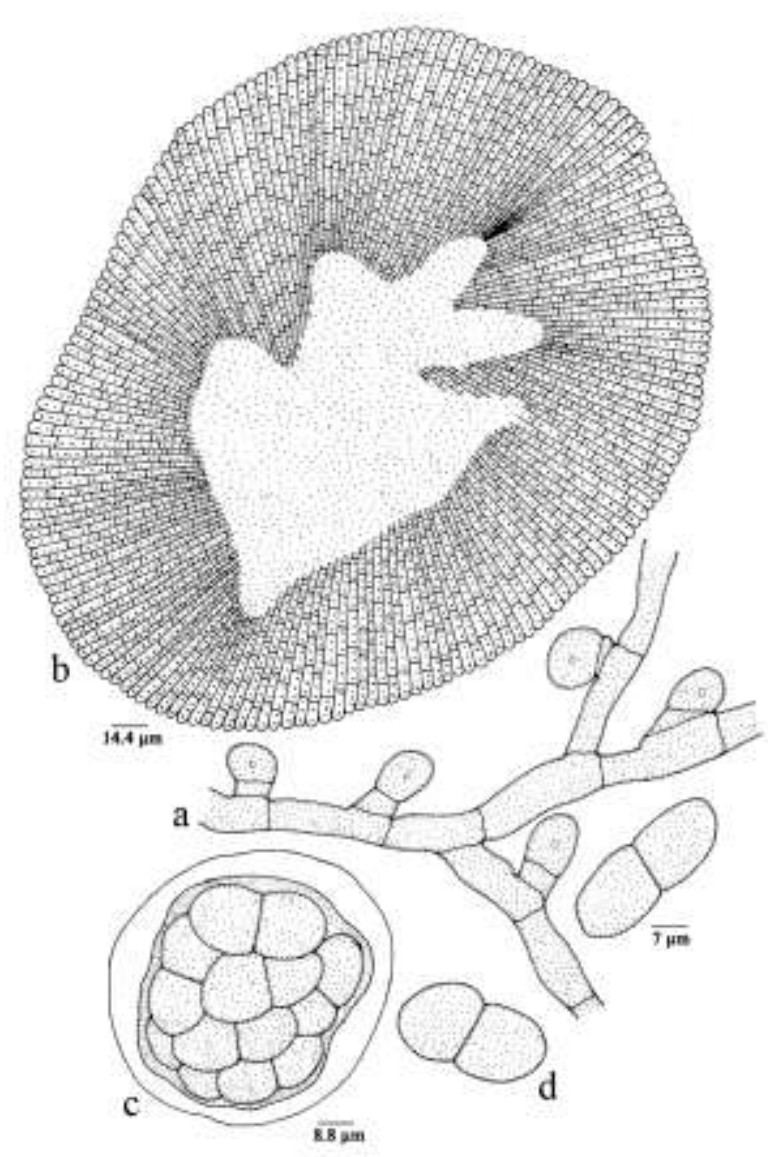

Figure 186 - Asterina rhodomyrti

Colonies amphigenous, mostly epiphyllous, dense, crustose to velvety, up to 2 $\mathrm{mm}$ in diameter, rarely confluent. Hyphae straight to substraight, branching irregular at acute angles, loosely to closely reticulate, cells 19-26 × 4-7 $\mu \mathrm{m}$. Appressoria alternate, about $2 \%$ opposite, closely antrorse, subantrorse to retrorse, two celled, 12-15 $\mu \mathrm{m}$ long; stalk cells cylindrical to cuneate, 3-7 $\mu \mathrm{m}$ long; head cells ovate to globose, entire, $8-10 \times 8-9 \mu \mathrm{m}$. Thyriothecia scattered, orbicular, up to $275 \mu \mathrm{m}$ in diameter, often 3-5 connate, and often elongated, margin crenate, stellately dehisced or dissolved at the centre; asci few to many, globose, octosporous, up to $60 \mu \mathrm{m}$ in diameter; ascospores oblong, conglobate, brown, 
uniseptate, constricted at the septum, 27-30 $\times$ 13-15 $\mu \mathrm{m}$, wall smooth.

Material examined: Kerala, Idukki, Munnar, Rajamala, on leaves of Rhodomyrtus tomentosa (Ait.) Hassk. (Myrtaceae), 20 January, 2003, A. Manoj Kumar \& H. Biju HCIO 44870 (holotype), TBGT 1098 (isotype); Palghat, Silent valley National Park, Sispara, Walakkad, 2 August 2008, Jacob Thomas \& al. HCIO 49037, TBGT 3292; HCIO 49038, TBGT 3293; Kottayam, Placheery, on leaves of Rhodomyrtus sp., 11 July 2007, Robin \& al. HCIO 49853, TBGT 4005.

About 32 species of Asterina are known on the members of the family Myrtaceae (Hosagoudar \& Abraham, 2000). Based on the host specificity and an endemic status of the host plant, a separate entity is maintained for this taxon.

Distribution: Kerala.

Asterina saccopetali Thite \& Kulkarni, Indian Phytopathol. 29: 169, 1976.

Colonies are amphiphyllous, circular, thin, up to $3 \mathrm{~mm}$ in diam. Hyphae sub straight, branched, loosely reticulate; cells mostly 15-20 $\times 3-5 \mu \mathrm{m}$. Appressoria one celled, alternate or opposite or irregular and are irregularly two to three lobed, superficial or subcuticular, 7-9 $\times 5$ $6 \mu \mathrm{m}$. thyriothecia are dark black, flat, dimidiate radiate, circular, up to $250-375 \mu \mathrm{m}$ in diam. asci are oblong, oval bitunicate, eight spored, 35-50 $\mu \mathrm{m}$ in diam. Ascospores irregularly arranged, obovate, elliptical, one septate, slightly constricted at the septum, 25$30 \times 10-12 \mu \mathrm{m}$.

Material examined: Karnataka, Castle Rock, on leaves of Saccopetalum tomentosum H.f. \& T. (Annonaceae), November 1969, A. N. Thite HCIO 31679 (holotype).

Distribution: Karnataka

Asterina sabiacearum Hosag. \& Goos, Mycotaxon 52: 469, 1994; Hosag. \& Abraham, J. Econ. Taxon. Bot. 4: 582,
2000; Hosag., Zoos' Print J. 18: 1284, 2003; Hosag., Zoos' Print J. 21: 2328, 2006; Singh, Duke, Bhandari \& Jain, J. Econ. Taxon. Bot. 30: 184, 2008; Hosag., Chandraprabha \& Agarwal, Asterinales of Kerala, p. 140, 2011. (Fig. 187)

Colonies epiphyllous, subdense, minute, up to $1 \mathrm{~mm}$ in diameter. Hyphae flexuous to slightly crooked, branching alternate to irregular at acute angles, loosely reticulate, cells 30-37 $\times$ 3-5 $\mu \mathrm{m}$. Appressoria alternate, scattered, mostly unicellular, rarely two celled, mammiform, entire to sublobate, 13-22 × 5-7 $\mu \mathrm{m}$. Thyriothecia grouped at the center of the colony, stellately dehisced and widely opened, margin crenate, up to $60 \mu \mathrm{m}$ in diameter; ascospores conglobate, brown, 1-septate, deeply constricted, upper cell larger, lower cell smaller, $18-22 \times 12-14 \mu \mathrm{m}$, wall smooth.

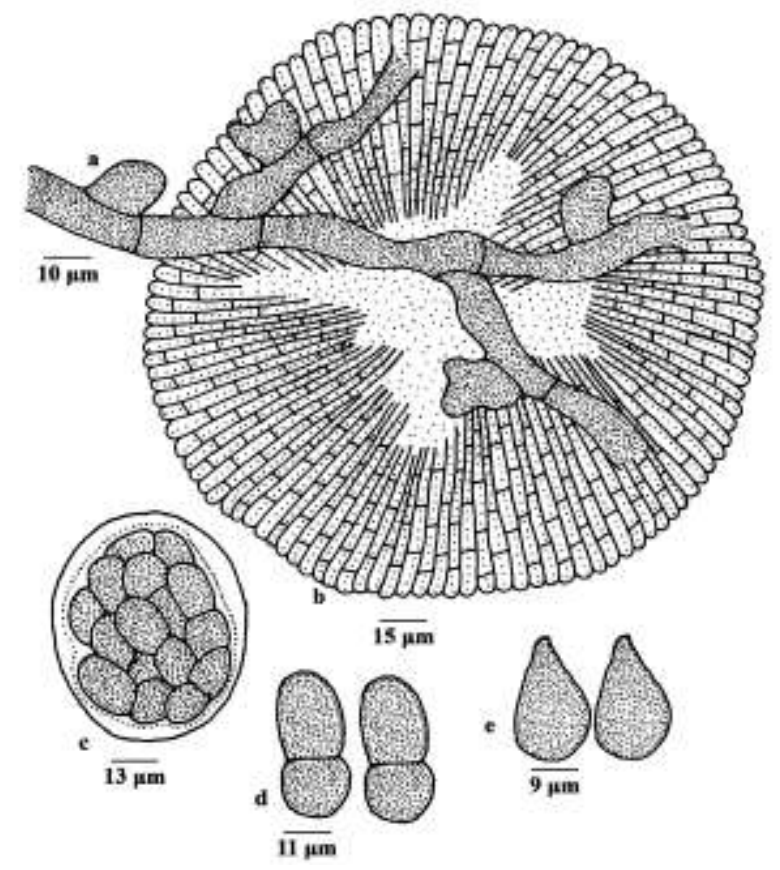

Figure 187 - Asterina sabiacearum

Material examined: Tamil Nadu, Kamarajar dist., Seithur hills, on leaves of Meliosma simplicifolia (Roxb.) Walp. ssp. pungens (Wall ex Wight \& Arn.) Beus (Sabiaceae), 14 November 1992, V.B. Hosagoudar HCIO 40850 (holotype); Kerala, Wayanad,Periya, 17 September 2008, Harish M. \& P.J. Robin HCIO 49070, TBGT 3325; Wayanad, Banasuran mala, on leaves of $M$. simplicifolia (Roxb.) Walp., 19 November 1999, C.K. Biju HCIO 43804, TBGT 371; 
Pathanamthitta, Uppupara, 16 December 2004, V.B. Hosagoudar \& al. HCIO 47712, TBGT 2734; Wyanad, Periya, 26 December 2002, M. Kamarudeen \& P. A. Jose HCIO 44882, TBGT 1110.

This species can be compared with Asterina meliosmaticola Petrak \& Cifferi, reported on Meliosma sp. from which it differs in having unicellular to bi-cellular appressoria, and smaller thyriothecia, asci and ascospores (Petrak \& Cifferi, 1932).

Distribution: Kerala, Tamil Nadu.

Asterina sabiana Kar \& Ghosh, Indian Phytopathol. 39:204, 1986.

Colonies epiphyllous scattered up to 5 mm. in diam.; confluent. Hyphae flexuous, branching irregular at acute angles, loosely reticulate, cells 11-36 × 3-6 $\mu \mathrm{m}$. Appressoria alternate to irregularly scattered, globose, broadly seated to slightly stipitate, straight to curved, deeply lobate 6-11 $\times$ 5-8 $\mu \mathrm{m}$. Thyriothecia scattered to grouped orbicular, up to $190 \mu \mathrm{m}$ in diam.; stellately dehisced at the centre, margin crenate; asci many round to oval, 8-spored, up to $44 \mu \mathrm{m}$. in diam.; ascospores oblong to elliptical, uniseptate, constricted at the septum, rounded at the ends, brown 23-28 $\mu \mathrm{m}$.

Material examined: West Bengal, Coochbehar, Chilapata forest, on leaves of Sabia sp., 30 November 1978, IMI 233946 (holotype).

\section{Distribution: West Bengal}

Asterina samaderae Hosag. \& Manoj. in Hosag., Zoos' Print J. 18: 1038, 2003; Hosag., Chandraprabha \& Agarwal, Asterinales of Kerala, p. 141, 2011.

(Fig. 188)

Colonies hypophyllous, subdense, water soaked, spreading, up to $5 \mathrm{~mm}$ in diameter, confluent. Hyphae straight, substraight to crooked, branching irregular at acute to wide angles, loosely reticulate, cells 20-28 $\times 3-5 \mu \mathrm{m}$. Appressoria scattered, alternate, unilateral, up to $5 \%$ opposite, antrorse, subantrorse, retrorse,
9-13 $\mu \mathrm{m}$ long; stalk cell cylindrical to cuneate, 3-5 $\mu \mathrm{m}$ long; head cells ovate, oblong, conoid, globose, entire, angular, bifid to variously and deeply lobate, straight to variously curved, 6-8 $\times$ 4-10 $\mu \mathrm{m}$. Thyriothecia scattered to loosely grouped, orbicular, up to $250 \mu \mathrm{m}$ in diameter, margin crenate to slightly fimbriate, fringed hyphae small, stellately dehisced at the centre; asci few, globose, octosporous, $30-40 \mu \mathrm{m}$ in diameter; ascospores oblong, conglobate, brown, 1-septate, slightly constricted at the septum, $25-30 \times 11-13 \mu \mathrm{m}$, wall smooth.

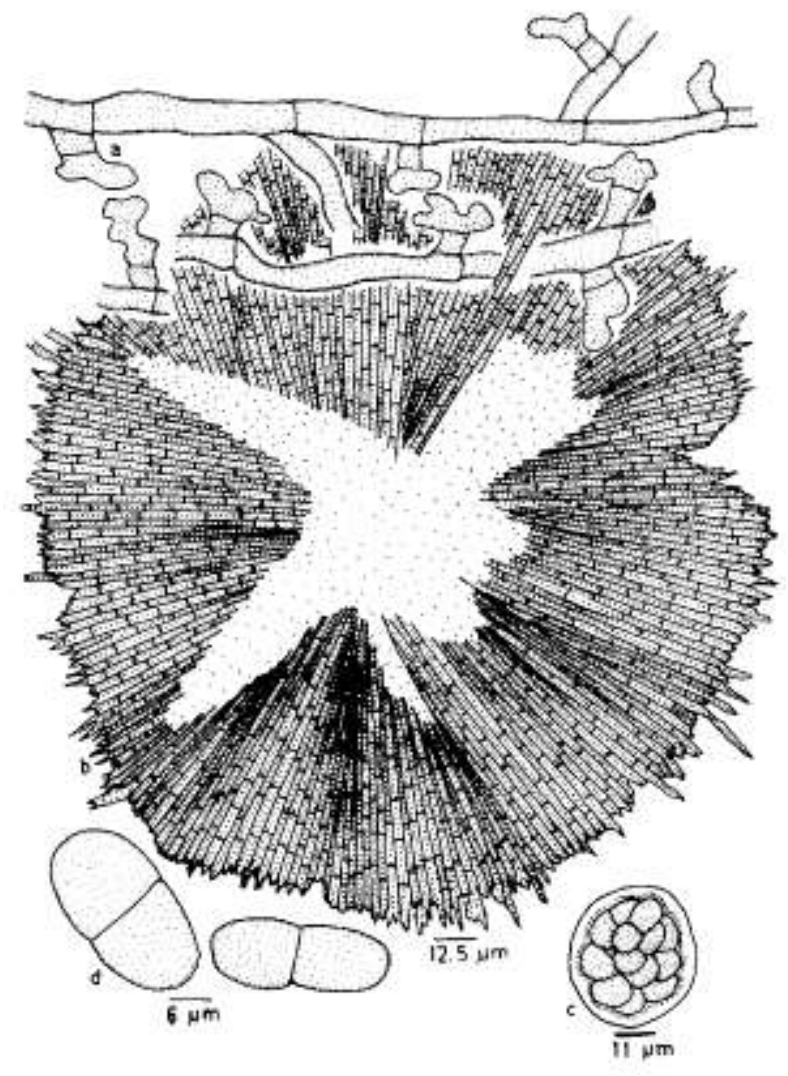

Figure 188 - Asterina samaderae

Material examined: Kerala, Alapuzha, Vandanam sacred grove, on leaves of Samadera indica Gaertn. (Simaroubaceae), 3 October 2002, A. Manojkumar HCIO 44615 (holotype), TBGT 897 (isotype).

Asterina lobata Sydow \& Sydow is the only Asterina species known on the host Picrasma philippensis of Simaroubaceae from Philippines (Sydow \& Sydow, 1912, Hosagoudar \& Abraham, 2000). However, Asterina samaderae differs from it in having two celled appressoria and larger ascospores.

Distribution: Kerala 
Asterina saracae Hosag., Abraham \& Crane, Mycotaxon 68: 19, 1998; Hosag., Zoos' Print J. 18: 1281, 2003; Hosag., Zoos' Print J. 21: 2328, 2006' Hosag., Chandraprabha \& Agarwal, Asterinales of Kerala, p. 141, 2011.

(Figs 189-190)

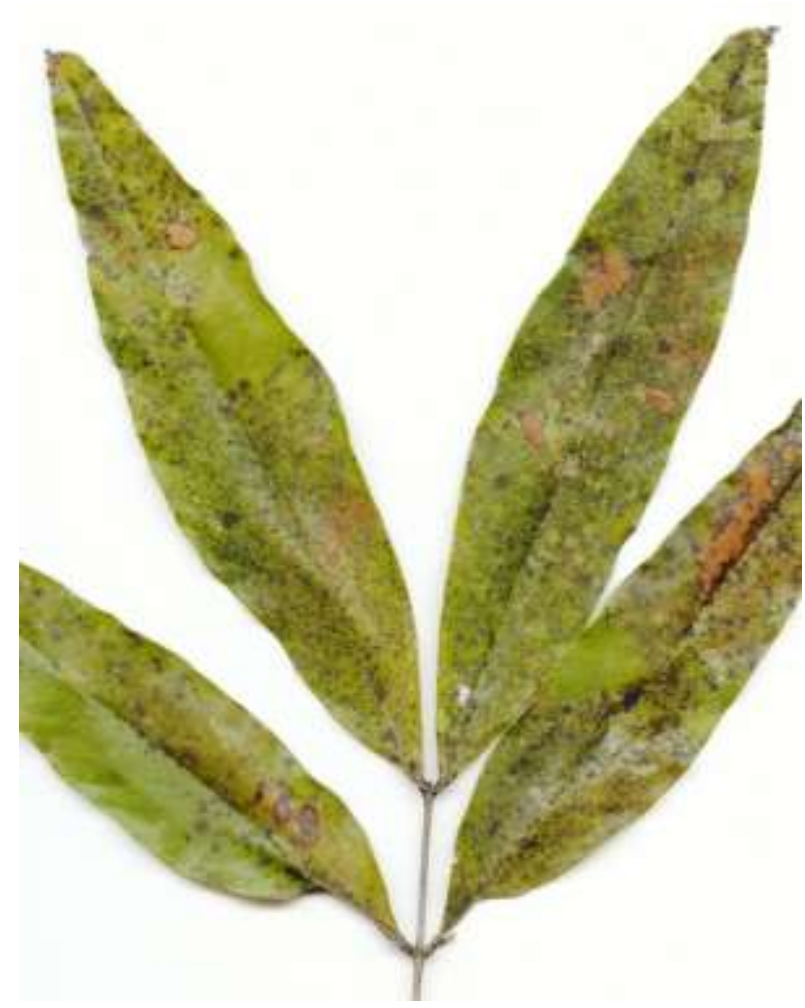

Figure 189 - Asterina saracae-Infected leaves

Colonies epiphyllous, subdense to dense, scattered, up to $2 \mathrm{~mm}$ in diameter, confluent. Hyphae straight to substraight, branching alternate, opposite to irregular at acute angles, loosely reticulate, cells 19-22.5 × 4.5-6.5 $\mu \mathrm{m}$. Appressoria alternate, about 20\% opposite, unicellular, conoid to ampulliform, tapering towards apex, broadly rounded at the apex, entire, 11-16 × 4-5 $\mu \mathrm{m}$. Thyriothecia scattered to loosely grouped, often connate, orbicular to oval, 196-294 $\times 176-196 \mu \mathrm{m}$, stellately dehiscence at the centre, margin crenate; asci globose to ovate, octosporous, 35$38 \times 27-30 \mu \mathrm{m}$; ascospores conglobate, 2celled, constricted at the septum, one cell smaller, $25-29 \times 11-13 \mu \mathrm{m}$, smooth walled.

Material examined: Kerala, Thiruvananthapuram, Palode, TBGRI Campus, on leaves of Saraca asoca (Roxb.) de Willd. (Caesalpiniaceae), 5 December 1996, V.B.
Hosagoudar HCIO 42413, TBGT 76; 23 October 2000, M. M. Shajivaz HCIO 44057, TBGT 488; 10 December 2002, M. Kamarudeen \& P. A. Jose HCIO 44735, TBGT 1016; Kottayam, Changanachery, Near S. B. College, 9 December 2002, A. Manojkumar HCIO 44731, TBGT 1012; Thiruvananthapuram, Peroorkada, 31 January 2003, G. Rajkumar HCIO 45232, TBGT 1269; Palode, TBGRI campus, 5 January 2001, H. Biju HCIO 46073, TBGT 1836; 6 July, 2006, R.Nithyatharani HCIO 47536, TBGT 2558; 25 October 2007, G.R. Archana TBGT 4210; 21 July 2010, A. Sabeena TBGT 4613; TBGT 4615; TBGT 4617; 10 April 2008, A. Sabeena \& M.C. Riju TBGT 4976; TBGT 4978; 14 May 2007, A. Sabeena \& al. TBGT 5007; TBGT 5009; 30 December 2010, C. Anil Kumar TBGT 5011; 27 May 2007, A. Sabeena TBGT 5038.

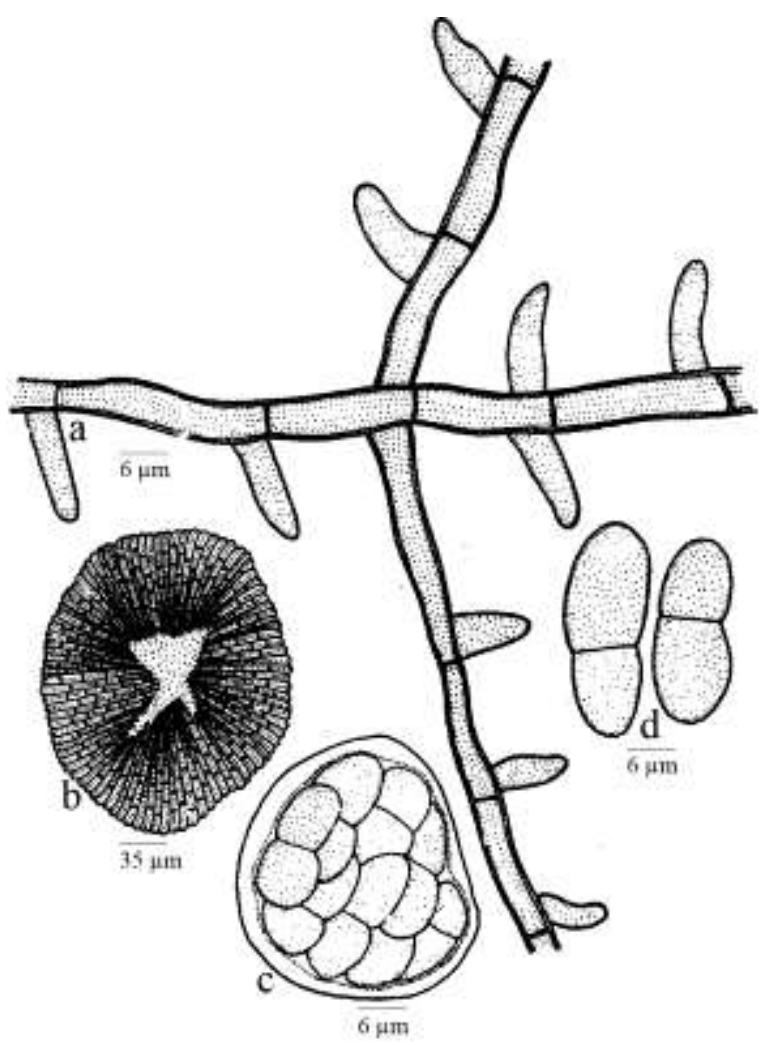

Figure 190 - Asterina saracae

Some thyriothecia were oval and had longitudinal slits reminiscent of the genus Lembosia Lev.; but most were orbicular and stellately dehisced at the centre. Hence, the present species is accommodated in the genus Asterina Lev. Asterina ciferriana Petrak and Asterina (Englerulaster) contigua Sydow are known on Caesalpinia crista L. from 
Dominican Republic and from Sierra Leone (Petrak \& Ciferri, 1932; Sydow, 1938). Asterina (Englerulaster) contigua Sydow appears to be a doubtful species of the genus Asterina because the mycelium is devoid of appressoria. The present species species differs from A. ciferriana Petrak in having 20\% opposite and longer appressoria, in the absence of primary and secondary hyphae, and in having smaller ascospores.

Distribution: Kerala

Asterina sarcandrae Hosag. \& Kamar. in Hosag. , Zoos' Print J. 21: 2305, 2006; Hosag., Chandraprabha \& Agarwal, Asterinales of Kerala, p. 143, 2011. (Fig. 191)

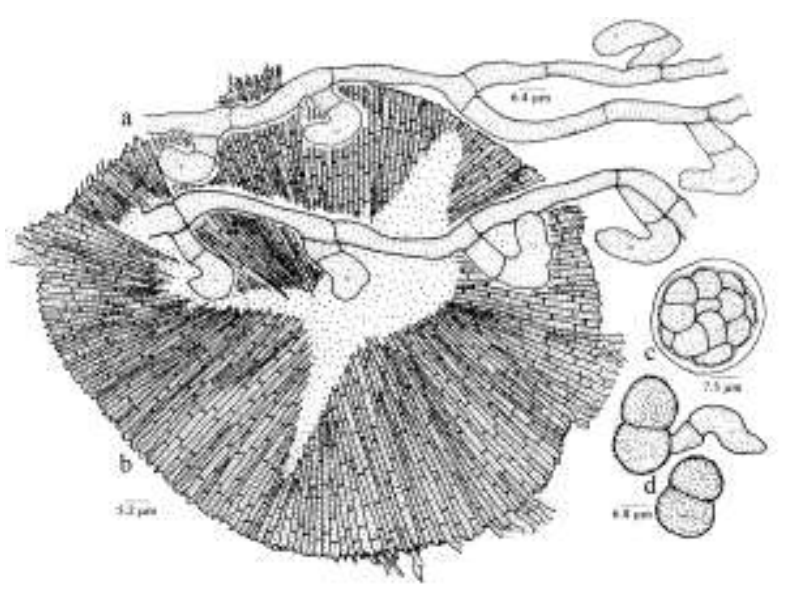

Figure 191 - Asterina sarcandrae

Colonies hypophyllous, very thin, up to $5 \mathrm{~mm}$ in diameter. Hyphae flexuous, branching irregular at acute to wide angles, form a circularly angular and irregular net, cells 16-21 $\times$ 4-7 $\mu \mathrm{m}$. Appressoria scattered, alternate to irregular, two celled, antrorse, retrorse, spreading, straight to curved, 12-32 $\mu \mathrm{m}$ long; stalk cells cylindrical to cuneate, 3-7 $\mu \mathrm{m}$ long; head cells ovate, oblong, mostly curved, hamate, twisted, rarely straight, entire, angular to rarely sublobate, $10-26 \times 6-10 \mu \mathrm{m}$. Thyriothecia scattered, orbicular, stellately dehisced at the centre, up to $104 \mu \mathrm{m}$ in diameter, margin crenate; asci few, globose, octosporous, up to $30 \mu \mathrm{m}$ in diameter; ascospores conglobate, uniseptate, strongly constricted at the septa, 20-22 × 7-9 $\mu \mathrm{m}$, wall echinulate.
Material examined: Kerala, WyanadPeriya, on leaves of Sarcandra chloranthoides Gard. (Chloranthaceae), 26 December 2002, M. Kamarudeen HCIO 44794 (holotype), TBGT 1031 (isotype); Palghat, Silent valley, Sairendhri, 13 December 2003, V.B. Hosagoudar \& al. HCIO 46347, TBGT 1993; 13 February 2007, M.C. Riju \& V. Gireesh Kumar TBGT 4965.

Asterina chloranthi Sydow is known on Chloranthus officinalis from Philippines (Sydow \& Petrak, 1931; Hosagoudar \& Abraham, 2000). However, Asterina sarcandrae differs from it in having very thin hypophyllous colonies, net forming mycelia and longer appressoria.

\section{Distribution: Kerala}

Asterina schimae Hosag., Jacob Thomas \& Robin, Indian J. Sci. Technol. 2(6):2, 2009. (Fig. 192)

Colonies epiphyllous, dense, scattered, up to $2 \mathrm{~mm}$ in diameter, confluent. Hyphae straight to undulate, branching opposite to alternate at acute to wide angles, closely reticulate and form a mycelial mat, cells 12-29 $\times \quad 5-7 \mu \mathrm{m}$. Appressoria opposite (70\%), alternate, unicellular, broad based, sessile, ovate, globose, mammiform, entire, 7-11 × 7$10 \mu \mathrm{m}$. Thyriothecia closely scattered, orbicular to rarely ovate, $130-220 \times 90-160 \mu \mathrm{m}$, dehiscing stellately at the centre, crenate to fimbriate at the margin; asci many, ovate to globose, octosporous, up to $58 \mu \mathrm{m}$ in diameter; ascospores conglobate, brown, 1-septate, constricted at the septum, broadly rounded at both apices, brown, 31-36 × 14-17 $\mu \mathrm{m}$, wall smooth. Pycnothyria similar to thyriothecia, smaller; pycnothyriospores pyriform, brown, $10-24 \times 5-10 \mu \mathrm{m}$.

Material examined: Meghalaya, Shillong, Lumshillong, on leaves of Schima wallachii (DC.) Korthals var. khasiana (Dyer) Bloem. (Theaceae), 18 January 2007, Jacob Thomas \& P.J. Robin HCIO 48018 (holotype), TBGT 2801 (isotype). 
Yamamoto (1957) proposed Asterina theae Yamam. on Thea sinensis from Taiwan and Katumoto (1975) synonymised it to Asterina camelliae Syd. \& Butler and narrated the total history of Schiffnerula camelliae (Sydow, Sydow \& Butler) Hughes. Subsequently, Hosagoudar (2005) have proposed Asterina cannonii Hosag. et al. However, the former two species have alternate appressoria and the latter has 5\% alternate appressoria. A. schimae is similar to Asterina cannonii Hosag. et al. in having alternate appressoria but differs from it in having 70\% opposite appressoria.

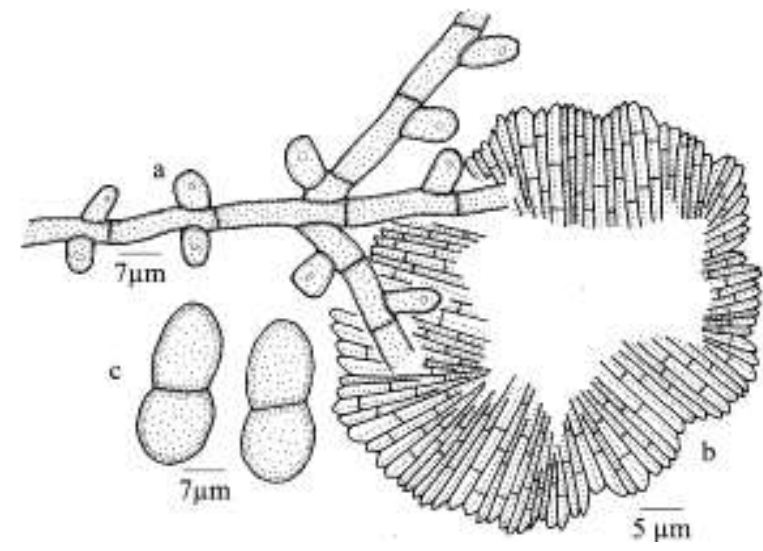

Figure 192 - Asterina schimae

Distribution: Meghalaya.

Asterina scleropyri Hosag. \& Chandra., Indian J. Sci. \& Techn. 2(6):16, 2009; Hosag., Chandraprabha \& Agarwal, Asterinales of Kerala, p. 145, 2011.

(Fig. 193-194)

Colonies amphigenous, mostly epiphyllous, dense, up to $2 \mathrm{~mm}$ in diameter, rarely confluent. Hyphae straight, branching mostly opposite at acute angles, loosely to closely reticulate, cells $15-22 \times 6-9 \mu \mathrm{m}$. Appressoria unicellular, opposite $(80 \%)$ to alternate $(20 \%)$, ovate, conoid, attenuated and broadly rounded at the tip, entire, $8-18 \times 6-9$ $\mu \mathrm{m}$. Thyriothecia loosely grouped at the center of the colony, orbicular, up to $210 \mu \mathrm{m}$ in diameter, stellately dehisced and the central portion dissolved by exposing inner contents, margin crenate; asci globose, octosporous, up to $30 \mu \mathrm{m}$ in diameter; ascospores oblong, conglobate, brown, uniseptate, constricted at the septum, 26-31 $\times 8-13 \mu \mathrm{m}$, wall smooth. Pycnothyria similar to thyriothecia, smaller;

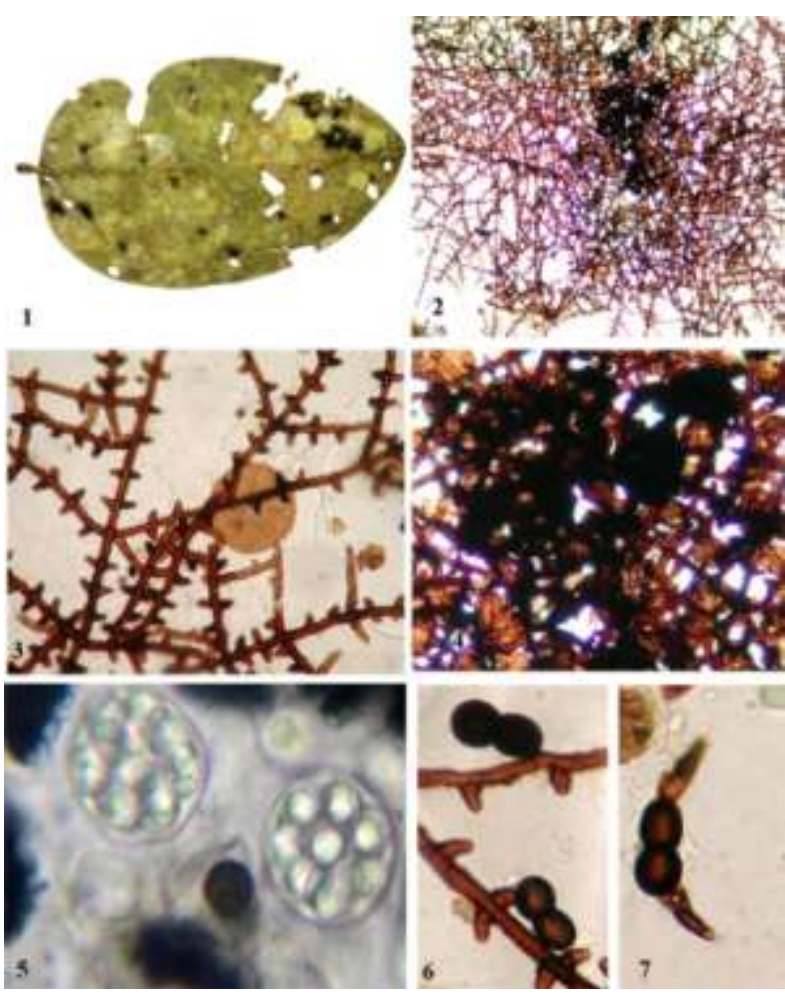

Figure 193 - Asterina scleropyri

1. Infected leaf, 2. Fungal colony with thyriothecia, 3. Appressoriate mycelium, 4. Dehisced thyriothecium, 5. Asci, 6. Ascospore, 7. Germinating Ascospore

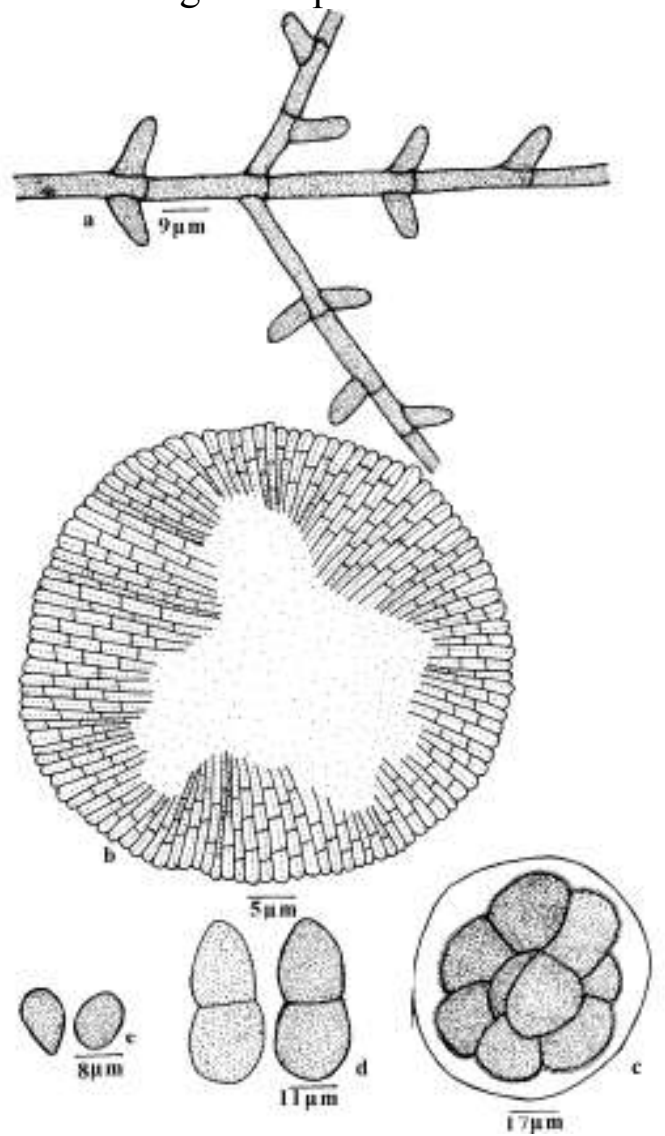

Figure 194 - Asterina scleropyri 
pycnothyriospores ovate, pyriform, brown, 8-13 $\times 4-7 \mu \mathrm{m}$, wall smooth

Material examined: Kerala, Palakkad, Silent Valley National Park, on the leaves of Scleropyrum pentandrum (Dennst.) Mabb. (Santalaceae), 15 May 2007, R. Rama Subbu HCIO 48240 (holotype), TBGT 2978 (isotype).

Asterina congesta Cooke, A. decipiens Sydow, A. elmeri Sydow and A. polythria Doidge are known on the members of the family Santalaceae (Hosagoudar \& Abraham, 2000). The present species differ from all in having unicellular, alternate and opposite, ovate but attenuated appressoria (Hansford \& Thirumalachar, 1948; Sydow \& Sydow, 1939; Doidge, 1942; Stevens \& Ryan, 1939).

\section{Distribution: Kerala}

\section{Asterina songii nom. nov.}

三 Asterina euryae Hosag \& C.K. Biju, Indian Phytopath. 58: 194, 2005; Hosag., Zoos' Print J. 21: 2327, 2006 (non Song, 2004). (Figs 195-196)

MycoBank 101025

Etymology: Named in the honour of Dr. B. Song for his notable contribution to Black Mildews of China

Colonies epiphyllous, dense, crustose, up to $2 \mathrm{~mm}$ diam.; rarely confluent. Hyphae crooked, branching irregular, often form a loose net, cells 12-21 × 4-6 $\mu \mathrm{m}$. Appressoria alternate to unilateral, scattered, ovate, globose, clavate, mostly entire, rarely furcate, $8-13 \times 6-7 \mu \mathrm{m}$. Thyriothecia scattered, orbicular, up to $130 \mu \mathrm{m}$ in diameter, stellately dehisced at the centre, margin crenate to fimbriate, fringed hyphae small, flexuous; asci few, octosporous, globose, up to $40 \mu \mathrm{m}$ in diam.; ascospores oblong, conglobate, uniseptate, deeply constricted, brown, 36-39 × 17-19 $\mu \mathrm{m}$, wall tubercled.

Material examined: Kerala, Idukki, Munnar, Mannavan Shola, on leaves of Eurya nitida Korth. (Theaceae), 26 June 2000, C.K. Biju HCIO 45277 (holotype), TBGT 1315 (isotype); 6 January 1999, C.K. Biju HCIO 45749, TBGT 1498; Palghat, Silent valley, on leaves of E. japonica Thunb., 29 September 2002, S. Shiburaj HCIO 47588, TBGT 2610; Tamil Nadu, Kodaikanal, Bombay shola, Eurya sp., 13 June 2006, R. Nithyatharani HCIO 47089, TBGT 2606.

Asterina euryae Song was proposed on Eurya acuminata from China (Song et al., 2004). A. euryae Hosag. \& C.K. Biju was proposed on Eurya nitida from India. The former species has clavate and angular appressoria in contrast to ovate and entire to rarely furcated appressoria. Hence, a new name has been proposed here to the latter species.

Based on the smooth outer surface of mycelia $A$. songii can be compared with Asterina theacearum Hosag. et al. known on Theaceae members from the same locality (Hosagoudar et al., 1997). However, differs from it in having crooked and net forming mycelia, ovate but shorter appressoria and larger ascospores.

Distribution: Kerala, Tamil Nadu

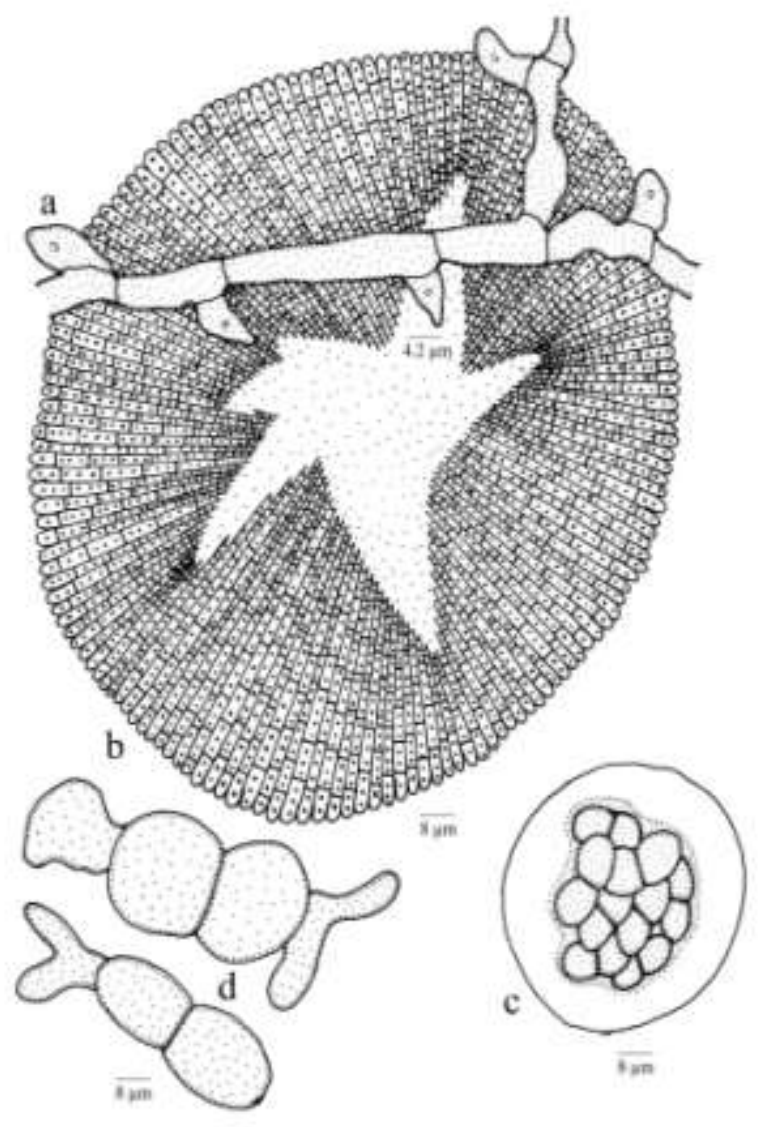

Figure 195 - Asterina songii 


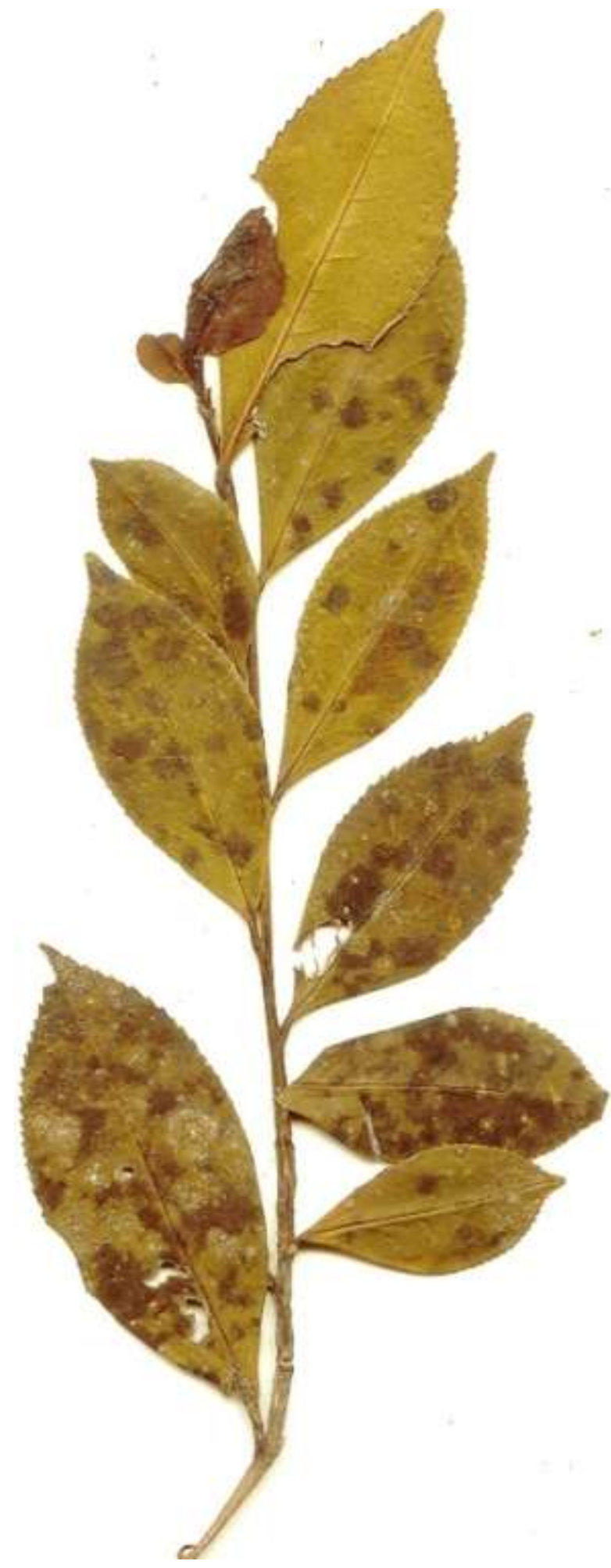

Figure 196 - Asterina songii - Infected leaves

Asterina suttonii Hosag., C.K. Biju \& Abraham, J. Mycopathol. Res. 40: 195, 2002; J. Econ. Taxon. Bot. 28: 181, 2004; Hosag., Chandraprabha \& Agarwal, Asterinales of Kerala, p. 146, 2011. (Fig. 197)

Colonies epiphyllous, minute, dense, up to $1 \mathrm{~mm}$ in diameter, rarely confluent. Hyphae straight, branching alternate, opposite to irregular at acute to wide angles, loosely reticulate, cells 12-15 × 5-6 $\mu \mathrm{m}$. Appressoria unicellular, alternate, ovate, clavate, cylindrical, entire to rarely angular, 11-13 $\times 8-12 \mu \mathrm{m}$. Thyriothecia scattered, orbicular, up to $300 \mu \mathrm{m}$ in diameter, stellately dehisced at the center, margin fimbriate, fringed hyphae elongated, crooked; asci ovate to ellipsoidal, octosporous, 60-65 × 28-32 $\mu \mathrm{m}$; ascospores ellipsoidal, uniseptate, slightly constricted at the septa, taper and broadly rounded at both the apices, 28-32 × 9-11 $\mu \mathrm{m}$, wall smooth. Pycnothyria not seen; Pycnothyriospores many, mostly pyriform, brown, 12-15 $\times$ 9-11 $\mu \mathrm{m}$, wall smooth.

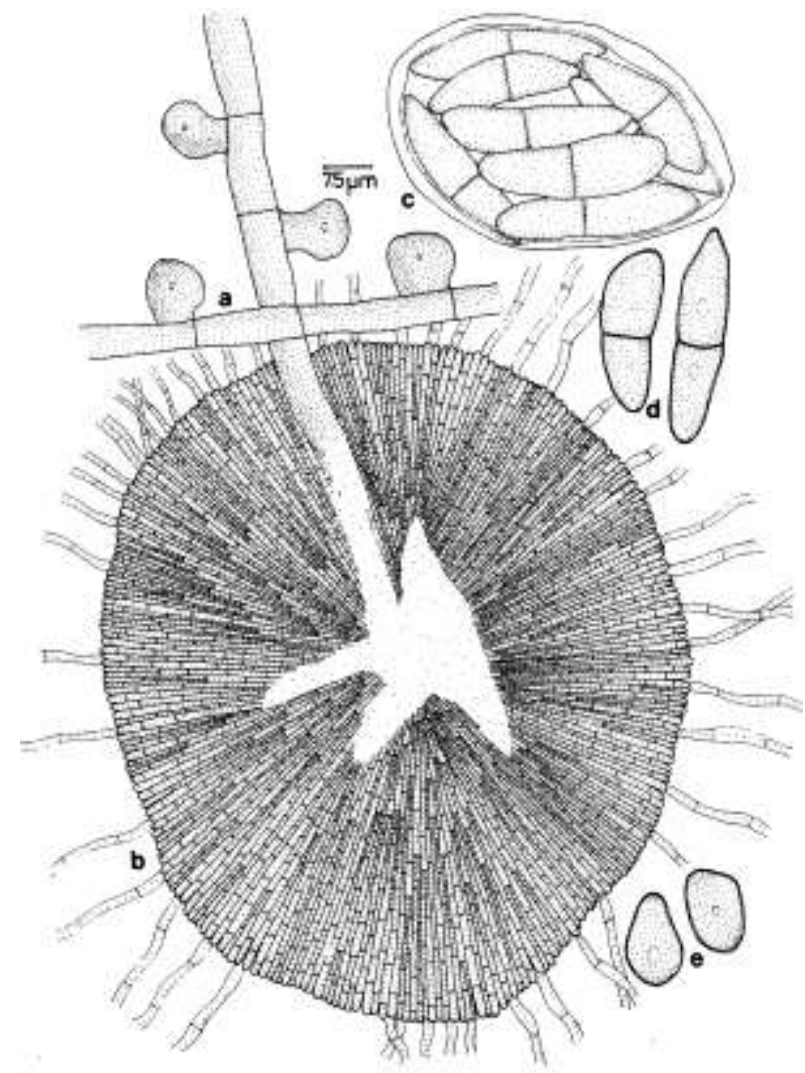

Figure 197 - Asterina suttonii

Material examined: Kerala, Idukki, Rajamala, on leaves of Symplocos sp. (Symplocaceae), 14 September 1999, C.K. Biju HCIO 43455 (holotype), TBGT 274 (isotype); Idukki, Kuttikanam, 17 March 2007, Robin \& al. HCIO 49493, TBGT 3735; Palghat, Silent Valley National Park, 1 March 2009, S.S. Shaji $\&$ al. HCIO 49455, TBGT 3797; Thiruvananthapuram, Neyyar Wildlife sanctuary, Kombe, 5 March 2008, Jacob Thomas HCIO 49593, TBGT 3835; Tamil Nadu, Kodaikanal, Vattakanal, 22 July 2006, 
R. Nithyatharani HCIO 47102, TBGT 2319; HCIO 49477, TBGT 3719; Kodaikanal, 3 January 2009, R. Nithyatharani TBGT 4353.

Asterina indica Sydow, $A$. grammocarpa Sydow and $A$. sodalis Sydow are known on this host genus from India Phillippines and Borneo, respectively (Sydow, 1928; Sydow \& Sydow, 1914; Sydow et al. 1911). However, the present new species differs from all in having larger ascospores tapering towards both ends.

Distribution: Kerala, Tamil Nadu

\section{Asterina talacauveriana Hosag., \\ Mycopathol. Res. 44: 11, 2006.}

(Fig. 198)

Colonies hypophyllous, dense, up to 2 $\mathrm{mm}$ in diameter, confluent. Hyphae straight to substraight, branching mostly opposite at acute to wide angles, loosely to closely reticulate, cells 20-24 × 3-5 $\mu \mathrm{m}$. Appressoria opposite, about $2 \%$ solitary and subopposite, antrorse, subantrorse to rarely retrorse, two celled, 10$16 \mu \mathrm{m}$ long; stalk cells cylindrical to cuneate, 3-5 $\mu \mathrm{m}$ long; head cells ovate, globose, oblong, shallowly and irregularly lobate, 9-11 $\times 6-10 \mu \mathrm{m}$. Thyriothecia scattered to connate, orbicular, stellately dehisced at the centre, up to $130 \mu \mathrm{m}$ in diameter, margin crenate to fimbriate, fringed hyphae very small; asci globose, octosporous, up to $30 \mu \mathrm{m}$ in diameter; ascospores brown, conglobate, uniseptate, constricted, 19-21 × 8-10 $\mu \mathrm{m}$, wall smooth. Pycnothyria mixed with thyriothecia, similar and smaller; pycnothyriospores oval, pyriform, brown, 13-15 × 11-13 $\mu \mathrm{m}$.

\section{Material examined: Karnataka}

Coorg, Madikeri, Talacauvery, Brahmagiri, Medicinal Plant Conservation Area, on leaves of Scolopia sp. (Flacourtiaceae), November 13, 2003, V.B. Hosagoudar HCIO 45700 (holotype), TBGT 1448 (isotype); Coorg, Galibeedu, on leaves of Scolopia crenata (Wight \& Arn.) D. Clos, 24 November 2008, V.B. Hosagoudar \& al. HCIO 49432, TBGT 3677; Talacauveri, 25 November 2008, V.B. Hosagoudar \& al. HCIO 49434, TBGT 3679; Kodagu, Vanachalu, 24 November 2008, C. Jagath Thimmaiah TBGT 5320.

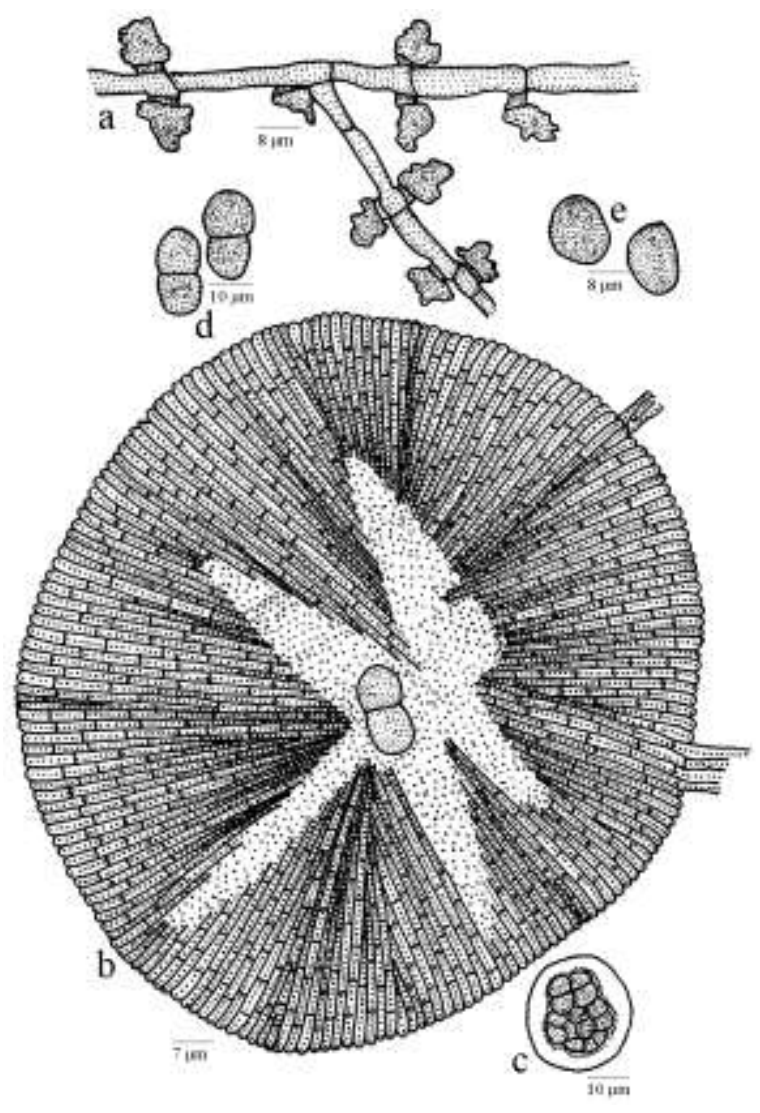

Figure 198 - Asterina talacauveriana

This species is close to Asterina cylindrophora Sydow and A. flacourtiae Petrak in having opposite appressoria. However, differs from both in having lobate head cells of the appressoria and smaller ascospores (Sydow \& Sydow, 1917; Sydow \& Petrak, 1931).

Distribution: Karnataka.

Asterina tertia Racib. in Theiss., Die Gattung Asterina 7:103, 1913; Sacc., Sylloge Fungorum 24: 443, 1926; Hosag. \& Abraham, J. Econ. Taxon. Bot. 4: 558, 2000; Hosag., H. Biju \& Appaiah, J. Mycopathol. Res. 43: 204, 2005; 44:12, 2006; Hosag., Zoos' Print J. 21: 2329, 2006; Hosag., Chandraprabha \& Agarwal, Asterinales of Kerala, p. 147, 2011. (Figs 199-200)

Colonies amphigenous, up to $3 \mathrm{~mm}$ in diameter, confluent. Hyphal cells up to $4 \mu \mathrm{m}$ broad. Appressoria sparse, continuous, 3-4 lobate, continuous, $5-8 \times 8-13 \mu \mathrm{m}$. Thyriothecia $120-160 \mu \mathrm{m}$ in diam.; margin fimbriate, stellately dehisced in the centre; asci 


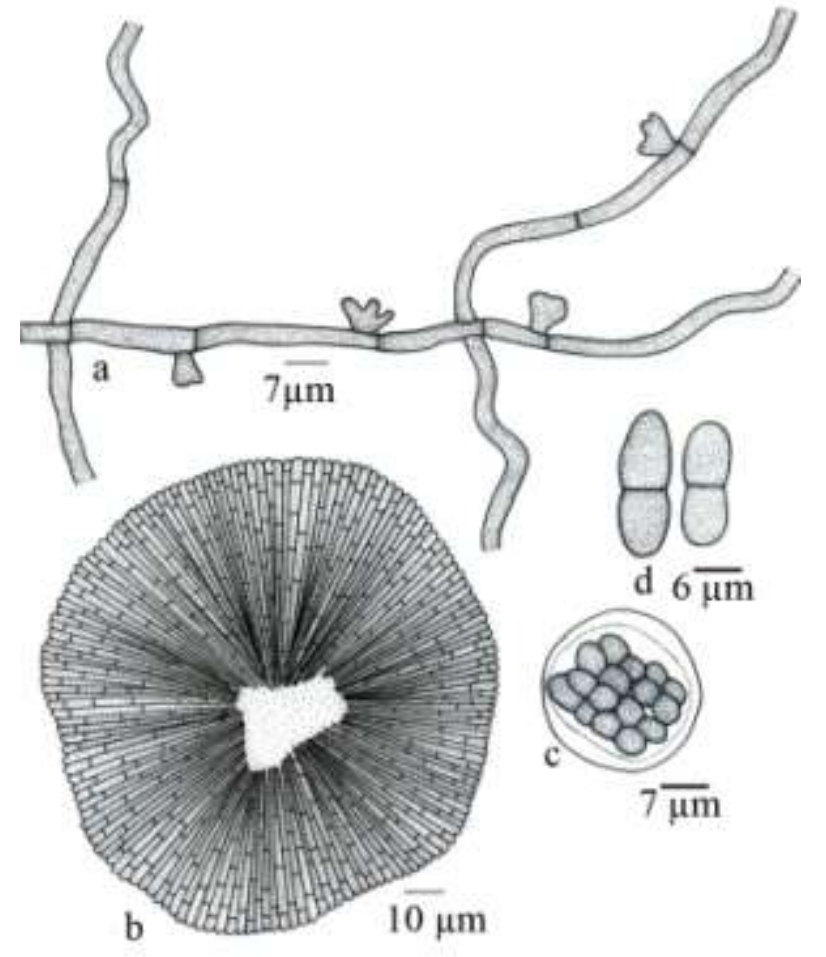

Figure 199 - Asterina tertia

octosporous, $40-48 \times 20-23 \mu \mathrm{m}$; ascospores brown, 1-septate, constricted, 16-20 $\times 8-10 \mu \mathrm{m}$. Pycnothyria 55-80 $\mu \mathrm{m}$; pycnothyriospores unicellular, ovate, brown, 17-20 × 12-15 $\mu \mathrm{m}$.

Material examined: Karnataka, South Canara, Kukke Subramanya, on leaves of Adhatoda zeylanica Medikus (A. vasica Nees) (Acanthaceae), 15 November 2003, V.B. Hosagoudar \& al. HCIO 45746, TBGT 1495; Kodagu, Hoddur, 29 December 2008, Jagath Thimmaiah TBGT 5386; Kodagu, Abbe falls, on leaves of Asystasia chelanoide Nees, 11 November 2003, V.B. Hosagoudar \& al. HCIO 45644, TBGT 1390; Kodagu, Madikeri, Jodupal, 12 November 2003, V.B. Hosagoudar \& al. HCIO 45611, TBGT 1354; HCIO 45671, TBGT 1418; Kodagu, Madikeri, Jodupal, on leaves of Barleria sp., 12 November 2003, V.B. Hosagoudar \& al. HCIO 45734, TBGT 1483; Crossandra sp. (Acanthaceae), Jodupal, Madikeri, Kodagu, Karnataka, 12 November 2003, V.B. Hosagoudar \& al. TBGT 1558; Jodupal, 12 November 2003, V.B. Hosagoudar \& al. HCIO 45732, TBGT 1481; Kodagu, Bhagamandala, on leaves of Strobilanthus sp., 25 November 2008, C. Jagath Thimmaiah TBGT 5348; Kodagu, Hoddur, on leaves of Justicia wyanadensis, 26 November 2008, C.
Jagath Thimmaiah TBGT 5378; Hoddur, Devarakadu,on leaves of Justicia wynaadensis (Nees) Heyne ex T. Anderson (Acanthaceae), 4 December 2009, C. Jagat Thimmaiah TBGT 5475; Kodagu, Hoddur, on leaves of Acanthaceae member, 23 August 2009, C. Jagath Thimmaiah TBGT 5406; Kerala, Kunnamkulam, 13 September 1999, C.K. Biju HCIO 43454, TBGT 275; Kollam, Shankily forest, 23 November 2004, A. Manojkumar HCIO 44520, TBGT 806; Thiruvananthapuram,

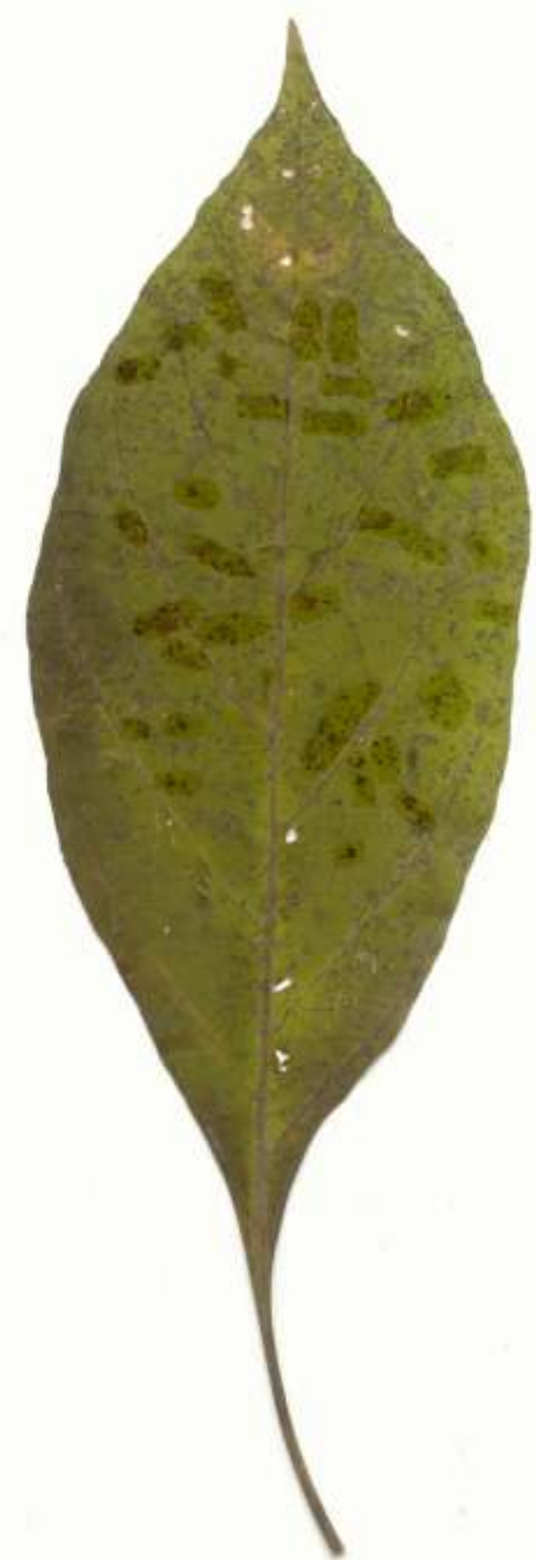

Figure 200 - Asterina tertia - Infected leaf 
Palode, TBGRI Campus, 2 March 2005, G. R. Archana \& al. HCIO 46001, TBGT 1765; Palode, Chippanchira, 4 October 2005, V. S. Sreelal HCIO 46975, TBGT 2192; Palode, TBGRI Campus, 11 November 2005, G. R. Archana \& A. Sabeena HCIO 47007, TBGT 2224; Palode, TBGRI Campus, 25 January 2005, G.R. Archana \& V.L. Sheeja HCIO 45924, TBGT 1686; 2 March, 2005, Archana \& al. HCIO 46003, TBGT 1767; Palode,TBGRI campus, Medicinal plants garden, 11 November 2005, G.R. Archana \& A. Sabeena HCIO 46813, TBGT 2154; Chippachinjira, 4 October 2005, V.S. Sreelal HCIO 46975, TBGT 2192; TBGRI campus, Palode, 5 January, 2001, H. Biju HCIO 46109, TBGT 1872; 11 November 2005, G.R. Archana \& A. Sabeena HCIO 47007, TBGT 2224; 17 September 2008, K. Anil Kumar TBGT 5122; Pathanamthitta, Kozhanchery, 9 January 2007, Jacob Thomas HCIO 47994, TBGT 2777; Thrissur, Vazhachal forest, 21 August 2007, Jacob Thomas \& al. HCIO 48190, TBGT 2926; Kottayam, Thazhathuvadakara, 27 December 2005, V.Gireesh Kumar \& P.J. Robin HCIO 46787, TBGT 2128; Kottayam, Ponthanpuzha, 22 November 2009, P.J. Robin \& al. TBGT 5217; 2 February 2009, K. Anil Kumar TBGT 5258; Pathanamthitta, Sabarimala forest division, Nilakal, 27 March 2009, P.J. Robin \& al. HCIO 49659, TBGT 3901; 5 July 2006, R. Anjugavairam HCIO 47645, TBGT 2667; Pathanamthitta, Thiruvalla, Kuttapuzha, 15 November 2006, Jacob Thomas HCIO 47981, TBGT 2764; TBGT 2677; Pathanamthitta, Kozhancherry, 9 January 2007, Jacob Thomas HCIO 47994, TBGT 2777; Thiruvananthapuram, Neyyar Wild Life Sanctuary, 21 August 2007, Jacob Thomas HCIO 48148, TBGT 2884; Thrissur, Vazhachal Forests, 21 August 2007, Jacob Thomas \& al. 48190, TBGT 2927; Thiruvananthapuram, Neyyar Wild Life Sanctuary, 22 August 2007, Jacob Thomas HCIO 48215, TBGT 2951; Thrissur, Vazhachal Forests, 21 August 2007, Jacob Thomas \& al. HCIO 48224, TBGT 2960; Wayanad, Padinharathara, 6 December 2009, A. Sabeena \& M.C. Riju TBGT 4643; TBGRI Campus, Palode Thiruvananthapuram, June 11, 2007, Sabeena \& al. HCIO 48350, TBGT 3071; Thiruvananthapuram, Ponmudi, 22 February 2007, Archana \& al. HCIO 48868, TBGT 3244;
Kollam, Arippa, 27 October 2006, V.B. Hosagoudar \& al. TBGT 4676; Kannur, on leaves of $A$. beddomii C. B. Clarke, 25 August 2002, M. Rajendraprasad HCIO 44639, TBGT 921; Thiruvananthapuram, Palode, Kusavoor, 9 September 2002, A. Manojkumar HCIO 44629, TBGT 911; Thiruvananthapuram, Kallara, 9 February 2006, A.Sabeena 49318, TBGT 3563; TBGRI Campus, Palode, 25 September 2005, G.R. Archana \& A. Sabeena HCIO 47389, TBGT 2427; 5 January 2001, H. Biju HCIO 46105, TBGT 1868; Kottayam, Wagamon, 5 December 2002, A. Manojkumar HCIO 44716, TBGT 997; Thiruvananthapuram, TBGRI campus, Palode, on leaves of Asystasia sp., 2 September 2009, K. Anil Kumar TBGT 5136; Thiruvananthapuram, Karakulam, 7 April, 2003, G. Rajkumar HCIO 45091, TBGT 1140; Idukki, Munnar, Mannavan shola, on leaves of Barleria involucrata Nees. (Acanthaceae), 5 January, 1999, C.K. Biju HCIO 45153, TBGT 1202; Kollam, Shankily forest, on leaves of Dipteracanthus patulus (Jacq.) Nees (Justicia betonica L.) (Acanthaceae), 23 November 2004, V.B. Hosagoudar HCIO 46208, TBGT 1620; Kollam, Sankily forest, on leaves of Justicia betonica, 23 November 2004, V.B. Hosagoudar HCIO 46216, TBGT 1628; HCIO 46023, TBGT 1787; Thiruvananthapuram, Palode, TBGRI Campus, on leaves of Justicia sp., 11 November 2005, G.R. Archana \& A. Sabeena HCIO 47070, TBGT 2287; HCIO 47072, TBGT 2289; Palode, TBGRI Campus, on leaves of Ruellia prostrate Poiret (Acanthaceae), 23 November, 2007, A. Chandraprabha HCIO 48256, TBGT 2995; Kottayam, Ponthanpuzha, on leaves of Ruellia sp., 25 November 2009, Robin \& al. TBGT 4244; Thiruvananthapuram, Palode, TBGRI campus, on leaves of Rhinacanthus nasuta (L.) Kurz (Acanthaceae), 29 January 2009, A. Sabeena TBGT 4270; 13 March 2009, A. Sabeena \& M.C. Riju TBGT 4697; 16 March 2009, A. Sabeena TBGT 4860; Wayanad, Padinharathara, on leaves of Asystasia violacea Dalz. ex C.B. Clarke (Acanthaceae), 6 November 2009, A. Sabeena \& M. C. Riju TBGT 4296; Wayanad, Padinharathara, $16^{\text {th }}$ mile, on leaves of Lepidagathis sp. (Acanthaceae), 6 November 2009, A. Sabeena \& M.C. Riju TBGT 4531; TBGT 4533; TBGT 4535; 4537; TBGT 4539; Thiruvananthapuram, 
Palode, TBGRI campus, on leaves of Asystasia sp., 21 October 2010, Nawaz TBGT 5004; Thiruvananthapuram, Palode, TBGRI Campus, on leaves of Rhinacanthus sp., 8 January 2008, K. Anil Kumar TBGT 5241; Kollam, Achenkovil, Amarakkathodu, 4 December 2008, V.B. Hosagoudar \& al. HCIO 49027, TBGT 3282; Achenkovil, Priya Estate, 4 December 2008, V.B. Hosagoudar \& al. HCIO 49028, TBGT 3283; Wayanad, Periya, 17 September 2008, M. Harish \& P.J. Robin HCIO 49634, TBGT 3976; HCIO 49646, TBGT 3888; Wayanad, Tirunelli, 18 September 2008, M. Harish \& P.J. Robin HCIO 49884, TBGT 4036; HCIO 49889, TBGT 4041; Wayanad, 14 March 2007, M.C. Riju HCIO 49981, TBGT 4133; Idukki, Munnar, Mattupatti, 1 January 2003, A. Manojkumar \& H. Biju HCIO 44889, TBGT 1117. Tamil Nadu, Tirunelveli, on the way to Manimuthar to Manjolai, Kalakkad Mundurai Tiger Reserve, on leaves of Lepidagathis scariosa Nees, 9 December 2006, K. Ravikumar HCIO 47655; Kodaikanal, Kuthuraiyar, on leaves of Peristrophe sp. (Acanthaceae), 21 November 2007, R. Nithyatharani TBGT 4419.

Alternate and lobate head cells of the appressoria distinguish this species from Asterina betonicae Hosag. \& Goose (Hosagoudar \& Goos, 1996).

Distribution: Kerala, Karnataka, Tamil Nadu

Asterina tertia Racib, var. africana Doidge, Trans. Royal Soc. South Africa 8: 264, 1920; Hosag. \& Goos, Mycotaxon 52: 470, 1994; Hosag., Balakr. \& Goos, Mycotaxon 59: 183, 1996; Hosag. \& Abraham, J. Econ. Taxon. Bot. 4: 558, 2000; Hosag., C.K. Biju \& Abraham, J. Mycopathol. Res. 40:195, 2002; Hosag., Zoos' Print J. 18: 1280, 2003; Hosag., Zoos' Print J. 21: 2329, 2006; Hosag., Chandraprabha \& Agarwal, Asterinales of Kerala, p. 149, 2011.

(Fig. 201)

Colonies epiphyllous, thin, up to $2 \mathrm{~mm}$ in diameter, confluent. Hyphae brown, straight to substraight, branching mostly opposite at wide angles, loosely reticulate, cells 15-25 × 3$5 \mu \mathrm{m}$. Appressoria predominantly sessile, rarely 1-septate, scattered, alternate, brown, globose, irregularly sublobate to deeply lobate and sometimes the lobes give finger like appearance, $5-7 \times 6-10 \mu \mathrm{m}$. Thyriothecia not seen. Pycnothyria scattered to connate, orbicular up to $76 \mu \mathrm{m}$ in diameter, stellately dehisced at the center, the dehisced portion later dissolve and exposed the pycnothyriospores, margin crenate to fimbriate, fringed hyphae radiating, tortuous, brown; pycnothyriospores cinnamon brown, ovate, ellipsoidal to pyriform, $15-19 \times 9-13 \mu \mathrm{m}$, wall smooth.

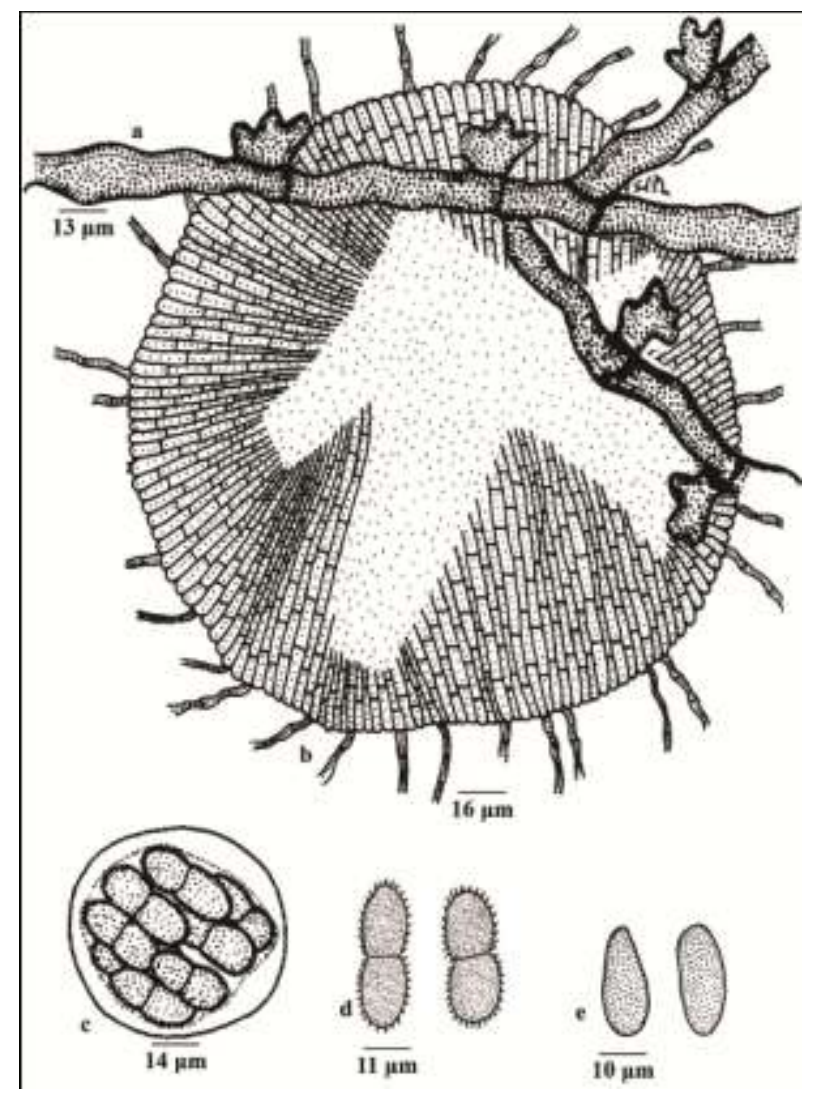

Figure 201 - Asterina tertia var. africana

Material examined: Kerala, Thiruvananthapuram, Palode, TBGRI Campus, on leaves of Adhatoda beddomei C. B. Clarke (Acanthaceae), 5 December 1996, V.B. Hosagoudar HCIO 42414, TBGT 77; Ernakulam, Vaikkom, on leaves of A. zeylanica Medikus (A. vasica Nees), 8 February 1997, S. Shiburaj HCIO 42538, TBGT 154; Thiruvananthapuram, Palode, Diotachanthus albiflorus (Bedd.) Benth. (Acanthaceae), 29 January 2002, M. Kamarudeen HCIO 44303, TBGT 731; Thiruvananthapuram, Palode, TBGRI campus, on leaves of Ruellia prostrata 
Poiret (Acanthaceae), 23 November 2007, A. Chandra Prabha HCIO 48256, TBGT 2995; Idukki, Mannavan Shola, 23 September 1998, C.K. Biju HCIO 42935, TBGT 218; Tamil Nadu, Nilgiris, Guddalur, Benne forest, on leaves of Eranthemum capense L. (Acanthaceae), 24 January 1990, V.B. Hosagoudar HCIO 30972; Kamarajar dist., Seithur hills, 14 November, 1992, V.B. Hosagoudar HCIO 40851;

This species differs from Asterina tertia in having shorter ascospores with echinulate wall.

Distribution: Kerala, Tamil Nadu.

Asterina theacearum Hosag., Abraham, C.K. Biju \& Shiburaj, New Botanist 24: 23, 1997; Hosag.,C.K. Biju \& Abraham, J. Mycopathol. Res. 40: 195, 2002; Hosag., Zoos' Print J. 18: 1285, 2003; Hosag., Chandraprabha \& Agarwal, Asterinales of Kerala, p. 151, 2011. (Fig. 202)

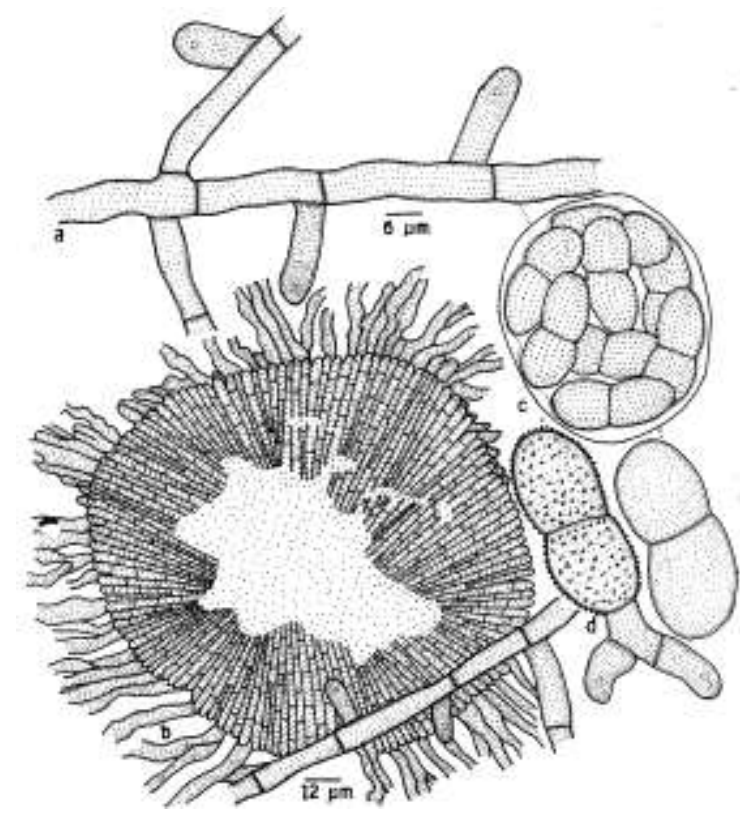

Figure 202 - Asterina theacearum

Colonies predominantly epiphyllous, rarely hypophyllous, thin to subdense, up to 5 $\mathrm{mm}$ in diameter, confluent. Hyphae straight, branching opposite to irregular at acute to wide angles, loosely reticulate, cells 12-34 × 5-8 $\mu \mathrm{m}$. Appressoria unicellular, alternate, about 5\% opposite, cylindrical, straight to slightly curved, mostly perpendicular to the hyphae, margin entire, $12-17 \times 5-8 \mu \mathrm{m}$. Thyriothecia scattered to mostly grouped, often joined marginally, orbicular, up to $150 \mu \mathrm{m}$ in diameter, stellately dehisced at the centre or widely opened, margin crenate to fimbriate, fringed hyphae yellowish brown and tortuous; asci many, globose, octosporus, 30-48 $\mu \mathrm{m}$ in diameter; ascospores oblong, brown, uniseptate, slightly constricted at the septum, 30-34 $\times 15-16 \mu \mathrm{m}$, wall tubercled.

Material examined: Kerala, Idukki, Munnar, Graval Banks, on leaves of Eurya sp. (Theaceae), 30 June 1998, S. Shiburaj HCIO 42886 (holotype), TBGT 209 (isotype); Munnar, Rajamala, 22 April 2008, A.Chandraprabha HCIO 48823, TBGT 3199; Munnar, Mannavan Shola, 16 September 1999, C.K. Biju HCIO 45290, TBGT 1328; 22 April 2008, A. Chandra Prabha HCIO 48825, TBGT 3199; Munnar, Vagapara, on leaves of Gordonia sp. (Theaceae), 22 January 2003, A. Manojkumar \& H. Biju HCIO 45218, TBGT 1255; Munnar, Poopara, on leaves of Eurya japonica Thunb., 23 January 2003, H. Biju \& A.Manoj Kumar HCIO 44844, TBGT 1081.

Sydow et al. (1911) proposed Asterina camallieae on Thea sinensis from Assam. After noticing conidia, Hansford (1954) transferred it to Clypeolella camelliae Sydow, Sydow \& Butler) Hughes. Yamamoto (1957) proposed Asterina theae on Thea sinensis from Taiwan. The present new species diffe5rs from it in having cylindrical appressoria measuring 12-17 $\times 5-7 \mu \mathrm{m}$ in contrast to hemispherical to subglobose appressoria measuring 11-18 $\times 12$ $17 \mu \mathrm{m}$. Further, ascospores are tubercled in the present new species.

Distribution: Kerala.

Asterina thotteae Hosag. \& Hanlin, New Botanist 22: 188, 1995; Hosag. \& Hosag., H. Biju \& Appaiah, J. Mycopathol. Res. 44:12, 2006; Hosag., Chandraprabha \& Agarwal, Asterinales of Kerala, p. 152, 2011.

(Figs 203-204)

Colonies epiphyllous, thin to subdense, spreading, up to $3 \mathrm{~mm}$ in diameter, confluent. 
Hyphae substraight to rarely crooked, branching alternate to opposite at acute to wide angles, loosely reticulate, cells 31-38 $\times 3-4 \mu \mathrm{m}$. Appressoria alternate and about $3 \%$ opposite, straight to curved, antrorse to recurved, two celled, 9-19 $\mu \mathrm{m}$ long; stalk cells cylindrical to cuneate, 3-7 $\mu \mathrm{m}$ long; head cells ovoid, globose, entire to sublobate, angular, straight to curved, $6-13 \times 6-10 \mu \mathrm{m}$. Thyriothecia scattered, rarely 1-2 connate, circular, up to $155 \mu \mathrm{m}$ in diameter, margin fimbriate, fringed hyphae flexuous to crooked, pale yellow, center carbonaceous black and stellately dehisced at the center; asci many, initially globose, slightly clavate at maturity, octosporous, 30-38 $\times$ 27-31 $\mu \mathrm{m}$; ascospores conglobate, oblong, deep brown, rounded at both ends, 1-septate, constricted at the septum, $18-20 \times 9-10 \mu \mathrm{m}$, wall verrucose.

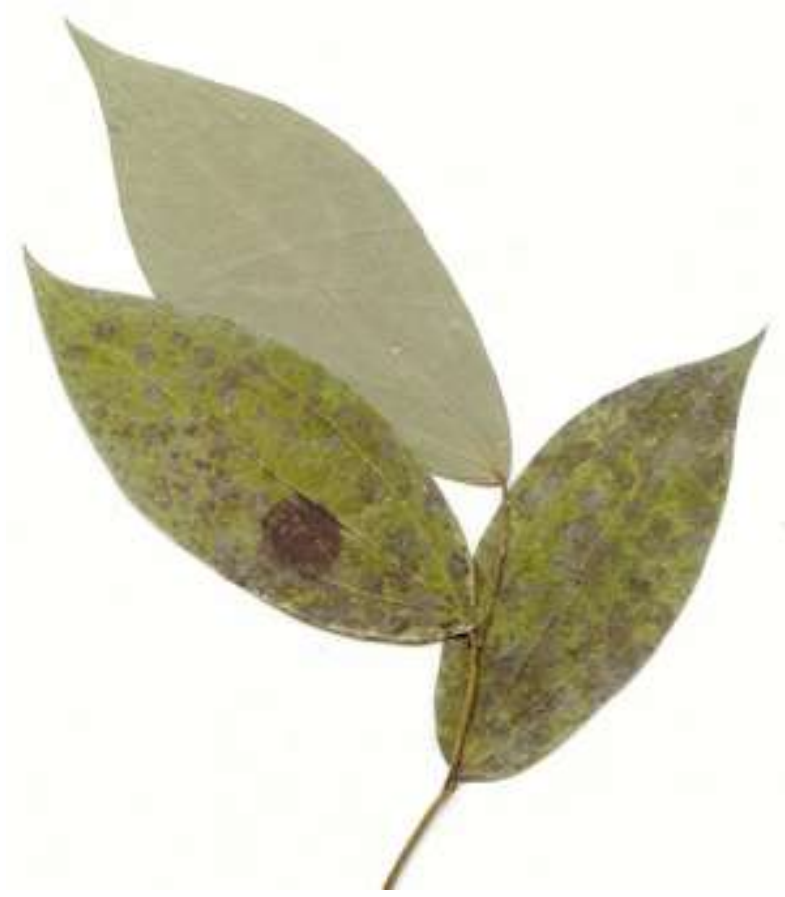

Figure 203 - Asterina thotteae

Material examined: Tamil Nadu, Coimbatore, Anamalai, Sheikalmudi, on the way to Manjaparai, on leaves of Thottea siliquosa (Lam.) Ding Hou. (Aristolochiaceae), 26 December 1991, V.B. Hosagoudar HCIO 40882; Kerala, Thiruvananthapuram, Attayar, 18 March 1997, V.B. Hosagoudar HCIO 43970, TBGT 417; Palode, TBGRI Campus, 7 March 2001, H. Biju HCIO 46071, TBGT 1834; Wayanad, Periya, 29 October 2007, A. Chandra Prabha HCIO 48238, TBGT 2976; Thiruvananthapuram, Attayar, on leaves of $T$. ponmudiana Sivarajan, 20 March 1997, V.B. Hosagoudar HCIO 44162, TBGT 557; Thiruvananthapuram, Bonacaud, 23 October 2001, G. Rajkumar HCIO 45233, TBGT 1270; Wayanad, Periya, T. sivarajanii Santhosh et al., 27 December 2002, M. Kamarudeen \& P. A. Jose HCIO 44800 ,

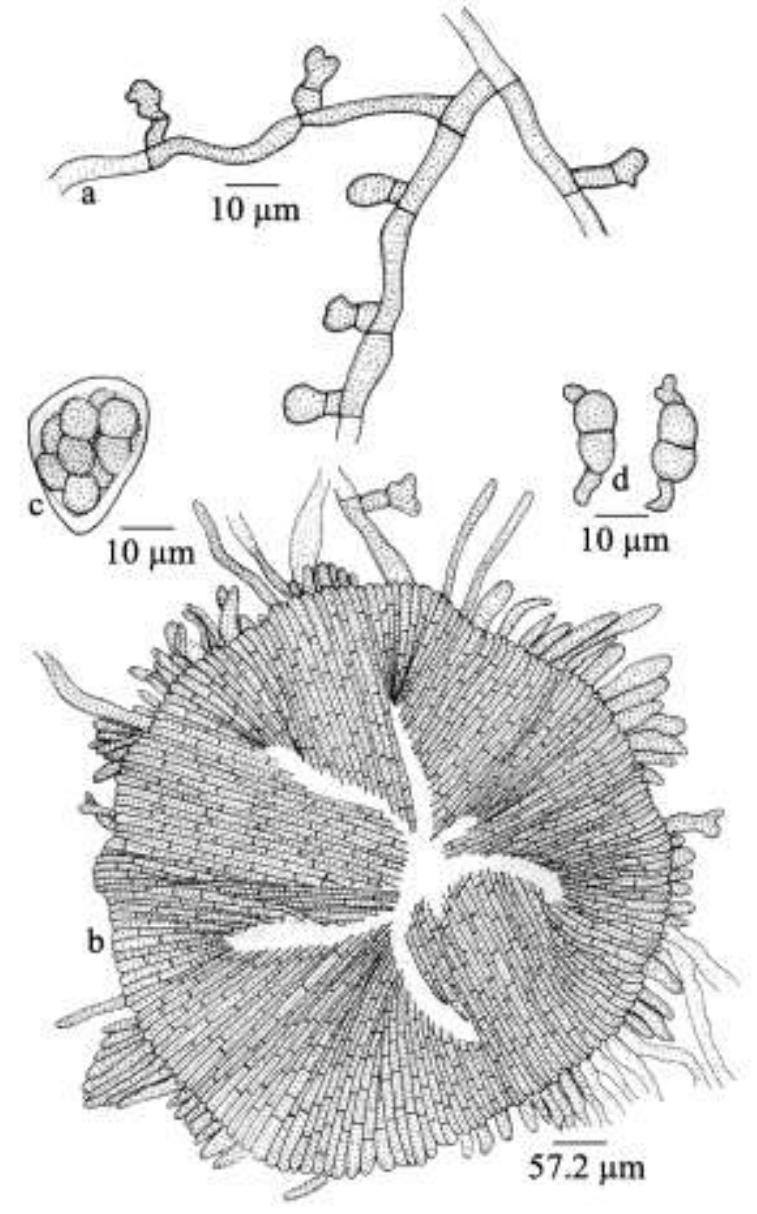

Figure 204 - Asterina thotteae

TBGT 1037; Kollam, Shendurney, T. siliquosa (Lam.) Ding Hou, 14 January 2003, V.B. Hosagoudar HCIO 44849, TBGT 1086; Thiruvananthapuram, Near Peppara dam, 18 November 2007, Jacob Thomas \& Vimalkumar HCIO 48213, TBGT 2949; Idukki, Thekkadi, Periyar Tiger Reserve, Shakuntala kadu, on leaves of Thottea sp., 22 March 2006, H. Biju \& V. Gireesh Kumar HCIO 47620, TBGT 2642; Thiruvananthapuram, Ponmudi, Thottea sp., 22 February 2007, Archana \& al. HCIO 48007, TBGT 2790; Kottayam, Ponthanpuzha forest, 14 February 2007, Robin \& al. HCIO 48326, TBGT 3047; Palghat, Silent Valley, Pulipara, 13 February 2007, M.C. Riju \& al. TBGT 4975; Kollam, Arippa, 3 January 2011, V.B. Hosagoudar \& al. TBGT 5053; Karnataka, Jodupal, Kodagu, Karnataka, 
November 12, 2003, V.B. Hosagoudar \& al. HCIO 45627, TBGT 1370.

This is the only species of the genus Asterina on the members of the family Aristolochiaceae (Steven \& Ryan, 1939; Diodge, 1942).

Distribution: Kerala, Karnataka, Tamil Nadu.

Asterina thunbergiicola Hansf. var. indica Hosag. \& Jacob-Thomas, Taprobanica 3: 16, 2011. (Figs 205-206)
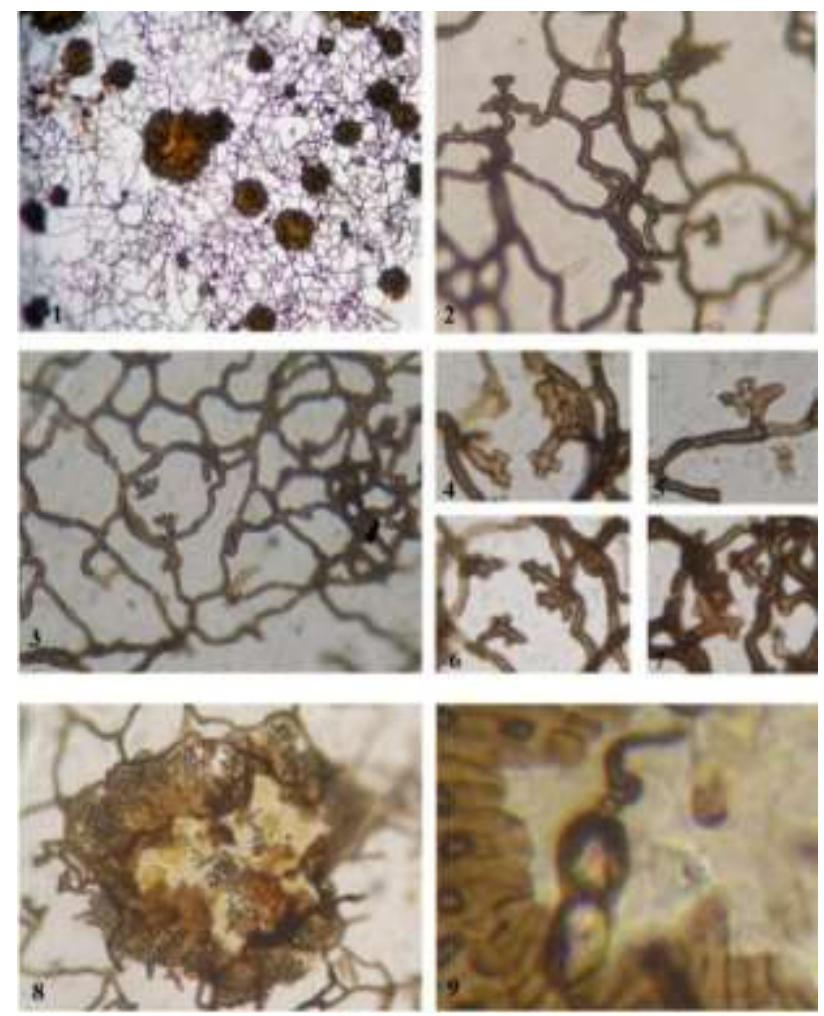

Figure 205 - Asterina thunbergiicola var. indica

1. Colony with thyriothecia, 2-3. Appressoriate mycelium formed the hyphal net, 4-7. Appressoria, 8. Dehisced thyriothecia showing asci, 9. Germinating ascospore

Colonies hypophyllous, thin, crustose, up to $5 \mathrm{~mm}$ in diameter, confluent. Hyphae crooked, branching irregular at various angles, loosely reticulate to form a mycelial net, cells 21-34 $\times 2-5 \mu \mathrm{m}$. Appressoria alternate, two celled, straight to curved, plugged around stomata of the host leaf, 12-24 $\mu \mathrm{m}$ long, stalk cells cylindrical, 7-12 $\mu \mathrm{m}$ long, head cells ovate, globose to hamate, subangular, angular, narrowly to deeply lobate, 4-12 × 9-15 $\mu \mathrm{m}$. Thyriothecia scattered, orbicular, often 1-2 connate, up to $180 \mu \mathrm{m}$ in diameter, stellately dehisce at the centre and dissolved later, margin crenate; asci few to many, globose, octosporous, up to $30 \mu \mathrm{m}$ in diam.; ascospores, conglobate, brown, uniseptate, constricted at the septum, 16-22 $\times 7-10 \mu \mathrm{m}$, wall smooth.

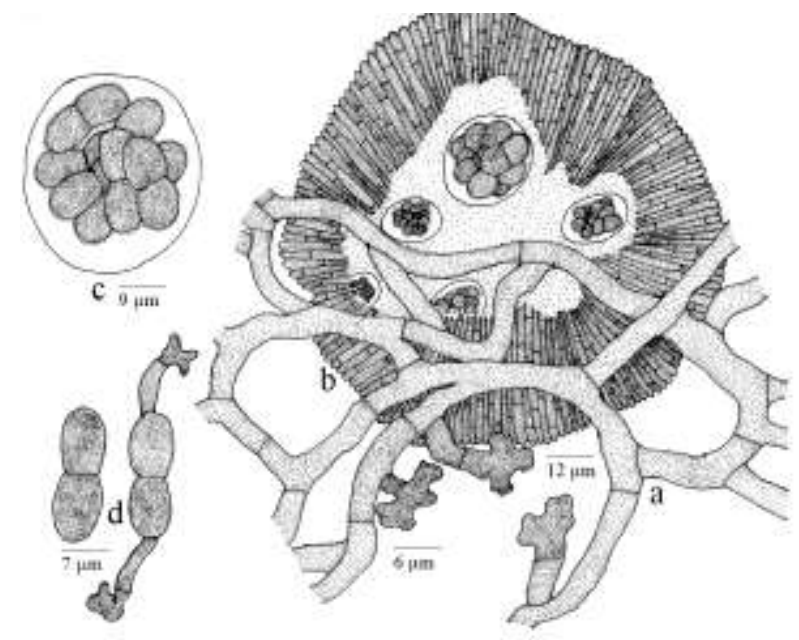

Figure 206 - Asterina thunbergiicola var. indica

Pycnothyria similar to thyriothecia, smaller; pycnothyriospores pyriform, brown, apiculate, broadly rounded at one end and, attenuated and truncate at the other, 16-22 $\times 9$ $15 \mu \mathrm{m}$, wall smooth.

Material examined: Kerala, Thiruvananthapuram, Athirumala, Peppara Wildlife Sanctuary, on leaves of Thunbergia sp. (Thunbergiaceae), 28 February 2008, Jacob Thomas HCIO 48870 (holotype), TBGT 3246 (isotype).

Asterina thunbergiicola Hansf. is known on Thunbergia chrysops from Sierra Leone, Uganda (Hansford, 1945). However, the new variety differs from the var. thunbergiicola in having longer appressoria and ascospores.

Distribution: Kerala.

Asterina tinosporae Hansf., Reinwardtia 3: 137, 954; Hosag., Chandraprabha \& Agarwal, Asterinales of Kerala, p. 154, 2011. (Fig. 207) 


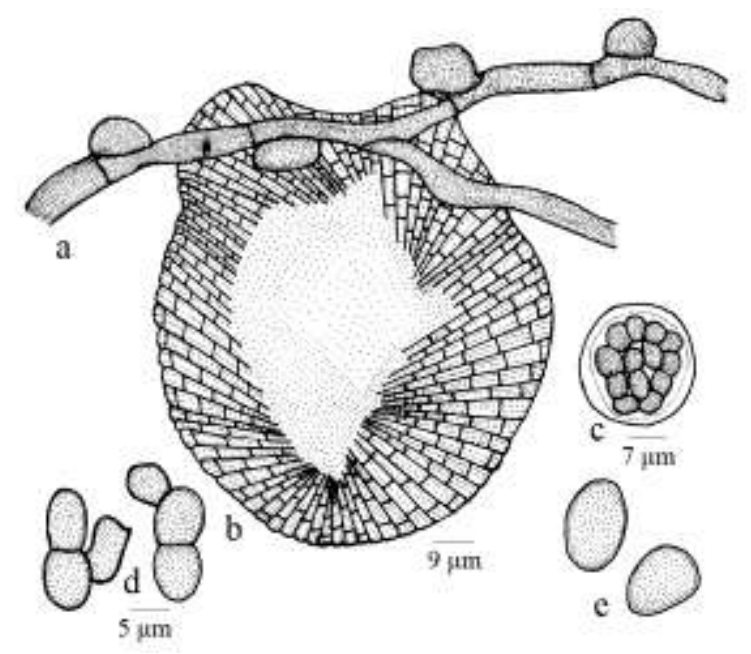

Figure 207 - Asterina tinosporae

Colonies epiphyllous, thin to subdense, up to $2 \mathrm{~mm}$. in diameter, confluent. Hyphae undulate, branching alternate at acute to wide angles, loosely reticulate, cells 22-28 × 4.4-6 $\mu \mathrm{m}$. Appressoria alternate, rarely opposite, scattered, unicellular, antrorse, sub antrorse to retrorse, entire, sub lobate to lobate, mostly broad based, $6-12 \times 4.4-9 \mu \mathrm{m}$. Thyriothecia closely scattered, flattened up to $88 \mu \mathrm{m}$ in diameter, stellately dehisced at the centre, margin fimbriate, asci globose up to $39 \mu \mathrm{m}$ in diameter, ascospores conglobate, uniseptate, constricted at the septa, 13-15 $\times$ 4.4-6 $\mu \mathrm{m}$. Pycnothyriospores ovate, globose, 9-20 × 4.4$11 \mu \mathrm{m}$.

Material examined: Kerala, Kozhikode, Malabar Botanic Garden, on leaves of Tinospora cordifolia (Willd.) Miers (Menispermaceae), 16 November 2006, A. Sabeena \& al. HCIO 48047, TBGT 2830. host genus.

This is the only species known on this

Distribution: Kerala.

Asterina toddaliae Kar \& Ghosh, Indian Phytopath. 39: 210, 1986; Hosag. \& Goos, Mycotaxon 52: 470, 1994; Hosag., Chandraprabha \& Agarwal, Asterinales of Kerala, p. 155, 2011.

(Figs 208-209)

Colonies epiphyllous, thin to subdense, up to $4 \mathrm{~mm}$ in diam., rarely confluent. Hyphae straight to flexuous. Branching irregular at acute to wide angles, loosely to closely reticulate, cells 19-32 × 4-6 $\mu \mathrm{m}$. Appressoria alternate to unilateral, unicellular, antrorse, retrorse, straight, flexuous to curved, ovate, oblong, cylindrical, broadly rounded at the tip, 11-18 × 4-6 $\mu \mathrm{m}$. Thyriothecia scattered, orbicular, up to $195 \mu \mathrm{m}$ in diam.., stellately dehisced at the centre, margin fimbriate, fringed Hyphae small, crooked; asci globose, 8 spored, up to $40 \mu \mathrm{m}$ in diam.; ascospores oblong, brown, uniseptate, constricted at the septum, 27-30 $\times 10-12 \mu \mathrm{m}$, margin tubercled.

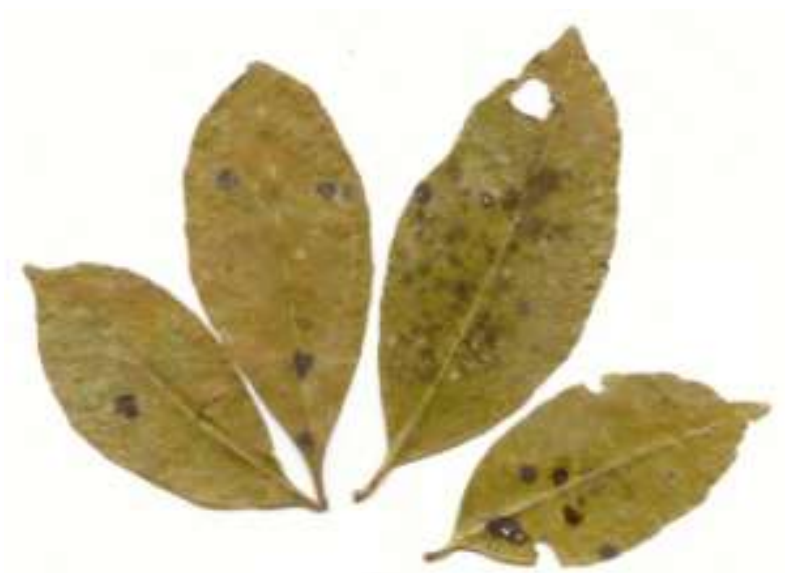

Figure 208 -Asterina toddaliae - Infected leaves

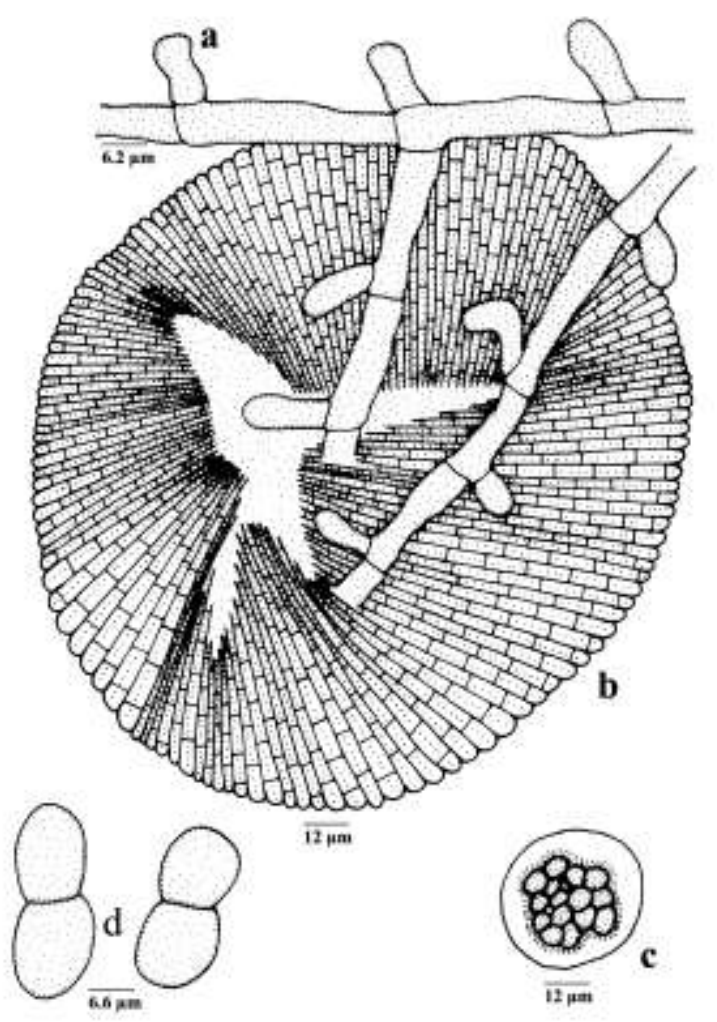

Figure 209 - Asterina toddaliae 
Material examined: Tamil Nadu, Kamarajar dist., Seithur hills, on leaves of Toddalia asiatica (L.) Lam. (Rutaceae), 12 November 1992, V.B. Hosagoudar HCIO 4085; Kerala, Pathanamthitta, Anathode Dam site, on leaves of Toddalia sp. (Rutaceae), 15 December 2004, V.B. Hosagoudar \& al. HCIO 46042, TBGT 1805; Idukki, Kuttikanam, 17 March 2007, Jacob Thomas \& al. HCIO 48161, TBGT 2897.

Kar \& Ghosh (1986) reported this species from Rangpo forest, Darjeeling, West Bengal

In the Western Ghats collection, colonies were hypophyllous.

Distribution: Kerala, West Bengal, Tamil Nadu.

Asterina toddaliicola Hosag., Agarwal, H. Biju \& Archana, Indian Phytopathol. 59: 525, 2006. (Fig. 210)

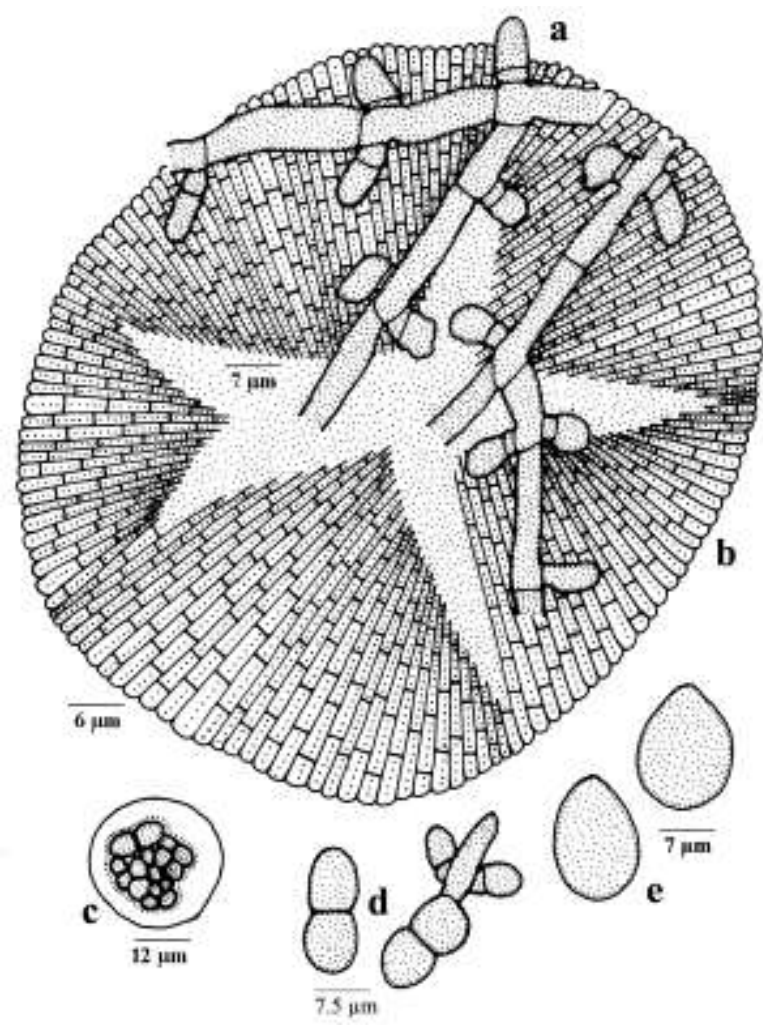

Figure 210 - Asterina toddaliicola

Colonies amphigenous, dense, velvety, up to $2 \mathrm{~mm}$ in diam., often confluent. Hyphae straight, substraight to rarely crooked, branching alternate, opposite to irregular at acute to wide angles, loosely to closely reticulate, cells $12-24 \times 3-5 \mu \mathrm{m}$. Appressoria about $90 \%$ opposite, often solitary and alternate, mostly perpendicular to the Hyphae, often slightly antrorse, two celled, 9-13 $\mu \mathrm{m}$ long; stalk cells cylindrical to cuneate, 3-4 $\mu \mathrm{m}$ long; head cells ovate, globose, entire, angular, rarely sublobate to lobate, $6-10 \times 6-8 \mu \mathrm{m}$. Thyriothecia scattered to loosely grouped, orbicular, up to $100 \mu \mathrm{m}$ in diam., stellately dehisced at the centre, margin crenate; asci very few, globose, octosporous, up to $35 \mu \mathrm{m}$ in diam.; ascospores oblong, conglobate, brown, uniseptate, constricted at the septum, 19-21 $\times$ 12-13 $\mu \mathrm{m}$, wall smooth. Pycnothyria similar to thyriothecia, smaller; pycnothyriospores pyriform, apiculate at one end, brown, 19-21 $\times$ 13-15 $\mu \mathrm{m}$, wall smooth.

\section{Material examined: Karnataka,} Coorg, Madikeri, Nishanimotta, on leaves of Toddalia sp. (Rutaceae), 11 November 2003, V.B. Hosagoudar et al. HCIO 46176, TBGT 1588 .

Asterina toddaliae Kar \& Ghosh (toddalae) is known on this host genus from West Bengal (Kar \& Ghosh, 1986). Asterina toddaliicola differs from it in having opposite, bicellular and straight appressoria.

Distribution: Karnataka.

Asterina toxocarpi Hosag. \& C.K. Biju, Indian Phytopath. 58: 200, 2005; Hosag., Chandraprabha \& Agarwal, Asterinales of Kerala, p. 156, 2011.

(Fig, 211)

Colonies amphigenous, mostly epiphyllous, subdense, up to $2 \mathrm{~mm}$ in diam., rarely confluent. Hyphae straight to flexuous, branching alternate to irregular at acute to wide angles, loosely reticulate, cells 12-20 × 3-4 $\mu \mathrm{m}$. Appressoria alternate, unilateral, two celled, antrorse to subantrorse, $8-13 \mu \mathrm{m}$ long; stalk cells cylindrical to cuneate, 3-5 $\mu \mathrm{m}$ long; head cells ovate, globose, entire to rarely slightly lobate, $4-8 \times 6-8 \mu \mathrm{m}$. Thyriothecia scattered to rarely connate, orbicular, up to $136 \mu \mathrm{m}$ in diam., stellately dehisced at the centre, margin crenate to fimbriate, fringed hyphae flexuous; asci globose, octosporous, up to $35 \mu \mathrm{m}$ in 
diam.; ascospores oblong, conglobate, uniseptate, deeply constricted, 17-23 × 10-12 $\mu \mathrm{m}$, wall echinulate.

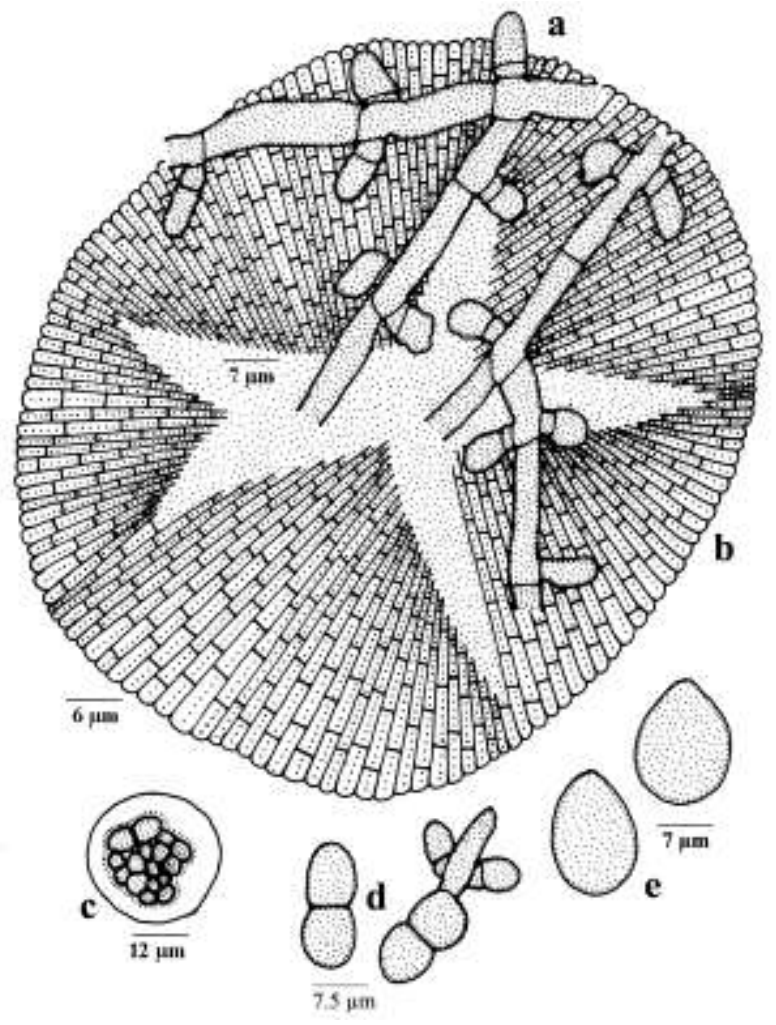

Figure 211 - Asterina toxocarpi

Material examined: Kerala, Idukki, Munnar, Mannavan Shola, on leaves of Toxocarpus sp. (Asclepiadaceae), 5 January 1999, C.K. Biju HCIO 45279 (holotype), TBGT 1317 (isotype).

Based on the morphology and arrangement of the appressoria, the present taxon is close to Asterina peraffinis Speg. Known on Tylophora flanagani from South Africa (Doidge, 1942). However, A. toxocarpi differs from it in having absence of one celled and shorter appressoria with mostly entire head cells.

Distribution: Kerala.

Asterina travancorensis Sydow \& Sydow, Ann. Mycol. 13: 38, 1915; Hosag. \& Goos, Mycotaxon 69: 160, 1996.

(Fig. 212)

Colonies foliicolous, epiphyllous, often surrounded by yellow haloes, scattered, dense, crustose to velvety, up to $2 \mathrm{~mm}$ in diameter, rarely confluent. Hyphae straight to flexuous, branching opposite to irregular at acute angles, loosely reticulate, cells $18-25 \times 5-7 \mu \mathrm{m}$. Appressoria one to two celled, alternate, about $1 \%$ opposite, antrorse to spreading, straight to curved, 12-25 $\mu \mathrm{m}$ long; stalk cells cylindrical to cuneate, 3-19 $\mu \mathrm{m}$ long; head cells ovate, globose, entire to sublobate, $6-10 \times 613 \mu \mathrm{m}$. Thyriothecia scattered to connate up to 5 , round, up to $110 \mu \mathrm{m}$ in diameter, dehisce stellately at the apex, upper cell radiating, margin crenate; asci globose, octosporous, bitunicate, 27-31 $\mu \mathrm{m}$ in diameter; ascospores conglobate, one septate, upper cell slightly larger, 21-25 × 9-13 $\mu \mathrm{m}$, wall smooth.

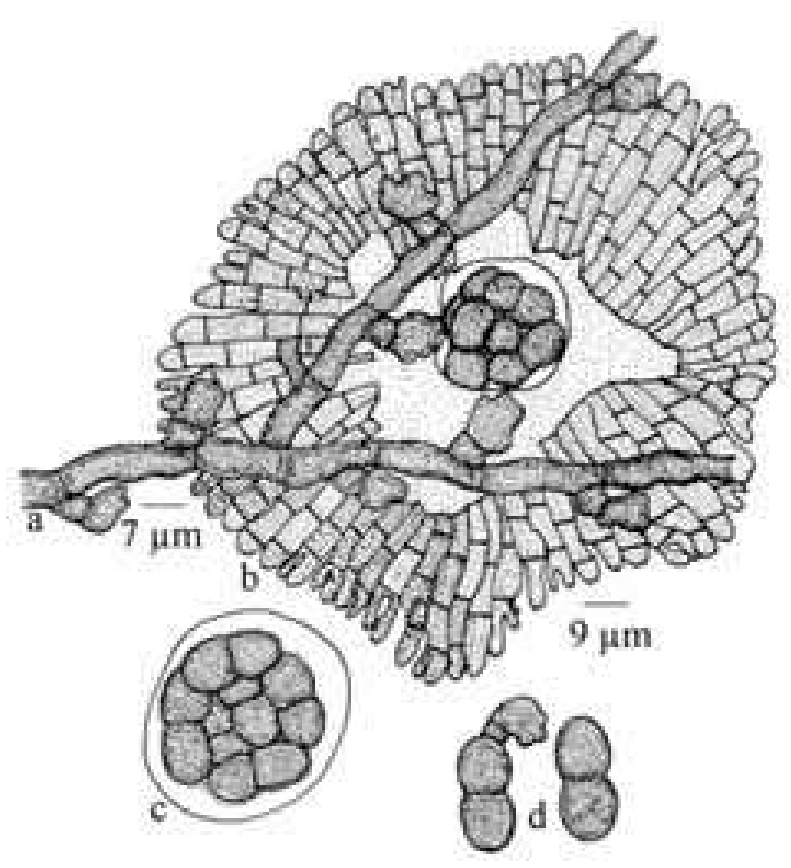

Figure 212 - Asterina travancorensis

Material examined: Tamil Nadu, Coimbatore, Valparai, Near dam, near Nooradi settlement, on leaves of Wattakaka volubilis (L. f.) Stapf. (Marsdenia volubilis (L. f.) Cooke) (Asclepiacaceae), 23 March 1990, V.B. Hosagoudar HCIO 30974; Kanniyakumari, Veerapuli Reserve Forest, 22 February 1994, V.B. Hosagoudar HCIO 41627.

Sydow \& Sydow (1915) described this species on Marsdenia sp., collected by E. J. Butler from Pulliyanur, Travancore of Kerala state on October 9, 1907.

This species is known only from the Southern Western Ghats.

Distribution: Tamil Nadu, Kerala 
Asterina trichiliae Doidge, Trans. Royal Soc. South Africa 8: 253, 1920. Hosag. \& Goos, Mycotaxon 60: 161, 1996.

(Fig. 213)

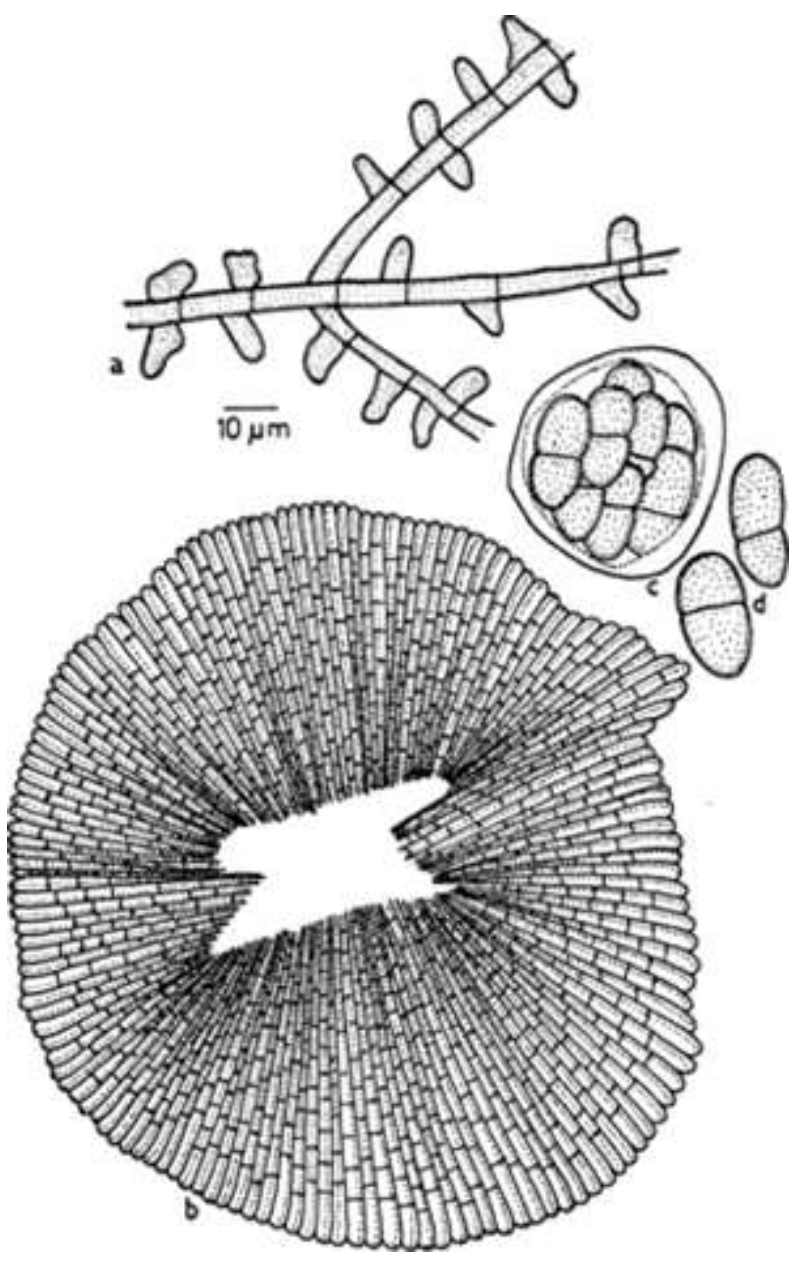

Figure 213 - Asterina trichiliae

Colonies epiphyllous, thin, up to $2 \mathrm{~mm}$ in diameter, confluent and covering the entire upper surface of the leaves. Hyphae straight, branching opposite to irregular at acute angles, loosely reticulate, cells 15-19 $\times$ 3-7 $\mu \mathrm{m}$. Appressoria opposite, subopposite, alternate and solitary, conoid, ovate, ampulliform, unicellular, entire, angular to rarely slightly lobate, 6-10 × 6-8 $\mu \mathrm{m}$. Thyriothecia loosely grouped, orbicular, up to $140 \mu \mathrm{m}$ in diameter, margin dentate to fringed, fringed hyphae tortuous, elongated and devoid of appressoria, such hyphae also emerge from the mycelia, thyriothecia dehiscing stellately at the center; asci many, globose, eight spored, $40-47 \mu \mathrm{m}$ in diameter; ascospores conglobate, brown, 1- septate, deeply constricted at septum, both cells unequal, 27-31 × 15-19 $\mu \mathrm{m}$, wall smooth.

Material examined: Tamil Nadu, Tirunelveli, Kakachi forest, on leaves of Trichilia connaroides (Wight \& Arn.) Bentv. (Meliaceae), 21 February 1994, V.B. Hosagoudar HCIO 41609.

This species was first reported from South Africa (Doidge, 1942).

Distribution: Tamil Nadu.

Asterina triumfetticola Yamam., sci. Rep. Hyogo Univ. Agric., Agric. Biol. Ser. 3: 29, 1957; Hosag. \& Abraham, J. Econ. Taxon. Bot. 4: 585, 2000; Hosag., Zoos' Print J. 17: 945, 2002; Hosag., Zoos' Print J. 21: 2329, 2006; Hosag., Chandraprabha \& Agarwal, Asterinales of Kerala, p. 158, 2011.

(Fig. 214)

Colonies epiphyllous, subdense to dense, up to $2 \mathrm{~mm}$ in diameter, confluent. Hyphae strongly appressed to the host surface, substraight to crooked, branching irregular at acute to wide angles, loosely reticulate, cells 16-24 $\times$ 3-4 $\mu \mathrm{m}$. Appressoria alternate, unilateral, unicellular, stipitate to sessile, globose, clavate, angular to deeply lobate, 4-7 $\mu \mathrm{m}$ long, 7-9 $\mu \mathrm{m}$ broad. Thyriothecia scattered, orbicular, up to $70 \mu \mathrm{m}$ in diameter, stellately dehisced at the centre, margin crenate; asci globose, octosporous, bitunicate, $20-28 \mu \mathrm{m}$ in diameter; ascospores pale-brown, conglobate, uniseptate, constricted at the septum, 14-16 × 6$8 \mu \mathrm{m}$, wall smooth to slightly punctate. Pycnothyria numerous, orbicular, smaller than thyriothecia; pycnothyriospores pyriform, deep brown, 14-16 × 11-12 $\mu \mathrm{m}$.

Material examined: Kerala, Kollam, Shankily forest, on leaves of Triumfetta sp. (Tiliaceae), 27 December 2001, V.B. Hosagoudar HCIO 44304, TBGT 679; HCIO 44305, TBGT 681; Thiruvananthapuram, Palode,TBGRI Campus, 15 November 2007, A. Sabeena \& al. HCIO 48341, TBGT 3062. 


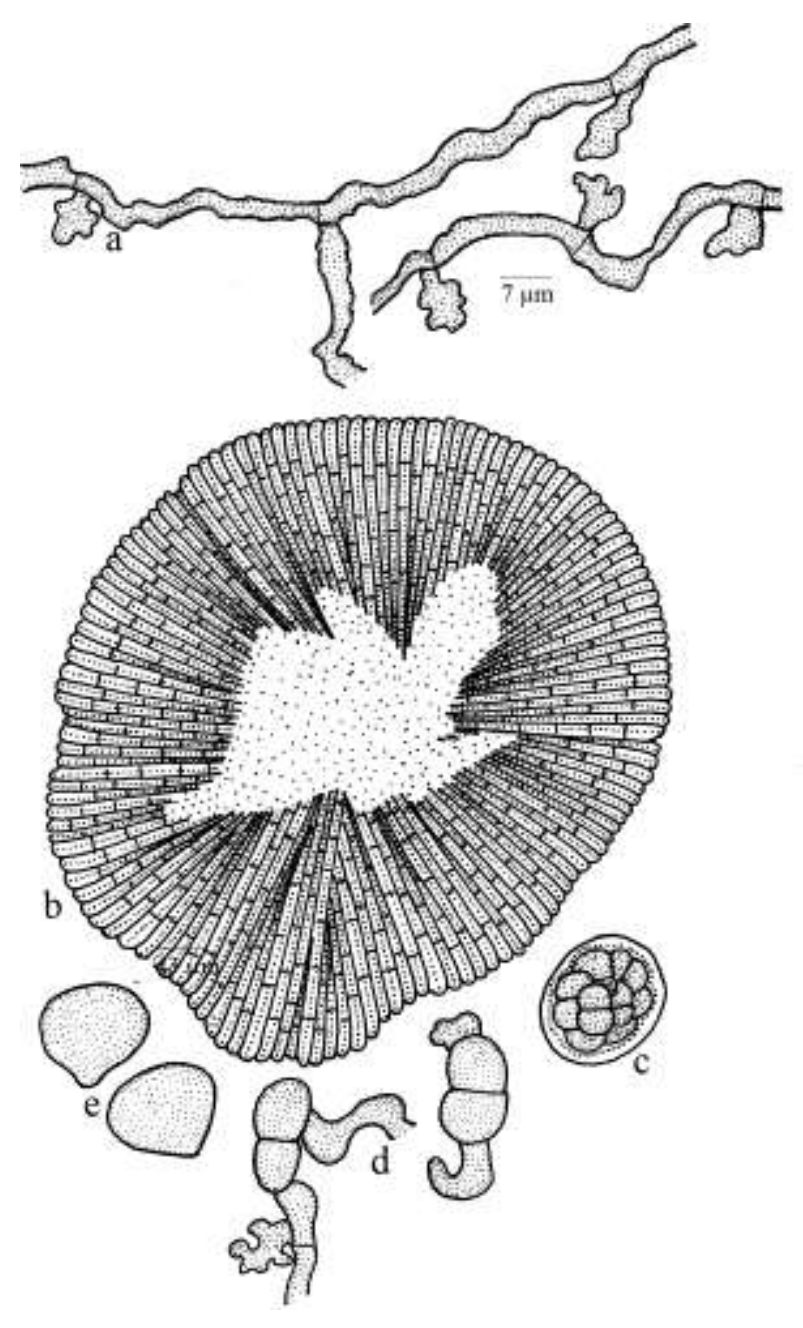

Figure 214 - Asterina triumfetticola

This species was known on Triumfetta bartamia from Taiwan (Yamamoto, 1956, 1957). This collection was associated with Irenopsis sp.

Distribution: Kerala.

Asterina tylophorae-indicae Hosag., H. Biju \& Manoj. in Hosag., Zoos' Print J. 21: 2336, 2006; Hosag., Chandraprabha \& Agarwal, Asterinales of Kerala, p. 158, 2011.

(Fig. 215)

Colonies epiphyllous, subdense to dense, up to $2 \mathrm{~mm}$ in diameter, rarely confluent. Hyphae substraight to crooked, curved irregularly, branching irregular at acute angles, loosely to closely reticulate, cells $14-18 \times 3-5$ $\mu \mathrm{m}$. Appressoria alternate, about 3\% opposite, unicellular, ovate, globose, straight to curved, entire, broad based, 8-10 $\times$ 4-7 $\mu \mathrm{m}$. Thyriothecia scattered to loosely grouped, orbicular, up to $100 \mu \mathrm{m}$ in diameter, crenate at the margin and stellately dehisced at the centre; asci few, globose, octosporous, up to $30 \mu \mathrm{m}$ in diameter; ascospores oblong, conglobate, brown, uniseptate, constricted at the septum, 19-21 × 9-11 $\mu \mathrm{m}$.

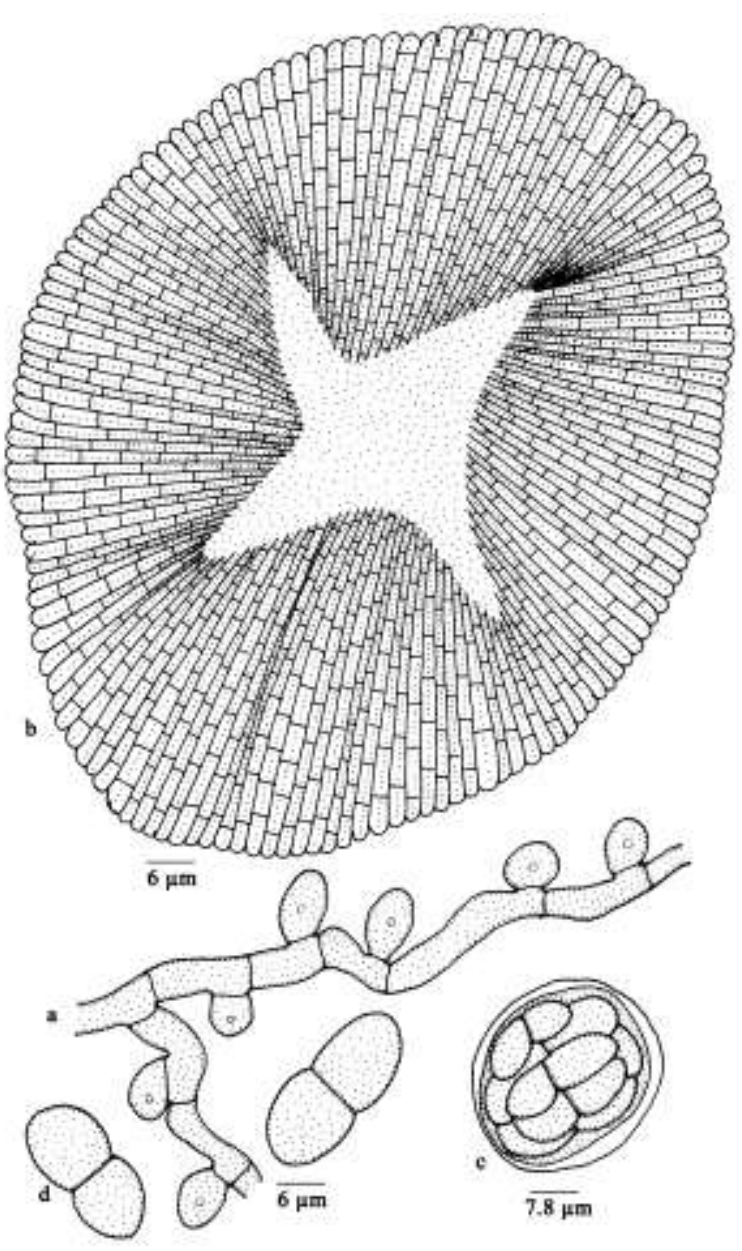

Figure 215 - Asterina tylophorae-indicae

Material examined: Kerala, Idukki, Munnar, Mannavan shola, on leaves of Tylophora indica (Burm. f.) Merr. (Asclepiadaceae), 21 January 2003, A. Manojkumar \& H. Biju HCIO 44848 (holotype), TBGT 1085 (isotype).

Asterina peraffinis Speg. is known on Tylophora flanagani from South Africa. The new species differs from it in having unicellular appressoria in contrast to bicellular (Doidge, 1942; Hosagoudar \& Abraham, 2000). 
Distribution: Kerala.

\section{Asterina viburnicola nom. nov.}

Asterina viburni Hosag., Dhivaharan

\& Nithytharani, J. Sci. Environ. \&

Technov. 4: 47, 2010. (Figs 216-217)

MycoBank 101026

Colonies amphigenous, mostly epiphyllous, dense, scattered to confluent, 2-5 $\mathrm{mm}$ in diameter. Hyphae flexuous, branching opposite at acute angle, loosely reticulate, cells 15-25 $\times$ 5-7 $\mu \mathrm{m}$. Appressoria alternate, two celled, antrorse to retrorse, straight to curved, 17-20 $\mu \mathrm{m}$ long; stalk cells cylindrical to cuneate, 7-10 $\mu \mathrm{m}$ long; head cells cylindrical, slightly angular to sublobate, $7-10 \times 5-10 \mu \mathrm{m}$. Thyriothecia grouped at the centre of the colony, orbicular, up to $95 \mu \mathrm{m}$ in diameter, dehisce stellately at the center, margin crenate; asci, globose, eight spored, 32-35 $\mu \mathrm{m}$ in diameter; ascospores conglobate, 1-septate, slightly constricted at the septum, 17-20 × 7-10 $\mu \mathrm{m}$, wall smooth.

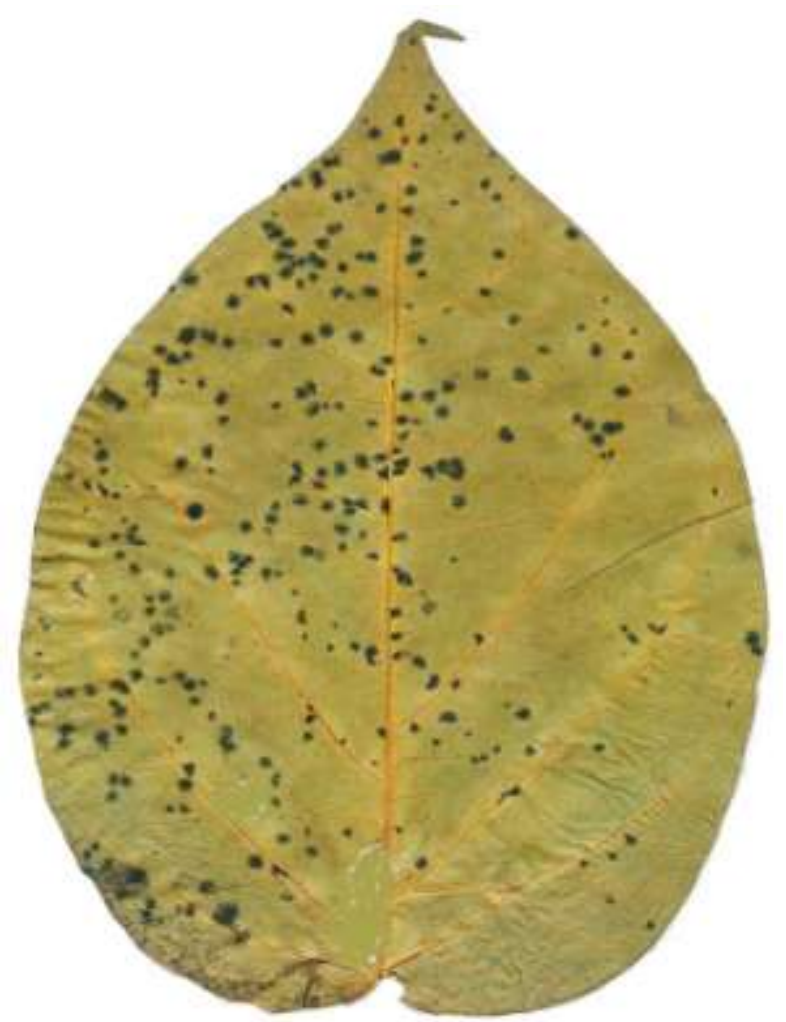

Figure 216 - Asterina viburnicola - Infected leaf

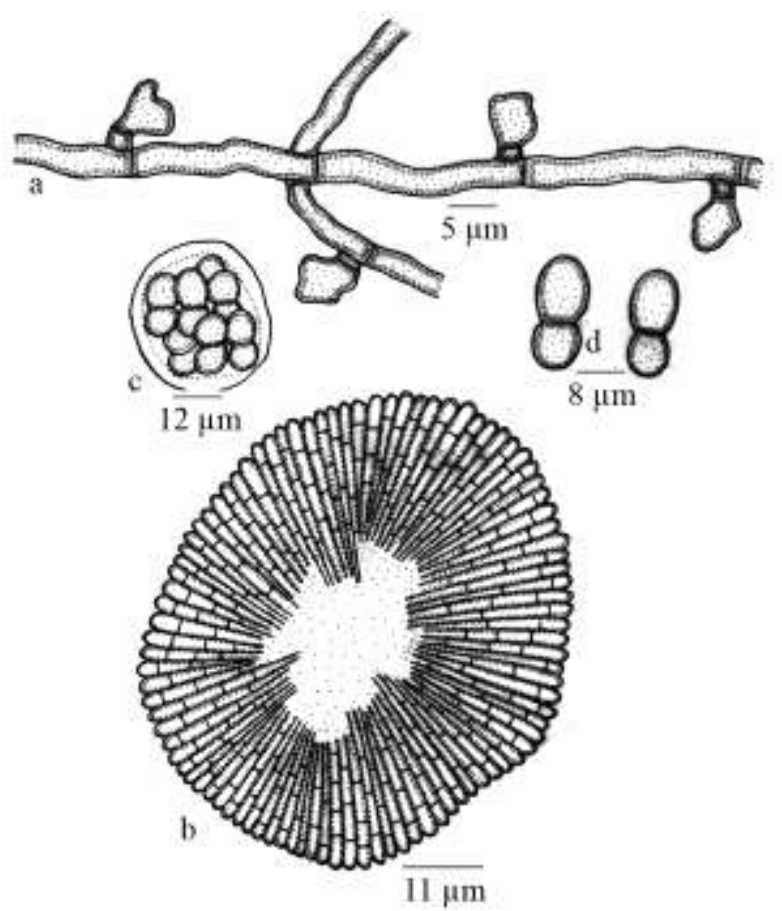

Figure 217 - Asterina viburnicola

Material examined: Tamil Nadu, Kodaikanal, Kukkal shola forest, on leaves of Viburnum cylindricum Buch.-Ham. ex D. Don (Caprifoliaceae), 4 December 2007, R. Nithyatharani TBGT 4263 (holotype).

This is the only species of the genus Asterina known on the members of the family Asteraceae (Hosagoudar \& Abraham, 2000; Stevens \& Ryan, 1939; Katumoto, 1975; Yamamoto, 1957).

Patouillard (J. Bot. 1888, p. 149) described Asteridium viburni on Viburnum sp. and later Saccardo (1891) named it as a species of the genus Asterina and Mycobank has entered it as Asterina viburni (Pat.) Sacc. This species is characterized by "sporidiis hyalinis, viridulis, primo 1 - dein 3 septatis, and 15-17? 5-6". This species is to be confirmed because of its hyaline and 1-3-septate ascospores. Subsequently, Nythyatharani et al. (2010) have proposed Asterina viburni Hosag. et al. as a new species on Viburnum cylindricum. However, the latter species, Asterina viburni Hosag. et al., differs from the former species, Asterina viburni (Pat.) Sacc.. in having brown and larger ascospores $(17-20 \times 7-10 \mu \mathrm{m})$. Hence, the new name has been proposed here. 
Distriubtion: Tamil Nadu

Asterina visci Hosag., Zoos' Print J. 17: 863, 2002; Hosag., Chandraprabha \& Agarwal, Asterinales of Kerala, p. 158, 2011.

(Fig. 218)

Colonies amphigenous, dense, velvety, up to $1 \mathrm{~mm}$ in diameter, rarely confluent. Hyphae substraight to flexuous, branching irregular at acute to wide angles, loosely to closely reticulate, cells $12-20 \times 3-4 \mu \mathrm{m}$. Appressoria alternate, about $2 \%$ opposite, ovate, unicellular, perpendicular to the mycelium, mostly entire, rarely sublobate at sides and furcate at the apex, 8-12 $\times 4-5 \mu \mathrm{m}$. Thyriothecia scattered to connate, orbicular, up to $150 \mu \mathrm{m}$ in diameter; ascospores conglobate, oblong, brown, uniseptate, constricted at the septum, $16-20 \times 8-10 \mu \mathrm{m}$, wall minutely echinulate in the matured ascospores. Pycnothyria similar to thyriothecia, smaller; pycnothyriospores globose, unicellular, brown, 11-18 × 11-16 $\mu \mathrm{m}$, wall smooth.

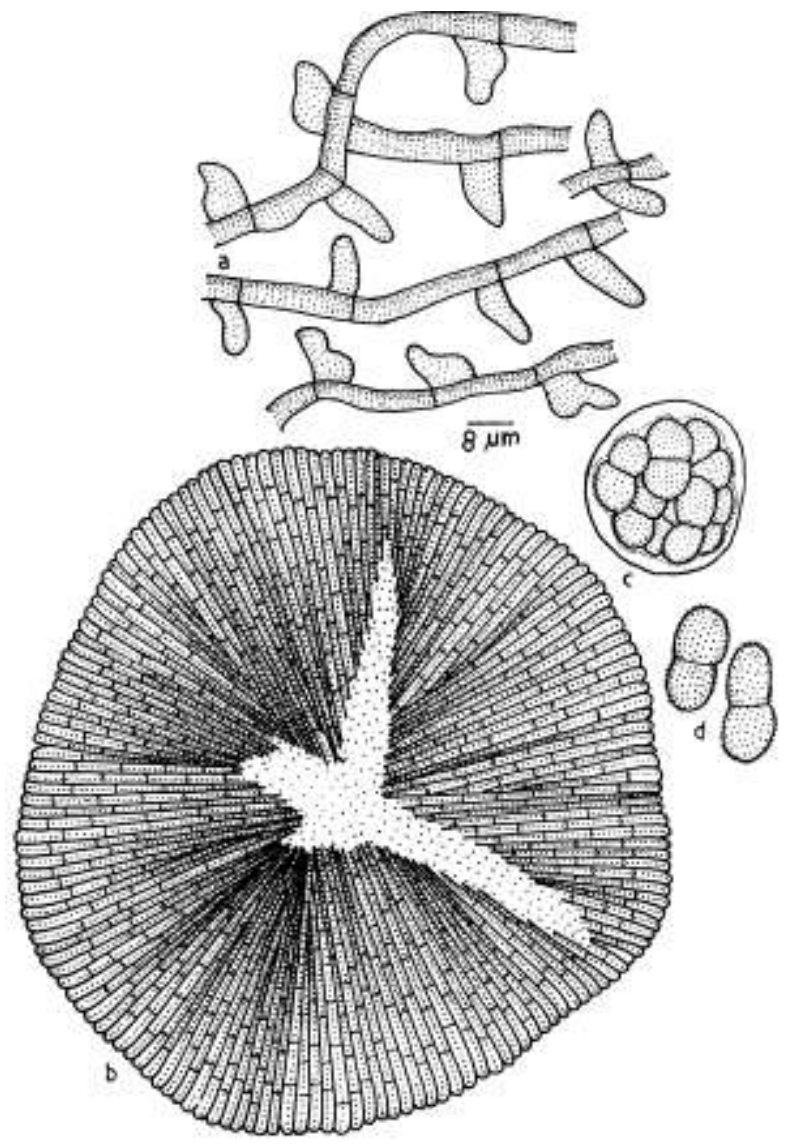

Figure 218 - Asterina visci

Material examined: Thiruvananthapuram, Peppara and Neyyar
Wildlife Sanctuary, Kombe, on leaves of Viscum sp. (Loranthaceae), 19 February 1997, V.B. Hosagoudar HCIO 44203 (holotype), TBGT 562 (isotype).

Based on the morphology of appressoria, Asterina visci can be compared with $A$. deightonii Sydow However, differs from it in having $2 \%$ opposite, ovate, entire to furcate appressoria (Hosagoudar \& Abraham, 2000 ; Sydow, 1938).

Distribution: Kerala.

Asterina viticola Kar \& Ghosh, Indian Phytopathol. 39: 207, 1986. (Fig. 219)

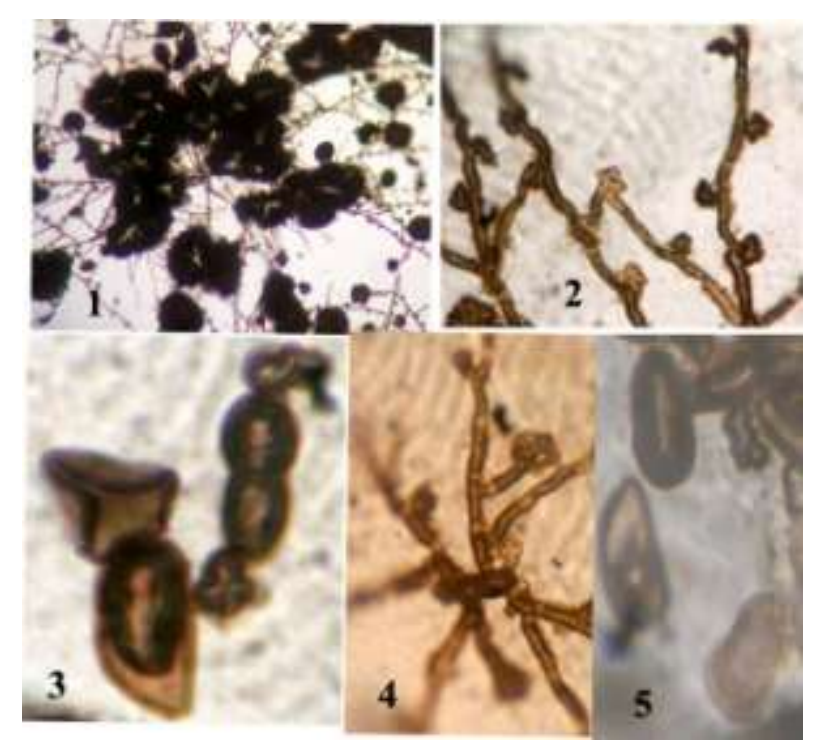

Figure 219 - Asterina viticola

1. Colony with thyriothecia, 2. Appressoriate mycelium, 3. Germinating ascospores, 4. Colony formed ascospore, 5. Germinating pycnothyriospores

Colonies epiphyllous, subdense, up to 4 $\mathrm{mm}$ in diameter. Hyphae straight, flexuous to crooked, branching alternate, opposite to irregular at acute to wide angles, loosely to closely reticulate, cells10-21 $\times$ 4-7 $\mu \mathrm{m}$. Appressoria scattered, alternate to unilateral, about (1\%) opposite, sessile to stipitate, unicellular, ovate, globose to mammiform, crenately to deeply lobate, often attenuated at the apex, 6-12 $\times 7-11 \mu \mathrm{m}$. Thyriothecia loosely to closely grouped, orbicular, stellately dehisced at the centre, up to $140 \mu \mathrm{m}$ in diam., 
margin crenate to fimbriate; asci globose, 8spored, up to $32 \mu \mathrm{m}$ in diam.; ascospores brown, conglobate, oblong, cylindrical, uniseptate, constricted at the septum, 15-20 × 8$11 \mu \mathrm{m}$, wall smooth. Pycnothyria numerous, scattered to closely grouped, orbicular, smaller than thyriothecia, stellately dehisced at the centre; pycnothyria numerous, ovate, oblong, pyriform, $11-16 \times 7-9 \mu \mathrm{m}$.

\section{Material examined: Karnataka,} Kodagu, Hoddur, on leaves of Vitaceae member, 23 August 2009, C. Jagath Thimmaih TBGT 5709.

This species was known on Vitis japonica from Darjeeling, West Bengal (Kar \& Ghosh, 1986) and the present locality reveals its extended distribution.

Distribution: Karnataka, West Bengal.

Asterina wrightiae Sydow in Sydow \& Petrak, Ann. Mycol. 29: 236, 1931; Hosag. \& Abraham, Indian Phytopath. 51: 390, 1998; J. Econ. Taxon. Bot. 4: 560, 2000; Hosag., C.K. Biju \& Abraham, J. Econ. Taxon. Bot. 25: 305, 2001; Hosag., Zoos' Print J. 18: 1280, 2003; Hosag., Zoos' Print J. 21: 2329, 2006; Hosag., Chandraprabha \& Agarwal, Asterinales of Kerala, p. 160, 2011. (Figs 220-222)

Colonies epiphyllous, dense, crustose, up to $5 \mathrm{~mm}$ in diameter, widely confluent and cover larger areas. Hyphae substraight to slightly flexuous, branching irregular at acute to wide angles, loosely reticulate, cells $6-13 \times 2.5$ $3.5 \mu \mathrm{m}$. Appressoria scattered, mostly 2 -celled, rarely unicellular, straight to curved, $11-13 \mu \mathrm{m}$ long; stalk cells cylindrical to cuneate, often flexuous and folding obscures its presence, 4-5 $\mu \mathrm{m}$ long; head cells globose, ovate, cylindrical, mostly 3-lobate, $6-8 \times 7-8 \mu \mathrm{m}$. Thyriothecia numerous, orbicular, up to $100 \mu \mathrm{m}$ in diameter, stellately dehisced at the

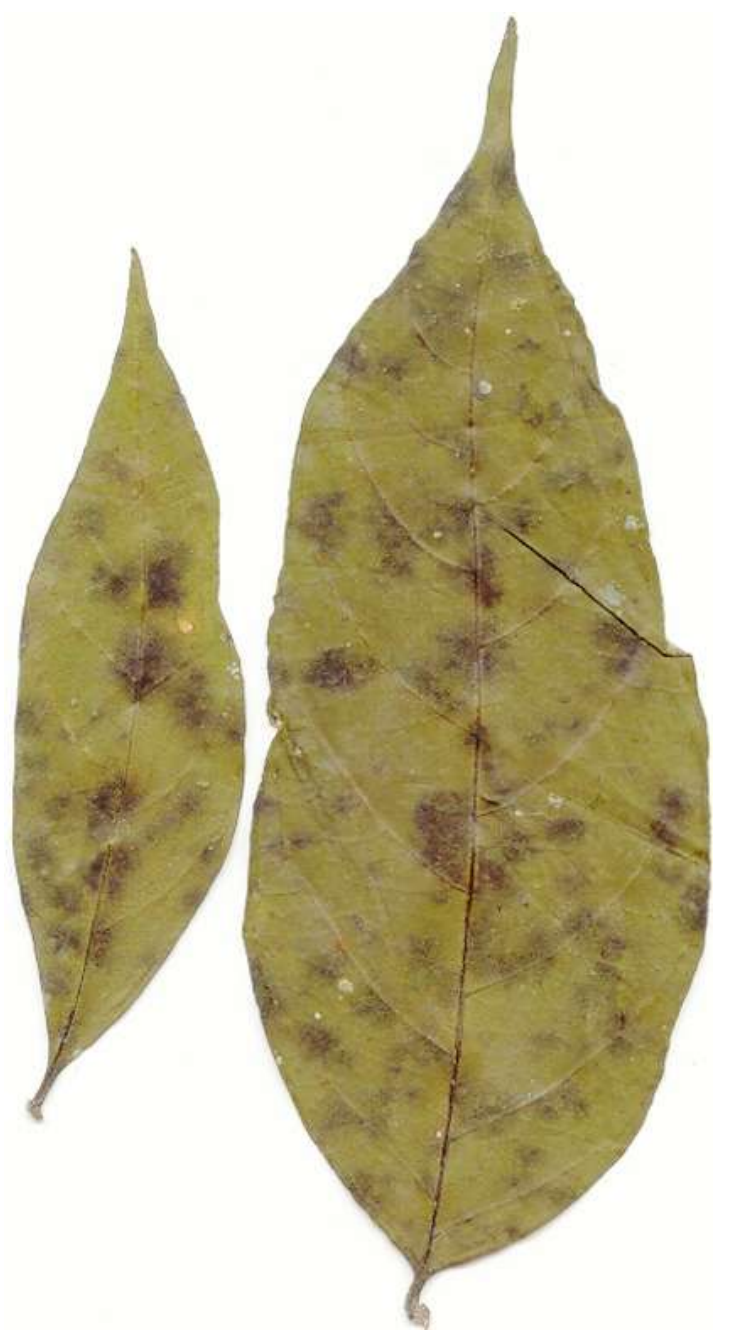

Figure 220 - Asterina wrightiae - Infected leaves

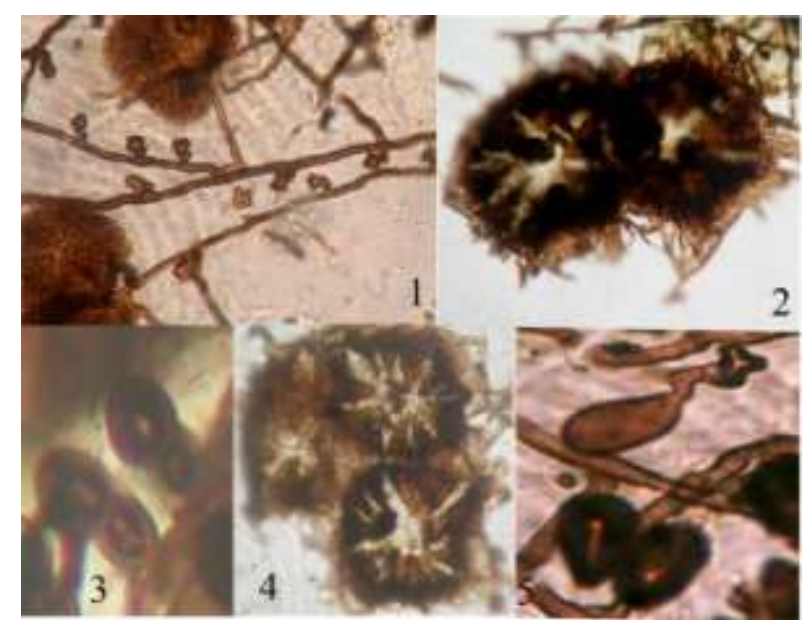

Figure 221 - Asterina wrightiae

1. Appressoriate mycelium, 2. Thyriothecia, 3.Ascospores, 4.Stellately dehisced thyriothecia, 5. Pycnothyriospores 

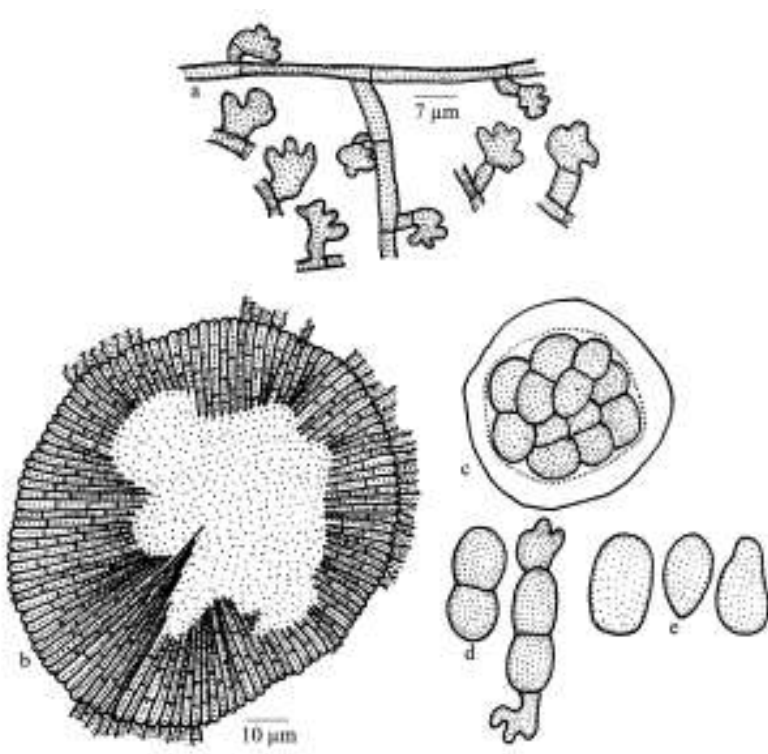

Figure 222 - Asterina wrightiae

centre, often central portion dissolve and exposed the inner content, margin fimbriate, rarely crenate, fringed hyphae small, flexuous to crooked; asci globose, octosporous, 25-35 $\mu \mathrm{m}$ in diameter; ascospores conglobate, brown, 1-septate, constricted at the septum, 14-18 $\times 4$ $10 \mu \mathrm{m}$, upper cell larger, globose, wall smooth. Pycnothyria numerous, similar to thyriothecia, smaller; Pycnothyriospores oblong, pyriform, kidney shaped, ellipsoidal, 12-16 × 6-8 $\mu \mathrm{m}$.

\section{Material examined: Kerala,} Thiruvananthapuram, Peppara Wildlife Sanctuary, on leaves of Wrightia tinctoria (Roxb.) R. Br. (Apocynaceae), 4 February 1997, V.B. Hosagoudar HCIO 42537, TBGT 155; Thiruvananthapuram, Neyyar Wildlife Sanctuary, 18 February 1997, V.B. Hosagoudar HCIO 42520, TBGT 156; Pathanamthitta, Ranni, Placherry forest, 4 October 2002, A. Manojkumar HCIO 44655, TBGT 937; Thrissur, Vazhachal, 23 October 2002, H. Biju \& A. Manojkumar HCIO 44666, TBGT 948; HCIO 44681, TBGT 962; Pathanamthitta, Erumeli, Cheruvalley, on leaves of Wrightia sp., December 29, 2005, V. Gireesh \& P.J. Robin HCIO 46993 (p. p. ), TBGT 2210 (p. p.).

This species was known from Philippines on Wrightia lanatus (Sydow \& Petrak, 1931).

Distribution: Kerala.
Asterina xanthophylli Hosag., Abraham \& C.K. Biju, J. Mycopathol. Res. 37: 25, 1999; Hosag., Chandraprabha \& Agarwal, Asterinales of Kerala, p. 162, 2011.

(Fig. 223)

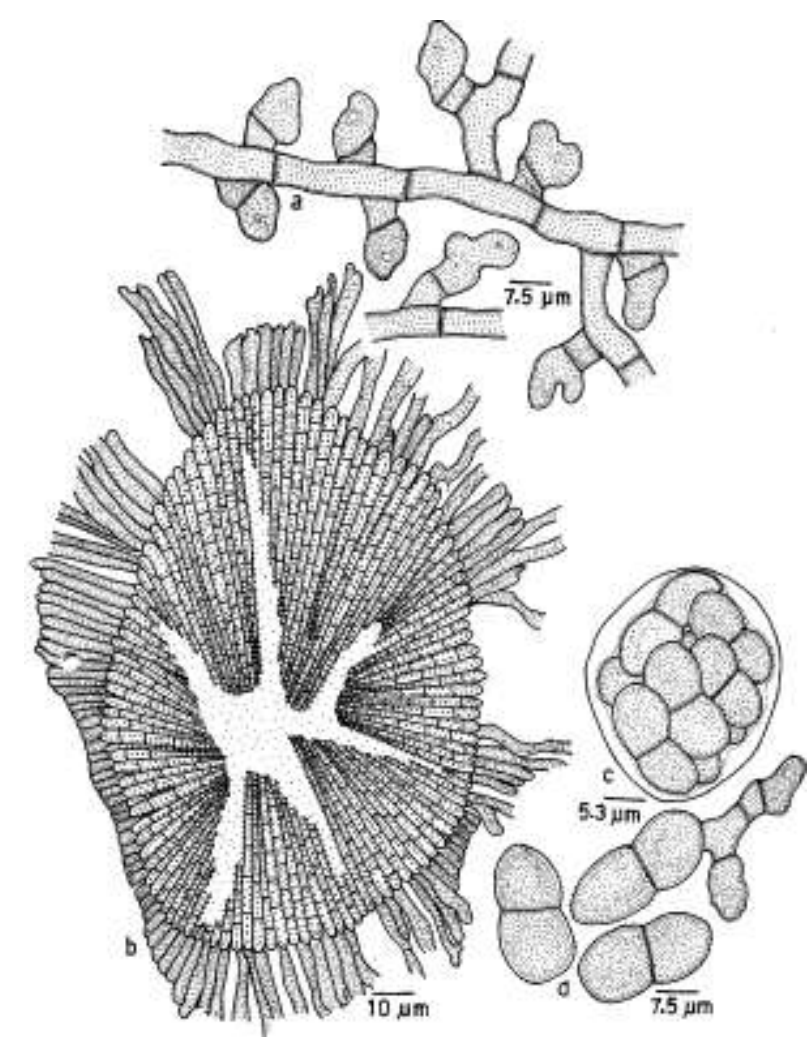

Figure 223 - Asterina xanthophylli

Colonies predominantly epiphyllous, rarely amphigenous, thin to subdense, up to 5 $\mathrm{mm}$ in diameter, often confluent. Hyphae straight to substraight, branching alternate to irregular at acute to wide angles, loosely to closely reticulate, cells $15-30 \times 4-5 \mu \mathrm{m}$. Appressoria alternate, about 5\% opposite, antrorse to subantrorse, 2-celled, 11-18 $\mu \mathrm{m}$ long; stalk cells cylindrical to mammiform, straight to curved, 2-6 $4 \mathrm{~m}$ long; head cells ovate, globose, entire, angular, sublobate, often hamate, $7-11 \times 6-8 \mu \mathrm{m}$. Thyriothecia scattered to rarely connate, orbicular, up to $147 \mu \mathrm{m}$ in diam.; margin fimbriate, fringed hyphae straight to crooked, exappressoriate, light brown, up to $100 \mu \mathrm{m}$. long; thyriothecia stellately dehisced at the center; asci globose, octosporous, $31-33 \mu \mathrm{m}$ in diameter; ascospores brown to dark brown, conglobate, oblong, 1septate, constricted at the septum, 23-26 $\times 11$ $13 \mu \mathrm{m}$. 
Material examined: Kerala, Idukki, Munnar, Chinnar, on leaves of Xanthophyllum arnottianum Wight (X. flavescens auct. non Roxb.) (Xanthophyllaceae), 1 July 1998, C.K. Biju HCIO 42892 (holoype), TBGT 203 (isotype).

This is the only species of the genus Asterina on the members of the family Xanthophyllaceae (Hosagoudar \& Abraham, 2000).

Distribution: Kerala.

Asterina wingfieldii Hosag., Balakr. \& Goos, Mycotaxon 59: 184, 1996. (Fig. 224)
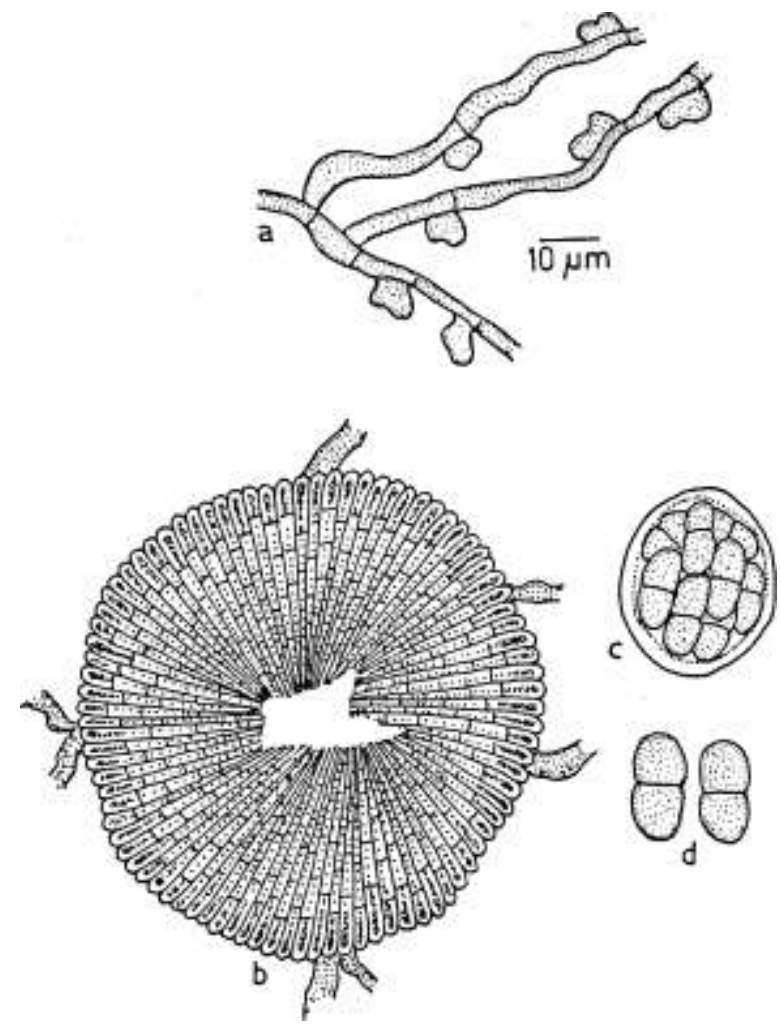

Figure 224 - Asterina wingfieldii

Colonies epiphyllous, thin, up to $2 \mathrm{~mm}$ in diameter, confluent. Hyphae cinnamon brown, flexuous to crooked, branching opposite to irregular at acute angles, loosely reticulate, cells 12-18 × 3-4 $\mu \mathrm{m}$. Appressoria cinnamon brown, unicellular, alternate to irregular, straight to variously curved, globose, versiform, entire, angular to sublobate, 3-7 × 6-8 $\mu \mathrm{m}$. Thyriothecia scattered to grouped, often connate, circular to ovate, up to $124 \mu \mathrm{m}$ in diameter, dehiscing stellately at the center, margin crenate to fringed, fringed hyphae exappressoriate, flexuous to crooked; asci many, globose, eight spored, 24-31 $\mu \mathrm{m}$ in diameter; ascospores conglobate, deep brown, 1-septate, deeply constricted at the septum, 15$19 \times 9-13 \mu \mathrm{m}$, upper cell slightly larger, wall smooth.

Material examined: Tamil Nadu, Coimbatore, Valparai, High forest, Shankaran kudi, on leaves of Grewia sp. (Tiliaceae), 27 October 1990, V.B. Hosagoudar HCIO 30975.

This species is similar to Asterina delicata Doidge (Doidge, 1942) but differs from it in having only epiphyllous colonies, flexuous to crooked hyphae, unicellular and smaller appressoria, and smaller thyriothecia, asci and ascospores.

This species was associated with Irenopsis sp.

\section{Distribution: Tamil Nadu.}

Asterina woodfordiae Sahni, Mycopath. Mycol. Appl. 23(4): 330, 1964; Patil \& Thite, J. Shivaji Univ. 17: 152, 1977.

(Fig. 225)

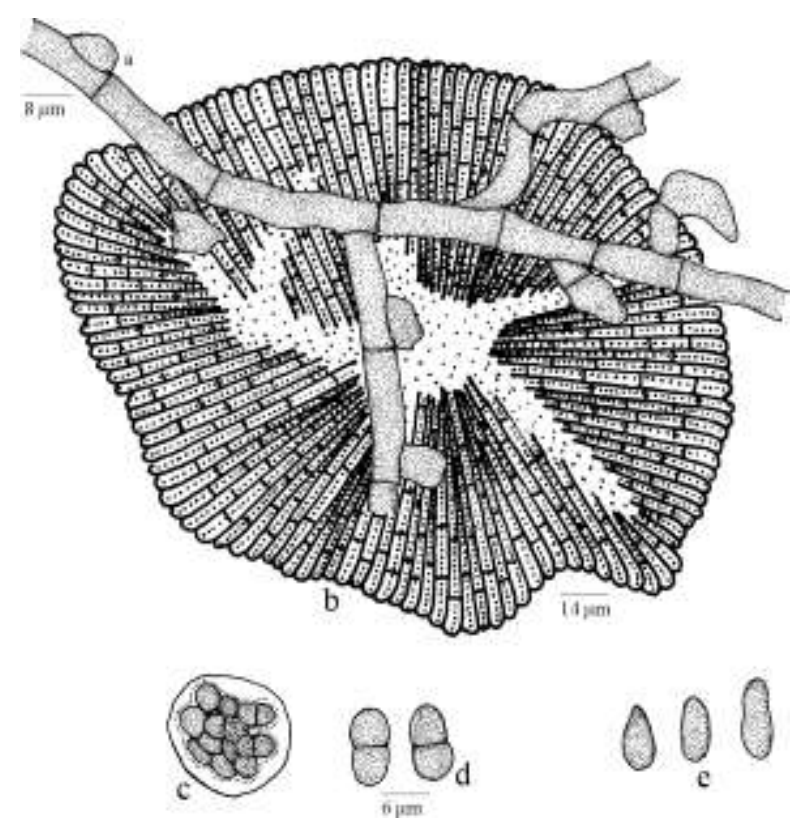

Figure 225 - Asterina woodfordiae

Colonies epiphyllous, thin, crustose, up to $2 \mathrm{~mm}$ in diameter, confluent. Hyphae substraight, flexuous, branching irregular at 
acute to wide angles, loosely to closely reticulate, cells 11-32 × 3-5 $\mu \mathrm{m}$. Appressoria alternate to unilateral, straight to curved, antrorse, subantrorse to retrorse, ovate, globose, 8-16 $\mu \mathrm{m}$ long; stalk cells cylindrical to cuneate, 3-6.5 $\mu \mathrm{m}$ long; head cells straight to variously curved, ovate, oblong, entire to angular, 4-10 $\times$ 4-8 $\mu \mathrm{m}$. Thyriothecia scattered to grouped, orbicular, up to $112 \mu \mathrm{m}$ in diam., stellately dehisced at the centre, margin crenate; ascospores conglobate, uniseptate, slightly constricted at the septum, 14-19 $\times 8-10 \mu \mathrm{m}$, wall smooth. Pycnothyria similar to thyriothecia, orbicular, smaller; pycnothyriospores unicellular, pyriform, ovate, $8-11 \times 4-6.5 \mu \mathrm{m}$.

Material examined: Maharashtra, Satara, Pateghar, on leaves of Woodfordia fruticosa (L.) Kurz (Lythraceae), 3 January 2009, V.B. Hosagoudar \& al. TBGT 4807.

\section{Distribution: Maharastra.}

Asterina zanthoxyli Yamamoto, Sci. Rep. Hyogo Univ. Agric. Biol. Ser. 3: 28, 1957; Kar \& Maity, Trans. Brit. Mycol. Soc. 54: 444, 1970; Hosag., Jacob Thomas \& Robin, Indian J. Sci. \& Techn. 2: 3, 2009. (Fig. 226)

Colonies epiphyllous, dense to subdense, minute $2 \mathrm{~mm}$ in diameter, scattered. Hyphae sub-straight, branching opposite to alternate at acute angles, closely reticulate, cells 10-26 $\times 5 \mu \mathrm{m}$. Appressoria unicellular, ovate to oblong, slightly curved, opposite, subantrorse, 7-10 $\times$ 5-10 $\mu \mathrm{m}$. Thyriothecia scattered, rounded, up to $192 \mu \mathrm{m}$ in diameter, margin fimbriate, stellately dehisce at the centre; asci globose, octosporous, $30-38 \mu \mathrm{m}$ in diameter; ascospores brown, oblong, 1-septate, constricted at the septum, 15-28 $\times 5-7 \mu \mathrm{m}$, wall smooth. Pycnothyriospores pyriform, dark brown in colour, 10-15 $\times 5-7 \mu \mathrm{m}$.

Material examined: Meghalaya, Shillong, Lumshillong, on leaves of Zanthoxylum khasianum Hk. f. (Rutaceae), 18 January 2007, Jacob Thomas \& P.J. Robin HCIO 48070, TBGT 2853.

Distribution: Meghalaya, West Bengal

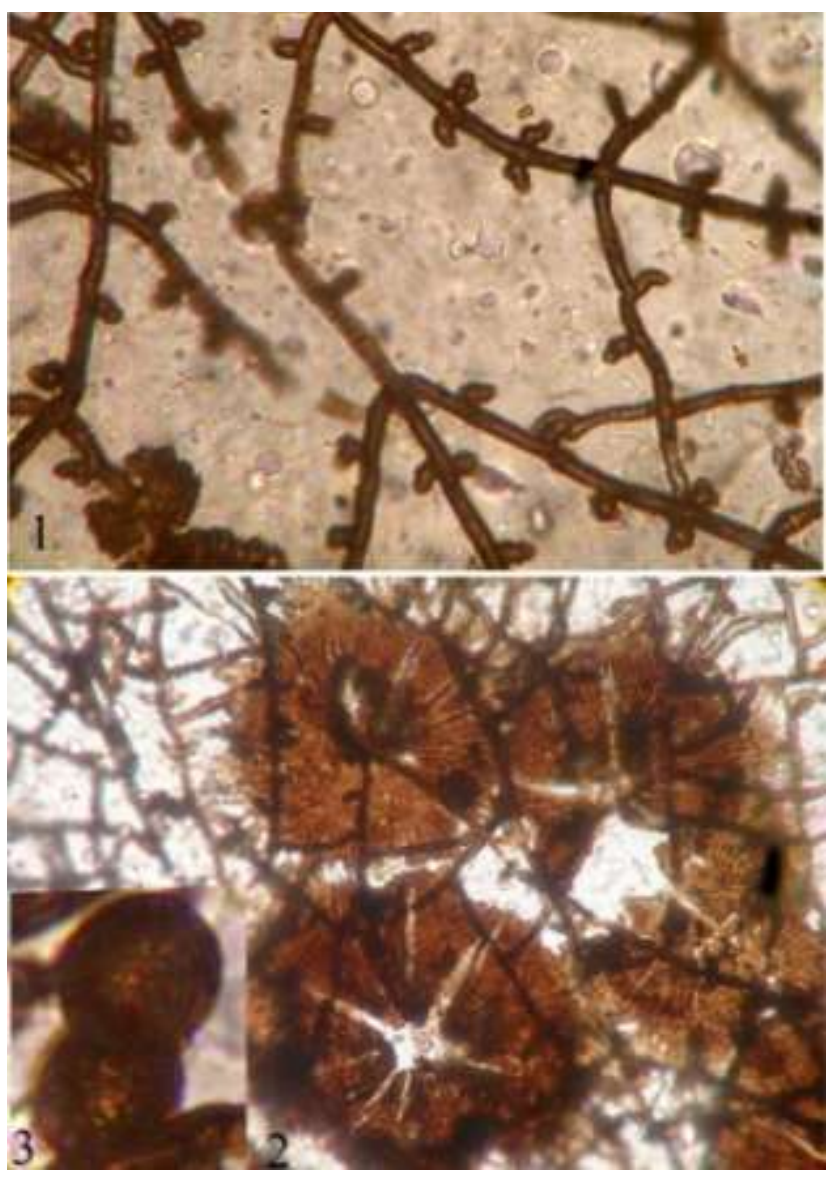

Figure 226 - Asterina zanthoxyli

1. Appressoriate mycelium, 2. Stellately dehisced thyriothecia, 3. Ascospore.

\section{THE GENUS Asterolibertia}

Asterolibertia Arn., Les Asterinees, 1: 161, 1918; Hansf., Mycol. Pap. 15: 189, 1946; Muller \& Arx, Beitr. Krypt. Schw. 11:97, 1962; Arx \& Muller, Stud. Mycol. 9: 43, 1975; Bilgrami, Jamaluddin \& Rizwi, Fungi of India p. 54, 1991; Hosag., Abraham \&C.K. Biju, J. Mycopathol. Res. 39: 61, 2001; Singh, Duke, Bhandari \& Jain, J. Econ. Taxon. Bot. 30: 185, 2008.

Steyaertia Bat. \& Maia, Univ. Recife, Inst. Mycol. Publ. 295:5, 1960. Wardina Arn., Les Asterinees 1:165, 1918.

Leaf parasites. Mycelium ectophytic, appressoria intercalary, setae absent. Thyriothecia orbicular with radiating cells, astomatous, dehisce stellately at the center; asci globose, octosporous, bitunicate; ascospores conglobate, uniseptate, brown. 
Type sp. : A. couepiae (Henn. ) Arn.

Abbreviations used in the line drawings:

a. Intercalary appressoriate mycelium,

b. Thyriothecium, c. Ascus, d. Ascospores

\section{DESCRIPTIONS OF SPECIES}

Asterolibertia hydnocarpi Hosag. \& Abraham, J. Mycopathol. Res. 35: 55, 1997; Hosag., Chandraprabha \& Agarwal, Asterinales of Kerala, p. 163, 2011.

(Fig. 227)

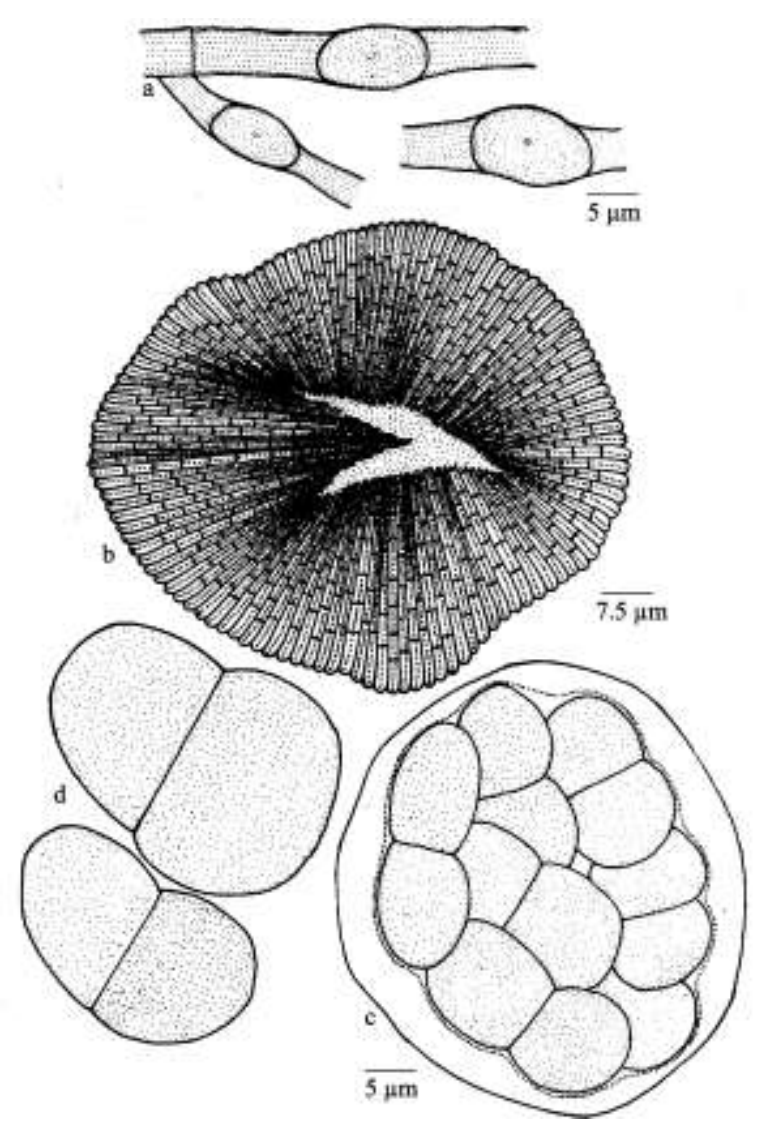

Figure 227 - Asterolibertia hydnocarpi

Colonies epiphyllous, dense, ectophytic, up to $5 \mathrm{~mm}$ in diameter, rarely confluent. Hyphae straight to substraight, distantly branched, loosely reticulate, cell 38-45.5 $\times 11$ $13.5 \mu \mathrm{m}$. Appressoria intercalary, scattered to distantly placed, globose to oval, 14-16 × 11-14 $\mu \mathrm{m}$. Thyriothecia scattered to rarely connate, orbicular, up to $264 \mu \mathrm{m}$ in diameter, stellately dehisced at the center, margin crenate; asci globose, rounded, bitunicate, octosporous, up to
$67 \mu \mathrm{m}$ in diameter, ascospores brown, conglobate, 1-septate, constricted at the septum upper cell smaller and lower cell larger, 49-51.5 $\times 26-32.5 \mu \mathrm{m}$, wall smooth.

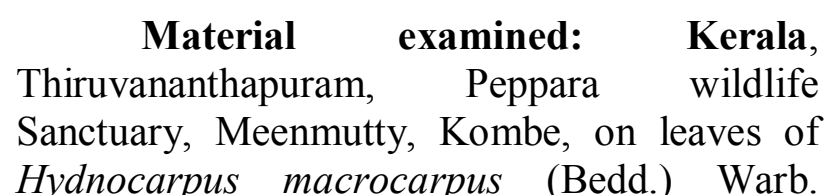
(Flacourtiaceae), 11 March 1996, V.B. Hosagoudar HCIO 42416, TBGT 76; on leaves of $H$. pentandra (Ham.) Oken., HCIO 43989, TBGT 418.

Ectophytic mycelium with intercalary appressoriaand rounded thyrothecia are the characteristics of the genus Asterolibertia. This genus is recorded on the members of Anacardiaceae, Bromeliaceae, Burseraceae, Dipterocarpaceae, Leguminosae, Lauraceae, Malphigiaceae, Melastomataceae, Polygalaceae, Rosaceae (Stevens and Ryan, 1939; Doidge, 1942; Hansford, 1949, 1954 and Mueller and Arx, 1962).

Distribution: Kerala.

Asterolibertia mangiferae Hansf. \& Thirum., Farlowia 3: 303, 1948; Hosag., Chandraprabha \& Agarwal, Asterinales of Kerala, p. 164, 2011.

(Fig. 228)

Colonies amphigenous, mostly epiphyllous, subdense to dense, up to $3 \mathrm{~mm}$ in diameter, confluent. Hyphae straight to substraight, branching opposite to irregular at acute to wide angles, loosely to closely reticulate, cells $8-32 \times 3-7 \mu \mathrm{m}$. Appressoria intercalary, oval to ellipsoidal with a hyaline pore, which indicate the presence of appressoria from the lower surface, $8-18 \times 6-8 \mu \mathrm{m}$. Thyriothecia scattered to loosely grouped, orbicular, up to $392 \mu \mathrm{m}$ in diameter, stellately dehisced at the centre, margin fringed, fringed hyphae small; asci not seen; ascospores brown, conglobate, uniseptate, rounded at both ends, constricted at the septum, $16-26 \times 11-13 \mu \mathrm{m}$, smooth walled.

Material examined: Kerala, Thiruvananthapuram, TBGRI Campus, Palode, 
on leaves of Mangifera indica L. (Anacardiaceae), 16 October 2005, Al-Ameen HCIO 46982, TBGT 2199; 12 October 2006, A. Sabeena \& M.C. Riju HCIO 47433 TBGT 2471; 11 June 2007, A. Sabeena HCIO 48346 TBGT 3067.

Asterolibertia mangiferae Hansf. \& Thirum. is the only species of the genus Asterolibertia known on Mangifera indica from India (Hansford \& Thirumalachar, 1948).

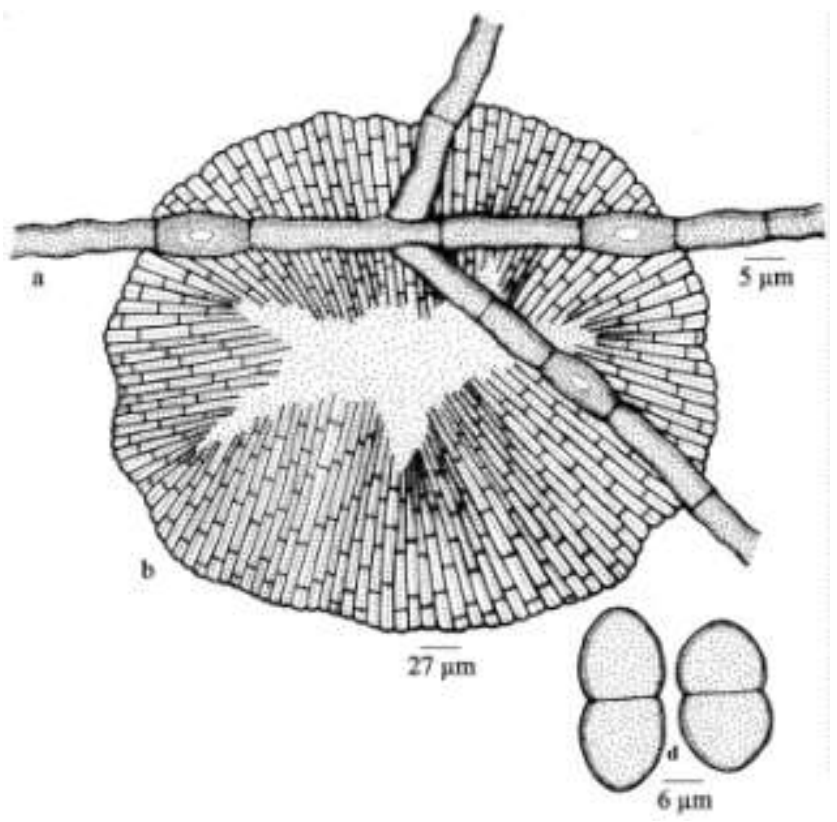

Figure 228 - Asterolibertia mangiferae

This species was collected from Thirthahalli, Western Ghats region of Karnataka, during the year 1945 (Hansford \& Thirumalachar, 1948).

Distribution: Kerala, Karnataka.

Asterolibertia nothopegiae Hosag. \& Abraham, New Botanist 24: 109, 1997; Hosag., J. Threatened Taxa 2: 1154, 2010; Hosag., Chandraprabha \& Agarwal, Asterinales of Kerala, p. 166, 2011.

(Fig. 229)

Colonies epiphyllous, subdense to dense, up to $3 \mathrm{~mm}$ in diameter, rarely confluent. Hyphae straight, branching opposite to irregular at wide angles, loosely reticulate, cells 24-37 × 5-7 $\mu \mathrm{m}$. Appressoria mostly distinct in the periphery of the colonies intercalary, scattered, rounded, central pale hyaline spot visible, 10-12 $\times 9.5-11 \mu \mathrm{m}$. Thyriothecia scattered to connate, orbicular, up to $265 \mu \mathrm{m}$ in diameter Stellately dehisced and later widely opened at the center, margin mostly crenate, often fimbriate, fringed hyphae small and run parallel; asci few, oval, octosporous, $32-35 \times 24-27 \mu \mathrm{m}$, ascospores brown, conglobate, 1-septate, constricted at the septum, 19-21 × 9-10 $\mu \mathrm{m}$, wall smooth.

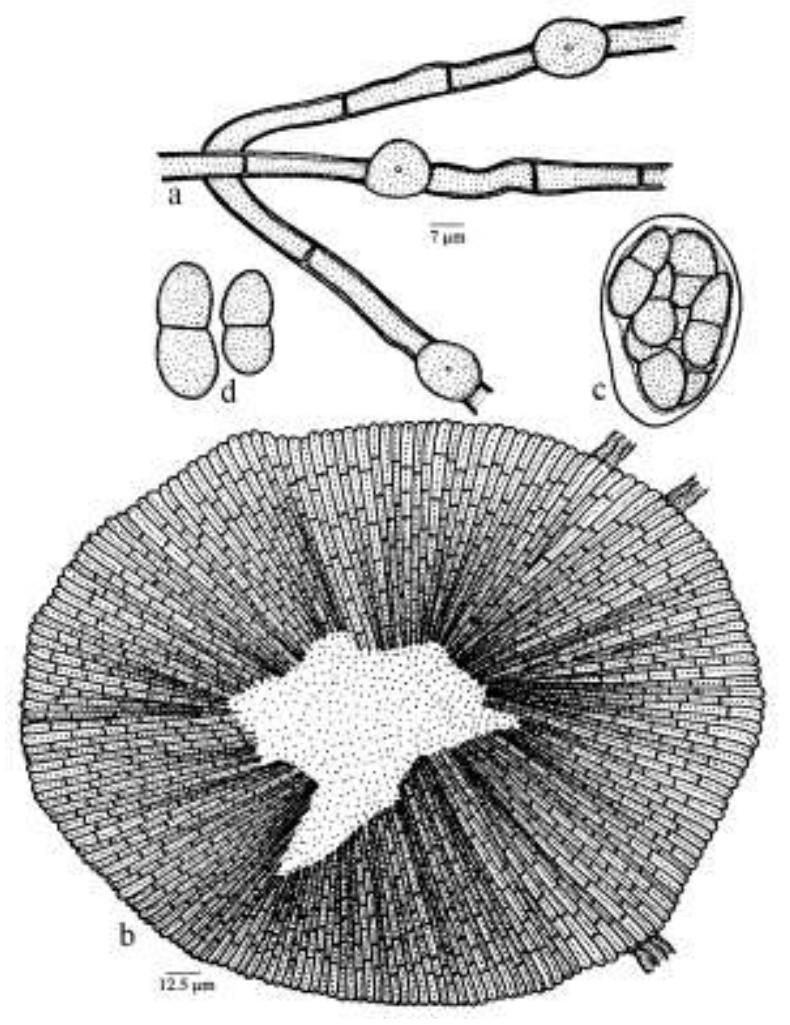

Figure 229 - Asterolibertia nothopegiae

\section{Material examined: Kerala, Thiruvananthapuram, Neyyar Wildlife Sanctuary, Attayar, on leaves of Nothopegia aureo-fulva Bedd. ex Hook. f. (Anacardiaceae), 19 March 1997, V.B. Hosagoudar HCIO 42475 (holoype), TBGT 115 (isotype).}

Asterolibertia mangiferae Hansf. \& Thirum. is the only species of the genus Asterolibertia known on Mangifera indica from India (Hansford \& Thirumalachar, 1948).

Distribution: Kerala.

Asterolibertia vateriae Hosag., J. Mycopathol. Res. 44: 13, 2006; Hosag., Chandraprabha \& Agarwal, Asterinales of Kerala, p. 168, 2011.

(Fig. 230) 


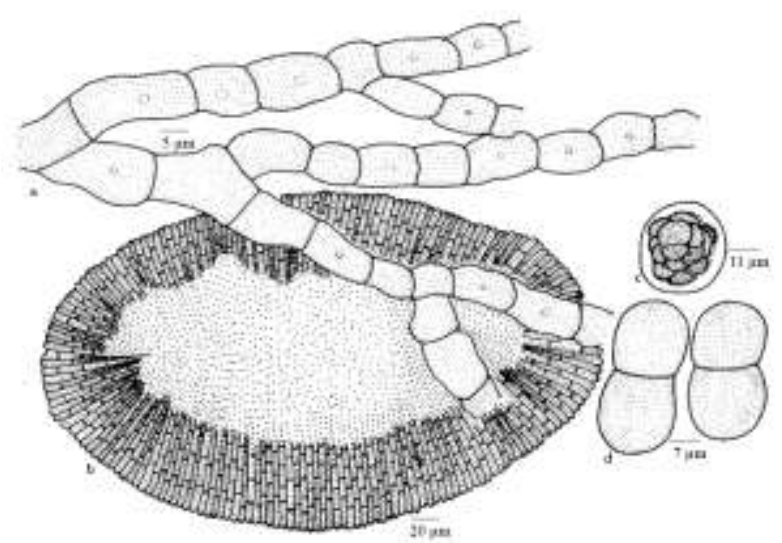

Figure 230 - Asterolibertia vateriae

Colonies amphigenous, mostly epiphyllous, dense, crustose, cause water soaked lesions on the corresponding opposite surface of the leaves, up to $10 \mathrm{~mm}$ in diameter, confluent. Hyphae substraight to crooked, branching opposite to irregular at acute to wide angles, loosely to closely reticulate, cells 12-21 $\times$ 11-13 $\mu \mathrm{m}$. Appressoria intercalary, ovate to oblong, located in the cell with a central marking, $10-15 \times 2-14 \mu \mathrm{m}$. Thyriothecia scattered, initially orbicular, later ellipsoidal, $300-400 \times 150-250 \mu \mathrm{m}$, vertically to irregularly dehisced at the centre, often central po0rtion dissolved, margin crenate to fimbriate, fringed hyphae flexuous, compact; asci globose, ovate, octosporous, up to $35 \mu \mathrm{m}$ in diameter; ascospores conglobate, brown, uniseptate, constricted at the septum, $36-39 \times 21-23 \mu \mathrm{m}$, wall smooth.

Material examined: Karnataka, Kodagu (Coorg), Madikeri, Jodupal, on leaves of Vateria indica L. (Dipterocarpaceae), 12 November 2003, V.B. Hosagoudar \& al. HCIO 45752 (holotype), TBGT 1501 (isotype); Kerala, Thiruvananthapuram, Bonacaud, 6 February 2003, G. Rajkumar HCIO 47698, TBGT 2720; TBGRI campus, 12 October 2006 A. Sabeena \& M.C. Riju HCIO 47543,TBGT 2565; 24 October 2008 A. Sabeena \& M.C. Riju HCIO 49137,TBGT 3392; Thiruvananthapuram, Peppara Wildlife Sanctuary, Athirumala, 1 March 2008, Jacob Thomas HCIO 48874, TBGT 2250.

Asterolibertia anisopterae (Sydow) Hansf. and A. flabellariae (Sydow) Hansf. are known on Anisoptera thursifera and Flabelliferia paniculata from Philippines and
Sierra Leone, respectively. A. vateriae differs from A. anisopterae in not forming polygonal meshes of hyphae, having smaller thyriothecia and in causing pathogenic effect on the host. It differs from $A$. fabelliferae in having distinctly larger ascospores (Hansford, 1947, 1949). Ascospores are smaller than $A$. hydnocarpi Hosag. \& Abraham (Hosagoudar \& Abraham, 1997a).

Distribution: Karnataka, Kerala.

\section{THE GENUS Bheemamyces}

Bheemamyces Hosag., J. Threatened Taxa 2(12): 1323, 2010.

(Fig. 231-233)

Colonies foliicolous, brown, branched, septate, appressoria both intercalary and lateral, often form mycelial ring. Thyriothecia orbicular, stellately dehisce at the centre; asci globose, octosporous; ascospores conglobate, brown, uniseptate.

Type sp.: B. argyreiae Hosag. \& Sabeena \& Riju
Abbreviations used in the line drawings:
a. Appressoriate mycelium, b. Thyriothecium
c. Ascus, d. Ascospores

\section{DESCRIPTIONS OF SPECIES}

Bheemamyces argyreicola Hosag. \& Sabeena \& Riju, J. Threatened Taxa 2(12): 1323, 2010. (Figs 231-235)

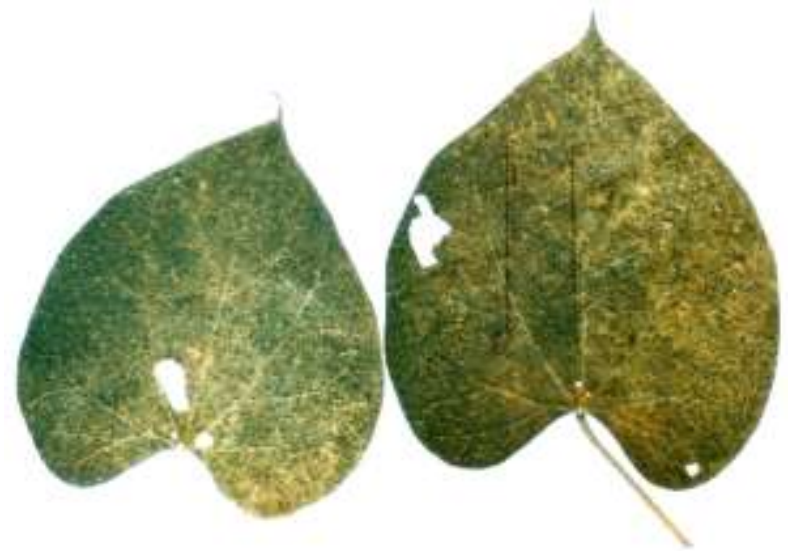

Figure 231 - Bheemamyces argyreicola Infected leaves 
Colonies epiphyllous, densely or closely scattered, up to $2 \mathrm{~mm}$ in diameter, often confluent. Hyphae of two types: substraight to crooked, branching irregular at acute to wide angles, loosely to closely reticulate, cells 12-32 $\times 4-5 \mu \mathrm{m}$. Appressoria scattered, alternate to unilateral, unicellular, ovate, oblong, globose, entire, angular to sublobate, 5-17 $\times 5-10 \mu \mathrm{m}$. The second type of hyphae originated from the main hyphae, narrower, curved, slightly elevated from the host surface and sharply pointed at the tip. These hyphae possess intercalary, sub intercalary and lateral appressoria. Hyphal rings are formed randomly but are devoid of appressoria. The intercalary and sub intercalary appressoria oval to globose, having a central slightly hyaline spot, 5-10 $\times 5$ $7 \mu \mathrm{m}$. Thyriothecia scattered to connate, orbicular, stellately dehisced at the centre, up to $90 \mu \mathrm{m}$ in diameter, margin crenate to fimbriate, fringed hyphae straight, flexuous to crooked; asci globose, octosporous, 32-35 $\mu \mathrm{m}$ in diameter; ascospores brown, conglobate, uniseptate, constricted at the septum, 15-25 × 7$12 \mu \mathrm{m}$, wall smooth, readily germinated.

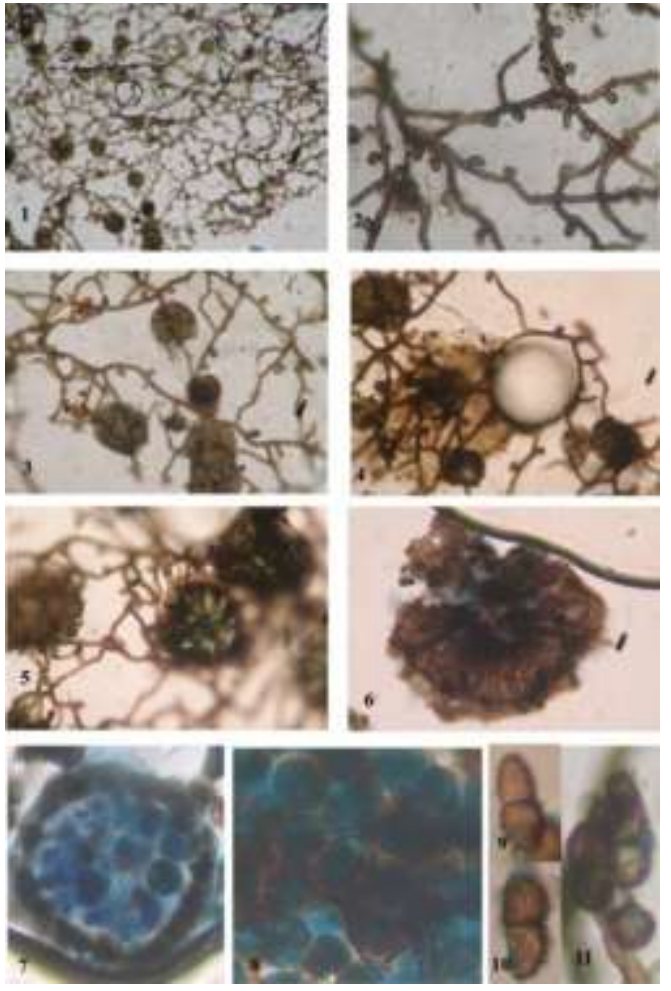

Figure 232 - Bheemamyces argyreicola

1. Fungal colony, 2.Appressoriate mycelium, 36. Thyriothecia, 7-8. Thyriothecia showing asci by dissolving the upper portion, 9-11. Ascospores
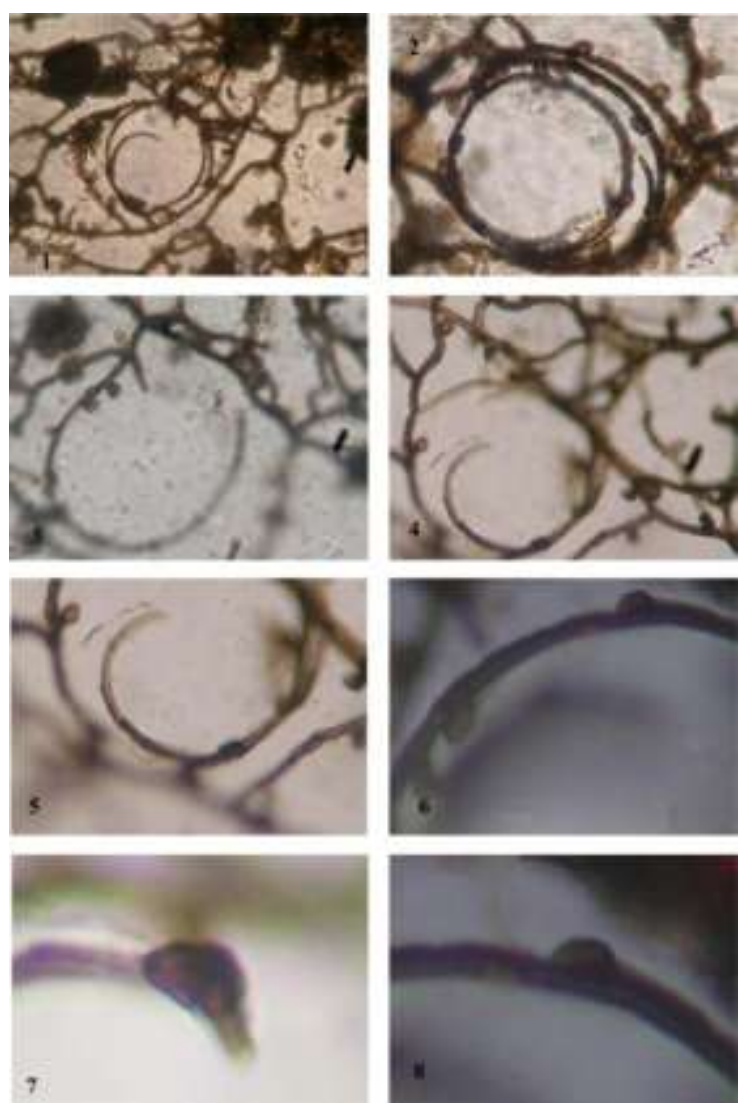

Figure 233 - Bheemamyces argyreicola

1-5. Coiled apical hyphae, 6-8. Intercalary appressoria in the coiled hyphae

Material examined: Kerala, Malabar Botanic Garden, Kozhikode, on leaves of Argyreia nervosa (Burm.f.) Bojer. (Convolvulaceae), 4 November 2009, A. Sabeena \& M.C Riju TBGT 4324 (holotype). Isotype is in HCIO, New Delhi.

The fungus revealed brown mycelium with the lateral appressoria and stellately dehisced orbicular thyriothecia. These are the characters of the genus Asterina. In addition to these typical characters, the mycelia originated from the main hyphae, lifted slightly above the host surface, appearing like a 'whip', possessing intercalary and sub intercalary or sub lateral appressoria, making it distinct from the genus Asterina. Because of the intercalary appressoria, it cannot be placed under the genus Asterolibertia because it also possesses the characters of the genus Asterina, having lateral appressoria (Muller \& Arx, 1962; Arx \& Muller, 1975; Hosagoudar et al., 2001a). Hence, to accommodate such fungi, the genus Bheemamyces is proposed here. 
This is a transitional genus between the genera Asterina and Asterolibertia.

Distribution: Kerala

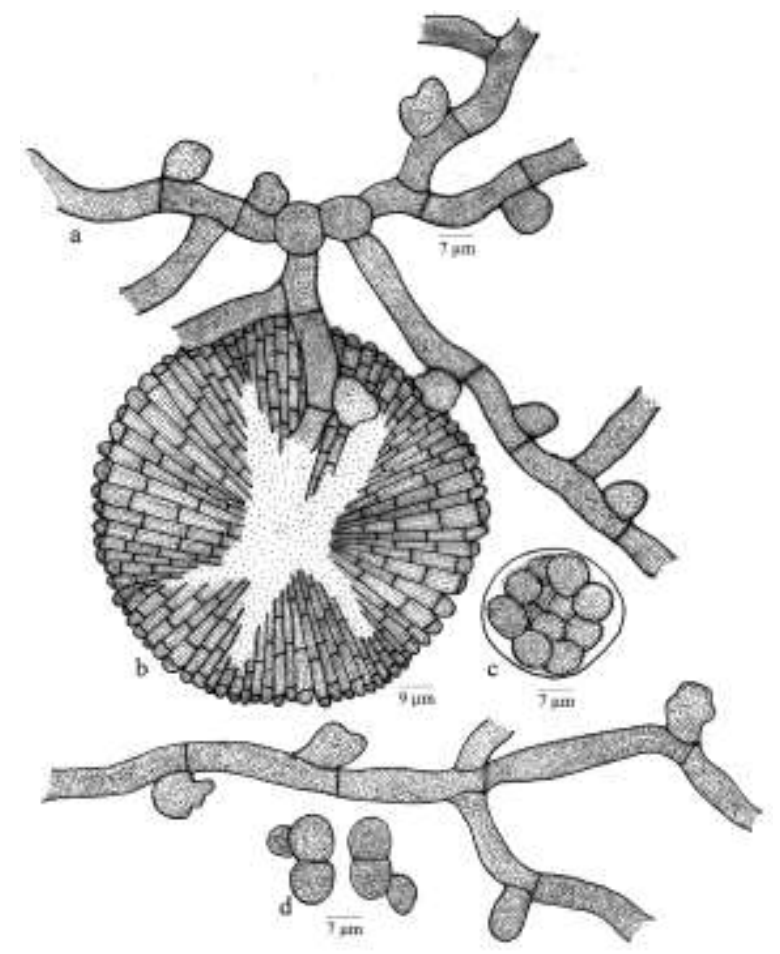

Figure 234 - Bheemamyces argyreicola

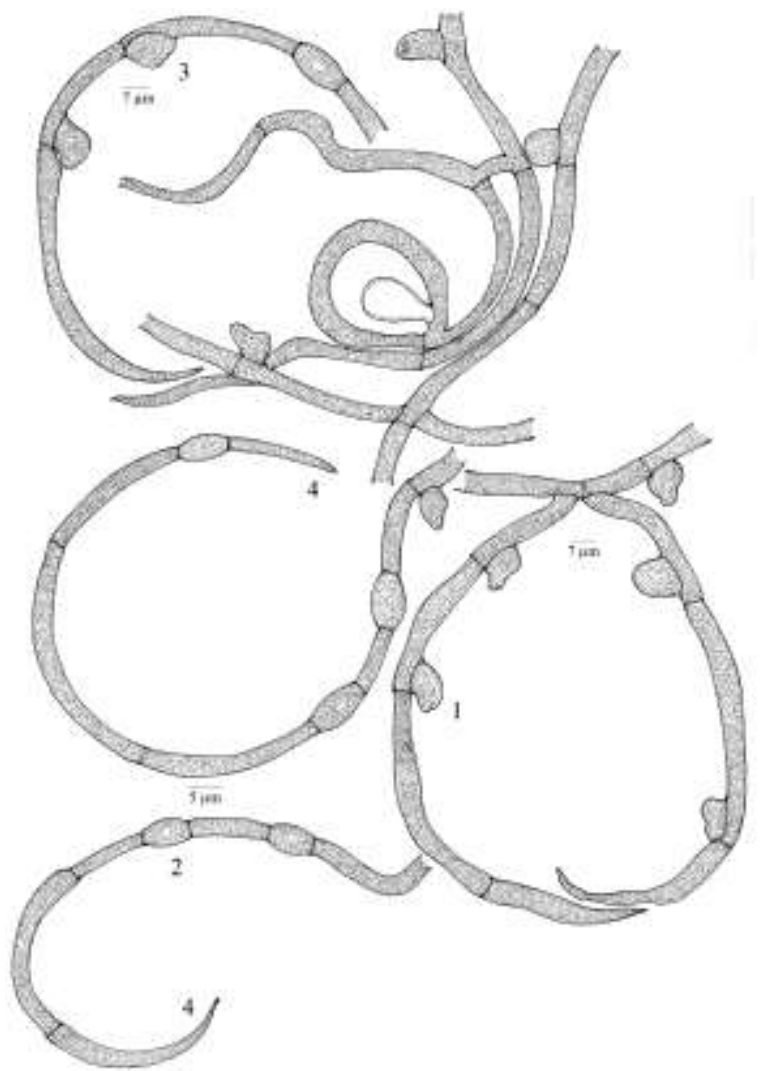

Figure 235 - Bheemamyces argyreicola Placement of appressoria:1. Lateral, 2. Intercalary, 3. Sublateral, 4. Coiled hyphal tip
Bheemamyces capparidis V.B. Hosagoudar \& A. Sabeena sp. nov. (Fig. 236)

MycoBank 101012

Etymology: Named after the host genus

Colonies amphigenous, subdense to dense, up to $3 \mathrm{~mm}$ in diameter, confluent. Hyphae of two types: straight to substraight, branching alternate to unilateral at acute to wide angles, loosely reticulate, cells $15-35 \times$ 2.5-5 $\mu \mathrm{m}$. Appressoria alternate $10 \%$ opposite, antrorse to subantrorse 7-12 $\mu \mathrm{m}$ long; stalk cells cylindrical to cuneate, $2-5 \mu \mathrm{m}$ long; head cells unicellular, ovate, entire to sublobate, 5-7 $\times$ 5-10 $\mu \mathrm{m}$. The second type of hyphae originated from the main hyphae, narrower, curved, slightly elevated from the host surface and sharply pointed at the tip. These hyphae possess intercalary, sub intercalary and lateral Appressoria. The intercalary and sub intercalary appressoria ovate, 5-12 $\times 5-7 \mu \mathrm{m}$. Thyriothecia scattered to connate, orbicular, stellately dehisced at the centre, up to $112 \mu \mathrm{m}$ in diameter, margin crenate; asci globose, octosporous, 17$25 \mu \mathrm{m}$ in diameter; ascospores, conglobate, uniseptate, constricted at the septum, 17-22 × 7$10 \mu \mathrm{m}$, wall slightly echinulate; Pycnothyria similar to thyriothecia; pycnothyriospores ovate to globose, $12-20 \times 10-12 \mu \mathrm{m}$.

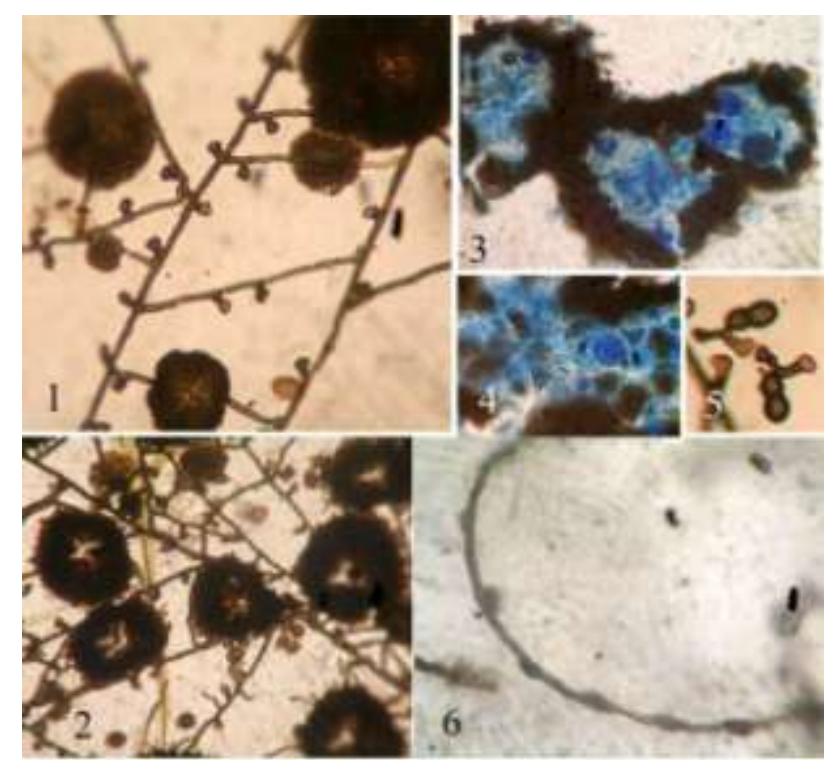

Figure 236 - Bheemamyces capparidis

1. Appressoriate mycelium, 2.Stellately dehisced thyriothecia, 3. Dehisced thyriothecia showing asci, 4. Asci, 5. Ascospores, 6. Apical portion of the hyphae with intercalary appressoria 
Material examined: Kerala, Thiruvananthapuram, Palode, TBGRI Medicinal Garden, on leaves of Capparis sp. (Capparaceae), 12 August 2010, A. Sabeena TBGT 5753 (holotype).

Distribution: Kerala

\section{THE GENUS Gangamyces}

Gangamyces V.B. Hosagoudar, gen. nov. MycoBank 800999

Etymology: Named after the holy river, Ganga

Leaf parasites. Mycelium ectophytic. Appressoria lateral in opposite couplet with a stellate lumen in the attached mycelial cell. Thyriothecia orbicular with radiating cells, astomatous, dehisce stellately at the center; asci globose, octosporous, bitunicate; ascospores conglobate, uniseptate, brown. Archana

Type sp.: G. miliusae Hosag. \&

This genus is similar to Asterina but differs from in having oppositely borne appressoria with a stellate lumen continuous with the parent hyphal cell.

\section{Abbreviations used in the line drawings:}

a. Appressoriate mycelium, b. Thyriothecium

c. Ascus, d. Ascospores, e. Pycnothyriospores

\section{DESCRIPTIONS OF SPECIES}

Gangamyces miliusae V.B. Hosagoudar \& G.R. Archana, sp. nov. (Figs 237-238)

MycoBank 801000

Etymology: Named after the host genus

Colonies amphigenous, mostly epiphyllous, subdense, up to $2 \mathrm{~mm}$ in diameter, confluent. Hyphae straight to substraight, branching opposite to irregular at acute to wide angles, closely to loosely reticulate, cells 12-36 $\times \quad 4-8 \mu \mathrm{m}$. Appressoria sessile in opposite couplet forming a stellate lumen in the cells on which they are born, scattered to closely scattered, ovate, globose, margin entire, couplet horizontally $18-22 \mu \mathrm{m}$ broad, appressoria individually $8-12 \mu \mathrm{m}$ broad, lumen quadrangular in shape (+-shaped) with its points towards upper and lower septa and the other two directed towards the centre of the appressoria, lumen 13-16 $\times$ 8-12 $\mu \mathrm{m}$. Thyriothecia scattered to loosely grouped, orbicular to oval, up to $400 \mu \mathrm{m}$ in diam., stellately dehisced in the centre, margin

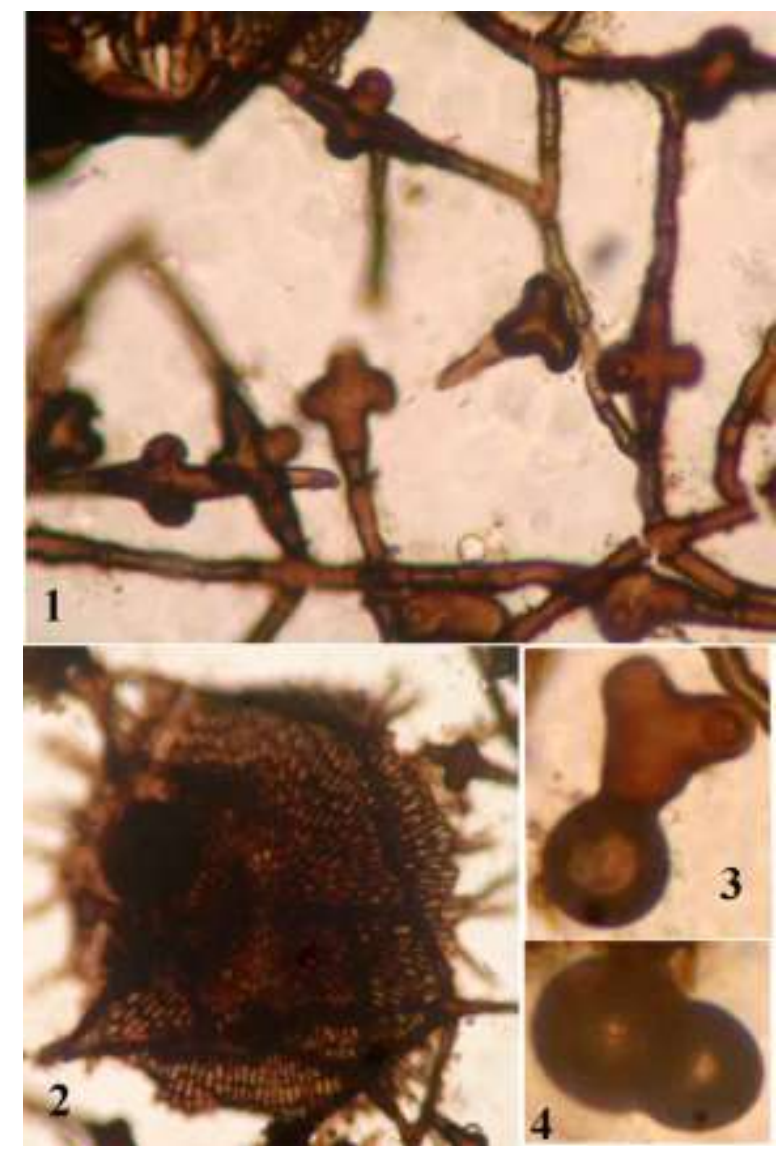

Figure 237 - Gangagamyces miliusae

1. Appressoria in couplet with stellate lumen, 2. Thyriothecium, 3. Germinating pycnothyriospore, 4. Ascospore

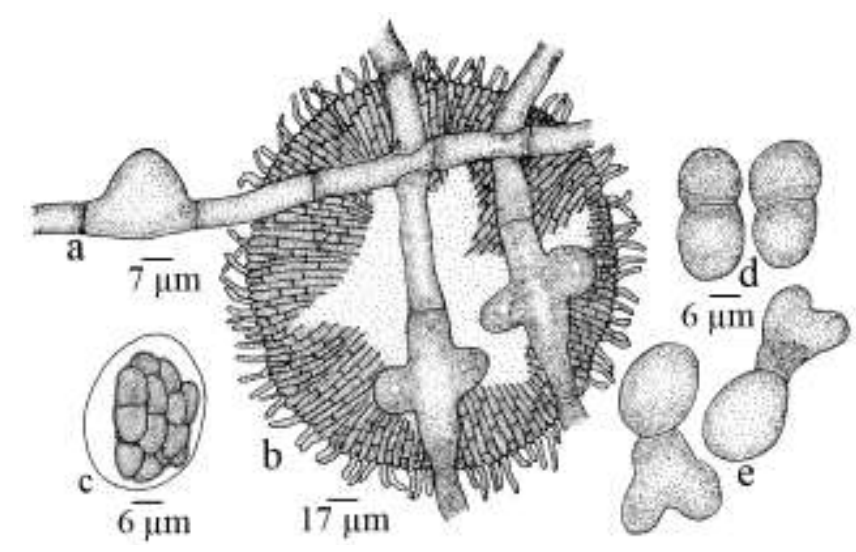

Figure 238 - Gangagamyces miliusae 
crenate to fimbriate, fringed hyphae flexuous; asci oval to globose, octosporous, up to $25 \mu \mathrm{m}$ in diam.; ascospores oblong to cylindrical, conglobate, dark brown to brown, 1-septate, constricted at the at the septum, 27-31 $\times 17-20$ $\mu \mathrm{m}$. Pycnothyriospores numerous, globose, pyriform, unicellular, often readily germinated, $16-20 \times 17-20 \mu \mathrm{m}$.

Material examined: Uttar Pradesh, on leaves of Miliusa tomentosa (Roxb.) Sinclair (Annonaceae), October 1999, Swadeep Srivastav HCIO 44215 (holotype).

\section{Distribution: Uttar Pradesh}

Gangamyces shoreae V.B. Hosagoudar, M.A. Nomani, Kamal \& G.R. Archana, sp. nov. (Figs 239-240)

MycoBank 801002

Etymology: Named after the host genus

Colonies epiphyllous, thin, up to $2 \mathrm{~mm}$ in diam., confluent. Hyphae straight, substraight to flexuous, branching opposite to irregular at acute to wide angles, cells 19-44 $\times$ 6-8 $\mu \mathrm{m}$. Appressoria sessile in opposite couplet forming a stellate lumen in the cells on which they are born, scattered to closely scattered, ovate, globose, margin entire, couplet horizontally 22-30 $\mu \mathrm{m}$ broad, appressoria individually $8-12 \mu \mathrm{m}$ broad, lumen quadrangular in shape (+-shaped) with its points towards upper and lower septa and two directed towards the centre of the appressoria, lumen $12-16 \times 9-11 \mu \mathrm{m}$. Thyriothecia scattered to loosely trouped, orbicular, stellately dehisced in the centre, up to $300 \mu \mathrm{m}$ in diam., margin crenate to fimbriate, fringed hyphae flexuous; asci oval to globose, octosporous, up to $25 \mu \mathrm{m}$ in diam.; ascospores oblong to cylindrical, conglobate, dark brown to brown, 1-sept僦, o constricted at the at the septum, 22-26 × 11-13 $\mu \mathrm{m}$. Pycnothyriospores numerous, globose, pyriform, unicellular, often readily germinated, $20-35 \times 19-23 \mu \mathrm{m}$.

Material examined: Uttar Pradesh, Maharganj, on leaves of Shorea robusta Roxb. ex Gaertner (Dipterocarpaceae), M.A. Nomani TBGT 3516 (holotype).
Distribution: Uttar Pradesh

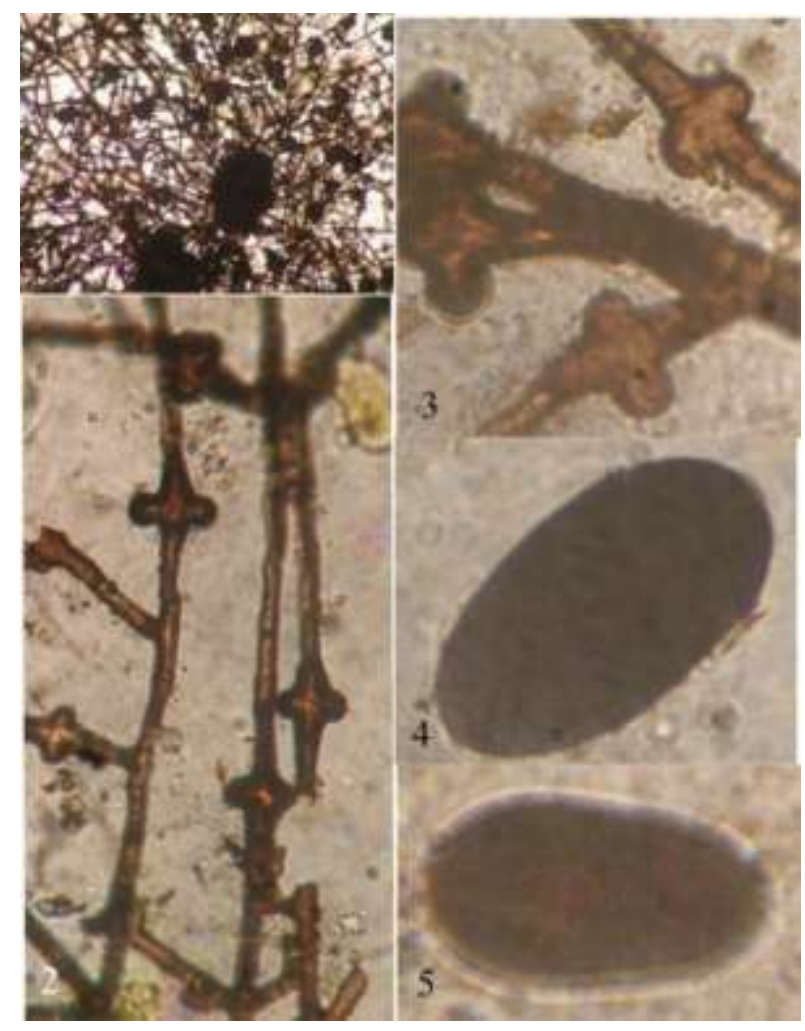

Figure 239 - Gangamyces shoreae

1. Mycelial colony, 2-3.Appressoria in couplet on the mycelial cells with stellate lumen, 4-5. Pycnothyriospores

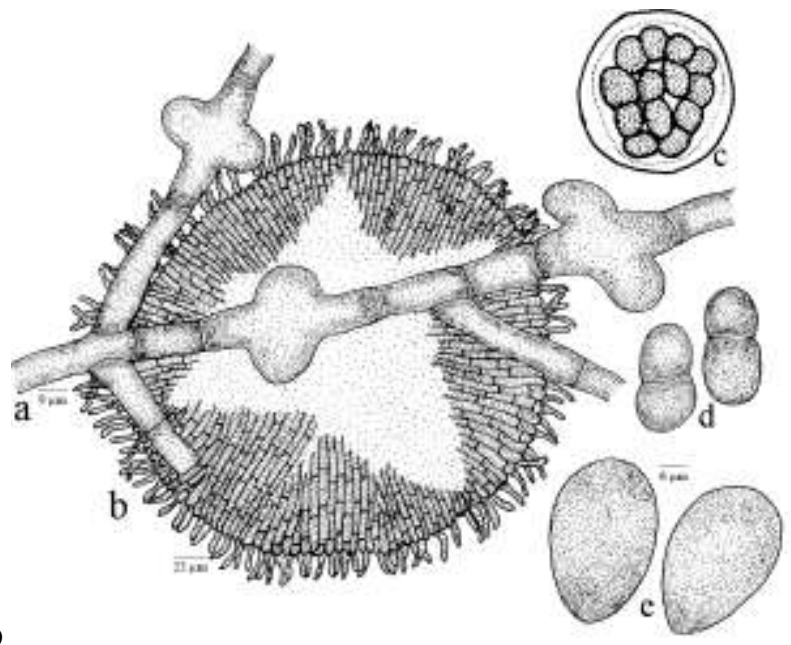

Figure 240 - Gangamyces shoreae

\section{THE GENUS Ishwaramyces}

Ishwaramyces Hosag., J. Econ. Taxon. Bot. 28: 183, 2004; Hosag., Chandraprabha \& Agarwal, Asterinales of Kerala, p. 169, 2011. 
Leaf parasites. Mycelium ectophytic, appressoria appears in clusters, setae absent. Thyriothecia orbicular with radiating cells, astomatous, dehisce stellately at the center; asci globose, octosporous, bitunicate; ascospores conglobate, uniseptate, brown.

Type sp.: I. flacourtiae Hosag., et al.

The genus Ishwaramyces differs from the genus Asterina in having axillary clusters of appressoria (Muller \& Arx, 1962; Arx \& Muller, 1975).

\section{Abbreviations used in the line drawings: \\ a. Appressoriate mycelium, b. Thyriothecium \\ c. Ascus, d. Ascospores}

\section{DESCRIPTIONS OF SPECIES}

Ishwaramyces flacourtiae Hosag., Kamar. \& Sabu in Hosag.,C.K. Biju \& Abraham, J. Econ. Taxon. Bot. 28: 183, 2004; Hosag., Chandraprabha \& Agarwal, Asterinales of Kerala, p. 169, 2011.

(Figs 241-242)

Colonies epiphyllous, subdense to dense, up to $2 \mathrm{~mm}$ in diameter, confluent and cover an entire upper surface of the leaves. Hyphae straight to substraight, branching opposite to closely reticulate, cells $16-20 \times 4-5$ $\mu \mathrm{m}$. Appressoria opposite, 1-2 smaller at the axilliary, antrorse, two celled, 8-12 $\mu \mathrm{m}$ long; stalk cells cylindrical to cuneate, 3-4 $\mu \mathrm{m}$ long; head cells ovate, globose, entire, 4-8 × 7-9 $\mu \mathrm{m}$. Thyriothecia descrete to cuneate, orbicular, up to $345 \mu \mathrm{m}$ in diameter, stellately dehisced at the center, margin crenate to fimbriate; asci numerous, globose, octosporous, 45-56 $\mu \mathrm{m}$ in diameter; ascospores conglobate, initially hyaline, brown at maturity, uniseptate, deeply constricted at the septum, 30-37 × 19-21 $\mu \mathrm{m}$, wall smooth. Pycnothyria similar to thyriothecia, smaller; Pycnothyriospores oblong to pyriform, brown, $26-30 \times 17-19 \mu \mathrm{m}$, wall smooth.

Material examined: Kerala, Thiruvananthapuram, Vithura, on leaves of Flacourtia montana Graham (Flacourtiaceae), 22 October 2000, K. P. Babu HCIO 43987,
TBGT 474; Thiruvananthapuram, Palode, TBGRI Campus, 23 October 2000, M. Shajivaz HCIO 44060, TBGT 493; 25 November 2000, T. Sabu HCIO 43805, TBGT 358; 18 December 2000, H. Biju HCIO 46103, TBGT 1866; 29 August 2005, G. R. Archana \& A. Sabeena HCIO 46702, TBGT 2043; 11 November 2003, T. Sabu HCIO 47077, TBGT 2294; Kannur, Aralam Wildlife Sanctuary, 27 February 2007, Jacob Thomas \& al. HCIO 48136, TBGT 2872; on leaves of $F$. indica (Burm. f. ) Merr., Idukki, Kulamavu, 5 September 2001, M. Kamarudeen HCIO 44186, TBGT 573; Thiruvananthapuram, Palode, TBGRI campus, 7 January 2008, H. Biju HCIO 48812, TBGT 3118; Idukki, Aruvillamchal, 13 March 2007 Jacob Thomas \& al. HCIO 48036, TBGT 2819.

\section{Distribution: Kerala.}
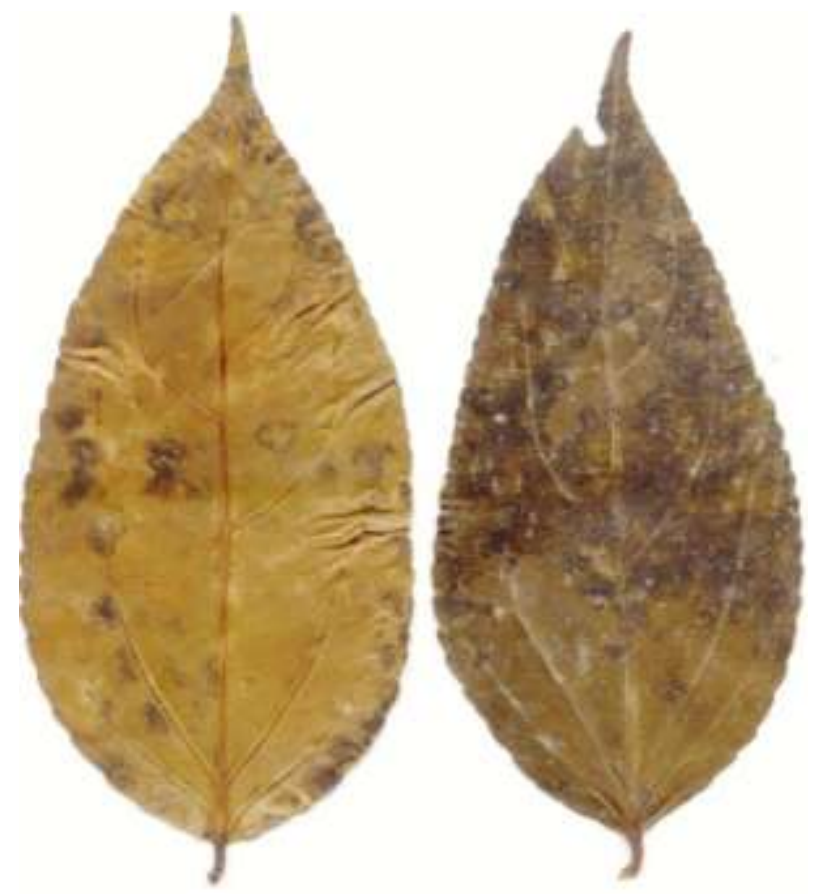

Figure 241 - Ishwaramyces flacourtiae Infected leaves

\section{THE GENUS Meliolaster}

Meliolaster Hohnel, Ber. Deutsch. Bot. Ges. 35:701, 1918; Hosag., Chandraprabha \& Agarwal, Asterinales of Kerala, p. 171, 2011.

Leaf parasites. Mycelium ectophytic, appressoria lateral. Thyriothecia orbicular with 
radiating cells, astomatous, dehisce stellately at the center; asci globose to ovate, octosporous, bitunicate; ascospores brown, 2- septate, upper cell globose, the lower two cells narrowed and tapering at the base.

Type sp.: M. clavisporus (Pat.) Hohn.

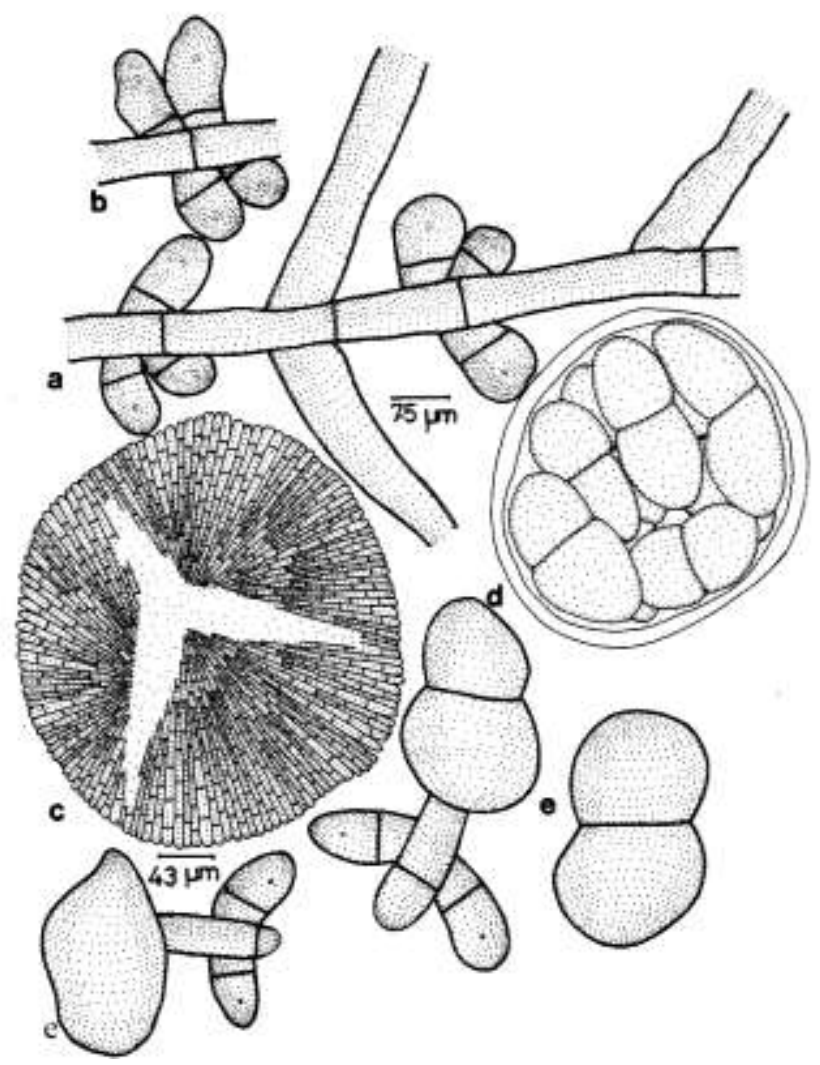

Figure 242 - Ishwaramyces flacourtiae

\section{DESCRIPTION OF SPECIES}

Meliolaster aporusae Hosag., Harish \& Archana, Indian Journal of Science andTechnology, Vol. 2, No 6., 2009; Hosag., Chandraprabha \& Agarwal, Asterinales of Kerala, p. 171, 2011.

(Figs 243-244)

Colonies epiphyllous, thin, crustose, up to $4 \mathrm{~mm}$ in diameter, confluent. Hyphae straight, rarely slightly undulate, branching opposite to irregular at acute to wide angles, loosely reticulate, cells $12-22 \times 5-7 \mu \mathrm{m}$. Appressoria alternate, unilateral, unicellular, sessile, cylindrical, straight to curved, antrorse, subantrorse, entire, rounded at the apex, 9-11 $\times$ 4-7 $\mu \mathrm{m}$. Thyriothecia uniformly scattered, orbicular, up to $250 \mu \mathrm{m}$ in diameter, margin crenate to fimbriate, fringed hyphae straight to slightly undulate and devoid of appressoria, thyriothecia stellately to irregularly dehisced at the centre; asci globose to ovate, octosporous, 54-61 × 49-55 $\mu \mathrm{m}$; ascospores brown, oblong, 2 -septate, upper cell globose, the lower two cells narrowed and tapering at base, constricted only at the upper septum, $41-45 \times 12-14 \mu \mathrm{m}$, wall smooth. Pycnothyriospores brown, oblong, unicellular, 9-13 $\times 4-7 \mu \mathrm{m}$.
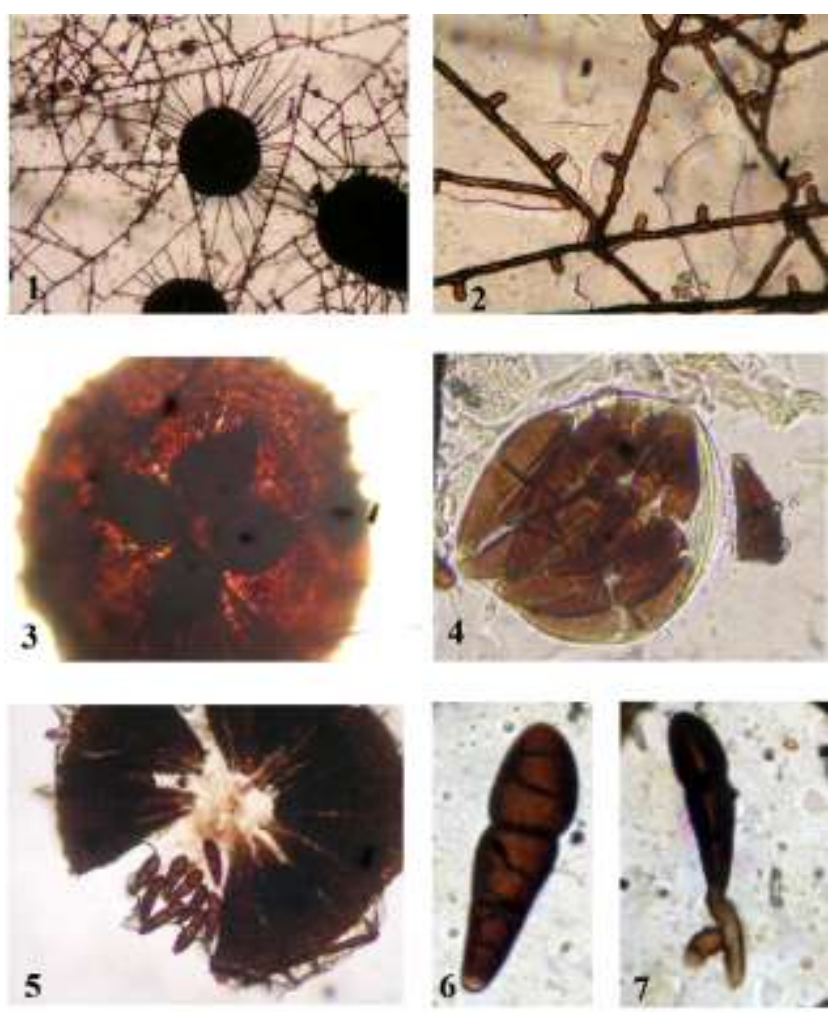

Figure 243 - Meliolaster aporusae

1. Fungal colony with thyriothecia, 2.Appressoriate mycelium, 3. Thyriothecium with asci, 4. Ascus, 5. Dehisced thyriothecium, 6. Ascospore, 7. Germinating ascospore

Material examined: Kerala, Wayanad, Periya, Kunkichira, on leaves of Aporusa lindleyana (Wight) Baill. (Euphorbiaceae), 6 December 2006, M. Harish \& al. HCIO 48296 (holotype), TBGT 3015 (isotype); Thiruvananthapuram, Palode, TBGRI Campus, 6 May 2006, G. R. Archana HCIO 48297, TBGT 3016; Kottayam, Ponthanpuzha forest, 12 October 2007, P.J. Robin HCIO 48315, TBGT 3036; Thiruvananthapuram, Palode, TBGRI Campus, 28 June 2007, A. Sabeena \& al. HCIO 49133, TBGT 3387. 


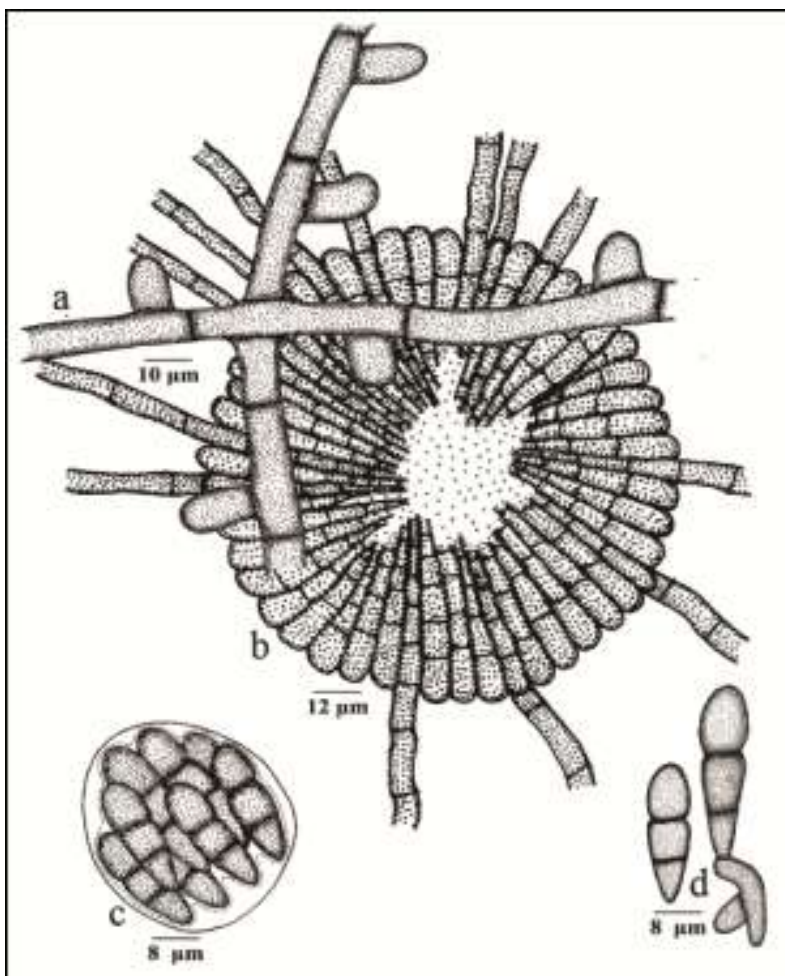

Figure 244 - Meliolaster aporusae

The genus Meliolaster is reported for the first time on the members of the family Euphorbiaceae.

\section{Distribution: Kerala}

\section{THE GENUS Prillieuxina}

Prillieuxina Arn., Ann. Ecol. Nat. Agric. Montpellier 16:161, 1918; Hansf., Mycol. Pap. 15: 169, 1946; Muller \& Arx, Beitr. Krypt. Schw. 11:132, 1962; Luttrell in Ainsworth et al. (eds.). The Fungi. An advanced Treatise 4: 207, 1973; Arx \& Muller, Stud. Mycol. 9: 44, 1975; Bilgrami, Jamaluddin \& Rizwi, Fungi of India p. 407, 1991; Hosag., Abraham \& C.K. Biju, J. Mycopathol. Res. 39: 62, 2001; Singh, Duke, Bhandari \& Jain, J. Econ. Taxon. Bot. 30: 191, 2008; Hosag., Chandraprabha \& Agarwal, Asterinales of Kerala, p. 173, 2011.

Leaf parasites. Mycelium ectophytic, appressoria and setae absent. Thyriothecia orbicular with radiating cells, astomatous, dehisce stellately at the center; asci globose, octosporous, bitunicate; ascospores brown, conglobate, uniseptate.
Type sp.: P. winteriana (Pazschke) Arn.
Abbreviations used in the line drawings:
a. Mycelium, b. Thyriothecium,
c. Pycnothyriospores

\section{DESCRIPTIONS OF SPECIES}

Prillieuxina anamirtae (Sydow \& Sydow) Ryan in Stevens \& Ryan, Illinois Biol. Monographs 17: 78, 1939; Hosag., Chandraprabha \& Agarwal, Asterinales of Kerala, p. 173, 2011.

Asterinella anamirtae Sydow \& Sydow, Ann. Mycol. 12: 558, 1914.

(Figs 245-246)

Colonies amphigenous, often epiphyllous or hypophyllous, dense, up to 5 $\mathrm{mm}$ in diameter, confluent. Hyphae flexuous to crooked, branching irregular at acute angles, loosely to closely reticulate, cells 3-4 $\mu \mathrm{m}$ broad. Appressoria absent. Thyriothecia few, orbicular, scattered to connate in a line, orbicular, up to $80 \mu \mathrm{m}$ in diameter, stellately dehisced at the centre, margin inconspicuously fimbriate; asci globose, ovate, octosporous, up to $40 \mu \mathrm{m}$ in diameter; ascospores oblong, brown, 1-septate, constricted at the septum, 24$31 \times 11-15 \mu \mathrm{m}$, wall echinulate in matured ascospores. Pycnothyria similar to thyriothecia, scattered, smaller than the thyriothecia; pycnothyriospores brown, unicellular, pyriform, $16-18 \times 12-15 \mu \mathrm{m}$, wall smooth.

Material examined: Kerala, Thiruvananthapuram, Palode, TBGRI Campus, on leaves of Anamirta cocculus (L.) Wight \& Arn. (Menispermaceae), 12 December 2000, M. Kamarudeen HCIO 44195, TBGT 566; 13 July 2001, M. Kamarudeen HCIO 44193, TBGT 567; 8 September 2003, A. Manojkumar HCIO 45231, TBGT 1268; 22 September 2005, Manu HCIO 47313, TBGT 2351; Thiruvananthapuram, Palode, TBGRI Campus, on leaves of Anamirta sp., 12 November 2001, M. Shajivaz HCIO 44401, TBGT 615, Thiruvananthapuram, Palode, TBGRI Campus, 5 October 2008, A. Sabeena \& M. C. Riju TBGT 3389, HCIO 49134. 


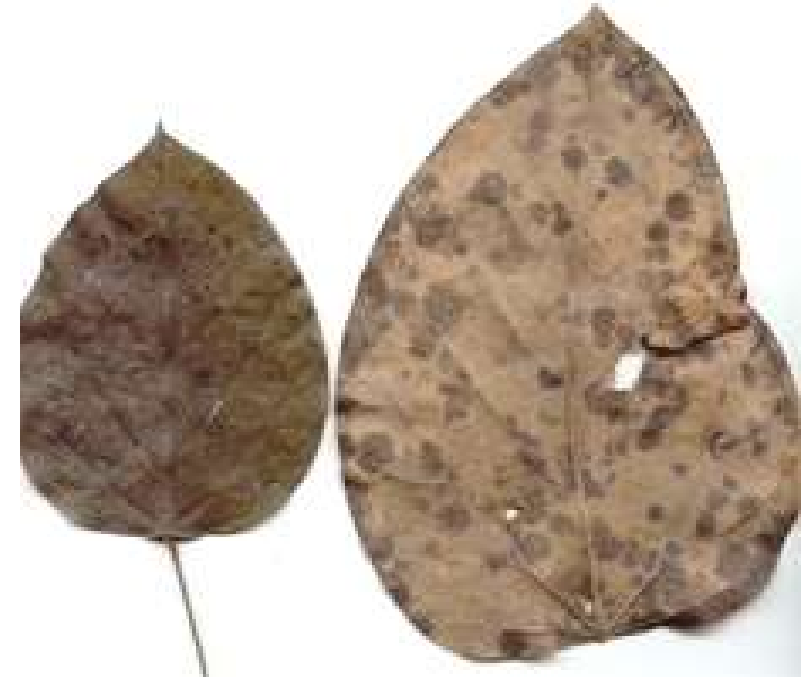

Figure 245 - Prillieuxina anamirtae - Infected leaves

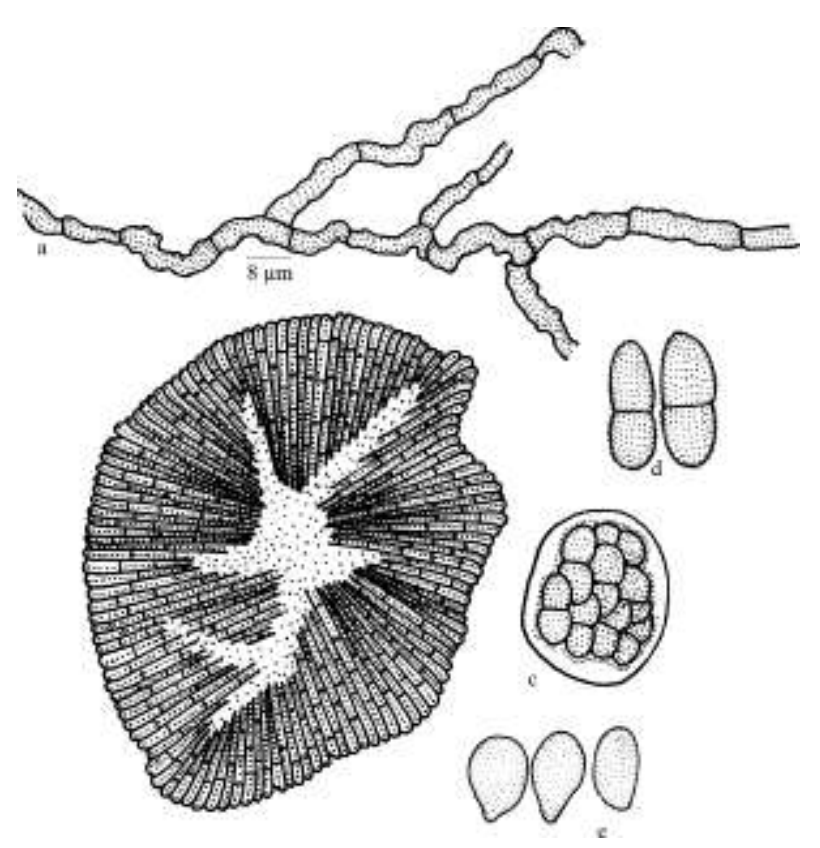

Figure 246 - Prillieuxina anamirtae

This species was known on the same host from Philippines and appears to be known only from the type collection (Muller \& Arx, 1962; Stevens \& Ryan, 1939).

However, ascospores and pycnothyriospores were larger in the present collections.

Distribution: Kerala.

Prillieuxina aquifoliacearum Hosag., Ravikumar \& Archana, Zoos Print J. 22 (9): 2832, 2007. (Fig.-247)

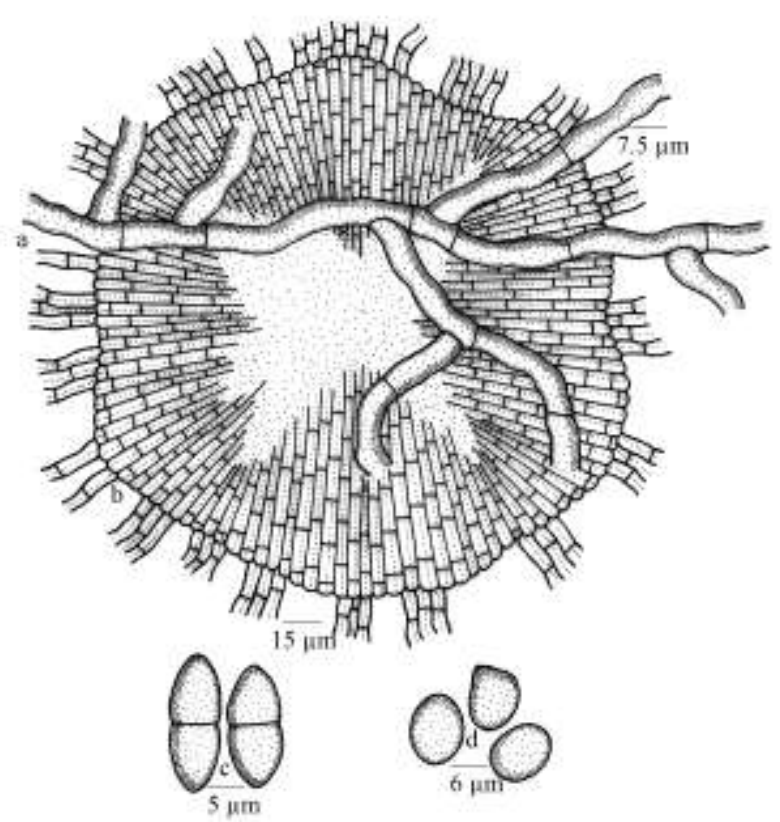

Figure 247 - Prillieuxina aquifoliacearum

Colonies hypophyllous, subdense, up to $2 \mathrm{~mm}$ in diameter, confluent. Hyphae substraight to crooked, branching opposite, alternate to irregular at acute angles, closely reticulate, cells $12-27 \times 3-5 \mu \mathrm{m}$. Thyriothecia scattered to grouped, orbicular, up to $192 \mu \mathrm{m}$ in diam., margin fringed with hyphae, fringed hyphae very short, thyriothecia stellately dehisced at the centre; asci not seen; ascospores conglobate, 1-septate, constricted at the septum, smooth walled, $16-19 \times 6-10 \mu \mathrm{m}$. Pycnothyria few, similar to thyriothecia, smaller; pycnothyriospores globose, pyriform, $8-10 \times 6$ $10 \mu \mathrm{m}$.

Material examined: Tamil Nadu, Mukurthi National Park, Nilgiri District, Ooty, Parson Valley, about 2100 MSL.On the leaves of Ilex denticulata Wall. ex Wight (Aquifoliaceae), K. Ravikumar HCIO (holoype), TBGT 2532 (isotype).

Prillieuxina ilicicola (Hansf.) Arx is known on Ilex odorata from Java (Hansford, 1954; Mueller and Arx, 1962).

Distribution: Tamil Nadu.

Prillieuxina ardisiae (Hansf.) Arx in Muller \& Arx, Beitr. Krypt. Flora 11: 135, 1962. Asterinella ardisiae Hansf., Proc. Linn. Soc. London, 1943-44: 102, 1944; 
Kamal \& Singh, Indian J. Mycol. Pl. Pathol. 9: 175, 1979.

Colonies hypophyllous, circular to irregular, later becoming effuse, mostly originating along the leaf margin, brown, up to $9 \mathrm{~mm}$ diam.; mycelium of hyphae free, not membranous, sinuous to flexuous, subhyaline, septate, smooth, 7-18 $\mu \mathrm{m}$ long, up to $4 \mu \mathrm{m}$ wide, dense reticulate, branching unilateral or irregular; setae and appressoria absent; hyphae bearing radiant scutellum; thyriothecia scattered, glabrous, dark brown, globose to subglobose, numerous, up to $130 \mu \mathrm{m}$ diam.; wall of the lower hyphae composited irregularly; wall of the upper hyphae composited radiantly, up to $45 \mu \mathrm{m}$ long; asci bitunicate, subhyaline, sessile, paraphysate dacryoid or lacrymoid, numerous, 8-spored, 20$30 \mu \mathrm{m}$ long, 18-25 $\mu \mathrm{m}$ diam.; spores heaped together, oblong club-shaped, slightly rounded on both ends, first subhyaline, later becoming pale olivaceous brown, uniseptate, constricted at the septum, with upper cell larger than the lower, smooth, 7.5-14 × 6.5-9 $\mu \mathrm{m}$; pycnothyria scattered, globose to subglobose, numerous, up to $90 \mu \mathrm{m}$ diam.; pycnothyriospores olive brown, smooth, one celled, dacryoid or lacrymoid, $6.0-10.5 \times 5.5-9 \mu \mathrm{m}$.

Material examined: Uttar Pradesh, Gorakhpur, on leavesof Ardisia solancea Roxb. (Myrsinaceae), February 1976, S. Singh, 495; IMI 200107.

Distribution: Uttar Pradesh

Prillieuxina argyreiae (Hosag., Balakr. \& Goos) comb. nov. (Fig.-248)

Asterinella argyreiae Hosag., Balakr. \& Goos, Mycotaxon 58: 489, 1996.

MycoBank 101027

Infected spots epiphyllous, black, gregarious, surrounded by yellow haloes, up to 8 $\mathrm{mm}$ in diameter, resulting in shot holes. Hyphae light brown, straight, branching irregular, loosely reticulate, cells 5-10 × 5-6.5 $\mu \mathrm{m}$. Appressoria absent. Thyriothecia arranged circularly in the colonies, circular, frequently connate, carbonaceous black, up to $130 \mu \mathrm{m}$ in diameter, dehiscing stellately at the centre, margin crenate to slightly fringed, fringed hyphae yellow; asci globose to slightly ovate, eight spored, 21-28 $\times$ 15-24 $\mu \mathrm{m}$; ascospores conglobate, cinnamon brown, 1-septate, constricted at septum, both cells equal, $21-23 \times 5-7 \mu \mathrm{m}$, wall smooth.

Material examined: Tamil Nadu, Coimbatore, Anamalai, near Manjaparai, on leaves of Argyereia sp. (Convolvulaceae), 26 March 1990, V.B. Hosagoudar HCIO 30976.

This species is distinct from other Asterinella species in that it causes a pathogenic Affect on the host (Stevens \& Ryan, 1939). Entire colonies with very little mycelia were covered with thyriothecia. Hyphal branches arising from the mycelia at the tip or in the centre, give the hyphae a bead-like appearance, and are the initials of thyriothecia. Several thyriothecia May coalesce (join together) and stellate splitting takes place.

Distribution: Tamil Nadu.

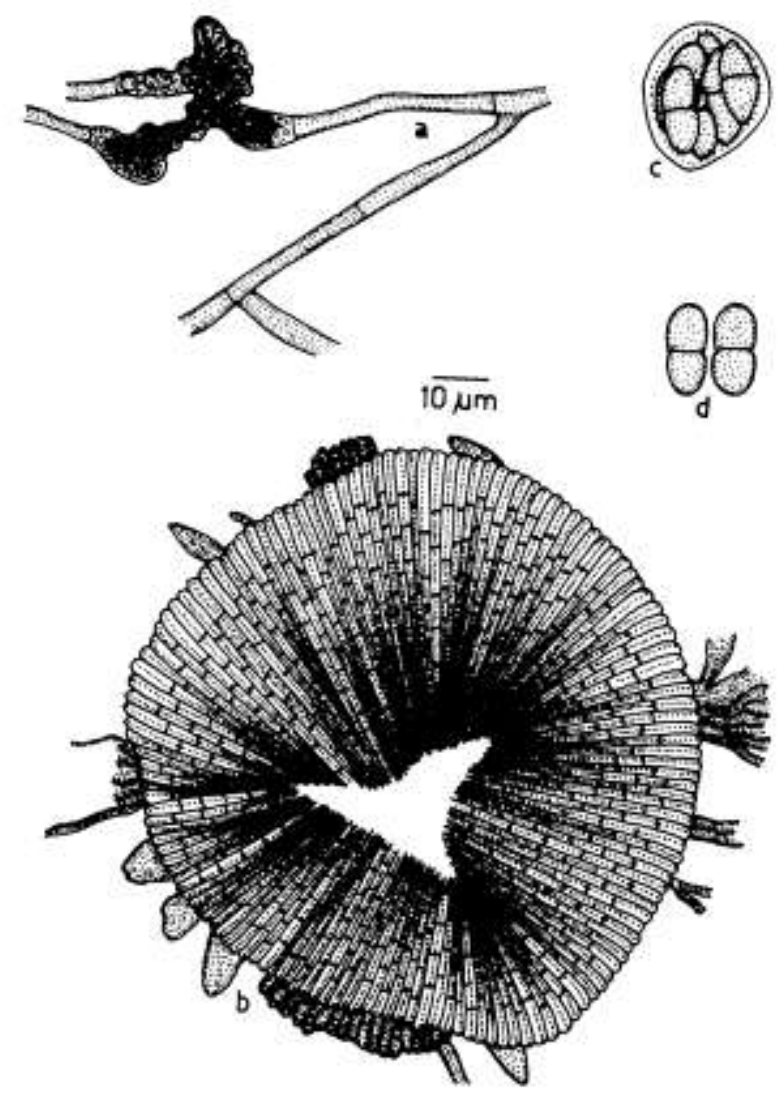

Figure 248 - Prillieuxina argyreiae

Prillieuxina diospyri Hosag. \& Chandra., Indian J. Sci. Technol. 2 (6):18, 2009; Hosag., Chandraprabha \& Agarwal, 
Asterinales of Kerala, p. 174, 2011. (Figs-249-250)

Colonies hypophyllous, dense, up to 3 $\mathrm{mm}$ in diameter. Hyphae crooked, branching irregular at acute to wide angles, loosely to closely reticulate, cells $18-26 \times 3-5 \mu \mathrm{m}$. Appressoria absent. Thyriothecia scattered to loosely grouped in the center of the colonies, orbicular, up to $121 \mu \mathrm{m}$ in diam., stellately dehisced at the center, margin crenate to fimbriate; asci globose, octosporous, up to 40 $\mu \mathrm{m}$ in diameter; ascospores oblong, conglobate, uniseptate, constricted at the septum, 20-26 $\times 9$ $11 \mu \mathrm{m}$, wall smooth. Pycnothyria similar to thyriothecia, smaller; pycnothyriospores ovate, pyriform, 9-24 × 4-13 $\mu \mathrm{m}$, wall smooth.

Material examined: Kerala, Thiruvananthapuram, Palode, TBGRI, on the leaves of Diospyros malabaricus (Desr.) Kostel. (Ebenaceae), 15 February 2008, A. Chandraprabha HCIO 48243 (holotype), TBGT 2981 (isotype); December 5, 2001, H. Biju HCIO 48314, TBGT 3035.

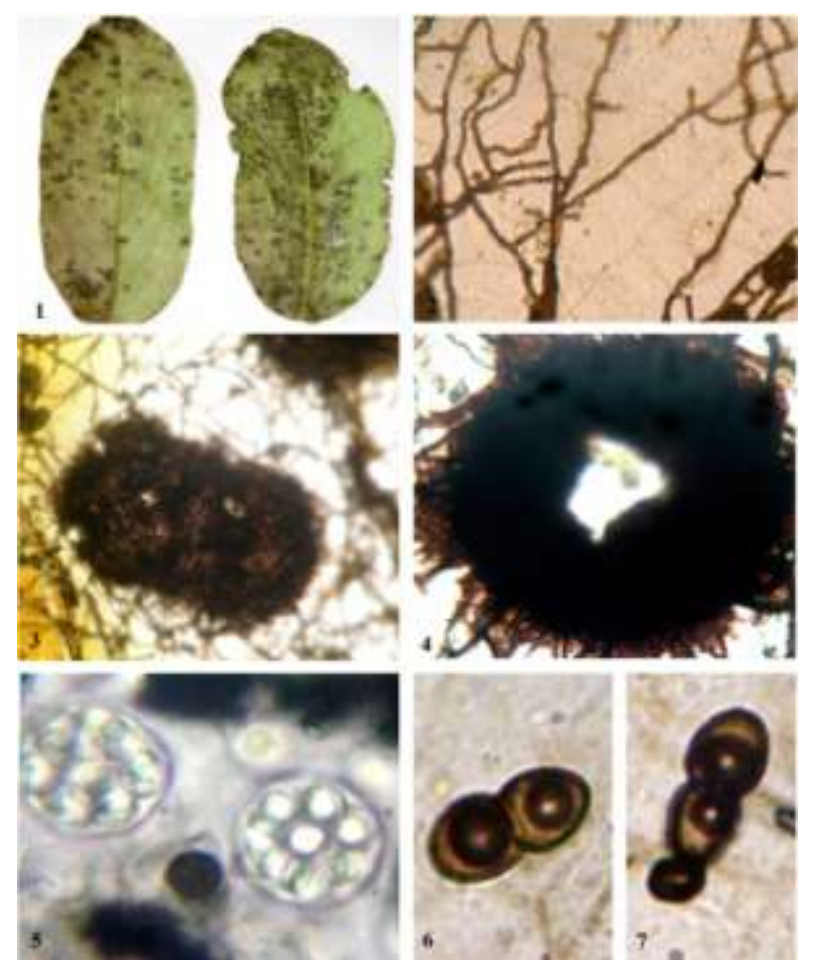

Figure 249 - Prillieuxina diospyri

1. Infected leaves, 2. Branched mycelium, 3. Fungal colony with thyriothecia,4. Dehisced thyriothecium, 5. Asci, 6. Ascospore, 7. Germinating ascospore

Prillieuxina mabae Ryan (Stevens \& Ryan, 1939) is known on members of the family Ebenaceae. Prillieuxina diospyri differs from it in having typically globose asci, larger ascospores $(20-26 \times 9-11 \mu \mathrm{m})$ in contrast to 12 $5 \mu \mathrm{m}$ (Stevens \& Ryan, 1939).
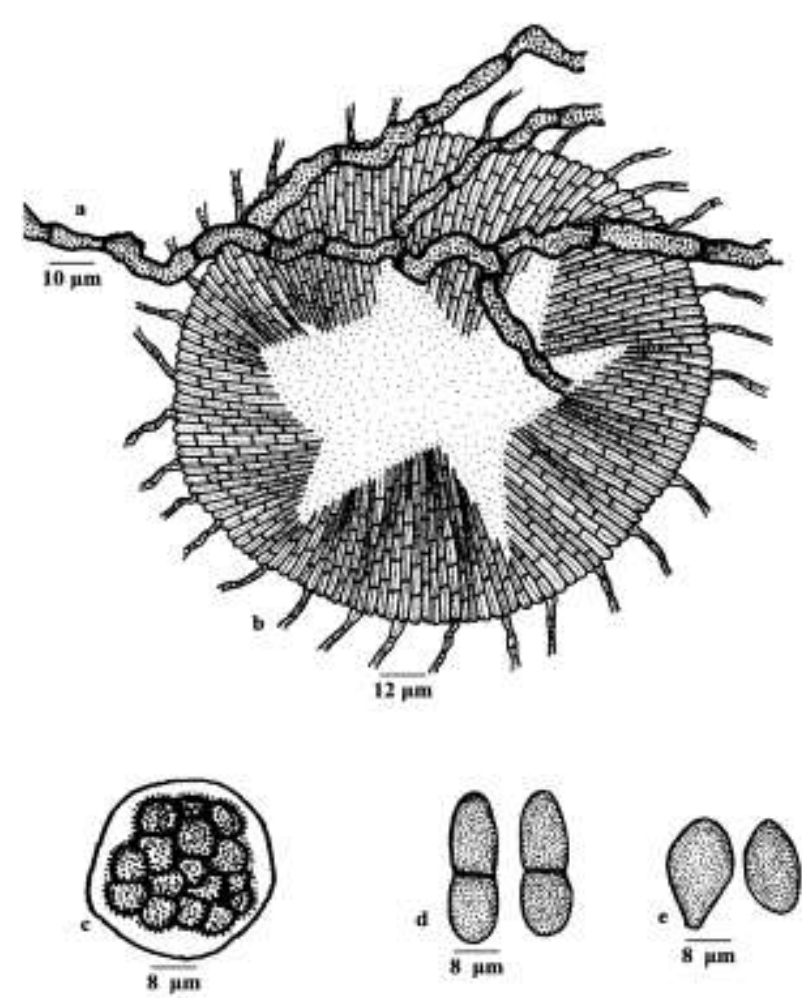

Figure 250 - Prillieuxina diospyri

Distribution: Kerala.

Prillieuxina elaegni Hosag. \& C.K. Biju in Hosag., C.K. Biju\& Abraham, J. Mycopathol. Res. 40:195, 2002; Hosag., C.K. Biju \& Abraham, Indian Phytopath. 57: 115, 2004; Hosag., Chandraprabha \& Agarwal, Asterinales of Kerala, p. 175, 2011.

(Figs 251-252)

Colonies epiphyllous, dense, minute, up to $1 \mathrm{~mm}$ in diameter. Hyphae straight to flexuous, branching irregular at acute to wide angles, loosely reticulate, cells 20-25 × 4-6 $\mu \mathrm{m}$. Thyriothecia mostly aggregated, 


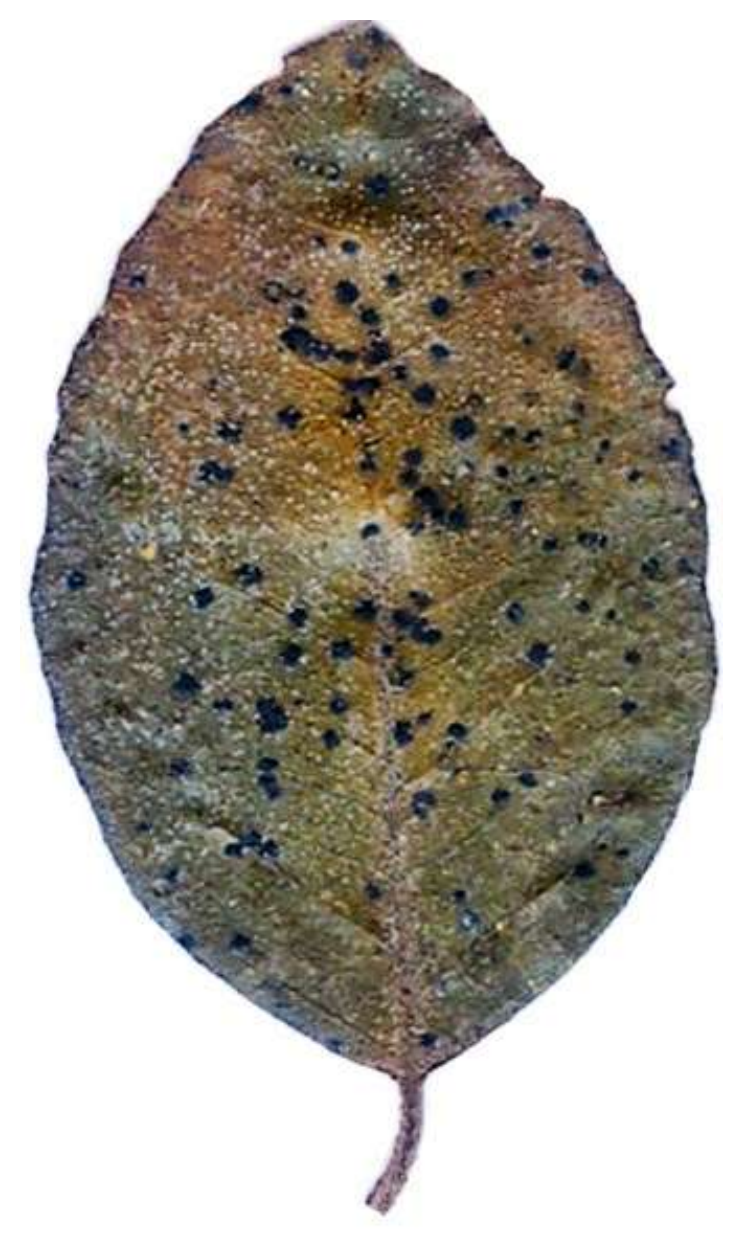

Figure 251 - Prillieuxina elaegni - Infected leaf

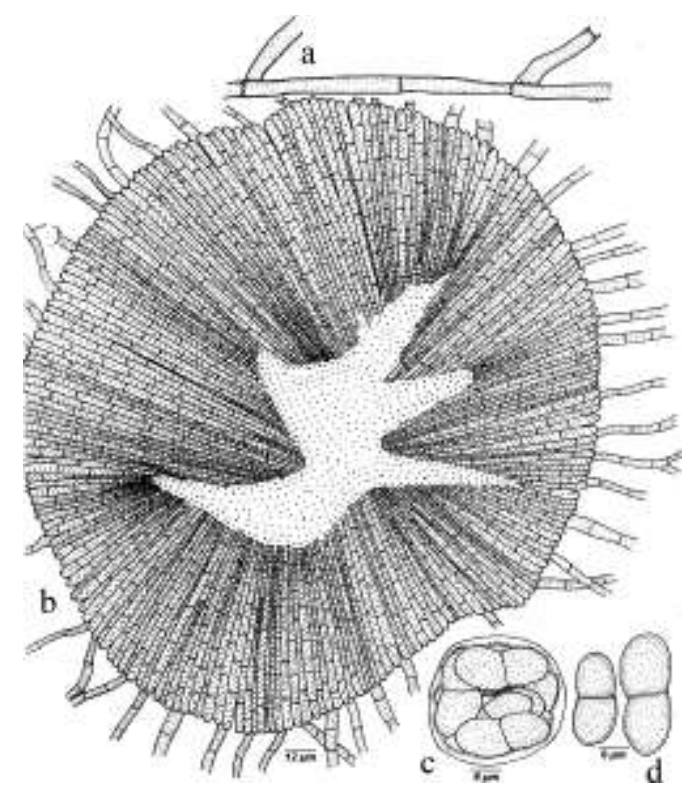

Figure 252 - Prillieuxina elaegni

orbicular, up to $225 \mu \mathrm{m}$ in diameter, stellately dehisced at the centre, margin crenate to fimbriate, fringed hyphae straight to substraight, run parallel; asci many, globose, octosporous, up to $30 \mu \mathrm{m}$ in diameter; ascospores conglobate, oblong, brown, 1- septate, constricted at the septum, 17-23 $\times 9-11$ $\mu \mathrm{m}$, wall smooth.

Material examined: Kerala, Idukki, near Pothundy Dam, on leaves of Elaeagnus kologa Schlecht. (Elaeagnaceae), 23 September 1998, C.K. Biju HCIO 42933 (holoype), TBGT 229 (isotype).

There is no record of the genus Prillieuxina on the members of the family Elaeagnaceae and hence, it is proposed here as new species.

Distribution: Kerala.

Prillieuxina garciniae Hosag. in Hosag., C.K. Biju \& Abraham, J. Econ. Taxon. Bot. 28: 191, 2004; Hosag., Zoos' Print J. 22: 2834, 2007; Hosag., Chandraprabha \& Agarwal, Asterinales of Kerala, p. 176, 2011. (Fig. 253)

Colonies hypophyllous, black, thin to subdense, orbicular, up to $10 \mathrm{~mm}$ in diameter, confluent. Hyphae flexuous to crooked, branching alternate to opposite at acute angles, loosely to closely reticulate, cells $12-19 \times 3-5$ $\mu \mathrm{m}$. Appressoria absent. Thyriothecia scattered, rarely 1-2 connate, more or less orbicular, up to $160 \mu \mathrm{m}$ in diameter, stellately dehisced at the centre, margin crenate to fimbriate; asci globose, octosporous, 32-38 $\mu \mathrm{m}$ in diameter; ascospores brown, conglobate, oblong, uniseptate, strongly constricted at the septum, upper cell larger than the lower, 27-32 × 14-16 $\mu \mathrm{m}$, wall echinulate in matured ascospores.

Material examined: Kerala, Thiruvananthapuram, Chemunji, on leaves of Garcinia imberti Bourd. (Clusiaceae), 17 March 1997, V.B. Hosagoudar HCIO 44124 (holoype), TBGT 526 (isotype); Thiruvananthapuram, Attayar, 18 March 1997, V.B. Hosagoudar HCIO 44160, TBGT 552; HCIO 44194, TBGT 568; Thiruvananthapuram, Chemunji, 23 April 1999, C.K. Biju HCIO 45208, TBGT 1244; 3 January 2001, G. Rajkumar HCIO 44859, TBGT 1087; HCIO 45140, TBGT 1195; 9 March 2003, G. Rajkumar HCIO 45226, TBGT 1263; Thiruvananthapuram, Chemunji Hills, 18 July 2001, M. Kamarudeen HCIO 44182, TBGT 529. 


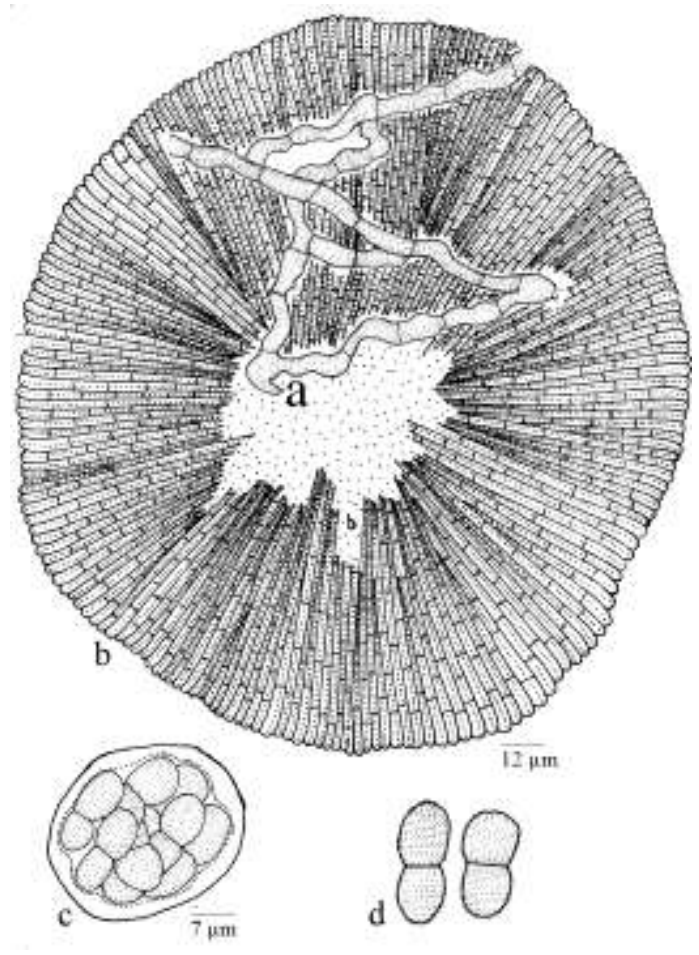

Figure 253 - Prillieuxina garciniae

This forms the first record of the genus Prillieuxina on the members of the family Clusiaceae (Muller \& Arx, 1962).

Distribution: Kerala.

Prillieuxina ixorigena Hosag. \& Chandra., Indian J. Sci. Technol. 2 (6):18, 2009; Hosag., Chandraprabha \& Agarwal, Asterinales of Kerala, p. 178, 2011.

(Figs 254-255)

Colonies amphigenous, dense, up to 2 $\mathrm{mm}$ in diam. Hyphae crooked, branching irregular at acute to wide angles, loosely to closely reticulate, cells $18-26 \mu \mathrm{m}$ long and up to $4 \mu \mathrm{m}$ broad. Appressoria absent. Thyriothecia scattered to grouped in the center of the colonies, orbicular, up to $100 \mu \mathrm{m}$ in diam., stellately dehisced at the center, margin crenate; asci globose, octosporous, up to $30 \mu \mathrm{m}$ in diameter; ascospores oblong, conglobate, uniseptate, constricted at the septum, 20-26 × 7$11 \mu \mathrm{m}$, wall smooth; pycnothyriospores ovate, pyriform, 11-26 × 7-13 $\mu \mathrm{m}$, wall smooth.

\section{Material examined: Kerala,} Thiruvananthapuram, Palode, on the leaves of Ixora coccinea L. (Rubiaceae), 18 November 2007, A. Chandraprabha HCIO 48242 (holoype), TBGT 2980 (isotype).

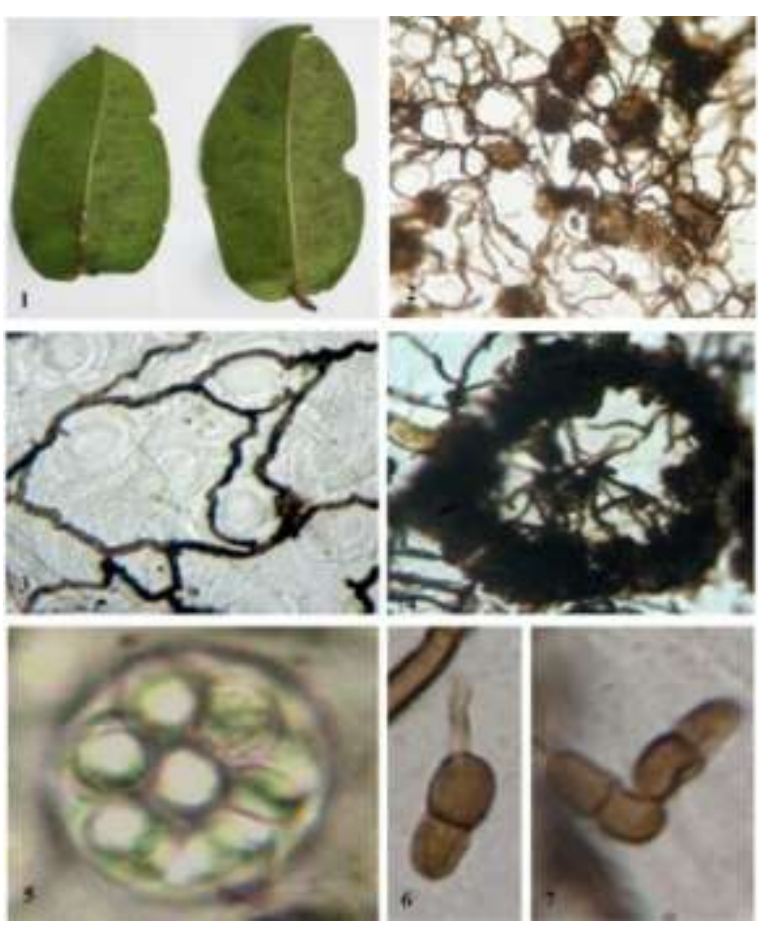

Figure 254 - Prillieuxina ixorigena

1. Infected leaves, 2. Fungal colony with thyriothecia, 3. Branched mycelium, 4. Dehisced thyriothecium, 5. Ascus, 6. Germinating ascospore, 7. Ascospores

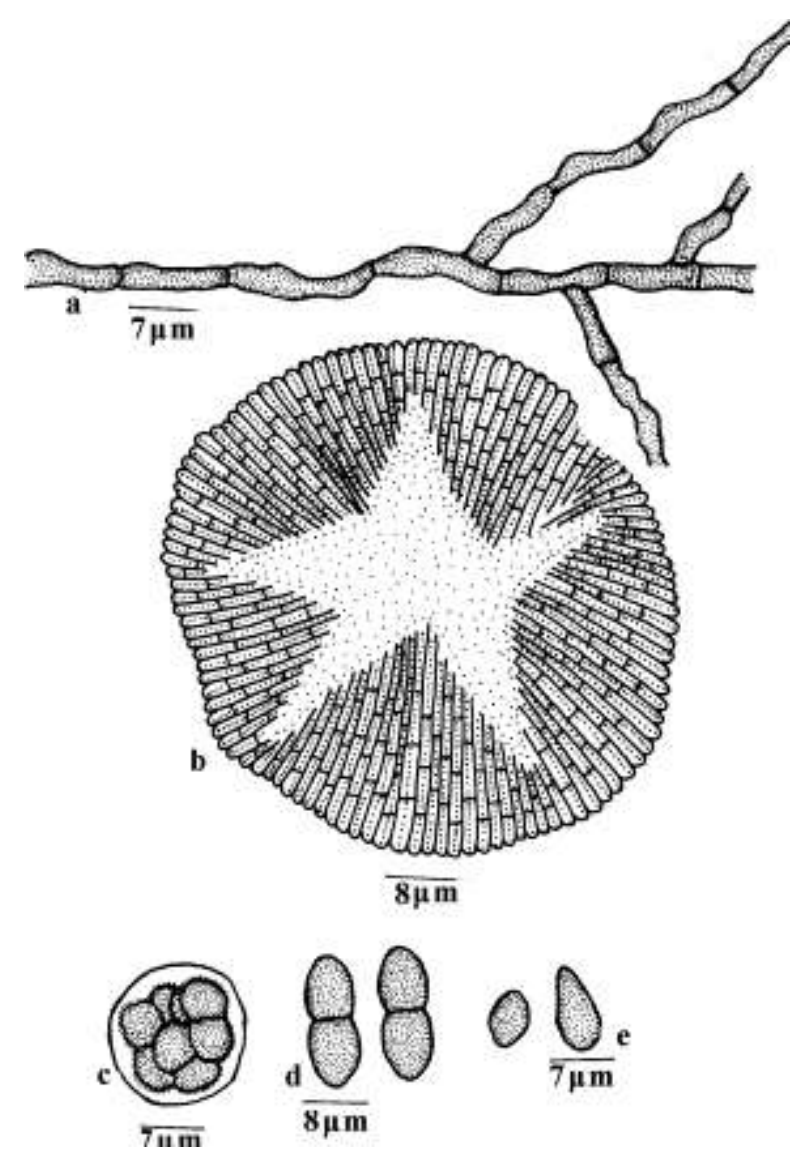

Figure 255 - Prillieuxina ixorigena 
Prillieuxina ixorae (Ryan) Ryan and Prillieuxinadistinguenda (Sydow) Ryan are known on the members of the family Rubiaceae (Stevens \& Ryan, 1939). However, the present species differs from Prillieuxina ixorae in having larger ascospores $(20-26 \times 7-11 \mu \mathrm{m})$ in contrast to $9 \times 2-7 \mu \mathrm{m}$. It also differs from Prillieuxina distinguenda in having smaller thyriothecia (up to $100 \mu \mathrm{m}$ in diameter) in contrast to $152-225 \times 100-170 \mu \mathrm{m}$ and larger ascospores $(20-6 \times 7-11 \mu \mathrm{m})$ in contrast to $12-14$ $\times 5-7 \mu \mathrm{m}$.

Distribution: Kerala.

Prillieuxina jasmini (Hosag. \& Abraham) Hosag. in Hosag., Chandraprabha \& Agarwal, Asterinales of Kerala, p. 179, 2011.

Asterinella jasmini Hosag. \& Abraham, Indian Phytopath. 50: 220, 1997; Hosag.,C.K. Biju\& Abraham, J. Econ. Taxon. Bot. 25: 306, 2001; Singh, Duke, Bhandari \& Jain, J. Econ. Taxon. Bot. 30: 74, 2008.

(Fig. 256)

Colonies hypophyllous, thin to subdense, carbonaceous, confluent and cover most of the leaf portion. Hyphae straight to substraight, branching irregular at acute angles, loosely reticulate, cells 9.5-14.5 $\times$ 2.5-3.5 $\mu \mathrm{m}$. Appressoria absent. Thyriothecia scattered, round, up to $120 \mu \mathrm{m}$ in diameter, stellately dehisced at the centre or diffused and widely opened, margin fimbriate, fringed hyphae flexuous; asci few, globose to ovate, octosporous, 41-46 × 29-34 urn; ascospores conglobate, brown, 1-septate, constricted at the septum, 20-22 × 9-10 $\mu \mathrm{m}$, upper cell globose, lower cell ovate, wall smooth. Pycnothyria numerous, scattered to rarely connate, orbicular, up to $75 \mu \mathrm{m}$ in diameter, stellately dehisced or widely opened at the centre, margin fimbriate, fringed hyphae flexuous; Pycnothyriospores brown, clavate, apiculate, 17-19.5 × 12-14.5 $\mu \mathrm{m}$.

Material

Thiruvananthapuram, examined:

Neyyar

Kerala, Sanctuary, Agastiamala, Pongalapara, on leaves of Jasminum flexile Vahl (Oleaceae), 26 March 1996, V.B. Hosagoudar HCIO 42292 (holoype), TBGT 78 (isotype).
About 37 species of the genus Asterinella are so far known on different members of the angiosperm families. These are obligate pathogens and are host specific, their host range is very narrow. The present report is the first report of the genus Asterinella on the member of the family Oleaceae and hence, it warrants its placement as a new species.

This species forms the first report of the genus Prillieuxina on the member of the family Oleaceae.

Distribution: Kerala.
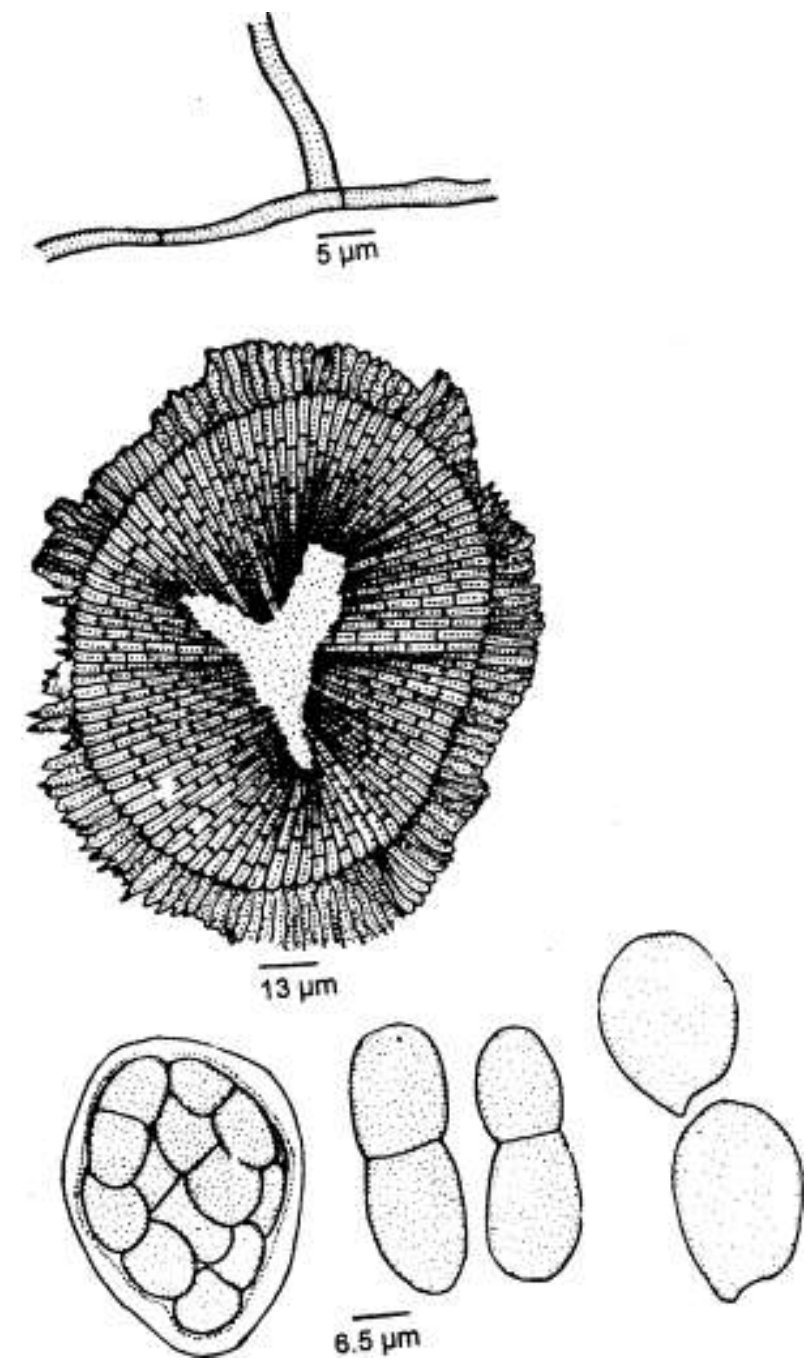

Figure 256 - Prillieuxina jasmini

Prillieuxina polyalthiae Hosag. \& Abraham, Indian Phytopath. 51: 391, 1998; Hosag.,C.K. Biju \& Abraham, J. Econ. Taxon. Bot. 25: 306, 2001; Hosag., Zoos' Print J. 18: 1280, 2003; Hosag., Zoos' Print J. 22: 2834, 2007; Singh, Duke, Bhandari \& Jain, J. Eco. Taxon. 
Bot. 30: 191, 2008; Hosag., Chandraprabha \& Agarwal, Asterinales of Kerala, p. 180, 2011. (Figs 257-258)

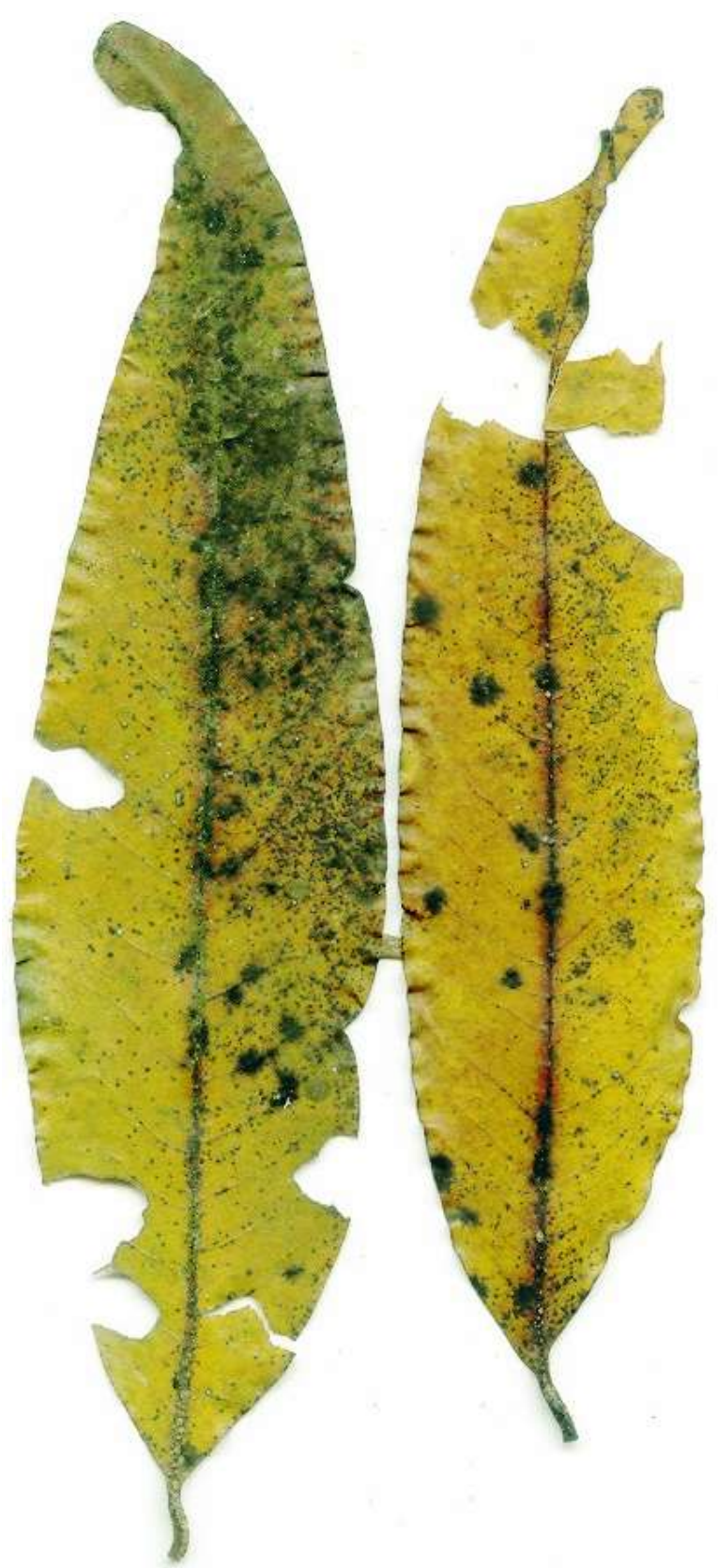

Figure 257 - Prillieuxina polyalthiae - Infected leaf

Colonies mostly epiphyllous, rarely amphigenous, dense, crustose to velvety, up to $2 \mathrm{~mm}$ in diameter, confluent. Hyphae straight, branching irregular, loosely reticulate, cells 12$16 \mu \mathrm{m}$ long and up to $2 \mu \mathrm{m}$ broad. Appressoria absent. Thyriothecia develop all along the hyphae, more or less round, carbonaceous black, up to $350 \mu \mathrm{m}$ in diameter, many thyriothecia join together marginally, dehisce stellately at the centre, margin fringed, fringed hyphae from the margin join with other thyriothecia and form a plate of thyriothecia but keep individuality; asci few to many per thyriothecia, globose, octosporous, up to $45 \mu \mathrm{m}$ in diameter; ascospores conglobate, brown, 1septate, constricted at the septum, 20-23 × 9.5$11 \mu \mathrm{m}$.
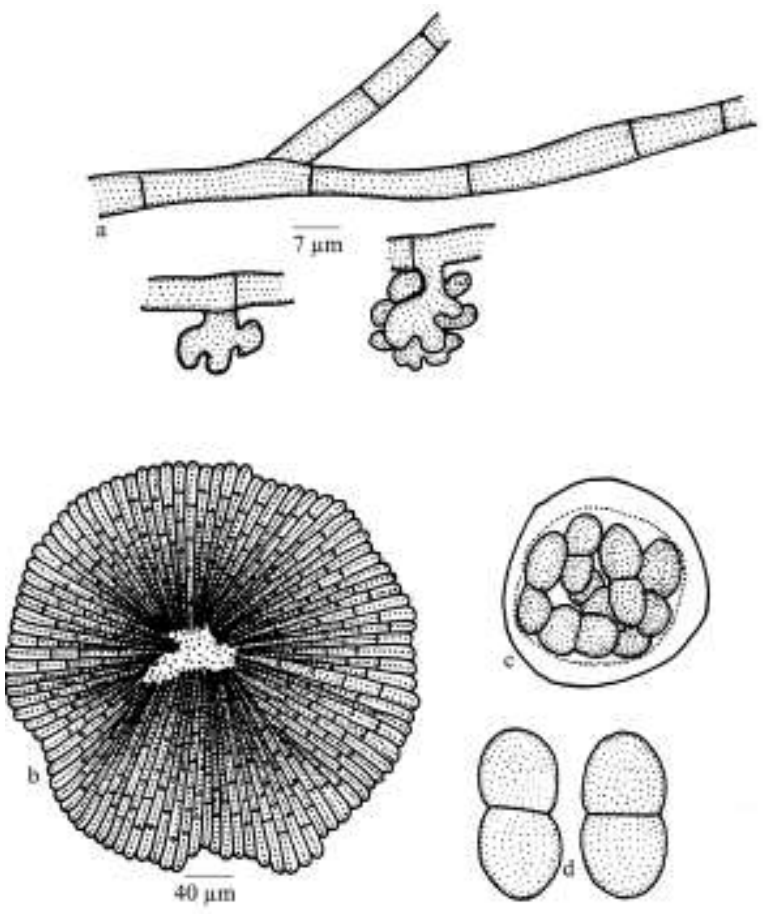

Figure 258 - Prillieuxina polyalthiae

Material examined: Kerala, Thiruvananthapuram, Neyyar Wildlife Sanctuary, on leaves of Polyalthia longifolia (Sonn.) Thawaites. (Annonaceae), 17 February 1997, V.B. Hosagoudar HCIO 42541 (holoype), TBGT 183 (isotype); Thiruvananthapuram, Palode, TBGRI Campus, 10 September 1997, C.K. Biju HCIO 44004, TBGT 460; 25 November 2000, T. Sabu HCIO 45101, TBGT 1156; Thrissur, 3 September 2001, P. A. Jose HCIO 44200, TBGT 581; Thiruvananthapuram, Kerala University campus, 28 November 2001, V.B. Hosagoudar HCIO 45203, TBGT 1233; Thiruvananthapuram, Palode, TBGRI Campus, 4 October 2005, V. S. Srilal HCIO 47314, TBGT 2358; Thiruvananthapuram, Nedumangad, Kallara, 8 October 2005, A. Sabeena HCIO 46821, TBGT 2162; Alapuzha, Mathilakam, 14 January 2006, V. Gireesh Kumar HCIO 46763, TBGT 2104; Thiruvananthapuram, Nedumangad, Kallara, 30 
June 2006, A. Sabeena HCIO 47619, TBGT 2641; Kozhikode, Malabar Botanic Garden, 16 November 2006, A. Sabeena \& al. HCIO 47590, TBGT 2612; Thiruvananthapuram, Kariyavattum campus, 4 December 2006, A. Sabeena \& G. R. Archana HCIO 47518, TBGT 2540; Pathanamthitta, Thiruvalla, Kuttapuzha, Marthoma College campus, 15 November 2007, Jacob Thomas HCIO 48077, TBGT 2860 .

Prillieuxina winteriana (Pazschke) Arn. is the type species of the genus known on Rollina and Annona species from South America. The present new species differs from it in forming the thyriothecial crust by joining the younger thyriothecia borne on the fringed hyphae and in having smaller ascospores.

Distribution: Kerala.

Prillieuxina pterigotae Hosag. \& Abraham, Mycol. Res. 102: 185, 1998; Hosag.,C.K. Biju\& Abraham, J. Econ. Taxon. Bot. 25: 306, 2001; Singh, Duke, Bhandari \& Jain, J. Econ. Taxon. Bot. 30: 184, 2008; Hosag., Chandraprabha \& Agarwal, Asterinales of Kerala, p. 181, 2011.

(Fig. 259)

Colonies hypophyllous, thin, spreading, up to $10 \mathrm{~mm}$ diameter, confluent and covering most of the leaf surface. Hyphae pale brown, straight, substraight to flexuous, branching opposite to irregular at acute to wide angles, loosely reticulate, cells 19-29 × 2.5-4.5 $\mu \mathrm{m}$. Appressoria absent. Conidiomata pycnothyrial, numerous, scattered to loosely grouped, orbicular, stellately dehisced at the centre, up to $55 \mu \mathrm{m}$ diameter Conidia obpyriform, papillate at one end, deep brown, smooth walled, 16-19.5 $\times 14-17 \mu \mathrm{m}$. Thyriothecia few, orbicular, up to $140 \mu \mathrm{m}$ diameter, stellately dehisced at the centre, mostly crenate and rarely fimbriate at the margin, fringed hyphae flexuous. Asci globose to subglobose, 8-spored, 38-44 $\mu \mathrm{m}$ diameter Ascospores dark brown, conglobate, 1-septate, strongly constricted at the septum, $26-29 \times 12-14.5 \mu \mathrm{m}$, wall smooth.

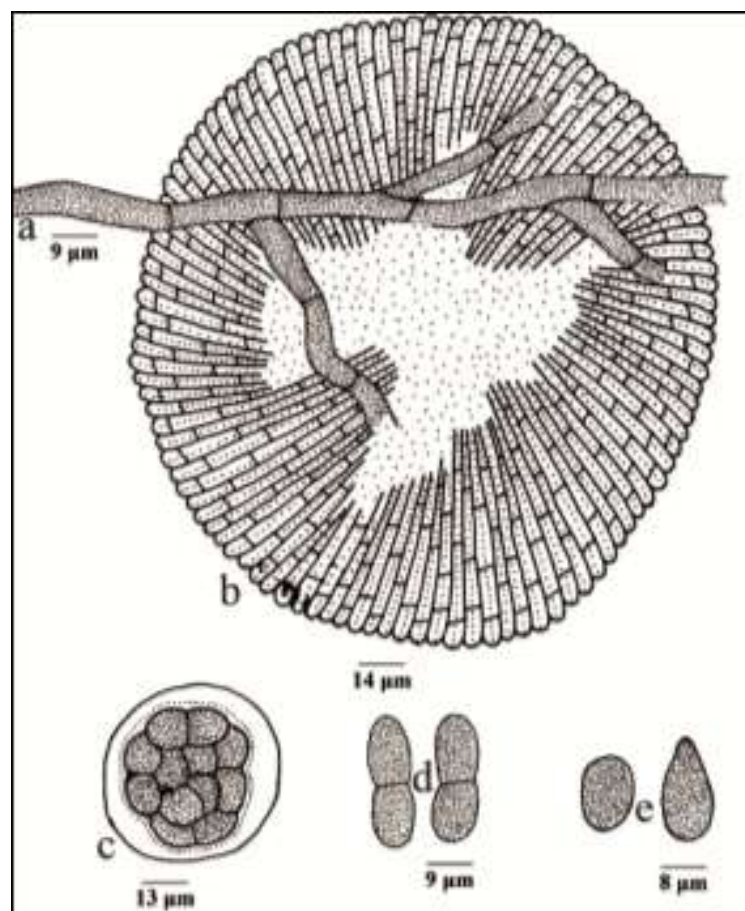

Figure 259 - Prillieuxina pterigotae

$\begin{array}{ccc}\text { Material } & \text { examined: } & \text { Kerala, } \\ \text { Thiruvananthapuram, } & \text { Neyyar } & \text { wildlife }\end{array}$ Sanctuary, Meenmutty forest, on leaves of Pterygota alata (Roxb.) R. Br. (Sterculiaceae), 11 March 1996, V.B. Hosagoudar HCIO 42143 (holotype), TBGT 7 (isotype).

This is the first report of a species of Asterinella on a member of the Sterculiaceae (Stevens \& Ryan, 1939, Doidge, 1942).

Distribution: Kerala.

\section{THE GENUS Symphaster}

Symphaster Theiss. \& Sydow, Ann. Mycol. 13: 217, 1915; Hansf., Mycol. Pap. 15: 189, 1946; Muller \& Arx, Beitr. Krypt. Schw. 11: 94, 1962; Luttrell in Ainsworth et al. (eds.). The Fungi. An advanced Treatise 4: 207, 1973; Arx \& Muller, Stud. Mycol. 9: 44, 1975; Hosag., Abraham \&C.K. Biju, J. Mycopathol. Res. 39: 61, 2001; Hosag., Chandraprabha \& Agarwal, Asterinales of Kerala, p. 183, 2011.

Isipinga Doidge, Bothalia 1: 15, 1921.

Leaf parasites. Mycelium ectophytic, appressoria formed only around the stomata. Thyriothecia formed below the mycelium, 
orbicular with radiating cells, astomatous, dehisce stellately at the center; asci globose, octosporous, bitunicate; ascospores conglobate, uniseptate, brown.

Type sp.: S. gesneraceae (Henn.) Theiss. \& Sydow

Anamorph: Bramhamyces Hosag.

Symphaster was devoid of lateral appressoria on its main hyphae but having at the growing tip of the mycelium to form 'areole' around the stomata with 1-4 appressoria are the characteristic feature of this genus.

\section{DESCRIPTION OF SPECIES}

Symphaster mimusopsidis Hosag., Sabeena \& Agarwal, Indian Phytopath. 62: 230, 2009; Hosag., Chandraprabha \& Agarwal, Asterinales of Kerala, p. 183, 2011. (Figs 260-261)

Colonies amphigenous, mostly hypophyllous, subdense, up to $3 \mathrm{~mm}$ in

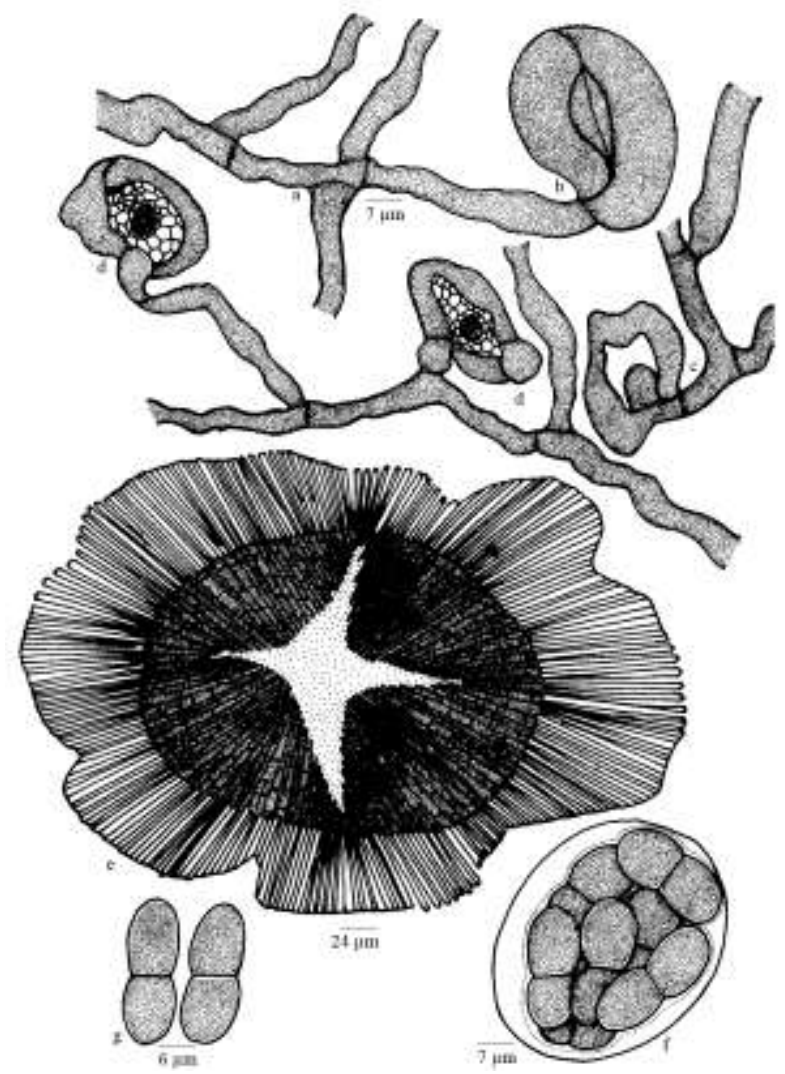

Figure 260 - Symphaster mimusopsidis a. Non-appressoriate branched mycelium, b. Areole formed around stoma, c. Areole with appressorium, d. Areole formed around stomata, plugged with mycelium, shows appressorium, e. Thyriothecium, f. Ascus, g. Ascospores

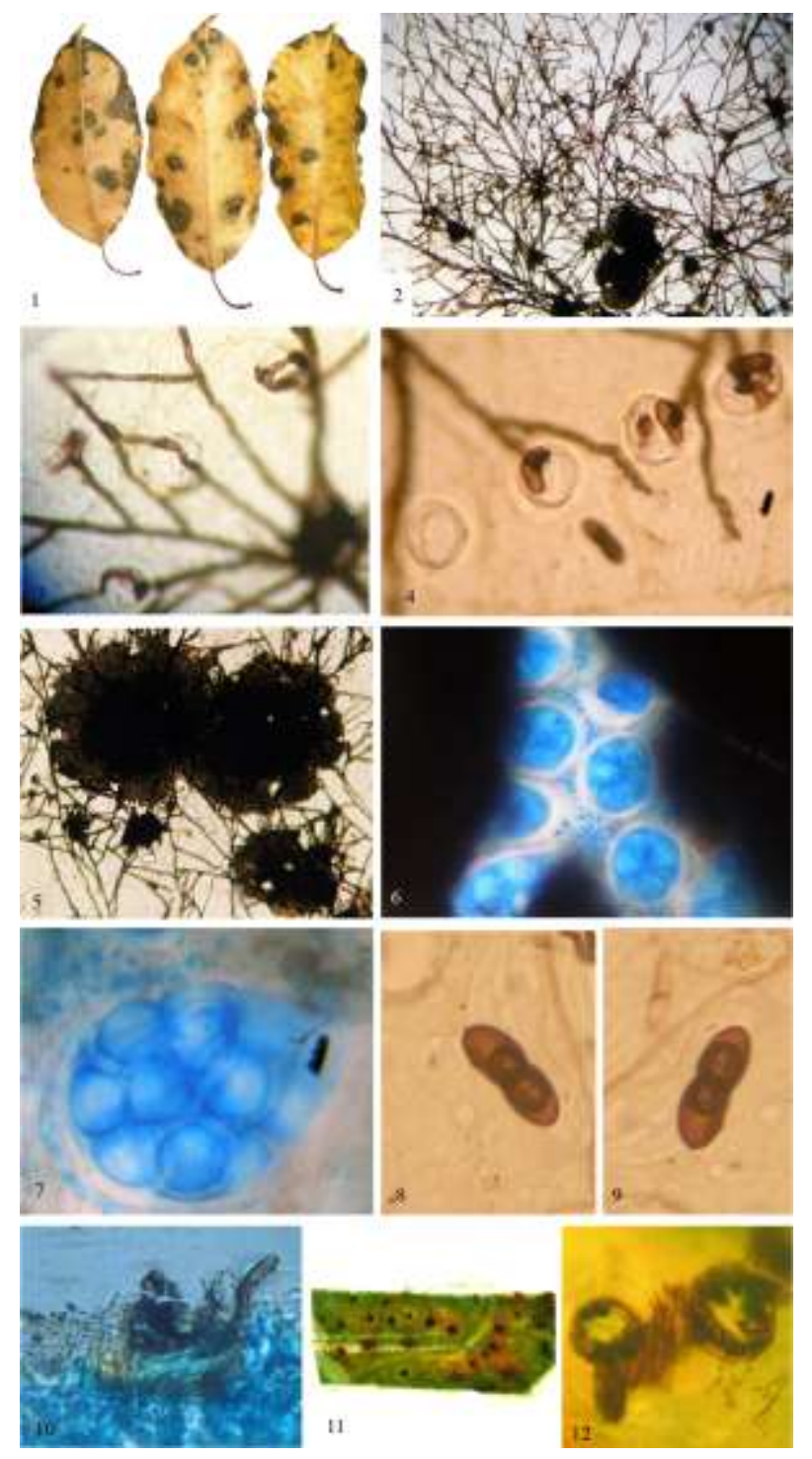

Figure 261. Symphaster mimusopsidis

1. Matured leaves with infection,2.Fungal colony, 3. Mycelium showing the formation of areole around stomata, 4\&12. Showing appressoria around stomata, 5. Connate thyriothecia, 6.Arrangement of asci in the dehisced thyriothecium, 7.Ascus, 8\&9. Ascospores, 10. T.S. shows entering of the hyphae through stomatum, 11. Hyphae plugged the stomatal opening

diameter, confluent. Hyphae ramifies in the grooves and between the convex part of the cuticle, forms an 'areole' around the stomata by 
producing 1-4 appressoria at the tip. Appressoria are produced on the main hyphae. Appressoria are produced on the adjacent cells of the guard cells. Stomata are being plugged with hyphae. However, the guard cells remain free from the mycelium. Appressoria ovoid, oblong, entire, $11-20 \times 6-13 \mu \mathrm{m}$. Thyriothecia mostly connate and form small stromata, individual thyriothecia are orbicular, up to 400 $\mu \mathrm{m}$ in diameter, stellately dehisced or dissolved at the centre, margin crenate; asci globose, octosporous, up to $44 \mu \mathrm{m}$ in diam.; ascospores conglobate, uniseptate, constricted at the septum, 20-24 × 9-13 $\mu \mathrm{m}$, wall smooth.

$\begin{aligned} & \text { Material examined: } \\ & \text { Kerala, } \\ & \text { Thiruvanaanthapuram, Palode, TBGRI }\end{aligned}$
Campus, on leaves of Mimusops elenji L.
(Sapotaceae), A. Sabeena HCIO 48271
(p.p.)(holotype), TBGT 3020, 3021 (isotypes);
Pathanamthitta, Thiruvalla, 23 August 2007,
Jacob Thomas HCIO 48413,TBGT 3134.

Stromatic thyriothecia and the formation of 'areole' is the characteristic of the genus Isipinga (Dodge, 1921). Subsequently, Hansford (1946) studied this fungus and provided additional characters of this genus. The genus Symphaster was established by Theissen \& Sydow (1915) with the same characters. Hence, the genus Isphingia merged with the genus Symphaster (Arx \& Muller, 1975). Now, the genus Symphaster has two species, namely Symphastergesneraceae (P. Henn.) Theissen \& Sydow known on the members of the family Gesneraceae and $S$. areolata (Dodge) Arx reported on Euclea natalensis. The present one forms the third species of the genus Symphaster on the members of family Sapotaceae.

Distribution: Kerala.

\section{THE GENUS Trichasterina}

Trichasterina Arn., Ann. Ecol. Nat. Agric. Montpellier 16: 172, 1918; Hansf., Mycol. Pap. 15: 189, 1946; Muller \& Arx, Beitr. Krypt. Schw. 11: 94, 1962; Luttrell in Ainsworth et al. (eds.). The Fungi. An advanced Treatise 4: 207, 1973; Arx \& Muller, Stud. Mycol. 9:
43, 1975; Hosag., Abraham \&C.K. Biju, J. Mycopathol. Res. 39: 62, 2001; Singh, Duke, Bhandari \& Jain, J. Econ. Taxon. Bot. 30: 192, 2008; Hosag., Chandraprabha \& Agarwal, Asterinales of Kerala, p. 185, 2011.

Dioguetia Bat. \& Lima., Inst. Mycol. Univ. Recife. Publ. 227: 21, 1960.

Leaf parasites. Mycelium ectophytic, appressoria lateral, mycelial and thyriothecial setae present. Thyriothecia orbicular with radiating cells, astomatous, dehisce stellately at the center; asci globose, octosporous, bitunicate; ascospores conglobate, uniseptate, brown.

Type sp.: T. styracis (Theiss. ) Arn.

\section{DESCRIPTION OF SPECIES}

Trichasterina goniothalami Hosag. \& Goos, Mycotaxon 59: 165, 1996; Hosag., Rajkumar, C.K. Biju \& Abraham, Mycotaxon 77: 433, 2001; Hosag. J. Appl. \&Nat. Sci. 1(1); 77, 2009' Hosag., Chandraprabha \& Agarwal, Asterinales of Kerala, p. 185, 2011.

(Fig. 262)

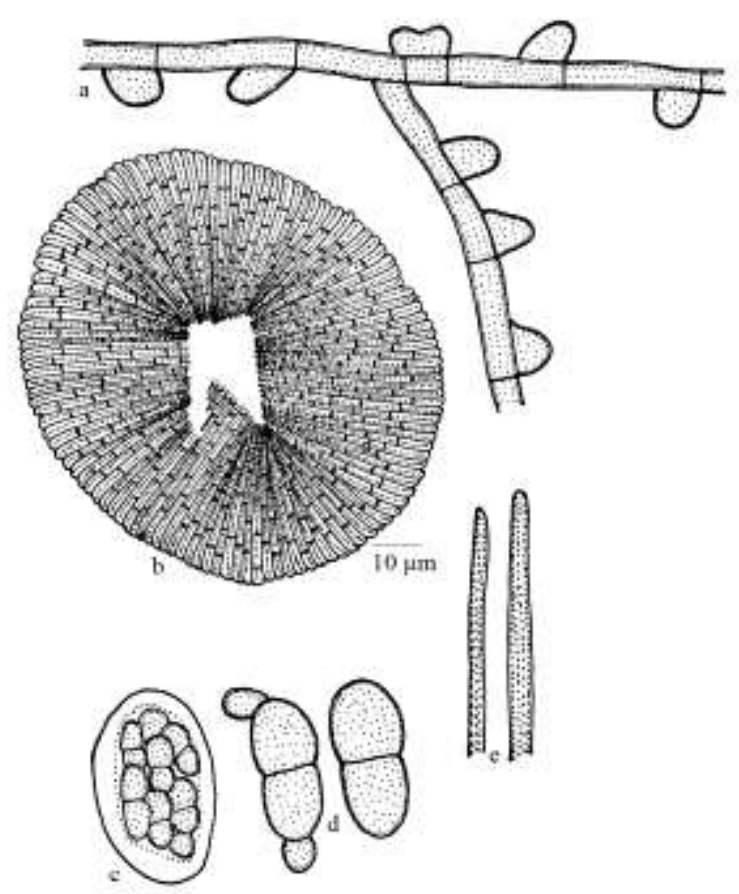

Figure 262 - Trichasterina goniothalami a. Appressoriate mycelium, b. Thyriothecium c. Ascus, d. Ascospores, e. Setae 
Colonies amphigenous, dense, velvety, up to $10 \mathrm{~mm}$ in diameter, rarely confluent. Hyphae straight to substraight, branching irregularly at acute angles, loosely to closely reticulate, cells 18-31 $\times$ 5-7 $\mu \mathrm{m}$. Appressoria unilateral, alternate and about 5\% opposite, seated on a broad base, 6-13 $\times 6-10 \mu \mathrm{m}$. Mycelial and thyriothecial setae numerous, simple, straight, erect, obtuse at the apex, up to $110 \mu \mathrm{m}$ long. Thyriothecia scattered, orbicular, carbonaceous black and opaque, up to $110 \mu \mathrm{m}$ in diameter; margin crenate, dehiscing stellately at the center; asci many, globose to slightly ovate, eight spored, 31-40 × 27-31 $\mu \mathrm{m}$; ascospores brown, conglobate, 1-septate, deeply constricted at the septum, 31-34 × 12-16 $\mu \mathrm{m}$.

Material examined: Tamil Nadu, Tirunelveli, Kudrevetti, on leaves of Goniothalmus wighti Hook. f. \& Thoms. (Annonaceae), 25 February 1994, V.B. Hosagoudar HCIO 41557; Kerala, Thiruvananthapuram, Chemunji hills, 11 March 1997, V.B. Hosagoudar HCIO 44076 (p. p.), TBGT 510 (p. p.); HCIO 44131(p. p.), TBGT 511(p. p.); 8 December 1998, G. Rajkumar HCIO 43367, TBGT 259; 23 April 1998, G. Rajkumar HCIO 43365, TBGT 260; 16 July 1998, G. Rajkumar HCIO 43366, TBGT 261; 12 April 1999, G. Rajkumar HCIO 43368, TBGT 258; Thiruvananthapuram, Chemunji hills, on leaves of $G$. rhyncantherus Dunn, 9 March 2003, G. Rajkumar HCIO 45122, TBGT 1177.

This species differs from Trichasterina polyalthiae Hansf. in having unilateral appressoria, smaller setae and larger ascospores (Hansford, 1955).

Distribution: Kerala, Tamil Nadu.

\section{THE GENUS Vishnumyces}

Vishnumyces Hosag. \& Harish, Indian Phytopath. 63: 85, 2010; Hosag., Chandraprabha \& Agarwal, Asterinales of Kerala, p. 187, 2011.

Leaf parasites. Mycelium ectophytic, appressoria lateral. Thyriothecia orbicular with radiating cells, astomatous, dehisce stellately at the center; asci ellipsoidal, octosporous, bitunicate; ascospores brown, conglobate, uniseptate, septa laid at the extreme end forming one pinch- off cell.

Type sp.: $V$. otonepheli Hosag. \& Harish

The genus is characterized by having orbicular thyriothecia, dehisce stellately at the center, ascospores conglobate, uniseptate, septa laid at the extreme end forming one pinch- off cell.

\section{DESCRIPTION OF SPECIES}

Vishnumyces otonephelii Hosag. \& Harish, Indian Phytopath. 63: 85, 2010; Hosag., Chandraprabha \& Agarwal, Asterinales of Kerala, p. 187, 2011.

(Figs 263-264)

Colonies epiphyllous, subdense to dense, crustose, up to $2 \mathrm{~mm}$ in diameter, confluent. Hyphae substraight to flexuous, branching alternate to irregular at acute to wide angles, loosely to rarely closely reticulate, cells 13-29 $\times 3-5 \mu \mathrm{m}$. Appressoria alternate, about $20 \%$ opposite, antrorse, subantrorse to spreading, $10-18 \mu \mathrm{m}$ long; stalk cells cylindrical to cuneate, 2-7 $\mu \mathrm{m}$ long; head cells ovate to globose, stellately sublobate, 8 -13 $\times 7$ $13 \mu \mathrm{m}$. Thyriothecia scattered, orbicular, stellately dehisced at the centre, up to $570 \mu \mathrm{m}$ in diam., margin fimbriate, fringed hyphae straight to flexuous; asci oblong to cylindrical, slightly stipitate, bitunicate, octosporous, 80-96 $\times$ 35-42 $\mu \mathrm{m}$; ascospores brown, 1-septate, septum at the distal end makes the spore proper "budding-off or pinching off" appearance, constricted at the septum to form one larger and one very smaller cell, $24-27 \times 7-18 \mu \mathrm{m}$; larger cell ovate to oblong, $16-21 \times 14-18 \mu \mathrm{m}$, smaller cell ovate to globose, often mammiform, 6-7 $\times$ 7-8 $\mu \mathrm{m}$, wall smooth.

Material examined: Kerala, Trissur, Vazhachal forest, on leaves of Otonephelium stipulaceum (Bedd.) Radlk. (Sapindaceae), 17 October 2007, M. Harish \& Jacob Thomas HCIO 48411 (holotype), TBGT 3132 (isotype).

Distribution: Kerala 


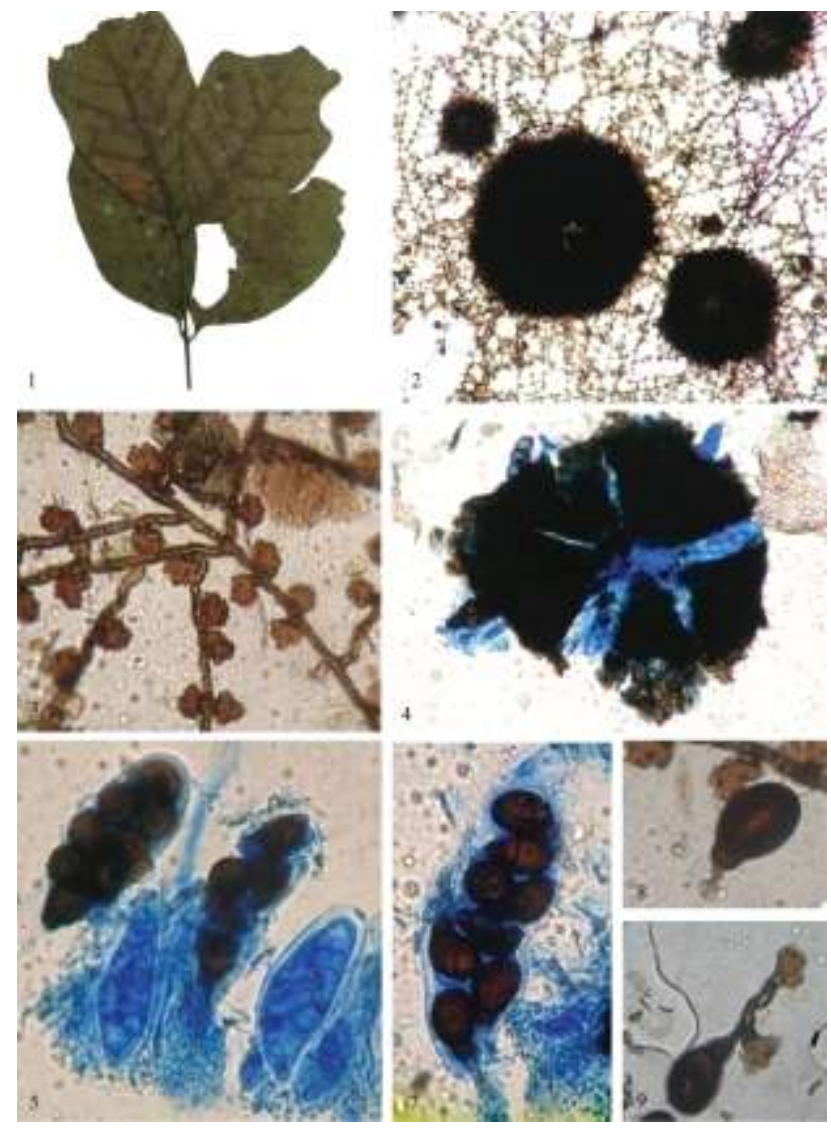

Figure 263 - Vishnumyces otonephelii

1. Infected leaves, 2. Colony, 3. Appressoriate mycelium, 4. Stellately dehisced thyriothecium, 5. Asci, 6. Ascus, 7-8. Germinating ascospores

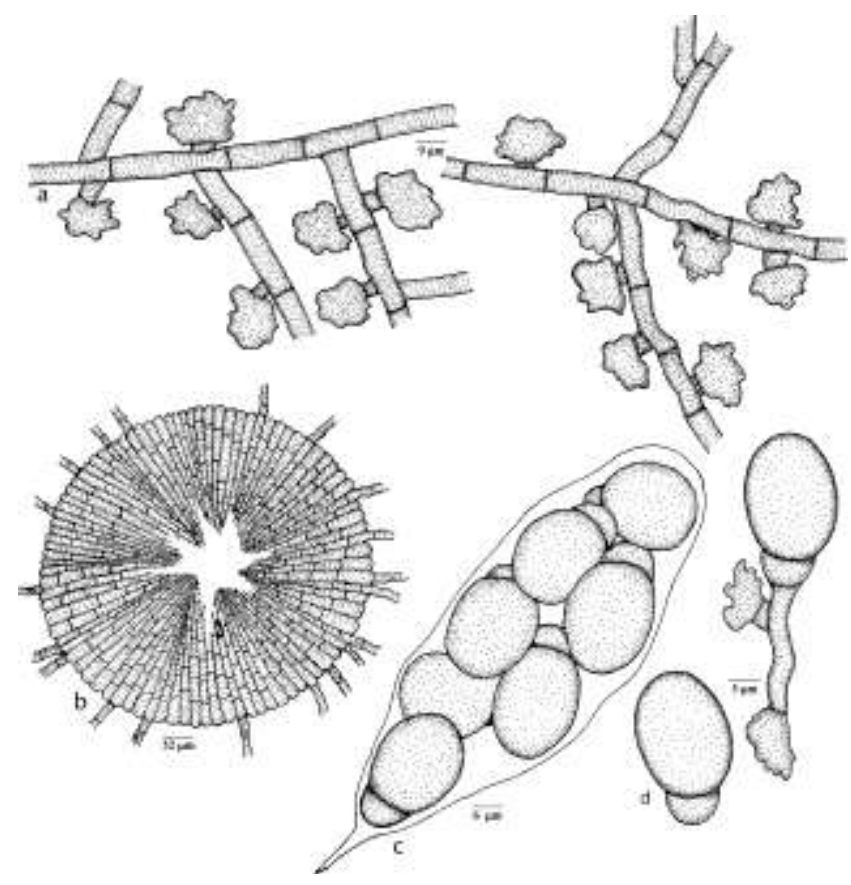

Figure 264 - Vishnumyces otonephelii

\section{THE FAMILY LEMBOSIACEAE}

\section{The family Lembosiaceae}

Lembosiaceae Höhn., Ann. Mycol. 16: 146, 1918. Lembosiaceae Hosag. in Abraham \& C.K. Biju, J. Mycopathol. Res. 39: 62, 2001.

Leaf parasites. Mycelium ectophytic, with or without appressoria, nutrient mycelium and leaf permeating stroma present. Ascomata ectophytic, dimidiate, oval, ellipsoidal, " $\mathrm{X}$ " or "Y" shaped, elongated with radiating cells, astomatous, dehisce longitudinally at the center; asci globose, spherical, octosporous, bitunicate; ascospores two to many septate, conglobate, hyaline to brown.

Type genus - Lembosia Lev.

\section{KEY TO THE GENERA OF LEMBOSIACEAE}

1. Appressoria present.................................. 2

1. Appressoria absent ...................................... 3

2. Appressoria intercalary ...................Cirsosia

2. Appressoria lateral .........................Lembosia

3. Conidia present .......................................... 4

3. Conidia absent ...................................... 5

4. Conidia 1-3 septate ......................... Eupelte

4. Conidia many septate..... Maheshwaramyces

5. Hypostroma present ...................Echidnodes

5. Hypostroma absent ................ Echidnodella

\section{KEY TO THE SPECIES}

\section{Annonaceae}

Echidnodella

Single species

E. polyalthiae

Araceae

\section{Lembosia}

Single species L. malabarensis

\section{Araliaceae}

Lembosia

Single species

L. araliacearum

\section{Arecaceae}

\section{Cirsosia}

1. Asci quadrisporous C. arecacearum

1. Asci octosporous C. globuliferae 
Lembosia

Single species

L. calamigena

\section{Caesalpiniaceae}

Lembosia

1. Appressoria oblong to elongated, 1-3 celled, straight, flexuous to crooked, cells of ascospores unequal...............L. humboldtiiae

1. Appressoria and ascospores not so. .. 2

2. Appressoria opposite, ovate to conoid L. humboldtiicola

2. Appressoria alternate, globose L. humboldtiigena

Clusiaceae

Lembosia

Single species L. garciniae

Combretaceae

Lembosia

Single species L. terminaliae-chebulae

\section{Dipterocarpaceae}

Cirsosia

1. On Hope

C. hopeae

1. On Vateria C. vateriae

\section{Echidnodella}

1.Thyriothecia less than $400 \mu \mathrm{m}$ long; on Vateria E. vateriae 1.Thyriothecia more than $400 \mu \mathrm{m}$ long; on Hopea E. hopeae

\section{Lembosia}

Single species

L. shoreae

Ebenaceae

Lembosia

Single species L. ormosiae

Fabaceae

\section{Lembosia}

Single species L. salaciae

\section{Fagaceae}

\section{Lembosia}

Single species L. perseae

\section{Hippocrataceae}

Lembosia

Single species
Lauraceae

Cirsosia

Single species

C. litseae

\section{Lembosia}

Single species

L. perseae

\section{Lythraceae}

\section{Lembosia}

Single species

L. lagerstroemiae

Melastomataceae

Echidnodella

Single species

E. memecyli

\section{Lembosia}

Single species L. memecylicola

\section{Menispermaceae}

Lembosia

Single species L. decalvans

\section{Maheshwaramyces}

1.on Coculus M. coculi 1.on Pachygone M. pachygones

Myrtaceae

Lembosia

Single species L. hosagoudarii

Oleaceae

Eupelte

Single species

E. amicta

\section{Lembosia}

Single species L. linocierae

\section{Pandanaceae}

Echidnoides

Single species E. pandanicola

\section{Lembosia}

Single species L. pandanacearum

Rubiaceae

Lembosia

Single species L. incisa

\section{Sapotaceae}

Echidnodella

Single species

E. manilkarae 
Cirsosia Arn., Ann. Ecol. Nat. Agric. Montpellier 16: 127, 1918; Hansf., Mycol. Pap. 15: 189, 1946; Muller \& Arx, Beitr. Krypt. Schw. 11:113, 1962; Luttrell in Ainsworth et al. (eds.). The Fungi. An advanced Treatise 4: 207, 1973; Arx \& Muller, Stud. Mycol. 9: 44, 1975; Hosag., Abraham \&C.K. Biju, J. Mycopathol. Res. 39: 62, 2001; Singh, Duke, Bhandari \& Jain, J. Econ. Taxon. Bot. 30: 186, 2008; Hosag., Chandraprabha \& Agarwal, Asterinales of Kerala, p. 192, 2011.

Cirsosiella Arn., Les Asterinees 1:127, 1918.

Halbania Arn., Les Asterinees 1: 163, 1918.

Leaf parasites. Mycelium ectophytic, appressoria intercalary. Thyriothecia oval, ellipsoidal, $\times$ or $\mathrm{Y}$ shaped, elongated with radiating cells, astomatous, dehisce longitudinally at the center; asci oval, octosporous, bitunicate; ascospores brown, conglobate, uniseptate.

Type sp.: C. manaosensis (Henn.) Arn.

\section{DESCRIPTIONS OF SPECIES}

Cirsosia arecacearum Hosag. \& Pillai, Mycol. Res. 98: 128, 1994; Hosag., J. Threatened Taxa 2: 1155, 2010; Hosag., Chandraprabha \& Agarwal, Asterinales of Kerala, p. 192, 2011. (Fig. 265)

Colonies epiphyllous dense, crustose, confluent and cover the entire upper surface of the leaves. Hyphae straight, branching opposite at acute angles, loosely to closely reticulate, cells $6-12.5 \times 3-5 \mu \mathrm{m}$. Appressoria intercalary, mostly near the septa, round, 9-9.5 $\times 8-9.5 \mu \mathrm{m}$. Thyriothecia initially orbicular with radiating cells, opaque and elongated to stellate at maturity, splits longitudinally and stellately at the center, 200-500 $\times 230-257 \mu \mathrm{m}$, margin fimbriate, fringed hyphae dense, deep brown, straight. Asci many, ovate, slightly papillate at the apex, quadrisporous, 43-59 $\times$ 24-28 $\mu \mathrm{m}$. Ascospores conglobate, brown, 1-septate, constricsted at the septum, $27-31 \times 12-14 \mu \mathrm{m}$, wall smooth.

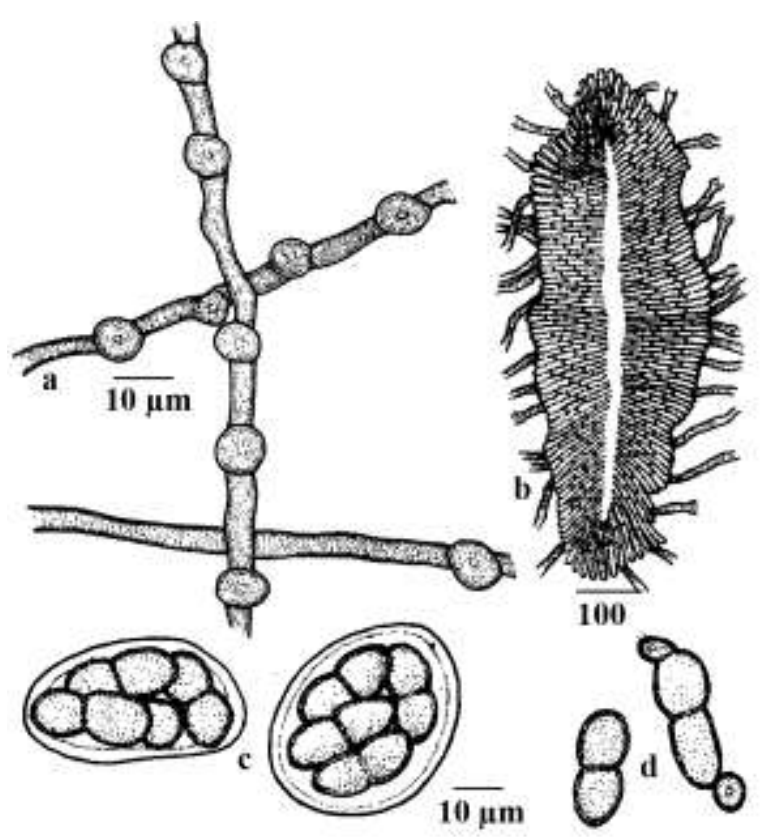

Figure 265 - Cirsosia arecacearum

Material examined: Karnataka, Gersoppa, on leaves of Calamus thwaitesii Beccary ex Hook f. (Arecaceae), 17 May 1992, Madhusoodhanan Pillai HCIO 30985 (holotype).

In addition to $C$. arecacearum described in the present paper, only two other species $C$. globulifera and $C$. transversalis are known to be host-specific on Araceae. Cirsosia transversalis differs from $C$. arecacearum in having larger asci $(60-80 \times 50-60 \mu \mathrm{m})$ and larger $(40-50 \times 18-22 \mu \mathrm{m})$ ascospores. This species also differs from $C$. globulifera in having quadrisporous asci (Muller \& Arx, 1962).

Distribution: Karnataka

Cirsosia globuliferae (Pat.) Arn. in Muller \& Arx, Beitr. Krypt. Schw. 11:114, 1962; Hosag. \& Pillai, J. Mycol. Res. 98: 128, 1994; Hosag., J. Threatened Taxa 2: 1155, 2010; Hosag., Chandraprabha \& Agarwal, Asterinales of Kerala, p. 192, 2011.

Lembosia globuliferae Pat., J. Bot. p65, 1890. 
Asterina globuliferae (Pat.) Theiss., Abn. Zool. Bot. Ges. Wein 7: 56, 1913. Cirsociella globuliferae (Pat.) Arn., Les. Asterinees 1: 128, 1916.

Asterina bakeri Sydow, Ann. Mycol. 14: 367, 1916.

Asterolibertia bakeri(Sydow) Hansf., Proc. Linn. Soc. London 160: 139, 1948. (Fig. 266)

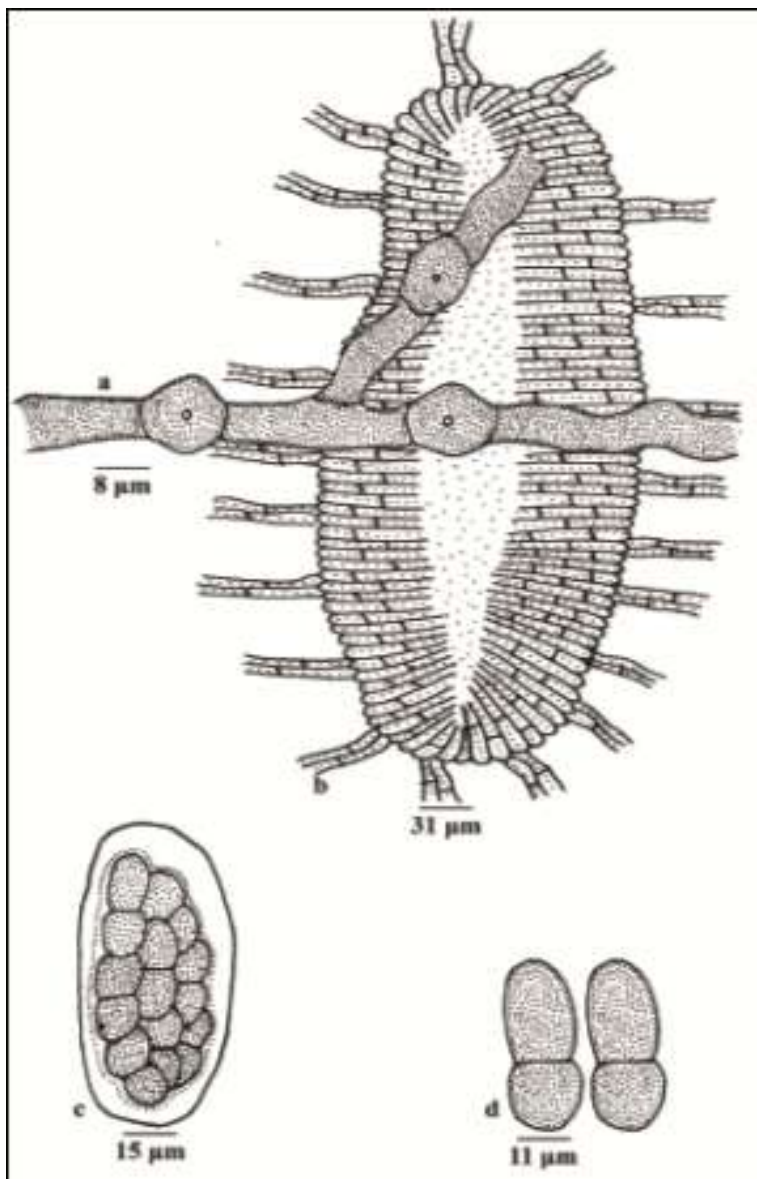

Figure 266 - Cirsosia globuliferae

Colonies epiphyllous subdense, black, up to $10 \mathrm{~mm}$ in diameter, confluent. Hyphae flexuous, brown to dark brown, branching irregular at acute angles, loosely reticulate, cells 18-31 × 3-6.5 $\mu \mathrm{m}$. Appressoria intercalary, distantly ovate, often slightly laterally, 9-12.5 × 7-9.5 $\mu \mathrm{m}$. Thyriothecia scattered, rarely connate, initially round and ovate, elongated at maturity with a longitudinal slit at the center, 240-350 $\times 150-200 \mu \mathrm{m}$, margin fimbriate, fringed devoid of intercalary appressoria. Asci many, subglobose to globate, 1-septate, initially hyaline to pale brown, late deep brown, 43-46.5 $\times$ 15-18.5 $\mu \mathrm{m}$. Ascospores conglobate, 1septate, initially hyaline to pale brown, later deep brown, 43-46.5 xf 15-18.5 $\mu \mathrm{m}$, upper cell slightly larger, wall smooth.

Material examined: Tamil Nadu, Coimbatore, Anamalai, Lower Sheikalmudy, Manjaparai forest, on leaves of Calamus pseudotenuis (Arecaceae), 26 March 1990, V.B. Hosagoudar HCIO 30986; Kerala, Thiruvananthapuram, Palode, TBGRI Campus, on leaves of Calamus sp. 20 February 1996, V.B. Hosagoudar HCIO 44143, TBGT 512.

This species was reported on Calamus sp. from Philippines (Muller \& Arx, 1962). Cirsosia globulifera matches with the assigned species in all the morphological characters except for the slightly larger ascospores (30-38 $\times 12-16 \mu \mathrm{m})$.

Distribution: Kerala, Tamil Nadu.

Cirsosia hopeae Hosag. \& Jacob-Thomas, Taprobanica 3: 17, 2011.

(Figs 267-268)

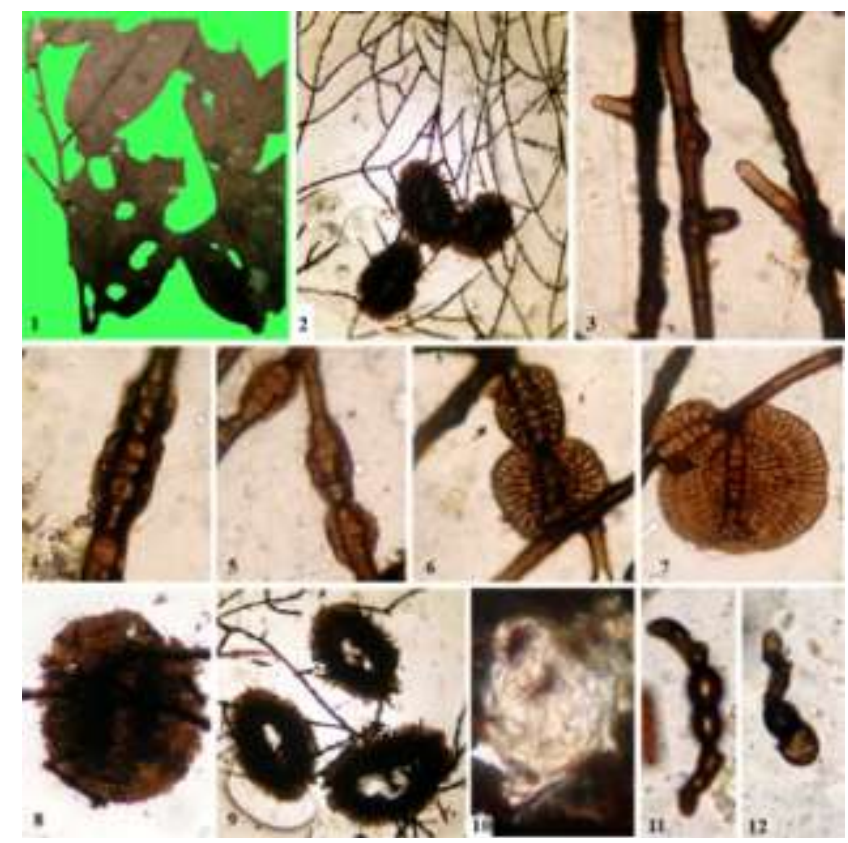

Figure 267 - Cirsosia hopeae

1. Infected leaves, 2. Thyriothecia in the colony, 3. Intercalary appressoria, 48.Developing thyriothecia, 9. Thyriothecia with dehiscence, 10. Dehisced thyriothecia with exposed asci, 11-12. Germinated ascospores

Colonies epiphyllous, subdense, up to 2 $\mathrm{mm}$ in diameter. Hyphae straight, branching 
mostly opposite at acute angles, loosely reticulate, cells $38-48 \times 9-12 \mu \mathrm{m}$. Appressoria intercalary, ovate, often slightly lateral, 9-15 $\mu \mathrm{m}$ in diam. Thyriothecia scattered, initially round to ovate, elongated at maturity with a longitudinal slit at the centre, 300-470 × 250$300 \mu \mathrm{m}$, margin crenate to fimbriate, fringed hyphae straight, closely aggregated and parallel, devoid of intercalary appressoria; asci many, globose, octosporous, 35-44 $\mu \mathrm{m}$ in diam.; ascospores obovate, conglobate, uniseptate, deeply constricted at the septum, cinnamon brown, 22-25 $\times$ 11-13 $\mu \mathrm{m}$, wall echinulate. Pycnothyria many, similar to thyriothecia; pycnothyriospores unicellular, deep brown, pyriform, slightly papillate, 18-20 $\times 11-13 \mu \mathrm{m}$.

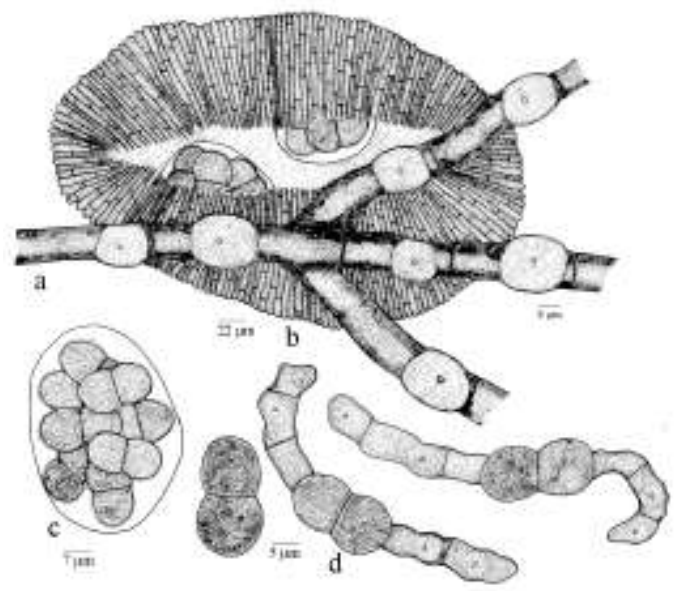

Figure 268. Cirsosia hopeae

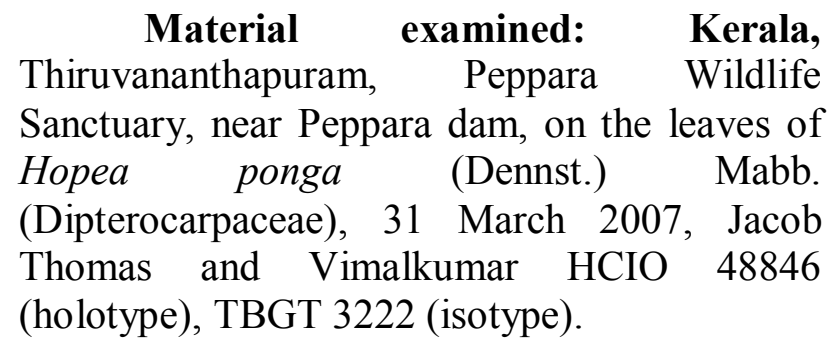

Intercalary appressoria, elliptical to elongated thyriothecia with longitudinal dehiscence are the characteristic of the genus Cirsosia. There are five species of the genus Cirsosia are known. Of these, $C$. arecacearum Hosag. \& Pillai, C. globuliferae (Pat. ) Arx. and C. transversalis Bat. \& Maia. are known on Arecaceae (Hosagoudar \& Pillai 1993), while, C. irregularis (Sydow) Arx is known on Vatica obtusifolia from Philippines (Muller \& Arx, 1962). Cirsosia hopeae differs from it in having epiphyllous colonies in contrast to the hypophyllous, straight mycelium in contrast to crooked, smaller thyriothecia against $750 \times$ 200-300 $\mu \mathrm{m}$ and smaller ascospores 23-25 $\times$ $11-12$ against $35-36 \times 15-16 \mu \mathrm{m}$.

Distribution: Kerala.

Cirsosia litseae V.B. Hosagoudar \& G.R. Archana, sp.nov. (Fig. 269)

MycoBank 801044

Etymology: Based on the host genus

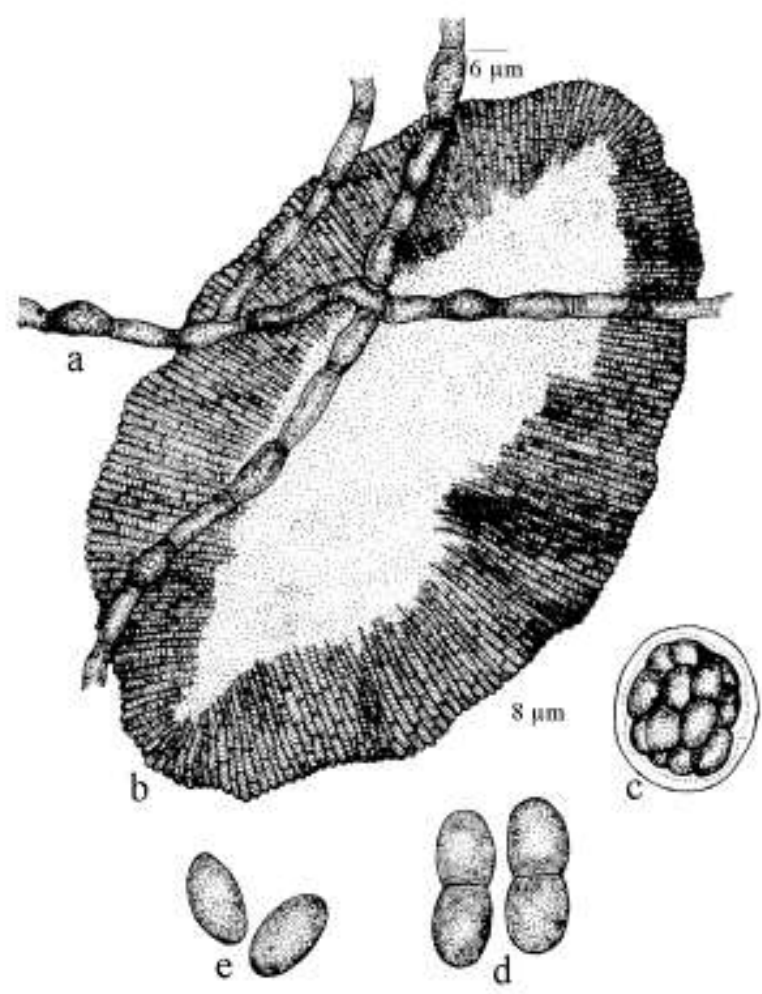

Figure 269 - Cirsosia litseae

Colonies hypophyllous, thin, up to 3 $\mathrm{mm}$ in diameter, confluent. Hyphae straight to substraight, branching opposite to irregular at acute to wide angles, loosely to closely reticulate, cells 14-21 × 3-5 $\mu \mathrm{m}$. Appressoria intercalary in position, $11-22 \times 3-7 \mu \mathrm{m}$. Thyriothecia scattered to grouped, initially orbicular, later elliptic to elongated, straight to curved, dehisce longitudinally at the centre, 156-392 × 78-196 $\mu \mathrm{m}$, margin fimbriate, fringed hyphae flexuous; asci globose, ovate, octosporous, $32-40 \times 35-40 \mu \mathrm{m}$; ascospores conglobate, uniseptate, slightly constricted at the septum, $17-26 \times 11-15 \mu \mathrm{m}$, wall smooth. Pycnothyria similar to thyriothecia, orbicular, smaller; pycnothyriospores unicellular, ovate, globose, pyriform, 16-19 $\times 9-11 \mu \mathrm{m}$. 
Material examined: Kerala, Thrissur, Adimali, on leaves of Litsea travancorica (Lauraceae), 14 February 2008, V.Gireesh Kumar TBGT 4815.

This is the first species of the genus Cirsosia on the members of Lauraceae.

Distribution: Kerala

Cirsosia vateriae V.B. Hosagoudar, sp. nov. (Fig. 270)

MycoBank 101013

Etymology: Named after the host genus

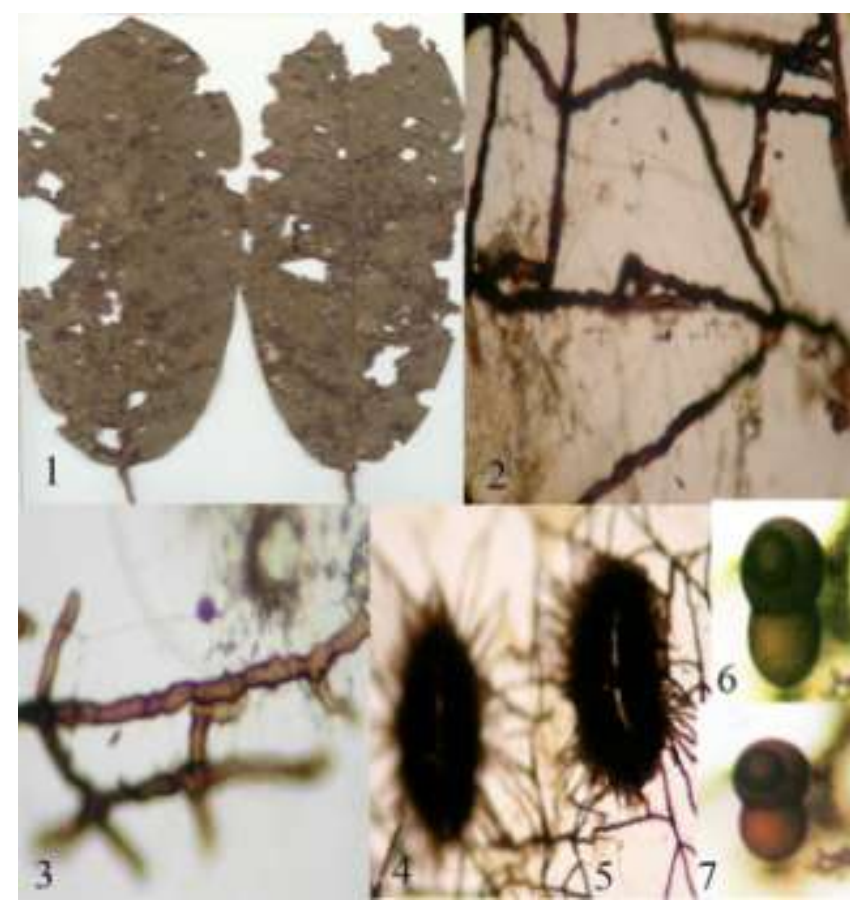

Figure 270 - Cirsosia vateriae

1. Infected leaves, 2.Mycelium, 3.Intercalary appressoria in the mycelium, 4-5.Thyriothecia, 6-7. Ascospores.

Colonies epiphyllous, rarely amphigenous, subdense, spreading, up to $5 \mathrm{~mm}$ in diameter, produced leaf spots later they were dry and tear off from the leaves leaving shot hole marks. Hyphae substraight, flexuous to crooked, branching irregular at acute to wide angles, loosely to closely reticulate, cells 14-18 $\times 7-9 \mu \mathrm{m}$. Appressoria intercalary, globose, marked with a round marking in the cells, oval, globose, $14-18 \times 9-11 \mu \mathrm{m}$ in diameter. Thyriothecia orbicular to oval, 245-345 × 90-
$245 \mu \mathrm{m}$, longitudinally dehisced at the centre, margin crenate to fimbriate, fringed hyphae small; asci globose, bitunicate, octosporous, 35$50 \mu \mathrm{m}$ in diameter; ascospores oblong, conglobate, deep brown, uniseptate, constricted at the septa, $28-32 \times 15-18 \mu \mathrm{m}$, wall smooth to tubercled.

Material examined: Karnataka, Coorg, Madikeri, Jodupal, on leaves of Vateria indica L. (Dipterocarpaceae), 12 November 2003, V.B. Hosagoudar \& al. HCIO 46332 (holotype), TBGT 1978 (isotype); Kerala, Kottayam, Ponthanpuzha, 22 November 2009, P.J. Robin \& Jayan TBGT 5752 (p.p.).

Cirsosia irregularis (Sydow) Arx is known on Vatica obtusifolia from Philippines. However, C. vateriae differs from it in having smaller thyriothecia (in contrast to 500-1000 $\times$ 190-280 $\mu \mathrm{m}$ ), asci (in contrast to 60-80 $\times 50-65$ $\mu \mathrm{m}$ ) and smaller ascospores (in contrast to 32$38 \times 15-18 \mu \mathrm{m})$.

Distribution: Karnataka, Kerala

\section{THE GENUS Echidnodella}

Echidnodella Theiss. \& Sydow, Ann. Mycol. 15: 422, 1917; Muller \& Arx, Beitr. Krypt. Schw. 11:118, 1962; Luttrell in Ainsworth et al. (eds.). The Fungi. An advanced Treatise 4: 207, 1973; Arx \& Muller, Stud. Mycol. 9: 46, 1975; Bilgrami, Jamaluddin \& Rizwi, Fungi of India p. 185, 1991; Hosag., Abraham \&C.K. Biju, J. Mycopathol. Res. 39: 62, 2001; Singh, Duke, Bhandari \& Jain, J. Econ. Taxon. Bot. 30: 187, 2008; Hosag., Chandraprabha \& Agarwal, Asterinales of Kerala, p. 194, 2011.

Leaf parasites. Mycelium ectophytic, appressoria absent, hypostroma absent. Thyriothecia oval, ellipsoidal, $\times$ or $Y$ shaped, elongated with radiating cells, astomatous, dehisce longitudinally at the center; asci oval, octosporous, bitunicate; ascospores brown, conglobate, uniseptate. Sydow

Type sp.: E. linearis (Sydow) Theiss. \& 


\section{DESCRIPTIONS OFTHE SPECIES}

Echidnodella hopeae Hosag., Balakr. \& Goos, Mycotaxon 58: 497, 1996.

(Fig. 271)

Colonies foliicolous, amphigenous, orbicular, up to $3 \mathrm{~mm}$ in diameter, rarely confluent. Hyphae straight, branching mostly opposite at acute angles, loosely reticulate, cells 18-28 $\times$ 6-8 $\mu \mathrm{m}$. Appressoria absent. Thyriothecia round, oval to elongated, scattered, rarely connate, up to $375 \mu \mathrm{m}$ in diameter, stellately to longitudinally dehisced at the center, margin crenate to fimbriate, fringed hyphae straight, closely aggregated and parallel; asci many, globose, eight spored, 46$50 \mu \mathrm{m}$ in diameter; ascospores obovate, conglobate, 1-septate, deeply constricted at the septum, cinnamon brown, 30-32 × 12-16 $\mu \mathrm{m}$. Pycnothyria many, same as thyriothecia; pycnothyriospores deep brown, pyriform, slightly papillate, $12-13 \times 9-10 \mu \mathrm{m}$.
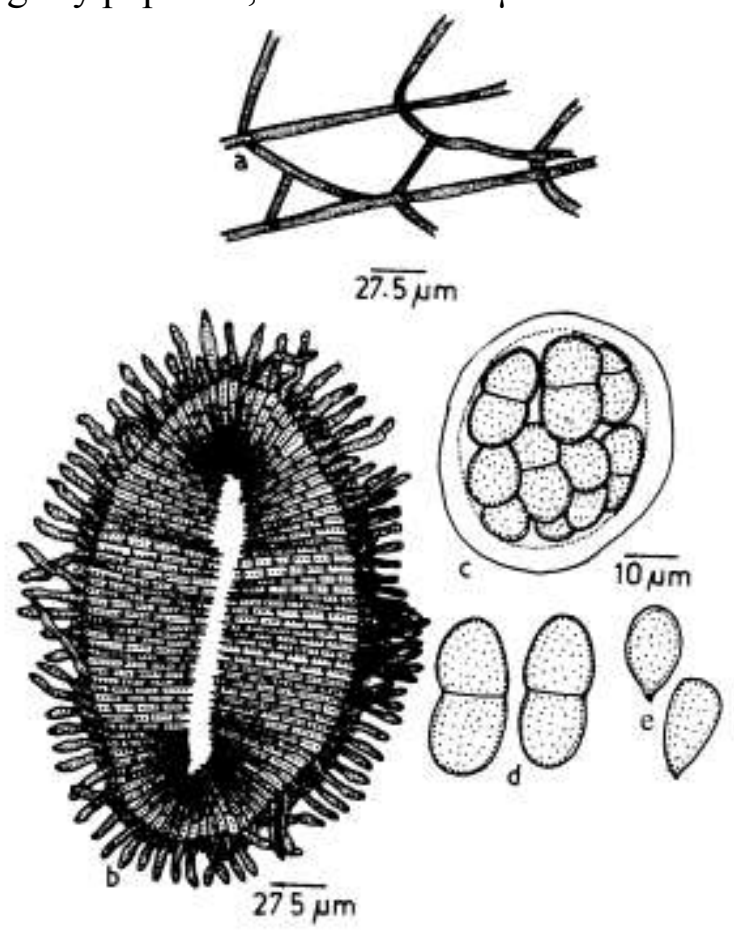

Figure 271. Echidnodella hopeae

Material examined: Karnataka, Mangalore, Konaje, on leaves of Hopea ponga (Dennst.) Mabberley (Dipterocarpaceae), 23 May 1992, B. Rajeshwari Dayal HCIO 30977 (holotype); on leaves of Hopea sp. 15 November 2003, V.B. Hosagoudar \& al. HCIO 45724, TBGT 1473.
This species differs from Echidnodella diptrocarpi (Henn.) Theiss. \& Sydow reported on Dipterocarpus sp. (Theissen \& Sydow, 1917) in having smaller perithecia, asci and ascospores.

Distribution: Karnataka.

Echidnodella manilkarae Hosag. \& T. Sabu, Indian Phytopathol. 58: 201, 2005; Hosag., Chandraprabha \& Agarwal, Asterinales of Kerala, p. 194, 2011.

(Fig. 272)

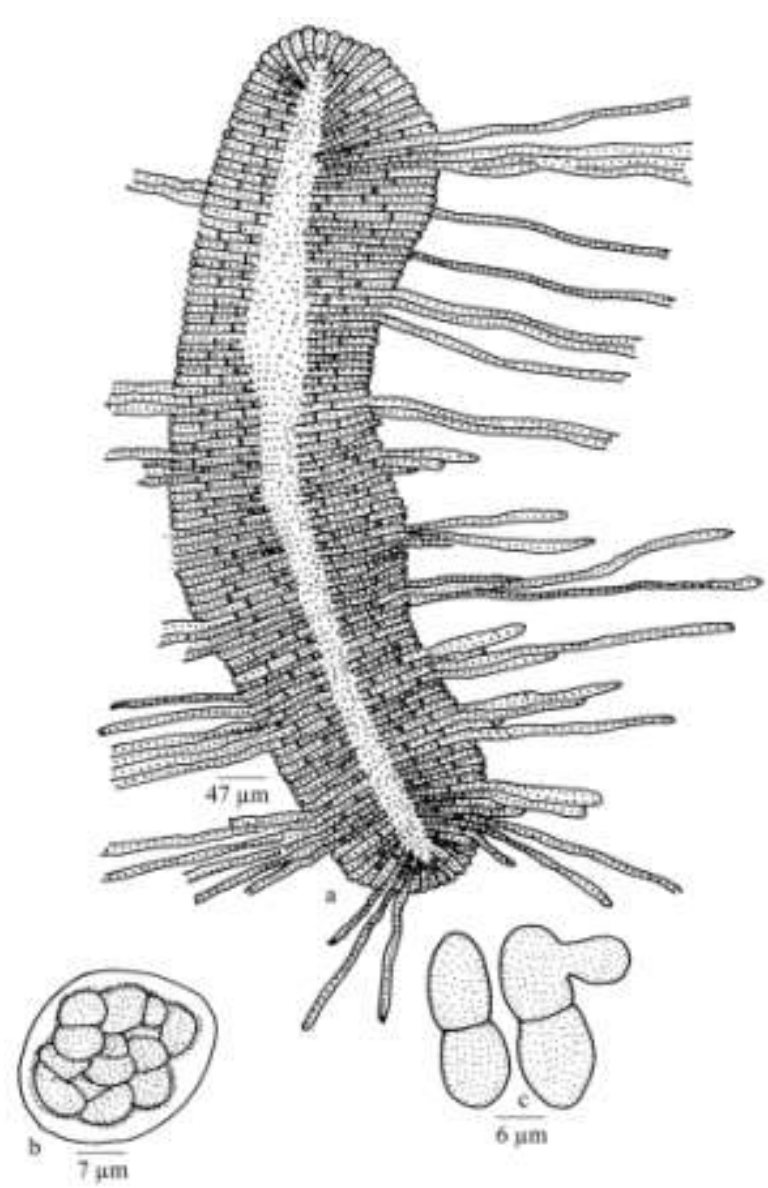

Figure 227. Echidnodella manilkarae

Colonies epiphyllous, dense, crustose, upto $1 \mathrm{~mm}$ in diam., often confluent, scanty, emerge from thyriothecia, straight to flexuous, irregularly less branched, cells 19-28 × 4-6 $\mu \mathrm{m}$. Appressoria absent. Thyriothecia elongated, connate, form a crust, up to $880 \mu \mathrm{m}$ long and up to $180 \mu \mathrm{m}$ broad, long and elongated mycelia spread from the margin of the thyriothecia, dehisce longitudinally at the centre, margin crenate; asci many, globose, 
octosporous, bitunicate, upto30 $\mu \mathrm{m}$ in diam.; ascospores oblong, conglobate, brown, uniseptate, constricted, $20-24 \times 9-11 \mu \mathrm{m}$, wall smooth.

Material examined: Kerala, TBGRI campus, Arboretum, on leaves of Manilkara hexandra (Roxb.) Dubard (Saportaceae), 8 October 2000, T. Sabu HCIO 45288 (holotype), TBGT 1326 (isotype).

Non-appressoriate mycelium with elongated thyriothecia dehisce longitudinally fits this taxon into the genera Echidnodes and Echidnodella. Lack of the stroma fits it to the latter genus. About a dozen species of the genus Echidnodella species are known on different members of the flowering plants. However, the present genus is known for the first time on the members of the family Sapotaceae. As such, there is no such free mycelium and mycelium radiates from the margin of the thyriothecia.

Distribution: Kerala.

Echidnodella memecyli Hosag. \& Abraham, J. Mycol. Res. 102: 185, 1998; Hosag., C.K. Biju \& Abraham, J. Econ. Taxon. Bot. 25: 306, 2001; Hosag., Zoos' Print J. 18: 1283, 2003; Hosag., Chandraprabha \& Agarwal, Asterinales of Kerala, p. 194, 2011.

(Fig. 273)

Colonies hypophyllous, subdense, spreading, up to $5 \mathrm{~mm}$ diameter, widely confluent; Hyphae substraight, flexuous to crooked, branching opposite to irregular at acute to wide angles, loosely reticulate, cells 24-29 $\times$ 2.5-3.5 $\mu \mathrm{m}$. Appressoria absent. Thyriothecia scattered, rarely connate, ovate, elongate, straight or curved to acutely sinuate or variously branched, $530-635 \times 31-36 \mu \mathrm{m}$, longitudinally split at the centre, mostly crenate at the margin and rarely fimbriate, fringed hyphae very small. Asci ovate, clavate, globose, 8-spored, 33-36 × 19-24 $\mu \mathrm{m}$. Ascospores conglobate, 1-septate, constricted at the septum, lower cell slightly attenuated and upper rounded, 12-14.5 × 4-6 $\mu \mathrm{m}$, wall smooth.
Material examined: Kerala, Thiruvananthapuram, Meenmutty, Neyyar Wildlife Sanctuary, Teerthankara, on leaves of Memecylon sp., 18 March 1996, V.B. Hosagoudar HCIO 42144 TBGT 8; Pathanamthitta, Anathode dam site, 15 February 2004, V.B. Hosagoudar \& al. HCIO 47297 TBGT 2335; Maharashtra, Mahabaleswar, M. A. Nomani TBGT 3532.

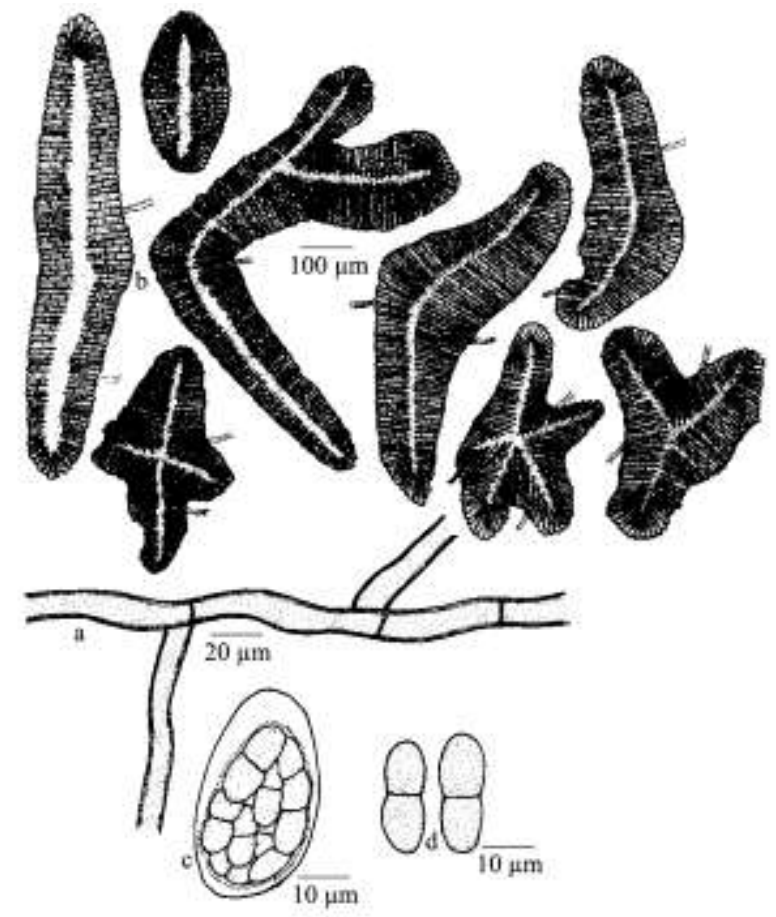

Figure 273. Echidnodella memecyli

Echidnodella miconiae R.W. Ryan and E. melastomatacearum R.W. Ryan have been reported on Miconia spp. From Puerto Rico (Stevens \& Ryan, 1939). E. memecyli differs from both in having different shaped, larger thyriothecia and smaller ascospores.

Distribution: Kerala, Maharashtra.

Echidnodella polyalthiae Hosag. in Hosag., C.K. Biju \& Abraham, J. Econ. Taxon. Bot. 28: 189, 2004; Hosag., Zoos' Print J. 21: 2413, 2006; Hosag., Chandraprabha \& Agarwal, Asterinales of Kerala, p. 196, 2011.

(Fig. 274)

Colonies restricted to the lower surface of the leaves, dense, crustose, granulose, up to 5 $\mathrm{mm}$ in diameter, confluent. Hyphae substraight 
to crooked, branching irregular at acute angles, loosely to closely reticulate, cells 12-19 × 3-5 $\mu \mathrm{m}$. Thyriothecia initially orbicular, elongated at maturity, mostly scattered, rarely connate, 400-1300 $\times 192-240 \mu \mathrm{m}$, fimbriate at the margin, fringed hyphae crooked, thyriothecia dehisce longitudinally at the center; asci ovate to globose, octosporous, $48-56 \times 32-40 \mu \mathrm{m}$. Ascospores conglobate, brown at maturity, two celled, constricted at the septum, 27-29 $\times 14-16$ $\mu \mathrm{m}$, upper cell ovate to globose, larger, lower cell smaller and measure 5-7 × 6-7 $\mu \mathrm{m}$, wall smooth.

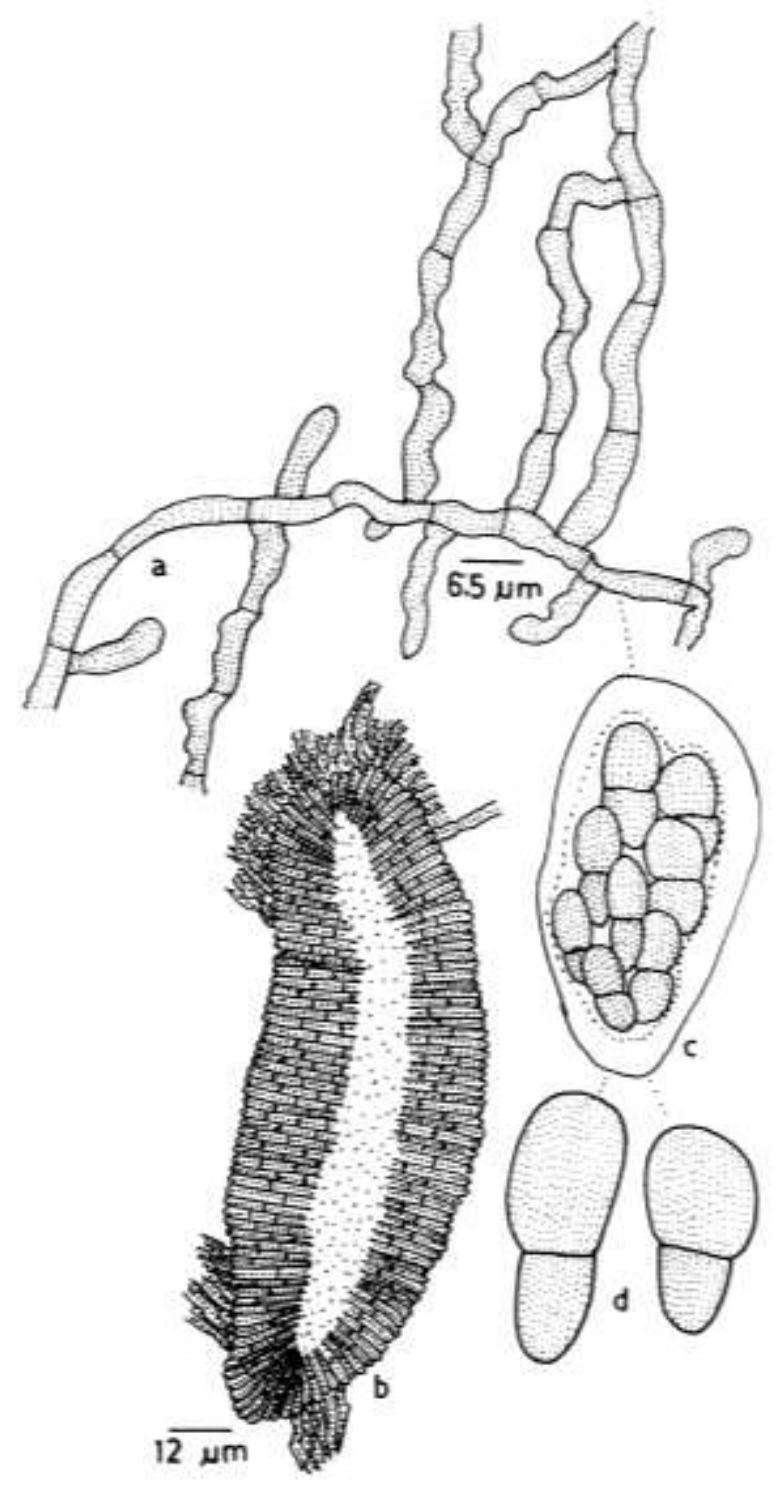

Figure 274 - Echidnodella polyalthiae

Material examined: Kerala, Thiruvananthapuram, Palode), near the main gate of TBGRI, on leaves of Polyalthia sp. (Annonaceae), 25 October 1996, V.B.
Hosagoudar HCIO 44156 (holotype), TBGT 527 (isotype); Thiruvananthapuram, Kariyavattum, in the campus of Botany Department, on leaves of $P$. longifolia (Sonn.) Thwaites, 5 November 2004, V.B. Hosagoudar \& H. Biju HCIO 46328, TBGT 1974.

The mycelium profusely branched and the tips of few branchlets enlarged and give an appearance of the head cells of appressoria but do not resemble like appressoria. Initially, thyriothecia were orbicular but elongated at maturity. However, longitudinal dehiscence is consistent. Interestingly, the upper cells of ascospores were very larger than the lower. Based on the non-appressoriate mycelia and elongated thyriothecia with longitudinal dehiscence, the present collection has been placed under the genus Echinodella. This species differs from E. guatemalensis Bat. et al. known on Anona sp. from Central America in having about three times larger thyriothecia (Muller \& Arx, 1962, Goos, 1999).

Distribution: Kerala.

Echidnodella vateriae Hosag. \& Kamar. in Hosag., Zoos' Print J. 17: 945, 2002; Hosag. \& Appaiah, J. Mycopathol. Res. 43:172, 2005; Hosag., Chandraprabha \& Agarwal, Asterinales of Kerala, p. 198, 2011.

(Fig. 275)

Colonies hypophyllous, thin to subdense, subvelvety, spreading, up to $10 \mathrm{~mm}$ in diameter, confluent. Hyphae flexuous to crooked, branching opposite at acute to wide angles, loosely to closely reticulate, cells $22-24$ $\times$ 5-7 $\mu \mathrm{m}$. Appressoria absent. Thyriothecia orbicular, oval, ellipsoidal to elongated, dehisce vertically at the centre, $450-784 \times 290-400 \mu \mathrm{m}$, margin fimbriate, fringed hyphae run parallel, compact; asci oval to globose, octosporous, bitunicate, 50-70 $\mu \mathrm{m}$ in diameter; ascospores conglobate, brown, uniseptate, deeply constricted at the septum, 35-40 × 20-24 $\mu \mathrm{m}$, wall punctate.

Material

examined:

Kerala, Thiruvananthapuram, Ponmudy, on leaves of Vateria indica L. (Diptocarpaceae), 26 July 
2001, M. Kamarudeen HCIO 44321 (holotype), TBGT 668 (isotype); Thiruvananthapuram, Palode, TBGRI Campus, 1 December 2004, V.B. Hosagoudar HCIO 46380, TBGT 2026; Kerala, Kottayam, Ponthanpuzha, 22 November 2009, P.J. Robin \& Jayan TBGT 5752 (p.p.).

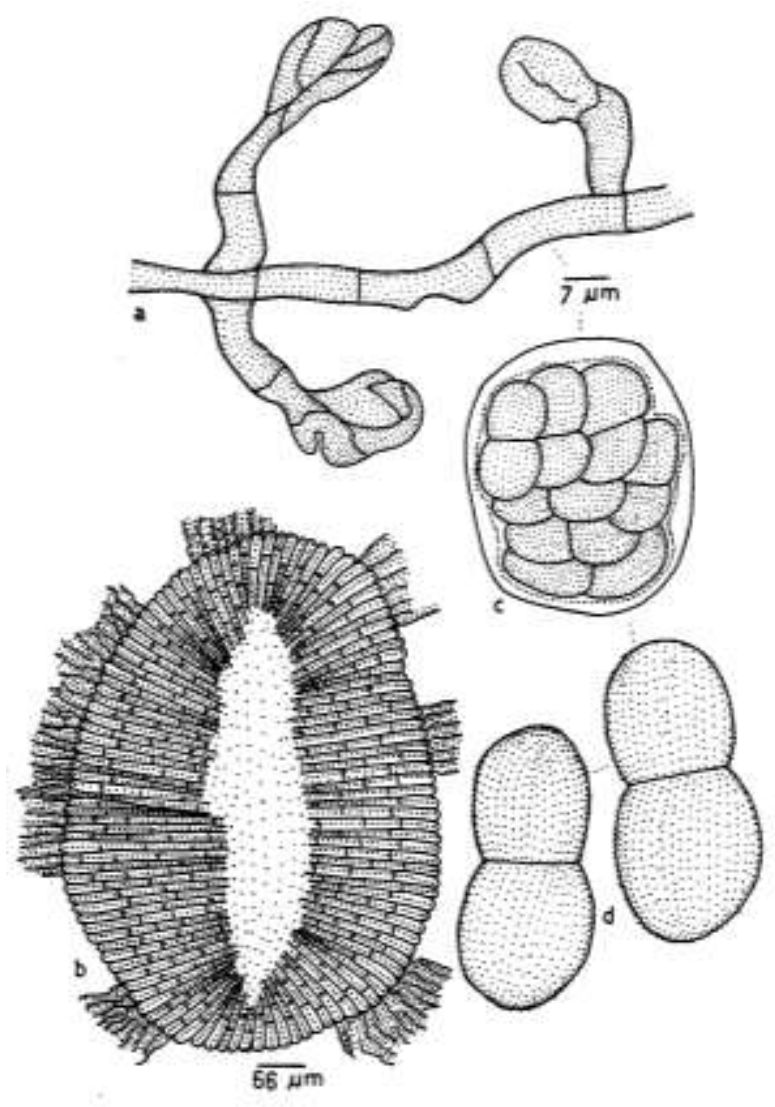

Figure 275 - Echidnodella vateriae

The tip of the hyphal branches reach the stomata, enlarge and divide. Probably, the nutritive hyphae would have formed through the stomata. Though many thyriothecia are orbicular to oval the dehiscence is verticle. Hence, this collection has been assigned to the genus Echidnodella. E. ramosii (Syd. ) Stev. and E. dipterocarpi (Henn. ) Theiss. \& Syd. are known on Pentacme sp. and Dipterocarpus sp. Echinodella veteriae differs from the former species in having larger thyriothecia and from the latter species in having smaller ascopores (Stevens \& Ryan, 1939).

Distribution: Kerala.

\section{THE GENUS Echidnodes}

Echidnodes Theiss. \& Sydow, Ann. Mycol. 15: 422, 1917; Hansf., Mycol. Pap. 15: 167, 1946; Arx \& Muller, Stud. Mycol. 9: 46, 1975; Hosag., Abraham \&C.K. Biju, J. Mycopathol. Res. 39: 62, 2001; Hosag., Chandraprabha \& Agarwal, Asterinales of Kerala, p. 200, 2011.

Lembosiodothis Hohn., Ann. Mycol. 15: 369, 1917.

Maurodothella Arn., Les Asterinees 1: 124, 1918.

Leaf parasites. Mycelium ectophytic, appressoria absent, hypostroma subcuticular or intra-epidermal. Thyriothecia oval, ellipsoidal, $\times$ or Y shaped, elongated with radiating cells, astomatous, dehisce longitudinally at the center; asci oval, octosporous, bitunicate; ascospores conglobate, brown, uniseptate.

\section{Type sp. : E. litura (Sydow) Theiss. \& Sydow}

This genus is represented here with a single species.

\section{DESCRIPTION OFTHE SPECIES}

Echidnoides pandanicola Hosag. \& Hanlin, New Botanist 22: 191, 1995; Hosag.,C.K. Biju\& Abraham, J. Econ. Taxon. Bot. 25: 306, 2001; Hosag., Zoos' Print J. 21: 2413, 2006; Hosag., Chandraprabha \& Agarwal, Asterinales of Kerala, p. 200, 2011. (Figs 276-277)

Colonies epiphyllous, dense, crustose, up to $\mathrm{mm}$ in diameter often confluent. Hyphae mostly undulate, branching alternate to irregular at wide angles, loosely reticulate, cells 45-80 × 4-6.5 $\mu \mathrm{m}$. Appressoria absent. Thyriothecia many, scattered to grouped, often connate, initially orbicular and elliptic linear at maturity, straight to curved, rarely forked, $600-800 \times 170-215 \mu \mathrm{m}$, margin crenate to slightly fimbriate, fringed hyphae flexuous, longitudinally dehisced at the centre, rarely dehisce stellately. Asci numerous, globose initially, slightly ovate to clavate at maturity, octosporous, 43-53 × 24-34 $\mu \mathrm{m}$; ascospores conglobate, oblong, brown at 
maturity, 1-septate, constricted at the septum, $24-28 \times 10-12.5 \mu \mathrm{m}$, wall smooth.

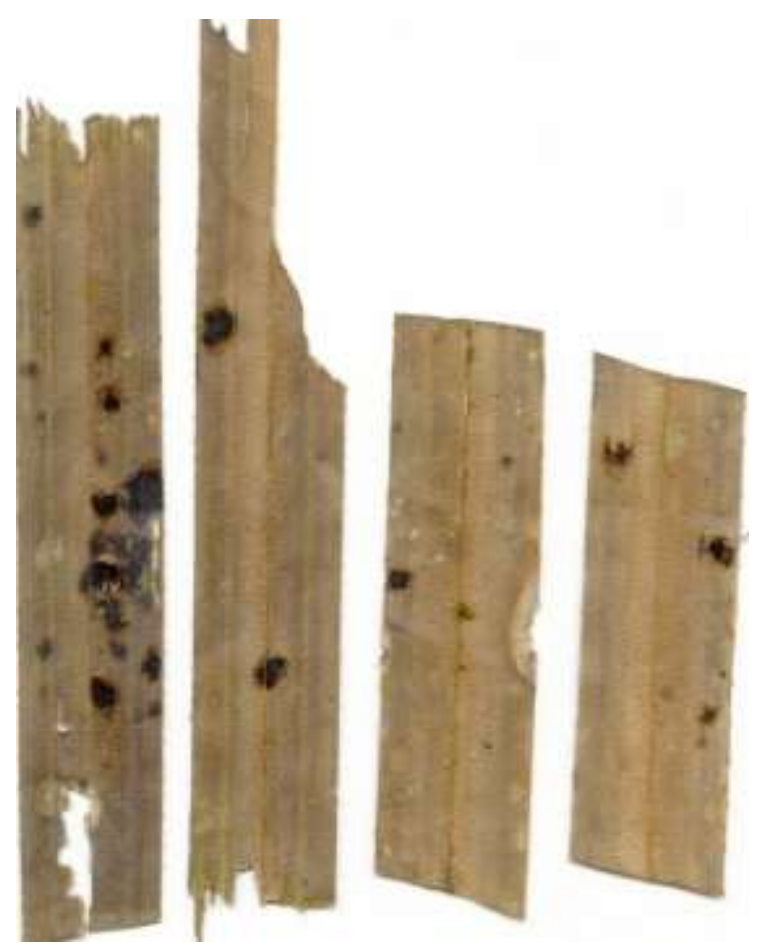

Figure 276 - Echidnoides pandanicola Infected leaves
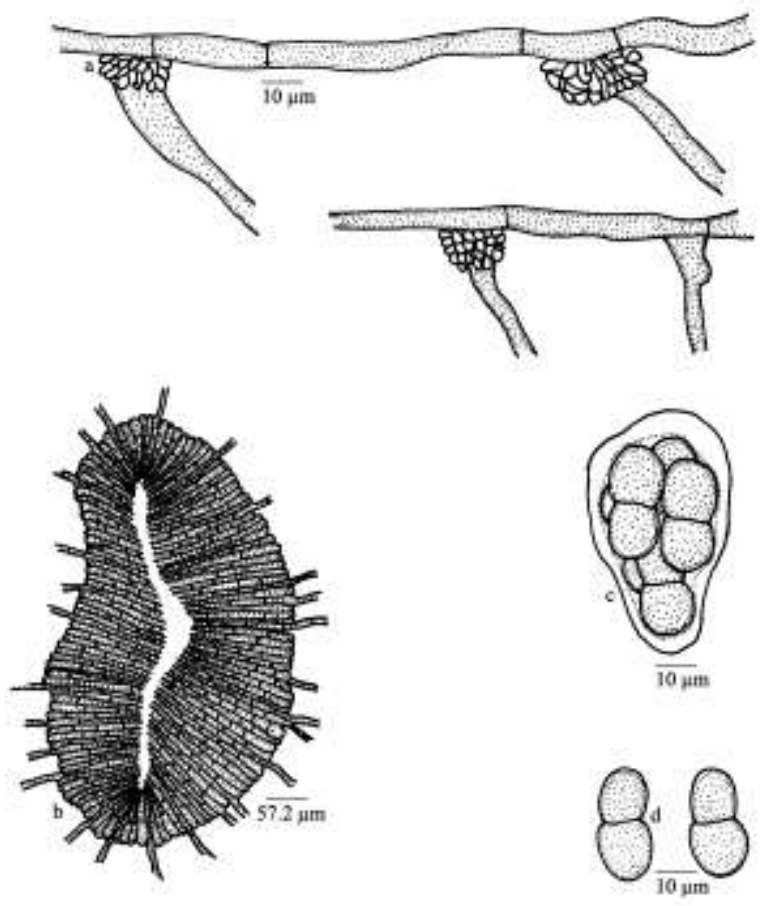

Figure 277 - Echidnoides pandanicola

Material examined: Tamil Nadu, Coimbatore, Anamalai, Sheikalmudy, Manjaparai, on leaves of Pandanus sp. (Pandanaceae), 29 December 1990, V.B.
Hosagoudar

HCIO

40884;

Thiruvananthapuram, Peppara Wildlife

Sanctuary, 4 February 1997, V.B. Hosagoudar HCIO 42508, TBGT 151 HCIO 42529, TBGT 158; Kollam, Shankily forest, Sasthanada, 23 November 2004, V.B. Hosagoudar HCIO 45969, TBGT 1733.

Theissen (1913c) proposed a new combination, Lembosia pandani (Rostr.) Theiss, based on Asterina pandani Rostr. because of the elongated thyrothecia with longitudinal slit at the centre. Later, Hansford (1946) transferred it to the genus Echidnodes because of the abscence of hyphopodia and haustoria, and made a new combination as E. pandani (Rostr.) Hansf, with the wrong basionym, Lembosia pandani (Rostr.) Theiss. The correct basionym for Echidnodes pandani (Rostr.) Hansf, should be Asterina pandani Rostr. However, the present new species differs from it in having larger asci and ascospores.

Distribution: Kerala, Tamil Nadu.

\section{THE GENUS Eupelte}

Eupelte Sydow, Ann. Mycol. 22: 426, 1924; Hansf., Mycol. Pap. 15: 168, 1946; Muller \& Arx, Beitr. Krypt. Schw. 11:137, 1962; Arx \& Muller, Stud. Mycol. 9: 43, 1975; Hosag., Abraham \& C.K. Biju, J. Mycopathol. Res. 39: 62, 2001; Hosag., Chandraprabha \& Agarwal, Asterinales of Kerala, p. 202, 2011 .

Maurodothina Arn. ex Piroz. \& Shoemaker, Can. J. Bot. 48: 1326, 1970. Anamorph: Sporidesmium sp.

Leaf parasites. Hyphae partly superficial and partly immersed. Conidia present, cylindrical, obclavate, broadly rounded at the apex, truncate at the base. Thyriothecia orbicular, elliptic to elongated, dehisce stellately, vertically at the center; asci clavate, spherical, octosporous, bitunicate; ascospores conglobate, uniseptate, brown.

Type sp.: E. amicta Syd. 
This genus is represented here with a single species.

\section{DESCRIPTION OFTHE SPECIES}

Eupelte amicta Sydow, Ann. Mycol. 22: 426, 1924; Hosag., Zoos' Print J. 21: 2413, 2006; Hosag., Chandraprabha \& Agarwal, Asterinales of Kerala, p. 202, 2011. (Fig. 278)

Colonies epiphyllous, dense, crustose, smooth, up to $10 \mathrm{~mm}$ in diameter, often cause yellow haloes around the colonies and also yellowing on the corresponding lower surface of the leaves. Hyphae partly superficial and partly immersed, superficial hyphae brown, septate, flexuous, irregularly branched at acute to wide angles, cells 12-35 $\times 3-4 \mu \mathrm{m}$. External mycelium enters the host through stomata extended up to palisade tissues. Conidiophores arise from the external mycelium, mostly deep brown to rarely dark, 0-1-septate, erect, often curved, simple, solitary, smooth, 25-32 $\mu \mathrm{m}$ long; conidiogenous cells terminal, integrated, monoblastic, determinate; conidia brown, 0-3septate, not constricted, straight to curved, cylindrical, obclavate, broadly rounded at the apex, truncate at the base, wall smooth, 20-48 $\times$ 8-10 $\mu \mathrm{m}$. Thyriothecia scattered to grouped, initially orbicular, later elliptic to elongated, simple, straight, curved, often $\times$ or $\mathrm{Y}$ shaped, astomatous, dehisce vertically at the centre, orbicular thyriothecia $100-120 \times 90-100 \mu \mathrm{m}$, ellipsoidal thyriothecia 441-700 × 196-245 $\mu \mathrm{m}$; asci born on the basal hymenium, clavate, bitunicate, become spherical at maturity, eight spored, $36-40 \times 14-18 \mu \mathrm{m}$; ascospores conglobate, oblong, brown, uniseptate, constricted at the septa, 17-20 ×9-11 $\mu \mathrm{m}$, wall smooth but becomes verrucose at maturity.

Material Examined: Kerala, Palghat, Silent Valley National Park, Chempatty, on leaves of Olea dioica Roxb. (Oleaceae), 14 December 2003, V.B. Hosagoudar \& al. HCIO 46291, TBGT 1937; HCIO 46303, TBGT 1949; Idukki, Periyar Tiger Reserve, 16 December 2003, V.B. Hosagoudar \& al. HCIO 46302, TBGT 1948; Karnataka, Coorg, Galibeedu, 24 November 2008, V.B. Hosagoudar \& al. HCIO 49153, TBGT 3408; Bagamandala, 25
November 2008, V.B. Hosagoudar \& al. HCIO 49172, TBGT 3427.

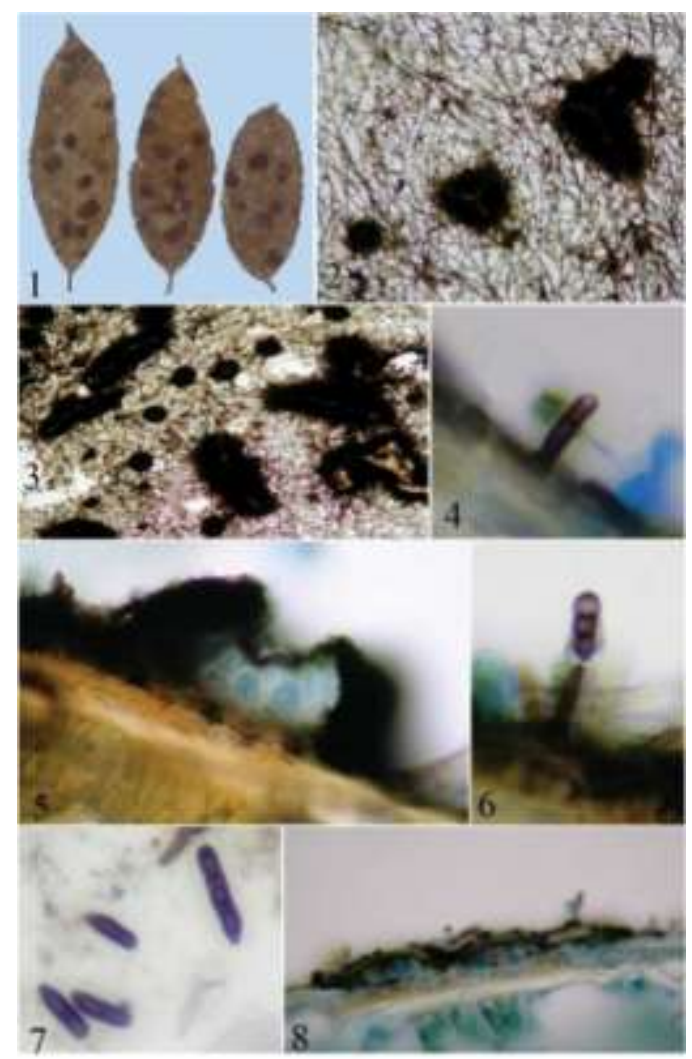

Figure 278 - Eupelte amicta

1. Infected leaves, 2-3.Thyriothecia, 4\&6. Conidiophores and to be released conidia, 5 . T.S. through thyriothecium to show the arrangement of asci, 7. Conidia, 8. Fungal mycelium in epidermal cells

Arx \& Muller (1975) have placed Maurodothina as synonym to Eupelte. Sivanesan (1984) has stated that the genus Eupelte is similar to Maurodothina but differs from it in absence of conidiogenous appressoria. Though this species was reported from India and South Africa (Sivanesan, 1984), a detailed study was not made from the Indian collections.

Distribution: Kerala, Karnataka.

\section{THE GENUS Lembosia}

Lembosia Lev., Ann. Sci. Nat. Bot. Ser., 3, 3: 58, 1845; Hansf., Mycol. Pap. 15: 189, 1946; Muller \& Arx, Beitr. Krypt. Schw. 11:111, 1962; Luttrell in 
Ainsworth et al. (eds.). The Fungi. An advanced Treatise 4: 207, 1973; Arx \& Muller, Stud. Mycol. 9: 43, 1975; Hosag., Chandraprabha \& Agarwal, Asterinales of Kerala, p. 204, 2011.

Heraldoa Bat., Att. Est. Bot. Lab. Critr. Univ. Pavia 16:105, 1959.

Lembosidium Speg., Biol. Acad. Nac. Cien. Cordova. 26:342, 1923.

Lembosiellina Bat. \& Maia, Atas Inst. Mycol. Recife 1:329, 1960. Morenoella Speg., Fungi Guar. 1: 258, 1883.

Leaf parasites. Mycelium ectophytic, appressoria lateral. Thyriothecia oval, ellipsoidal, $\times$ or $\mathrm{Y}$ shaped, elongated with radiating cells, astomatous, dehisce longitudinally at the center; asci oval, octosporous, bitunicate; ascospores conglobate, uniseptate, brown.

Type sp. : L. melastomatum Mont.

\section{DESCRIPTIONS OFTHE SPECIES}

Lembosia araliacearum Hosag. \& Kamar. in Hosag., Zoos' Print J. 17: 946, 2002; Hosag., Chandraprabha \& Agarwal, Asterinales of Kerala, p. 204, 2011.

(Fig. 279)

Colonies epiphyllous, thin, up to $3 \mathrm{~mm}$ in diameter, rarely confluent. Hyphae straight, branching alternate to irregular at acute angles, loosely reticulate, septa not clear, mycelium 3$5 \mu \mathrm{m}$ wide. Appressoria scattered, mostly in the axils of branches, oval, oblong to globose, entire to slightly lobate, 6-10 $\times$ 5-7 $\mu \mathrm{m}$. Thyriothecia scattered, oval to ellipsoidal, 146$400 \times 140-200 \mu \mathrm{m}$, dehisce longitudinally at the centre, margin fimbriate, fringed hyphae run parallel, often compact; asci globose, octosporous, $30-35 \mu \mathrm{m}$ in diameter; ascospores conglobate, oblong, uniseptate, constricted at the septum, 16-18 $\times$ 7-9 $\mu \mathrm{m}$, wall smooth.

Material examined: Kerala, Thiruvananthapuram, Ponmudy, on leaves of Araliaceae member, 26 July 2001, M.
Kamarudeen HCIO 44324 (holotype), TBGT 640 (isotype).

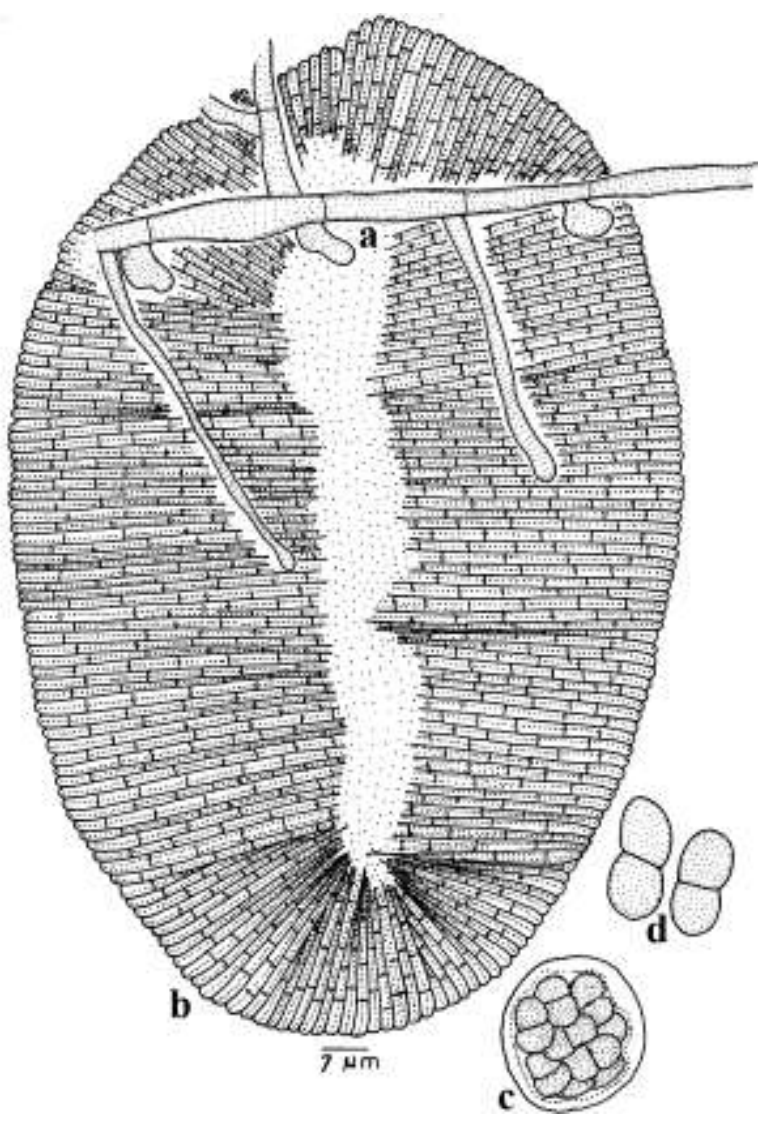

Figure 279 - Lembosia araliacearum

Lembosia heptapluri Sacc. and $L$. morototoni Petrak \& Ciferri are known on Heptaplurum sp. and Didymophanax morototoni from Singapore and San Domingo. Lembosia heptapluri does not bear appressoria and has been synonymised with Lembosina (Müller \& Arx, 1962). L. araliacearum differs from $L$. morototoni in having larger and entire to slightly lobate appressoria and larger ascospores. This species stands distinct from all in having appressoria mostly in an axillary position of the branches.

Distribution: Kerala

Lembosia calamigena V.B. Hosagoudar, M.A. Nomani, Kamal \& A. Sabeena, sp. nov. (Fig. 280)

MycoBank 101014

Etymology: Named after the host genus

Colonies amphigenous, subdense to dense, up to $3 \mathrm{~mm}$ in diam., confluent. Hyphae 
straight, branching opposite at acute to wide angels, loosely reticulate, cells 30-40 $\times 2-3 \mu \mathrm{m}$. Appressoria alternate, more scattered, unicellular, mammiform, broad based, crenately lobate with a hyaline circular spot at the center indicating haustorium produced into the epidermal cells of the host plants, 8-12 × 12-14 $\mu \mathrm{m}$. Thyriothecia scattered, elliptic to elongated, $300-600 \times 150-300 \mu \mathrm{m}$, dehisced vertically at the centre, margin fimbriate to fringed, fringed hyphae compact and run parallel; asci globose, octosporous, up to $32 \mu \mathrm{m}$ in diam.; ascospores oblong, conglobate, uniseptate, constricted at the septum, 37-40 $\times$ 15-17 $\mu \mathrm{m}$.

Material examined: Uttar Pradesh, Bahraich, on leaves of Calamus tenues (Arecaceae), M.A. Nomani TBGT 3502.

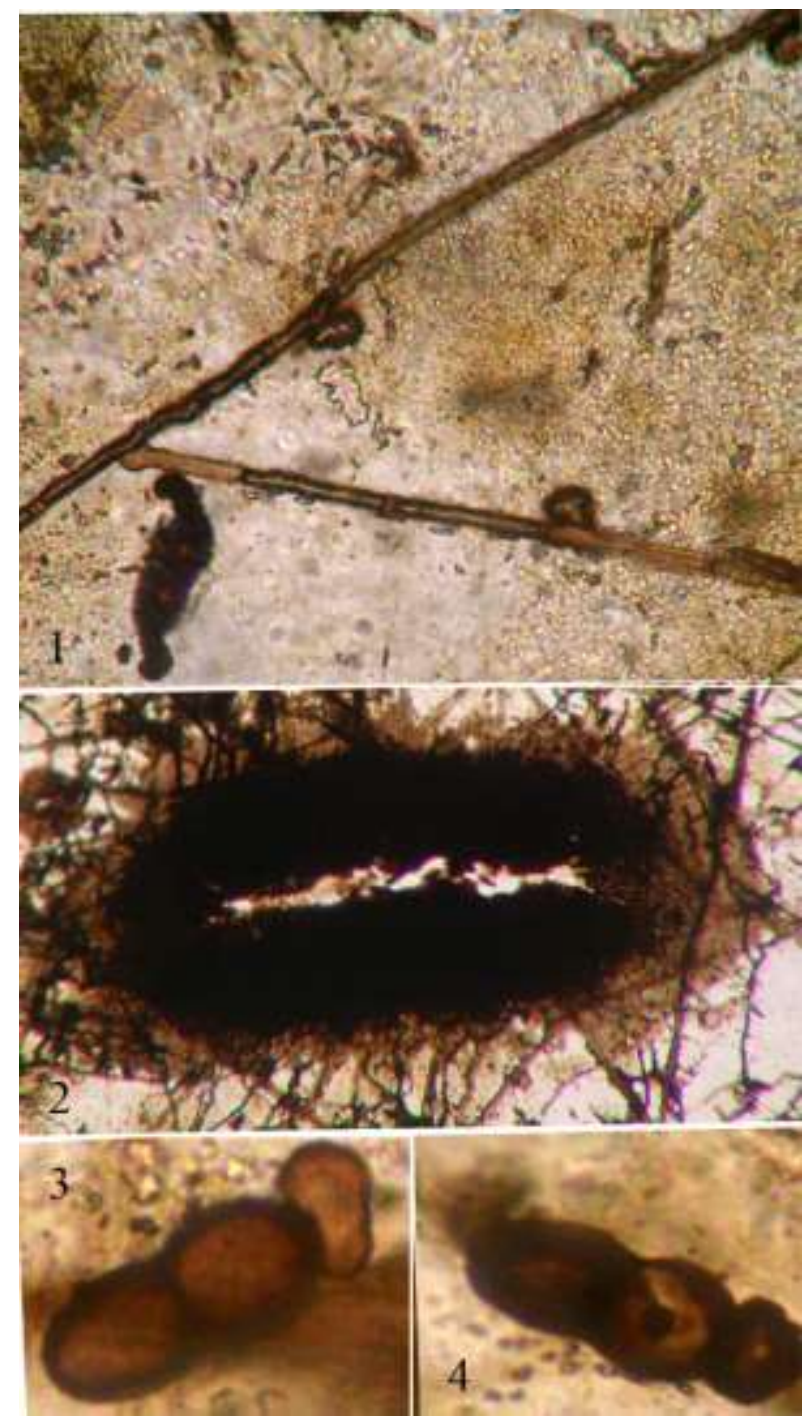

Figure 280 - Lembosia calamigena
1. Appressoriate mycelium, 2. Thyriothecium, 3-4. Ascospores

Lembosia calamigena differs from Lembosia microcarpa Sydow known on Calamus sp. from Philippines in having crenately lobate appressoria, larger thyriothecia and ascospores (Sydow \& Sydow, 1917).

Distribution: Uttar Pradesh

Lembosia decalvans Pat., Ann. Jard. Bot. Buitenzorg, P. 122, 1897; Hosag. Indian J. Forestry 18: 275, 1995. Morenoella decalvans (Pat.) Theiss., Ann. Mycol. 11: 452, 1913. (Fig. 281)

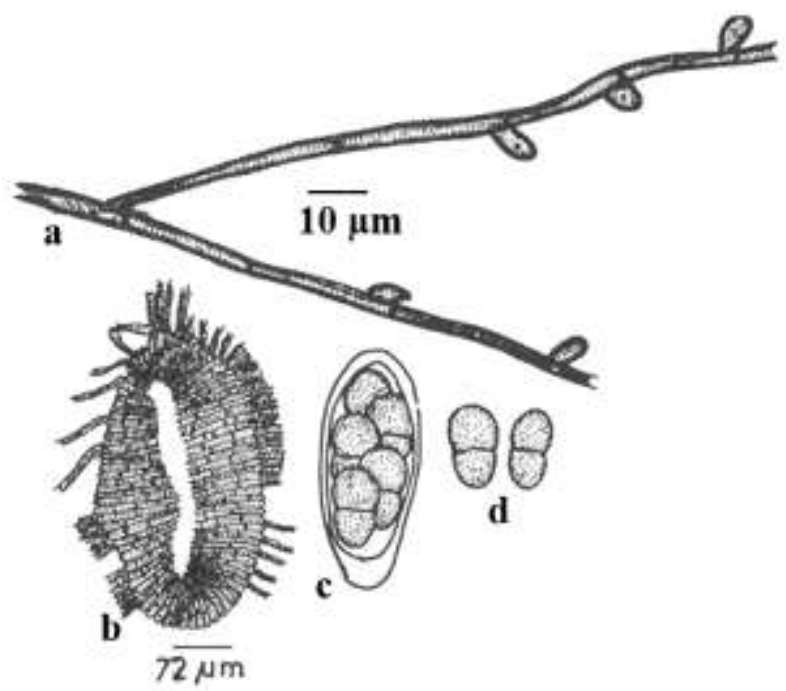

Figure 281 - Lembosia decalvans

Colonies amphigenous, carbonaceous black, dense, woolly, raised, up to $3 \mathrm{~mm}$ in diameter, rarely confluent. Hyphae straight to slightly flexuous, branching irregular at acute angles, loosely reticulate, cells $24-31 \times 3-4 \mu \mathrm{m}$. Appressoria few, continuous, alternate or unilateral, very distantly placed, globose, ovate, conoid to truncate at the apex, 4-6.5 $\times 3$ $5 \mu \mathrm{m}$. Thyriothecia mostly connate, initially circular, elliptical to elongated at maturity, up to $300 \mu \mathrm{m}$ long and $200-250 \mu \mathrm{m}$ broad, margin fringed, fringed hyphae flexuous, rupture collectively and stellately at the center; asci many, globose to oblong, octosporous, 43-46.5 $\times 18-22 \mu \mathrm{m}$; ascospores initially hyaline, brown at maturity, conglobate, oblong,, 1septate, constricted at the septum, one cell 
globose and another ovate, sometimes both cells oblong, 15-18.5 ×9-10 $\mu \mathrm{m}$, wall smooth.

\section{Material examined: Tamil Nadu,} Nilgiris, Mudumalai, near Mavana Halla, on leaves of Pachygone ovata (Poir.) Miers ex Hook. f. \& Thoms. (Menispermaceae), 21 January 1990, V.B. Hosagoudar HCIO.

The present collection slightly varies from the species description in having larger thyriothecia and slightly smaller ascospores (Theissen, 1913).

Distribution: Tamil Nadu.

Lembosia garciniae V.B. Hosagoudar and C. Jagath Thimmaiah, sp. nov. (Fig. 282)

MycoBank 101015

Etymology: Named after the host genus

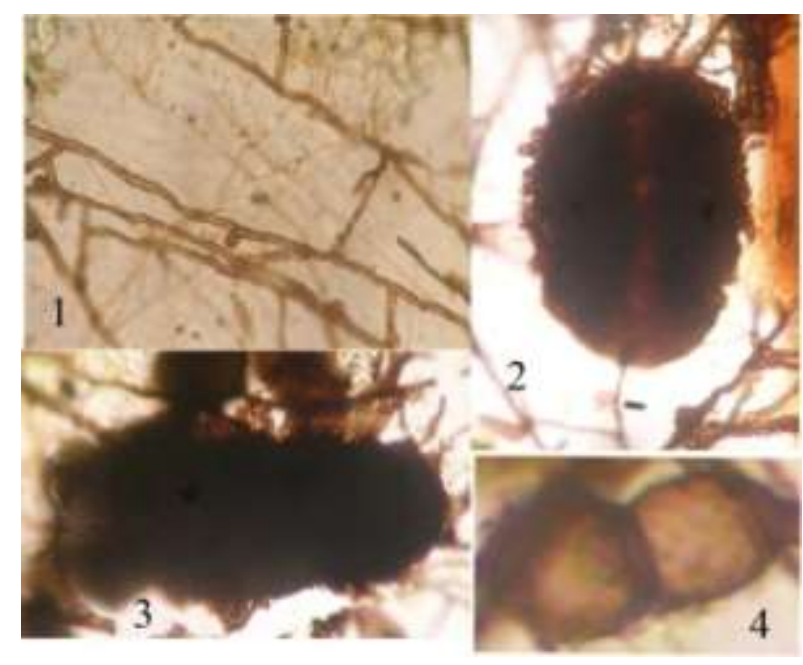

Figure 282 - Lembosia garciniae

1. Appressoriate mycelium, 2-3. Thyriothecia, 4. Ascospore

Colonies epiphyllous, thin, up to $3 \mathrm{~mm}$ in diameter. Hyphae straight to flexuous, branching irregular at acute to wide angles, loosely reticulate, cells $25-30 \times 5-7 \mu \mathrm{m}$. Appressoria more scattered, globose, mammiform, broad based, often ovate, mostly entire, rarely sublobate, $7-10 \times 5-7 \mu \mathrm{m}$. Thyriothecia initially ovate with hyaline suture in the centre, later elongated, simple, dehisce vertically at the centre, $200-400 \times 115-125 \mu \mathrm{m}$, margin mostly crenate, often fimbriate, fringed hyphae singular, straight to flexuous; asci globose, 8-spored, up to $20 \mu \mathrm{m}$ in diameter; ascospores conglobate, oblong, uniseptate, constricted at the septum, 25-30 $\times 1013 \mu \mathrm{m}$, wall smooth.

Material examined: Karnataka, Kodagu, Mandrane, Hoddur, on leaves of Garcinia gummigutta (L.) Robs. (Clusiaceae), 22 February 2009, C. Jagath Thimmaiah TBGT 5702 (holotype).

This is the first report of the Lembosia species on the members of the family Clusiaceae (Song \& Hosagoudar, 2003).

This species was associated with Meliola garciniae and Asterina clusiacearum.

Distribution: Karnataka

Lembosia hosagoudarii Sivanesan \& Shivas, Fungal Diversity 11: 163, 2002; Hosag., Chandraprabha \& Agarwal, Asterinales of Kerala, p. 205, 2011.

Lembosia syzygiicola Hosag., Indian J. Forestry 18: 276, 1995. (Fig. 283)

Colonies amphigenous, mostly epiphyllous, dense, crustose, up to $3 \mathrm{~mm}$ in diameter, rarely confluent. Hyphae straight to substraight, branching alternate to irregular at acute to wide angles, loosely reticulate, cells 31-38 $\times 3-5 \mu \mathrm{m}$. Appressoria two celled, scattered, alternate, straight to curved, 9-18 $\mu \mathrm{m}$ long; stalk cells cylindrical to cuneate, $4-7 \mu \mathrm{m}$ long; head cells ovate, globose, entire, 4-11 $\times$ 4-7 $\mu \mathrm{m}$. Thyriothecia scattered to grouped, rarely connate, initially circular, linear to elliptical at maturity, carbonaceous black, margin crenate to fimbriate, fringed hyphae flexuous, thyriothecia dehiscing by a longitudinal slit at the center, 640-930 × 214$286 \mu \mathrm{m}$; asci globose initially, cylindrical to clavate at maturity, octosporous, 71-84 × 37$46.5 \mu \mathrm{m}$; ascospores deep brown, conglobate, uniseptate, constricted at the septum, both cells mostly equal in size and shape, 21-28 $\times$ 9-12.5 $\mu \mathrm{m}$, wall smooth.

Material examined: Karnataka, Uttara Kannada, Gersoppa, on leaves of 
Syzygium tamilnadensis Rathakr. \& Chitra (Myrtaceae), 20 September 1992, P. A. Raghu HCIO; Kerala, Thiruvananthapuram, Palode, TBGRI Campus, on leaves of Syzygium cumini (L.) Skeels, 16 June 1997, V.B. Hosagoudar HCIO 42524, TBGT 163; Idukki, Munnar, Rajamala, on leaves of $S$. arnottianum Walp., 15 February 2008, A. Chandra Prabha HCIO 48292, TBGT 3011; Thiruvananthapuram, Chemunji, on leaves of Syzygium sp., 11 March 1998, V.B. Hosagoudar HCIO 42934, TBGT 219; Thiruvananthapuram, Palode Forest, 27 September 2006, Archana G. R. HCIO 49095, TBGT 3350; Thrissur, Vazhachal forest, 21 September 2007, Jacob Thomas \& al. HCIO 48220, TBGT 2956.

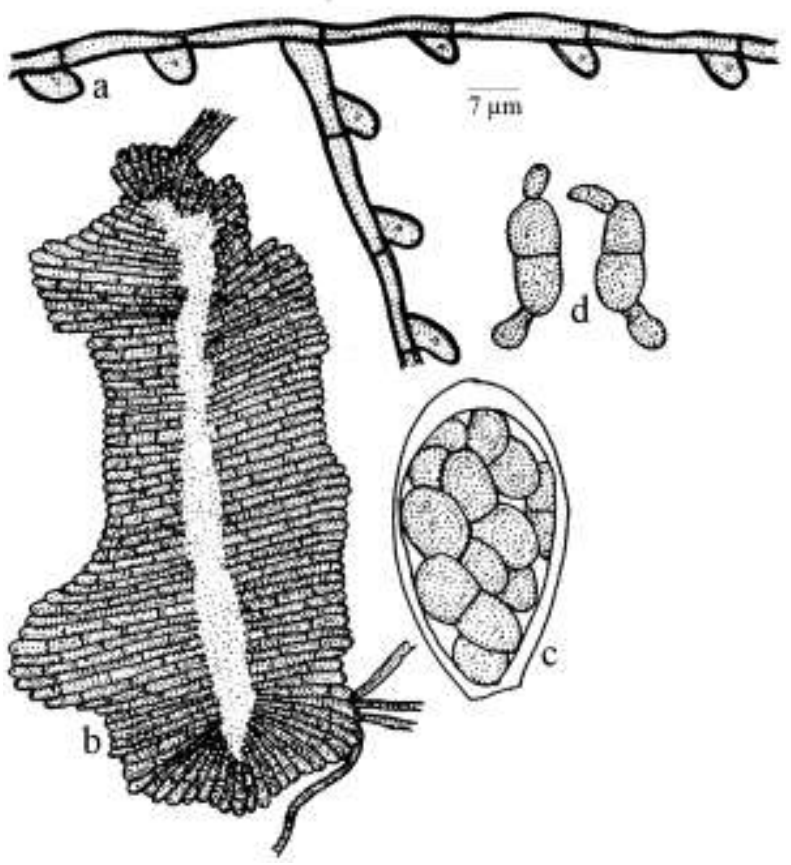

Figure 283 - Lembosia hosagoudarii

Many species of the genus Eugenia have been brought under the genus Syzygium. So far, three species, namely, Lembosia eugeniae Rehm, L. robinsonii Sydow and $L$. tenella Lev. are reported on the host species of the genus Eugenia. The present species differs from L. eugeniae Rehm in having larger thyriothecia, asci and ascospores; L. robinsonii Sydow in having smaller ascospores and from L. tenella Lev. in having larger asci and narrower ascospores (Stevens \& Ryan, 1939).
Distribution: Karnataka, Kerala, Uttar Pradesh

Lembosia humboldtiae Hosag. \& Abraham, Mycol. Res. 102: 186, 1996 (humboltiae); Song Bin \& Hosag., Guizhou Science 21: 95, 2003; Hosag., Chandraprabha \& Agarwal, Asterinales of Kerala, p. 207, 2011.

(Figs 284-285)

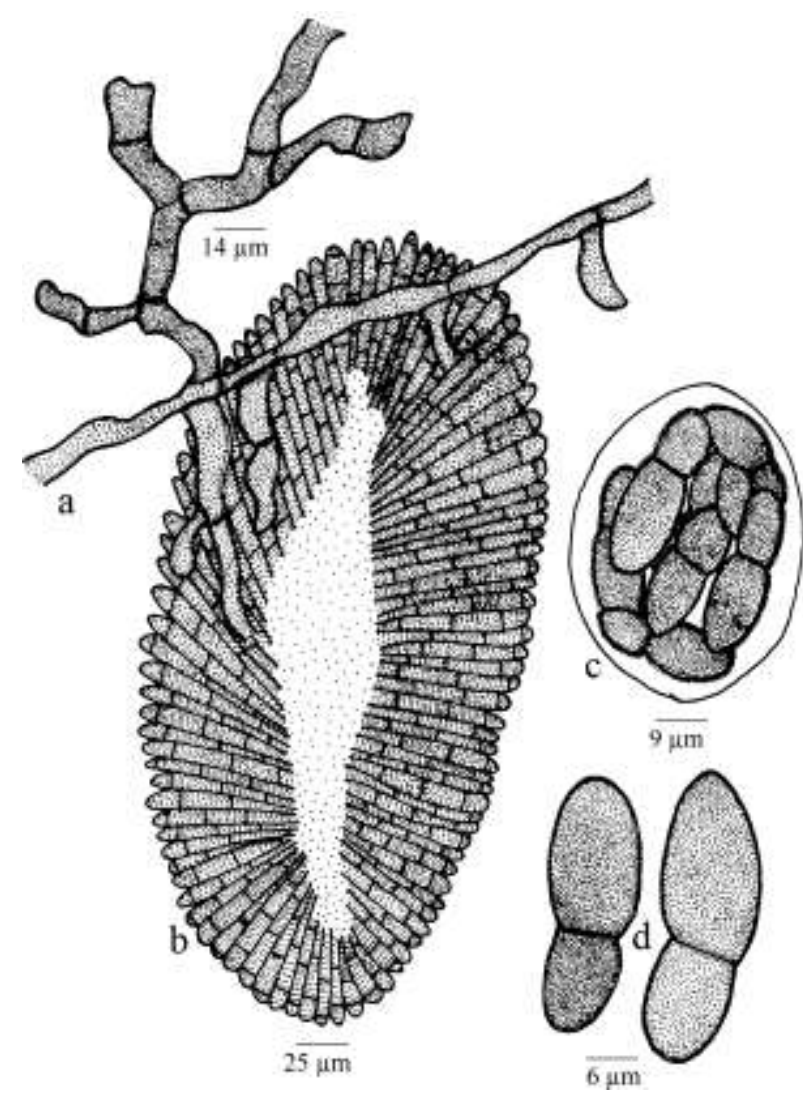

Figure 284 - Lembosia humboldtiae

Colonies amphigenous, dense, crustose, up to $5 \mathrm{~mm}$ in diameter, confluent. Hyphae flexuous to crooked, branching opposite at acute to wide angles, closely reticulate, cells 15-44 × 2-4 $\mu \mathrm{m}$. Appressoria scattered, some part of the mycelium devoid of appressoria, alternate to opposite, straight, curved, flexuous to crooked, 1-3 celled, 11-44 $\mu \mathrm{m}$ long; stalk cells 1-2 septate, 12-22 $\mu \mathrm{m}$ long; head cells ovate, straight, curved, entire to rarely lobate, 6-22 $\times 2-4 \mu \mathrm{m}$. Thyriothecia scattered, rounded to elongated, longitudinally fissured at the centre, $200-1000 \times 180-250 \mu \mathrm{m}$, margin crenate to fimbriate, fringed hyphae flexuous to crooked; asci octosporous, subglobose to ovoid, 22-42 $\mu \mathrm{m}$ in diameter; ascospores 1-septate, brown, constricted at the septum, 22-35 $\mu \mathrm{m}$ 
long, lower cell smaller, ovate, 9-15 × 6-11 $\mu \mathrm{m}$, upper cell larger than the lower, ovate, 13-20 $\times$ 11-13 $\mu \mathrm{m}$, wall smooth.
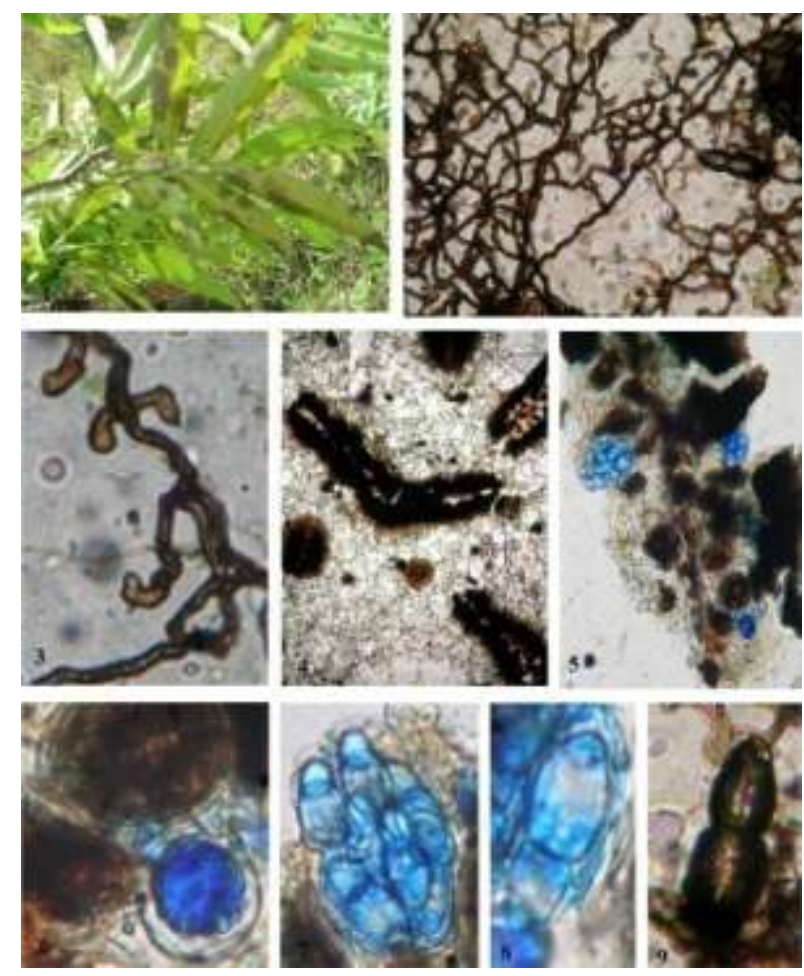

Figure 221 - Lembosia humboldtiae

1. Infected leaves, 2. Mycelium, 3. Mycelium with opposite and many celled appressoria, 4. Thyriothecia, 5. Broken thyriothecium with exposed asci, 6. Ascus, 7. Ascus having ascospores showing unequal cells, 8-9. Ascospores having unequal cells.

\section{Material examined: Kerala,} Thiruvananthapuram, Neyyar Wildlife Sanctuary, Meenmutty, Kombe, on leaves of Humboldtia vahliana Wight (Caesalpiniaceae), 9 March 1996, V.B. Hosagoudar HCIO 42179 (holotype), TBGT 51 (isotype); Thiruvananthapuram, Palode, TBGRI Campus, on leaves of Humboldtia sp., 25 November 2000, T. Sabu HCIO 43829, TBGT 379; 28 December 2006, A. Sabeena \& M. C. Riju HCIO 48289, TBGT 3008; Arboretum, 4 November 2004, T. Sabu HCIO 47318, TBGT 2356; Peppara Wildlife Sanctuary, near Peppara Dam, 18 November 2007, Jacob Thomas and Vimalkumar HCIO 48290, TBGT 3009. Idukki, Kulamavu, 5 September 2001, M. Kamarudeen HCIO 45109, TBGT 1164.

This species is distinct from rest of the two in having unicellular to multi cellular, flexuous to crooked stalk cells of the appressoria and unequal cells in the ascospores (Hosagoudar \& Abraham, 1998).

Lembosia sclerolobii Henn. and L. sophorae (Rehm) Ryan have been reported on the members of the Leguminosae (Stevens \& Ryan, 1939), L. humboldtiae differs from them in having 1-2 septate appressoria. The host is endemic to Southern Western Ghats.

Distribution: Kerala.

Lembosia humboldtiicola Hosag. Jacobe \& Sabeena., Indian J. Sci. echnol. 2 (6):19, 2009; Hosag., Chandraprabha \& Agarwal, Asterinales of Kerala, p. 208, 2011. (Figs 286-287)

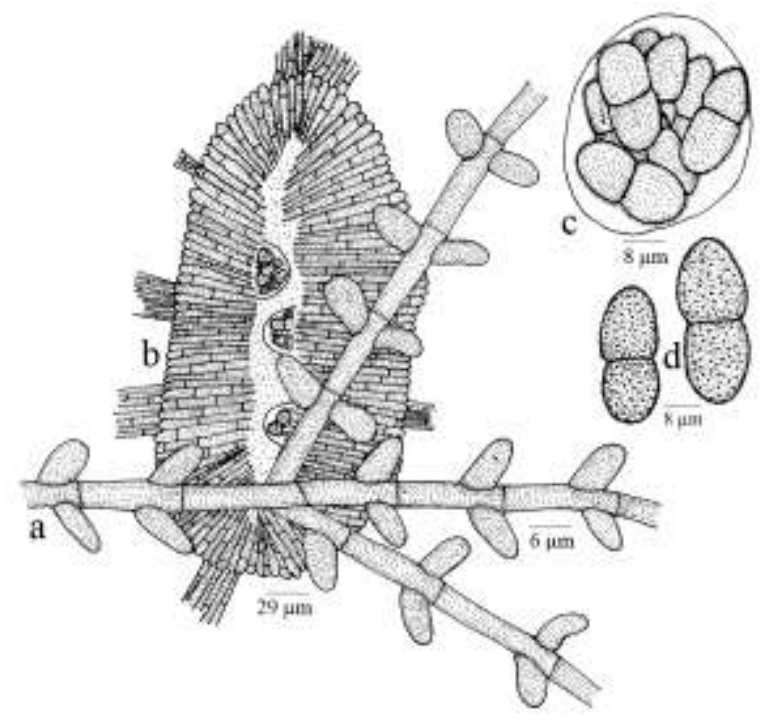

Figure 286 - Lembosia humboldtiicola

Colonies hypophyllous, thin, crustose, up to $2 \mathrm{~mm}$ in diameter, confluent. Hyphae straight to substraight, branching opposite to irregular at acute to wide angles, loosely reticulate, cells 9-14 × 2-5 $\mu \mathrm{m}$. Appressoria mostly opposite, sub opposite, few unilateral, unicellular, ovate, conoid to cylindrical, broad based, sessile, entire, 5-7 × 4-10 $\mu \mathrm{m}$. Thyriothecia scattered, initially rounded, elongated at maturity, longitudinally dehisced at the centre, $250-400 \times 150-200 \mu \mathrm{m}$, margin crenate to fimbriate, fringed hyphae substraight to flexuous, appressoriate; asci octosporous, globose, up to $48 \mu \mathrm{m}$ diameter; ascospores initially hyaline, deep brown at maturity, 
uniseptate, conglobate, constricted at the septum, $31-36 \times 11-13 \mu \mathrm{m}$ long, wall echinulate.
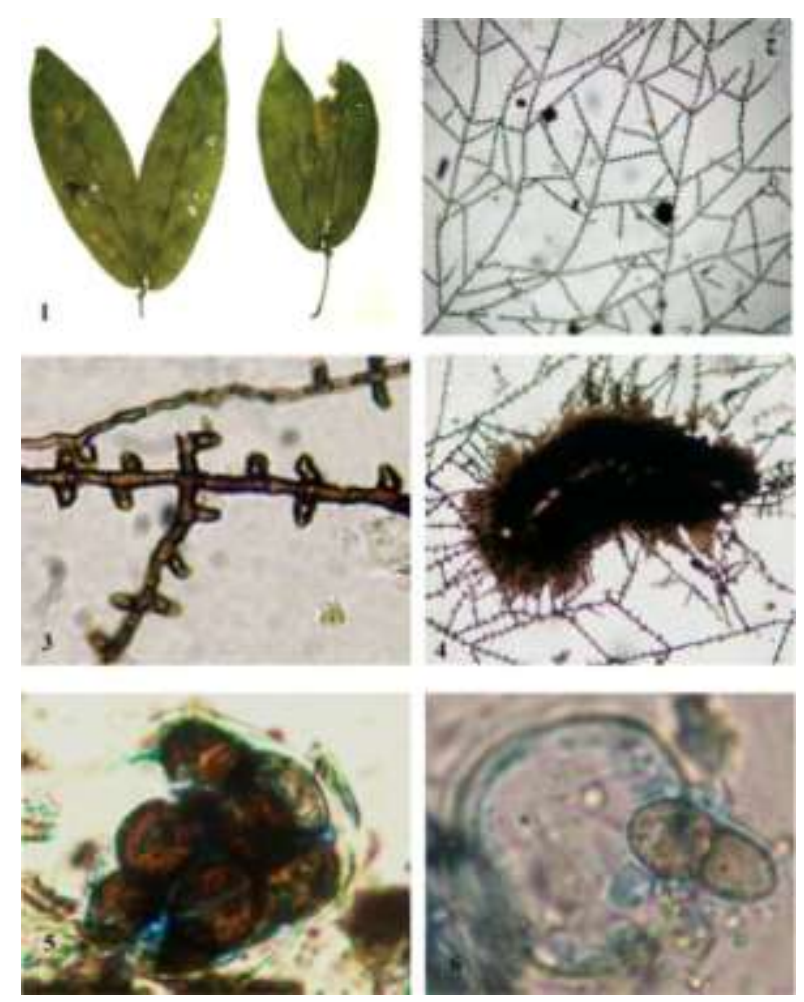

Figure 287 - Lembosia humboldtiicola

1. Infected leaves, 2. Mycelium, 3. Appressoriate mycelium, 4. Thyriothecium, 5. Broken ascus, 6. Ascospore emerging from the ascus.

\section{Material examined: Kerala, Thiruvananthapuram, Athirumala, Peppara Wildlife Sanctuary, on leaves of Humboldtia unijuga Bedd. (Caesalpiniaceae), 28 February 2008, Jacob Thomas \& al HCIO (holotype), TBGT 3010 (isotype).}

Opposite to sub opposite, ovate to conoid appressoria distinguishes this species from the other two species.

\section{Distribution: Kerala.}

Lembosia humboldtiigena Hosag. Jacob \& Sabeena., Indian J. Sci. Technol. 2(6):19, 2009; Hosag., Chandraprabha \& Agarwal, Asterinales of Kerala, p. 210, 2011. (Figs 288-289)

Colonies amphigenous, dense, up to 5 $\mathrm{mm}$ diameter, confluent. Hyphae substraight to flexuous, branching alternate to irregular at acute to wide angles, loosely to closely reticulate, cells $44-55 \times 3-5 \mu \mathrm{m}$. Appressoria scattered, alternate to unilateral, unicellular, broad based, ovate, globose, entire to

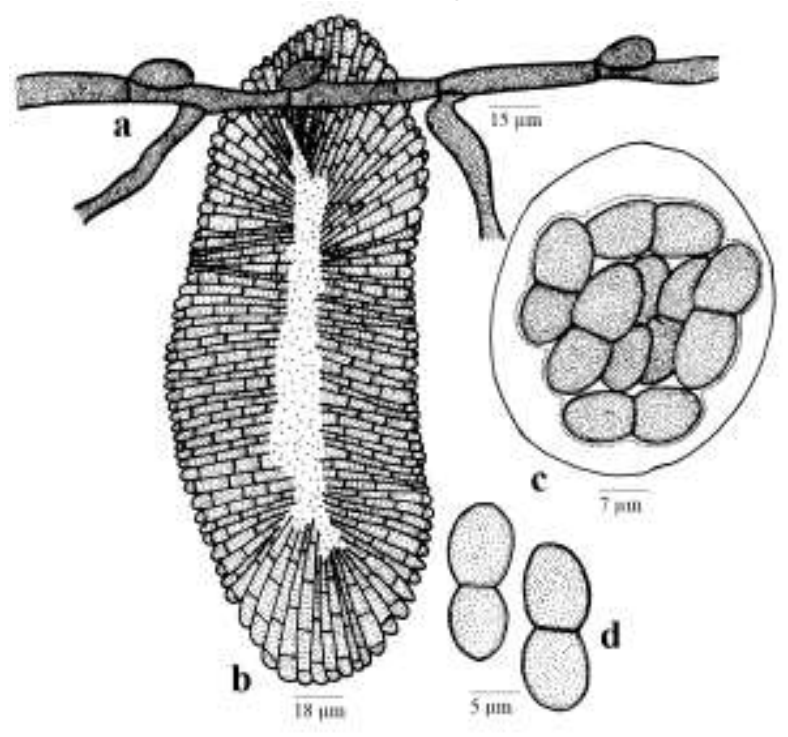

Figure 288 - Lembosia humboldtiigena
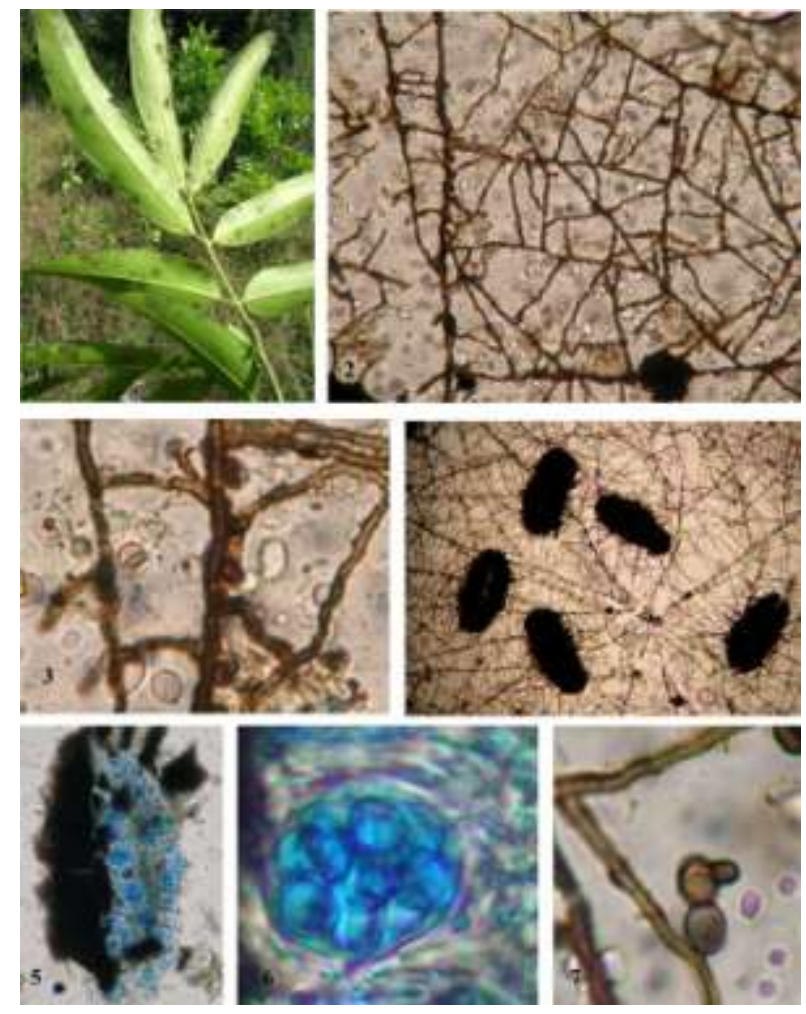

Figure 289 - Lembosia humboldtiigena

1. Infected leaves, 2. Branched mycelium, 3. Appressoriate mycelium, 4. Elongated thyriothecia in the colony, 5. Dehisced thyriothecium with exposed asci, 6. Ascus, 7. Ascospore. 
sublobate, $2-9 \times 2-6 \mu \mathrm{m}$. Thyriothecia scattered, rounded to elongated, longitudinally fissured at the centre, $150-480 \times 100-330 \mu \mathrm{m}$, margin crenate to fimbriate, fringed hyphae flexuous, exappressoriate; asci sub globose, octosporous, 31-44 $\mu \mathrm{m}$ in diameter; ascospores initially hyaline, brown at maturity, 1-septate, conglobate, constricted at the septum, 13-17 $\times$ 4-9 $\mu \mathrm{m}$, wall smooth.

Material examined: Kerala, Thiruvananthapuram, Palode, TBGRI Campus, on leaves of Humboldtia vahliana Wight (Caesalpiniaceae), 28 December 2006, A. Sabeena and M. C. Riju HCIO (holotype), TBGT 3008 (isotype).

Unicellular, scattered, ovate, globose, entire to sublobate appressoria distinguishes this from the other two species.

Distribution: Kerala.

Lembosia incisa (Sydow) Theiss. Ann. Mycol. 11: 443, 1913; Stev. \& Ryan, The Microthyriaceae, p. 89, 1939.

Asterina incise Sydow, Ann. Mycol. 9:390, 1911.

Colonies epiphyllous. Appressoria few. Thyriothecia scattered to aggregated, oblong to elongated, $300-500 \times 100-175 \mu \mathrm{m}$; asci ovate to clavate, $38-54 \times 26-30 \mu \mathrm{m}$; ascospores conglobate, ellipsoidal to oblong-ellipsoidal, 1septate, constricted at the septum, rounded at both ends, 17-20 $\times 8-9 \mu \mathrm{m}$, wall smooth.

Karnataka, Balehonnur, on leaves of Webera corymbosa Willd. (Rubiaceae), 19 September 1919, E.J. Butler HCIO 1184.

Lembosia lagerstroemiae Hosag. \& Abraham, New Botanist 24: 115, 1997; Hosag., C.K. Biju \& Abraham, J. Econ. Taxon. Bot. 25: 306, 2001; Hosag., Chandraprabha \& Agarwal, Asterinales of Kerala, p. 211, 2011.

(Fig. 290)

Colonies epiphyllous, crustose, dense, up to $2 \mathrm{~mm}$ in diameter, confluent. Hyphae substraight to flexuous, branching irregular at acute to wide angles, loosely reticulate, cells 16-24 $\times$ 3-4 $\mu \mathrm{m}$. Appressoria scattered, alternate, unicellular, globose, broad based, mostly entire, rarely slightly angular, 6-7 $\mu \mathrm{m}$ in high, 4-8 $\mu \mathrm{m}$ broad. Thyriothecia initially rounded, oval, ellipsoidal to elongated at maturity, $350-450 \times 160-200 \mu \mathrm{m}$, longitudinally dehisced at the center, contents reddish brown, margin fimbriate, fringed hyphae flexuous; asci, many, initially rounded, ovate at maturity, octosporous, paraphysate, 30$42 \times 19-24 \mu \mathrm{m}$, paraphyses hyaline, filiform, septate; ascospores conglobate, brown, 1septate, slightly constricted at the septum, 22$24 \times 9-11 \mu \mathrm{m}$, one cell slightly larger, wall smooth but echinulate in matured spores.

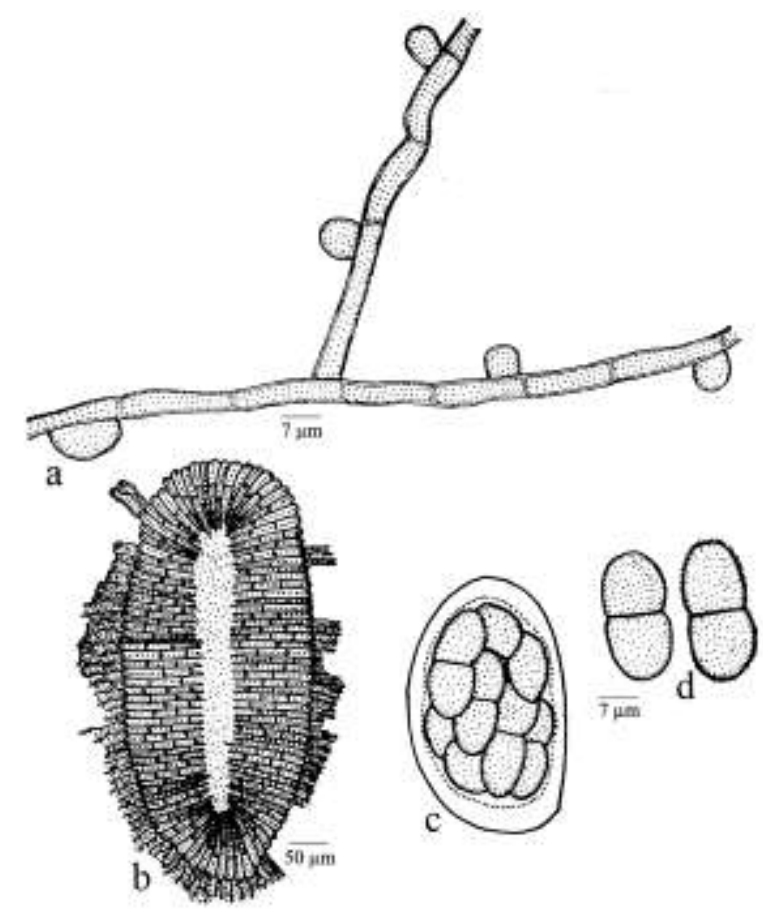

Figure 290 - Lembosia lagerstroemiae

Material examined: Kerala, Thiruvananthapuram, Peppara Wildlife Sanctuary, on leaves of Lagerstroemia microcarpa Wight (Lytheracae), 5 February 1997, V.B. Hosagoudar HCIO 42504 (holoype), TBGT 134 (isotype).

This forms the first species of the genus Lembosia on the members of the family Lythraceae and hence, it is proposed here as a new species.

Distribution: Kerala. 
Lembosia linocierae Hosag., Zoos' Print J. 17: 947, 2002; Hosag., Chandraprabha \& Agarwal, Asterinales of Kerala, p. 213, 2011. (Fig. 291)

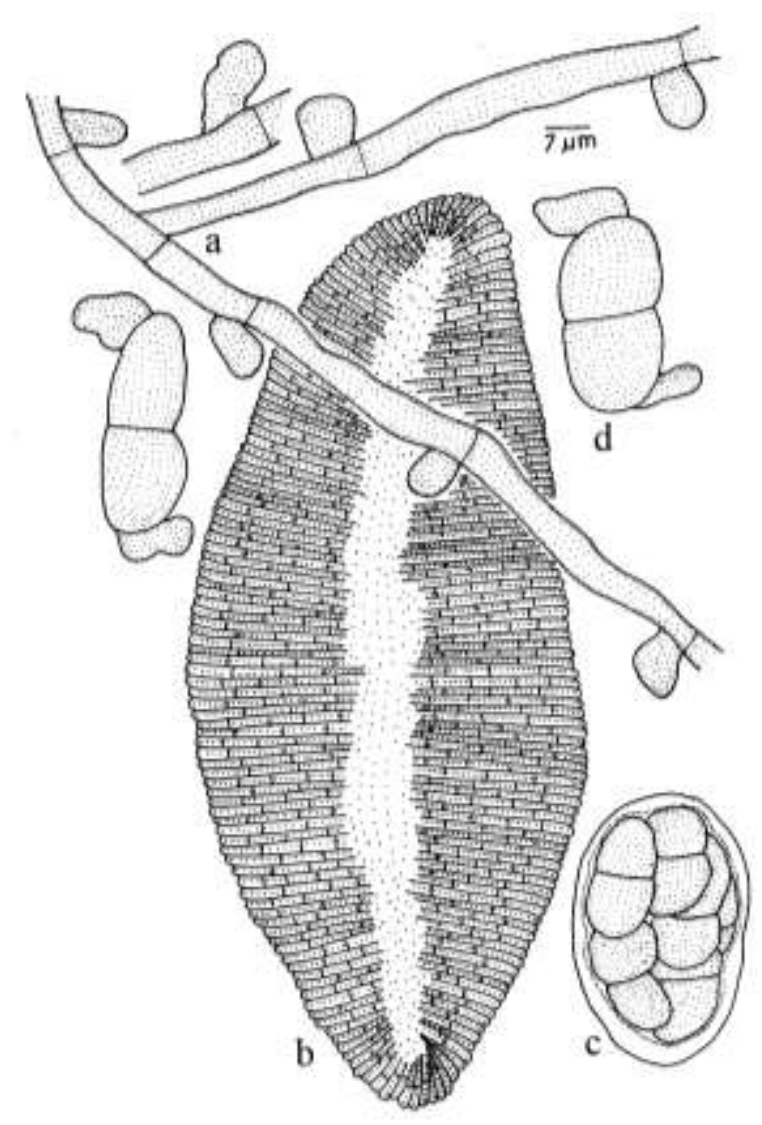

Figure 291 - Lembosia linocierae

Colonies epiphyllous, dense, crustose, granulose, up to $2 \mathrm{~mm}$ in diameter, confluent. Hyphae straight to substraight, branching irregular at acute angles, loosely reticulate, cells 24-32 × 5-7 $\mu \mathrm{m}$. Appressoria unicellular, more scattered, alternate to unilateral, ovate, conoid, globose, attenuated and broadly rounded to truncate at the apex, entire to rarely slightly lobate at sides or at the apex, straight to curved, antrorse to retrorse, $9-16 \times 4-11 \mu \mathrm{m}$. Thyriothecia scattered to aggregated, oblong to elongated, $\times$ or $Y$ or star shaped, 350-1400 $\mu \mathrm{m}$ long, 200-300 $\mu \mathrm{m}$ broad, dehisce vertically at the centre, margin fimbriate, fringed hyphae mostly run parallel and devoid of appressoria; asci ovate to globose, octosporous, bitunicate, 40-60 $\mu \mathrm{m}$ in diameter; ascospores brown, conglobate, oblong, uniseptate, constricted at the septum, $27-30 \times 12-15 \mu \mathrm{m}$, wall slightly verrucose.
Material examined Kerala, Kollam, Shendurney Wildlife Santuary, Sankili forest, on leaves of Linociera sp. (Oleaceae), 27 December 2001, V.B. Hosagoudar HCIO 44325 (holotype), TBGT 688 (isotype).

Lembosia rizalensis Petrak is known on Linociera ramiflora from Philippines (Petrak, 1955). However, L. linocierae differs from it in having larger thyriothecia and ascospores.

Distribution: Kerala.

Lembosia malabarensis (Sydow \& Sydow) Hosag. \& Goos, Mycotaxon 52: 472, 1994; Song Bin \& Hosag., Guizhou Science 21: 94, 2003; Hosag., Chandraprabha \& Agarwal, Asterinales of Kerala, p. 213, 2011.

Asterina malabreansis Sydow \& Sydow in Sydow, Sydow \& Butler, Ann. Mycol. 9: 391, 1911.

Asterinla malabarensis (Sydow \& Sydow) Theiss., Broteria 10: 106, 1912. Prilliexina malabarensis (Sydow \& Sydow) Ryan in Stevens \& Ryan, Illinois Biol. Monographs 17: 80, 1939.

(Figs 292-294)

Colonies hypophyllous, on sheaths, up to $3 \mathrm{~mm}$ in diameter, confluent. Hyphae flexuous to crooked, branching irregular at acute to wide angles, loosely to closely reticulate, cells 15-23 × 3-4 $\mu \mathrm{m}$. Appressoria alternate, unilateral, opposite, unicellular, cylindrical, straight, curved, flexuous, zig-zag to uncinate, broadly rounded at the apex, 19-24 $\times 4-5 \mu \mathrm{m}$. Thyriothecia initially orbicular, later elongated, straight to curved, 500-1000 $\times 290$ $400 \mu \mathrm{m}$, dehisce longitudinally at the centre or sub centre, margin crenate to fimbriate, fringed hyphae compact, flexuous; asci few, globose to ovate, octosporous, $40-60 \times 25-40 \mu \mathrm{m}$; ascospores oblong, conglobate, brown, uniseptate, constricted at the septum, 26-30 $\times$ 16-18 $\mu \mathrm{m}$, wall smooth. Pycnothyria mixed with thyriothecia, similar to thyriothecia, smaller; pycnothyriospores oval, oblong, brown, $28-30 \times 16-18 \mu \mathrm{m}$.

Material examined: Kerala, Thiruvananthapuram, Palode, TBGRI Campus, 


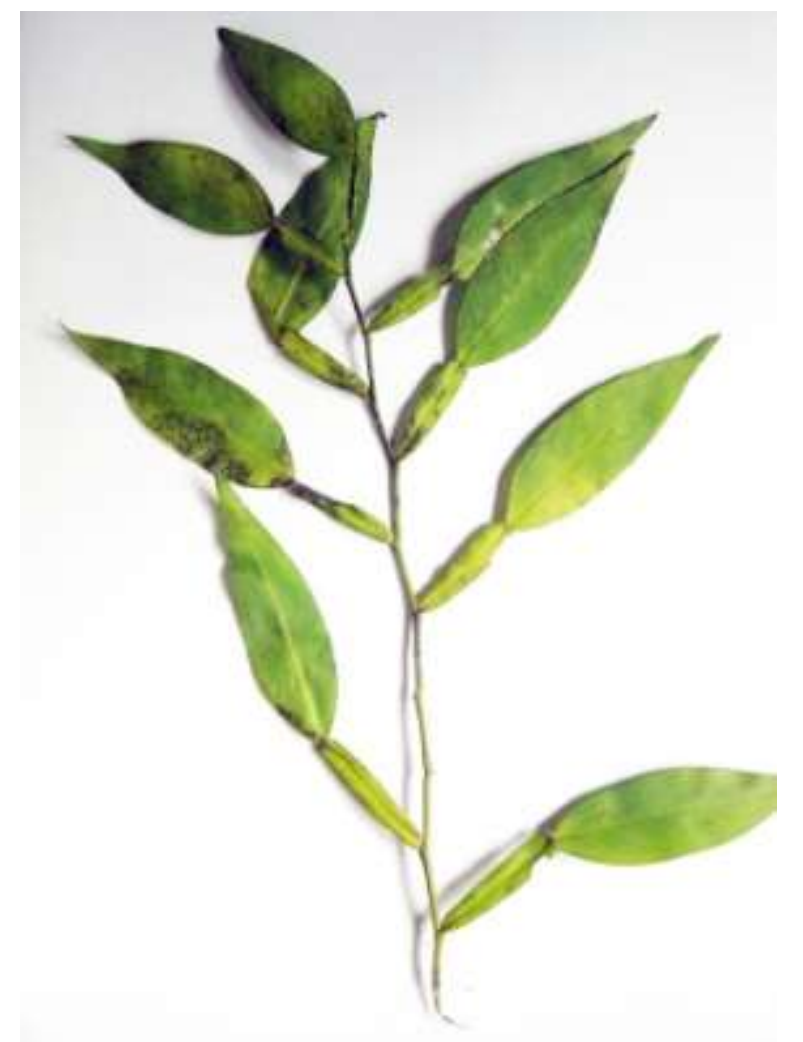

Figure 298 - Lembosia malabarensis - Infected leaves

on leaves of Pothosscandens L. (Araceae), 4 December 1996, V.B. Hosagoudar HCIO 42418, TBGT 82; Thiruvananthapuram, Attayar, 19 March 1997, V.B. Hosagoudar HCIO 43992, TBGT 426; Kollam, Shankily forest, Sasthanada tribal colony, 23 November 2004, V.B. Hosagoudar HCIO 46163, TBGT 1575; HCIO 46168, TBGT 1580; Kottayam, Ponthanpuzha, 14 February 2007, P.J. Robin HCIO 48293, TBGT 3012; Kannur, Talassery, Aralam Wildlife Sanctuary, Valavanchal, 28 February 2007, P.J. Robin \& al. HCIO 48214, TBGT 2950.

This is the only species of the genus known on the members of the family Araceae. Malabar is the type locality of this fungus. Pycnothyriospores are reported for the first time for this species.

This species was collected by E. J. Butler from Kanouth of Malabar region. Elongated thyriothecia with a central longitudinal slit and the presence of appressoria are characteristic of the genus Lembosia.
Distribution: Kerala.

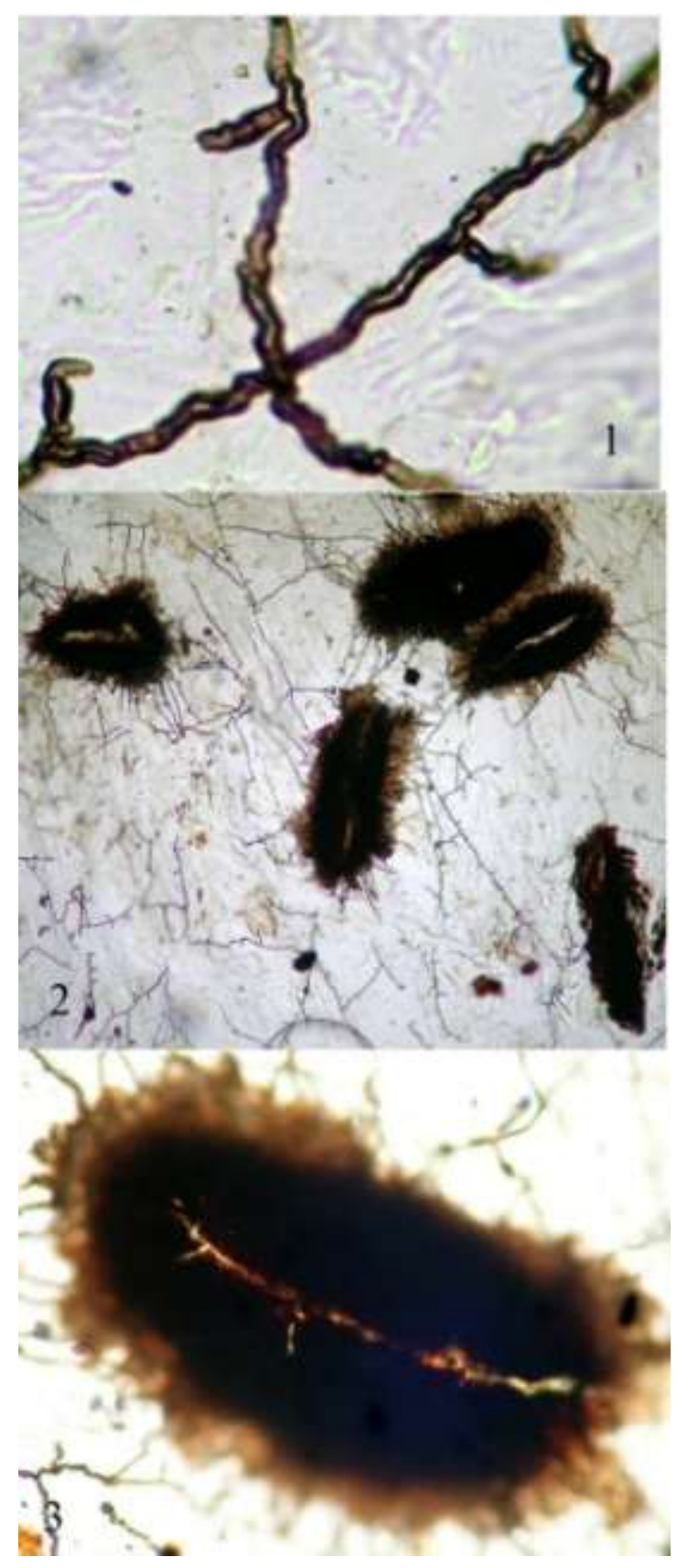

Figure 293 - Lembosia malabarensis

1. Appressoriate mycelium, b. Scattered thyriothecia, 3. Thyriothecium

Lembosia memecylicola Hosag., J. Mycopathol. Res. 43(2): 204, 2005.

(Fig. 295)

Colonies hypophyllous, dense, crustose, up to $5 \mathrm{~mm}$ in diameter, confluent. Hyphae straight to flexuous, branching alternate to irregular at acute to wide angles, loosely to 
closely reticulate, cells $28-35 \times 6-8 \mu \mathrm{m}$. Appressoria scattered, distantly placed, unicellular, cylindrical, ovate, oblong, clavate, antrorse, subantrorse to rarely recurved, entire, attenuated to broadly rounded at the apex, 4$12 \times 6-8 \mu \mathrm{m}$. Thyriothecia scattered, oval, oblong, elongated, elliptical, straight to curved, dehisce vertically along the centre, 294-882 × 176-300 $\mu \mathrm{m}$, margin fimbriate, fringed hyphae long, flexuous and compact; asci globose, octosporous, up to $45 \mu \mathrm{m}$ in diameter; ascospores oblong, conglobate, brown, uniseptate, slightly constricted, 22-26 $\times 11-13 \mu \mathrm{m}$, wall smooth.

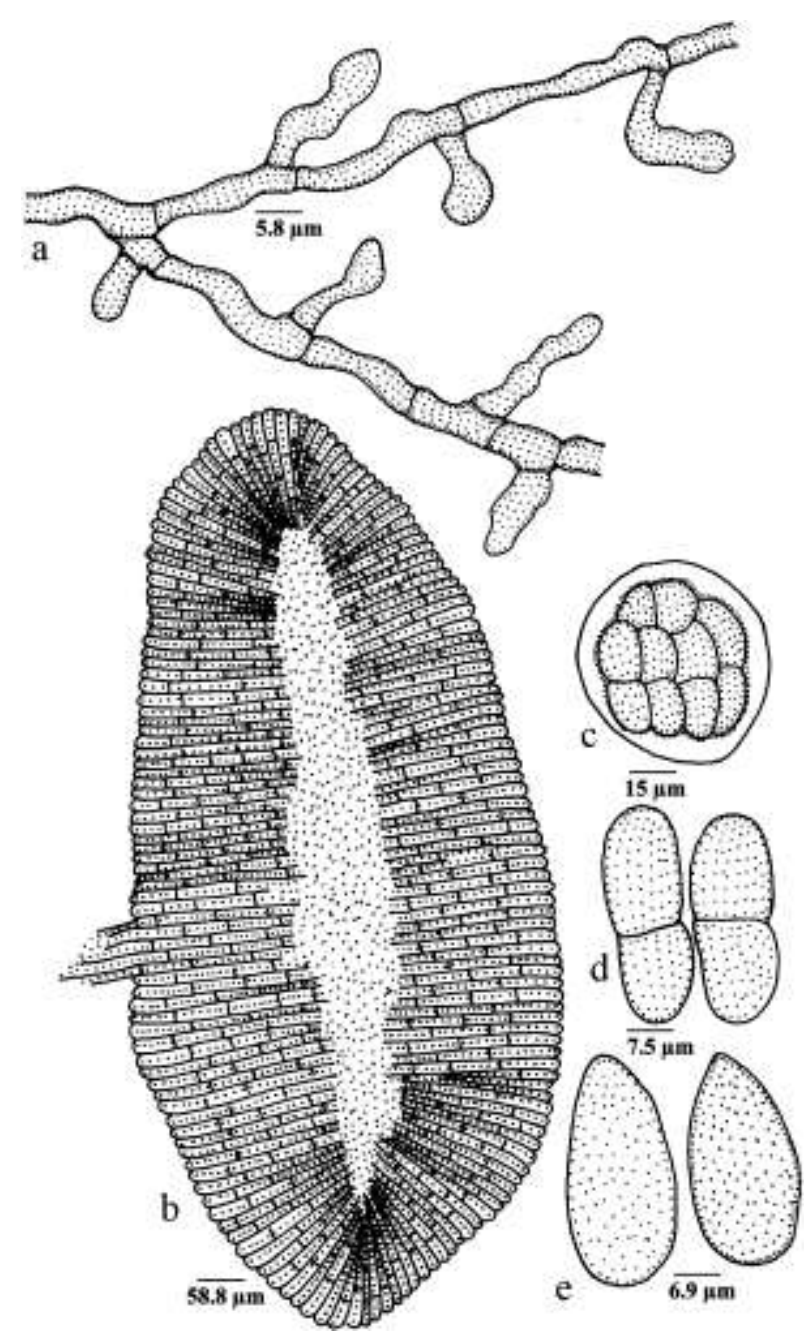

Figure 294 - Lembosia malabarensis

Material examined: Karnataka, South Canara, Kukke Subramanya, on leaves of Memecylon sp. (Melastomataceae), 15 November 2003, V.B. Hosagoudar HCIO 45783 (holotype), TBGT 1532 (isotype).
Morenoella memecyli Sydow and Lembosia memecyli Sydow \& Sydow are known on this host genus (Sydow \& Sydow, 1914; Sydow \& Petrak, 1931). Since the genus Morenoella is synonymous to Lembosia, Stevens \& Ryan (1939) synonymised the latter species with the former. Lembosia memecyli Sydow is the only species known on the genus Memecylon. Lembosia memecyli differs from $L$. memecylicola in having larger thyriothecia and ascospores.

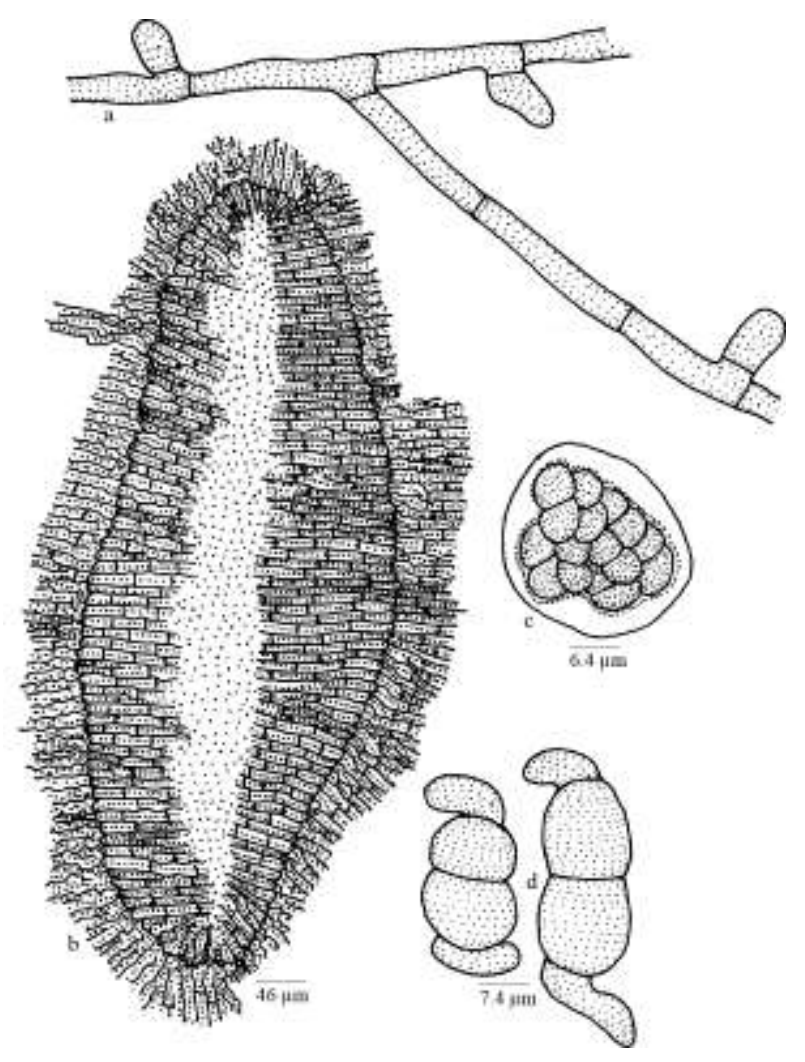

Figure 295 - Lembosia memecylicola

Distribution: India.

Lembosia ormosiae Yamam., Sci. Rep. Hyogo Univ. Agric. Agric. Biol. Ser. II. 3: 28, 1957; Song Bin \& Hosag., Guizhou Science 21: 96, 2003; Hosag., Zoos' Print J. 21: 2414, 2006; Hosag., Chandraprabha \& Agarwal, Asterinales of Kerala, p. 215, 2011.

(Fig. 296)

Colonies epiphyllous, dense, crustose, up to $2 \mathrm{~mm}$ in diameter, confluent. Hyphae straight to substraight, branching opposite to irregular at acute to wide angles, loosely to closely reticulate, cells $19-24 \times 4-6 \mu \mathrm{m}$. 
Appressoria alternate, unilateral, rarely about $1 \%$ opposite, ovate, globose, mammiform, truncate to attenuated at the apex, straight to curved, entire to angularly to rarely slightly sublobate, 6-10 $\times$ 6-8 $\mu \mathrm{m}$. Thyriothecia scattered, loosely grouped to rarely connate, straight to curved, $400-800 \times 70-90 \mu \mathrm{m}$, dehisce vertically at the centre, margin fimbriate to crenate; asci globose, octosporous, 35-40 $\mu \mathrm{m}$ in diameter; ascospores oblong, conglobate, brown, uniseptate, slightly constricted at the septum, 19-21 × 7-9 $\mu \mathrm{m}$, wall smooth.

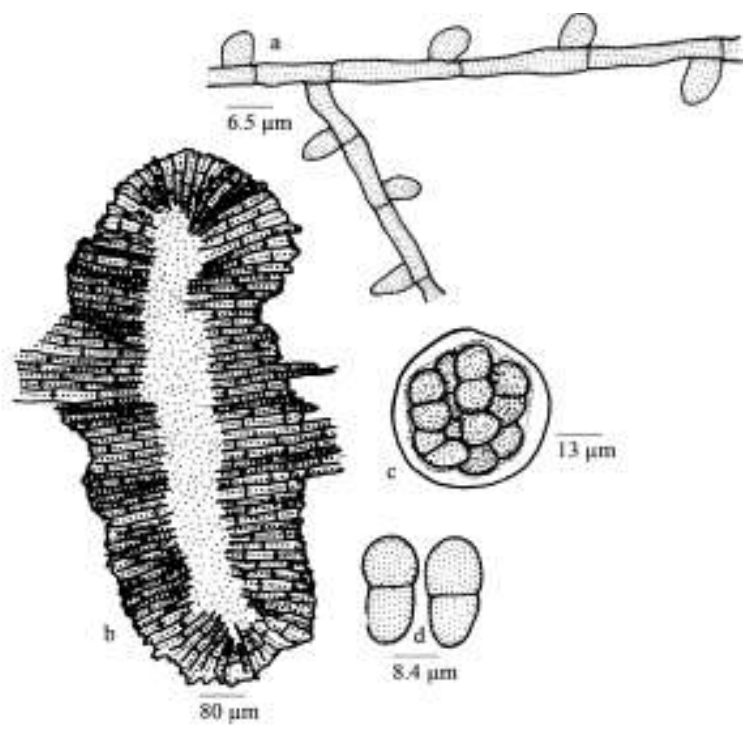

Figure 296 - Lembosia ormosiae

Material examined: Kerala, Thiruvananthapuram, Palode, in the campus of Tropical Botanic Garden and Research Institute, on leaves of Ormosia travancorica Bedd. (Fabaceae), 5 September 2002, A. Manoj Kumar \& H. Biju HCIO 44597, TBGT 884; 24 April 2002, T. Sabu HCIO 44643, TBGT 925.

This species was known on Ormosia formosana from Taiwan (Yamamoto, 1957) and is reported here for the first time from India on hitherto unrecorded host.

Distribution: Kerala.

\section{Lembosia pandanacearum V.B.}

Hosagoudar \& C. Jagath

Thimmaiah, sp. nov. (Fig. 297)

MycoBank 101016

Etymology: Named after the host genus
Colonies amphigenous, mostly epiphyllous, subdense to dense, scattered, up to $4 \mathrm{~mm}$ in diameter. Hyphae straight, branching irregular at wide angles, loosely reticulate, cells 17-35 $\times$ 4-8 $\mu \mathrm{m}$. Appressoria scattered, distantly placed, unicellular, unilateral to alternate, globose, broad based, entire, often covered with a membrane continuous with hyphal cell wall, $8-13 \times 9-12 \mu \mathrm{m}$. Thyriothecia scattered, oval, elongated, $350-550 \times 200-300$ $\mu \mathrm{m}$, margin crenate to fimbriate, fringed hyphae small, flexuous; asci many, globose, 28$35 \mu \mathrm{m}$; ascospores conglobate, uniseptate, constricted at the septum, $23-27 \times 11-14 \mu \mathrm{m}$, wall smooth.

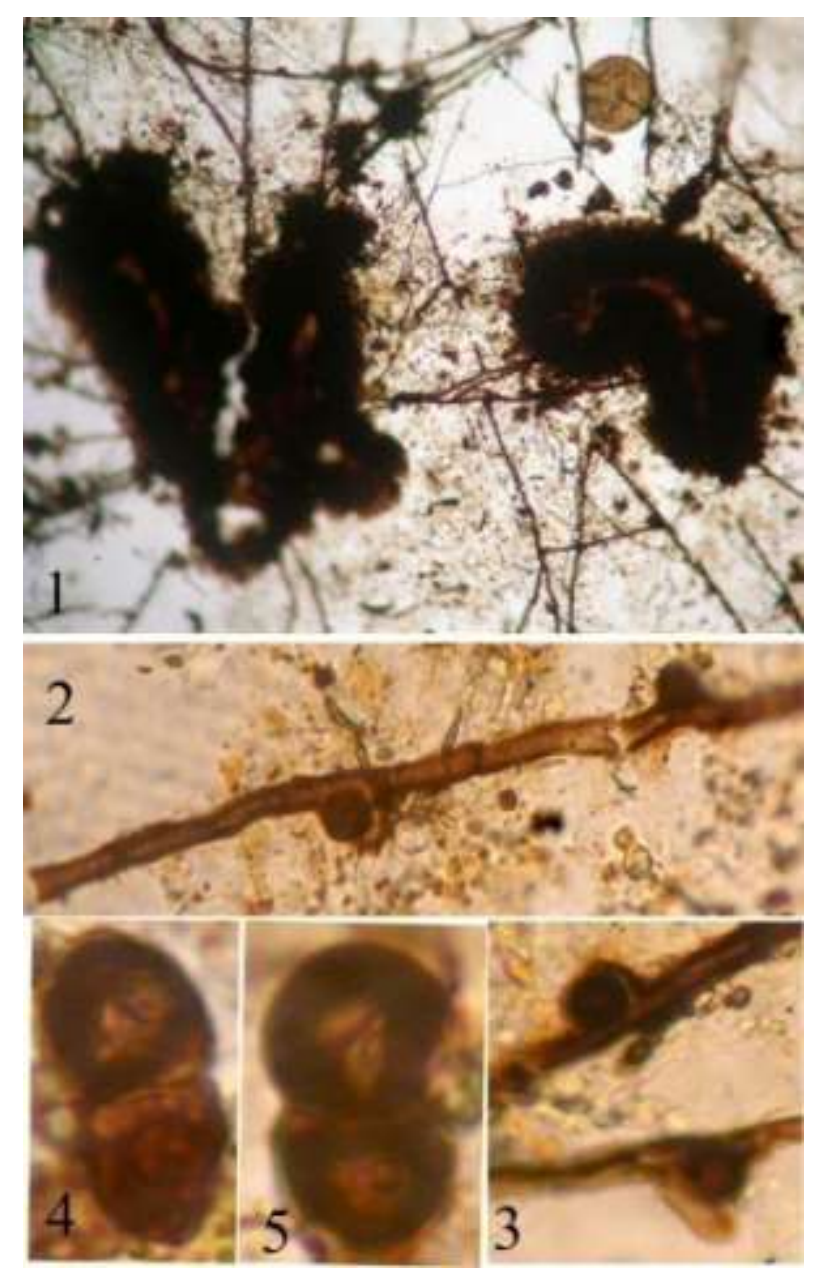

Figure 297 - Lembosia pandanacearum

1. Mycelium with thyriothecia, 2.Appressoriate mycelium, 3. Appressoria partly covered with mycelial cap, 4-5. Ascospores

Material examined: On infected leaves of Pandanus sp. (Pandanaceae), Hoddur, 
Kodagu, Karnataka, 23 August 2009, C. Jagath Thimmaiah TBGT 5729 (holotype).

This is the only species known on this host genus.

Lembosia perseae Orejuela, Mycologia 36: 449, 1944; Hosag., Indian Phytopath. 58: 202, 2005; Song Bin \& Hosag., Guizhou Science 21: 97, 2003; Hosag., Chandraprabha \& Agarwal, Asterinales of Kerala, p. 216, 2011.

(Fig. 298)

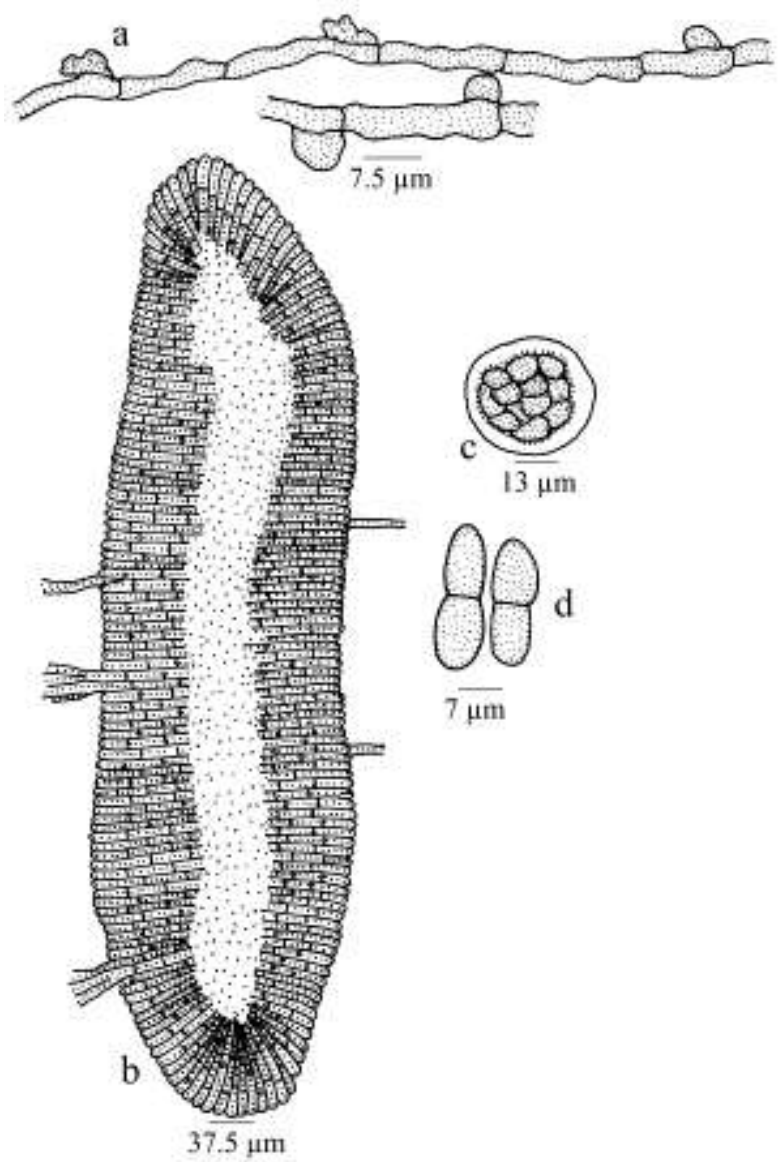

Figure 298 - Lembosia perseae

Colonies epiphyllous, dense, crustose, up to $2 \mathrm{~mm}$ in diameter, rarely confluent. Hyphae substraight to slightly crooked, branching irregular at acute angles, loosely reticulate, mostly non parallel, cells 16-24 × 3$5 \mu \mathrm{m}$. Appressoria few, scattered, alternate to unilateral, oval, globose, broad based, 3-8 $\times 4$ $8 \mu \mathrm{m}$. Thyriothecia grouped, oval, elongate, of different shapes like X, Y, 450-550 × 140-200 $\mu \mathrm{m}$; asci few, globose to ovate, up to $35 \mu \mathrm{m}$ in diam.; ascospores oblong, conglobate, brown, uniseptate, constricted, $10-21 \times 7-9 \mu \mathrm{m}$, wall smooth.

Material examined: Kerala, Idukki, Munnar, Rajamala, on leaves of Persea macrantha (Nees) Kosterm. (Lauraceae) 20 January 2003, A. Manoj Kumar \& H. Biju HCIO 45215 (holotype), TBGT 1252 (isotype).

This species was known from Columbia on Persea sp. (Orejula, 1944).

\section{Distribution: Kerala}

Lembosia salaciae Hosag. \& Archana., Indian J. Sci. Technol. 2 (6):1, 2009' Hosag., Chandraprabha \& Agarwal, Asterinales of Kerala, p. 216, 2011.

(Fig. 299)

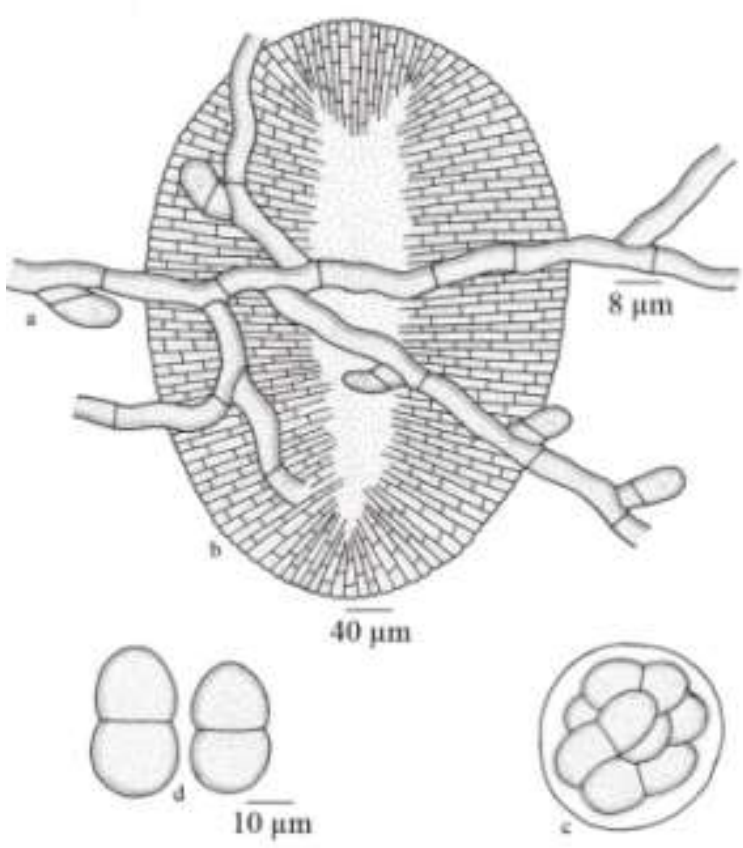

Figure 299 - Lembosia salaciae

Colonies amphigenous, subdense, up to $2 \mathrm{~mm}$ in diameter, confluent. Hyphae profusely branched, flexuous to crooked, branching opposite, alternate to irregular at acute to wide angles, loosely to closely reticulate, cells 16-32 $\times 4-8 \mu \mathrm{m}$. Appressoria few, 2-celled, unilateral to alternate, straight, curved, 9-18 $\mu \mathrm{m}$ long; stalk cells cylindrical to cuneate, 3-6 $\mu \mathrm{m}$ long; head cells ovate, oblong, straight, curved, entire 
to slightly angular, $4-11 \times 4.8-10 \mu \mathrm{m}$. Thyriothecia scattered to grouped, initially rounded, elongated at maturity, 500-640 × 250$300 \mu \mathrm{m}$, longitudinally fissured at the centre, margin crenate to fimbriate, fringed hyphae flexuous to crooked; asci globose to ovate, octosporous, $32-42 \times 26-35 \mu \mathrm{m}$; ascospores ovate, oblong, conglobate, brown, uniseptate, constricted at the septum, $28-35 \times 12-18 \mu \mathrm{m}$, smooth walled.

Material examined: Kerala, Palakkad, Silent Valley National Park, Sairandhiri, on leaves of Salacia sp. (Hippocrataceae), 13 December 2003, V.B. Hosagoudar \& al. HCIO 46307 (holotype), TBGT 1953 (isotype).

This is the only report of the genus Lembosia on the members of the family Hippocrataceae (Song \& Hosagoudar, 2003).

Distribution: Kerala.

Lembosia shoreae (Ryan) Hosag. \& B. Song, Guizhou Science 21: 96, 2003.

Morenolella shoreae Ryan, Mem. Dept. Agric. India 15: 104.1928.

Colonies epiphyllous, form small irregular spots. Hyphae brown, nodulose, cells up to $6 \mu \mathrm{m}$ broad. Thyriothecia linear, carbonaceous, with a longitudinal fissure, 330$346 \times 347-544 \mu \mathrm{m}$, margin irregular, fimbriate and distinctly radiate; asci quadrisporous, oval to globose, aparaphysate, $40 \mathrm{x}$ 48-52 $\mu \mathrm{m}$; ascospores brown, 2-celled, 20x 36-40 $\mu \mathrm{m}$.

Material examined: Karnataka, North Canara, on leaves of Shorea talura Roxb. (Dipterocarpaceae), October 1919, L.J. Sedwick HCIO 1975b (holotype).

\section{Distribution: Karnataka}

\section{Lembosia terminaliae-chebulae Hosag.,} Abraham \& Crane, Mycotaxon 68: 20, 1998; Song Bin \& Hosag., Guizhou Science 21: 96, 2003; Hosag., Chandraprabha \& Agarwal, Asterinales of Kerala, p. 218, 2011.

(Figs 300-301)
Colonies epiphyllous, dense, up to 2 $\mathrm{mm}$ in diameter, confluent forming large patches. Hyphae substraight, branching irregularly, loosely reticulate; cells 14-18 × 3-4 $\mu \mathrm{m}$. Appressoria scattered, unicellular, mammiform, entire, broad based, 7-9 $\mu \mathrm{m}$ broad and 6-7 $\mu \mathrm{m}$ high. Thyriothecia oval, ellipsoidal, elongated, of various shapes like $\times$ and $Y$, closely confluent, split longitudinally and also along arms, 390-540 × 190-250 $\mu \mathrm{m}$, margin crenate to fimbriate; asci globose to ovate, octosporous, $48-65 \times 19-24 \mu \mathrm{m}$; ascospores oblong, conglobate, 1-septate, slightly constricted at the septum, initially hyaline, later becoming brown, 17-22.5 $\times 9-11 \mu \mathrm{m}$, wall verrucose to tuberculate.

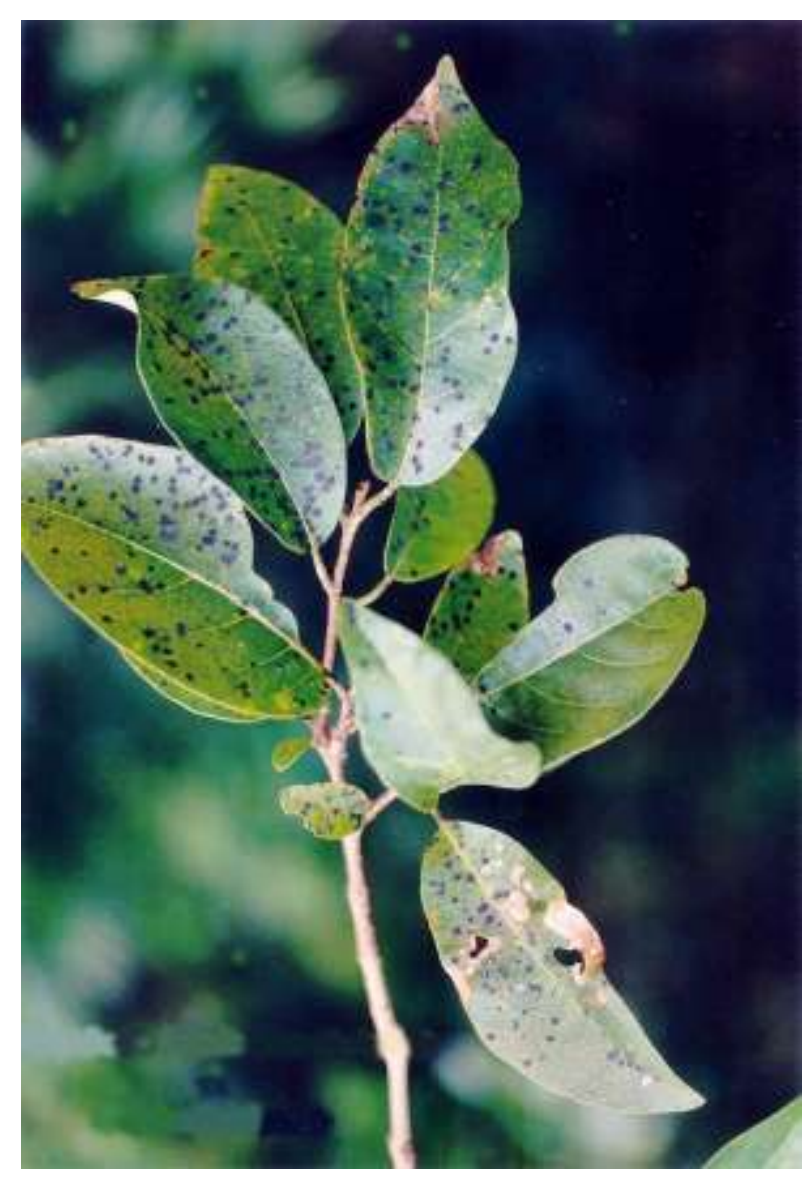

Figure 300. Lembosia terminaliae-chebulae Infected leaves

Material examined: Kerala, Thiruvananthapuram, Peppara Wild life Sanctuary, on leaves of Terminalia chebula Retz. (Combretaceae), 4 February 1997, V.B. Hosagoudar HCIO 42503 (holoype), TBGT 135 (isotype); Thiruvananthapuram, Near 
Karamanayar, 4 December 2001, G. Rajkumar TBGT 1148, HCIO 45093; Thiruvananthapuram, Palode, TBGRI Campus, 22 November 2000, C.K. Biju HCIO 44062, TBGT 496; 17 January 2001, H. Biju HCIO 46104, TBGT 1867
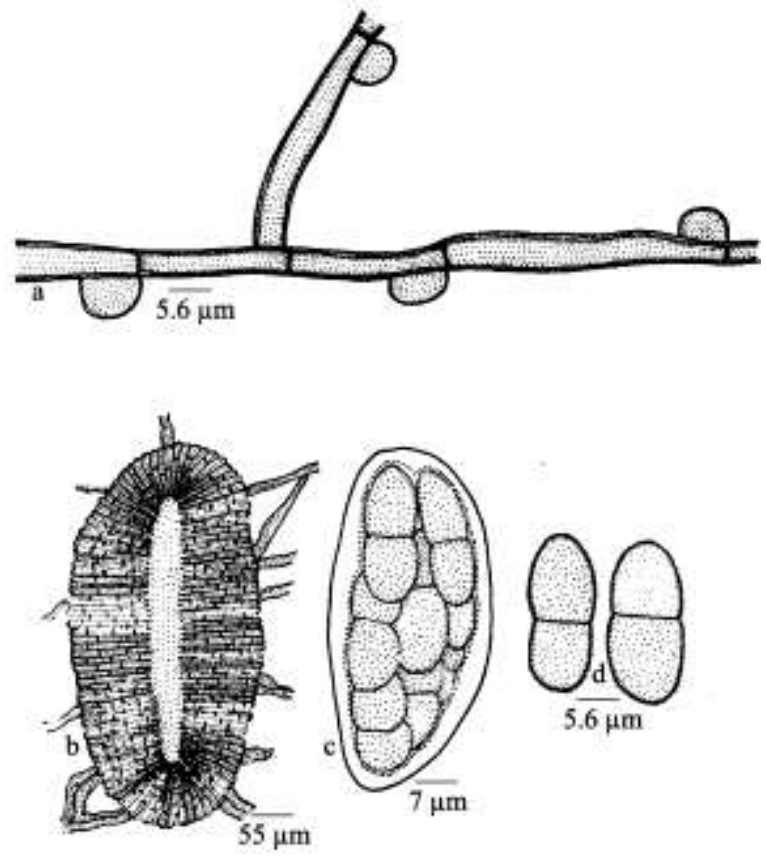

Figure 301 - Lembosia terminaliae-chebulae

Epiphyllous colonies, larger thyriothecia and smaller ascospores distinguish the present new species from Lembosia terminaliae Hansf., reported on Terminalia sp. from New Guinea (Hansford, 1957).

Distribution: Kerala.

\section{THE GENUS Maheshwaramyces}

Maheshwaramyces Hosag., Indian J. Sci. \& Techn. 2(6), 12, 2009; Hosag., Chandraprabha \& Agarwal, Asterinales of Kerala, p. 220, 2011.

Leaf parasites. Hyphae partly superficial and partly immersed in the epidermis. Superficial hyphae brown, branched. Conidiogenous cells borne directly on the mycelial cells, globose. Conidia phragmosporous, one to many septate, brown, constricted at the septa. Thyriothecia mostly connate, elongated, dehisce longitudinally; asci ovate, clavate, globose, bitunicate, octosporous; ascospores brown, two celled, constricted at the septum.
Type sp. : $M$. pachygones Hosag. et al.

The characters of the fungus is similar to that of the genus Lembosia, a type genus of the family Lembosiaceae (Hosagoudar et al., 2001a) but differs from it in having the conidiogenous cells borne directly on the mycelial cells.

\section{DESCRIPTIONS OFTHE SPECIES}

Maheshwaramyces coculi V.B. Hosagoudar \& G.R. Archana, sp.nov. (Fig. 302)

MycoBank 801017

Etymology: Named after the host genus

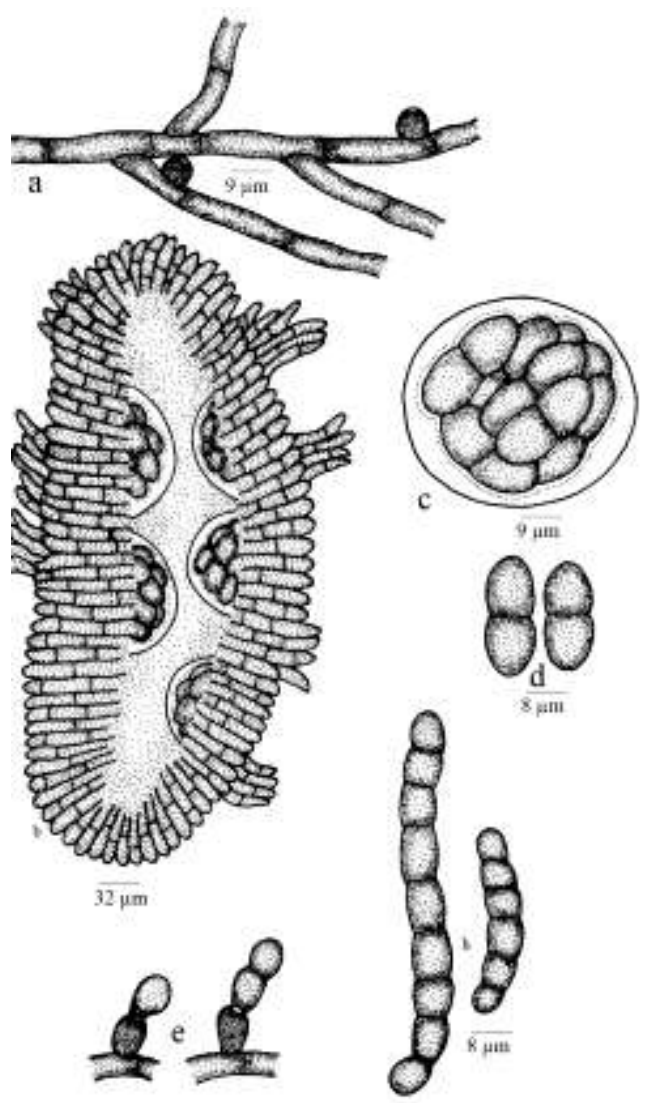

Figure 302 - Maheshwaramyces coculi

a. Appressoriate mycelium, b. Thyriothecium, c. Ascus, d. Ascospores, e. Conidiophore, f. Conidia

Colonies hypophyllous, subdense, crustose, up to $2 \mathrm{~mm}$ in diameter, confluent. Hyphae partly superficial and partly immersed in the epidermis. Superficial hyphae straight, substraight, flexuous, irregularly branched, bearing conidiogenous cells, cells $12-37 \times 2-5$ $\mu \mathrm{m}$. Conidiogenous cells globose to cylindrical, monotretic, $8-19 \times 4-7 \mu \mathrm{m}$. 
Conidia phragmosporous, brown, one to many septate, constricted at the septa, straight to variously curved, rounded at both the ends, wall smooth, 36-400 $\times 8-10 \mu \mathrm{m}$. Thyriothecia mostly grouped, oval, elongate, $\times$ or $Y$-shaped, straight to curved, vertically dehisced at the center, margin fimbriate, fringed hyphae small, compact, $490-735 \times 176-490 \mu \mathrm{m}$; asci many, initially globose, subglobose, clavate, octosporous, paraphysate, bitunicate, 19-43 $\times$ 16-26 $\mu \mathrm{m}$, paraphyses hyaline, filiform; ascospores initially hyaline, brown at maturity, conglobate, uniseptate, constricted at the septum, 17-23 × 9-12 $\mu \mathrm{m}$, wall smooth.

Material examined: Kerala, Idukki, on leaves of Coculus laurifolius (Menispermaceae), 11 May 1999, C.K. Biju TBGT 4816 (holotype).

Conidia longer than that of Maheshwaramyces pachygones Hosag. et. al.

Distribution: Kerala

Maheshwaramyces pachygones Hosag. Archana \& Dan., Indian J. Sci. Technol. 2 (6):12, 2009; Hosag., Chandraprabha \& Agarwal, Asterinales of Kerala, p. 220, 2011. (Figs 303-305)

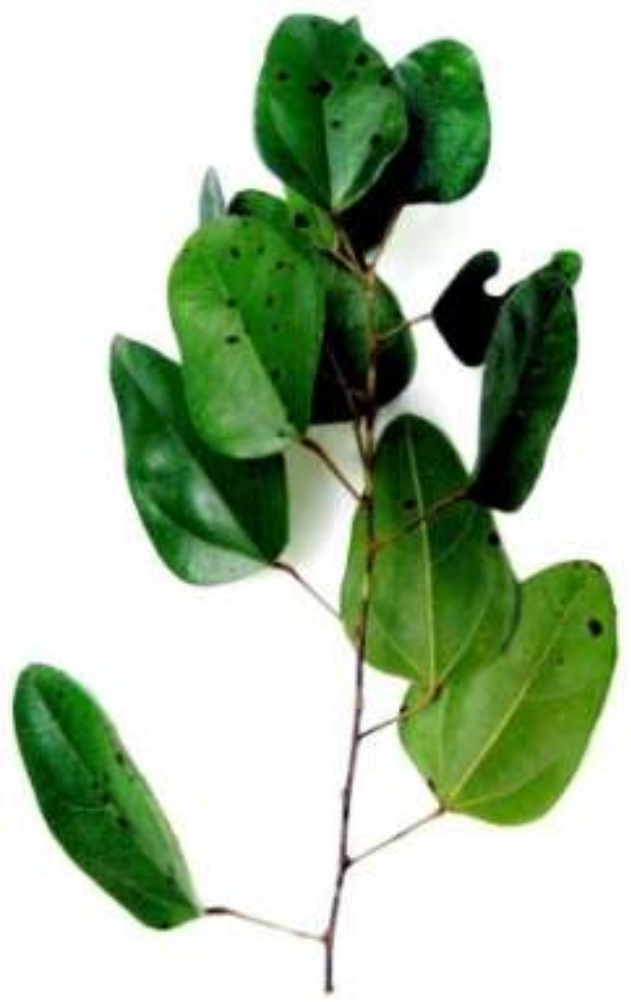

Figure 303 - Maheshwaramyces pachygonesInfected leaves
Colonies amphigenous, caulicolous, dense, up to $2 \mathrm{~mm}$ in diam. Hyphae partly superficial and partly immersed in the epidermis. Superficial hyphae substraight, flexuous, irregularly branched, bearing conidiogenous cells, cells 11-28 $\times 3-5 \mu \mathrm{m}$. Conidiogenous cells globose to cylindrical, monotretic, $4-10 \times 4-8 \mu \mathrm{m}$. Conidia phragmosporous, brown, one to many septate, constricted at the septa, straight to variously curved, rounded at both the ends, wall smooth, 19-72 $\times 12-48 \mu \mathrm{m}$. Thyriothecia mostly grouped, oval, elongate, $\times$ or $Y$ shaped, straight to curved, astomatous, dehisce vertically at the centre, margin fimbriate, fringed hyphae small, compact, thyriothecia $195-690 \times 100-252 \mu \mathrm{m}$; asci many, initially globose, subglobose, clavate, octosporous, paraphysate, bitunicate, 20-51 × 12-37 $\mu \mathrm{m}$, paraphyses hyaline, filiform; ascospores initially hyaline, brown at maturity, conglobate, uniseptate, constricted at the

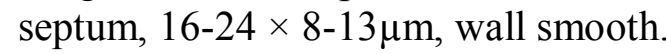

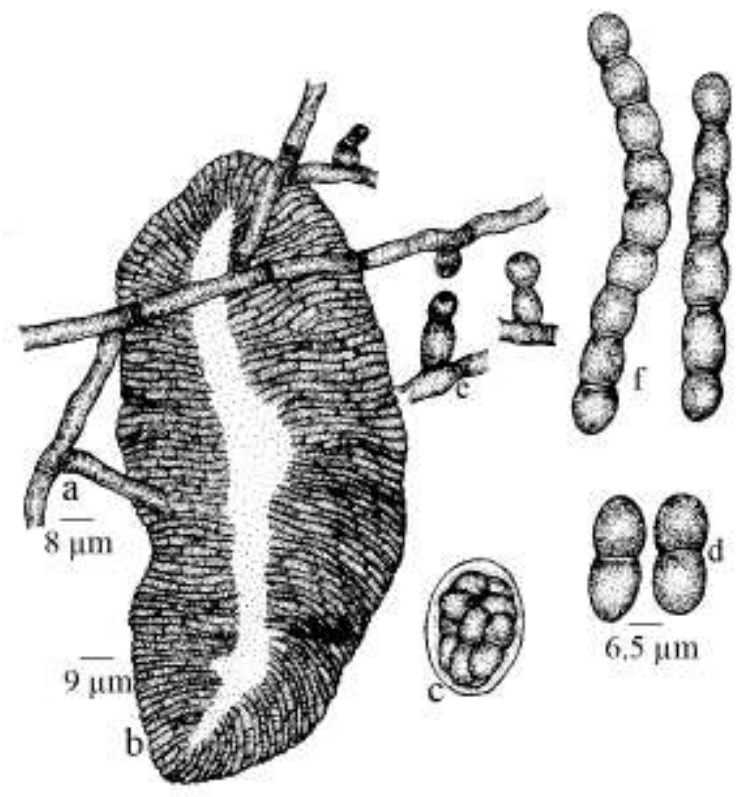

Figure 304 - Maheshwaramyces pachygones

a.Appressoriate mycelium, b.Thyriothecium, c. Ascus, d. Ascospores, e. Conidiophore, f.Conidia

Material examined: Kerala, Thiruvananthapuram, Palode, TBGRI Campus, on leaves of Pachygone ovata (Poir.) Miers ex Hook. f. \& Thoms. (Menispermaceae), 4 April 2008, Mathew Dan HCIO (holotype), TBGT 3017 (isotype); Thiruvananthapuram, Palode, 
Mycosphere Doi 10.5943/mycosphere/3/5/9

TBGRI Campus, 4 February 2004, Mathew Dan TBGT 3122, HCIO 48401; Thiruvananthapuram, Peppara Wildlife Sanctuary, MPCA, Bonocaud, 6 March 2008, Jacob Thomas, TBGT 3237, HCIO 48861; Palode, TBGRI Campus, 21 July 2008, Anilkumar TBGT 3349, HCIO 49094; 26 June 2008, A. Sabeena TBGT 3371, HCIO 49116; Tamil Nadu, Chennai, Madras Christian College, 29 December 2007, V.B. Hosagoudar \& al. TBGT 3038, HCIO 48317.

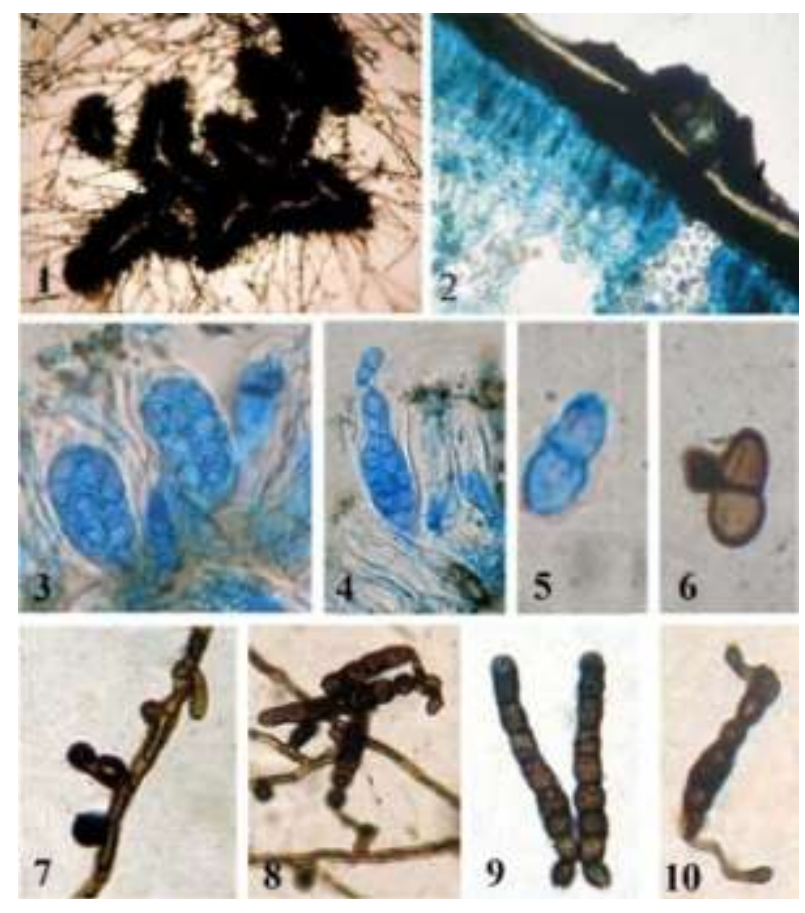

Figure 305 - Maheshwaramyces pachygones

1. Connate thyriothecium, 2. T.S. Showing subepidermal stromata, 3. Asci flanked with paraphyses, 4. Ascospores getting released from ascus, 5-6. Ascospores, 7. Conidiogenous cells, 8. Conidia produced from the conidiogenous cell, 9. A pair of conidia, 10. Germinating conidium

Distribution: Kerala, Tamil Nadu.

\section{KEY TO THE ANAMORPHIC GENERA}

1. Appressoria absent Asterostomula

1. Appressoria present 2

2. Appressoria formed only around the stomata ..Bramhamyces

2. Appressoria not so .. 2

3 Pycnothyriospores pyriform, ovate. Asterostomella
3. Pycnothyriospores angular, truncate at the base Mahanteshamyces

\section{KEY TO THE SPECIES}

\section{Annonaceae}

Asterostomella

Single species A. miliusae

\section{Aquifoliaceae}

Bramhamyces

Single species B. ilecis

\section{Asclepiadaceae \\ Asterostomella}

Single species A. ceropegiae

\section{Bignoniaceae} Asterostomella

Single species A. radermacherae

\section{Combretaceae} Asterostomella
1. On Terminalia
A. terminaliae
1. On Anogeissus A. anogeissi

\section{Daphniphyllaceae} Asterostomella

Single species A. daphniphylli

\section{Dilleniaceae}

Asterostomella

Single species A. dilleniacearum

\section{Dipterocarpaceae}

Asterostomella

Single species A. shoreae

\section{Elaeocarpaceae} Asterostomella

Single species A. elaeocarpi-serrati

\section{Euphorbiaceae} Asterostomella

1. Appressoria unicellular .........A. baliospermi 1. Appressoria not so .......................................2 2. Appressoria two-celled, head cells ovate, globose, entire to deeply and irregularly lobate A. excoecariicola 2. Appressoria two to many celled, head cells oval to hemispherical.........A. kushinagarensis 
Mahanteshamyces

Single species M. agrostachydis

Fabaceae

Asterostomella

Single species

A. pongamiae

\section{Flacourtiaceae}

Asterostomella

1. On Scolopia A. scolopiae-crenatae

1. On other hosts A. xylosmae

2. On Xylosma.

1. On Flacourtia montana

A. flacourtiae-montanae

Loranthaceae

Asterostomula

Single species A. loranthi

Magnoliaceae

Asterostomella

Single species A. micheliae

Myrtaceae

Asterostomula

Single species A. syzygii

Olacaceae

Asterostomella

Single species A. strombosiae

Oleaceae

Asterostomella

Single species

A. ligustri

Rhamnaceae

Single species A. ziziphina

\section{Rubiaceae}

\section{Asterostomula}

Single species A. pavettae

Sabiaceae

Asterostomella

Single species A. meliosmigena

\section{Sapindaceae}

Asterostomella

Single species A. otonephelii

Sapotaceae Asterostomella
Single species

A. isonadrae

\section{Urticaceae}

Asterostomella

Single species

A. boehmeriae

\section{THE GENUS Asterostomella}

Asterostomella Speg., Ann. Soc. Cien. Arg. 22: 198, 1886; Hosag., Chandraprabha \& Agarwal, Asterinales of Kerala, p. 224, 2011.

Leaf parasites. Mycelium ectophytic, appressoria lateral, setae absent. Pycnothyria orbicular with radiating cells, astomatous, dehisce stellately at the center; pycnothyriospores ovate, pyriform, brown.

Type sp.: A. paraguayensis Speg.

Abbreviations used in the line drawings:

a. Appressoriate mycelium, b. Thyriothecium, c. Pycnothyriospores

\section{DESCRIPTIONS TOTHE SPECIES}

Asterostomella anogeissi V.B. Hosagoudar \&

G.R. Archana, sp.nov. (Fig. 306)

MycoBank 101018

Etymology: Named after the host genus

Colonies amphigenous, mostly epiphyllous, thin, crustose, up to $4 \mathrm{~mm}$ in diameter, confluent. Hyphae undulating, branching opposite at acute to wide angles, loosely reticulate, cells $16-22 \times 3-5 \mu \mathrm{m}$. Appressoria alternate, unilateral, subantrorse, 9$17 \mu \mathrm{m}$ long; stalk cells cylindrical to cuneate, 3 $9 \mu \mathrm{m}$ long; head cells straight to variously curved, angular, sublobate, 6-8 $\times 6-9 \mu \mathrm{m}$. Pycnothyria scattered, orbicular, up to $68 \mu \mathrm{m}$ in diameter, stellately dehisced at the centre, margin fringed with hyphae; pycnothyriospores pyriform, ovate, $16-21 \times 11-14 \mu \mathrm{m}$.

Material examined: Maharashtra, Satara, Pateghar, on leaves of Anogeissus latifolia (Roxb. ex DC.) Wall. ex Guill. \& Perr. 
(Combretaceae), 3 January 2009, V.B. Hosagoudar \& al. TBGT 4806 (holotype).

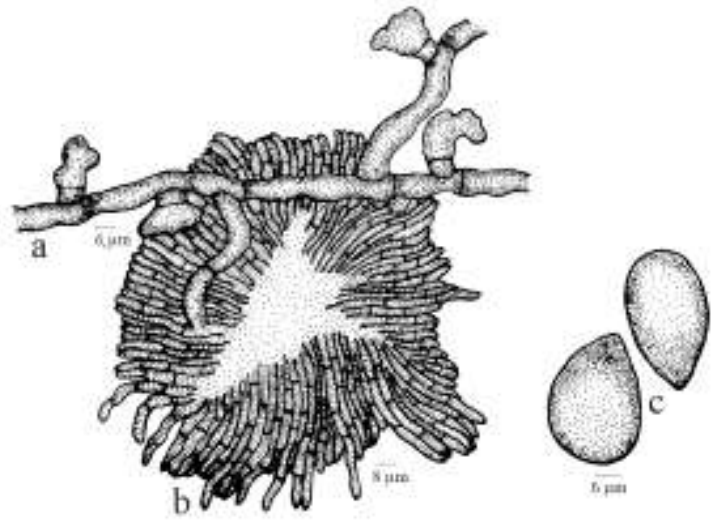

Figure 306 - Asterostomella anogeissi

This is the only species on this host in anamorph state.

Distribution: Maharashtra

Asterostomella baliospermi Hosag., Arachana \& Agarwal, Indian Phytopath. 60: 345, 2007; Hosag., Chandraprabha \& Agarwal, Asterinales of Kerala, p. 224, 2011. (Fig. 307)

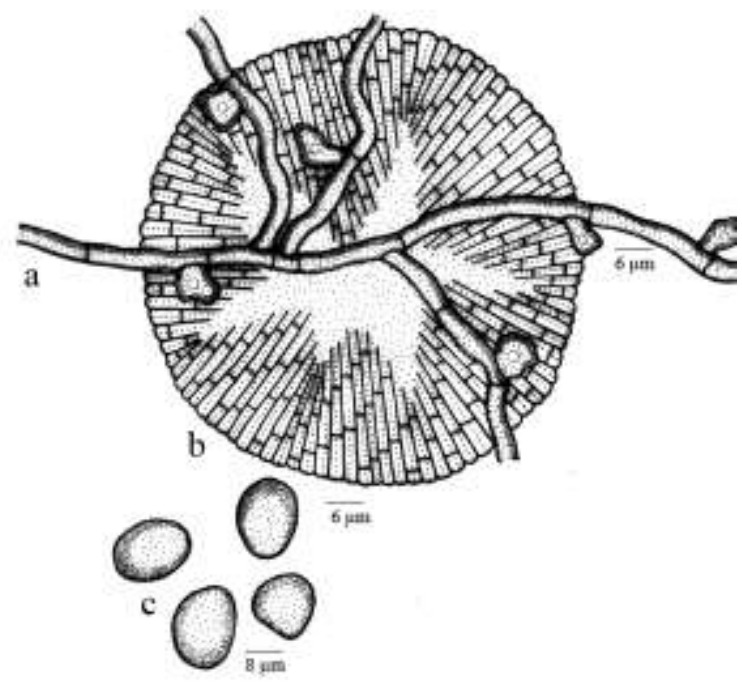

Figure 307 - Asterostomella baliospermi

Colonies amphigenous, sub-dense, up to $2 \mathrm{~mm}$ in diameter, confluent. Hyphae substraight, branching opposite, alternate to irregular, at acute to wide angles, loosely reticulate, cells $8-32 \times 3-5 \mu \mathrm{m}$. Appressoria alternate, unilateral, sessile, angular, broad based, $5-10 \times 5-11 \mu \mathrm{m}$. Pycnothyria scattered to grouped, orbicular, up to $120 \mu \mathrm{m}$ in diameter, stellately dehisced at the centre, margin fringed with hyphae; pycnothyriospores pyriform, globose to ovate, $9-18 \times 8-12 \mu \mathrm{m}$.

\section{Material examined: Kerala, Thiruvananthapuram, Palode, TBGRI, Medicinal Plants Garden, on leaves of Baliospermum montanum (Willd. ) Muell. - Arg. (Euphorbiaceae), 5 January 2001, H. Biju HCIO 46780 (holotype), TBGT 2121 (isotype).}

This forms the first report of the asterinaceous fungus on this host genus (Stevens \& Ryan, 1939; Doidge, 1942; Hosagoudar \& Abraham, 2000). Hence, it is described here as a new species.

Distribution: Kerala.

Asterostomella boehmeriae Hosag., Balakr. \& Goos, Mycotaxon 58: 491, 1996; Hosag., Zoos' Print J. 18: 1285, 2003; Hosag., Zoos' Print J. 21: 2412, 2006; Hosag., Chandraprabha \& Agarwal, Asterinales of Kerala, p. 226, 2011.

(Fig. 308)
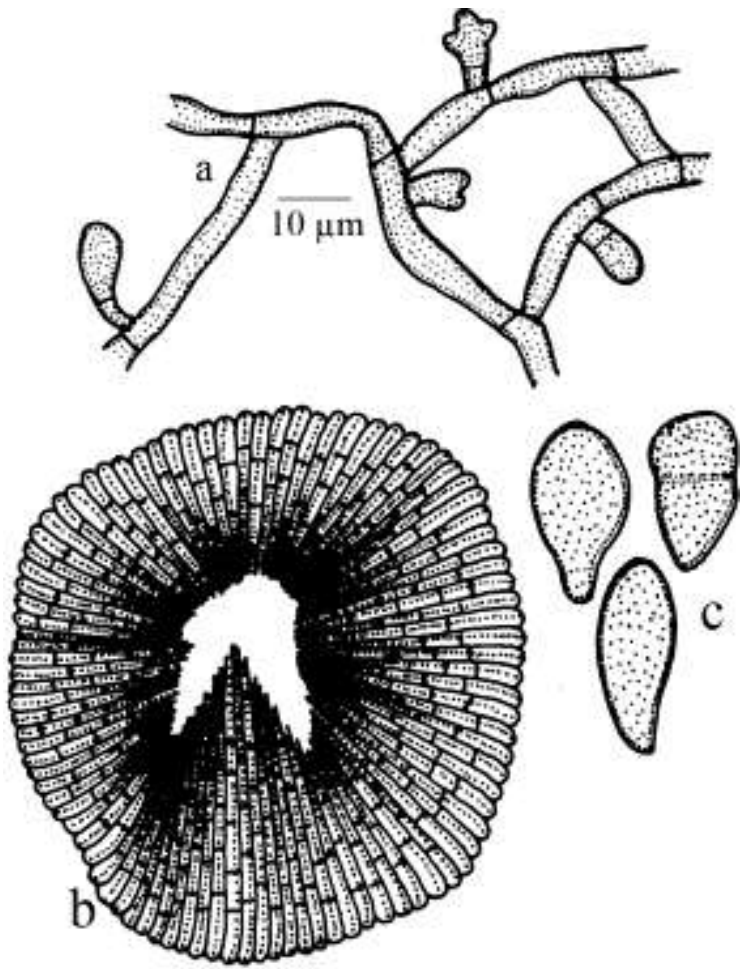

Figure 308 - Asterostomella boehmeriae

Colonies hypophyllous, black, velvety, later ash-coloured, mostly confluent, giving a 
dusty appearance, corresponding upper surface of the infected leaf portion turn brick red, severely infected leaves roll dorsally so as to cover the infected parts by exposing the ventral surface. Hyphae pale yellow, strongly appressed to the host epidermis, straight to flexuous, branching opposite to irregular at acute angles, loosely reticulate, cells 12-18.5 $\times$ 5-6.5 $\mu \mathrm{m}$. Appressoria one to two celled, sessile to stalked, alternate, $6-18.5 \mu \mathrm{m}$ long; stalk cells (when two celled) cylindrical to cuneate, 6-6.5 $\mu \mathrm{m}$ long; head cells ovate, globose, entire to angular (in sessile), 9-12.5 $\times$ 6-8 $\mu \mathrm{m}$. Pycnothyria scattered to mostly grouped, orbicular, 90-155 $\mu \mathrm{m}$ in diameter, margin crenate, dehiscing stellately at the centre, Pycnothyriospores brown, numerous, ellipsoidal, unicellular, tapered to broadly rounded at the apex, straight to slightly curved, a hyaline band often present in the middle, 18$31 \times 12-15.5 \mu \mathrm{m}$, wall smooth.

Material examined: Tamil Nadu, Coimbatore, Valparai, Near Sholayar dam, on leaves of Boehmeria glomerulifera Miq. (Urticaceae), 23 March 1990, V.B. Hosagoudar HCIO 30978 (holotype); Kerala, Wyanad, Chandanathode, on leaves of Boehmeria sp., 27 December 2002, M. Kamarudeen \& P.A. Jose HCIO 44791, TBGT 1028. the host.

This species has a pathogenic effect on

Distribution: Kerala, Tamil Nadu.

Asterostomella ceropegiae Hosag., H. Biju \& Manoj. in Hosag., Zoos' Print J. 21: 2337, 2006; Hosag., Chandraprabha \& Agarwal, Asterinales of Kerala, p. 227, 2011. (Fig. 309)

Colonies amphigenous, dense, crustose, up to $2 \mathrm{~mm}$ in diameter. Hyphae substraight to flexuous, branching irregular at acute to wide angles, loosely reticulate, cells 19-23 × 3-4 $\mu \mathrm{m}$. Appressoria scattered, alternate, unilateral, unicellular, ovate, globose, cylindrical, irregularly sublobate to deeply lobate, gibbous at the base, $8-16 \times 8-10 \mu \mathrm{m}$. Pycnothyria scattered, often connate, orbicular, up to 138 $\mu \mathrm{m}$ in diameter, margin crenate to fimbriate, fringed hyphae flexuous, small, pycnothyria scattered, often connate, orbicular, up to 136 $\mu \mathrm{m}$ in diameter, margin crenate to fimbriate, fringed hyphae flexuous, small, pycnothyria stellately dehisced at the centre; pycnothyriospores unicellular, brown, pyriform, $16-20 \times 8-10 \mu \mathrm{m}$, wall smooth.

Material examined: Kerala, Idukki, Munnar, Kundala, on leaves of Ceropegia sp. (Asclepiadaceae), 23 January 2003, A. Manojkumar \& H. Biju HCIO 44833 (holotype), TBGT 1070 (isotype).

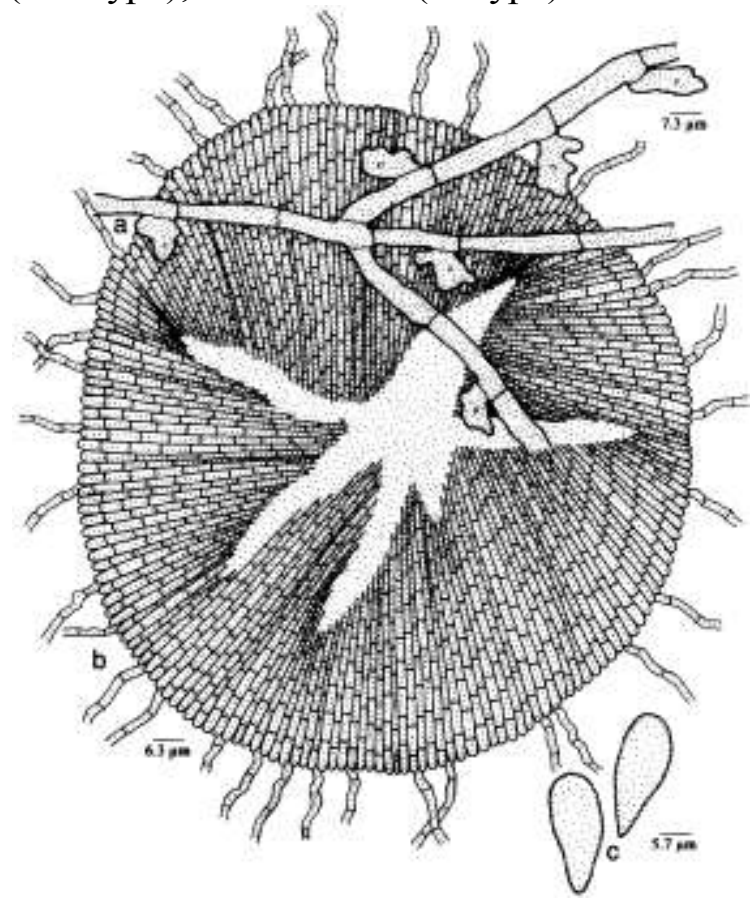

Figure 309 - Asterostomella ceropegiae

Basal portion of some of the appressoria is gibbous and very firmly appressed to the hyphae and impart that they are septate. Few thyriothecia were observed but were empty. Numerous pycnothyriospores were scattered in the colonies.

Of the known six species of the genus Asterina on the members of Asclepiadaceae, the present collection is closer to Asterina secamonicola, which is having unicellular appressoria. The present species differs from it in having only pycnothyrial state and the gibbous nature of the basal portion of the appressoria.

Distribution: Kerala. 
Asterostomella daphniphylli Hosag. \& Ravikumar in Hosag. \& Goos, Mycotaxon 52: 471, 1994; Hosag., Chandraprabha \& Agarwal, Asterinales of Kerala, p. 229, 2011.

(Fig. 310)

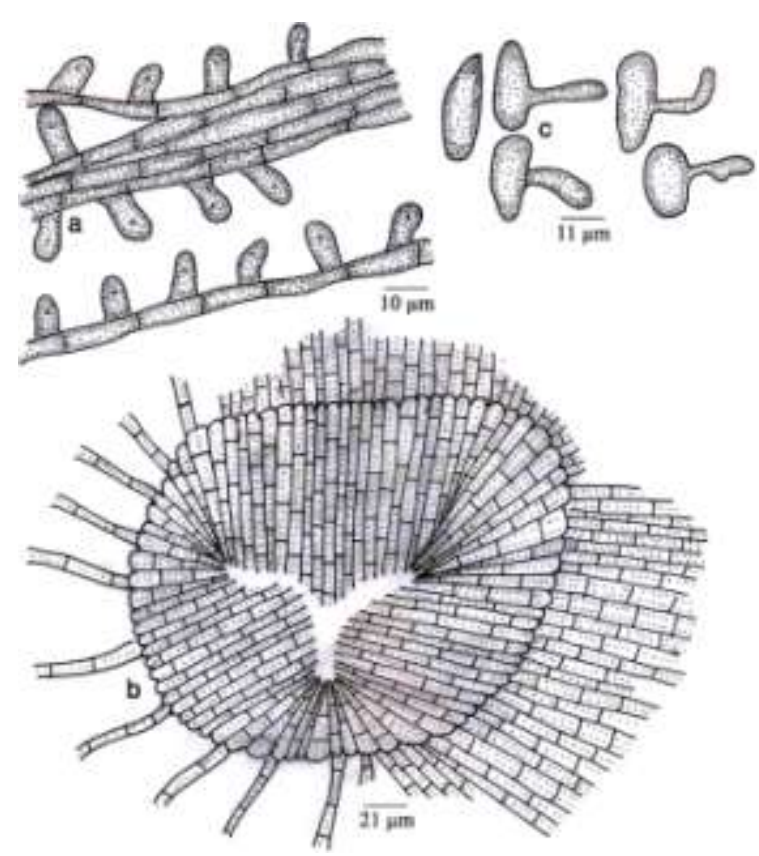

Figure 310 - Asterostomella daphniphylli

Colonies amphigenous, mostly epiphyllous, crustose to velvety, up to $2 \mathrm{~mm}$ in diameter, confluent and covering the entire upper surface of the leaves. Hyphae straight, flexuous, often crooked when solitary, branching alternate to irregular at acute angles, several hyphae running closely parallel and forming a compact mycelial mat, cells 9-15.5 $\times$ 4.5-7.5 $\mu \mathrm{m}$. Appressoria alternate and produced only on the outer surface of the compact hyphae, mostly straight but rarely curved, unicellular ovate to globose, entire, 6-12.5 × 6$9.5 \mu \mathrm{m}$. Pycnothyria numerous, loosely crowded, circular in outline, often ovate, 130$190 \mu \mathrm{m}$ in diameter, covering membrane initially brown, later becoming dark and opaque, splitting stellately at the center or having a wide opening. Pycnothyriospores oval, ellipsoidal, pyriform, straight to slightly curved, pale brown to deep brown, often with a hyaline band at the center, $18-28 \times 9-12.5 \mu \mathrm{m}$.

Material examined: Tamil Nadu, Kodaikanal, on leaves of Daphniphyllum neilgherrense (Wight) K. Rosenthal
(Daphniphyllaceae), 18 October 1991, K. Ravikumar HCIO 40859 (holotype); Kodaikanal, Gundan shola, 23 July 2006, R. Nithyatharani TBGT 2261, HCIO 47044; 21 July 2006, R. Nithyatharani, TBGT 2257, 2259, 2704, HCIO 47040, 47042, 47682; Mathikettan shola, 18 July 2006, B. Bhairavi TBGT 2322, HCIO 47105; Kerala, Idukki, Mannavan shoal, 23 September 1998, C.K. Biju HCIO 42950, TBGT 237; Idukki, Munnar, Gravel Banks, 18 December 2001, S. Shiburaj HCIO 44307, TBGT 627; Idukki, Munnar, Pooppara, 24 January 2003, H. Biju \& A. Manojkumar HCIO 44836, TBGT 1073; Idukki, Mannavan shola, on leaves of Daphniphyllum sp., 5 January 1999, C.K. Biju HCIO 44000, 43830, TBGT 402, 375; Idukki, Mannavan shola, 2 June 2000, C.K. Biju HCIO 43803, TBGT 376; Munnar, Gravel Banks, 10 October 2001, S. Shiburaj TBGT 604, HCIO 44306; Palghat, Silent valley National Park, Cheriya Walakkad, 2 August 2008, Jacob Thomas \& al. TBGT 3471, HCIO 49232.

Asterina daphniphylli Yamam. has been recorded on Daphniphyllum species from Taiwan and Japan (Hino \& Katumoto, 1957). The new species differs from it in having a very compactly reticulate mycelium and the presence of an anamorph.

Distribution: Kerala, Tamil Nadu.

Asterostomella dilleniacearum Hosag., Abraham \& C.K. Biju, New Botanist 24: 19, 1997; Hosag.,C.K. Biju\& Abraham, J. Econ. Taxon. Bot. 25: 307, 2001; Hosag., Zoos' Print J. 18: 1282, 2003; Hosag., Zoos' Print J. 21: 2412, 2006; Hosag., Chandraprabha \& Agarwal, Asterinales of Kerala, p. 231, 2011. (Fig. 311)

Colonies hypophyllous, or epiphyllous, thin to very thin, spreading, up to $10 \mathrm{~mm}$ in diameter, confluent. Hyphae flexuous, branching alternate to irregular at acute to wide angles, loosely to closely reticulate, cells $12-20$ $\times$ 4-5 $\mu \mathrm{m}$. Appressoria scattered, alternate, cylindrical to mammiform, entire to sublobate, 6-8 $\times 8-9.5 \mu \mathrm{m}$. Pycnothyria scattered, rounded, up to $72 \mu \mathrm{m}$ in diameter, margin crenate, 
stellately dehisce at the center and later widely opened; Pycnothyriospores cinnamon brown, oval to slightly oblong, $14-17 \times 9-12 \mu \mathrm{m}$; wall smooth.

\section{Material examined: Kerala, Thiruvananthapuram, Peppara Wildlife} Sanctuary, Meenmutty, Kombe, on leaves of Dillenia pentagyna Roxb. (Dilleniaceae) 9 March 1998, V.B. Hosagoudar HCIO 42890, TBGT 201; 18 November 2007, Jacob Thomas \& Vimal HCIO 48294, TBGT 3013; Thiruvananthapuram, Palode, TBGRI Campus, 7 December 2001, H. Biju HCIO 46067, TBGT 1830; 12 October 2006, A. Sabeena \& M. C. Riju HCIO 47545, TBGT 2567; Pathanamthitta, Moozhiyar Forest, 14 December 2004, V.B. Hosagoudar HCIO 46338, TBGT 1984; Thiruvananthapuram, Peppara Wildlife Sanctuary, on leaves of Dillenia sp., 5 February 1997, V.B. Hosagoudar HCIO 44309, TBGT 666; Kollam, Shankily forest, 27 December 2001 V.B. Hosagoudar HCIO 44308, TBGT 728; Thrissur, Vazhachal, 23 October 2002, A. Manojkumar \& H. Biju HCIO 45173, TBGT 1334.

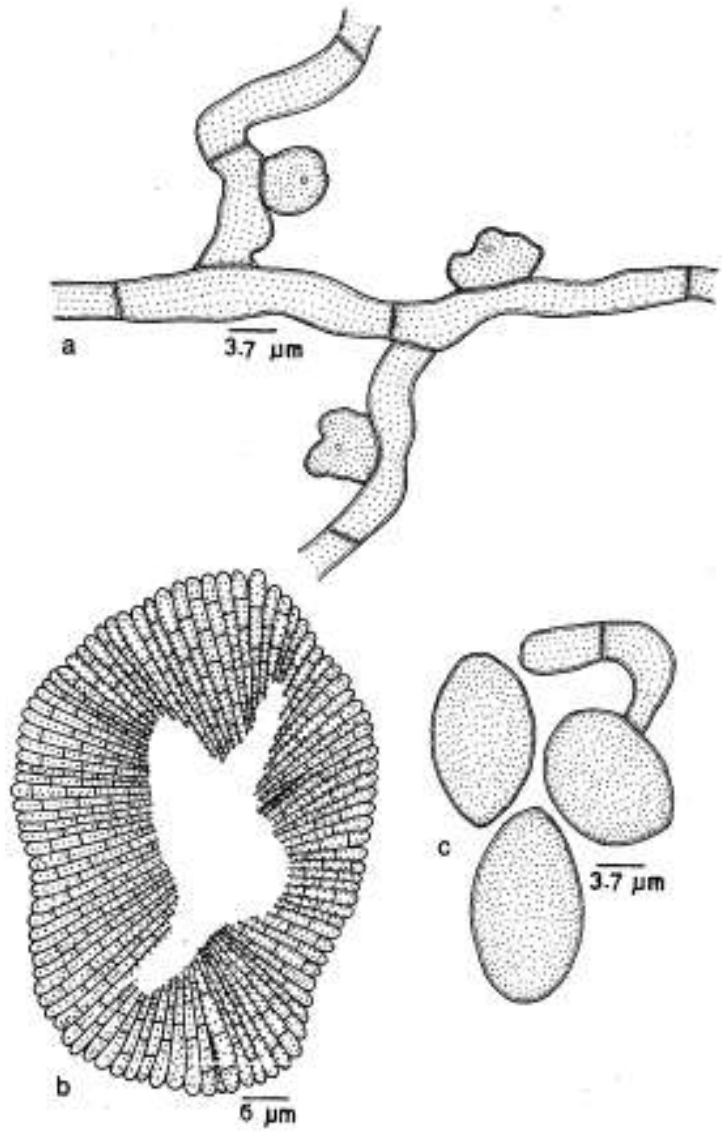

Figure 311 -Asterostomella dilleniacearum
Repeated collections of this fungus from the Western Ghats persisted only in its anamorph state, Asterostomella and are close to A. dilleniicola Farr but differs from it in the morphology of the appressoria and smaller pycnothyriospores.

\section{Distribution: Kerala.}

Asterostomella elaeocarpi-serrati Hosag. in Hosag., H. Biju \& Appaiah, J.Mycopathol. Res. 44: 13， 2006; Hosag. J. Appl. \& Nat. Sci. 1(1):29, 2009; Hosag., Chandraprabha \& Agarwal, Asterinales of Kerala, p. 231, 2011. (Fig. 312)

Colonies amphigenous, dense, up to 1 $\mathrm{mm}$ in diameter, rarely confluent. Hyphae straight to flexuous, branching mostly opposite at acute angles, loosely to closely reticulate, cells $8-24 \times 5-7 \mu \mathrm{m}$. Appressoria alternate, about $30 \%$ opposite, unicellular, conoid, ovate, entire, straight, attenuated and broadly rounded at the apex, 8-13 $\times 6-8 \mu \mathrm{m}$. Thyriothecia scattered, orbicular, up to $258 \mu \mathrm{m}$ in diam., stellately dehisced at the centre, margin crenate; pycnothyriospores pyriform, brown, 20-26 $\times$ 19-21 $\mu \mathrm{m}$, wall smooth.

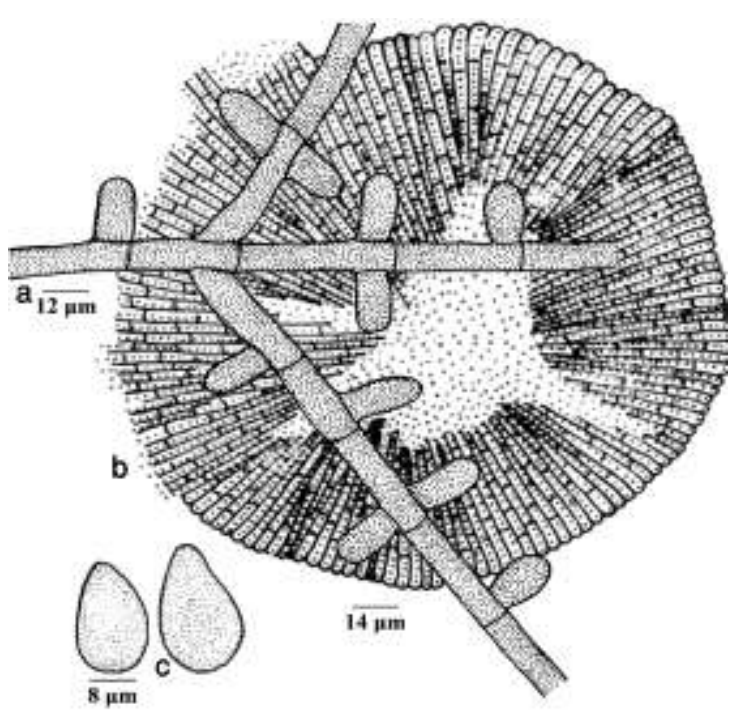

Figure 312 - Asterostomella elaeocarpi-serrati

Material examined: Karnataka, Coorg, Madikeri, Abbe falls, on leaves of Elaeocarpus serratus L. (Elaeocarpaceae), 11 November 2003, V.B. Hosagoudar \& al. HCIO 45817 (holotype), TBGT 1567 (isotype); 
Kerala, Thiruvananthapuram, Palode, TBGRI Campus, 12 October 2006, A. Sabeena \& M. C. Riju HCIO 48021, TBGT 2804; 12 October 2006, A. Sabeena \& al. HCIO 48020, TBGT 2803.

This is an anamorph of the genus Asterina and is close to A. borneensis Hansf. in having smaller and alternate to opposite appressoria. However, differs from it in having conoid but $30 \%$ opposite appressoria (Hansford, 1954).

Distribution: Kerala, Karnataka.

Asterostomella excoecariicola Hosag. \& Goos, Mycotaxon 60: 161, 1996.

(Fig. 313)

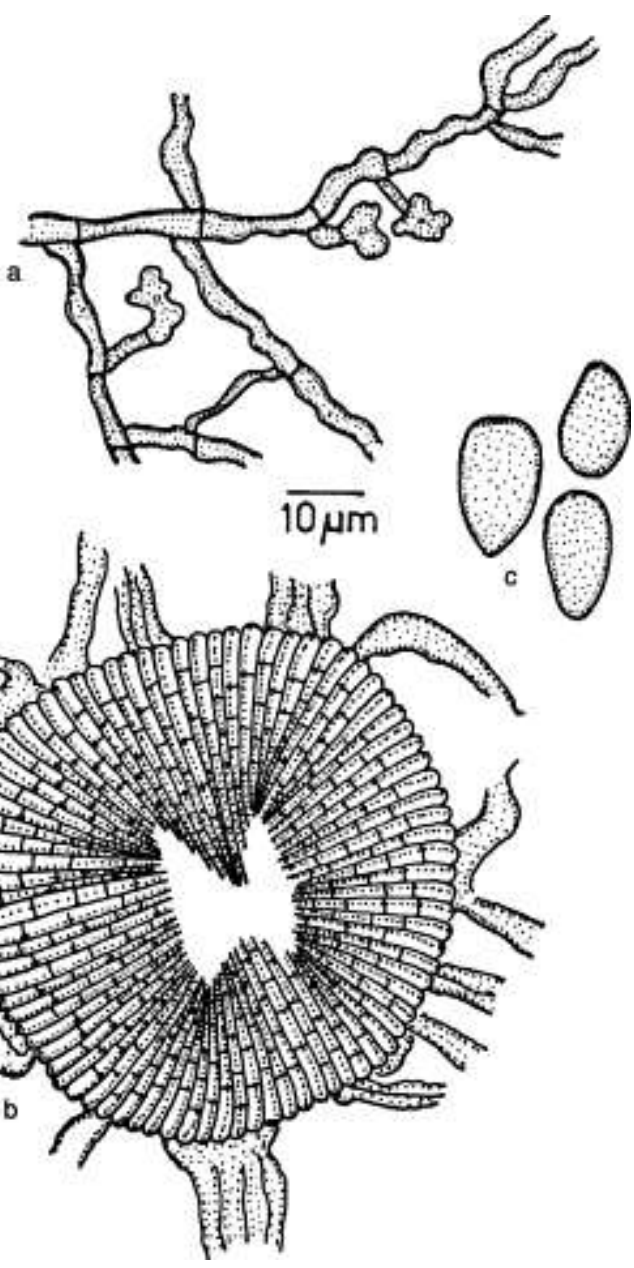

Figure 313 - Asterostomella excoecariicola

Colonies hypophyllous, thin, spreading, up to $10 \mathrm{~mm}$ in diameter. Hyphae pale brown, flexuous to crooked, branching profuse, loosely reticulate, cells $18-25 \times 2-3 \mu \mathrm{m}$. Appressoria few, scattered, two to many celled, stipitate, 6$40 \mu \mathrm{m}$ long; stalk cells unicellular to multicellular, straight, flexuous to crooked, 6$25 \mu \mathrm{m}$ long; head cells ovate, globose, entire to deeply and irregularly lobate, 6-13 × 6-10 $\mu \mathrm{m}$. Pycnothyria scattered, orbicular, up to $85 \mu \mathrm{m}$ in diameter, margin fimbriate, fringed hyphae crooked, dehiscing stellately at the center; pycnothyria unicellular, pyriform, brown, 8-25 $\times 9-13 \mu \mathrm{m}$.

Material examined: Tamil Nadu, Kanniyakumari, Valve house forest, on leaves of Excoecaria crenulata Wight (Euphorbaceae), 28 February, 1994, V.B. Hosagoudar HCIO 41632.

This species differs from Asterina excoecariae Doidge in having multicellular stalk cells of the appressoria with lobate head cells.

Distribution: Tamil Nadu.

Asterostomella flacourtiae-montanae V.B. Hosagoudar \& A. Sabeena, sp. nov. (Fig. 314)

MycoBank 101019

Etymology: Named after the host plant

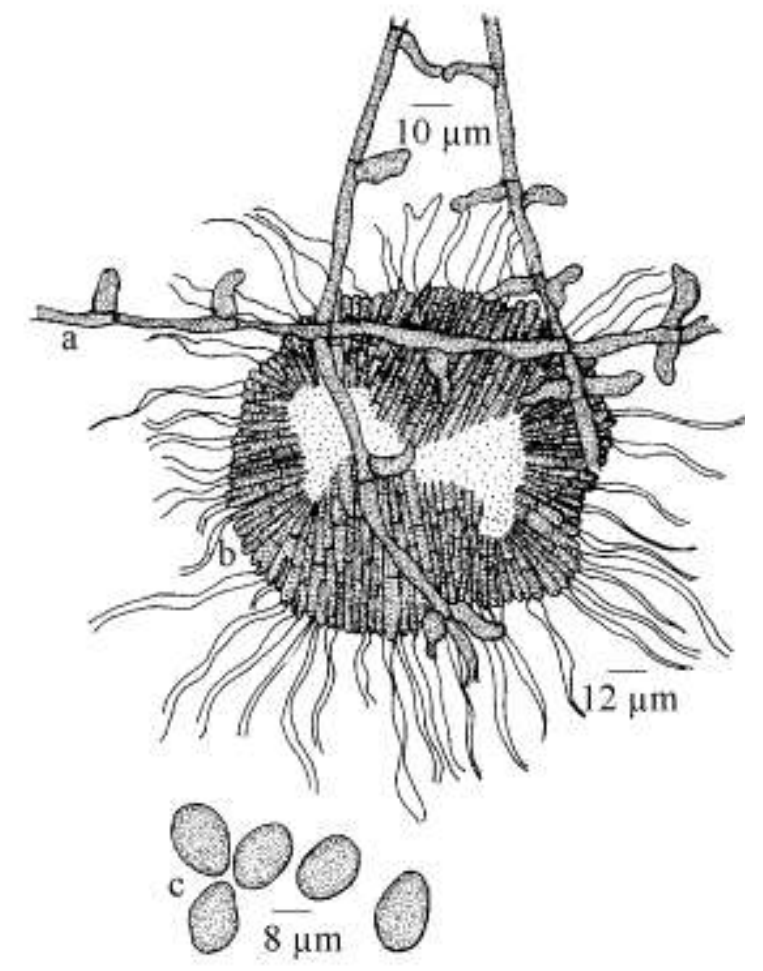

Figure 314 - Asterostomella flacourtiaemontanae 
Colonies amphigenous, thin to subdense, up to $2 \mathrm{~mm}$ in diameter, confluent. Hyphae substraight to flexuous, branching irregular at acute to wide angles, loosely to closely reticulate, cells $15-27 \times 2-5 \mu \mathrm{m}$. Appressoria mostly alternate, subopposite to opposite, pyriform narrowly ovate, entire to sublobate straight to variously curved, 7-15 $\times$ 2-5 $\mu \mathrm{m}$. Pycnothyria, scattered, orbicular, up to $120 \mu \mathrm{m}$ in diameter, margin crenate to fimbriate, fringed hyphae flexuous, stellately dehisced at the centre; pycnothyriospores ovate, $12-17 \times 7-12 \mu \mathrm{m}$.

\section{Material examined: Kerala, Thiruvananthapuram, Palode, JNTBGRI campus, on leaves of Flacourtia montana Graham (Flacourtiaceae), 24 June 2008, K. Anilkumar TBGT 5737 (holotype).}

Based on the morphology of appressoria this species is similar to Astrostomella scolopiae-crenulatae but differs from it in having unicellular and smaller appressoria.

Asterostomella isonadrae Hosag. \& Goos, Mycotaxon 52; 470, 1994. (Fig. 315)

Colonies hypophyllous, subdense, spreading. Hyphae crooked, branching irregular at acute angles, closely reticulate, cells 12-15 $\times$ 3-4.5 $\mu \mathrm{m}$. Appressoria unicellular, often scattered, ovate, ampulliform, mammiform, entire to sublobate, 9-12.5 $\times$ 6-8 $\mu \mathrm{m}$. Pycnothyria $\mu \mathrm{m}$ in diameter, margin crenate. Pycnothyriospores unicellular, ovoid, ellipsoid, brown, acutely rounded at both ends, 24-26 $\times$ 9-15.5 $\mu \mathrm{m}$.

Material examined: Tamil Nadu, Kamarajar dist., Seithur Hills, on leaves of Isonandra lanceolata Wight forma anfractuosa (Clarke) Jewken (Sapotaceae), 14 November 1992, V.B. Hosagoudar HCIO 40858 (holotype).

This new species differs from other anamorphs of Asterina species reported on Sapotaceae in the infection pattern and in the morphology of hyphae, appressoria and Pycnothyriospores (Stevens \& Ryan, 1939; Doidge, 1942).
Distribution: Tamil Nadu.

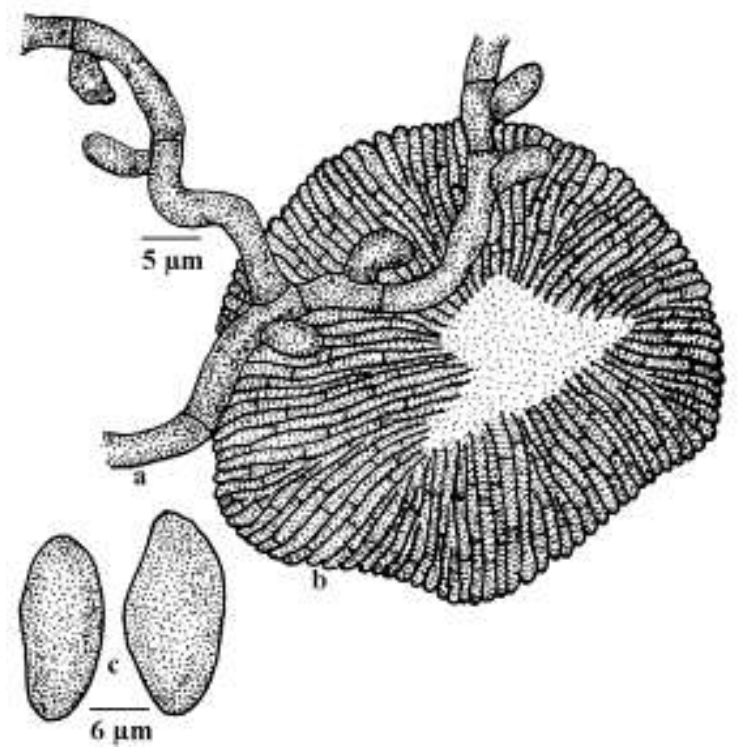

Figure 315 - Asterostomella isonadrae

\section{Asterostomella kushinagarensis Verma, Tripathi \& Chaudhary, Indian Phytopathol. 52:378, 1999.}

Infection spots epigenous, irregular, variable in size, mostly brown, coalescing to from an irregular shape. Mycelium superficial, to subimmeresed, pale olivaceous brown, septate, hyphal branching alternate measuring 2.1-6.0 $\mu \mathrm{m}$ in diameter. Hyphopodia capitata, alternate, sometimes one sided, 2 celled ,head cell larger ,oval to hemispherical, lower cell small cuneate to cylindrical, pale brown, lateral, measuring 6.7-14.5 $\times$ 3.5-7.8 $\mu \mathrm{m}$. pycnidia superficial gregarious, olivaceous to olivaceous brown, globose to sub-globose radiate, ostiole absent,about 79.4-95.2 $\mu \mathrm{m}$. Conidiophores invisible; Conidiogeous cells smooth, holoblastic, determinate hyaline, conidia dry, simple, unicellular, obovoidal to pyriform or balloon shaped, reddish brown, smooth, thick walled, measuring 18.2-26.4 × 9.5-18.5 $\mu \mathrm{m}$.

Material examined: Uttar Pradesh, Kushinagar, on leaves of Croton roxburghii Balakar. (Euphorbiaceae), December 1996, R. C. Verma, GPU/RCV-4/97(isotypus) HCIO 42575 (holotypus).

A survey of the pertinent literature concerning Asterostomella species shows that no species of this genus has been described on 
this host species (Hosagoudar el al., 1996 a). A perusal of the morphotaxonomic features indicated that the fungus in question is distinctly different from Asterostomella excoeariiola in having epiphyllous and irregular infection spots, wider hyphal cells which are alternatively branched, bicellular capitates appressoria with smaller and oval to hemispherical head, gregarious, globose, larger pycnidia and ovoidal to pyriform reddish brown and wider conidia.

Distribution: Utter Pradesh.

Asterostomella ligustri Hosag., Balakr. \& Goos, Mycotaxon 58: 493, 1996. (Fig. 316)

Infection spots hypophyllous, thin, dirty grey, up to $10 \mathrm{~mm}$ in diameter. Hyphae crooked, branching irregular at acute angles, loosely reticulate, cells 9-18.5 $\times 9-9.5 \mu \mathrm{m}$. Appressoria alternate, straight to curved, sessile to stipitate, irregularly lobed at the apex, 6-6.5 $\times$ 9-9.5 $\mu \mathrm{m}$. Pycnothyria scattered, round, 90$100 \times 68-90 \mu \mathrm{m}$, stellately dehisced at the center, margin crenate; Pycnothyriospores deep brown, oval, tapering towards both ends, ends broadly rounded, $15-18.5 \times 9-9.5 \mu \mathrm{m}$.

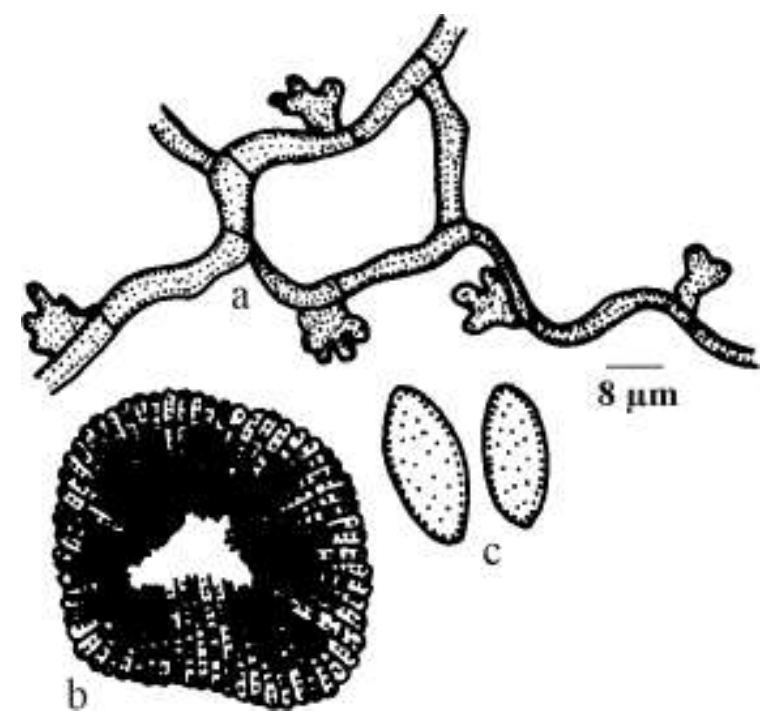

Figure 316 - Asterostomella ligustri

Material examined: Tamil Nadu, Coimbatore, Anamalai, Near Nirar dam, on leaves of Ligustrum bamlei Ramam. (Oleaceae), 27 December 1990, V.B. Hosagoudar HCIO 30981 (holotype).
This species resembles the Pycnothyrial form of Asterina erysiphoides Kalch. \& Cooke (Doidge, 1942; Hansford \& Thirumalachar, 1948) but differs from it in the absence of a central median hyaline band in the Pycnothyriospores.

Distribution: Tamil Nadu.

Asterostomella meliosmigena Hosag. in Hosag., Chandraprabha \& Agarwal, Asterinales of Kerala, p. 232, 2011. Asterostomella meliosmaticola Hosag., Balakr. \& Goos, Mycotaxon 58: 495, 1996 (meliosmaticola) (nom. illeg. Art. 53. 1), non. Petr. \& Ciff., 1932; Hosag., Zoos' Print J. 18: 1284, 2003; Hosag., Zoos' Print J. 21: 2412, 2006. (Fig. 317)

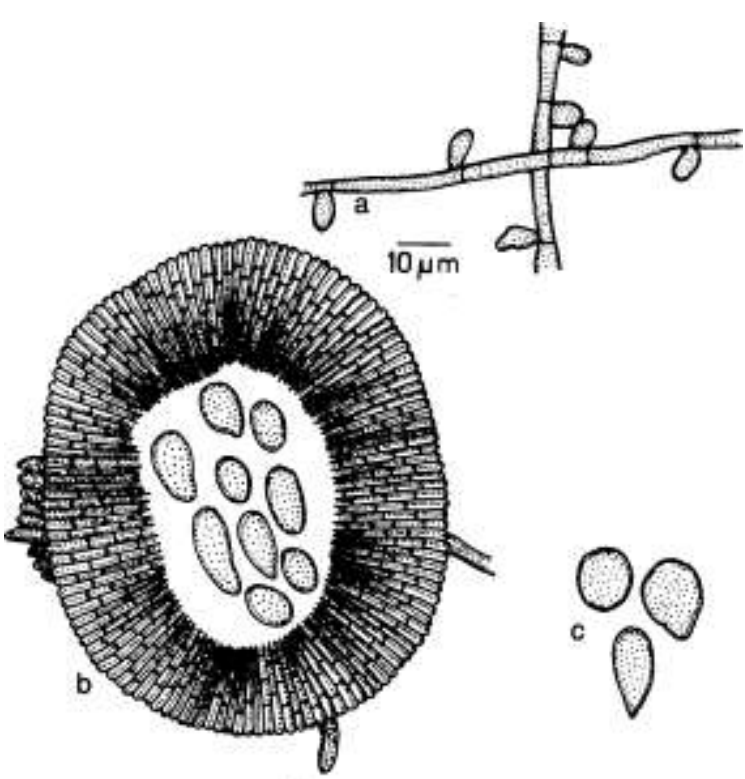

Figure 317 - Asterostomella meliosmigena

Colonies amphigenous, mostly epiphyllous, thin to dense, crustose, up to $2 \mathrm{~mm}$ in diameter, confluent. Hyphae flexuous to slightly crooked, branching alternate to irregular at acute angles, loosely reticulate, cells 15-34 $\times$ 3-4.5 $\mu \mathrm{m}$. Appressoria alternate, scattered, unicellular, straight to curved, oval to globose, entire to rarely lobate, 6-12.5 × 4-6.5 $\mu \mathrm{m}$. Pycnothyria scattered, round to slightly irregular, rarely connate, up to $140 \mu \mathrm{m}$ in diameter, stellately dehisced at the centre, margin fimbriate, fringed hyphae flexuous; pycnothyriospores ovoid, ellipsoidal to predominantly pyriform, $15-18.5 \times 12-13 \mu \mathrm{m}$. 
Material examined: Tamil Nadu, Nilgiris, Benne forest, on leaves of Meliosma simplicifolia (Roxb.) Walp. (Sabiaceae), 24 January 1990, V.B. Hosagoudar HCIO 30982; Meliosma sp., Pachakanam, Pathanamthitta, 14 December 2004, V.B. Hosagoudar HCIO 46337, TBGT 1983; 16 December 2004, Hosagoudar \& al TBGT, 2203, 2734, HCIO 46986, 47712; 21 July 2006, R. Anjugavairam TBGT 2324, HCIO 47107.

Repeated collections from the Western Ghats region of southern India yielded only the pycnothyrial state. The new species differs from the anamorph of Asterina meliosmaticola Petr. \& Ciff, in the morphology and measurements of appressoria and pycnothyriospores (Petrak \& Cifferi, 1932).

Distribution: Kerala, Tamil Nadu.

Asterostomella micheliae Hosag. \& Goos, Mycotaxon 60: 162, 1996; Hosag., Zoos' Print J. 18: 1283, 2003; 21: 2412, 2006; Hosag., Chandraprabha \& Agarwal, Asterinales of Kerala, p. 234, 2011. (Fig. 318)

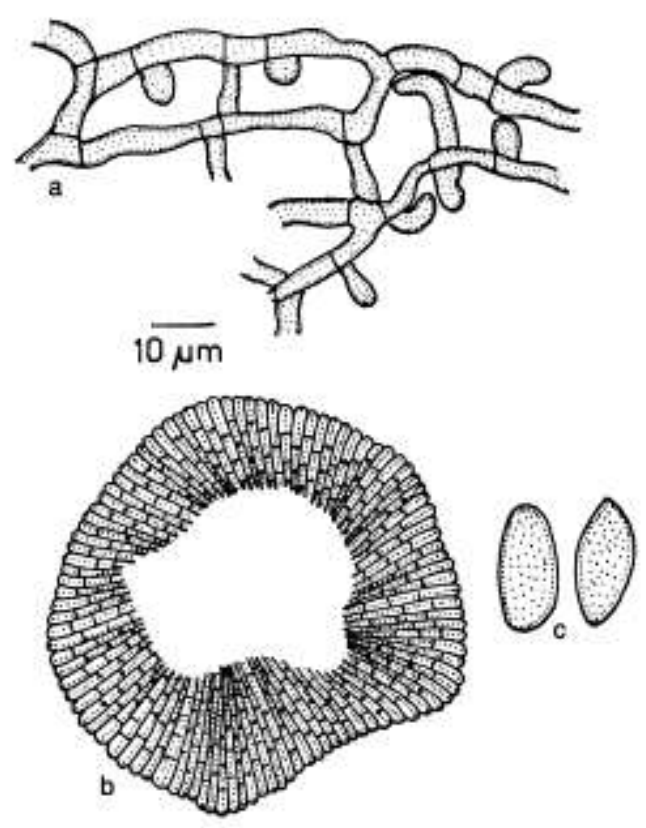

Figure 318 - Asterostomella micheliae

Colonies hypophyllous, dense, up to 2 $\mathrm{mm}$ in diameter, confluent and cover the entire lower surface of the leaves. Hyphae straight, branching irregular at acute to wide angles, forming closely reticulate rings, cells $15-22 \times$ 3-5 $\mu \mathrm{m}$. Appressoria few, distantly placed, unicellular, ovate, mammiform, entire to rarely sinuate, $6-13 \mu \mathrm{m}$. Pycnothyria closely scattered, orbicular, $\mathrm{u}[\mathrm{p}$ to $80 \mu \mathrm{m}$ in diameter, margin crenate, dissolved and widely opened at the center; pycnothyriospores oval to slightly clavate, often attenuated to broadly rounded at both ends, $21-25 \times 12-16 \mu \mathrm{m}$, wall smooth.

Material examined: Tamil Nadu, Tirunelveli, Kakachi forest, on leaves of Michelia nilagirica Zenker (Magnoliaceae), 21 February 1994, V.B. Hosagoudar HCIO 41584 (holotype); Kerala, Idukki, Munnar, Mannavan shola, 11 May 1999, C.K. Biju HCIO 45255, TBGT 1293.

This species is close to Asterina drymidis (Lev.) Speg. but differs from it in having unicellular appressoria and ring-like network formation of the hyphae (Stevens \& Ryan, 1939).

Distribution: Tamil Nadu.

Asterostomella miliusae A.K. Singh, Kamal \& S.K. Singh, Curr. Sci. 51: 787, 1982.

Colonies epiphyllous, irregular. Hyphae branched, cells 2-3 $\mu \mathrm{m}$ broad. Appressoria mostly two celled, 6-8 $\times 5-6 \mu \mathrm{m}$. Pycnothyria scattered, 58-120 $\mu \mathrm{m}$ in diameter; pycnothyriospores dry, simple, unicellular, obovoid to pyriform, reddish brown, truncate at the base, $18-25 \times 11-13 \mu \mathrm{m}$.

Uttar Pradesh, Gorakhapur, on leaves of Miliusa tomentosa (Roxb.) Cinclair (Annonaceae), December 1979, A.K. Singh KA-55, IMI 244909.

Though the description states that appressoria are celled, the line drawings depict that they are unicellular, globose, clavate and bilobate at the apex.

Distribution: Uttar Pradesh

Asterostomella otonephelii Hosag., Agarwal, H. Biju \& Archana, Indian Phytopathol. 59: 526, 2006; Hosag., Chandraprabha \& Agarwal, Asterinales of Kerala, p. 235, 2011. (Figs 319-320) 


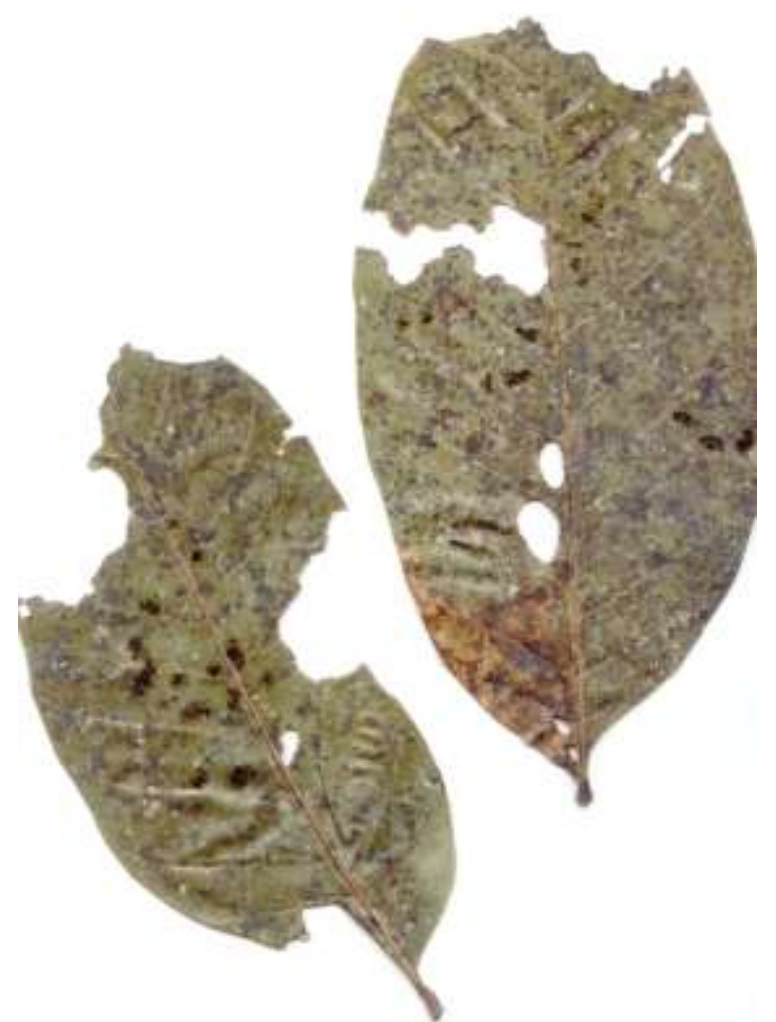

Figure 319 - Asterostomella otonephelii Infected leaves

Colonies epiphyllous, thin to dense, up to $3 \mathrm{~mm}$ in diam., confluent. Hyphae substraight to flexuous, branching alternate to irregular at acute to wide angles, loosely reticulate, cells 24-32 × 4-6 $\mu \mathrm{m}$. Appressoria alternate, about $15 \%$ opposite, antrorse, subantrorse to perpendicular to the hyphae, 12$16 \mu \mathrm{m}$ long; stalk cells cylindrical to cuneate, 3-4 $\mu \mathrm{m}$ long; head cells ovate to globose, stellately sublobate, $9-13 \times 12-15 \mu \mathrm{m}$. Pycnothyria scattered, rarely connate, orbicular, up to $220 \mu \mathrm{m}$ diam., margin fimbriate, fringed hyphae very small, stellately dehisced at the centre; pycnothyriospores pyriform, brown, 24$26 \times 16-18 \mu \mathrm{m}$.

Material examined: Kerala, Pathanamthitta, Pachakanam, Uppupara, on leaves of Otonephelium stipulaceum (Bedd.) Radlk. (Sapindaceae), 15 December 2004, V.B. Hosagoudar HCIO 45988 TBGT 1752; Pathanamthitta, Gavi, 16 December 2004, V.B. Hosagoudar \& al. HCIO 46092, TBGT 1855; Idukki, Adimali, 14 February 2006, V. Gireesh Kumar \& H. Biju HCIO 47513, 47517, TBGT 2537, 2539.
These colonies were associated with Meliola otonepheli Hosag.

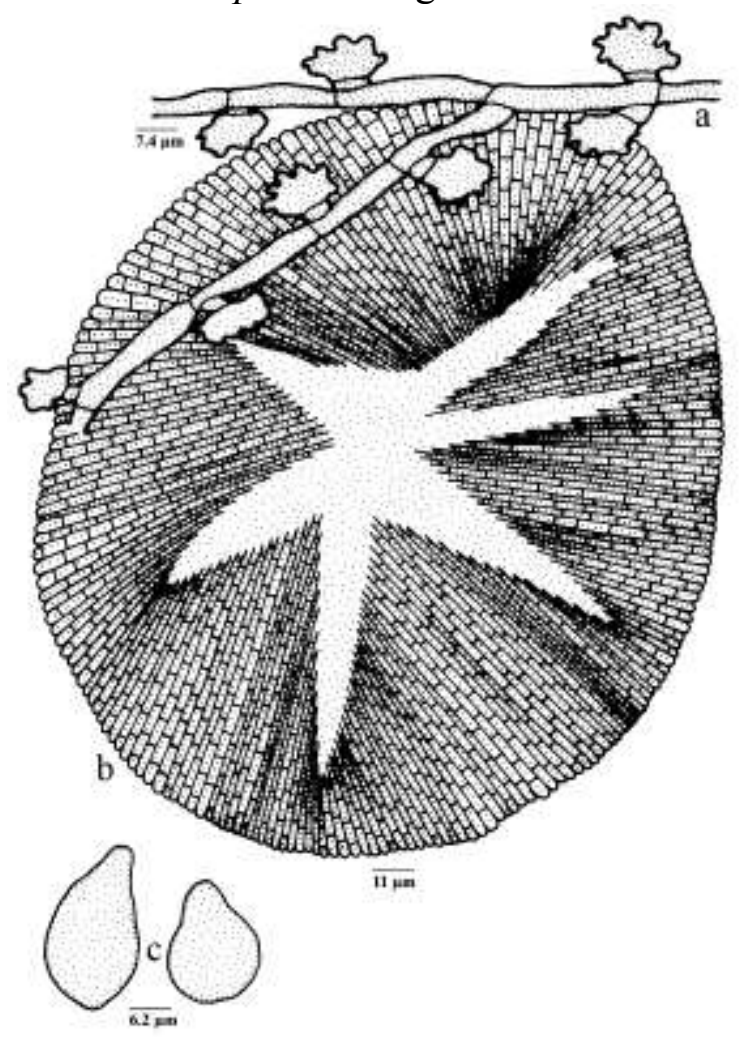

Figure 320 - Asterostomella otonephelii

Distribution: Kerala.

Asterostomella pongamiae K. V. Rao, Gopal \& Manoharachary, Asian J. P1. Sci. 1: 93, 1989.

Infection spots epiphyllous, irregular, variable in size, punctiform, mostly black; mycelium or hyphae superficial or semiimmersed, pale brown, septate branched 3-4 $\mu \mathrm{m}$; appressoria capitates, mostly 2 lobed, sessile, 7-9 × 4-5 $\mu \mathrm{m}$; pycnidia superficial, gregarious, olivaceous brown, globose to subglobose, unilocular, radiate, without ostiole, 46-105 $\mu \mathrm{m}$ diam.; conidiophores invisible; conidiogenous cells holoblastic, determinate, hyaline smooth, formed from the inner cells of the pycnothyrial wall, conidia dry, simple, unicellular, obovoid or pyriform, reddish brown, smooth and thick -walled with truncate base. $25-32 \times 18-20 \mu \mathrm{m}$.

Material examined: Andhra Pradesh, Vikarbad, Anantagiri hills, on leaves of pongamia glabra Vent. (Fabaceae), 18 March 1979, IMI 2395546 (holotype), OUSFH 1/A 
Distribution: Andhra Pradesh.

Asterostomella radermacherae Hosag., Balakr. \& Goos, Mycotaxon 58: 495, 1996; Hosag., Zoos' Print J. 18: 1280, 2003; Hosag., Chandraprabha \& Agarwal, Asterinales of Kerala, p. 236, 2011. (Fig. 321)

Colonies epiphyllous, dense, up to 2 $\mathrm{mm}$ in diameter, rarely confluent. Hyphae flexuous, branching alternate to irregular at acute to wide angles, loosely reticulate, cells $24-28 \times 3-5 \mu \mathrm{m}$. Appressoria alternate, about $1 \%$ opposite, straight to curved, sessile to stipitate, 6-15.5 $\mu \mathrm{m}$ long; stalk cells (where stipitate) cylindrical to cuneate, 2-12.5 $\mu \mathrm{m}$ long; head cells ovate, globose, entire, angular to sublobate, $3-8 \times 5-8.5 \mu \mathrm{m}$. Pycnothyria mostly scattered, round, 37-46.5 $\mu \mathrm{m}$ in diameter, central portion dissolved and Pycnothyriospores exposed, margin crenate; Pycnothyriospores deep brown, globose to pyriform, $15-18.5 \times 12-14 \mu \mathrm{m}$.

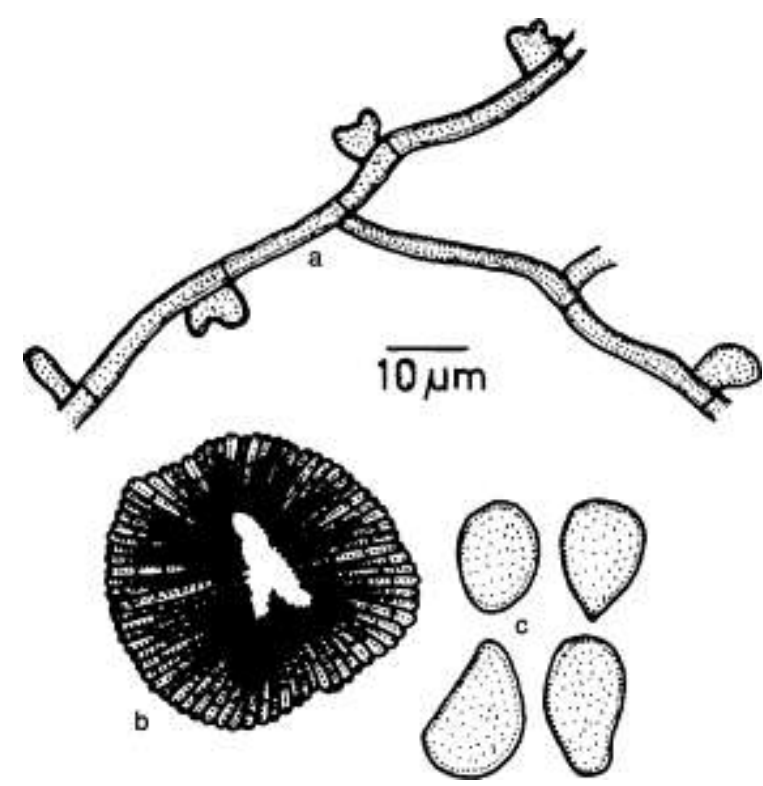

Figure 321 - Asterostomella radermacherae

Material examined: Kerala, Idukki, in the forest between Painavu and Kulamavu, on leaves of Radermachera xylocarpa (Roxb.) K. Schum. (Bignonaceae), 25 December 1984, V.B. Hosagoudar HCIO 30983 (holotype).

This species is similar to Asterina memorae Henn. and A. inaequalis Mont. var. nodulosa Theiss. but differs from then in having entire to lobate appressoria and smaller pycnothyriospores (Hennings, 1904; Theissen, 1913).

Distribution: Kerala.

Asterostomella scolopiae-crenatae Hosag. \& Abraham, New Botanist 24: 111, 1997; Hosag.,C.K. Biju\& Abraham, J. Econ. Taxon. Bot. 25: 307, 2001; Hosag., Zoos' Print J. 18: 1283, 2003; Hosag., Zoos' Print J. 21: 2412, 2006; Hosag., H. Biju \& Appaiah, J. Mycopathol. Res. 44:14, 2006; Hosag. \& H. Biju, J. Mycopathol. Res. 44: 43, 2006; Hosag., Chandraprabha \& Agarwal, Asterinales of Kerala, p. 237, 2011.

(Fig. 322)

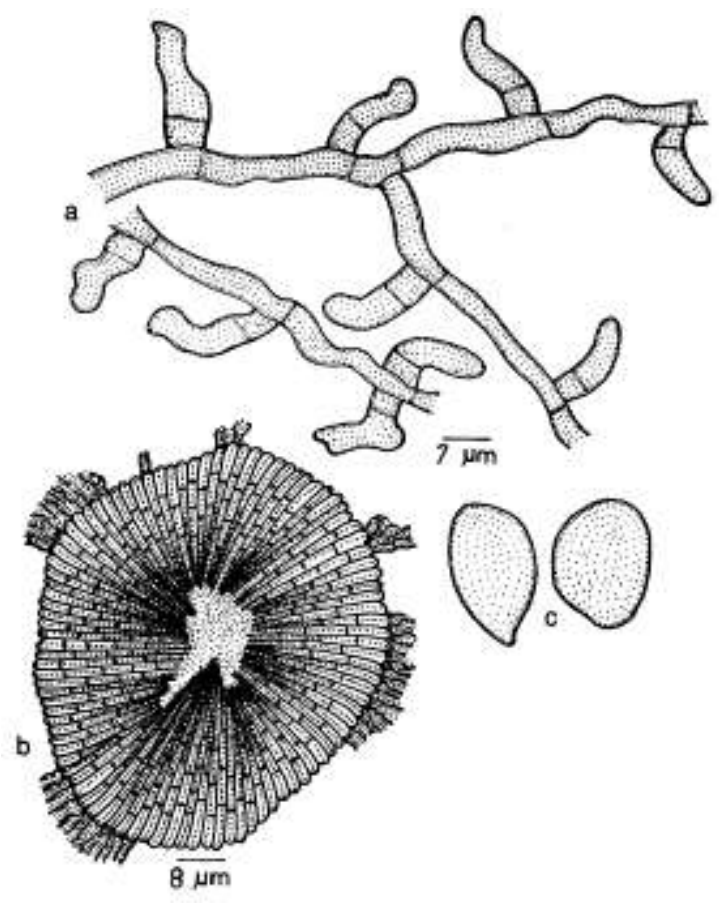

Figure 322 - Asterostomella scolopiaecrenatae

Colonies hypophyllous, very thin, spreading, up to $10 \mathrm{~mm}$ in diameter, confluent. Hyphae fledxuous to rarely crooked, branching irregular at acute to wide angles, loosely reticulate, cells $24-30 \times 3-3.5 \mu \mathrm{m}$. Appressoria alternate, about $30 \%$ opposite, 2-celled, straight, flexuous, curved, uncinate, $12-19.5 \mu \mathrm{m}$ long; stalk cells cylindrical, 3-5 $\mu \mathrm{m}$ long; head cells cylindrical, straight, curved, uncinate, flexuous, crooked, entire, angular, hamate, 9- 
$14.5 \times 4-5 \mu \mathrm{m}$. Pycnothyria scattered, orbicular, up to $75 \mu \mathrm{m}$ in diameter, stellately dehisce at the center, margin crenate to fimbriate, fringed hyphae small, flexuous to crooked; Pycnothyriospores pyriform, acute at one end and broadly rounded at another, cinnamon brown, 19-22.5 × 14-16 $\mu \mathrm{m}$, wall smooth, germ pore distinct in some spores.

\section{Material examined: Kerala,} Thiruvananthapuram, Peppara and Neyyar Wildlife Sanctuaries, Attayar, on leaves of Scolopia crenata (Wight \& Arn.) D. Clos (Flacourtiaceae), 18 April 1997, V.B. Hosagoudar HCIO 42476 (holotype), TBGT 116 (isotype); Palghat, Sairandhri, on leaves of Flacourtiaceae member, 13 December 2003, V.B. Hosagoudar \& al. HCIO 45761, TBGT 1510; Idukki, Munnar, Rajamala, 14 September 1999, C.K. Biju HCIO 45222 TBGT 1259; Idukki, Munnar, Rajamala, 5 January 1999, C.K. Biju HCIO 45271, TBGT 1309; Palghat, Silent Valley National Park, on leaves of 14 February 2007, M.C. Riju \& al. TBGT 5511; Karnataka, Kodagu, Nishane motta, 11 November 2003, V.B. Hosagoudar \& al. HCIO 45793, TBGT 1542; Kodagu, Nishane motta, on leaves of Scolopia sp., 13 November 2003, V.B. Hosagoudar \& al. HCIO 45813, TBGT 1563; Coorg, Madikeri, Talacauvery, MPCA, 13 November 2003, V.B. Hosagoudar HCIO 46269, TBGT 1915.

These colonies were associated with the colonies of Asteridiella scolopiae Hosag. and Meliola scolopiae Doidge var. indica Hosag.

This is an anamorph of the genus Asterina. Asterina cylindrophora Sydow \& Sydow, A. flacourtiacearum Hosag. \& Ravikumar and A. granulosa (Hansf.) Hosag. et al. (Sydow \& Sydow, 1917; Hosagoudar et al. 1996) are known on this host genus. However, the present new species differs from all in having very thin hypophyllous colonies and long cylindrical head cells of the appressoria.

Distribution: Kerala, Karnataka.

Asterostomella shoreae K.K. Soni, V.B. Hosagoudar, Abhishekh Pyasi \& R. K. Verma, sp. nov. (Fig. 323)
MycoBank 101020

Etymology: Named after the host genus

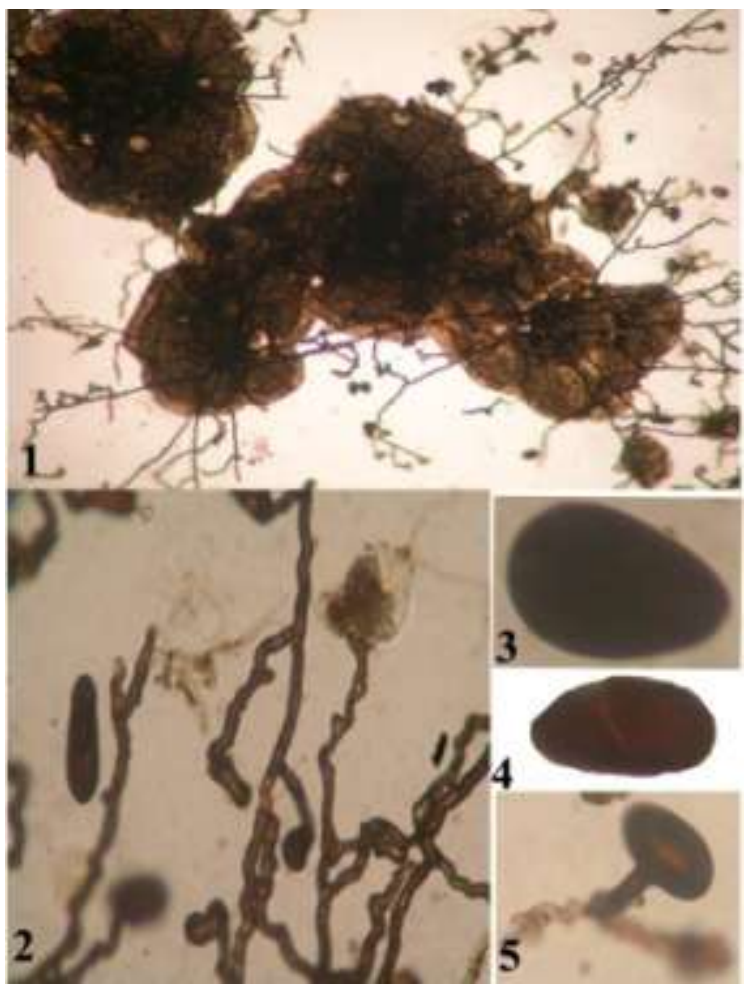

Figure 323 - Asterostomella shoreae

1. Mycelial colony with aggregated pycnothyria. 2. Multicellular stalk cells of appressoria with oblong pycnothyriospores, 34. Pycnothyriospores, 5. Germinating pycnothyriospores.

Colonies hypophyllous, minute, dense, scattered to coalesced, up to $1 \mathrm{~mm}$ in diam. Hyphae mostly flexuous, cross connecting hyphae crooked, branching mostly opposite at acute to wide angles, loosely to closely reticulate, cells 21-26 × 5-7 $\mu \mathrm{m}$. Appressoria scattered, alternate to opposite, antrorse to retrorse, arched, irregularly uncinate, 32-126 $\mu \mathrm{m}$ long; stalk cells unicellular to multicellular, cuneate, flexuous, crooked, arched, uncinate, 9$110 \mu \mathrm{m}$ long; head cells ovate, globose, oblong, boat shaped, shallowly lobate to deeply and irregularly lobate, $26-34 \times 16-19 \mu \mathrm{m}$, wall smooth.

Material examined: Chattisgarh, Achanakmar Biosphere Reserve, on leaves of Shorea robusta Gaertn.f. (Dipterocarpaceae), 18 March 2010, K.K. Soni TBGT 4264 (holotype), TF (2014 isotype). 
This is an anamorph of the genus Asterina. A. camarinensis Sydow \& Sydow and A. shoreana Sacc. Are known on Parashorea plicata from Philippines (Saccardo, 1924; Hosagoudar \& Abraham, 2000). However, the present anamorph differs from both in having multicellular appressoria. Asterina plurisporus Ryan is known on Shorea talura from Siddapur, North Canara, Karnataka (Ryan, 1928). However, Asterostomella shoreae differs from it in having multicellular stalk cells of the appressoria.

Distribution: Chhattisgarh.

Asterostomella strombosiae Hosag., D.K. Agarwal, H.Biju and Archana, Indian Phytopathology 59(4): 527, 2006.

(Fig. 324)

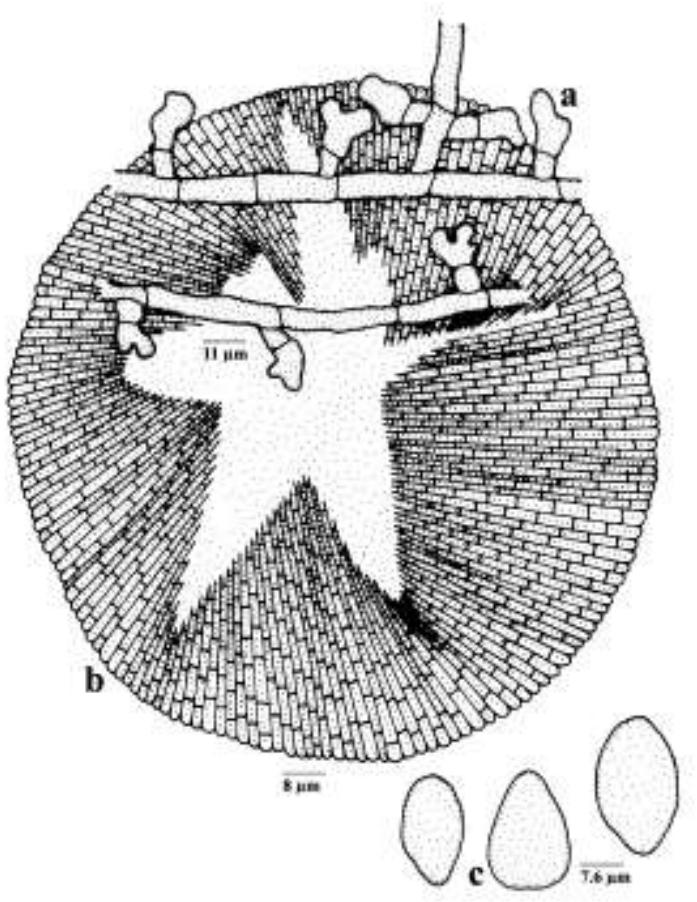

Figure 324 - Asterostomella strombosiae

Colonies epiphyllous, thin to dense, up to $2 \mathrm{~mm}$ in diam., Hyphae straight to substraight, branching irregular at wide angles, loosely reticulate, cells $27-35 \times 4-8 \mu \mathrm{m}$. Appressoria alternate, about $10 \%$ opposite, subantrorse to perpendicular to hyphae, two celled, 12-20 $\mu \mathrm{m}$ long; stalk cells cylindrical to cuneate, 4-13 $\mu \mathrm{m}$ long; head cells ovate, cylindrical, clavate, angular, sublobate to deeply lobate, 6-8 $\times 6-11 \mu \mathrm{m}$. Pycnothyria scattered, orbicular, up to $140 \mu \mathrm{m}$ in diam., stellately dehisce at the centre, margin crenate to fimbriate, fringed hyphae small; pycnothyriospores pyriform, ovate to ellipsoidal, brown, $16-26 \times 12-15 \mu \mathrm{m}$.

Material examined: Karnataka, South Canara, Kukke Subramanya, on leaves of Strombosia ceylanica Garden. (Olaceae), 15 November 2003, V.B. Hosagoudar \& al. HCIO 45972 (holotype), TBGT 1736 (isotype).

This is the only report of the genus Asterostomella on this host genus.

Distribution: Karnataka.

Asterostomella terminaliae Hosag., Balakr. \& Goos, Mycotaxon 58: 496, 1996.

(Fig. 325)

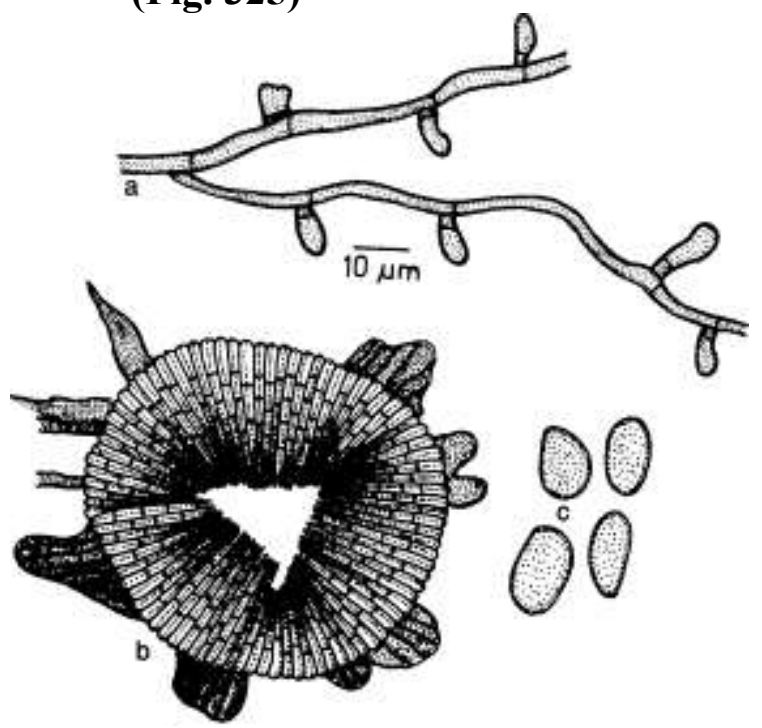

Figure 325 - Asterostomella terminaliae

Colonies epiphyllous, thin, crustose, up to $5 \mathrm{~mm}$ in diameter, confluent. Hyphae flexuous to crooked, branching opposite, alternate to irregular at acute angles, loosely reticulate, cells 15-37 × 3-4.5 $\mu \mathrm{m}$. Appressoria alternate and unilateral, 2-celled, straight to curved, 9-12.5 $\mu \mathrm{m}$ long; stalk cells cylindrical to cuneate, 2-3.5 $\mu \mathrm{m}$ long; head cells ovate, globose, pointed to truncate at apex, entire to rarely lobate, 4-6.5 × 4.5-6.5 $\mu \mathrm{m}$. Pycnothyria scattered, roughly rounded, up to $62 \mu \mathrm{m}$ in diameter, stellately dehisced at the center, margin crenate; pycnothyriospores oval to ellipsoidal, brown, unicellular, cent4ral lighter median band present, $15-17 \times 5-7 \mu \mathrm{m}$. 
Material examined: Tamil Nadu, Nilgiris, Gudalur, Benne forest, on leaves of Terminalia paniculata Roth. (Combertaceae), 24 January 1990, V.B. Hosagoudar HCIO 30984 (holotype); Uttar Pradesh, Maharaganj, on leaves of T. arjuna (Roxb. ex DC.) Wight \& Arn., A.W. Nomani TBGT 3521.

Based on the lighter median band in the Pycnothyriospores, the new species is similar to the anamorph of Asterina combreti Sydow reported on combretum species (Doidge, 1942) but differs from it inhaving smaller capitate appressoriaand Pycnothyriospores. It also differs from Asterina terminaliae Thite in morphology and size.

\section{Pradesh}

Distribution: Tamil Nadu, Uttar

Asterostomella xylosmae V.B. Hosagoudar, M.A. Nomani \& Kamal, sp. nov.

(Fig. 326)

MycoBank 101021

Etymology: Named after the host genus

Colonies amphigenous, thin to dense, up to $5 \mathrm{~mm}$ in diameter, confluent and cover entire both the upper and lower surfaces of the leaves. Hyphae substraight to flexuous, branching mostly opposite at acute to wide angles, loosely to closely reticulate, cells $16-23 \times 3-5 \mu \mathrm{m}$. Appressoria mostly opposite, often alternate to solitary, two celled, straight to irregularly curved, antrorse to retrorse, 11-23 $\mu \mathrm{m}$ long; stalk cells cylindrical to cuneate, 1-3 $\mu \mathrm{m}$ long; head cells ovate, oblong, cylindrical, entire, straight, uncinate to irregularly curved, $8-12 \times$ 2-5 $\mu \mathrm{m}$. Thyriothecia scattered, orbicular, up to $100 \mu \mathrm{m}$ in diam., stellately dehisced at the centre, margin mostly fimbriate, fringed hyphae straight to crooked; pycnothyriospores unicellular, long-pyriform, broadly rounded at one end and attenuated at the other, 19-23 $\times 8$ $13 \mu \mathrm{m}$.

Material examined: Uttar Pradesh, Kataranya Ghat, on leaves of Xylosma longifolium Hook.f. \& Thomson
(Flacourtiaceae), M.A. Nomani TBGT 3526 (holotype).

This is the only species known on this host under the form genus Asterostomella.

Distribution: Uttar Pradesh

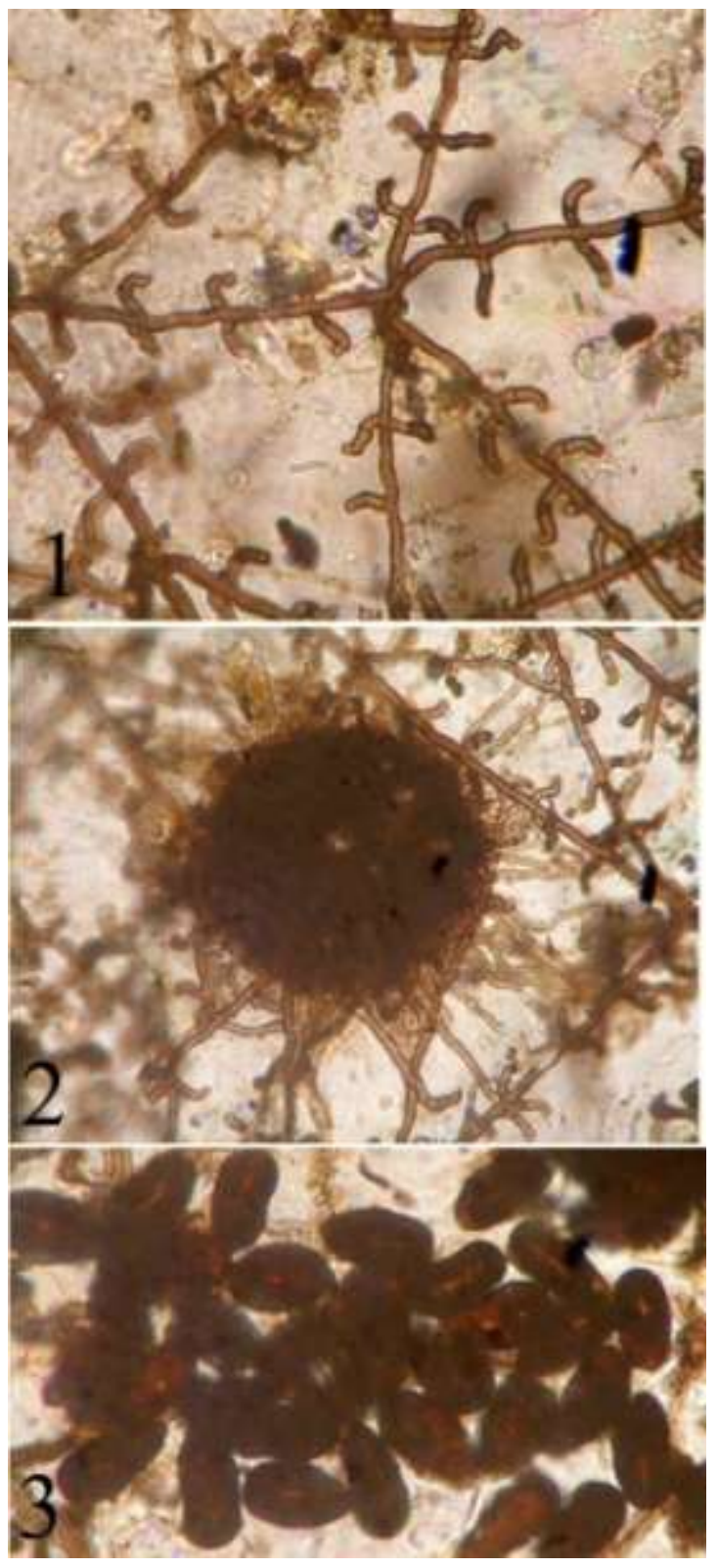

Figure 326 - Asterostomella xylosmae

1. Appressoriate mycelium, 2. Pycnothyrium, 3. Pycnothyriospores

Asterostomella ziziphina V.B. Hosagoudar \& G.R. Archana, sp.nov. (Fig. 327)

MycoBank 101022

Etymology: Named after the host genus 


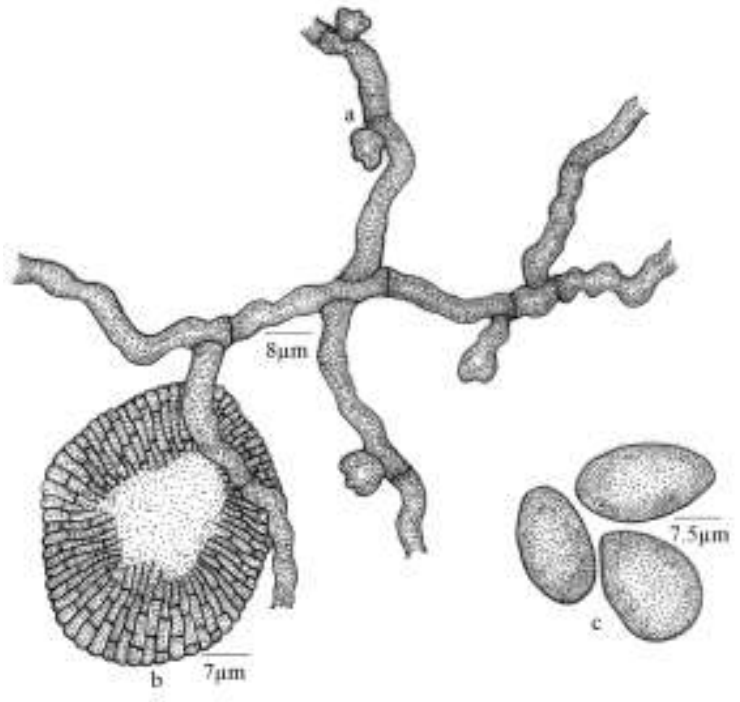

Figure 327 - Asterostomella ziziphina

Colonies amphigenous, thin, associated with colonies of Meliola zizyphi, up to $2 \mathrm{~mm}$ in diameter, confluent. Hyphae undulating to crooked, branching irregular at acute to wide angles, loosely to closely reticulate, cells 12-24 $\times$ 1-3 $\mu \mathrm{m}$. Appressoria alternate, unilateral, sessile, straight to curved, angular to sublobate 4-8 $\times$ 4-7 $\mu \mathrm{m}$. Pycnothyria scattered to grouped, orbicular, up to $36 \mu \mathrm{m}$ in diam.; pycnothyriospores pyriform, globose to ovate, $9-13 \times 8-10 \mu \mathrm{m}$.

\section{Material examined: Kerala,} Thiruvananthapuram, Palode, on leaves of Zizyphus rugosa Lam. (Rhamnaceae), 6 June 2009, G.R. Archana. TBGT 4813 (holotype).

Mycelium having very thin hyphae.

Distribution: Kerala

\section{THE GENUS Asterostomula}

Mycelium superficial, septate, lacking appressoria (Batista \& Ciferri 1959). Pycnothyria scutate, orbicular, with radiating cells on the upper surface, stellately dehiscing at the centre, with a crenate to fimbriate margin (Batista \& Ciferri 1959). Pycnothyriospores brown, unicellular, ovate, clavate, and pyriform to cylindrical.

Type sp.: A. loranthi Theiss.

\section{Abbreviations used in the line drawings:}

a. Mycelium, b. Thyriothecium, c. Pycnothyriospores

\section{DESCRIPTIONS OFTHE SPECIES}

Asterostomula loranthi Theiss., Ann. Mycol. 14: 270, 1916; Hosag, Sabeena \& Jacob-Thomas, Plant Pathology \& Quarantine 1(1): 7, 2011. (Fig. 328)

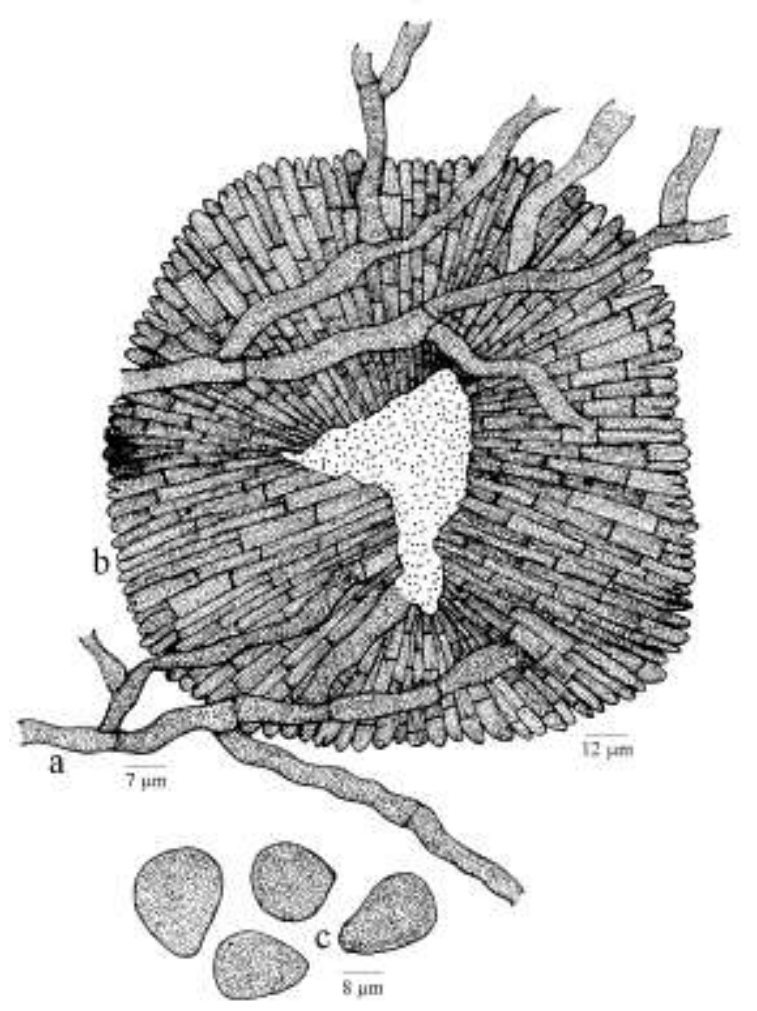

Figure 328 - Asterostomula loranthi

Colonies amphigenous, subdense to dense, up to $4 \mathrm{~mm}$ in diameter, confluent. Hyphae flexuous to crooked, branching irregular at acute to wide angles, closely reticulate, cells $25-40 \times 3-5 \mu \mathrm{m}$. Appressoria absent. Pycnothyria many, orbicular, joined together marginally, up to $180 \mu \mathrm{m}$ in diameter, stellately dehisced at the centre, margin crenate to fimbriate, fringed hyphae flexuous; pycnothyriospores unicellular, pyriform, ovate, $17-25 \times 12-17 \mu \mathrm{m}$, wall smooth.

Material examined: Kerala, Thiruvananthapuram, Bonacaud, on leaves of Loranthus sp. (Loranthaceae), 14 February 2008, Jacob Thomas \& K. Anilkumar TBGT 4564 (holotype). Part of the collection has been deposited in HCIO, New Delhi. 
This is the type species of the genus

Distribution: Kerala

Asterostomula pavettae V.B. Hosagoudar \& A.

Sabeena, sp. nov. (Fig. 329)

MycoBank 101023

Etymology: Named after the host genus

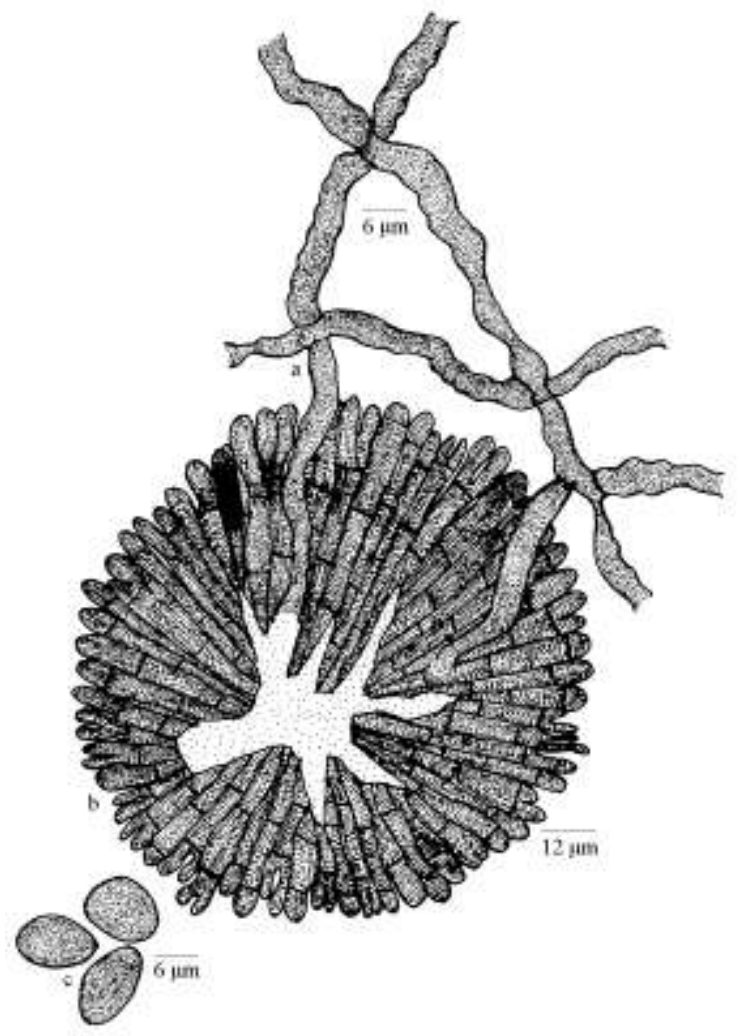

Figure 329 - Asterostomula pavettae

Colonies amphigenous, thin, up to 2 $\mathrm{mm}$ in diameter, confluent. Hyphae flexuous to crooked, branching irregular at acute to wide angles, cells 20-37 × 5-7 $\mu \mathrm{m}$. Pycnothyria scattered to connate, orbicular, up to $140 \mu \mathrm{m}$ in diameter, stellately dehisced at the centre, margin mostly crenate; pycnothyriospores ovate to pyriform, $17-27 \times 10-12 \mu \mathrm{m}$.

\section{Material examined: Kerala,} Thiruvananthapuram, Palode, TBGRI Campus, on leaves of Pavetta sp. (Rubiaceae), 10 October, 2008, A. Anil Kumar TBGT 5755 (holotype).

This the only species known on this host genus

Asterostomula syzygii Hosag,, Sabeena \& Jacob-Thomas, Plant Pathology \& Quarantine 1(1): 8, 2011. (Fig. 330)
Colonies hypophyllous, thin to dense, up to $2 \mathrm{~mm}$ in diameter, confluent. Hyphae flexuous to crooked, branching irregular at acute to wide angles, loosely to closely reticulate, cells 13-40 × 2-3 $\mu \mathrm{m}$. Appressoria absent. Pycnothyria scattered, orbicular, up to $125 \mu \mathrm{m}$ in diameter, stellately dehisced at the centre; pycnothyriospores globose to ovate, brown, $14-19 \times 11-14 \mu \mathrm{m}$.

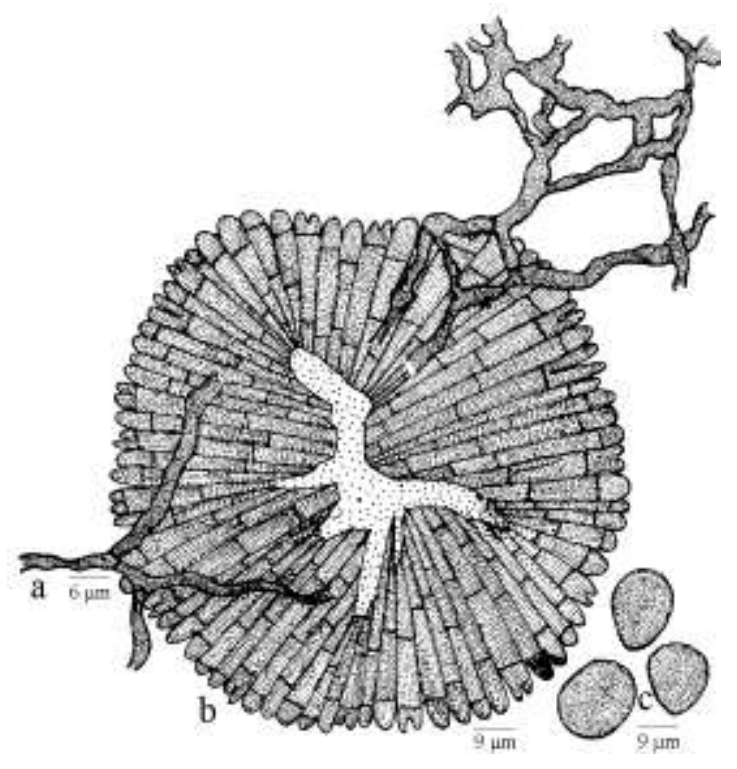

Figure 330 - Asterostomula syzygii

$\begin{array}{ccc}\text { Material } & \text { examined: } & \text { Kerala, } \\ \text { Thiruvananthapuram, } & \text { Peppara } & \text { Wildlife }\end{array}$ Sanctuary, on leaves of Syzygium sp. (Myrtaceae), 18 November 2007, Jacob Thomas TBGT 3474 (holotype). Part of the collection has been deposited in HCIO, New Delhi.

This is the only species of the genus Asterostomulaon the members of the family Myrtaceae.

Distribution: Kerala

\section{THE GENUS Bramhamyces}

Bramhamyces Hosag., Indian J. Sci. Technol. 2 (6):17, 2009; Hosag., Chandraprabha \& Agarwal, Asterinales of Kerala, p. 239, 2011.

Leaf parasites. Hyphae brown, branched, septate, ramify in the grooves only around stomata to form 'areole' to produce 1-3appressoria. Remaining hyphae devoid of 
appressoria. Appressoria produced on the guard cells of the stoma produce corolloid haustoria in the neighboring cells. Stomata often plugged with mycelium. Pycnothyria grown below the mycelium, orbicular, connate; pycnothyriospores, unicellular, brown, oval, pyriform.

Type sp.: B. ilecis Hosag. \& Chandra.

Mycelium devoid of appressoria but are produced around the stomata of the host plant by forming 'areole' is the character of this anamorph genus.

\section{DESCRIPTION OFTHE SPECIES}

Bramhamyces ilecis Hosag. \& Chandraprabha., Indian J. Sci. Technol. 2 (6):17, 2009; Hosag., Chandraprabha \& Agarwal, Asterinales of Kerala, p. 239, 2011.

(Fig. 331, 332)
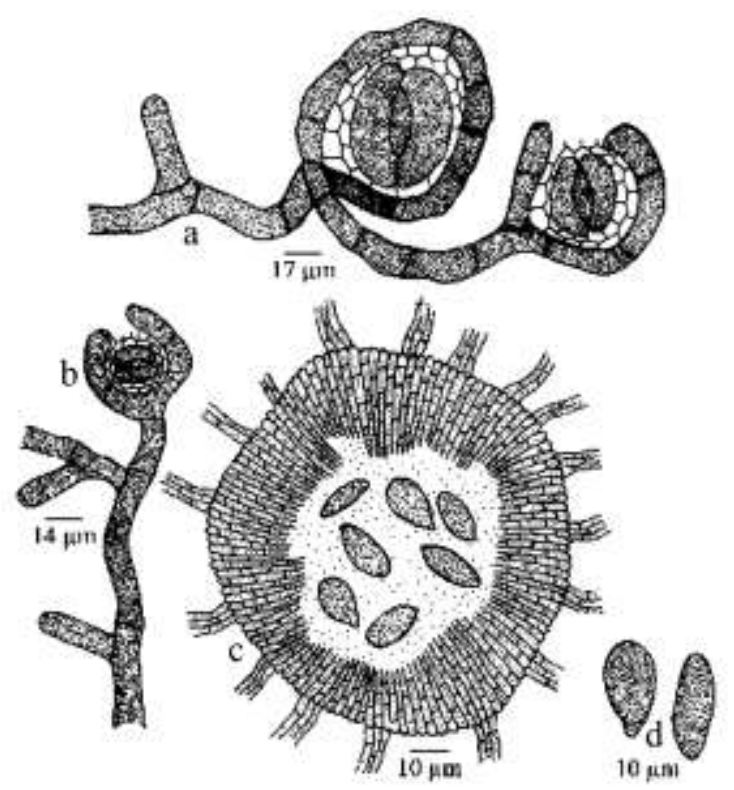

Figure 331 - Bramhamyces ilecis

$a-b$. Branched mycelium formed areole around stomata and showing appressoria at the tip of the mycelium near the guard cells, c. Pycnothyrium, d. Pycnothyriospores
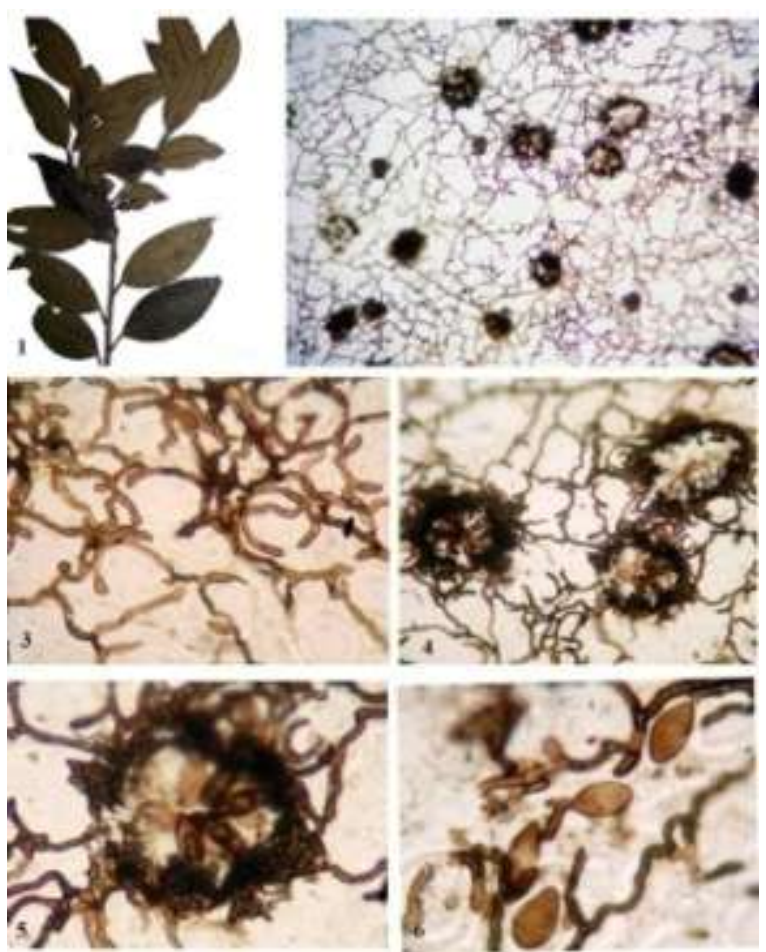

Figure 332 - Bramhamyces ilecis

1. Infected leaves, 2.Mycelial colony with Pycnothyria, 3. Hyphae coiled around stomata and ended with appressoria, 4. Pycnothyria, 5. Pycnothyrium with pycnothyriospores, 6 . Pycnothyriospores.

Colonies amphigenous, dense, up to 2 $\mathrm{mm}$ in diameter, rarely confluent. Hyphae straight to flexuous, branching at acute to wide angles, loosely to closely reticulate, cells 22-33 $\times$ 4-7 $\mu \mathrm{m}$, devoid of appressoria but hyphae form an 'areole' around the stoma by producing one to three appressoria. Appressoria produced on the tip of the 'areolar' net unicellular, ovate, oblong, entire, 9-15 × 4-7 $\mu \mathrm{m}$. Pycnothyria connate, formed below the mycelium, orbicular, up to $110 \mu \mathrm{m}$ in diameter, stellately dehisce at the center, margins crenate to fimbriate; pycnothyriospores brown oval to ellipsoidal, pyriform, unicellular, $20-26 \times 8-11 \mu \mathrm{m}$, wall smooth.

Material examined: Kerala, Idukki, Munnar, Mannavan shola, on the leaves of Ilex wightiana Wall. (Aquifoliaceae), 11 May 1999, C.K. Biju HCIO (holotype), TBGT 3019 (isotype).

The genus Bramhamyces is reported for the first time on the members of the family Aquifoliaceae. 
Distribution: Kerala.

\section{THE GENUS Mahanteshamyces}

Mahanteshamyces Hosag. J. Econ. Taxon. Bot. 28: 189, 2004; Hosag., Chandraprabha \& Agarwal, Asterinales of Kerala, p. 241, 2011.

Foliicolous, ectophytic, parasitic. Mycelium brown, superficial, appressoriate. Pycnothyria scutate, dimidiate, radiate, orbicular, stellately dehisced at the center; pycnothyriospores brown, angular, wall straight to sinuate.

Type sp.: M. agrostistachydis Hosag. \& C.K. Biju

The genus Mahanteshamyces differs from the genus Asterostomella in having roundedly projected and shallowly lobate, angular and thick walled pycnothyriospores (Batista and Cifferri, 1959; Sivanesan, 1981; Sutton, 1980). Hofmann \& Pipenbaring (2008) showed that this is an anamorph of the genus Asterina.

Abbreviations used in the line drawings:

a. Appressoriate mycelium, b. Thyriothecium, c. Pycnothyriospores

\section{DESCRIPTIONS OFTHE SPECIES}

Mahanteshamyces agrostachydis Hosag. \& C.K. Biju in Hosag.,C.K. Biju \& Abraham, J. Econ. Taxon. Bot. 28: 189, 2004; Hosag., Chandraprabha \& Agarwal, Asterinales of Kerala, p. 241, 2011. (Fig. 333)

Colonies hypophyllous, thin to subdense, spreading, up to $5 \mathrm{~mm}$ in diameter, confluent. Hyphae straight to substraight, branching alternate to opposite at acute angles, loosely to closely reticulate, cells 4-16 $\times 1.5$ $2.5 \mu \mathrm{m}$. Appressoria unicellular, alternate, about $20 \%$ opposite, ovate, globose, oblong, irregularly sublobate to lobate, $6-8 \times 4-6.5 \mu \mathrm{m}$. Pycnothyria scattered, orbicular, up to $80 \mu \mathrm{m}$ in diameter, margin crenate, stellately dehisced to widely opened at the center; pycnothyriospores brown, unicellular, truncate at the base, apex more or less flattened, 1-3 rounded projects or shallowly lobate, angular, $12-15 \times 11-13 \mu \mathrm{m}$, smooth, thick walled.

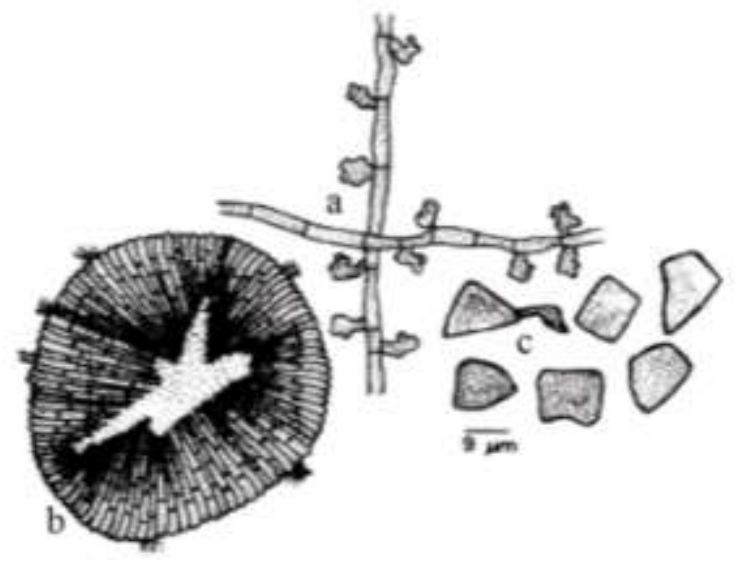

Figure 333 - Mahanteshamyces agrostachydis

Material examined: Kerala, Wayanad, towards the stream side of Chembra hills, on leaves of Agrostistachys indica Dalz. (Euphorbiaceae), 14 April 1999, C.K. Biju HCIO 43993 (holotype), TBGT 403 (isotype).

Distribution: Kerala.

\begin{tabular}{|l|l|}
\hline \multicolumn{2}{|c|}{ Host - Fungus - Index } \\
\hline Abutilon sp. & Asterina diplocarpa \\
\hline Acronychia laurifolia & $\begin{array}{l}\text { Asterina } \\
\text { rhabdodendri var. } \\
\text { levibus }\end{array}$ \\
\hline $\begin{array}{l}\text { Acronychia } \\
\text { longipedunculata }\end{array}$ & $\begin{array}{l}\text { Asterina } \\
\text { acronychiae }\end{array}$ \\
\hline $\begin{array}{l}\text { Acronychia } \\
\text { pedunculata }\end{array}$ & $\begin{array}{l}\text { Asterina } \\
\text { acronychiae }\end{array}$ \\
\hline Acronychia sp. & $\begin{array}{l}\text { Asterina } \\
\text { acronychiae }\end{array}$ \\
\hline Acrotrema arnottianum & Asterina acrotremae \\
\hline Adenia hondala & Asterina adeniicola \\
\hline Adenostemma viscosum & $\begin{array}{l}\text { Asterina } \\
\text { adenostemmatis }\end{array}$ \\
\hline Adhatoda beddomei & $\begin{array}{l}\text { Asterina tertia var. } \\
\text { africana }\end{array}$ \\
\hline Adhatoda beddomii & Asterina tertia \\
\hline Adhatoda vasica & Asterina tertia \\
\hline Adhatoda zeylanica & $\begin{array}{l}\text { Asterina tertia var. } \\
\text { africana }\end{array}$ \\
\hline Adhatoda zeylanica & Asterina tertia \\
\hline Aegle marmelos & Asterina delicatula \\
\hline Aganosma cymosum & Asterina aganosmae \\
\hline Aglaia sp. & Asterina aglaiae \\
\hline Agrostistachys indica & $\begin{array}{l}\text { Mahanteshamyces } \\
\text { agrostachydis }\end{array}$ \\
\hline Alangium lamarckii & Asterina balii \\
\hline
\end{tabular}




\begin{tabular}{|c|c|}
\hline Host - Fungus - Index & \\
\hline Alangium salvifolium & Asterina perpusilla \\
\hline Alangium sp. & Asterina perpusilla \\
\hline Anamirta cocculus & Asterina anamirtae \\
\hline Anamirta cocculus & $\begin{array}{l}\text { Prillieuxina } \\
\text { anamirtae }\end{array}$ \\
\hline Anamirta sp. & $\begin{array}{l}\text { Prillieuxina } \\
\text { anamirtae }\end{array}$ \\
\hline Anogeissus latifolia & Asterina anogeissi \\
\hline Anogeissus latifolia & $\begin{array}{l}\text { Asterostomella } \\
\text { anogeissi }\end{array}$ \\
\hline Aporusa bourdillonii & Asterina aporusae \\
\hline Aporusa lindleyana & Asterina aporusae \\
\hline Aporusa lindleyana & $\begin{array}{l}\text { Meliolaster } \\
\text { aporusae }\end{array}$ \\
\hline Aporusa roxburghii & $\begin{array}{l}\text { Asterina aporusae } \\
\text { var. cylindrica }\end{array}$ \\
\hline Araliaceae & $\begin{array}{l}\text { Lembosia } \\
\text { araliacearum }\end{array}$ \\
\hline Ardisia solanacea & Asterina ardisiae \\
\hline Ardisia solancea & Prillieuxina ardisiae \\
\hline Ardisia sonchifolia & Asterina ardisiicola \\
\hline Argyeria sp. & $\begin{array}{l}\text { Prillieuxina } \\
\text { argyreiae }\end{array}$ \\
\hline Argyreia nervosa & $\begin{array}{l}\text { Bheemamyces } \\
\text { argyreicola }\end{array}$ \\
\hline Aristolochia tagala & $\begin{array}{l}\text { Asterina } \\
\text { aristolochiae }\end{array}$ \\
\hline Asclepias curassavica & Asterina asclepiadis \\
\hline Asystasia chelanoide & Asterina tertia \\
\hline Asystasia sp. & Asterina tertia \\
\hline Asystasia violacea & Asterina tertia \\
\hline Atalantia rotundifolia & Asterina atalantiae \\
\hline Atalantia wightii & Asterina atalantiae \\
\hline Atalantia sp. & Asterina atalantiae \\
\hline Averrhoa carambola & Asterina averrhoae \\
\hline $\begin{array}{l}\text { Baliospermum } \\
\text { montanum }\end{array}$ & $\begin{array}{l}\text { Asterostomella } \\
\text { baliospermi }\end{array}$ \\
\hline Barleria involucrata & Asterina tertia \\
\hline Blumea sp. & Asterina blumeae \\
\hline $\begin{array}{l}\text { Boehmeria } \\
\text { glomerulifera }\end{array}$ & $\begin{array}{l}\text { Asterostomella } \\
\text { boehmeriae }\end{array}$ \\
\hline Boehmeria sp. & $\begin{array}{l}\text { Asterostomella } \\
\text { boehmeriae }\end{array}$ \\
\hline $\begin{array}{l}\text { Brassaiopsis } \\
\text { actinophila }\end{array}$ & Asterina araliae \\
\hline Calamus pseudotenuis & $\begin{array}{l}\text { Cirsosia } \\
\text { globuliferae }\end{array}$ \\
\hline Calamus sp. & $\begin{array}{l}\text { Cirsosia } \\
\text { globuliferae }\end{array}$ \\
\hline
\end{tabular}

\begin{tabular}{|c|c|}
\hline Calamus sp. & $\begin{array}{l}\text { Asterina } \\
\text { arecacearum }\end{array}$ \\
\hline Calamus tenues & $\begin{array}{l}\text { Lembosia } \\
\text { calamigena }\end{array}$ \\
\hline Calamus thwaitesii & $\begin{array}{l}\text { Cirsosia } \\
\text { arecacearum }\end{array}$ \\
\hline Calycopteris floribunda & Asterina combreti \\
\hline Calycopteris sp. & Asterina combreti \\
\hline Cansjera rheedii & Asterina cansjerae \\
\hline Cansjera rheedii & $\begin{array}{l}\text { Asterina } \\
\text { cansjericola }\end{array}$ \\
\hline Cansjera rheedii & $\begin{array}{l}\text { Asterina } \\
\text { cansjericola var. } \\
\text { indica }\end{array}$ \\
\hline Canthium dicoccum & $\begin{array}{l}\text { Asterina canthii- } \\
\text { dicocci }\end{array}$ \\
\hline Canthium sp. & $\begin{array}{l}\text { Asterina } \\
\text { canthiigena }\end{array}$ \\
\hline Capparis clegghornii & $\begin{array}{l}\text { Asterina } \\
\text { kodajadriensis }\end{array}$ \\
\hline Capparis horrida & Asterina capparidis \\
\hline Capparis sp. & $\begin{array}{l}\text { Bheemamyces } \\
\text { capparidis }\end{array}$ \\
\hline Capparis spinosa & Asterina capparidis \\
\hline Capparis zeylanica & Asterina capparidis \\
\hline Casearia esculenta & $\begin{array}{l}\text { Asterina caseariae- } \\
\text { esculentae }\end{array}$ \\
\hline Casearia tomentosa & Asterina caseariae \\
\hline Cassia fistula & Asterina cassiicola \\
\hline Ceropegia sp. & $\begin{array}{l}\text { Asterostomella } \\
\text { ceropegiae }\end{array}$ \\
\hline Chukrasia tabularis & Asterina chukrasiae \\
\hline Cinnamomum wightii & $\begin{array}{l}\text { Asterina } \\
\text { cinnamomicola }\end{array}$ \\
\hline Cinnamomum sp. & $\begin{array}{l}\text { Asterina } \\
\text { munnarensis }\end{array}$ \\
\hline Cinnamomum sp. & Asterina cinnamomi \\
\hline Cinnamomum sp. & $\begin{array}{l}\text { Asterina } \\
\text { cinnamomicola }\end{array}$ \\
\hline Cipadessa baccifera & Asterina cipadessae \\
\hline Cissus sp. & Asterina cissi \\
\hline Clematis sp. & Asterina clematidis \\
\hline $\begin{array}{l}\text { Clerodendrum } \\
\text { viscosum }\end{array}$ & $\begin{array}{l}\text { Asterina } \\
\text { kannurensis }\end{array}$ \\
\hline Coculus laurifolius & $\begin{array}{l}\text { Maheshwaramyces } \\
\text { coculi }\end{array}$ \\
\hline Combretum sp. & $\begin{array}{l}\text { Asterina combreti } \\
\text { var. cylindrica }\end{array}$ \\
\hline
\end{tabular}


Mycosphere Doi 10.5943/mycosphere/3/5/9

\begin{tabular}{|c|c|}
\hline Host - Fungus - Index & \\
\hline Crossandra sp. & Asterina tertia \\
\hline Croton roxburghii & $\begin{array}{l}\text { Asterostomella } \\
\text { kushinagarensis }\end{array}$ \\
\hline $\begin{array}{l}\text { Cryptocarya } \\
\text { bourdillonii }\end{array}$ & $\begin{array}{l}\text { Asterina } \\
\text { cryptocariicola }\end{array}$ \\
\hline Cryptocarya wightiana & $\begin{array}{l}\text { Asterina } \\
\text { cryptocariicola }\end{array}$ \\
\hline Cryptocarya sp. & $\begin{array}{l}\text { Asterina } \\
\text { cryptocariicola }\end{array}$ \\
\hline $\begin{array}{l}\text { Cyananchum } \\
\text { callialatum }\end{array}$ & Asterina cynanchi \\
\hline Cynanchum alatum & $\begin{array}{l}\text { Asterina } \\
\text { cynanchicola }\end{array}$ \\
\hline $\begin{array}{l}\text { Daphniphyllum } \\
\text { neilgherrense }\end{array}$ & $\begin{array}{l}\text { Asterostomella } \\
\text { daphniphylli }\end{array}$ \\
\hline Daphniphyllum sp. & $\begin{array}{l}\text { Asterostomella } \\
\text { daphniphylli }\end{array}$ \\
\hline Dendrophthoe falcata & Asterina deightonii \\
\hline $\begin{array}{l}\text { Dendrophthoe sp. } \\
\text { Loranthus sp. }\end{array}$ & $\begin{array}{l}\text { Asterina } \\
\text { loranthigena }\end{array}$ \\
\hline Dendrophthoe sp. & Asterina deightonii \\
\hline $\begin{array}{l}\text { Dichapetalum } \\
\text { gelaniodes }\end{array}$ & Asterina dichapetali \\
\hline Dillenia pentagyna & $\begin{array}{l}\text { Asterostomella } \\
\text { dilleniacearum }\end{array}$ \\
\hline Dillenia sp. & $\begin{array}{l}\text { Asterostomella } \\
\text { dilleniacearum }\end{array}$ \\
\hline Diospyros malabaricus & Prillieuxina diospyri \\
\hline Diospyros sp. & Asterina diospyri \\
\hline $\begin{array}{l}\text { Diotachanthus } \\
\text { albiflorus }\end{array}$ & $\begin{array}{ll}\text { Asterina } & \text { tertia } \\
\text { var.africana } & \end{array}$ \\
\hline Dipteracanthus patulus & Asterina tertia \\
\hline $\begin{array}{l}\text { Drimycarpus } \\
\text { racemosus }\end{array}$ & Asterina drimycarpi \\
\hline Elaeagnus kologa & Prillieuxina elaegni \\
\hline Elaegnus kologa & Asterina elaegni \\
\hline $\begin{array}{l}\text { Elaeocarpus } \\
\text { glandulosus }\end{array}$ & Asterina gamsii \\
\hline Elaeocarpus munronii & $\begin{array}{l}\text { Asterina } \\
\text { elaeocarpicola }\end{array}$ \\
\hline Elaeocarpus sp. & $\begin{array}{l}\text { Asterina elaeocarpi } \\
\text { var. ovalis }\end{array}$ \\
\hline Elaeocarpus serratus & $\begin{array}{l}\text { Asterostomella } \\
\text { elaeocarpi-serrati }\end{array}$ \\
\hline Elaeocarpus sp. & $\begin{array}{l}\text { Asterina elaeocarpi } \\
\text { var. ovalis }\end{array}$ \\
\hline Elaeocarpus tectorius & Asterina gamsii \\
\hline $\begin{array}{l}\text { Elaeocarpus } \\
\text { tuberculatus }\end{array}$ & $\begin{array}{l}\text { Asterina elaeocarpi } \\
\text { var. ovalis }\end{array}$ \\
\hline Elaeocarpus variabilis & Asterina gamsii \\
\hline
\end{tabular}

\begin{tabular}{|c|c|}
\hline Elatostema lineolatum & $\begin{array}{l}\text { Asterina } \\
\text { elatostematis }\end{array}$ \\
\hline Enicostema axillare & $\begin{array}{l}\text { Asterina } \\
\text { enicostematis }\end{array}$ \\
\hline Eranthemum capense & $\begin{array}{l}\text { Asterina tertia var. } \\
\text { africana }\end{array}$ \\
\hline $\begin{array}{l}\text { Erythropalum } \\
\text { populifolium }\end{array}$ & $\begin{array}{l}\text { Asterina } \\
\text { erythropalicola }\end{array}$ \\
\hline Eugenia discifera & Asterina disciferae \\
\hline Euodia luna-ankenda & $\begin{array}{l}\text { Asterina } \\
\text { melicopecola }\end{array}$ \\
\hline Euonymous crenulatus & Asterina euonymi \\
\hline $\begin{array}{l}\text { Eupatorium } \\
\text { glandulosum }\end{array}$ & Asterina eupatorii \\
\hline Eurya japonica & Asterina cannonii \\
\hline Eurya japonica & $\begin{array}{l}\text { Asterina } \\
\text { theacearum }\end{array}$ \\
\hline Eurya nitida & Asterina songii \\
\hline Eurya sp. & Asterina songii \\
\hline Eurya japonica & Asterina songii \\
\hline Eurya sp. & $\begin{array}{l}\text { Asterina } \\
\text { theacearum }\end{array}$ \\
\hline Excoecaria crenulata & $\begin{array}{l}\text { Asterostomella } \\
\text { excoecariicola }\end{array}$ \\
\hline Ficus sp. & Asterina mysorensis \\
\hline Flacourtia indica & $\begin{array}{l}\text { Ishwaramyces } \\
\text { flacourtiae }\end{array}$ \\
\hline Flacourtia montana & $\begin{array}{l}\text { Ishwaramyces } \\
\text { flacourtiae }\end{array}$ \\
\hline Flacourtia montana & $\begin{array}{l}\text { Asterostomella } \\
\text { flacourtiae- } \\
\text { montanae }\end{array}$ \\
\hline Garcinia gummigutta & $\begin{array}{l}\text { Asterina } \\
\text { clusiacearum }\end{array}$ \\
\hline Garcinia gummigutta & Lembosia garciniae \\
\hline Garcinia imberti & $\begin{array}{l}\text { Prillieuxina } \\
\text { garciniae }\end{array}$ \\
\hline Garcinia morella & Asterina morellae \\
\hline Garcinia travancorica & Asterina garciniae \\
\hline Garcinia sp. & $\begin{array}{l}\text { Asterina } \\
\text { garciniicola }\end{array}$ \\
\hline Garcinia sp. & Asterina garciniae \\
\hline Girardinia diversifolia & Asterina girardiniae \\
\hline Glochidion sp. & $\begin{array}{l}\text { Asterina lobulifera } \\
\text { var. indica }\end{array}$ \\
\hline Glochidionsp. & Asterina lobulifera \\
\hline Glycosmis pentaphylla & $\begin{array}{l}\text { Asterina } \\
\text { banguiensis }\end{array}$ \\
\hline
\end{tabular}




\begin{tabular}{|c|c|}
\hline Host - Fungus - Index & \\
\hline Glycosmis pentaphylla & $\begin{array}{l}\text { Asterina } \\
\text { glycosmidigena }\end{array}$ \\
\hline Glycosmis sp. & $\begin{array}{l}\text { Asterina } \\
\text { glycosmidigena }\end{array}$ \\
\hline Glycosmis sp. & $\begin{array}{l}\text { Asterina } \\
\text { glycosmidis }\end{array}$ \\
\hline $\begin{array}{l}\text { Glyptopetalum } \\
\text { zeylanicum }\end{array}$ & $\begin{array}{l}\text { Asterina } \\
\text { glyptopetali }\end{array}$ \\
\hline Gomphandra sp. & $\begin{array}{l}\text { Asterina } \\
\text { gomphandrae }\end{array}$ \\
\hline $\begin{array}{l}\text { Goniothalmus } \\
\text { rhyncantherus }\end{array}$ & $\begin{array}{l}\text { Trichasterina } \\
\text { goniothalami }\end{array}$ \\
\hline Goniothalmus wighti & $\begin{array}{l}\text { Trichasterina } \\
\text { goniothalami }\end{array}$ \\
\hline Gordonia sp. & $\begin{array}{l}\text { Asterina } \\
\text { theacearum }\end{array}$ \\
\hline Grewia sp. & Asterina wingfieldii \\
\hline Gymnema sylvestre & Asterina gymnemae \\
\hline Gymnema sp. & Asterina gymnemae \\
\hline Gymnosporia puberula & $\begin{array}{l}\text { Asterina } \\
\text { gymnosporiae }\end{array}$ \\
\hline Gymnosporia rothiana & Asterina dissiliens \\
\hline Helicteres isora & Asterina helicteris \\
\hline Helixanthera sp. & Asterina deightonii \\
\hline Hibiscus rosa-sinensis & Asterina hibisci \\
\hline Hibiscus sp. & Asterina hibisci \\
\hline Homalium zeylanica & $\begin{array}{l}\text { Asterina } \\
\text { homaligena }\end{array}$ \\
\hline Homonoia riparia & Asterina homonoiae \\
\hline Hopea parviflora & Asterina hopiicola \\
\hline Hopea ponga & Asterina hopeae \\
\hline Hopea ponga & Cirsosia hopeae \\
\hline Hopea ponga & $\begin{array}{l}\text { Echidnodella } \\
\text { hopeae }\end{array}$ \\
\hline Hugonia mystax & Asterina hugoniae \\
\hline Humboldtia unijuga & $\begin{array}{l}\text { Lembosia } \\
\text { humboldtiicola }\end{array}$ \\
\hline Humboldtia vahliana & $\begin{array}{l}\text { Lembosia } \\
\text { humboldtiae }\end{array}$ \\
\hline Humboldtia sp. & $\begin{array}{l}\text { Lembosia } \\
\text { humboldtiae }\end{array}$ \\
\hline Humboldtia vahliana & $\begin{array}{l}\text { Lembosia } \\
\text { humboldtiigena }\end{array}$ \\
\hline $\begin{array}{l}\text { Hydnocarpus } \\
\text { macrocarpa }\end{array}$ & Asterina hydnocarpi \\
\hline $\begin{array}{l}\text { Hydnocarpus } \\
\text { macrocarpus }\end{array}$ & $\begin{array}{l}\text { Asterolibertia } \\
\text { hydnocarpi }\end{array}$ \\
\hline $\begin{array}{l}\text { Hydnocarpus } \\
\text { pentandra }\end{array}$ & $\begin{array}{l}\text { Asterolibertia } \\
\text { hydnocarpi }\end{array}$ \\
\hline
\end{tabular}

\begin{tabular}{|c|c|}
\hline Hydnocarpus sp. & Asterina hydnocarpi \\
\hline Hydrocotyle javanica & $\begin{array}{l}\text { Asterina } \\
\text { hydrocotyles }\end{array}$ \\
\hline Hydrocotyle sp. & $\begin{array}{l}\text { Asterina } \\
\text { hydrocotyles }\end{array}$ \\
\hline Hyptis sauveolense & Asterina hyptidicola \\
\hline Ilex denticulata & $\begin{array}{l}\text { Prillieuxina } \\
\text { aquifoliacearum }\end{array}$ \\
\hline Ilex walker & $\begin{array}{l}\text { Asterina } \\
\text { bottomleyae }\end{array}$ \\
\hline Ilex wightiana & $\begin{array}{l}\text { Asterina } \\
\text { bottomleyae }\end{array}$ \\
\hline Ilex wightiana & Bramhamyces ilecis \\
\hline Impatiens viscida & $\begin{array}{l}\text { Asterina } \\
\text { dhivaharanii }\end{array}$ \\
\hline Isonandra lanceolata & $\begin{array}{l}\text { Asterostomella } \\
\text { isonadrae }\end{array}$ \\
\hline Ixora coccinea & $\begin{array}{l}\text { Prillieuxina } \\
\text { ixorigena }\end{array}$ \\
\hline Ixora lanceolariae & Asterina ixorae \\
\hline $\begin{array}{l}\text { Jasminum } \\
\text { angustifolium }\end{array}$ & $\begin{array}{l}\text { Asterina } \\
\text { erysiphoides }\end{array}$ \\
\hline $\begin{array}{l}\text { Jasminum } \\
\text { bignoniacearum }\end{array}$ & $\begin{array}{l}\text { Asterina jasmini } \\
\text { var. indica }\end{array}$ \\
\hline Jasminum cordifolium & $\begin{array}{l}\text { Asterina } \\
\text { erysiphoides }\end{array}$ \\
\hline Jasminum cordifolium & $\begin{array}{l}\text { Asterina } \\
\text { erysiphoides }\end{array}$ \\
\hline Jasminum flexile & Prillieuxina jasmini \\
\hline Jasminum pubescens & Asterina jasminicola \\
\hline $\begin{array}{l}\text { Jasminum retchiei } \\
\text { Jasminum rigidum }\end{array}$ & $\begin{array}{l}\text { Asterina } \\
\text { erysiphoides }\end{array}$ \\
\hline \multicolumn{2}{|l|}{ Jasminum sp. } \\
\hline Jasminum sp. & $\begin{array}{l}\text { Asterina } \\
\text { erysiphoides }\end{array}$ \\
\hline $\begin{array}{l}\text { Jasminum } \mathrm{sp} . \\
\text { Jasminum sambac }\end{array}$ & $\begin{array}{l}\text { Asterina } \\
\text { pongalaparensis }\end{array}$ \\
\hline Justicia betonica & Asterina tertia \\
\hline Justicia betonica & Asterina betonicae \\
\hline Justicia sp. & Asterina tertia \\
\hline Justicia wynaadensis & Asterina tertia \\
\hline Knema attenuata & $\begin{array}{l}\text { Asterina knemae- } \\
\text { attenuatae }\end{array}$ \\
\hline $\begin{array}{l}\text { Lagerstroemia } \\
\text { microcarpa }\end{array}$ & $\begin{array}{l}\text { Lembosia } \\
\text { lagerstroemiae }\end{array}$ \\
\hline Lannea coromandelica & Asterina lanneae \\
\hline Lawsonia inermis & Asterina lawsoniae \\
\hline
\end{tabular}


Mycosphere Doi 10.5943/mycosphere/3/5/9

\begin{tabular}{|c|c|}
\hline Host - Fungus - Index & \\
\hline $\begin{array}{l}\text { Lepianthes umbellata } \\
\text { Hackeria subpeltata }\end{array}$ & Asterina lepianthis \\
\hline Lepidagathis scariosa & Asterina tertia \\
\hline Lepidagathis sp. & Asterina tertia \\
\hline Leucas sp. & Asterina leucadis \\
\hline Ligustrum gamblei & $\begin{array}{l}\text { Asterostomella } \\
\text { ligustri }\end{array}$ \\
\hline $\begin{array}{l}\text { Ligustrum } \\
\text { travencoricum }\end{array}$ & Asterina ligustricola \\
\hline $\begin{array}{l}\text { Ligustrum walkeri } \\
\text { Ligustrum } \mathrm{sp} .\end{array}$ & $\begin{array}{l}\text { Asterina ligustricola } \\
\text { Asterina ligustricola }\end{array}$ \\
\hline Linociera $\mathrm{sp}$ & Lembosia linocierae \\
\hline Litsea deccanensis & Asterina litseae \\
\hline Litsea chinensis & Asterina litseae \\
\hline Litsea ligustrina & $\begin{array}{ll}\text { Asterina } & \text { litseae- } \\
\text { ligustrinae } & \\
\end{array}$ \\
\hline Litsea travancorica & Cirsosia litseae \\
\hline Litsea floribunda & $\begin{array}{l}\text { Asterina } \\
\text { cryptocariicola }\end{array}$ \\
\hline Lobelia nicotinifolia & $\begin{array}{l}\text { Asterina } \\
\text { lobeliacearum }\end{array}$ \\
\hline $\begin{array}{l}\text { Loeseneriella } \\
\text { obtusifolia }\end{array}$ & $\begin{array}{l}\text { Asterina } \\
\text { loeseneriellae }\end{array}$ \\
\hline Loranthus falcatus & Asterina deightonii \\
\hline Loranthus sp. & Asterina deightonii \\
\hline Loranthus sp. & Asterina deightonii \\
\hline Loranthus sp. & Asterina deightonii \\
\hline Loranthus sp. & $\begin{array}{l}\text { Asterostomula } \\
\text { loranthi }\end{array}$ \\
\hline Lycianthes laevis & $\begin{array}{l}\text { Asterina } \\
\text { lycianthedis }\end{array}$ \\
\hline Maba nigrescens & Asterina mabae \\
\hline Maerua apetala & Asterina emciciana \\
\hline Mahonia leschenaultii & Asterina goosii \\
\hline Mallotus philippensis & Asterina malloticola \\
\hline Malotus sp. & $\begin{array}{l}\text { Asterina } \\
\text { mallotigena }\end{array}$ \\
\hline Mangifera indica & $\begin{array}{l}\text { Asterolibertia } \\
\text { mangiferae }\end{array}$ \\
\hline Manilkara hexandra & $\begin{array}{l}\text { Echidnodella } \\
\text { manilkarae }\end{array}$ \\
\hline Marsdenia volubilis & $\begin{array}{l}\text { Asterina } \\
\text { travancorensis }\end{array}$ \\
\hline Maytenus ovata & Asterina dissiliens \\
\hline Melicope lunuankanda & $\begin{array}{l}\text { Asterina } \\
\text { melicopecola }\end{array}$ \\
\hline Melicope lunu-ankenda & $\begin{array}{l}\text { Asterina } \\
\text { clausenicola }\end{array}$ \\
\hline Meliosma simplicifolia & $\begin{array}{l}\text { Asterina meliosmae- } \\
\text { simplicifoliae }\end{array}$ \\
\hline
\end{tabular}

\begin{tabular}{|c|c|}
\hline Meliosma simplicifolia & $\begin{array}{l}\text { Asterostomella } \\
\text { meliosmigena }\end{array}$ \\
\hline Meliosma sp. & $\begin{array}{l}\text { Asterostomella } \\
\text { meliosmigena }\end{array}$ \\
\hline Meliosma simplicifolia & \begin{tabular}{|l} 
Asterina \\
sabiacearum
\end{tabular} \\
\hline $\begin{array}{l}\text { Meliosma simplicifolia } \\
\text { ssp.pungens }\end{array}$ & $\begin{array}{l}\text { Asterina } \\
\text { sabiacearum }\end{array}$ \\
\hline Memecylon edule & $\begin{array}{l}\text { Asterina } \\
\text { memecylonis }\end{array}$ \\
\hline Memecylon sp. & $\begin{array}{l}\text { Asterina } \\
\text { memecylonis }\end{array}$ \\
\hline Memecylon sp. & $\begin{array}{l}\text { Lembosia } \\
\text { memecylicola }\end{array}$ \\
\hline Memecylon sp. & $\begin{array}{l}\text { Echidnodella } \\
\text { memecyli }\end{array}$ \\
\hline Memecylon sylvaticum & $\begin{array}{l}\text { Asterina } \\
\text { memecylonis }\end{array}$ \\
\hline Memecylonsp. & $\begin{array}{l}\text { Asterina } \\
\text { madikeriensis }\end{array}$ \\
\hline Merremiasp. & Asterina argyreiae \\
\hline $\begin{array}{l}\text { Mezonevron } \\
\text { cucullatum }\end{array}$ & \begin{tabular}{|l} 
Asterina \\
mezonevronis
\end{tabular} \\
\hline Michelia chempaka & $\begin{array}{l}\text { Asterina } \\
\text { micheliifolia }\end{array}$ \\
\hline Michelia chempaka & $\begin{array}{l}\text { Asterina } \\
\text { micheliigena }\end{array}$ \\
\hline Michelia nilagirica & $\begin{array}{l}\text { Asterostomella } \\
\text { micheliae }\end{array}$ \\
\hline Microtropis latifolia & $\begin{array}{l}\text { Asterina } \\
\text { microtropidicola }\end{array}$ \\
\hline Microtropis ovalifolia & $\begin{array}{l}\text { Asterina } \\
\text { microtropidis }\end{array}$ \\
\hline Miliusa tomentosa & $\begin{array}{l}\text { Gangamyces } \\
\text { miliusae }\end{array}$ \\
\hline Miliusa tomentosa & $\begin{array}{l}\text { Asterostomella } \\
\text { miliusae }\end{array}$ \\
\hline Miliusa sp. & Asterina miliusae \\
\hline Millettia rubiginosa & Asterina millettiae \\
\hline Millettia sp. & Asterina millettiae \\
\hline Mimusops elengi & $\begin{array}{l}\text { Asterina } \\
\text { mimusopsidicola }\end{array}$ \\
\hline Mimuspos elenji & $\begin{array}{l}\text { Symphaster } \\
\text { mimusopsidis }\end{array}$ \\
\hline Murraya exotica & $\begin{array}{l}\text { Asterina } \\
\text { murrayicola }\end{array}$ \\
\hline Murraya koenigii & Asterina murrayae \\
\hline Myristica malabarica & $\begin{array}{l}\text { Asterina } \\
\text { myristicacearum }\end{array}$ \\
\hline
\end{tabular}




\begin{tabular}{|c|c|}
\hline Host - Fungus - Index & \\
\hline Myristica malabarica & Asterina myristicae \\
\hline Myristica sp. & Asterina myristicae \\
\hline Myristica sp. & Asterina myristicae \\
\hline $\begin{array}{l}\text { Myrsine semiserrata } \\
\text { var. subsinosa }\end{array}$ & Asterina myrsines \\
\hline Naravelia zeylanica & Asterina naraveliae \\
\hline Natsiatum hypericum & Asterina natsiati \\
\hline Neolitsea scrobiculata & $\begin{array}{l}\text { Asterina } \\
\text { neolitsiicola }\end{array}$ \\
\hline Neolitsea sp. & $\begin{array}{l}\text { Asterina } \\
\text { neolitsiicola }\end{array}$ \\
\hline Nothopegia aureo-fulva & $\begin{array}{l}\text { Asterolibertia } \\
\text { nothopegiae }\end{array}$ \\
\hline Nothopegia aureo-fulva & $\begin{array}{l}\text { Asterina } \\
\text { nothopegiae }\end{array}$ \\
\hline $\begin{array}{l}\text { Nothopegia } \\
\text { colebrookiana }\end{array}$ & $\begin{array}{l}\text { Asterina } \\
\text { nothopegiae }\end{array}$ \\
\hline $\begin{array}{l}\text { Nothopegia } \\
\text { travancorica }\end{array}$ & $\begin{array}{l}\text { Asterina } \\
\text { nothopegiae }\end{array}$ \\
\hline Olax scandens & Asterina olacicola \\
\hline Olea dioica & Eupelte amicta \\
\hline Opilia amentacea & Asterina crebra \\
\hline Oreocnide integrefolia & $\begin{array}{l}\text { Asterina } \\
\text { oreocnidecola }\end{array}$ \\
\hline Oreocnide integrefolia & $\begin{array}{l}\text { Asterina } \\
\text { oreocnidecola }\end{array}$ \\
\hline Oreocnide integrifolia & $\begin{array}{l}\text { Asterina } \\
\text { oreocnidegena }\end{array}$ \\
\hline Oreocnide sp. & $\begin{array}{l}\text { Asterina } \\
\text { oreocnidecola }\end{array}$ \\
\hline Ormosia travancorica & Lembosia ormosiae \\
\hline $\begin{array}{l}\text { Otonephelium } \\
\text { stipulaceum }\end{array}$ & $\begin{array}{l}\text { Vishnumyces } \\
\text { otonephelii }\end{array}$ \\
\hline $\begin{array}{l}\text { Otonephelium } \\
\text { stipulaceum }\end{array}$ & $\begin{array}{l}\text { Asterostomella } \\
\text { otonephelii }\end{array}$ \\
\hline Pachygone ovata & Lembosia decalvans \\
\hline Pachygone ovata & $\begin{array}{l}\text { Maheshwaramyces } \\
\text { pachygones }\end{array}$ \\
\hline Palaquium ellipticum & Asterina palaquii \\
\hline Pandanus sp. & $\begin{array}{l}\text { Echidnoides } \\
\text { pandanicola }\end{array}$ \\
\hline Pandanus sp. & $\begin{array}{l}\text { Lembosia } \\
\text { pandanacearum }\end{array}$ \\
\hline $\begin{array}{l}\text { Parsonsia } \\
\text { alboflavescens }\end{array}$ & Asterina parsonsiae \\
\hline Pavetta sp. & $\begin{array}{l}\text { Asterostomula } \\
\text { pavettae }\end{array}$ \\
\hline Peristrophe sp. & Asterina tertia \\
\hline Persea macrantha & Lembosia perseae \\
\hline
\end{tabular}

\begin{tabular}{|c|c|}
\hline $\begin{array}{l}\text { Phlogacanthus } \\
\text { curviflorus }\end{array}$ & $\begin{array}{l}\text { Asterina } \\
\text { phlogacanthi }\end{array}$ \\
\hline Phyllanthus beddomi & $\begin{array}{l}\text { Asterina phyllanthi- } \\
\text { beddomei }\end{array}$ \\
\hline Phyllanthus reticulates & $\begin{array}{l}\text { Asterina } \\
\text { phyllanthicola }\end{array}$ \\
\hline Phyllanthus sp. & $\begin{array}{l}\text { Asterina } \\
\text { phyllanthigena }\end{array}$ \\
\hline Physalis sp. & Asterina physalidis \\
\hline Piper sp. & Asterina piperina \\
\hline $\begin{array}{l}\text { Pittosporum } \\
\text { dasycaulon }\end{array}$ & Asterina pittospori \\
\hline Plectranthus sp. & Asterina plectranthi \\
\hline Pleurostylia opposita & Asterina dissiliens \\
\hline Pleurostyliasp. & $\begin{array}{l}\text { Asterina } \\
\text { microtropidicola }\end{array}$ \\
\hline Pogostemon sp. & $\begin{array}{l}\text { Asterina } \\
\text { pogostemonis }\end{array}$ \\
\hline Polyalthia longifolia & $\begin{array}{l}\text { Prillieuxina } \\
\text { polyalthiae }\end{array}$ \\
\hline $\begin{array}{l}\text { Polyalthia sp. } \\
\text { Polyalthia longifolia }\end{array}$ & $\begin{array}{l}\text { Echidnodella } \\
\text { polyalthiae }\end{array}$ \\
\hline Polygala arillata & Asterina polygalae \\
\hline pongamia glabra & $\begin{array}{l}\text { Asterostomella } \\
\text { pongamiae }\end{array}$ \\
\hline Pothos scandens & $\begin{array}{l}\text { Lembosia } \\
\text { malabarensis }\end{array}$ \\
\hline Premna corymbosa & Asterina pusilla \\
\hline Premna serratifolia & Asterina pusilla \\
\hline Premna sp. & Asterina pusilla \\
\hline Premnalatifolia & Asterina pusilla \\
\hline Premna sp. & Asterina kukkalensis \\
\hline Psychotria sp. & $\begin{array}{l}\text { Asterina } \\
\text { psychotriicola }\end{array}$ \\
\hline Pterygota alata & $\begin{array}{l}\text { Prillieuxina } \\
\text { pterigotae }\end{array}$ \\
\hline Quisqualis indica & $\begin{array}{l}\text { Asterina } \\
\text { escharoides }\end{array}$ \\
\hline $\begin{array}{l}\text { Radermachera } \\
\text { xylocarpa }\end{array}$ & $\begin{array}{l}\text { Asterostomella } \\
\text { radermacherae }\end{array}$ \\
\hline Rhamnus sp. & Asterina rhamni \\
\hline Rhinacanthus nasuta & Asterina tertia \\
\hline Rhinacanthus sp. & Asterina tertia \\
\hline $\begin{array}{l}\text { Rhododendron } \\
\text { arboreum }\end{array}$ & $\begin{array}{l}\text { Asterina } \\
\text { hakgalensis }\end{array}$ \\
\hline $\begin{array}{l}\text { Rhododendron } \\
\text { arboretum } \\
\text { nilagiricum }\end{array}$ & $\begin{array}{l}\text { Asterina } \\
\text { hakgalensis }\end{array}$ \\
\hline
\end{tabular}


Mycosphere Doi 10.5943/mycosphere/3/5/9

\begin{tabular}{|c|c|}
\hline Host - Fungus - Index & \\
\hline Rhododendron sp. & $\begin{array}{l}\text { Asterina } \\
\text { hakgalensis }\end{array}$ \\
\hline Rhodomyrtus sp. & Asterina rhodomyrti \\
\hline $\begin{array}{l}\text { Rhodomyrtus } \\
\text { tomentosa }\end{array}$ & Asterina rhodomyrti \\
\hline Ruellia prostrata & $\begin{array}{l}\text { Asterina tertia var. } \\
\text { africana }\end{array}$ \\
\hline Ruellia prostrate & Asterina tertia \\
\hline Ruellia sp. & Asterina tertia \\
\hline Sabia sp. & Asterina sabiana \\
\hline $\begin{array}{l}\text { Saccopetalum } \\
\text { tomentossum }\end{array}$ & Asterina saccopetali \\
\hline Salacia sp. & Lembosia salaciae \\
\hline Samadera indica & Asterina samaderae \\
\hline $\begin{array}{l}\text { Santalum sp. } \\
\text { Santalum album }\end{array}$ & Asterina congesta \\
\hline Saraca asoca & Asterina saracae \\
\hline $\begin{array}{l}\text { Sarcandra } \\
\text { chloranthoides }\end{array}$ & Asterina sarcandrae \\
\hline $\begin{array}{l}\text { Schima wallachii var. } \\
\text { khasiana }\end{array}$ & Asterina schimae \\
\hline $\begin{array}{l}\text { Scleropyrum } \\
\text { pentandrum }\end{array}$ & Asterina scleropyri \\
\hline Scolopia crenata & $\begin{array}{l}\text { Asterina } \\
\text { flacourtiacearum }\end{array}$ \\
\hline Scolopia crenata & Asterina granulosa \\
\hline Scolopia crenata & $\begin{array}{l}\text { Asterostomella } \\
\text { scolopiae-crenatae }\end{array}$ \\
\hline Scolopia crenata & $\begin{array}{l}\text { Asterina } \\
\text { talacauveriana }\end{array}$ \\
\hline Scolopia sp. & Asterina granulosa \\
\hline Scolopia sp. & $\begin{array}{l}\text { Asterostomella } \\
\text { scolopiae-crenatae }\end{array}$ \\
\hline Scolopia sp. & $\begin{array}{l}\text { Asterina } \\
\text { talacauveriana }\end{array}$ \\
\hline $\begin{array}{l}\text { Sentellaria violacea } \\
\text { Pogostemonbengalensis }\end{array}$ & $\begin{array}{l}\text { Asterina } \\
\text { pogostemonis }\end{array}$ \\
\hline Shorea robusta & $\begin{array}{l}\text { Gangamyces } \\
\text { shoreae }\end{array}$ \\
\hline Shorea robusta & $\begin{array}{l}\text { Asterostomella } \\
\text { shoreae }\end{array}$ \\
\hline Shorea talura & Asterina plurisporus \\
\hline Shorea talura & Lembosia shoreae \\
\hline Sida cordata & Asterina diplocarpa \\
\hline Sida glutinosa & Asterina diplocarpa \\
\hline Sida rhombifolia & Asterina pavoniae \\
\hline Solanum ferox & Asterina diplopoda \\
\hline Solanum torvum & $\begin{array}{l}\text { Asterina } \\
\text { balakrishnanii }\end{array}$ \\
\hline
\end{tabular}

\begin{tabular}{|c|c|}
\hline Strombosia ceylanica & $\begin{array}{l}\text { Asterostomella } \\
\text { strombosiae }\end{array}$ \\
\hline $\begin{array}{l}\text { Symplocos } \\
\text { cochinchinensis } \\
\text { ssp.laurina }\end{array}$ & Asterina indica \\
\hline Symplocos sp. & Asterina indica \\
\hline Symplocos theacifolia & Asterina indica \\
\hline Symplocos sp. & Asterina indica \\
\hline Symplocos sp. & Asterina suttonii \\
\hline Syzygium arnottianum & $\begin{array}{l}\text { Lembosia } \\
\text { hosagoudarii }\end{array}$ \\
\hline Syzygium cumini & $\begin{array}{l}\text { Asterina } \\
\text { gopalakrishnanii }\end{array}$ \\
\hline Syzygium cumini & Asterina jambolana \\
\hline Syzygium cumini & Asterina claviflori \\
\hline Syzygium cumini & $\begin{array}{l}\text { Lembosia } \\
\text { hosagoudarii }\end{array}$ \\
\hline Syzygium densiflorum & Asterina jambolana \\
\hline Syzygium henianum & Asterina jambolana \\
\hline Syzygium henianum & Asterina henianii \\
\hline Syzygium jambolana & Asterina jambolana \\
\hline Syzygium jambolana & Asterina claviflori \\
\hline Syzygium mundagam & Asterina claviflori \\
\hline $\begin{array}{l}\text { Syzygium } \\
\text { tamilnadensis }\end{array}$ & $\begin{array}{l}\text { Lembosia } \\
\text { hosagoudarii }\end{array}$ \\
\hline Syzygium zeylanicum & Asterina claviflori \\
\hline Syzygium sp. & $\begin{array}{l}\text { Asterostomula } \\
\text { syzygii }\end{array}$ \\
\hline Taxillus sp. & Asterina deightonii \\
\hline Taxillus sp. & Asterina deightonii \\
\hline Terminalia arjuna & Asterina combreti \\
\hline Terminalia arjuna & $\begin{array}{l}\text { Asterostomella } \\
\text { terminaliae }\end{array}$ \\
\hline Terminalia chebula & $\begin{array}{l}\text { Lembosia } \\
\text { terminaliae- } \\
\text { chebulae }\end{array}$ \\
\hline Terminalia cuneata & Asterina combreti \\
\hline Terminalia paniculata & $\begin{array}{l}\text { Asterostomella } \\
\text { terminaliae }\end{array}$ \\
\hline Terminaliasp. & Asterina combreti \\
\hline Thottea ponmudiana & Asterina thotteae \\
\hline Thottea siliquosa & Asterina thotteae \\
\hline Thottea siliquosa & Asterina thotteae \\
\hline Thottea sivarajanii & Asterina thotteae \\
\hline Thotteasp. & Asterina thotteae \\
\hline Thunbergia sp. & $\begin{array}{l}\text { Asterina } \\
\text { thunbergiicola } \\
\text { var.indica }\end{array}$ \\
\hline Tinospora cordifolia & Asterinatinosporae \\
\hline
\end{tabular}




\begin{tabular}{|c|c|}
\hline Host - Fungus - Index & \\
\hline Toddalia asiatica & Asterina toddaliae \\
\hline Toddalia sp. & Asterina toddaliae \\
\hline Toddalia sp. & $\begin{array}{l}\text { Asterina } \\
\text { toddaliicola }\end{array}$ \\
\hline Toxocarpus sp. & Asterina toxocarpi \\
\hline Trema orientalis & Asterina dallasica \\
\hline Trichilia connaroides & Asterina trichiliae \\
\hline Triumfetta sp. & $\begin{array}{l}\text { Asterina } \\
\text { triumfetticola }\end{array}$ \\
\hline Tylophora dalzelli & $\begin{array}{l}\text { Asterina } \\
\text { prataparajii }\end{array}$ \\
\hline Tylophora indica & $\begin{array}{l}\text { Asterina tylophorae- } \\
\text { indicae }\end{array}$ \\
\hline Vateria indica & $\begin{array}{l}\text { Asterolibertia } \\
\text { vateriae }\end{array}$ \\
\hline Vateria indica & Cirsosia vateriae \\
\hline Vateria indica & $\begin{array}{l}\text { Echidnodella } \\
\text { vateriae }\end{array}$ \\
\hline Viburnum cylindricum & Asterina viburnicola \\
\hline Villebrunea integrifolia & $\begin{array}{l}\text { Asterina } \\
\text { oreocnidecola }\end{array}$ \\
\hline Villebrunea sp. & $\begin{array}{l}\text { Asterina } \\
\text { oreocnidecola }\end{array}$ \\
\hline Viscum sp. & Asterina visci \\
\hline Vitaceae & Asterina viticola \\
\hline Vitis sp. & Asterina cissi \\
\hline Wattakaka volubilis & $\begin{array}{l}\text { Asterina } \\
\text { travancorensis }\end{array}$ \\
\hline Webera corymbosa & Lembosia incisa \\
\hline Woodfordia fruticosa & $\begin{array}{l}\text { Asterina } \\
\text { woodfordiae }\end{array}$ \\
\hline Wrightia tinctoria & Asterina wrightiae \\
\hline Xanotis tomentosum & Asterina laxiuscula \\
\hline $\begin{array}{l}\text { Xanthophyllum } \\
\text { arnottianum }\end{array}$ & $\begin{array}{l}\text { Asterina } \\
\text { xanthophylli }\end{array}$ \\
\hline $\begin{array}{l}\text { Xanthophyllum } \\
\text { flavescens }\end{array}$ & $\begin{array}{l}\text { Asterina } \\
\text { xanthophylli }\end{array}$ \\
\hline Xylosma longifolium & $\begin{array}{l}\text { Asterostomella } \\
\text { xylosmae }\end{array}$ \\
\hline $\begin{array}{l}\text { Zanthoxylum } \\
\text { khasianum }\end{array}$ & Asterina zanthoxyli \\
\hline Ziziphus rugosa & $\begin{array}{l}\text { Asterostomella } \\
\text { ziziphina }\end{array}$ \\
\hline
\end{tabular}

\section{Discussion}

This is my persistent effort in bringing out this work but the collections are mainly concentrated in the Western Ghats. The result would have been still amazing if the entire Western Ghats, Eastern Ghats, North East and Andaman \& Nicobar Islands were thoroughly studied. The morpho-taxonomy flourished till 1960 but still it was up to 1980 . Since then, the number of students of taxonomy started diminishing and now it has become hard to identify even common fungi. Morphotaxonomy has a strong foot base but is being strongly tilted by the molecular taxonomy by one side and diminishing in the number of morpho-taxonomists in another. However, molecular taxonomy would have been moved with morpho till completing the molecular study of all the morphologically identified material. Now, the movement of material from one place to another is being restricted. Now we are neither in a position to identify the material morphologically or by using molecular taxonomy because of our poor knowledge in it in the tropics. In spite of all these, we have CBD (Convention on Biological Diversity), GTC (Global Taxonomy Initiative), etc. If both these studies supplement and complement each other and one another, we will achieve something in taxonomy. Otherwise, it will be: Tax-on-me.

\section{Acknowledgements}

It is my pleasure to thank Mr. K. Vivekananthan, Drs. C.N. Mohanan, M. Mohanan, R. Gopalan and K. Ravikumar, Botanical Survey of India, Southern Circle, Coimbatore and Director and Drs. N. Mohanan and E. Santhosh Kumar of JNTBGRI for the facilities and their help in identification of host plants, respectively. Curators of $\mathrm{HCIO}$ and $\mathrm{AMH}$ are greatly acknowledged for permitting me to examine the collections. Forest Department, Govt. of Kerala and Ministry of Environment \& Forests, New Delhi, Govt. of India are acknowledged for the forest permission and financial support. Dr. Jacob Thomas, Messrs. P.J. Robin, Shiju and Jayakumar assisted during the progress of this work. 


\section{References}

Arx JA von, Muller E. 1975 - A re-evaluation of the bitunicate ascomycetes with key to the families and genera.Studies in Mycology 9, 1-159.

Bakshi BK. 1971- Indian Polyporaceae. ICAR, New Delhi.

Castellani E. 1942 - Micromiceti dell' Africa orientale Italiana. Nuovo Giornale botanico Italiano 49, 1-31.

Chen Z. 1974 - Notes on new Formosan forest fungi. I. Black mildews on Abieskawakamii. Taiwania 2, 115-122.

Cooke MC. 1884 - Some exotic fungi. Grevillea 12,85 .

Das P, Alikhan M, De B, SamajpatiN, BeraS. 2007 - Evidence of relationship between Asterina (Asterinaceae) and Chonemorpha (Apocynaceae) from the upper Siwalik sediments of Arunachal subhimalaya, India. Journal of Mycopathological Research 45, 225-230.

Doidge EM. 1921 - South African Ascomycetes in the National Herbarium I. Bothalia1, 5-32.

Doidge EM. 1942 - A revision of the South African Microthyriaceae. Bothalia 4, 273-344.

Goos RD. 1999 - Notes on the genus Echidnodella (Asterinaceae). Mycotaxon 73, 455-464.

Hansford CG.1944 - Contributions towards the fungus flora of Uganda-VI. New records. Proceedings of the Linnean Society of London 156, 102-124.

Hansford CG. 1945 - Contribution towards the fungus flora of Uganda-VII. New records and revision. Proceedings of the Linnean Society of London 157, 20-212.

Hansford CG. 1946a - The foliicolous Ascomycetes, their parasites and associated fungi. Mycological Papers $15,1-240$.

Hansford CG. 1946b - Contribution towards the fungus flora of Uganda-VIII.New records. Proceedings of the Linnean Society of London 157, 132-212.

Hansford CG. 1946b -The foliicolous Ascomycetes, their parasites and associated fungi. Mycological Papers $15,1-240$.
Hansford CG. 1947 - New or interesting tropical fungi-I. Proceedings of the Linnean Society of London 158, 28-50.

Hansford CG. 1948 - Chinese fungi collected byCheo. Farlowia 3, 260-283.

Hansford CG. 1949 - Tropical fungi-III.New species and revision. Proceedings of the Linnean Society of London 160, 116-153.

Hansford CG. 1954a - Some Microthyriales and other fungi from Indonesia. Reinwardtia 3, 113-144.

Hansford CG. 1954b - Australian fungi-II. New records and revisions. Proceedings of the Linnean Society of New South Wales 79(3-4), 97-141.

Hansford CG. 1955 - Tropical fungi-V.New species and revision. Sydowia 9, 1-88.

Hansford CG. 1957 - Australian fungi-IV.New records and revision. Proceedings of the Linnean Society of New South Wales 82, 209-229.

Hansford CG. 1961 - The Meliolineae. A monograph. Sydowia 2, 1-806.

Hansford CG, Thirumalachar MJ.1948 -Fungi of South India. Farlowia 3, 285-314.

Hennings P. 1904 - Fungi Amazonia. Hedwigia 43, 350-400.

Hino I, Katumoto K. 1957 - Notes on fungi from Western Japan (2). Bulletin of the Faculty of Agriculture Yamaguti University8, 637-648.

Hosagoudar VB. 1995 - Some Lembosia and Asterina species from Southern India. Indian Journal of Forestry 18, 274-277.

Hosagoudar VB. 1996 - Meliolales of India. Botanical Survey of India, Calcutta, pp.363.

Hosagoudar VB. 2002 - Studies on foliicolous fungi-X. Five new species and a new record. Zoos' Print Journal 17, 943-948.

Hosagoudar VB. 2002a -Studies on foliicolous fungi-IV. A new species of Asterinaand key to other species on Dipterocarpaceae. Zoos' Print Journal $17,815-816$.

Hosagoudar VB. 2002c -Studies on foliicolous fungi-VI. Two new species from Kerala and a new record from India. Zoos' Print Journal 17, 863-866. 
Hosagoudar VB. 2002d - Studies on foliicolous fungi-X. Five new species and a new record. Zoos' Print Journal 17, 943-948.

Hosagoudar VB. 2003a - Asterinaceae of India.Zoos' Print Journal 18, 1280-1285.

Hosagoudar VB. 2003b - Studies on foliicolous fungi-XII. New species, new records and hyperparasites. Zoos' Print Journal 18, 1037-1040.

Hosagoudar VB. 2003 - Studies on foliicolous fungi-XV. New species, new records and rare fungus. Zoos' Print Journal 19, 1386-1389.

Hosagoudar VB. 2004 - A new Asterina species from Kerala, India.Zoos' Print Journal 19, 1522.

Hosagoudar VB. 2004a - Studies on foliicolous fungi-VII. Journal of Economc \& Taxonomic Botany 28, 187-195.

Hosagoudar VB. 2004b - Studies on foliicolous fungi $-\mathrm{XV}$. New species, new records and rare fungus. Zoos' Print Journal 19, 1386-1389.

Hosagoudar VB. 2005 - Studies on foliicolous fungi-XIX. Indian Phytopathology 58, 194-204.

Hosagoudar VB. 2006 - Studies on foliicolous fungi-XXV. New species and new records. Zoos' Print Journal 21, 2335-2338.

Hosagoudar VB. 2008 - Meliolales of India. Vol. II. Botanical Survey of India, Calcutta, pp. 390.

Hosagoudar VB. 2009 - The genus Asterina and its anamorph on Elaeocarpus species in southern Western Ghats in peninsular India. Journal of Applied and Natural Science 1, 27-30.

Hosagoudar VB. 2010 - Notes on the genera Asterolibertia and Cirsosia.Journal of Threatened Taxa 2(9), 1153-1157.

Hosagoudar VB. 2010 - Anamorphs of Asterinales. Journal of Theoretical and Experimental Biology 6, 199-211.

Hosagoudar VB, Abraham TK. 1997a - A new species of Asterolibertia from Kerala, India. Journal of Mycopathological Research 35, 55-56.

Hosagoudar VB, Abraham TK. 1997b - Some interesting foliicolous fungi from
Kerala, India. New Botanist 24, 109-119.

Hosagoudar VB, Abraham TK. 1997c - Three new foliicolous Ascomycetes from Kerala. Indian Phytopathology 50, 216-221.

Hosagoudar VB, Abraham TK.1998a - Four new foliicolous Ascomycetes from Kerala, India. Mycological Research 102, 184-186.

Hosagoudar VB, Abraham TK. 1998b-Some interesting foliicolous thyriotheceous Ascomycetes from Kerala. Indian Phytopathology 51, 389-392.

Hosagoudar VB, Abraham TK.2000 - A list of Asterina Lev. species based on the literature. Journal of Economc \& Taxonomic Botany 24, 557-587.

Hosagoudar VB, Agarwal DK. 2003 - Studies on foliicolous fungi - IX. Indian Phytopathology 58, 98-101.

Hosagoudar VB, Agarwal DK. 2008 Taxonomic studies of Meliolales. Identification Manual. International Book Distributors, Dehra Dun, pp. 263.

Hosagoudar VB, Biju H. 2006 - Studies on foliicolous fungi-XXII. Microfungi of Silent Valley National Park, Palghat District in Kerala State. Journal of Mycopathological Research 44, 39-48.

Hosagoudar VB, Chandraprabha A. 2009 New Asterinaceae members from Kerala, India. Indian Journal of Science \& Technology 2(6), 15-16.

Hosagoudar VB, Chandraprabha A. 2009 Brahmamyces, a new anomorphic genus from India. Indian Journal of Science \& Technology 2(6), 17.

Hosagoudar VB, Chandraprabha A. 2009 Two new Prillieuxina species from Kerala, India. Indian Journal of Science \& Technology 2 (6), 18.

Hosagoudar VB, Chandraprabha A. 2009 Two new Asterina species from Kerala, India. Pollen Spore and Aerobiology 27, $155-158$.

Hosagoudar VB, Goos RD 1994 - Some Asterina, Asterostomella and Lembosia species from Southern India. Mycotaxon 52, 467-473. 
Hosagoudar VB, Goos RD. 1996 - Some foliicolous fungi from Southern India. Mycotaxon 59, 149-166.

Hosagoudar VB, Hanlin RT. 1995 - New species of Asterina and Echinoides from India. New Botanist 22, 187-192.

Hosagoudar VB, Harish M. 2010 Vishnumyces, a new genus of the family Asterinaceae from India. Indian Phytopathology 63, 85-86.

Hosagoudar VB, Kapoor JN. 1984 - New technique of mounting Meliolaceous fungi. Indian Phytopathology 38, 548-549.

Hosagoudar VB, Mohanan M. 1996 Asterostomella alangii. a new species from Andhra Pradesh, India. Indian Journal of Forestry 19, 371-372.

Hosagoudar VB, Riju MC. 2011 - Two new Asterina species on Michelia champaca from Kerala, India. Journal of Threatened Taxa 3(7), 1942-1946.

Hosagoudar VB, Sabeena A. 2007 - Two interesting fungi from Malabar Botanic Garden, Kozhikode, Kerala. Zoos' Print Journal 22, 2786-2787.

Hosagoudar VB, Sabeena A. 2011 -The genus Asterina (Asterinaceae) on the members of Myristicaceae in Kerala State, India. Journal of Threatened Taxa 3 (10), 2143-2146.

Hosagoudar VB, Abraham TK, Pushpangadan P. 1996a - Fungi of Kerala. Tropical Botanic Garden and Research Institute, Palode, Thiruvananthapuram, pp. 151.

Hosagoudar VB, Balakrishnan NP, Goos RD. 1996b - Some Asterina species from Southern India. Mycotaxon 59, 167-187.

Hosagoudar VB, Balakrishnan NP, Goos RD. 1996c - Some Asterinella, Asterostomella and Echidnodella species from Southern India. Mycotaxon 58, 489-498.

Hosagoudar VB, Abraham TK, Pushpangadan P. $1997-$ The Meliolineae-A Supplement. Tropical Botanic Garden and Research Institute, Palode, Thiruvananthapuram, Kerala, India, pp. 201.

Hosagoudar VB, Abraham TK, Biju CK. 1997a - A new species of Asterostomella from
Southern India. New Botanist 24, 19-22.

Hosagoudar VB, Abraham TK, Biju CK, Shiburaj S. 1997b - Asterina theacearum from Kerala, India.New Botanist 24, 23-25.

Hosagoudar VB, Abraham TK, Crane JL. 1998a - Two new Asterinaceous fungi from Kerala, India. Mycotaxon 68, 19-22.

Hosagoudar VB, Abraham TK, Nayar TS.1998b - Asterina knemae attenuatae from India. Mycotaxon 67, 487-488.

Hosagoudar VB, Abraham TK, Biju CK. 1999 - Notes on some foliicolous fungi from Kerala, India. Journal of Mycopathological Research 37, 25-28.

Hosagoudar VB, Biju CK, Abraham TK. 2001

- Some interesting Asterina species from Kerala. Indian Phytopathology 54, 137-139.

Hosagoudar VB, Abraham TK, Biju CK. 2001a - Re-evaluation of the family Asterinaceae. Journal of Mycopathological Research 39, 61-63.

Hosagoudar VB, Abraham TK, Biju CK, Hyde KD. 2001b - Fungi from palms-XLVII.A new species of Asterinaon palms from India. Fungal Diversity6, 69-73.

Hosagoudar VB, Biju CK, Abraham TK. 2001c. Diversity in the foliicolous micromycobionts in Peppara and Neyyar Wildlife Sanctuaries, Thiruvananthapuram, Kerala, India. Journal of Econome \& Taxonomic Botany 25, 297-307.

Hosagoudar VB, Biju CK, Abraham TK, Pradeep CK. 2001e - Asterina diospyri from Kerala, India. Journal of Economc \& Taxonomic Botany 25, 279-280.

Hosagoudar VB, Biju CK, Abraham TK. 2002a - Diversity of foliicolous micro mycobionts in Munnar and Wyanad forest regions of Kerala. Journal of Mycopathological Research 40, 191-196.

Hosagoudar VB, Biju CK, Abraham TK, Agarwal DK. 2002b - Studies on foliicolous fungi - III. Indian Phytopathology 55, 497-502. 
Hosagoudar VB, Biju CK, Abraham TK. 2004a - Additions to Asterinaceae. Indian Phytopathology 57, 114-116.

Hosagoudar VB, Biju CK, Abraham TK. 2004b

- Studies on foliicolous fungi. Journal of Economc \& Taxonomic Botany 28, $175-182$.

Hosagoudar VB, Biju CK, Abraham TK. 2004c - Studies on foliicolous fungi-II. Journal of Economc \& Taxonomic Botany 28, 183-186.

Hosagoudar VB, Agarwal DK, Biju H, Archana GR. 2006a - Studies on foliicolous fungi - XXIII. Indian Phytopathology $59,525-528$.

Hosagoudar VB, Biju H, Anu Appaiah KA. $2006 \mathrm{~b}$ - Studies on foliicolous fungi XX. Microfungi of Coorg, Karnataka. Journal of Mycopathological Research $44,1-25$.

Hosagoudar VB, Archana GR, Dan M. 2008a -Maheshwaramyces, a new genus of the family Lembosiaceae. Indian Journal of Sciience \& Technology 2(6), 12-13.

Hosagoudar VB, Riju MC, Uma Maheswari C. 2008c - Asterina dallasica Petrak, a new record to India.Indian Journal of

Science \& Techology 1, 1-2.

Hosagoudar VB, Sabeena A, Agarwal DK.2009

- Two new species and a new generic record of Asterinaceae on Mimusops elengi from Kerala.Indian Phytopathology 62, 229-232.

Hosagoudar VB, Jacob Thomas, Sabeena A.2009 - The genus Lembosia on Humboldtia species in Kerala, India. Indian Journal of Science \& Technology 2(6), 19-21.

Hosagoudar VB, Harish M, ArchanaGR. 2009b

- Meliolaster aporusae, a new species and a new generic record from Kerala, India. Indian Journal of Science \& Technology 2(6), 14.

Hosagoudar VB. Sabeena A, Riju MC. $2010-$ Bheemamyces, a new genus of the family Asterinaceae. Journal of Threatened Taxa 2 (12), 1323-1324.

Hosagoudar VB, Jacob Thomas, Agarwal DK. 2011 - Four new asterinaceous members from Kerala, India. Taprobanica 3(1), 15-17.
Hosagoudar VB, Riju MC, Uma Maheshwari C. 2011 - Status of Asterina exsiccatae in Herbarium Cryptogamae Indiae Orientalis (HCIO), New Delhi, India. Indian Phytopathology 64, 56-70.

Hosagoudar VB, Sabeena A, Jacob-Thomas. 2011- The genus Asterostomula from Kerala, India, including one new species. Plant Pathology \& Quarantine 1, 7-10.

Hosagoudar VB, Chandraprabha A, Agarwal DK. 2011 - Asterinales of Kerala. Associated Publishing Company, pp. $270+$ pl.23.

Hosagoudar VB, Sabeena A, Riju MC. 2011 Asterina hugoniae sp. nov. (Dothideomycetes, Asterinaceae) from Kerala, India. Journal of Threatened Taxa 3(6), 1880-1881.

Hughes SJ. 1952 - Fungi from the Gold Coast. Mycological Papers 48, 1-91.

Hughes SJ. 1953 - Fungi from the Gold Coast-II. Mycological Papers 50, 1104.

Kamat MN, Seshadri VS, Pande AA.1978Monographic study of Indian species of Phyllachora. UAS Monograph, Bangalore.

Kar AK, Ghosh SN. 1986 - New Asterina species from West Bengal. Indian Phytopathology 39, 204-220.

Kar AK, Maity MK. 1970 - New Asterina species from West Bengal. Transactions of the British Mycological Society 54, 435-444.

Katumoto K. 1975 - The Hemisphaeriales in Japan. Bulletin of Faculty of Agriculture Yamaguti University 26, 45-122.

Katumoto K. 1991- Three Ascomycetous fungi from the Ryukyu Islands, Japan. Transactions of the Mycological Society of Japan 32, 37-43.

Kaul VP, Nair LN. 1988 -Some new reports of Asterina from India. Acta Botanica Indica 16, 227-229.

Manoharachary C, Sridhar K, Singh R, Adholeya A, Suryanarayanan TS, Rawat S, Johri BN. 2005. Fungal biodiversity: distribution, conservation and prospecting of fungi from India. Current Science 89, 58-71.

Mitra S, Bera S, BanerjeeM.2002 -On a new epiphyllous fungus Palaeoasterina 
siwalika from the Siwalik sediment of Darjeeling foothills, India with remarks on environment. Phytomorphology 52, 285-292.

Muller E, Arx JA von. 1962- Die Gattungen der didymosporen Pyrenomyceten. Beitr. Kryptogamenfl. Schweiz 11, $1-922$.

Mundkur BB, Thirumalachar MJ. 1952 Ustilaginales of India. CMI, Kew, Surrey, England.

Nayar TS, Rasiya Beegum A, Mohanan N, Rajkumar G. 2006 - Flowering plants of Kerala. TBGTR, Palode, Thiruvananthapuram, Kerala, pp. 1069.

Nithyatharani R, Dhivaharan V. Hosagoudar VB. 2010 - Three new Asterina species from Kodaikanal hills in Tamil Nadu. Scientific Transactions in Environment and Technovation 4, 46-48.

Orejuela CG. 1944 - New and hitherto unreported species of higher Ascomycetes from Columbia and Venezuela. Mycologia 36, 429-529.

Ouyang Y, Song B, Hu Y. 1995 - Studies on the taxonomy of Asterina in China.Acta Mycologica Sinica 14, 241-247.

PatilMS, Pawar AB. 1989 - Studies on foliicolous fungi - IV. Indian Phytopathology 42, 247-252.

Patil MS, Thite AN. 1977 - Fungal flora of Rathnagiri (Kolhapur). Journal of ShivajiUniversity Science 17, 149-162.

Patil MS, Thite AN. 1978 - Fungal flora of Amboli (Rathnagiri). Journal of Shivaji University Science 18, 219-224.

Patil MS, Thite AN. 1981 - Investigations of fungi of Mahabaleshwar (M. S.).Journal of Shivaji University Science 20, 61-67.

Petrak F. 1954 - Beitrage zur Pilzflora von Britisch Nord, Borneo. Sydowia 8, 12-26.

Petrak F. 1955 - Beitrage zur Pilzflora von Sud und Ostasien. Sydowia 9, 530-538.

Petrak F. 1958 - Beitrage zur Kenntnis einiger Asterineen der Philippinen. Sydowia 12, 472-486.

Petrak F, Cifferri R. 1930 - Fungi dominicani. Annales Mycologici 28, 377-420.
Petrak F, Cifferri R. 1932 - Fungi dominicani-II. Annales Mycologici 30, 149-353.

Petrak F, Sydow H 1931. Micromycetes philippinensis. Annales Mycologici 29, 145-279.

Ramanujam CGK. 1963 - Thyriothecia of Asterina from the South Arcot Lignite Madras. Current Science 32, 327-328.

Ramanujam CGK, Rao KP. 1973 -On some Microthyriaceous fungi from atertiary lignite of South India. Palaeobotanist 20, 203-209.

Rangaswamy G. 1975 - Diseases of crop plants in India. Prentice-Hall, India, New Delhi.

Ryan R. 1928 - Asterina species from India. Memoirs of the Department of Agriculture, India, Botany 15, 103-105

Saccardo PA. 1924 - Sylloge fungorum omnium hucusque cognitorum 4, 450-451.

Santapau H, Henry AN. 1984 -A Dictionary of the flowering plants in India. CSIR, New Delhi, pp.198.

Sawada K. 1959 - Descriptive catalogue of Taiwan (Formosan) fungi. Part XI. Special Publication of College of Agriculture Nat. Taiwan University, Taipei, Taiwan 8, 43-45.

Singh S. 1980 -Asterina phyllanthicola from India. Transactions of the British Mycological Society 74, 204-205.

Sivanesan A. 1984 - The Bitunicate Ascomycetes. International Books \& Periodical Supply Service, New Delhi.

Song B, Hosagoudar VB. 2003 - A list of Lembosia species based on the literature. Guizhou Science 21, 93-101.

Song B, Li TH, Shen YH. 2004 - New species of Asterina from Guangdong, China. Mycotaxon 90, 29-34.

Stevens FL, Ryan MH. 1939 - The Microthyriaceae. Illinois Biological Monographs 17, 1-138.

Subramanian CV. 1971 - Hyphomycetes. An account of Indian species except Cercosporae. ICAR, New Delhi.

Sydow H. 1913 - Enumeration of Philippine fungi with notes and descriptions of new species. Philippine Journal of Science 8, 475-508. 
Sydow H.1923 - Ein neuer Beitrag zur Kenntnis der Pilzflora der PhilippinenInseln. Annales Mycologici 21, 93-106.

Sydow H. 1928 - Fungi borneenses. Annales Mycologici 26, 85-99.

Sydow H. 1930 - Fungi venezuelani. Annales Mycologici 28, 29-224.

Sydow H. 1938 - Novae fungorum species-XXVI. Annales Mycologici 36, 156-197.

Sydow H. 1939 - Novae fungorum species-XXVII. Annales Mycologici 37, 197-253.

Sydow H, Petrak F. 1931 - Micromycetes Philippinensis II. Annales Mycologici 29, 145-279.

Sydow H, Sydow P. 1912 - Fungi from the island of Palawan. Leaflets of Philippine Botany 5, 1533-1547.

Sydow H, Sydow P. 1913 - Enumeration of Philippines fungi with notes and description of new species. Philippine Journal of Science8, 475-508.

Sydow H, Sydow P. 1914 - Fungi from Northern Palawan. Philippine Journal of Science 9, 157-189.

Sydow H, Sydow P. 1915 - Novae fungorum species-XIII. Annales Mycologici 13, 35-43.

Sydow H, Sydow P. 1917 - Beitrage zur Kenntnis der Pilzflora der PhilippinenInseln. Annales Mycologici 15, 165268.

Sydow H, Sydow P. 1939 - Fungi from the island of Palawan. Leaflets of Philippine Botany5, 1533-1547.

Sydow H, Sydow P, Butler EJ 1911 -Fungi Indiae Orientalis-II. Microthyriaceae. Annales Mycologici 9, 389-392.

Tandon RN. 1968 - Mucorales of India. ICAR, New Delhi.

Theissen F. 1913 - Die Gattung Asterina. Bibliotheca Mycologica10, 1-130.

Theissen F. 1913b - Hemisphariales. Annales Mycologici1 1, 468-469.

Theissen F. 1913c - Lembosia - Studien. Annales Mycologici 11, 425-467.

Theissen F, Sydow H. 1915 - Die Dothideales. Annales Mycologici 13, 149-746.

Theissen F, Sydow H. 1917 -Synoptische Tafeln. Annales Mycologici 15, 389-491.
Thind KS. 1961- The Clavariaceae of India. Indian Department of Agriculture, New Delhi.

Thind KS. 1977 - The Myxomycetes of India. ICAR, New Delhi.

Thite AN, Kulkarni AR. 1976 - Fungal flora of Panhala. Journal of Bombay Natural History Society 73, 456-463.

Vasudeva RS. 1963 - Indian Cercosporae. ICAR, New Delhi.

Werdermann E. 1923 - Einige neue Pilze aus Natal. Repertorium specierum novarum regni vegetabilis $19,49-54$.

Yamamoto W. 1956 - The Formosan species of Microthyriaceae - I. Science Reporter Hyogo University Agriculture Series, Agriculture Biology 2, 33-36.

Yamamoto W. 1957 -The Formosan species of Microthyriaceae - II. Science Reporter Hyogo University Agriculture Series, Agriculture Biology 3, 23-31.

Yates HS. 1918a - Some recently collected Philippine fungi - I. Philippine Journal of Science 12, 61-80.

Yates HS. 1918b - Some recently collected Philippine fungi - II. Philippine Journal of Science13, 361-384. 